


\section{An Album of Map Projections}

By John P. Snyder, U.S. Geological Survey, and Philip M. Voxland, University of Minnesota 3 Introduction by Joel L: Morrison, ff U.S. Geological Survey

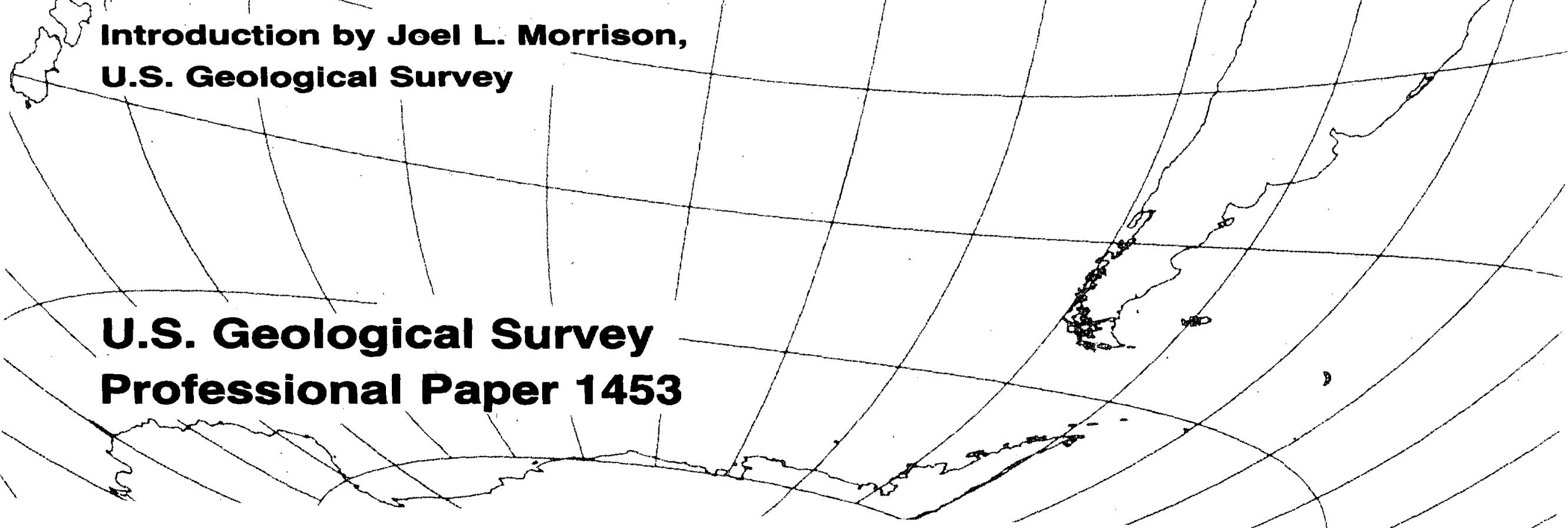




\section{Department of the Interior \\ BRUCE BABBITT Secretary}

\section{U.S. Geological Survey}

Gordon P. Eaton, Director

First printing 1989

Second printing 1994

Any use of trade names in this publication is for descriptive purposes only and does not imply endorsement by the U.S. Geological Survey

\section{Library of Congress Cataloging in Publication Data}

Snyder, John Parr, 1926-

An album of map projections.

(U.S. Geological Survey professional paper ; 1453)

Bibliography: $p$.

Includes index.

Supt. of Docs. no.: I 19.16:1453

1. Map-projection. I. Voxland, Philip M. II. Title. III. Series.

GA110.S575 $1989 \quad 526.8 \quad 86-600253$

For sale by Superintendent of Documents,

U.S. Government Printing Office

Washington, DC 20401

United States Government Printing Office : 1989 


\section{CONTENTS}

\section{Preface vii}

Introduction 1

Glossary 2

Guide to selecting map projections $\mathbf{5}$

Distortion diagrams 8

Cylindrical projections

Mercator 10

Transverse Mercator 12

Oblique Mercator 14

Lambert Cylindrical Equal-Area 16

Behrmann Cylindrical Equal-Area 19

Plate Carrée 22

Equirectangular 24

Cassini 26

Oblique Plate Carrée $\mathbf{2 8}$

Central Cylindrical $\mathbf{3 0}$

Gall 33

Miller Cylindrical 35

Pseudocylindrical projections

Sinusoidal 37

McBryde-Thomas Flat-Polar Sinusoidal

Eckert V 46

Winkel I 48

Eckert VI 50

McBryde S3 52

Mollweide 54

Eckert III $\mathbf{5 8}$

Eckert IV 60

Wagner IV 62

Hatano Asymmetrical Equal-Area 64

Goode Homolosine 66

Boggs Eumorphic 68

Craster Parabolic $\mathbf{7 0}$
McBryde-Thomas Flat-Polar Parabolic 72

Quartic Authalic $\mathbf{7 4}$

McBryde-Thomas Flat-Polar Quartic 76

Putniṇss $P_{5} 78$

DeNoyer Semi-Elliptical 80

Robinson 82

Collignon 84

Eckert 186

Eckert II 88

Loximuthal 90

Conic projections

Equidistant Conic $\mathbf{9 2}$

Lambert Conformal Conic 95

Bipolar Oblique Conic Conformal 99

Albers Equal-Area Conic 100

Lambert Equal-Area Conic 102

Perspective Conic 104

Polyconic 106

Rectangular Polyconic 110

Modified Polyconic, International Map of the World series 111

Bonne 112

Werner 114

Azimuthal projections

Perspective

Gnomonic 116

Stereographic 120

Orthographic 124

General Vertical Perspective 128

Nonperspective

Azimuthal Equidistant 132

Lambert Azimuthal Equal-Area 136

Airy 140 


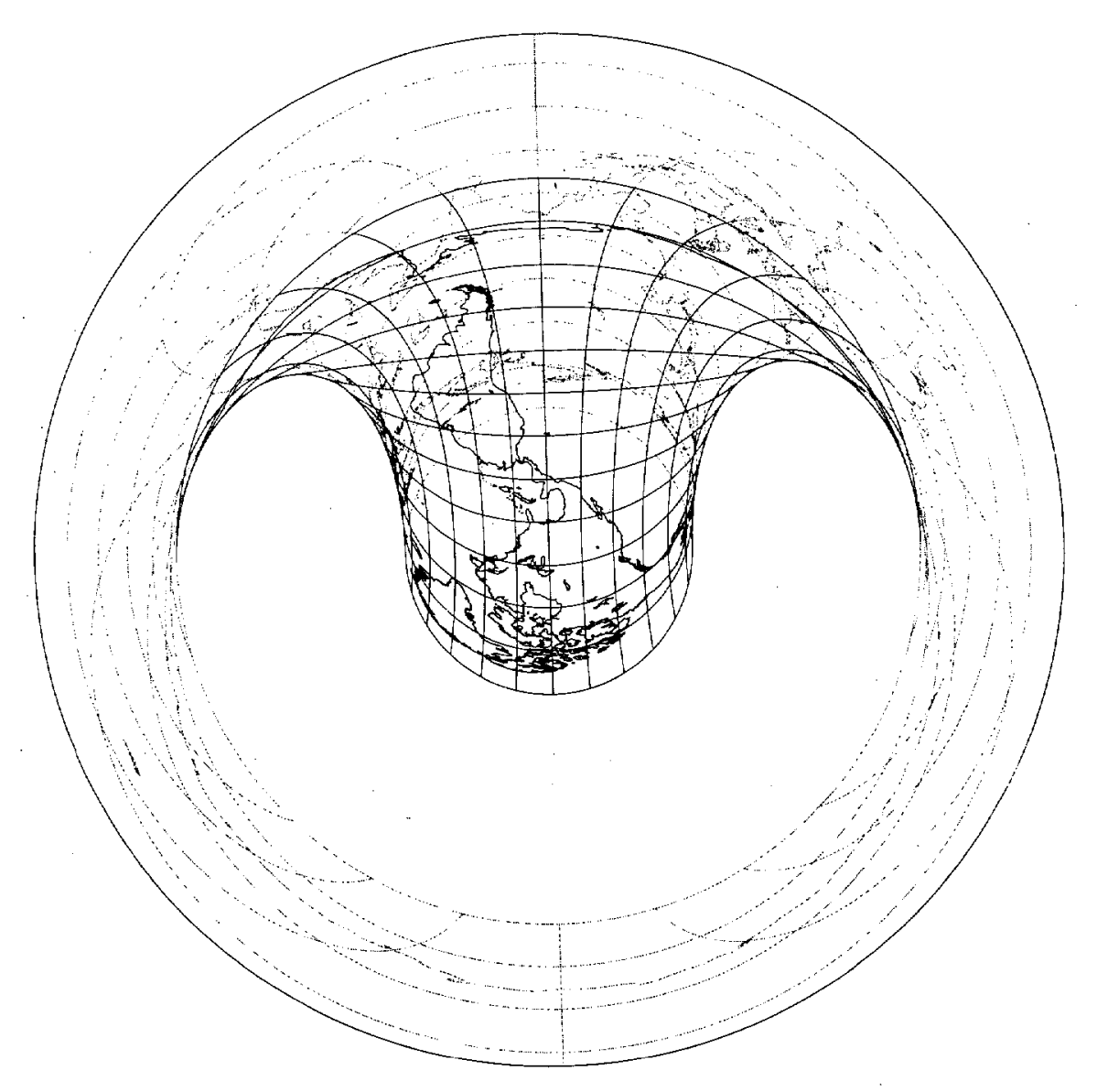




\section{CONTENTS}

Modified

Two-Point Azimuthal 144

Two-Point Equidistant 146

Miller Oblated Stereographic 148

Wiechel 149

Craig Retroazimuthal 150

Hammer Retroazimuthal 152

Littrow 154

Berghaus Star 156

Aitoff 158

Hammer 160

Briesemeister 162

Winkel Tripel 164

Eckert-Greifendorff 166

Wagner VII 168

Chamberlin Trimetric 170

Tilted Perspective 172

Miscellaneous

Bacon Globular 174 .

Fournier Globular 175

Nicolosi Globular 176

Apian Globular 1177

Ortelius Oval 178

Lagrange 180

Eisenlohr 184

August Epicycloidal 186

\section{Guyou 188}

Peirce Quincuncial 190

Adams Hemisphere in a Square 192

Adams World in a Square I 194

Adams World in a Square II 195

Lee Conformal World in a Triangle 196

Conformal World in an Ellipse 198

Van der Grinten I 200

Van der Grinten II 202

Van der Grinten III 203

Van der Grinten IV 204

Armadillo 206

GS50 208

Space Oblique Mercator 211

Satellite Tracking 213

Appendix: Formulas for projections 216

Cylindrical projections $\mathbf{2 1 8}$

Pseudocylindrical projections $\mathbf{2 2 0}$

Conic projections 224

Pseudoconic projections $\mathbf{2 2 5}$

Azimuthal projections

Perspective 226

Nonperspective 228

Modified $\mathbf{2 3 0}$

Miscellaneous projections $\mathbf{2 3 4}$

Selected references $\mathbf{2 4 0}$

Index 242 



\section{Preface}

Every map must begin, either consciously or unconsciously, with the choice of a map projection, the method of transforming the spherelike surface being mapped onto a flat plane. Because this transformation can become complicated, there is a tendency to choose the projection that has been used for a suitable available base map, even if it does not emphasize the desired properties.

This album has been prepared to acquaint those in the cartographic profession and other cartophiles with the wide range of map projections that have been developed during the past few centuries. Because including all the hundreds of known projection systems would be both difficult and expensive, this volume presents some 90 basic projections in over 130 different modifications and aspects. Dozens of other projections are mentioned briefly. All the popular projections have been included, along with many others that have interesting appearances or features, even if their usefulness for any serious mapping is limited.

The most important question to raise about a map projection concerns distortion. How accurately does the flat projection show the desired portion of the spherelike Earth (or heavens or planetary body)? Since accuracy is desired in area, shape, and distance but cannot be achieved in all three simultaneously and since the region mapped may vary in overall extent and shape, the answer is not clear cut. Because this album emphasizes visual comparisons rather than tables of scale variation, the Tissot indicatrix has been adopted as the most common means of comparing distortion on different parts of the same projection and on different projections.

Computerized plotting has facilitated the preparation of many outline map projections on a standardized basis, so that comparisons are more realistic. These maps have been plotted on film as lines 0.005 in. wide by means of a photohead computer-driven plotter and World Data

Bank I shoreline files (national boundaries included where appropriate). Central meridians and other parameters have been given the same values in so far as it was practical.

Some smaller maps have been prepared with the Dahlgren (Hershey) shoreline file. The map projection software for any given map consisted of one of three programs. WORLD was developed for a mainframe computer by Philip Voxland at the University of Minnesota. CYLIND and AZIMUTH were developed for a personal microcomputer and converted to mainframe computer programs at the U. S. Geological Survey (USGS) by John Snyder, with the guidance of Michael K. Linck, Jr., and John F. Waananen. The authors thank Joel L. Morrison, past president of the International Cartographic Association and Assistant

Division Chief for Research, National Mapping Division, USGS, for writing the introduction to this book, as well as Tau Rho Alpha (USGS) and Arthur $\mathrm{H}$. Robinson (University of Wisconsin/ Madison) for many constructive comments on the manuscript. The authors also thank Christopher $\mathrm{J}$. Cialek, Kathie R. Fraser, Carolyn S. Hulett, and

Ann Yaeger of the USGS for their assistance.

With this reprint, several corrections have been incorporated. We thank Daniel Strebe for reporting most of the errors in the formulas.

\section{John P. Snyder}

Philip M. Voxland 



\section{Introduction}

The puzzling world of map projections has fascinated humans for at least 2,500 years. Perhaps no single aspect of the cartographic discipline has held the cartographer's interest so consistently throughout history. How does one portray on a nonspherical surface the essentially spherical shape of the planet on which we live? It cannot be done without distortion, but the number of ways in which that distortion can be handled is infinite.

Although the subject is inherently mathematical and very complex in some instances, it also is highly visual and readily calls forth human reactions ranging from near disbelief to unquestioned acceptance as truth, from confusion to a clear understanding of information. Map projections have been devised for prop aganda purposes, as evidence in court, for use in detailed planning, and to attract attention.

Most people are affected more than they realize by map projections. Cognition of the relative areas and shapes on the Earth's surface and knowledge (correct or incorrect) of angles, sizes, distances, and other relationships among places on the globe are determined by the cartographer's choice of map projections. "An Album of Map Projections" should enable readers to sharpen and in some cases to correct their knowledge of the distances, directions, and relative areas of the world.

Although there has been no dearth of valuable books describing a wide range of map projec tions. "An Album of Map Projections" is the first attempt to set forth in a comprehensive manner most of the useful and commonly known map projections. Consistent and concise nonmathematical textural descriptions are accompanied by standardized visual protrayals. Descriptions of graticules, scale, distortion char acteristics, and aspects are carefully worded. Supplemental information on usage, origin, and similar projections is also presented.
No other collection of world portrayals is comparable, because of the standard-format illustrations and the accompanying distortion diagrams presented in this volume. These il. lustrations will be useful for classroom teaching, for selecting a projection to fit an output format, and for making visual comparisons between projections.

An appendix contains forward formulas for all but a few highly complex projections to transform from latitude and longitude positions on a sphere to rectangular coordinates. Inverse formulas and formulas treating the Earth as an ellipsoid have not been included. Standardized notation is introduced and used throughout.

Finally, it must be noted that this volume could not have been realistically produced with out the aid of a computer. Although a compendium of this nature has been needed for decades (to avoid some of the confusion resulting from identical projections' being called by different names, if nothing else), the enormity of manually compiling the necessary information prevented its production. The computer's ability. to calculate and plot the portrayals made this album possible.

\section{Joel L. Morrison}




\section{Glossary}

Aspect Conceptual placement of a projection system in relation to the Earth's axis (direct, normal, polar, equatorial, oblique, and so on).

Authalic projection See Equal-area projection.

Azimuthal projection Projection on which the azimuth or direction from a given central point to any other point is shown correctly. Also called a zenithal projection. When a pole is the central point, all meridians are spaced at their true angles and are straight radii of concentric circles that represent the parallels.

Central meridian Meridian passing through the center of a projection, often a straight line about which the projection is symmetrical.

Central projection Projection in which the Earth is projected geometrically from the center of the Earth onto a plane or other surface. The Gnomonic and Central Cylindrical pro jections are examples.

Complex algebra Branch of algebra that deals with complex numbers (combinations of real and imaginary numbers using the square root of -1 ).

Complex curves Curves that are not elementary forms such as circles, ellipses, hyperbolas parabolas, and sine curves.

Composite projection Projection formed by connecting two or more projections along common lines such as parallels of latitude necessary adjustments being made to achieve fit. The Goode Homolosine projection is an example.

Conformal projection Projection on which al angles at each point are preserved. Also called an orthomorphic projection.

Conceptually projected Convenient way to visu alize a projection system, although it may not correspond to the actual mathematical projection method.

Conic projection Projection resulting from the conceptual projection of the Earth onto a tangent or secant cone, which is then cut lengthwise and laid flat. When the axis of the cone coincides with the polar axis of the Earth, all meridians are straight equidis tant radii of concentric circular arcs representing the parallels, but the meridians are spaced at less than their true angles. Mathematically, the projection is often only partially geometric.

Constant scale Linear scale that remains the same along a particular line on a map. although that scale may not be the same as the stated or nominal scale of the map.

Conventional aspect See Direct aspect.

Correct scale Linear scale having exactly the same value as the stated or nominal scale of the map, or a scale factor of 1.0. Also called true scale.

Cylindrical projection Projection resulting from the conceptual projection of the Earth onto a tangent or secant cylinder, which is then cut lengthwise and laid flat. When the axis of the cylinder coincides with the axis of the Earth, the meridians are straight, paral lel, and equidistant, while the parallels of latitude are straight, parallel, and perpen. dicular to the meridians. Mathematically, the projection is often only partially geometric.

Direct aspect Form of a projection that provides the simplest graticule and calculations. It is the polar aspect for azimuthal projections the aspect having a straight Equator for cylindrical and pseudocylindrical projections, and the aspect showing straight meridians for conic projections. Also called conventional or normal aspect.

Distortion Variation of the area or linear scale on a map from that indicated by the stated map scale, or the variation of a shape or angle on a map from the corresponding shape or angle on the Earth.

Ellipsoid When used to represent the Earth, a solid geometric figure formed by rotating an ellipse about its minor (shorter) axis. Also called spheroid.

Equal-area projection Projection on which the areas of all regions are shown in the same proportion to their true areas. Also called an equivalent or authalic projection. Shapes may be greatly distorted.

Equatorial aspect Aspect of an azimuthal projection on which the center of projection or origin is some point along the Equator. For cylindrical and pseudocylindrical projections, this aspect is usually called conventional, direct, normal, or regular rather than equatorial.

Equidistant projection Projection that maintains constant scale along all great circles from one or two points. When the projection is centered on a pole, the parallels are spaced in proportion to their true distances along each meridian.

Equivalent projection See Equal-area projection.

Flat-polar projection Projection on which, in direct aspect, the pole is shown as a line rather than as a point.

Free of distortion Having no distortion of shape area, or linear scale. On a flat map, this condition can exist only at certain points or along certain lines.

Geometric projection See Perspective projection.

Globular projection Generally, a nonazimuthal projection developed before 1700 on which a hemisphere is enclosed in a circle and meridians and parallels are simple curves or straight lines.

Graticule Network of lines representing a selec tion of the Earth's parallels and meridians.

Great circle Any circle on the surface of a sphere, especially when the sphere represents the Earth, formed by the intersection of the surface with a plane passing through 
the center of the sphere. It is the shortest path between any two points along the circle and therefore important for navigation. All meridians and the Equator are great circles on the Earth taken as a sphere.

Indicatrix Circle or ellipse having the same shape as that of an infinitesimally small circle (having differential dimensions) on the Earth when it is plotted with finite dimensions on a map projection. Its axes lie in the directions of and are proportional to the maximum and minimum scales at that point. Often called a Tissot indicatrix after the originator of the concept.

Interrupted projection Projection designed to reduce peripheral distortion by making use of separate sections joined at certain points or along certain lines, usually the Equator in the normal aspect, and split along lines that are usually meridians. There is normally a central meridian for each section.

Large-scale mapping Mapping at a scale larger than about $1: 75,000$, although this limit is somewhat flexible.

Latitude (geographic) Angle made by a perpendicular to a given point on the surface of a sphere or ellipsoid representing the Earth and the plane of the Equator ( + if the point is north of the Equator, - if it is south). One of the two common geographic coordinates of a point on the Earth.

Latitude of opposite sign See Parallel of opposite sign.

Limiting forms Form taken by a system of projection when the parameters of the formulas defining that projection are allowed to reach limits that cause it to be identical with another separately defined projection.

Longitude Angle made by the plane of a meridian passing through a given point on the Earth's surface and the plane of the (prime) meridian passing through Greenwich, England, east or west to $180^{\circ}$ ( + if the point is east, - if it is west). One of the two common geographic coordinates of a point on the Earth.

Loxodrome Complex curve (a spherical helix) on the Earth's surface that crosses every meridian at the same oblique angle; in navigation, called a rhumb line. A navigator can proceed between any two points along a rhumb line by maintaining a constant bearing. A loxodrome is a straight line on the Mercator projection.

Meridian Reference line on the Earth's surface formed by the intersection of the surface with a plane passing through both poles and some third point on the surface. This line is identified by its longitude. On the Earth as a sphere, this line is half a great circle; on the Earth as an ellipsoid, it is half an ellipse.

Minimum-error projection Projection having the least possible total error of any projection in the designated classification, according to a given mathematical criterion. Usually, this criterion calls for the minimum sum of squares of deviations of linear scale from true scale throughout the map ("least squares").

Nominal scale Stated scale at which a map projection is constructed.

Normal aspect See Direct aspect.

Oblique aspect Aspect of a projection on which the axis of the Earth is rotated so it is neither aligned with nor perpendicular to the conceptual axis of the map projection.

Orthoapsidal projection Projection on which the surface of the Earth taken as a sphere is transformed onto a solid other than the sphere and then projected orthographically and obliquely onto a plane for the map.

Orthographic projection Specific azimuthal projection or a type of projection in which the Earth is projected geometrically onto a surface by means of parallel projection lines.

Orthomorphic projection See Conformal projection.

Parallel Small circle on the surface of the Earth formed by the intersection of the surface of the reference sphere or ellipsoid with a plane parallel to the plane of the Equator. This line is identified by its latitude. The Equator (a great circle) is usually also treated as a parallel.

Paraliel of opposite sign Parallel that is equally distant from but on the opposite side of the Equator. For example, for lat $30^{\circ} \mathrm{N}$. (or $+30^{\circ}$ ), the parallel of opposite sign is lat $30^{\circ} \mathrm{S}$. (or $-30^{\circ}$ ). Also called latitude of opposite sign.

Parameters Values of constants as applied to a map projection for a specific map; examples are the values of the scale, the latitudes of the standard parallels, and the central meridian. The required parameters vary with the projection.

Perspective projection Projection produced by projecting straight lines radiating from a selected point (or from infinity) through points on the surface of a sphere or ellipsoid and then onto a tangent or secant plane. Other perspective maps are projected onto a tangent or secant cylinder or cone by using straight lines passing through a single axis of the sphere or ellipsoid. Also called geometric projection.

Planar projection Projection resulting from the conceptual projection of the Earth onto a tangent or secant plane. Usually, a planar projection is the same as an azimuthal projection. Mathematically, the projection is often only partially geometric.

Planimetric map Map representing only the horizontal positions of features (without their elevations). 


\section{Glossary}

Polar aspect Aspect of a projection, especially an azimuthal one, on which the Earth is viewed from the polar axis. For cylindrical or pseudocylindrical projections, this aspect is called transverse.

Polyconic projection Specific projection or member of a class of projections in which, in the normal aspect, all the parallels of latitude are nonconcentric circular arcs, except for a straight Equator, and the centers of these circles lie along a central axis.

Pseudoconic projection Projection that, in the normal aspect, has concentric circular arcs for parallels and on which the meridians are equally spaced along the parallels, like those on a conic projection, but on which meridians are curved.

Pseudocylindrical projection Projection that, in the normal aspect, has straight paraliel lines for parallels and on which the meridians are (usually) equally spaced along parallels, as they are on a cylindrical projection, but on which the meridians are curved.

Regional map Small-scale map of an area covering at least 5 or 10 degrees of latitude and Iongitude but less than a hemisphere.

Regular aspect See Direct aspect.

Retroazimuthal projection Projection on which the direction or azimuth from every point on the map to a given central point is shown correctly with respect to a vertical line parallel to the central meridian. The reverse of an azimuthal projection.

Rhumb line See Loxodrome.

Scale Ratio of the distance on a map or globe to the corresponding distance on the Earth; usually stated in the form 1:5,000,000, for example.

Scale factor Ratio of the scale at a particular location and direction on a map to the stated scale of the map. At a standard parallel, or other standard line, the scale factor is 1.0 .

Secant cone, cylinder, or plane A secant cone or cylinder intersects the sphere or ellipsoid along two separate lines; these lines are parallels of latitude if the axes of the geometric figures coincide. A secant plane inter sects the sphere or ellipsoid along a line that is a parallel of latitude if the plane is at right angles to the axis.

Similar (projection) Subjective and qualitative term indicating a moderate or strong re. semblance.

Singular points Certain points on most but not all conformal projections at which conformality fails, such as the poles on the normal aspect of the Mercator projection.

Small circle Circle on the surface of a sphere formed by intersection with a plane that does not pass through the center of the sphere. Parallels of latitude other than the Equator are small circles on the Earth taken as a sphere.

Small-scale mapping Mapping at a scale smaller than about $1: 1,000,000$, although the limiting scale sometimes has been made as large as $1: 250,000$.

Spheroid See Ellipsoid.

Standard parallel In the normal aspect of a projection, a parallel of latitude along which the scale is as stated for that map. There are one or two standard parallels on most cylindrical and conic map projections and one on many polar stereographic projections.

Stereographic projection Specific azimuthal projection or type of projection in which the Earth is projected geometrically onto a surface from a fixed (or moving) point on the opposite face of the Earth.

Tangent cone or cylinder Cone or cylinder that just touches the sphere or ellipsoid along a single line. This line is a parallel of latitude if the axes of the geometric figures coin cide.

Thematic map Map designed to portray primarily a particular subject, such as population, railroads, or croplands.

Tissot indicatrix See Indicatrix.

Topographic map Map that usually represents the vertical positions of features as well as their horizontal positions.

\section{Transformed latitudes, longitudes, or poles} Graticule of meridians and parallels on a projection after the Earth has been turned with respect to the projection so that the Earth's axis no longer coincides with the conceptual axis of the projection. Used for oblique and transverse aspects of many projections.

Transverse aspect Aspect of a map projection on which the axis of the Earth is rotated so that it is at right angles to the conceptual axis of the map projection. For azimuthal projections, this aspect is usually called equatorial rather than transverse.

True scale See Correct scale.

Zenithal projection See Azimuthal projection. 


\section{Guide to Selecting Map Projections}

This publication displays the great variety of map projections from which the choice for a particular map can be made. Although most of the projections illustrated in this album have been in existence for many years, cartographers have been content with using only a few of them.

This past lack of innovation is easily understood. The difficulty and expense of creating a new base map using a different projection often outweighed the perceived benefits. The advent of computer-assisted cartography has now made it much easier to prepare base maps. A map can now be centered anywhere on the globe, can be drawn according to any one of many projection formulas, and can use cartographic data files (coastline boundaries, for example) having a level of generalization appropriate to the scale of the map.

However, now that more choices can be made the actual decision may be more difficult. Some of the traditional rules for selection are now merely guidelines. This chapter discusses clas. sifications of maps and presents well-established principles for their evaluation. Technical and mathematical methods also exist for determining exactly the characteristics of a specific projection, but those methods are not used here.

First, we should define what is meant by a map projection. A projection is a systematic transformation of the latitudes and longitudes of locations on the surface of a sphere or an ellipsoid into locations on a plane. That is, locations in three-dimensional space are made to correspond to a two-dimensional representation.

If we assume for simplicity that a globe, which is a sphere, can perfectly represent the surface of the Earth, then all of the following characteristics must be true of that globe: 1. Areas are everywhere correctly represented.
2. All distances are correctly represented.

3. All angles are correctly represented.

4. The shape of any area is faithfully represented.

When the sphere is projected onto a plane, the map will no longer have all of these characteristics simultaneously. Indeed, the map may have none of them. One way of classifying maps and their projections is through terms describing the extent to which the map preserves any of those properties.

\section{Properties of Map Projections}

An equal-area map projection correctly represents areas of the sphere on the map. If a coin is placed on any area of such a map, it will cover as much of the area of the surface of the sphere as it would if it were placed else where on the map. When this type of projection is used for small-scale maps showing larger regions, the distortion of angles and shapes increases as the distance of an area from the projection origin increases.

An equidistant map projection is possible only in a limited sense. That is, distances can be shown at the nominal map scale along a line from only one or two points to any other point on the map. The focal points usually are at the map center or some central location. The term is also often used to describe maps on which the scale is shown correctly along all meri dians.

An azimuthal map likewise is limited in the sense that it can correctly show directions or angles to all other points on the map only with respect to one (or rarely two) central point(s).

A conformal map is technically defined as a map on which all angles at infinitely small locations are correctly depicted. A conformal projection increasingly distorts areas away from the map's point or lines of true scale and increasingly distorts shapes as the region be comes larger but distorts the shapes of moderately small areas only slightly.

Consistent with these definitions, maps simultaneously exhibiting several of these properties can be devised:

\begin{tabular}{|c|c|c|c|c|}
\hline & $\begin{array}{c}\text { Conformal } \\
-\end{array}$ & $\begin{array}{c}\text { Equal } \\
\text { area } \\
\text { No }\end{array}$ & $\begin{array}{c}\text { Equidistant } \\
\text { No }\end{array}$ & $\begin{array}{c}\text { Azimuthal } \\
\text { Yes }\end{array}$ \\
\hline & No & - & Yes & Yes \\
\hline & No & Yes & - & Yes \\
\hline zim & Yes & Yes & Yes & - \\
\hline
\end{tabular}

In summary, any map will distort areas, angles, directions, or distances to some extent. Since all of these distortions can be measured or estimated, one rigorous selection rule would be "to select a projection in which the extreme distortions are smaller than would occur in any other projection used to map the same area" (Maling, 1973, p. 159).

A map projection may have none of these general properties and still be satisfactory. A map projection possessing one of these proper

ties may nevertheless be a poor choice. As an example, the Mercator projection continues to be used inappropriately for worldwide thematic data. The Mercator map projection is conformal and has a valid use in navigation but very seriously distorts areas near the poles, which it cannot even show. It should not be used (although it frequently is) for depicting general information or any area-related subjects.

\section{Classification Based on Construction}

From the perspective of design as well as distortion reduction, a projection may be selected because of the characteristic curves formed by the meridians and parallels. L.P. Lee preferred terms based on the pattern formed by the meridians and parallels in the normal aspect or orientation. The following definitions 


\section{Guide to Selecting Map Projections}

are quoted from his 1944 paper, "The nomenclature and classification of map projections" (Lee, 1944, p. 193):

Cylindric: Projections in which the meridians are represented by a system of equidistant parallel straight lines, and the parallels by a system of parallel straight lines at right angles to the meridians.

Pseudocylindric: Projections in which the parallels are represented by a system of parallel straight lines, and the meridians by concurrent curves.

Conic: Projections in which the meridians are represented by a system of equally inclined concurrent straight lines, and the parallels by concentric circular arcs, the angle between any two meridians being less than their true difference of longitude.

Pseudoconic: Projections in which the parallels are represented by concentric circular arcs, and the meridians by concurrent curves.

Polyconic: Projections in which the parallels are represented by a system of nonconcentric circular arcs with their centres lying on the straight line representing the central meridian. Azimuthal: Projections in which the meridians are represented by a system of concurrent straight lines inclined to each other at their true difference of longitude, and the parallels by a system of concentric circles with their common centre at the point of concurrency of the meridians.

D.H. Maling used an additional term consistent with Lee's definitions:

Pseudoazimuthal: Projections comprised of "concentric circular parallels and curved meridians which converge at the pole at their true angular values" (Maling, 1960, p. 209).

Finally, a small class of projections has also been identified by the term retroazimuthal, denoting projections in which the direction to a central point from every point on the map is shown correctly.

\section{Philosophy of Map-Projection Selection}

On the basis of these concepts, three traditional rules for choosing a map projection were at one time recommended:

1. For low-latitude areas: cylindrical.

2. For middle-latitude areas: conical.

3. For polar regions: azimuthal.

Inherent in these guidelines was the idea that it would be difficult to recenter a map so that the area of main interest was near the area of the map that has the least areal or angular distortion. On the other hand, it is now much less difficult than it formerly was to rotate latitude and longitude coordinates so that any point is moved to any other new location. As a result, a projection does not need to be rejected merely because the only available copy is centered inappropriately for a new application. In fact, many projections permit the user to alter the form of the map to reduce the distortions within a certain area. Most commonly, such alteration is accomplished by establishing standard lines along which distortion is absent; often, these lines are parallels of latitude. However, most properties of the map projection are affected when a standard line is changed. Another way to alter the relationships on a map is by using different aspects, which involves moving the center of a projection from the normal position at a pole or along the Equator to some other position.

"Map makers must first define the purpose of a map which in turn can provide answers to many questions essential to decisions concerning the projections" (Hsu, 1981, p. 170). When a map requires a general property, the choice of a projection becomes limited. For example, because conformal projections correctly show angles at every location, they are advisable for maps displaying the flow of oceanic or atmospheric currents. The risk of using a conformal projection for a worldwide map is that the distortion of areas greatly enlarges the outer boundaries, and a phenomenon may seem to take on an importance that the mapmaker did not intend. Equal-area maps should be considered for displaying area-related subjects or themes, such as crop-growing regions.

"Once the purpose of a map has been decided, the geographical area to be included on the map must be determined. Let us call it the map area for brevity. The map area can be a region or the entire world. The larger the area covered, the greater is the earth's curvature involved on the map.... If the map area is a region, then its shape, size, and location are important determinants in making decisions concerning projections" (Hsu, 1981, p. 172).

Bearing in mind these determinants, mapmakers can apply traditional rules of choice, such as those mentioned above, or they can study the patterns of distortion associated with particular projections. For example, azimuthal projections have a circular pattern for lines of constant distortion characteristics, centered on the map origin or projection center. Thus, if an area is approximately circular and if its center is made the origin of the projection, it is possible to create a map that minimizes distortion for that map area. Ideally, the general shape of a geographic region should be matched with the distortion pattern of a specific projection.

An appropriate map projection can be selected on the basis of these principles and classifications. Although there may be no absolutely correct choice, it is clearly possible to make a bad judgment.

\section{Edge Matching}

One problem encountered by many map users is the edge matching of adjacent regions. For two or more maps to fit exactly along their 
edges, whether these be common meridians,

parallels, or rectangular coordinate gridlines,

not only must they be cast on the same projec-

tion and at the same scale, but the projection

for each map must also have the same critical

parameters. The critical parameters, which

vary somewhat with each projection, are those

specifications that affect the shape and size but not the position or orientation of a projection.

The Mercator projection, which is commonly used in edge-matching operations, must be based on the same ellipsoid or sphere and must use the same scale at the same latitude for

each map. Conic projections such as the Albers, Lambert Conformal, or Equidistant will match only if the standard parallels and the ellipsoid are the same for each map. For the Mercator and conic projections, the central meridian and the latitude of origin do not have to be identical for edge matching. For the Transverse Mercator projection, however, the ellipsoid, the central

meridian, and the scale along the central meridian must be the same, although the latitude of origin may vary.

In practice, edge matching is hampered by the dimensional instability of the paper or other material that the maps are printed on and also by cartographic or surveying errors in extending roads and streams to the edges of the maps. The differences between the projections used for large-scale maps may be small enough to permit a satisfactory fit, depending on the required accuracy and purpose of the maps. 


\section{Distortion Diagrams}

The most important characteristics of a map projection are the magnitude of distortion and the effect of that distortion on the intended use of a map. To assist in evaluating these features, many of the graticules for the projections described herein are presented on two illustrations.

The larger of the two plots uses the World Data Bank I shoreline file (occasionally includ ing national boundaries). It also uses the same central meridian $\left(90^{\circ} \mathrm{W}\right.$.), unless doing so would defeat the purpose of the projection, as it would with the Briesemeister. Some evaluation of distortion can be achieved by comparing familiar shapes of islands and shorelines. A more uniform comparison can be made by using the smaller plots, which include no shorelines but which show Tissot indicatrices at every $30^{\circ}$ of latitude and longitude, except at the poles (arbitrarily omitted because of plotting complications).

The Tissot indicatrix, devised by French cartographer Nicolas Auguste Tissot in the 19th century, shows the shape of infinitesimally small circles on the Earth as they appear when they are plotted by using a fixed finite scale at the same locations on a map. Every circle is plotted as a circle or an ellipse or, in extreme cases, as a straight line. On an equal-area pro jection, all these ellipses and circles are shown as having the same area. The flattening of the ellipse shows the extent of local shape distortion and how much the scale is changed and in what direction. On conformal map projections, all indicatrices remain circles, but the areas change. On other projections, both the areas and the shapes of the indicatrices change.

For example, for the conformal Mercator projection, all indicatrices are circles (fig. $1 A$, p. 10). Scale is constant along every parallel; thus, every circle along a given parallel is the same size. On the other hand, the farther the parallel from the Equator, the greater the size of the circle. Quantitatively, the diameter of the circle is proportional to the linear scale at that point, and the area of the circle is proportional to the area scale. The shape of the circle also indicates that the (linear) scale is the same in every direction at a given point.

Figure $13 A$ (p. 37) represents indicatrices for the equal-area Sinusoidal projection. The indicatrices are circles along the Equator and along the central meridian, because there is no local shape distortion there. As it did in figure $1 A$, the shape of the circles indicates that the scale is the same in all directions at each location. The indicatrices elsewhere are ellipses, charac terized by varying degrees of flattening and by axes pointed in various directions. Because the areas of all these ellipses are equal, the projection is equal area. The orientation and flattening of a given ellipse provide additional information. The major axis represents the magnitude and direction of the greatest scale at that point. The magnitude can be determined by multiplying the map scale by the ratio of the length of the major axis of the ellipse to the diameter of the circle at a point of no distortion, such as a point along the Equator of this projection. The minor axis represents the magnitude and direction of the smallest scale at that point, determined in the same manner. The angular distortion at each point is similarly indicated by the indicatrix. At every point, there is no angular distortion in plotting the intersection of two particular lines on the Earth, one in the direction of the major axis of the ellipse and the other in the direction of the minor axis. These two lines intersect at right angles on the Earth (lines $A B$ and $C D$ ) and also at right angles on the map (lines $A^{\prime} B^{\prime}$ and $\left.C^{\prime} D^{\prime}\right)$. A pair of perpendicular lines inclined at $45^{\circ}$ to these lines on the Earth (lines EF and $\mathrm{GH}$ ) will not intersect at right angles on the map if the indicatrix is an ellipse (lines $E^{\prime} F$ and $G^{\prime} H^{\prime}$ ). This statement is true for every pair of lines other than the two axes mentioned. The scale in these various directions is propor. tional to the length of the diameter across the ellipse in the same direction. In two particular

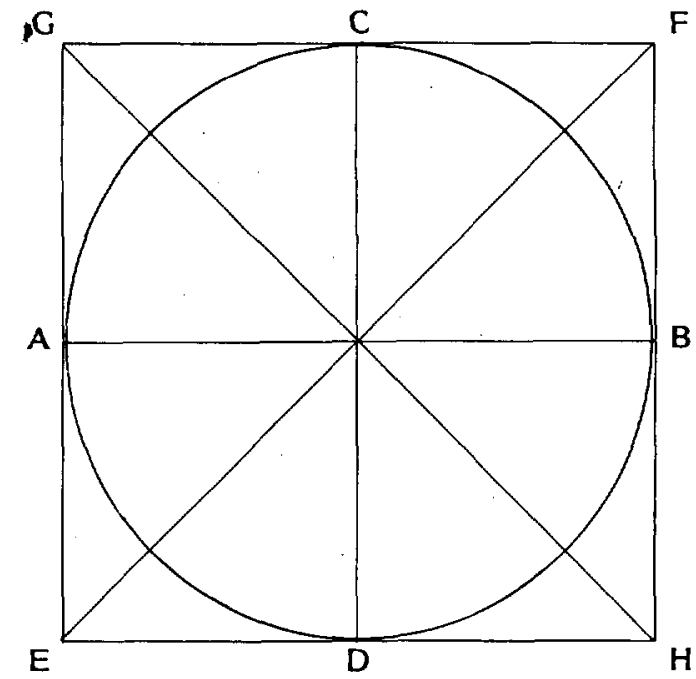

An infinitely small circle on the Earth, circumscribed by a square. 


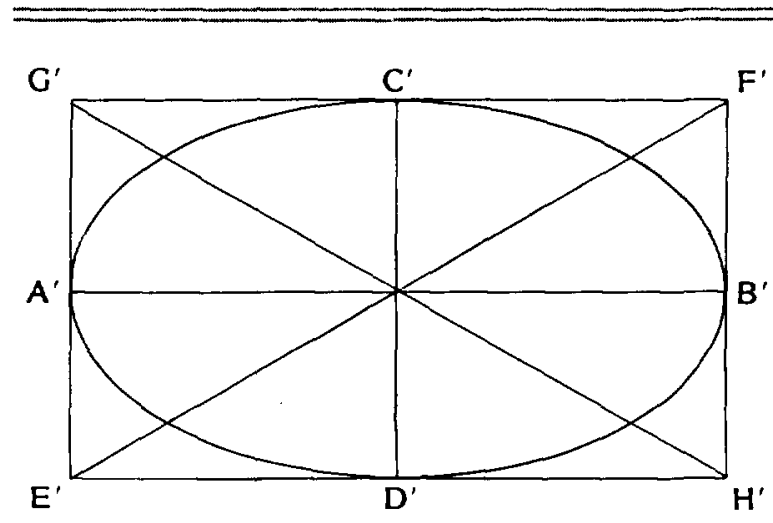

The circle shown on the preceding page as plotted on a noncontormal map, circumscribed by a rectangle.

directions, between the directions of the major and minor axes on an elliptical indicatrix of an equal-area map projection, the scale is the same as that of the map.

Figure $32 A$ (p. 82) illustrates distortions on the Robinson, a projection that is neither equal area nor conformal. Both the size and the shape of the indicatrices change. No point is completely free of distortion; therefore, all indicatrices are at least slightly elliptical. Their areas are proportional to the area scale, and linear scale and angular distortion can be interpreted just as they were for the ellipses of the Sinusoidal plot, except that there is no circular indicatrix. The indicatrices as shown in this volume are obviously too small to permit effective quantitative scale and distortion measurements. Nevertheless, they provide more certainty in this evaluation than do the larger plots of shorelines. 


\section{MERCATOR Projection}

\section{Classifications}

Cylindrical

Conformal

\section{Graticule}

Meridians: Equally spaced straight parallel

lines

Parallels: Unequally spaced straight parallel lines, closest near the Equator, perpendicular to meridians

Poles: Cannot be shown

Symmetry: About any meridian or the Equator

\section{Scale}

True along the Equator or along two parallels equidistant from the Equator

Increases with distance from the Equator to infinity at the poles

Constant along any given parallel; same scale at parallel of opposite sign (north + , south -) Same in all directions near any given point

\section{Distortion}

Infinitesimally small circles (indicatrices) of equal size on the globe appear as circles on the map (indicating conformality) but increase in size away from the Equator (indicating area distortion) (fig. 1A). Great distortion of area in polar regions. Conformality (and therefore local angle preservation) fails at the poles.

\section{Other features}

All loxodromes or rhumb lines (lines that make equal angles with all meridians and are therefore lines of constant true bearing) are straight lines.
Meridians can be geometrically projected onto a cylinder, the axis of which is the same as that of the globe.

Parallels cannot be geometrically (or perspectively) projected.

Meridians cannot be compressed relative to parallels, as they can on Cylindrical Equal-

Area and Equirectangular projections, since conformality would be lost.

\section{Usage}

Designed and recommended for navigational usage because of straight rhumb lines; standard for marine charts

Recommended and used for conformal mapping of regions predominantly bordering the

Equator

Often and inappropriately used as a world map in atlases and for wall charts. It presents a misleading view of the world because of the excessive distortion of area.

\section{Origin}

Presented by Gerardus Mercator (1512-94) of Flanders in 1569 on a large world map "for use in navigation"

\section{Aspects}

Normal is described here.

Transverse and Oblique aspects are listed separately (p. 12-15) because of importance and common treatment as separate projections.

\section{Other names}

Wright (rare) (after Edward Wright of England, who developed the mathematics in 1599)

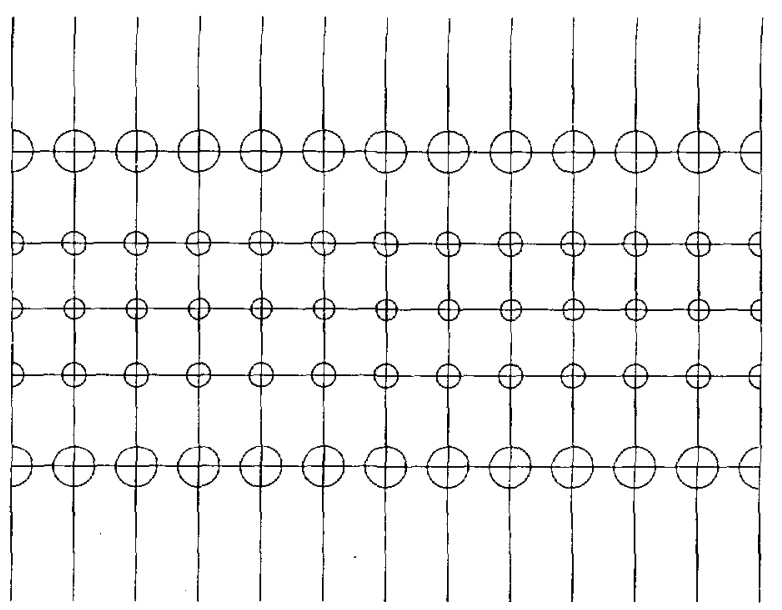

\section{Similar projections}

Central Cylindrical projection (p. 30) also cannot show poles, but it is not conformal, and the spacing of parallels changes much more rapidly.

Miller Cylindrical projection (p. 35) shows the poles, is not conformal, and has more gradual spacing of parallels.

Gall projection (p. 33) shows the poles, is not conformal, and has more gradual spacing of parallels. 


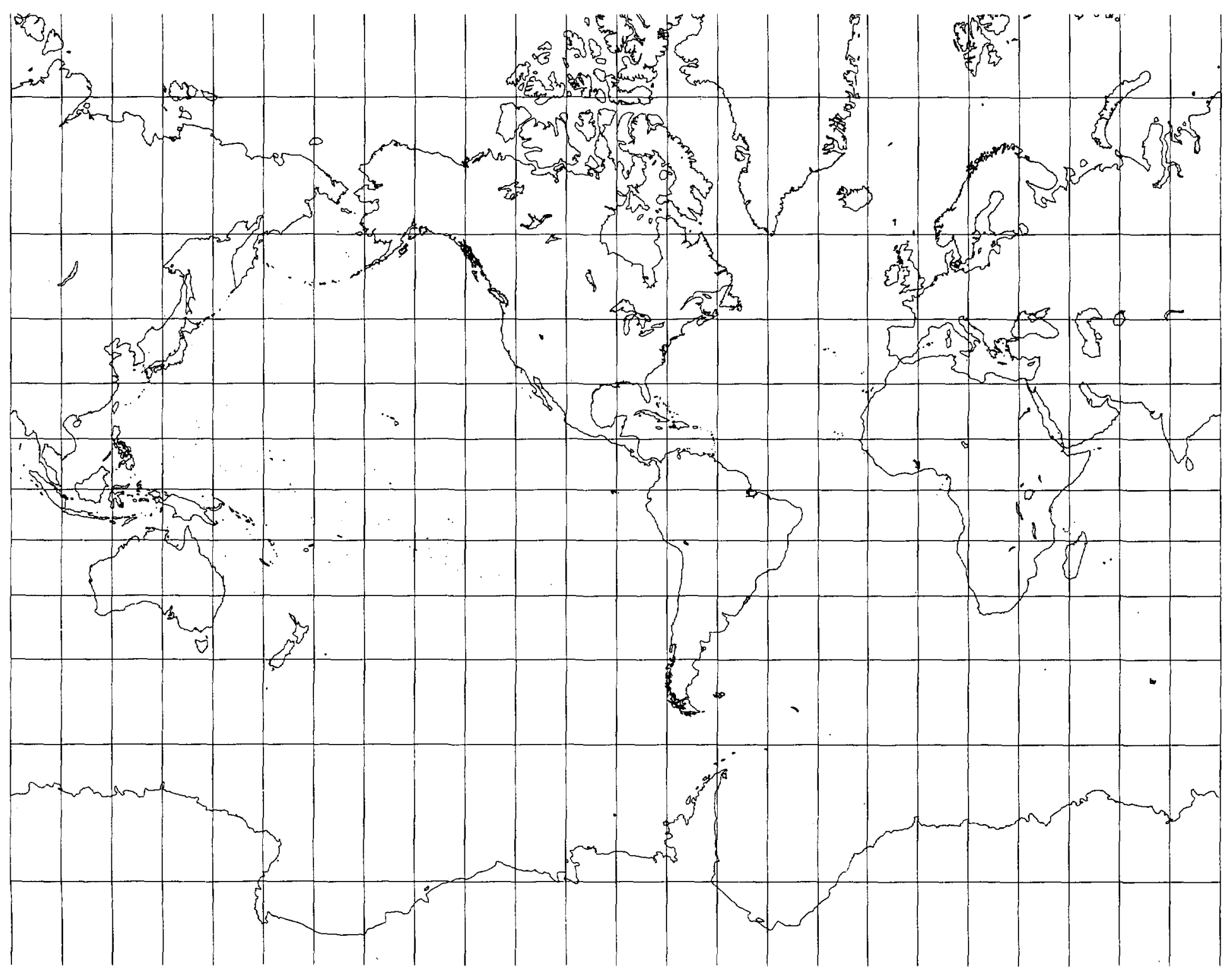




\section{Classifications}

Transverse aspect of Mercator projection

Cylindrical

Conformal

\section{Graticule}

Meridians and parallels: Central meridian, each meridian $90^{\circ}$ from central meridian, and the Equator are straight lines. Other meridians and parallels are complex curves, concave toward the central meridian and the nearest pole, respectively.

Poles: Points along the central meridian

Symmetry: About any straight meridian or the Equator

\section{Scale}

True along the central meridian or along two straight lines on the map equidistant from and parallel to the central meridian

Constant along any straight line on the map parallel to the central meridian. (These lines are only approximately straight for the projection of the ellipsoid.)

Increases with distance from the central meridian

Becomes infinite $90^{\circ}$ from the central meridian

\section{Distortion}

At a given distance from the central meridian in figure $2 A$, the distortion in area is identical with that at the same distance from the Equator in figure $1 \mathrm{~A}$.

\section{Other features}

Conceptually projected onto a cylinder wrapped around the globe tangent to the central meridian or secant along two small circles equidistant from the central meridian

Cannot be geometrically (or perspectively) projected

Rhumb lines generally are not straight lines.

\section{Usage}

Many of the topographic and planimetric map quadrangles throughout the world at scales of $1: 24,000$ to $1: 250,000$

Basis for Universal Transverse Mercator

(UTM) grid and projection

Basis for State Plane Coordinate System in U.S. States having predominantly north-south extent

Recommended for conformal mapping of regions having predominantly north-south extent

\section{Origin}

Presented by Johann Heinrich Lambert (172877) of Alsace in 1772. Formulas for ellipsoidal use developed by Carl Friedrich Gauss of Germany in 1822 and by L. Krüger of Germany, L.P. Lee of New Zealand, and others in the 20th century.

\section{Other names}

Gauss Conformal (ellipsoidal form only)

Gauss-Krüger (ellipsoidal form only) Transverse Cylindrical Orthomorphic

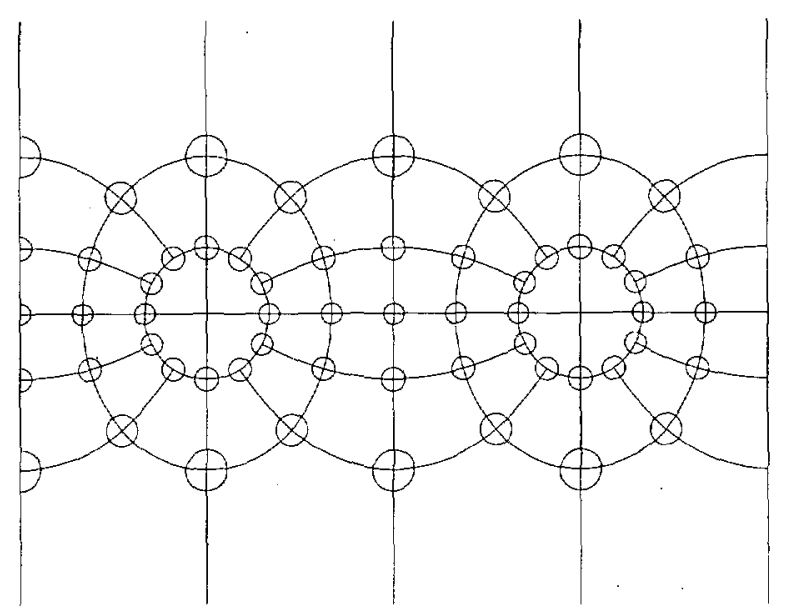


Figure 2B.-Transverse Mercator projection, with shorelines, $15^{\circ}$ graticule. Central meridian $90^{\circ} \mathrm{E}$. and W. North Pole at $-90^{\circ}$ longitude on base projection.

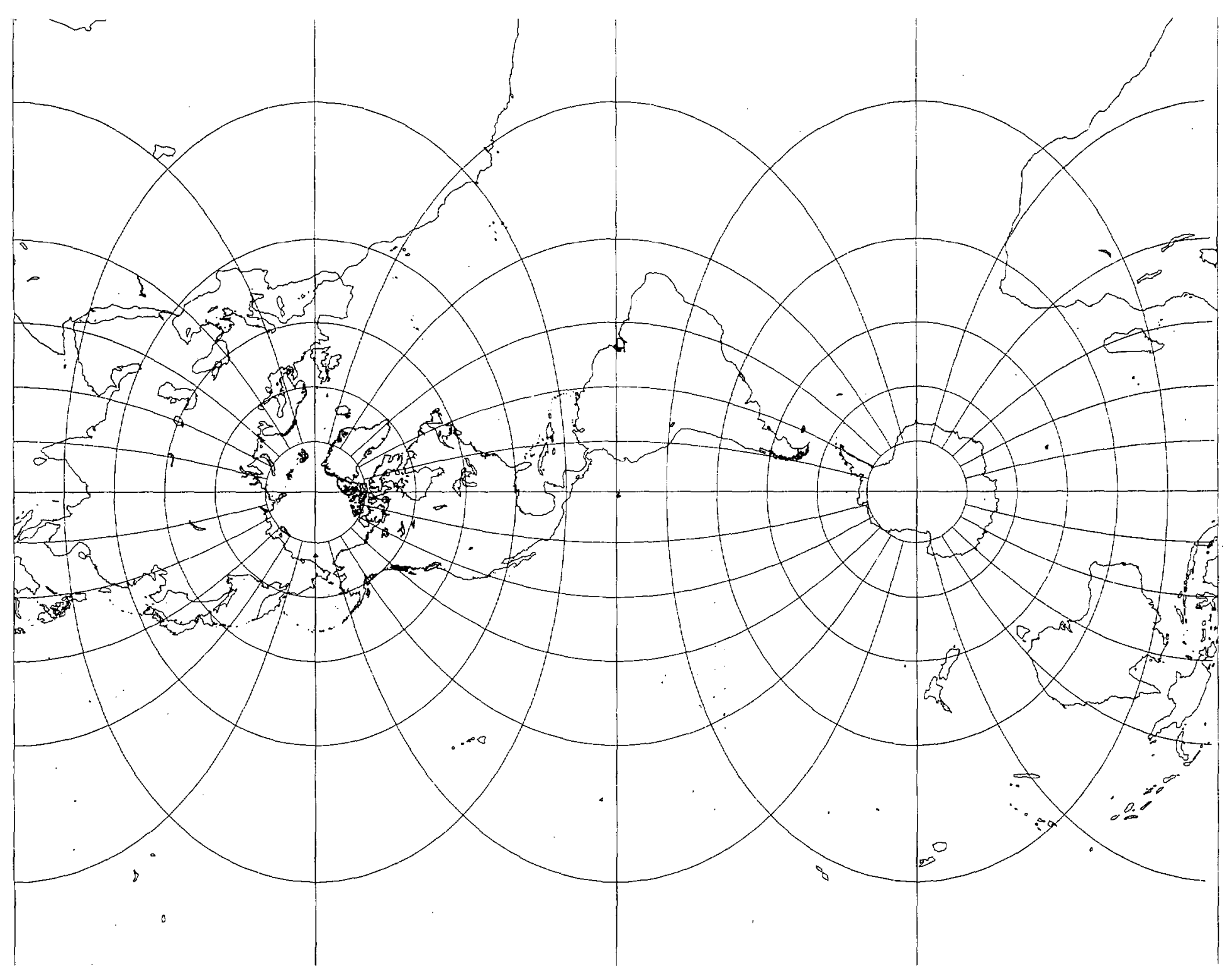




\section{Classifications}

Oblique aspect of Mercator projection

Cylindrical

Conformal

\section{Graticule}

Meridians and parallels: Two meridians $180^{\circ}$ apart are straight lines. Other meridians and parallels are complex curves.

Poles: Points not on the central line

Symmetry: About either straight meridian

\section{Scale}

True along a chosen central line (a great circle at an oblique angle) or along two straight lines on the map parallel to the central line

Constant along any straight line parallel to the central line

(The scale for the projection of the ellipsoid varies slightly from these patterns.)

Increases with distance from the central line Becomes infinite $90^{\circ}$ from the central line

\section{Distortion}

At a given distance from the central line, distortion on figure $3 A$ is the same as that on the regular Mercator projection (fig. 1A).

\section{Other features}

Conceptually projected onto a cylinder wrapped around the globe tangent to an oblique great circle or secant along two small circles equidistant from and on each side of the central great circle
Cannot be geometrically (or perspectively) projected

There are various means of adapting to the ellipsoid, but none can simultaneously maintain both perfect conformality and constant scale along the central line.

\section{Usage}

Large-scale mapping in Switzerland, Madagascar, and Borneo

Atlas maps of regions having greater extent in an oblique direction, such as Hawail Recommended for conformal mapping of regions having predominant extent in oblique direction, neither east-west nor north-south

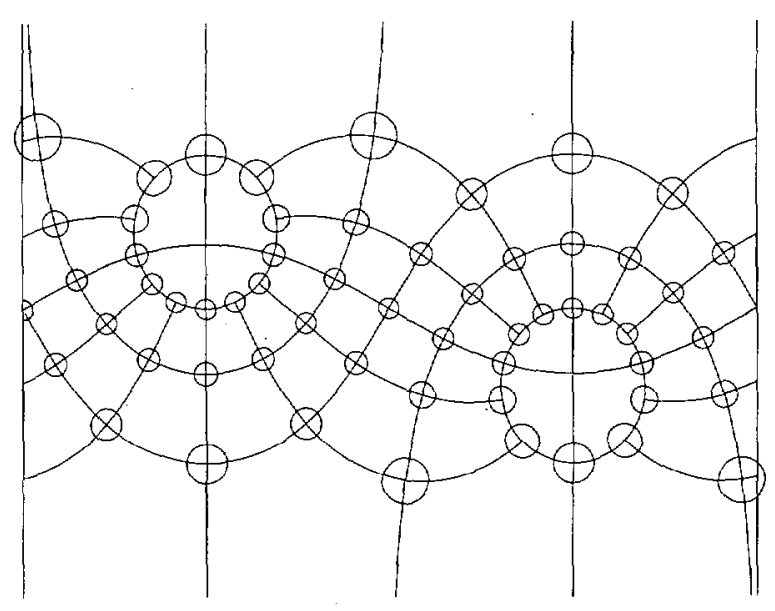

\section{Origin}

Developed for various applications, chiefly large-scale mapping of the ellipsoid, by $M$. Rosenmund of Switzerland in 1903, J. Laborde of France in 1928, Martin Hotine of England in 1947, and others during the 20th century.

\section{Other names}

Rectified Skew Orthomorphic (when using Hotine's formulas)

Laborde (when using Laborde's formulas)

Hotine Oblique Mercator (when using Hotine's formulas)

Oblique Cylindrical Orthomorphic

\section{Limiting forms}

Mercator (p. 10), if the Equator is the central line

Transverse Mercator (p. 12), if a meridian is the central line 


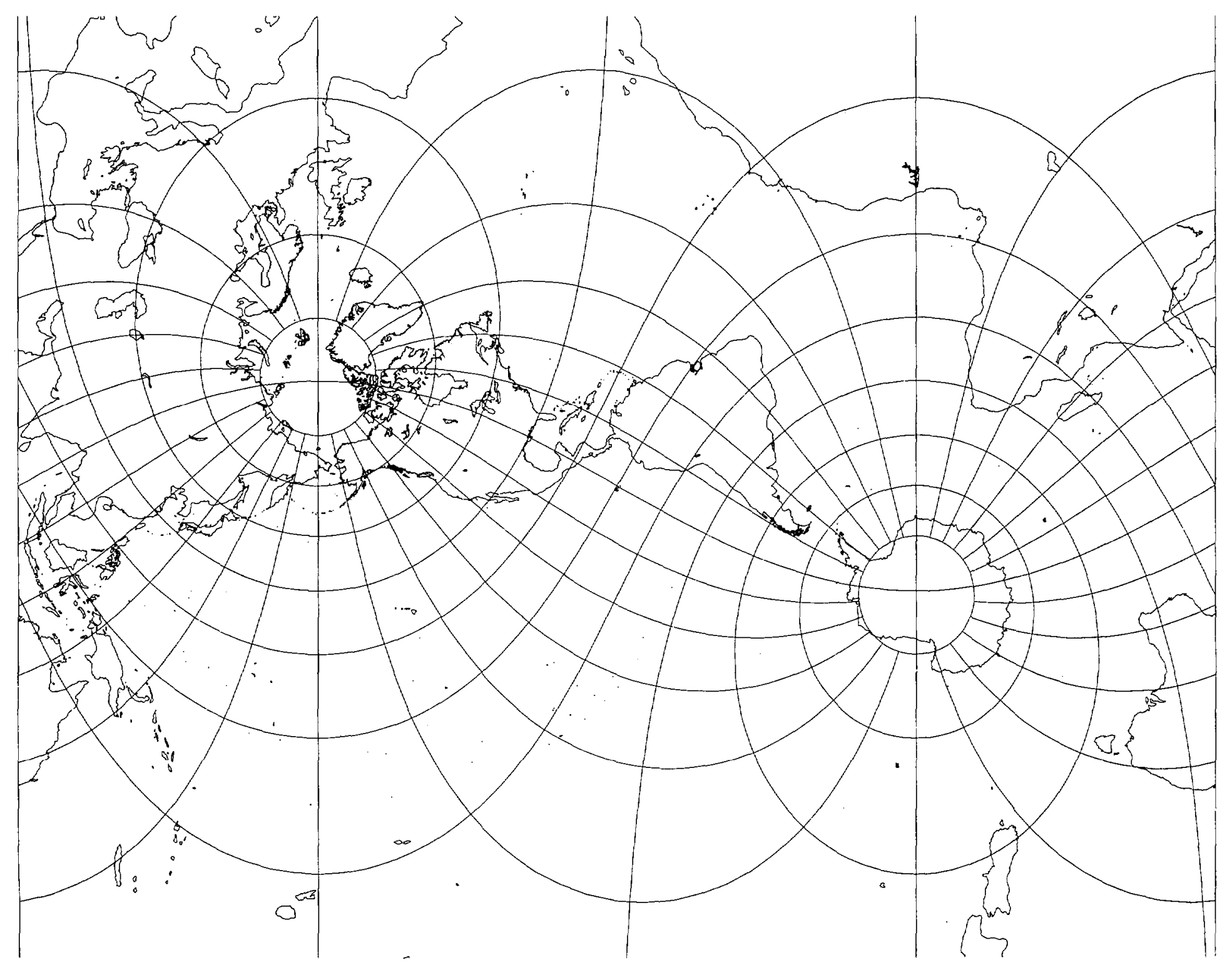




\section{LAMBERT CYLINDRICAL EQUAL-AREA Projection}

\section{Classifications \\ Cylindrical \\ Equal area \\ Perspective}

\section{Graticule}

Meridians: Equally spaced straight parallel lines 0.32 as long as the Equator.

Parallels: Unequally spaced straight parallel lines, farthest apart near the Equator, perpendicular to meridians

Poles: Straight lines equal in length to the Equator

Symmetry: About any meridian or the Equator

\section{Scale}

True along the Equator

Increases with distance from the Equator in the direction of parallels and decreases in the direction of meridians to maintain equal area Same scale at the parallel of opposite sign

\section{Distortion}

Infinitesimally small circles (indicatrices) of equal size on the globe are ellipses except at the Equator, where they are circles (fig. $4 A$ ). The areas of all the indicatrices are the same. Thus, there is shape distortion but no area distortion. Shape distortion in polar regions is extreme.

\section{Other features}

Simple graticule, perspectively projected in lines perpendicular to the axis onto a cylinder wrapped around the globe tangent to the Equator

\section{Usage}

Minimal except to describe basic principles in map projection texts

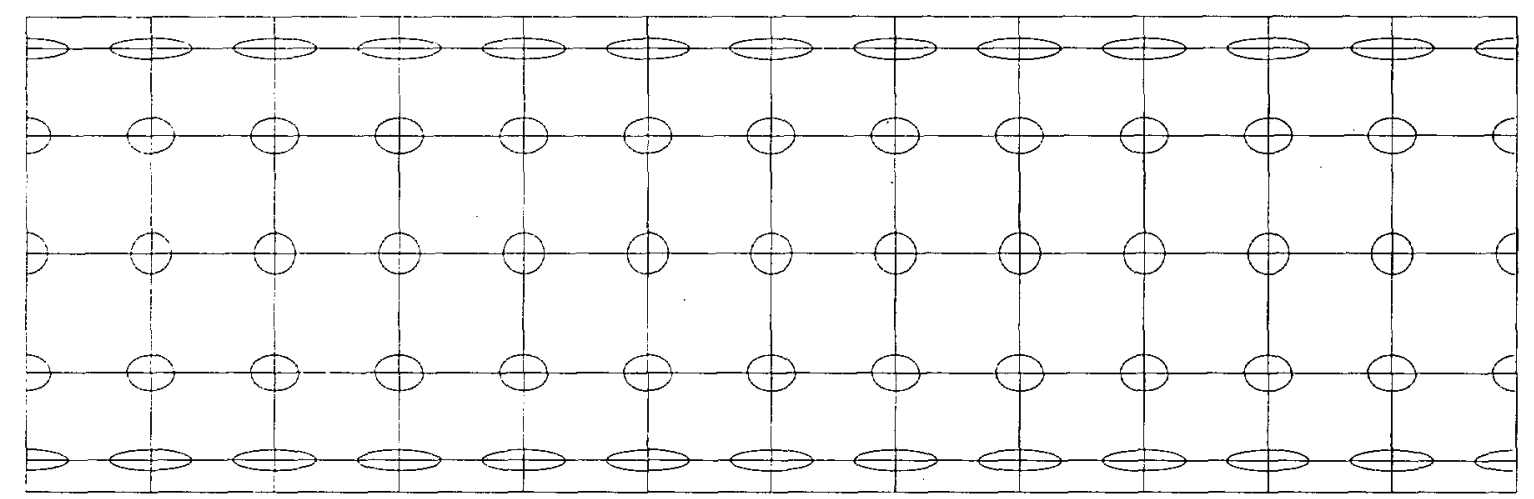

Figure 4A. - Lambert Cylindrical Equal-Area projection with Tissot indicatrices, $30^{\circ}$ graticule. Standard parallel $0^{\circ}$. All ellipses have the same area, but shapes vary.

Prototype for Behrmann and other modified cylindrical equal-area projections (fig. $5 B$ )

Recommended for equal-area mapping of regions predominantly bordering the Equator

\section{Origin}

Presented by Johann Heinrich Lambert (172877) of Alsace in 1772

\section{Aspects}

Normal is described here.

Transverse and oblique aspects are rarely used (figs. $4 C, 4 D$ ) but are recommended for equalarea mapping of predominantly north-south regions or regions extending obliquely.

\section{Other names}

Cylindrical Equal-Area

\section{Similar projections}

If meridians are compressed relative to parallels and if the spacing of parallels is increased in inverse proportion, other cylindrical equal-area projections result (fig.
$5 B$ ), and the standard parallel changes. The extreme case, in which the poles are standard parallels, consists of a single vertical line, infinitely long.

Named examples are as follows:

Behrmann

Gall Orthographic

Trystan Edwards

Peters

(See Behrmann Cylindrical Equal-Area projection, p. 19, for the differences.) 
Figure 4B.-Lambert Cylindrical Equal-Area projection with shorelines, $15^{\circ}$ graticule. Standard parallet $0^{\circ}$. Central meridian $90^{\circ} \mathrm{W}$.

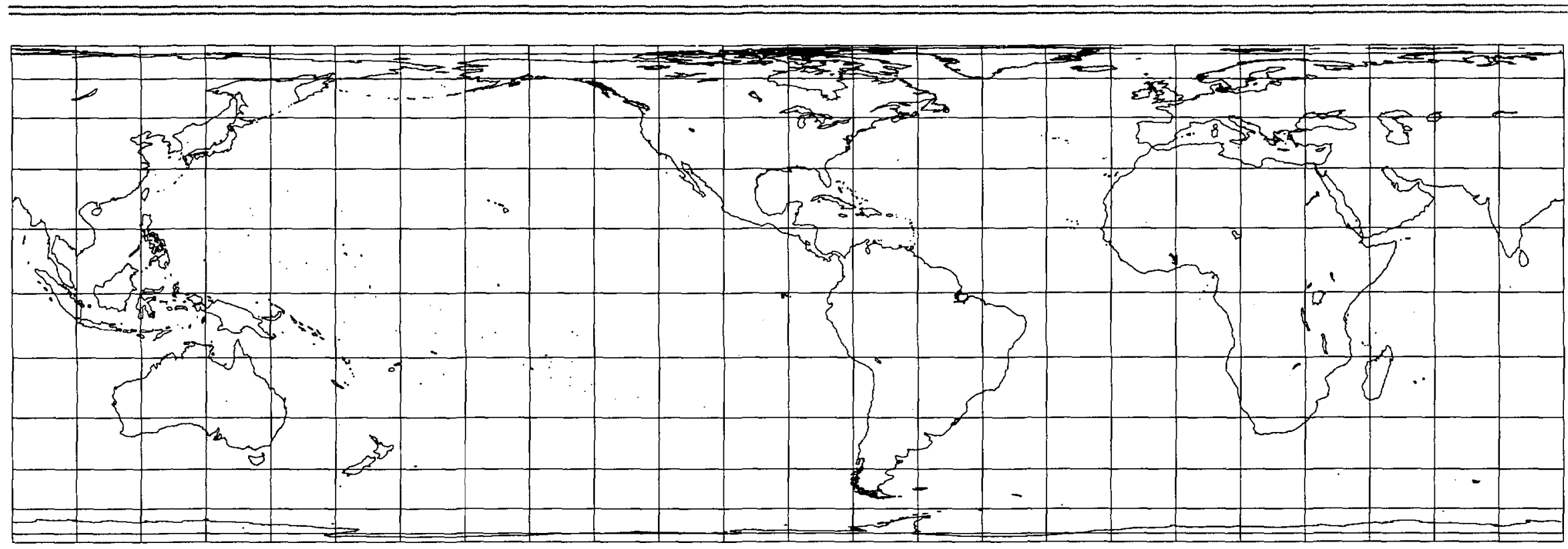




\section{LAMBERT CYLINDRICAL EQUAL-AREA Projection}

Figure $4 C$.-Transverse Lambert Cylindrical Equal-Area projection with shorelines, $15^{\circ}$ graticule. Central meridians $90^{\circ} E$. and W., at true scale. North Pole at $+90^{\circ}$ longitude on base projection.

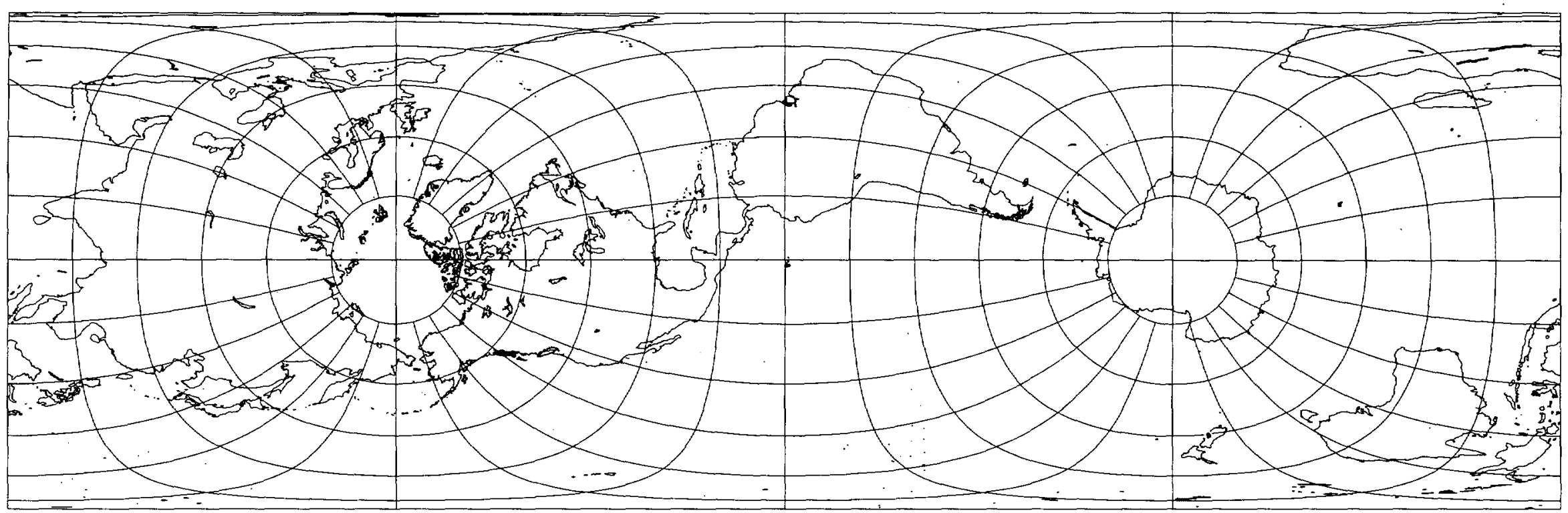

Figure 4D.-Oblique Lambert Cylindrical Equal-Area projection with shorelines, $15^{\circ}$ graticule. Central meridian $90^{\circ} \mathrm{W}$. Central great circle at true scale through latitude $60^{\circ} \mathrm{N}$., longitude $180^{\circ}$ and latilude $60^{\circ} \mathrm{S}$., longitude $0^{\circ}$. North Pole at $+30^{\circ}$ latitude, $-90^{\circ}$ longitude on base projection.

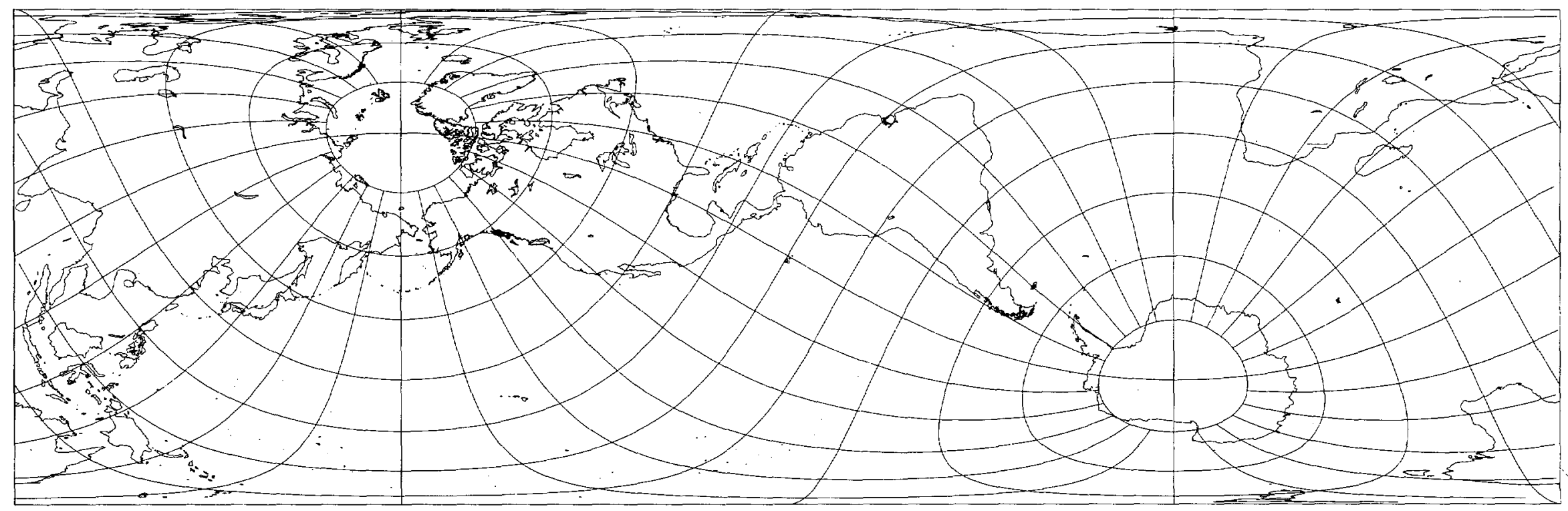




\section{BEHRMANN CYLINDRICAL EQUAL-AREA Projection}

\section{Classifications \\ Cylindrical \\ Equal area \\ Perspective}

\section{Graticule}

Meridians: Equally spaced straight parallel lines 0.42 as long as the Equator.

Parallels: Unequally spaced straight lines, farthest apart near the Equator, perpendicular to meridians

Poles: Straight lines equal in length to the Equator

Symmetry: About any meridian or the Equator

\section{Scale}

True along latitudes $30^{\circ} \mathrm{N}$. and S.

Too small along the Equator but too large at the Equator along meridians

Increases with distance from the Equator in the direction of parallels and decreases in the direction of meridians to maintain equal area Same scale at the parallel of opposite sign

\section{Distortion}

In contrast to figure $4 A$, figure $5 A$ shows

indicatrices as circles at latitudes $30^{\circ} \mathrm{N}$. and S., where there is no distortion, instead of at the Equator. All others appear as ellipses, but their areas remain the same. They are compressed east to west and lengthened north to south between latitudes $30^{\circ} \mathrm{N}$. and $\mathrm{S}$. The opposite is true poleward of these latitudes.

\section{Other features}

Same as the Lambert Cylindrical Equal-Area projection except for horizontal compression and vertical expansion to achieve no distortion

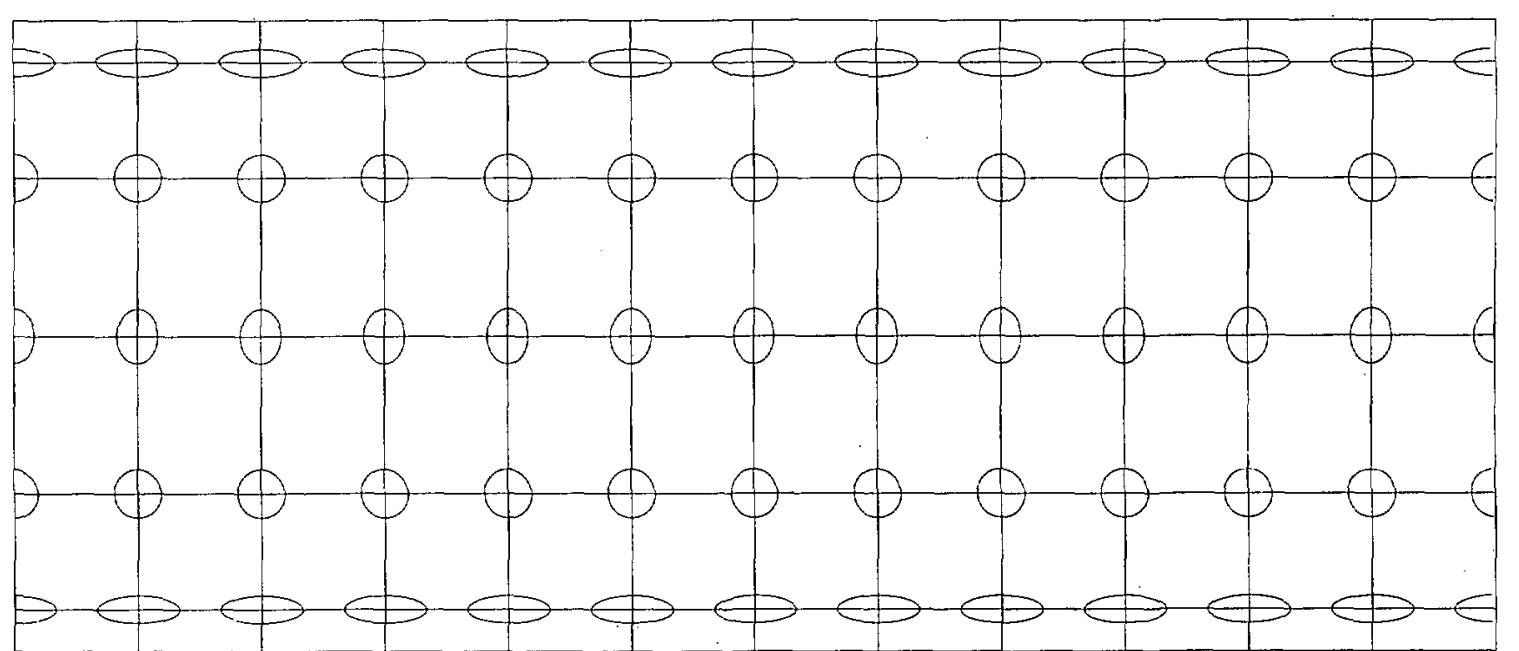

Figure 5A.-Behrmann Cylindrical Equal-Area projection with Tissot indicatrices, $30^{\circ}$ graticule. Standard parallels $30^{\circ} \mathrm{N}$. and $\mathrm{S}$.

at latitudes $30^{\circ} \mathrm{N}$. and S. instead of at the

Equator

Equivalent to a projection of the globe using

parallel lines of projection onto a cylinder

secant at $30^{\circ} \mathrm{N}$. and $\mathrm{S}$.

\section{Origin}

Presented by Walter Behrmann (1882-1955) of

Berlin in 1910

\section{Similar projections}

Lambert Cylindrical Equal-Area (p. 16) by Johann Heinrich Lambert in 1772 (Standard parallel: Equator)

Gall Orthographic (fig. $5 C$ ) by James Gall in 1855 (Standard parallels: $45^{\circ} \mathrm{N}$. and S.)

Trystan Edwards in 1953 (Standard parallels $37^{\circ} 24^{\prime}$ N. and $S$.)

Peters (fig. 5C) by Arno Peters in 1967

(Standard parallels: approximately $45^{\circ} \mathrm{N}$. and S., thus essentially identical with the Gall 


\section{BEHRMANN CYLINDRICAL EQUAL-AREA Projection}

Figure 5B.-Behrmann Cylindrical Equal-Area projection with shorelines, $15^{\circ}$ graticule. Central meridian $90^{\circ} \mathrm{W}$. Standard parallels $30^{\circ}$ $\mathrm{N}$. and $\mathrm{S}$.

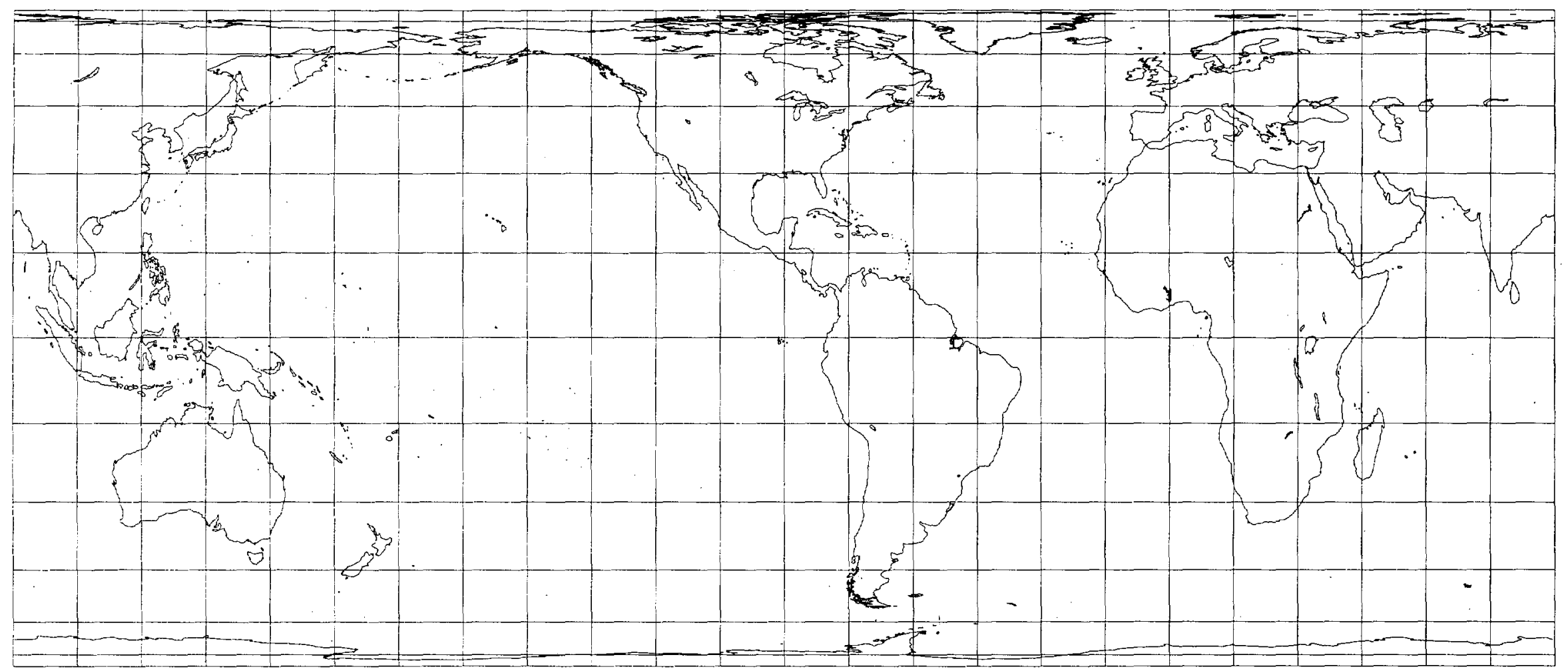




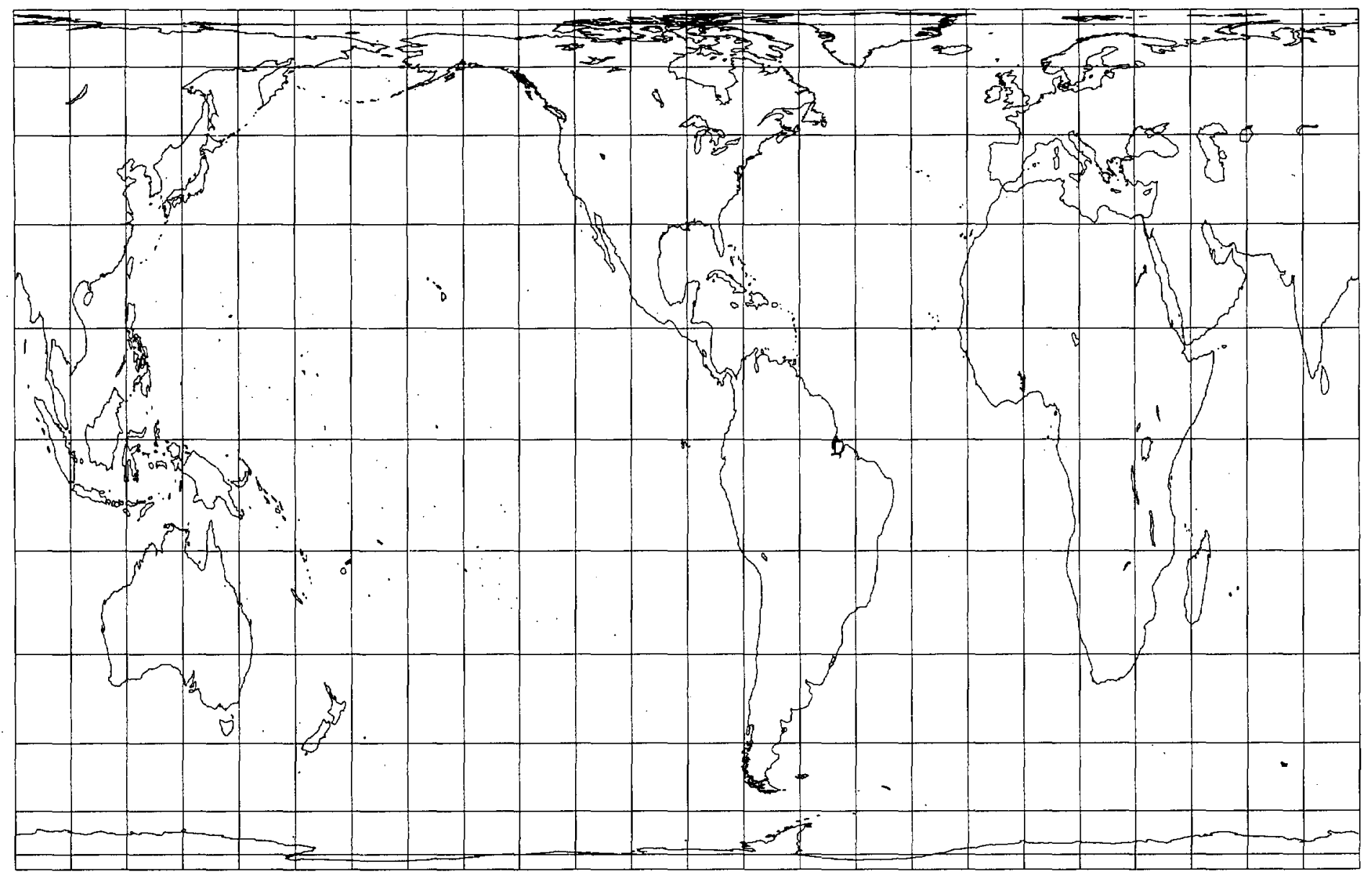




\section{Classifications \\ Cylindrical \\ Equidistant}

\section{Graticule}

Meridians: Equally spaced straight parallel lines half as long as the Equator.

Parallels: Equally spaced straight parallel lines, perpendicular to and having same spacing as meridians

Poles: Straight lines equal in length to the Equator

Symmetry: About any meridian or the Equator

\section{Scale}

True along the Equator and along all meridians

Increases with the distance from the Equator along parallels

Constant along any given parallel; same scale at the parallel of opposite sign

\section{Distortion}

Infinitesimally small circles of equal size on the globe (indicatrices) are ellipses except along the Equator, where they remain circles (fig. 6A). Areas of the ellipses also vary. Thus, there is distortion of both shape and area.

\section{Other features}

Most simply constructed graticule of any projection

Conceptually projected onto a cylinder wrapped around the globe tangent to the Equator Not perspective

\section{Usage}

Many maps during the 15th and 16 th centuries

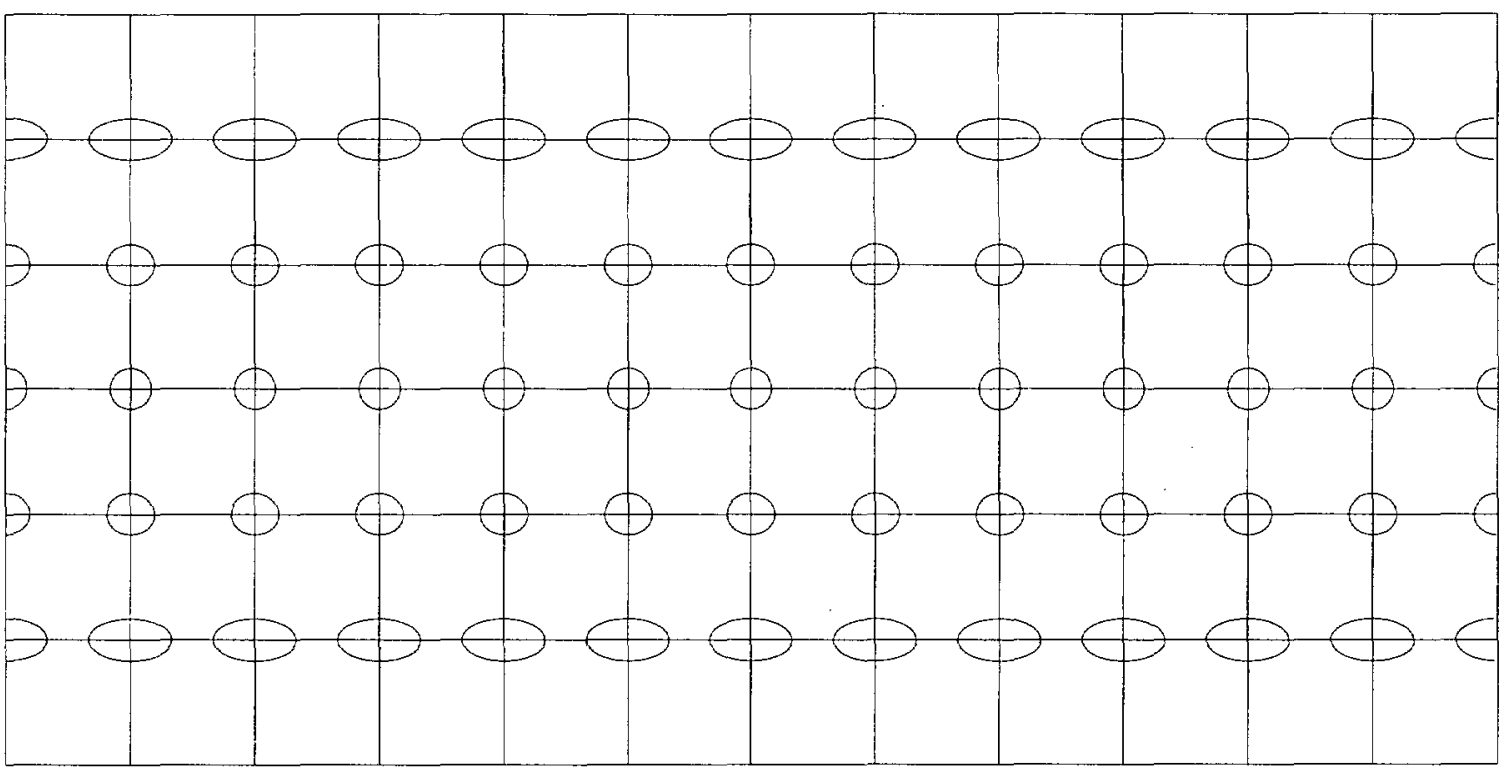

Simple outline maps of regions or of the world or index maps

Used only for the Earth taken as a sphere

\section{Similar projections}

If meridians are compressed relative to parallels, the Equirectangular projection (p. 24) results.

\section{Origin}

May have been originated by Eratosthenes (275? -195 ? B.C.)

Marinus of Tyre also credited with its invention about A.D. 100

\section{Aspects}

Normal is described here.

Transverse aspect is the Cassini projection (figs. $8 A, 8 B$ ), which is also applied to the ellipsoid.

Oblique aspect is rarely used (see fig. 9B).

\section{Other names}

Simple Cylindrical

Equidistant Cylindrical (particular form) 


\section{Figure 68.-Plate Carrée projection with shorelines, $15^{\circ}$ graticule. Central meridian $90^{\circ} \mathrm{W}$}

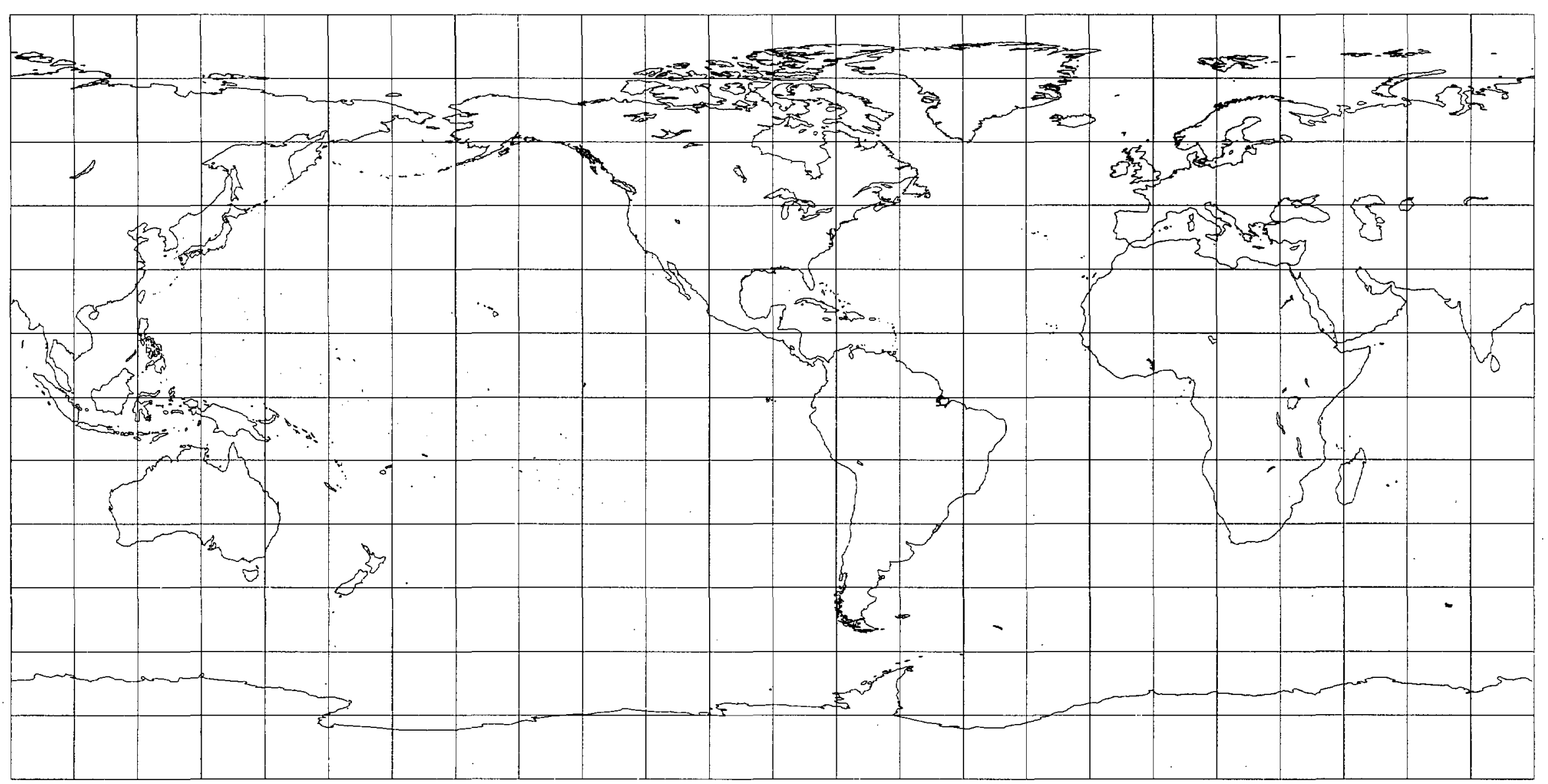




\section{EQUIRECTANGULAR Projection}

\section{Classifications \\ Cylindrical \\ Equidistant}

\section{Graticule}

Meridians: Equally spaced straight parallel lines more than half as long as the Equator. Parallels: Equally spaced straight parallel lines, perpendicular to and having wider spacing than meridians

Poles: Straight lines equal in length to the Equator

Symmetry: About any meridian or the Equator

\section{Scale}

True along two standard parallels equidistant from the Equator and along all meridians Too small along the Equator but increases with distance from the Equator along the parallels Constant along any given parallel; same scale at the parallel of opposite sign

\section{Distortion}

Infinitesimally small circles on the globe (indicatrices) are circles on the map at latitudes $30^{\circ} \mathrm{N}$. and $\mathrm{S}$. for this choice of standard parallels (fig. 7A). Elsewhere, area and local shape are distorted.

\section{Other features}

Simple modification of Plate Carrée (p. 22)

having east-west compression

Conceptually projected onto a cylinder secant

to the globe along the chosen standard parallels

Not perspective

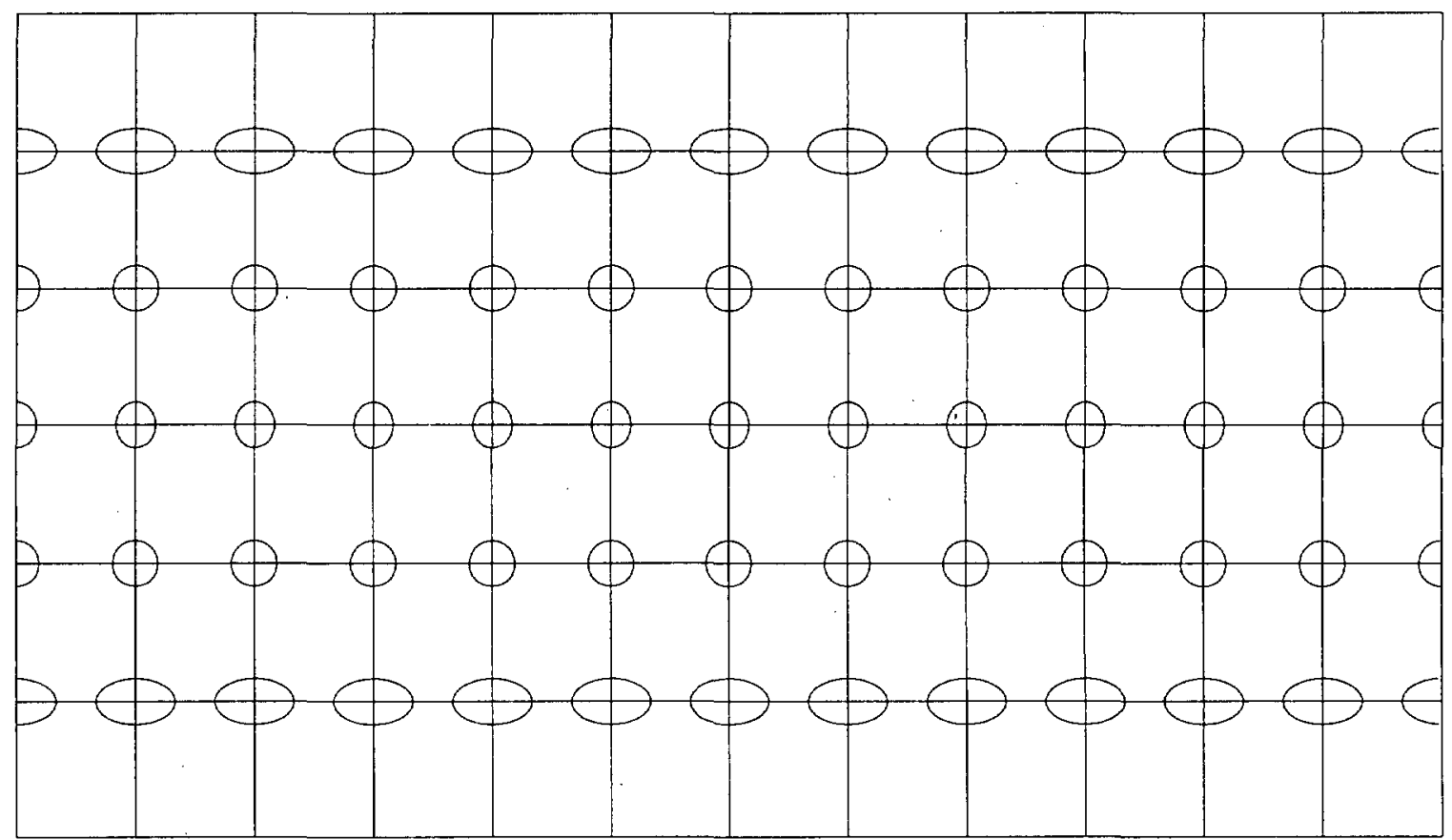

Figure 7A.-Equirectangular projection with Tissot indicatrices, $30^{\circ}$ graticule. Standard parallels $30^{\circ} \mathrm{N}$. and S.

\section{Usage}

Simple outline maps of regions or of the world or for index maps

Used only in the spherical form

Origin

Marinus of Tyre about A.D. 100

\section{Other names}

Equidistant Cylindrical

Rectangular

La Carte Parallélogrammatique
Die Rechteckige Plattkarte Projection of Marinus

Gall Isographic (if standard parallels are latitudes $45^{\circ} \mathrm{N}$. and S.) 


\section{Figure 78.-Equirectangular projection with shorelines, $15^{\circ}$ graticule. Central meridian $90^{\circ} \mathrm{W}$. Standard parallels $30^{\circ} \mathrm{N}$. and S}

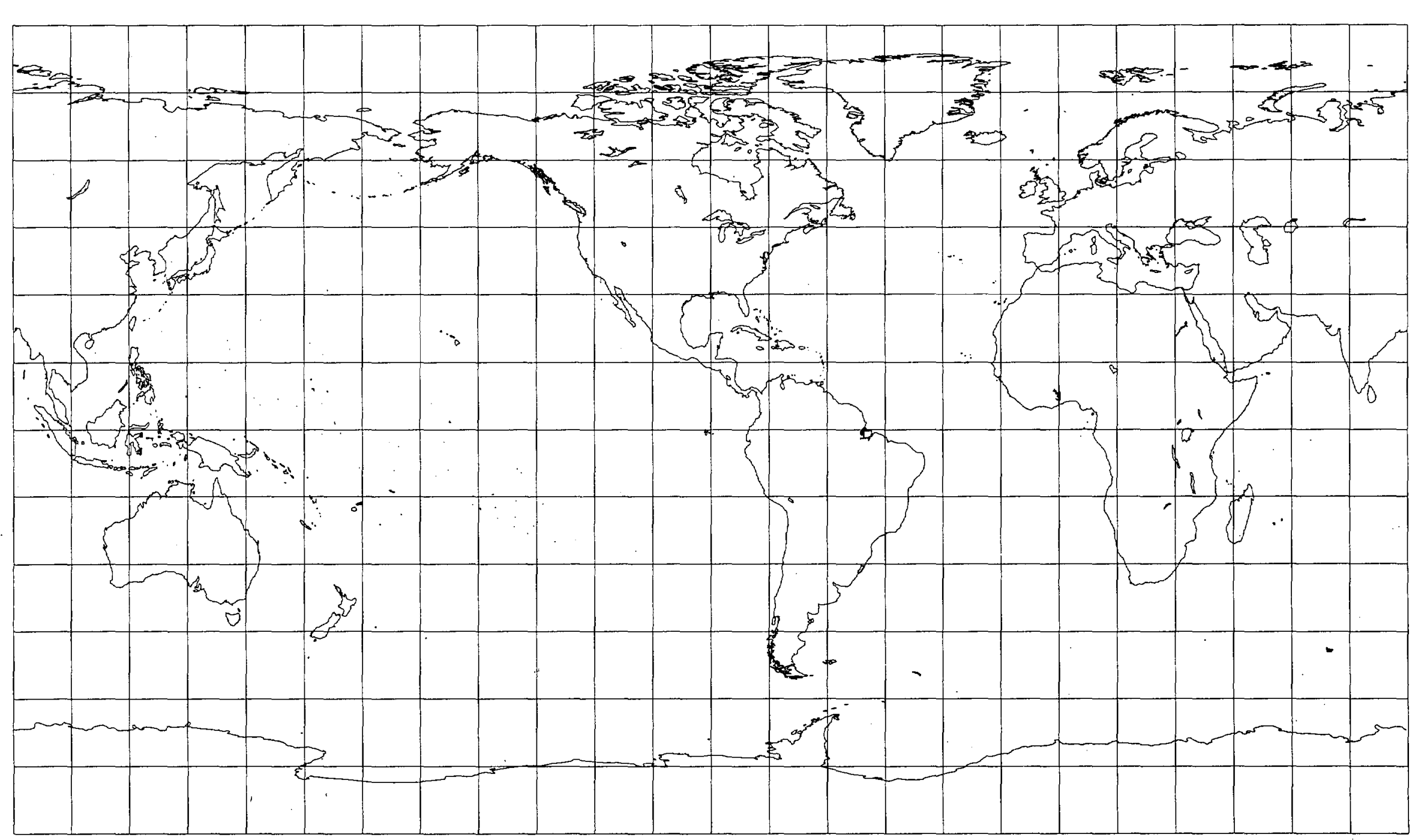




\section{Classifications}

Transverse aspect of Plate Carrée (p. 22)

Cylindrical

Equidistant

\section{Graticule}

Central meridian, each meridian $90^{\circ}$ from central meridian, and the Equator are straight lines.

Other meridians and parallels are complex curves, concave toward the central meridian and the nearest pole, respectively.

Poles: Points along the central meridian Symmetry: About any straight meridian or the Equator

\section{Scale}

True along the central meridian and along any straight line perpendicular to the central meridian

Increases with distance from the central meridian, along a direction parallel to the central meridian

\section{Distortion}

Function of the distance from the central meridian. No distortion occurs along the centra meridian, but there is both area and local shape distortion elsewhere. Long horizontal straight lines near the upper and lower limits of figure $8 A$ represent infinitesimal circles on the globe $90^{\circ}$ from the central meridian.

\section{Other features}

Conceptually projected onto a cylinder tangent to the globe at the central meridian

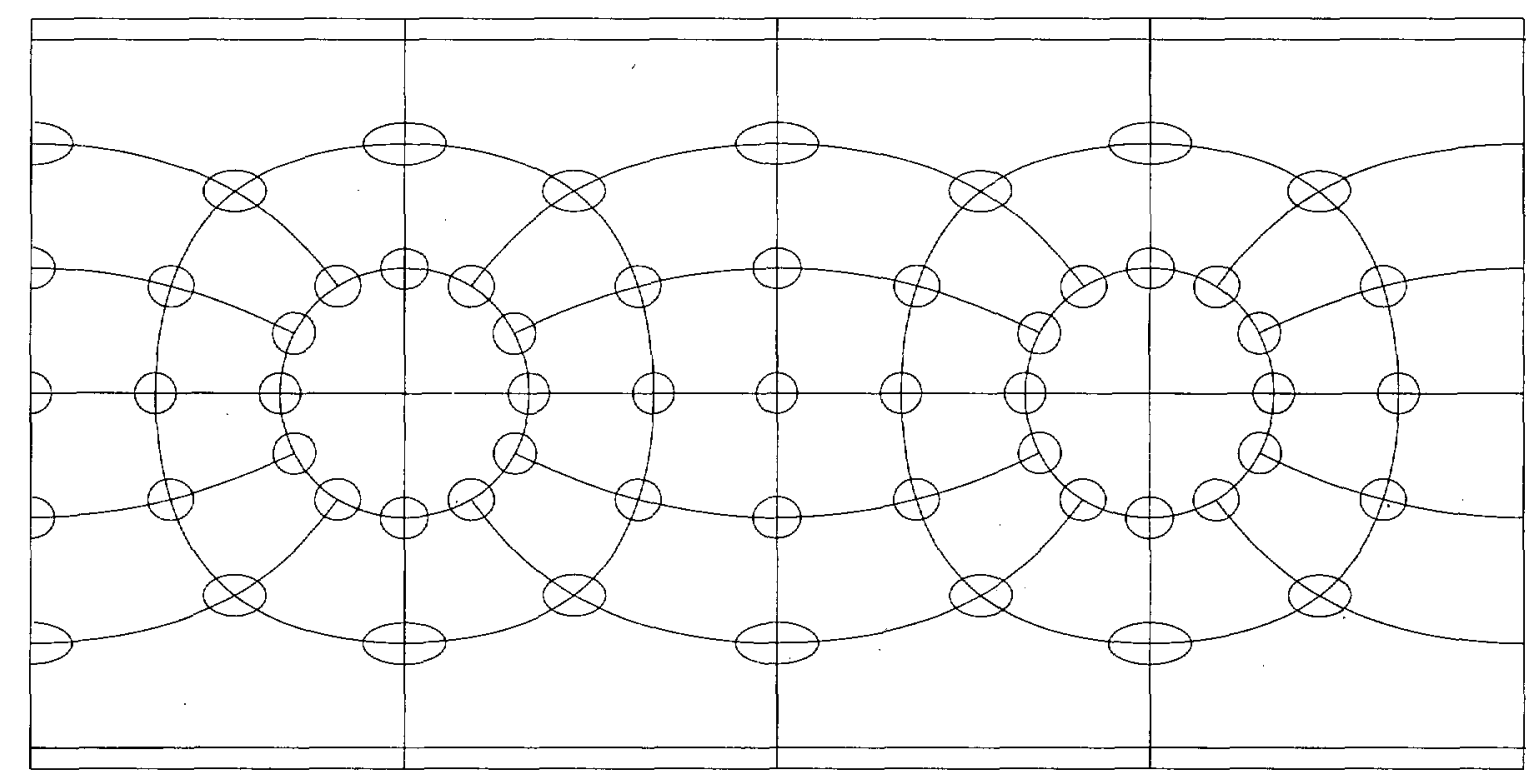

Can be compressed north-south to provide a Transverse Equirectangular projection, but rarely done

\section{Usage}

Topographic mapping (ellipsoidal form) of British Isles before the 1920's; replaced by the Transverse Mercator

Topographic mapping of a few countries currently

\section{Origin}

Developed by César François Cassini de Thury

(1714-84) for topographic mapping of France

in the middle 18th century 
Figure 88 . Cassini projection with shorelines, $15^{\circ}$ graticule. Central meridian $90^{\circ} \mathrm{W}$.

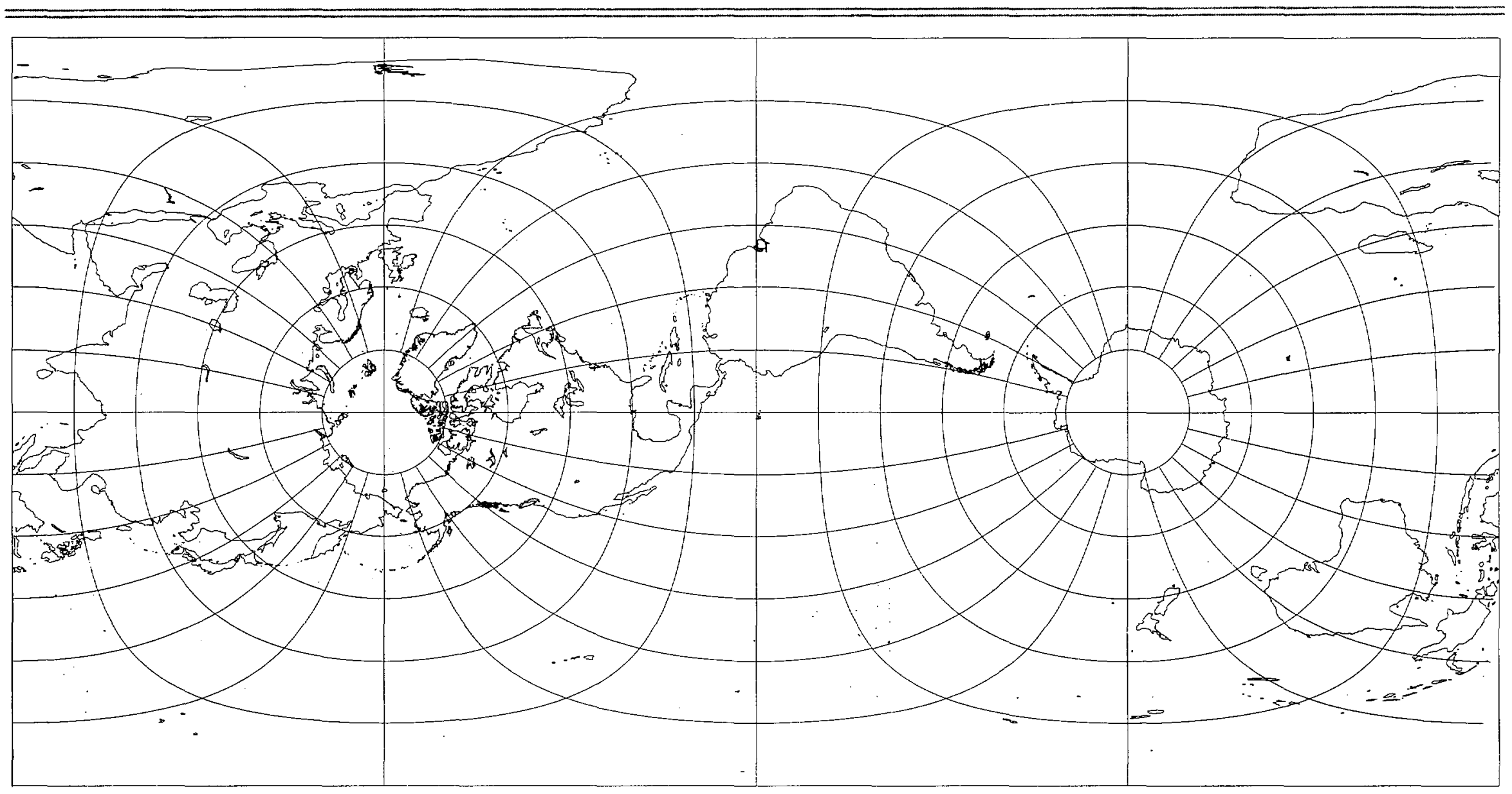




\section{OBLIQUe PLATE CARREe Projection}

\section{Classifications}

Oblique aspect of Plate Carree

Cylindrical

Equidistant

\section{Graticule}

Two meridians $180^{\circ}$ apart are straight lines. Other meridians and parallels are complex curves.

Poles: Points away from central line Symmetry: About either of the straight meridians

\section{Scale}

True along the chosen central line (a great circle at an oblique angle) and along any straight line perpendicular to the central line. Increases with distance from the central line along a direction parallel to the central line.

\section{Distortion}

At a given distance from the central line,

distortion on figure $9 A$ is the same as that on the Plate Carree (fig. $6 A$ ) .

\section{Limiting forms}

Plate Carree (p. 22), if the Equator is the central line

Cassini (p. 26), if a meridian is the central line

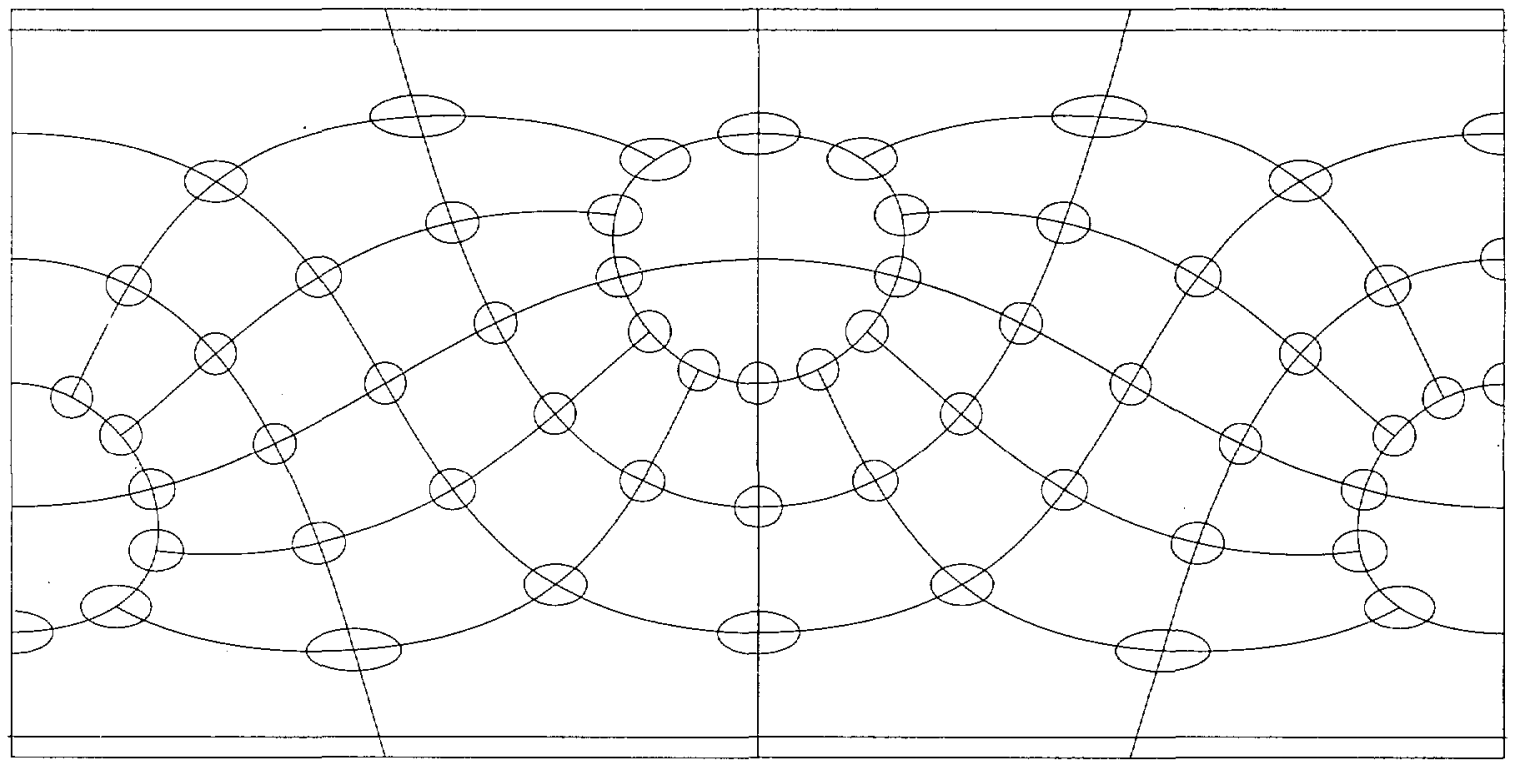

Figure 9A.-Oblique Plate Carre projection with Tissot indicatrices, $30^{\circ}$ graticule. North Pole at $+30^{\circ}$ latitude, $0^{\circ}$ longitude on base projection. 
Figure 9B.-Oblique Plate Carrée projection with shorelines, $15^{\circ}$ graticule. Central meridian $90^{\circ} \mathrm{W}$. North Pole at $+30^{\circ}$ latitude, $0^{\circ}$ longitude on base projection.

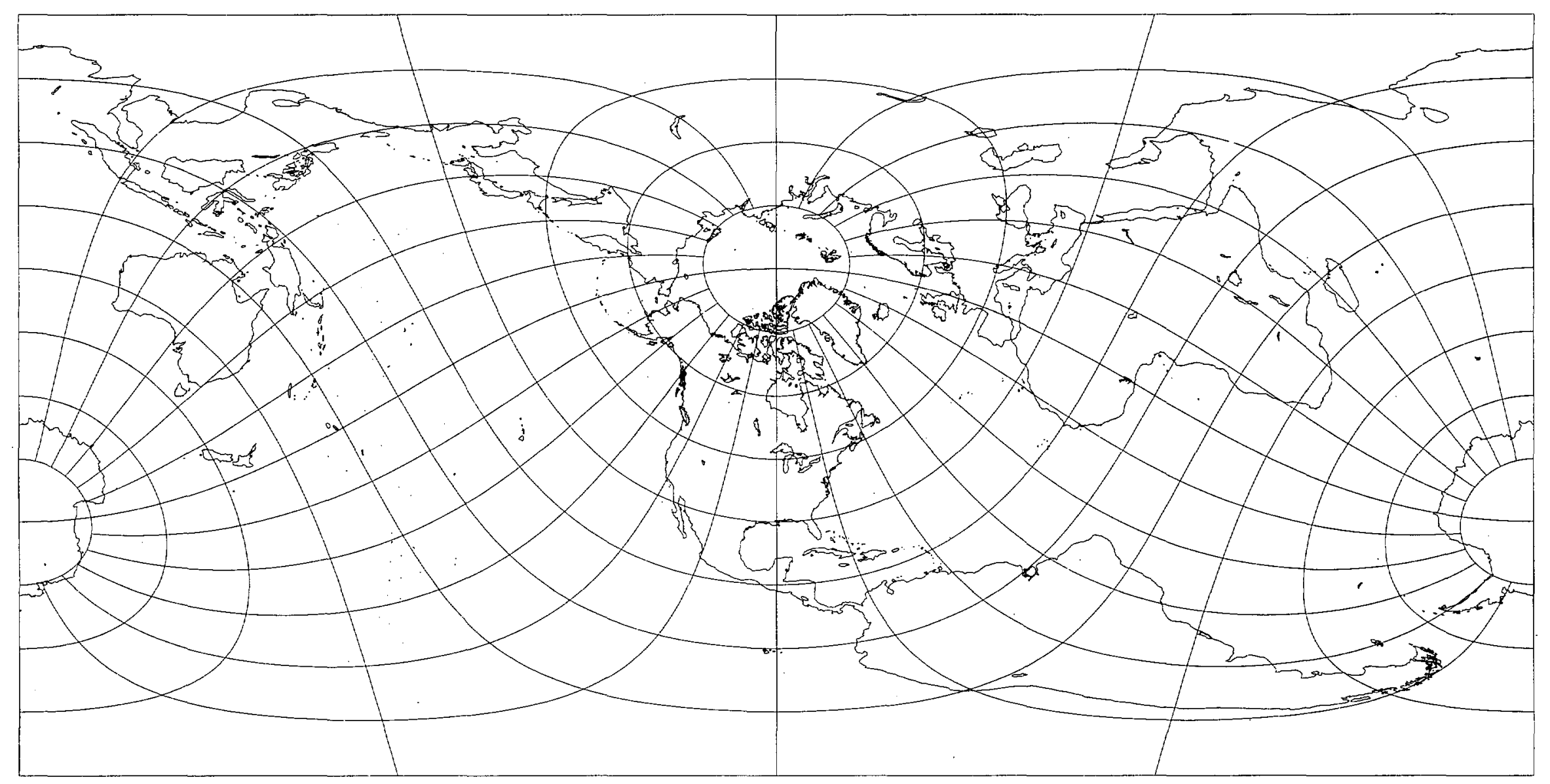




\section{CENTRAL CYLINDRICAL Projection}

\section{Classifications}

Cylindrical

Perspective

Neither conformal nor equal area

\section{Graticule}

Meridians: Equally spaced straight parallel lines

Parallels: Unequally spaced straight parallel lines, closest near the Equator, but spacing increases poleward at a much greater rate than it does on the Mercator. Perpendicular to meridians.

Poles: Cannot be shown

Symmetry: About any meridian or the Equator

\section{Scale}

True along the Equator

Increases with distance from the Equator to infinity at the poles

Changes with direction at any given point, except at the Equator, but scale in a given direction is constant at any given latitude or at the latitude of opposite sign

\section{Distortion}

Shape, area, and scale distortion increase rapidly away from the Equator, where there is no distortion (fig. 10A).

\section{Other features}

Projection is produced geometrically by projecting the Earth's surface perspectively from its center onto a cylinder tangent at the Equator. Should not be confused with the Mercator (p. 10), which is not perspective.

\section{Usage}

Distortion is too great for any usage except showing the appearance of the Earth when so projected and contrasting with the Mercator.

\section{Origin}

Uncertain

\section{Aspects}

Normal is described above.

Transverse aspect (fig. $10 \mathrm{C}$ ) is called the Wetch projection, since it was discussed by J. Wetch in the early 19th century.

\section{Other names}

Simple Perspective Cylindrical

\section{Similar projections}

Mercator projection (p. 10), which is not perspective, also cannot show the poles, but the poleward increase in the spacing of the parallels does not occur as rapidly as it does on the Central Cylindrical projection.

Gall (p. 33) and other perspective cylindrical projections can be produced by moving the point of perspective away from the center of the Earth. The poles can then be shown. Gnomonic projection (p. 116) is projected perspectively from the center of the Earth onto a tangent plane rather than a cylinder and is very different in appearance.
Figure 10A.-Central Cylindrical projection with Tissof indicatrices, $30^{\circ}$ graticule.

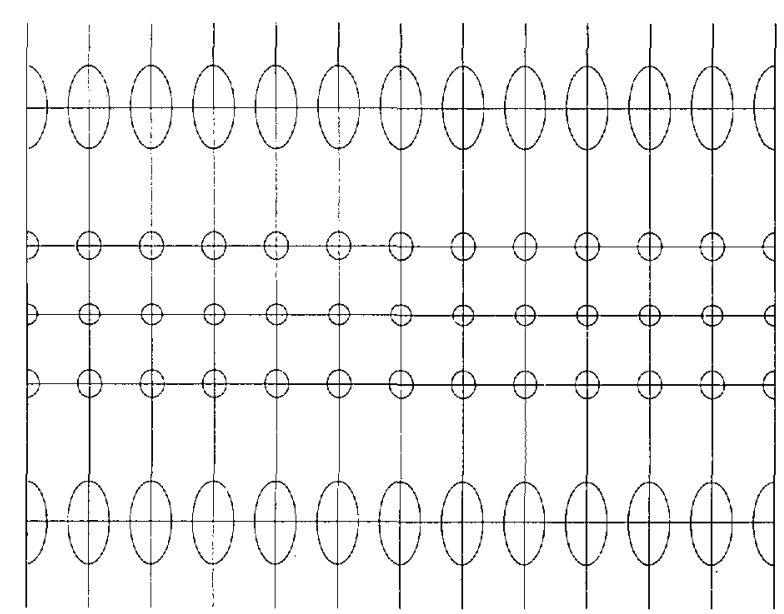


Figure 108.-Central Cylindrical projection with shorelines, $15^{\circ}$ graticule. Central meridian $90^{\circ} \mathrm{W}$.

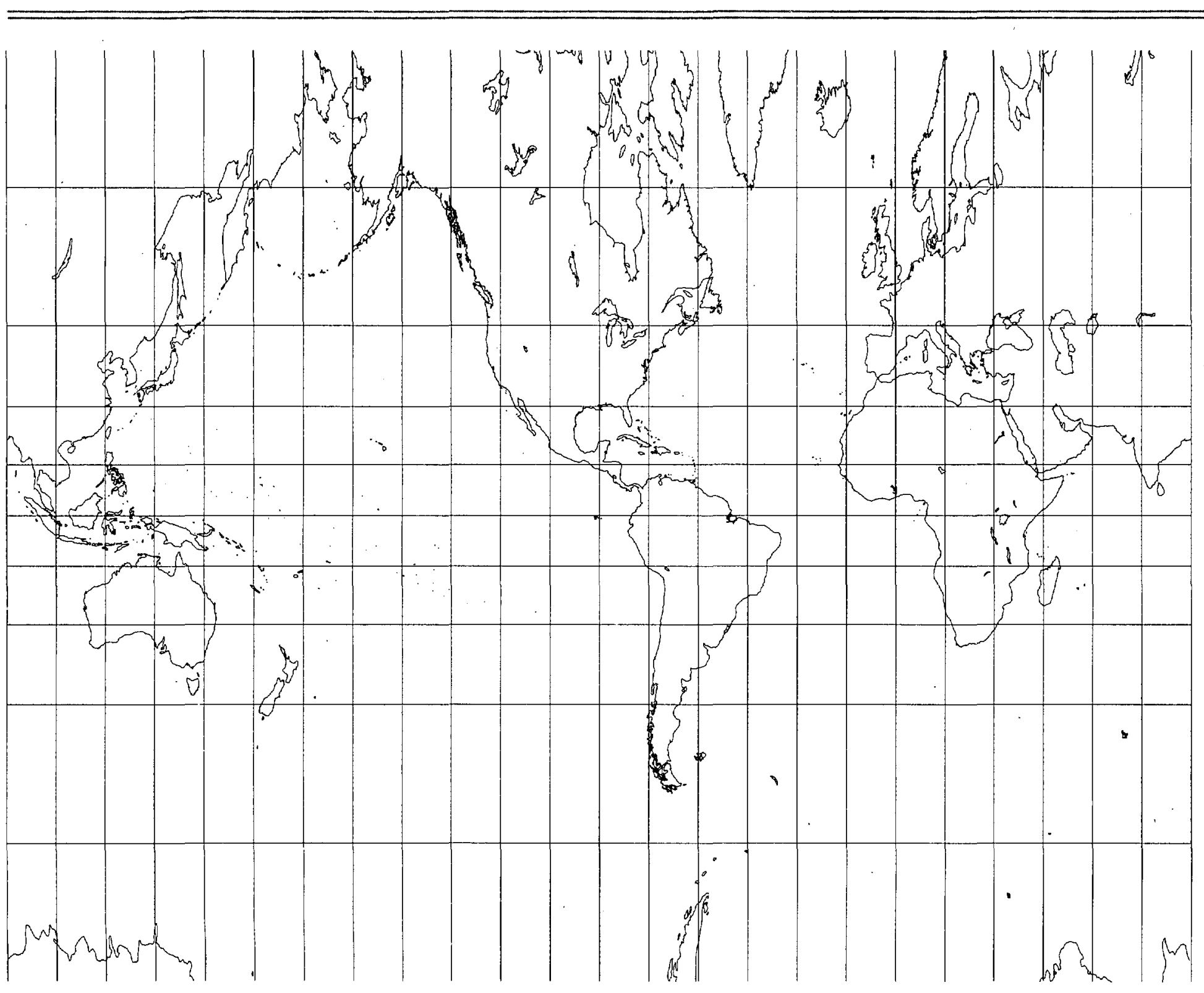




\section{CENTRAL CYLINDRICAL Projection}

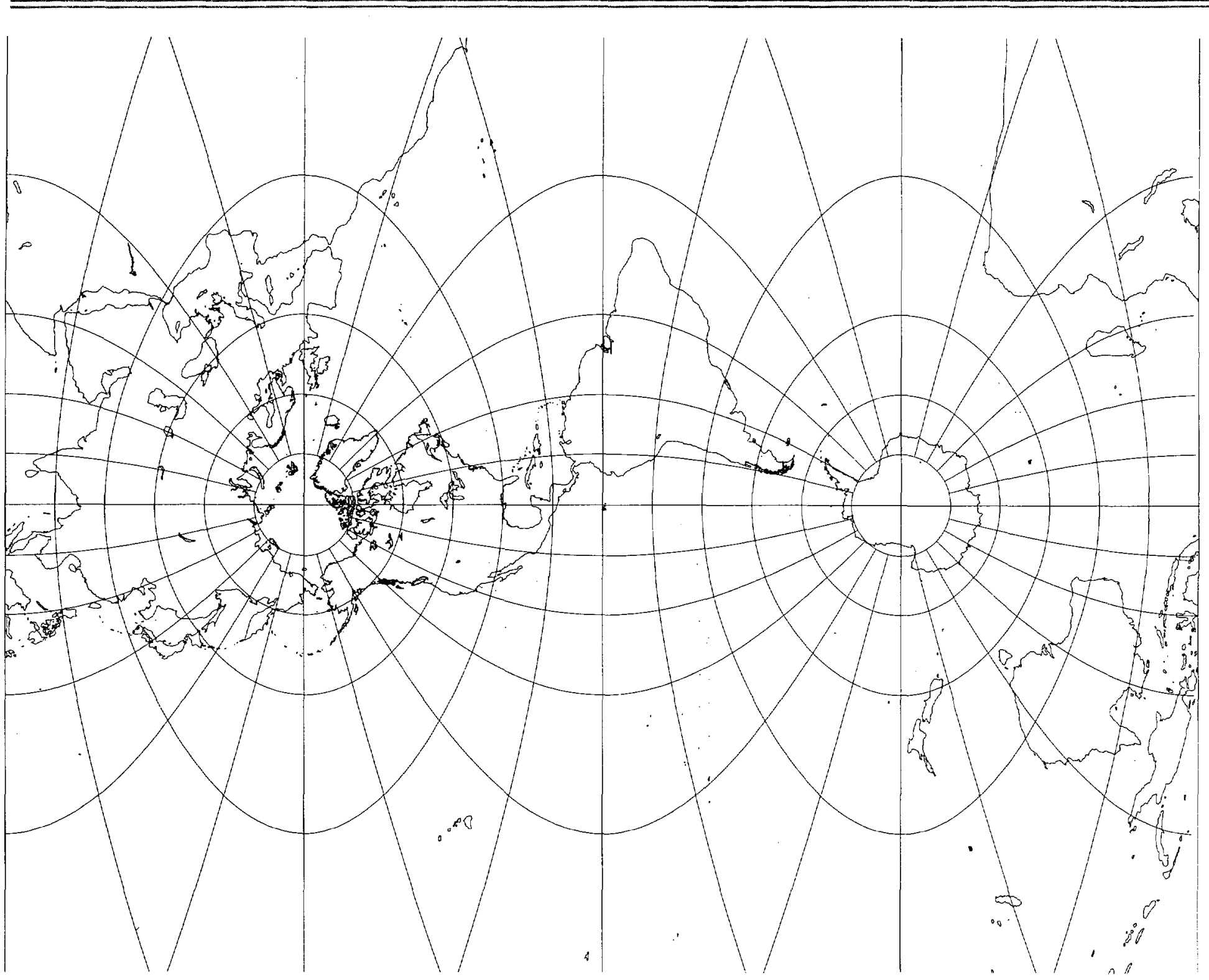

Figure $10 \mathrm{C}$. - Transverse Central Cylindrical projection with shorelines, $15^{\circ}$ graticule. Central meridian $90^{\circ} \mathrm{E}$. and $\mathrm{W}$. at true scale. 


\section{Classifications}

Cylindrical

Perspective

Neither conformal nor equal area

\section{Graticule}

Meridians: Equally spaced straight parallel lines 0.77 as long as the Equator

Parallels: Unequally spaced straight parallel lines, closest near the Equator, but spacing does not increase poleward as fast as it does on the Mercator. Perpendicular to meridians.

Poles: Straight lines equal in length to the Equator

Symmetry: About any meridian or the Equator

\section{Scale}

True along latitudes $45^{\circ} \mathrm{N}$. and $\mathrm{S}$. in all directions.

Constant in any given direction along any other given latitude or the latitude of opposite sign Changes with latitude and direction but is always too small between latitudes $45^{\circ} \mathrm{N}$. and $\mathrm{S}$. and too large beyond them

\section{Distortion}

None at latitudes $45^{\circ} \mathrm{N}$. and S. Shape, area, and scale distortion increases moderately away from these latitudes but becomes severe at poles (fig. 11A).

\section{Other features}

Projection is produced geometrically by projecting the Earth perspectively from the point on the Equator opposite a given meridian onto a secant cylinder cutting the globe at latitudes $45^{\circ} \mathrm{N}$. and $\mathrm{S}$.

\section{Usage}

World maps in British atlases and some other atlases, as a projection somewhat resembling the Mercator but having less distortion of area and scale near the poles

\section{Origin}

Presented by James Gall of Edinburgh in 1855 as his Stereographic, which he preferred to his Orthographic (equal-area) and Isographic (equidistant) cylindrical projections presented at the same time and also based on cylinders secant at latitudes $45^{\circ} \mathrm{N}$. and $\mathrm{S}$.

\section{Aspects}

Only the normal aspect is used.

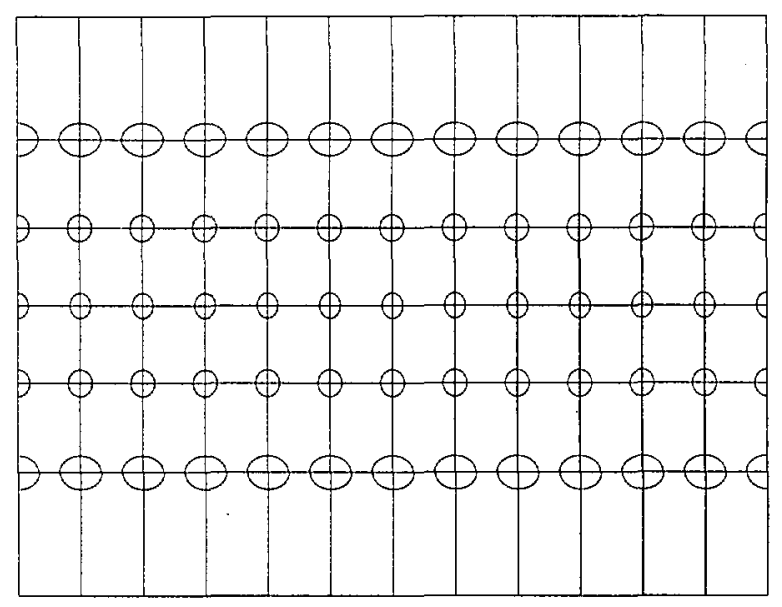

\section{Other names}

\section{Gall Stereographic}

\section{Similar projections}

Miller Cylindrical projection (p. 35) has different spacing of the parallels, and the line of no distortion is the Equator rather than latitudes $45^{\circ} \mathrm{N}$. and $\mathrm{S}$.

B.S.A.M. (Great Soviet World Atlas) projection of 1937 is the same, except that the cylinder is secant at latitudes $30^{\circ} \mathrm{N}$. and $\mathrm{S}$.

V.A. Kamenetskiy used an identical projection for Russian population density in 1929, except that the cylinder was made secant at latitudes $55^{\circ} \mathrm{N}$. and $\mathrm{S}$.

Guy Bomford of Oxford University in England about 1950 devised a Modified Gall projection, which is like the regular Gall in spacing except that meridians are slightly curved at higher latitudes to decrease scale exaggeration at the poles.

Moir devised "The Times" projection in which the straight parallels are spaced as they are on the Gall projection but the meridians are distinctly curved. 


\section{GALL Projection}

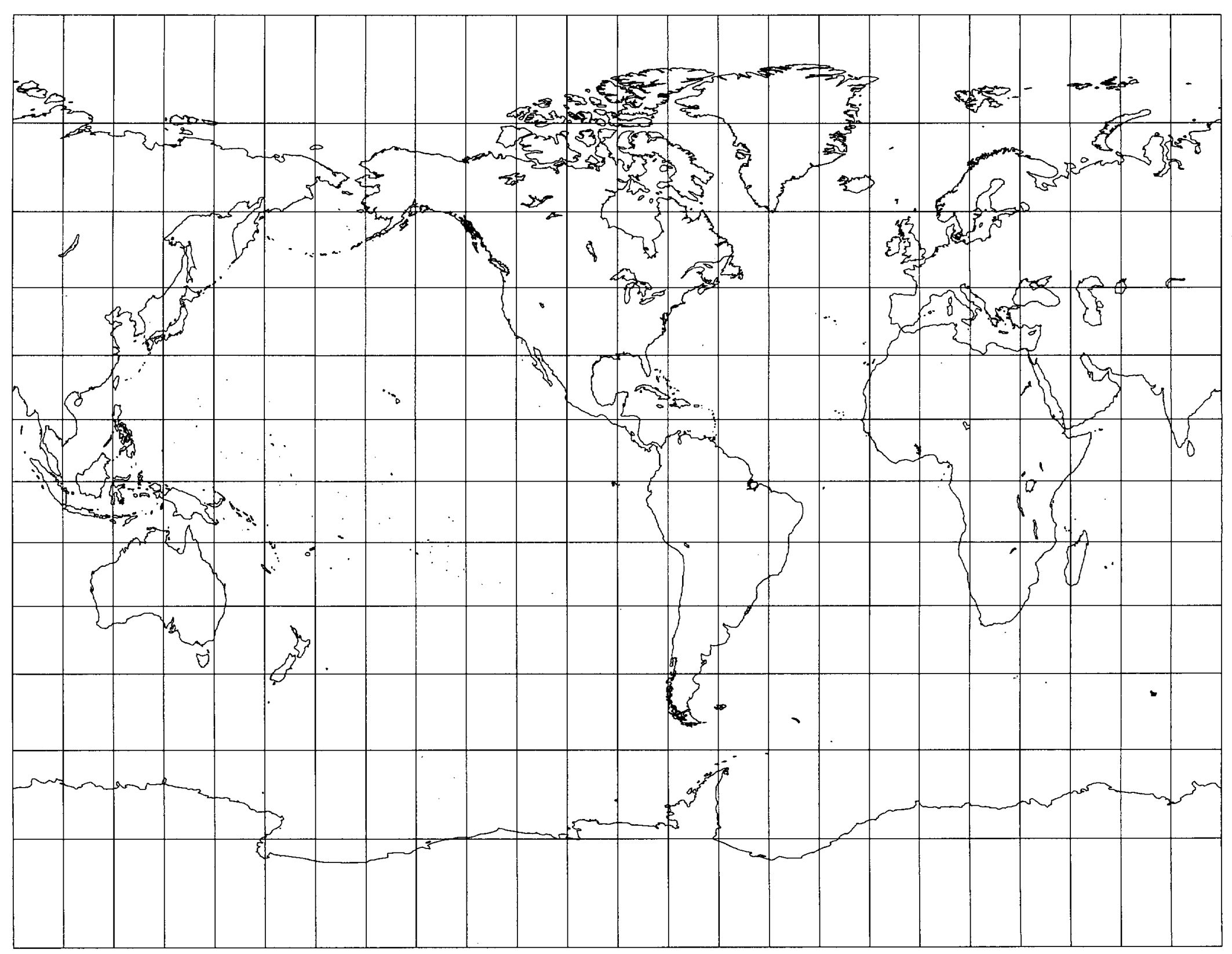

Figure 11B.-Gall projection with shorelines, $15^{\circ}$ graticule. Central meridian $90^{\circ} \mathrm{W}$. 


\section{MILLER CYLINDRICAL Projection}

Figure 12A.-Miller Cylindrical projection with Tissot indicatrices, $30^{\circ}$ graticule.

\section{Classifications}

\section{Cylindrical}

Neither conformal nor equal area

\section{Graticule}

Meridians: Equally spaced straight parallel lines 0.73 as long as the Equator

Parallels: Unequally spaced straight parallel lines, closest near the Equator, but spacing does not increase poleward as fast as it does on the Mercator. Perpendicular to meridians. Poles: Straight lines equal in length to the Equator

Symmetry: About any meridian or the

Equator

\section{Scale}

True along the Equator in all directions

Constant in any given direction along any other given latitude; same scale at the latitude of opposite sign

Changes with latitude and direction

\section{Distortion}

None at the Equator. Shape, area, and scale distortion increases moderately away from the Equator but becomes severe at the poles (fig. 12A).

\section{Other features}

Parallels are spaced from the Equator by calculating the distance for 0.8 of the same latitude on the Mercator and dividing the result by 0.8 . Therefore, the two projections are almost identical near the Equator.

\section{Usage}

World maps in numerous American atlases and some other atlases, as a projection resembling the Mercator but having less distortion of area and scale, especially near the poles

\section{Origin}

Presented by Osborn Maitland Miller (18971979) of the American Geographical Society in 1942

\section{Aspects}

Normal aspect is commonly used.

Oblique aspect has been used by the National Geographic Society.

\section{Similar Projections}

Gall projection (p. 33) has different spacing of parallels. The lines of no distortion are at latitudes $45^{\circ} \mathrm{N}$. and $\mathrm{S}$. rather than at the

\section{Equator.}

Miller proposed other alternates in 1942 . including one identical with his preferred cylindrical but using two-thirds instead of $\mathbf{0 . 8}$

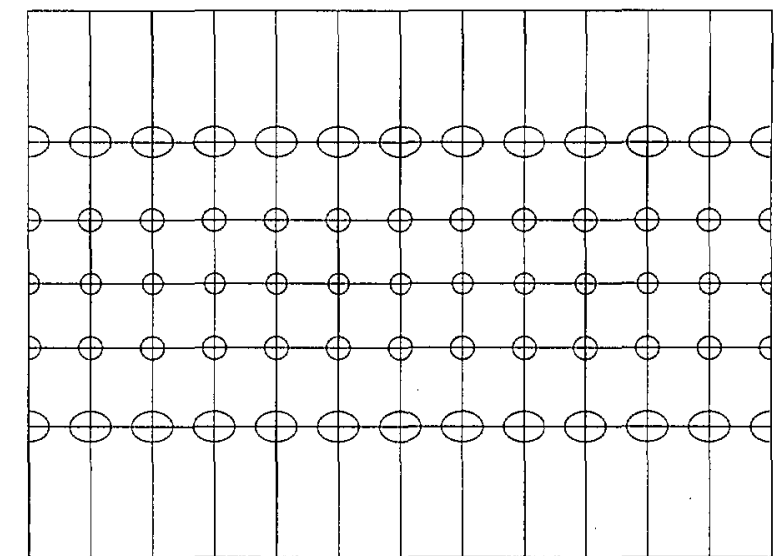




\section{MILLER CYLINDRICAL Projection}

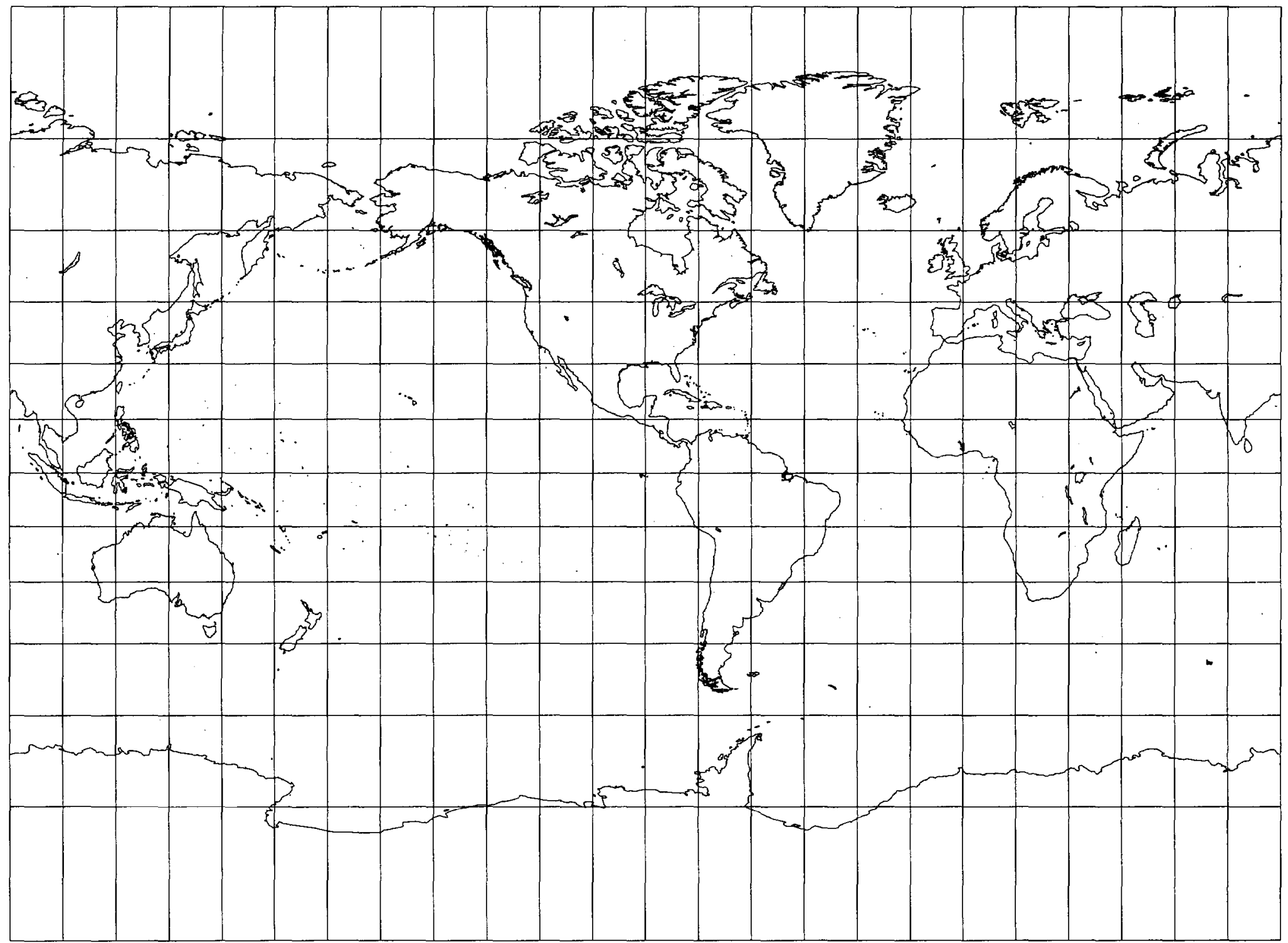

Figure 12B.-Miller Cylindrical projection with shorelines, $15^{\circ}$ graticule. Central meridian $90^{\circ} \mathrm{W}$. 


\section{Classifications \\ Pseudocylindrical \\ Equal area \\ Equally spaced parallels}

\section{Graticule}

Meridians: Central meridian is a straight line half as long as the Equator. Other meridians are equally spaced sinusoidal curves intersecting at the poles and concave toward the central meridian.

Parallels: Equally spaced straight parallel lines perpendicular to the central meridian Poles: Points

Symmetry: About the central meridian or the Equator

\section{Scale}

True along every parallel and along the central meridian

\section{Distortion}

Severe near outer meridians at high latitudes (fig. 13A) but can be substantially reduced by interruption with several central meridians

(fig. 13G). Free of distortion along the Equator and along the central meridian.

\section{Usage}

Atlas maps of South America and Africa. Occasionally used for world maps. Formerly used for other continental maps and star maps. Combined with Mollweide projection to develop other projections such as the Homolosine and the Boggs.

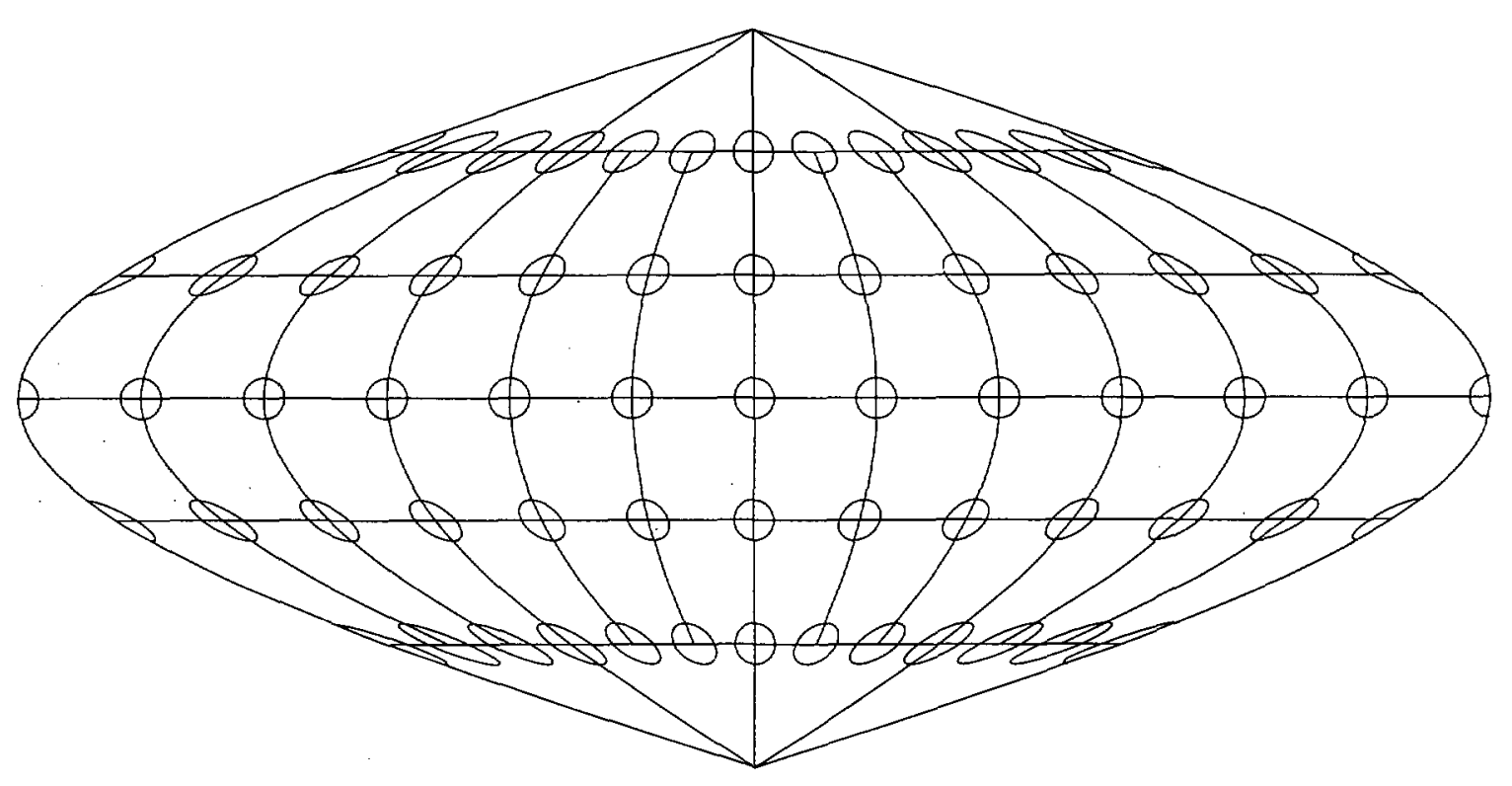

\section{Origin}

Developed in the 16th century. Used by J. Cossin in 1570 and by $\mathrm{J}$. Hondius in Mercator atlases of the early 17th century. Often called Sanson-Flamsteed projection after later users. Oldest current pseudocylindrical projection.

\section{Aspects}

For educational purposes, it has been shown in various aspects as examples of normal, transverse, and oblique aspects of almost any pseudocylindrical projection (figs. 13C-13F).

\section{Other names}

Sanson-Flamsteed

Mercator Equal-Area

\section{Similar projections}

Several other pseudocylindrical projections, such as Craster Parabolic (p. 70) and Boggs Eumorphic (p. 68), are very similar, but parallels are not equally spaced, and meridians are curved differently. Eckert $\vee(p .46)$ and VI (p. 50) have sinusoidal meridians but have lines for poles. 


\section{SINUSOIDAL Projection}

Figure 13B.-Sinusoidal projection with shorelines, $15^{\circ}$ graticule. Central meridian $90^{\circ} \mathrm{W}$.

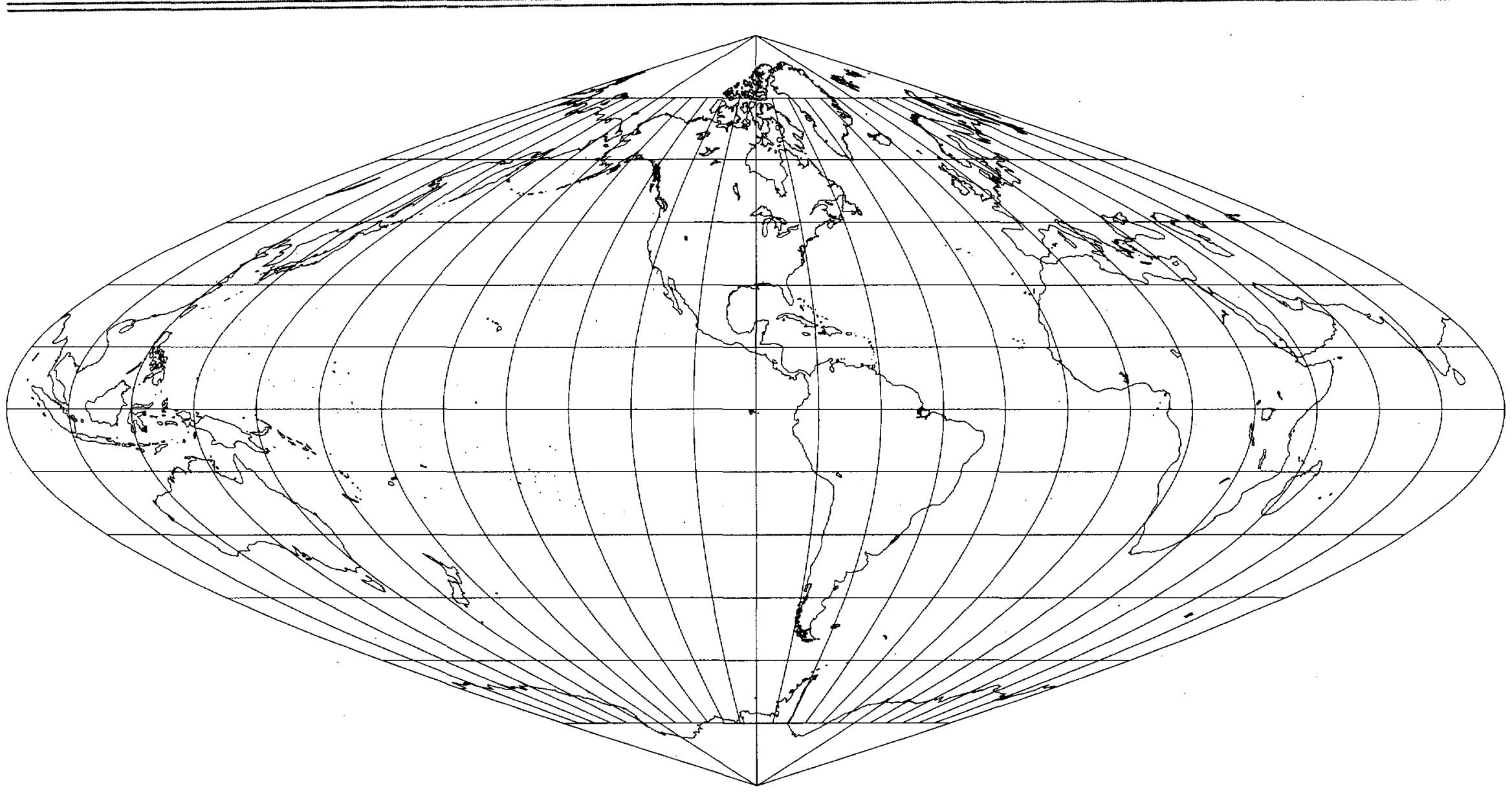


Figure 13C.-Transverse Sinusoidal projection with shorelines, $15^{\circ}$ graticule. Central meridian $90^{\circ} \mathrm{W}$. North Pole centered on base projection (at $0^{\circ}$ longitude).

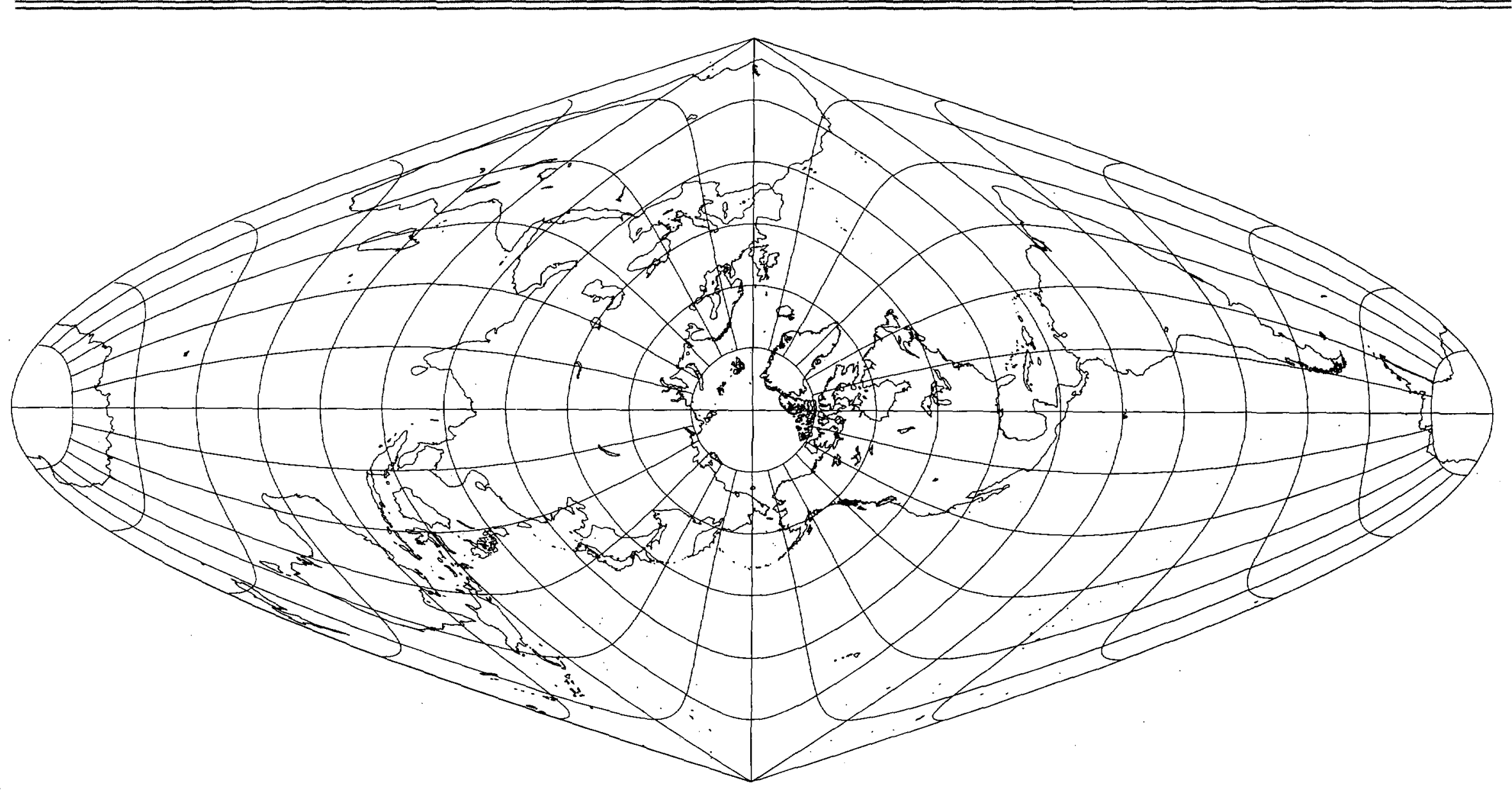




\section{SINUSOIDAL Projection}

Figure 130 .-Transverse Sinusoidal projection with shorelines, $15^{\circ}$ graticule. Central meridian $90^{\circ} \mathrm{W}$. North Pole at $-90^{\circ}$ longitude on base projection.

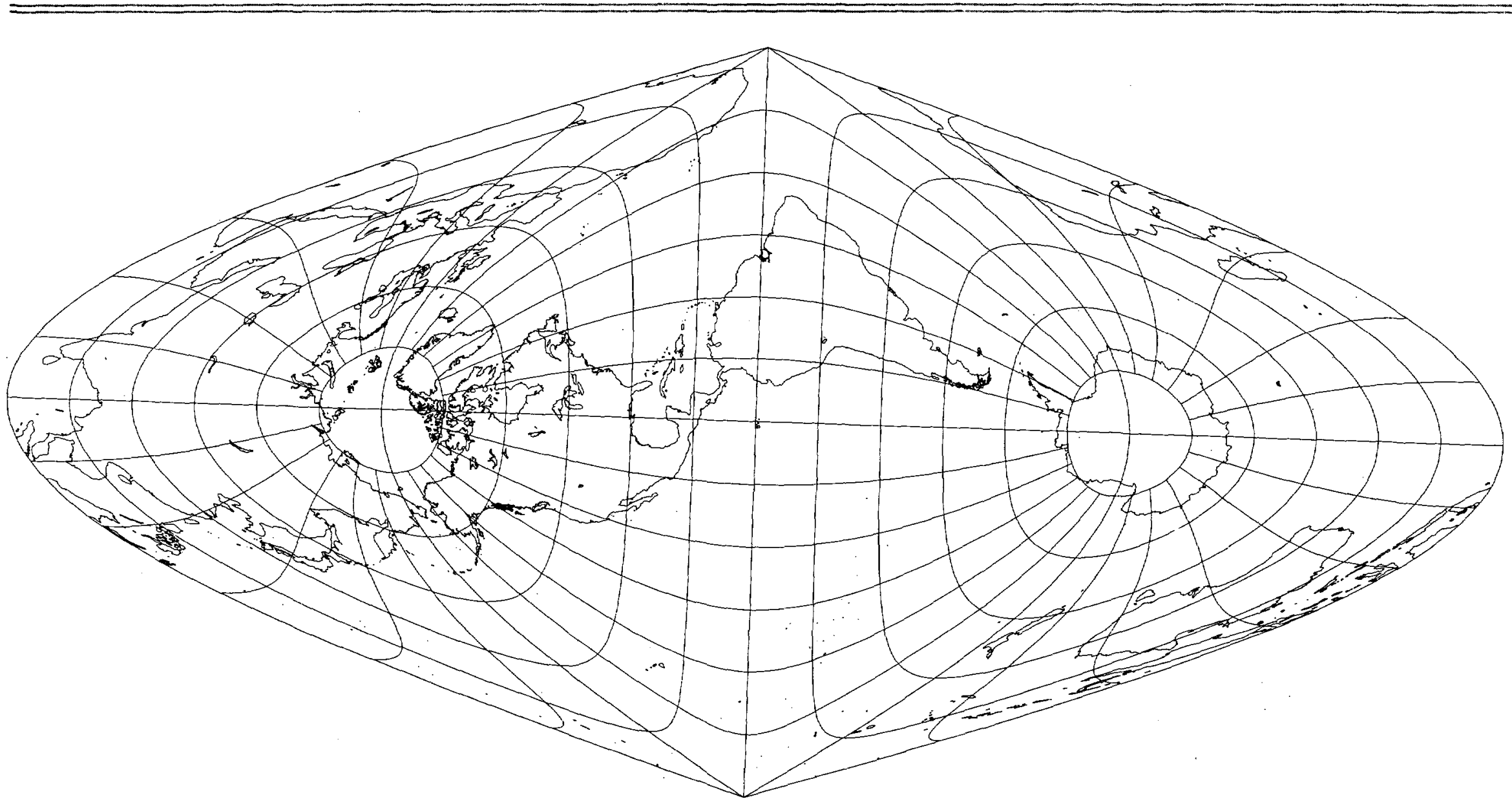




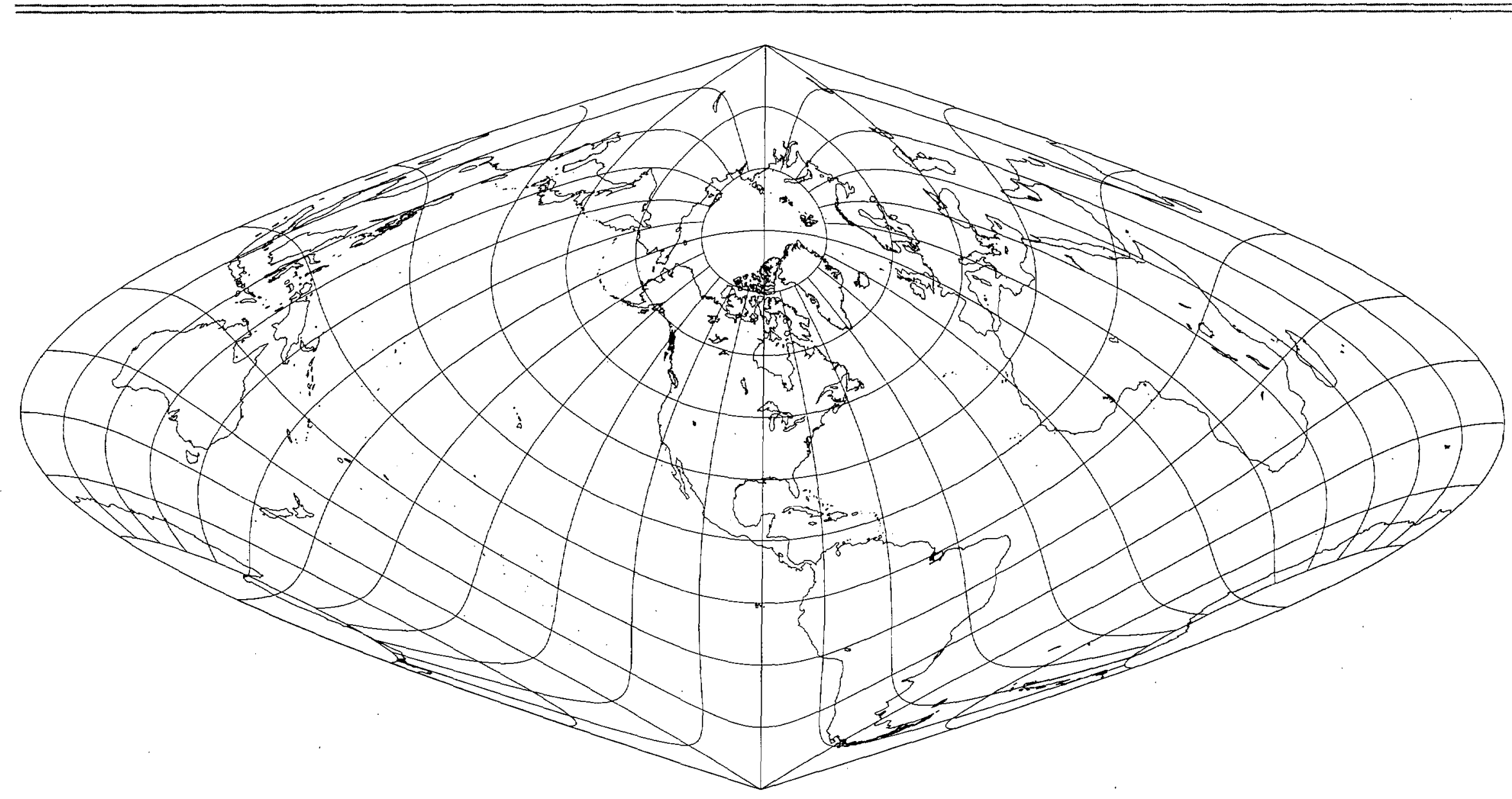




\section{SINUSOIDAL Projection}

Figure $13 F$.-Oblique Sinusoidal projection with shorelines, $15^{\circ}$ graticule. Central meridian $90^{\circ} \mathrm{W}$. North Pole at $+45^{\circ}$ latitude, $-90^{\circ}$ longitude on base projection.

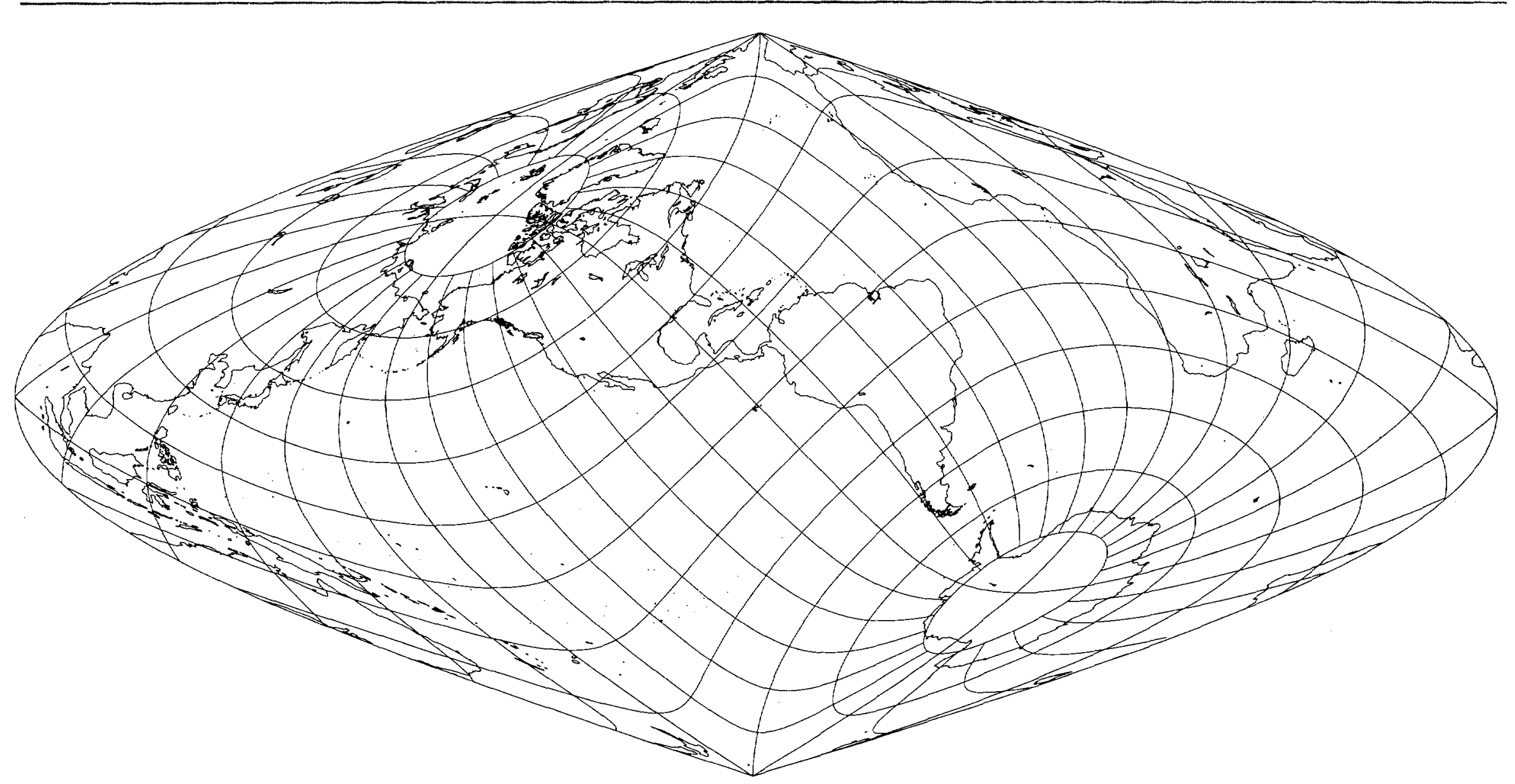


Figure 13G.-Interrupted Sinusoidal projection, with shorelines, $10^{\circ}$ graticule. Interruptions symmetrical about Equator.

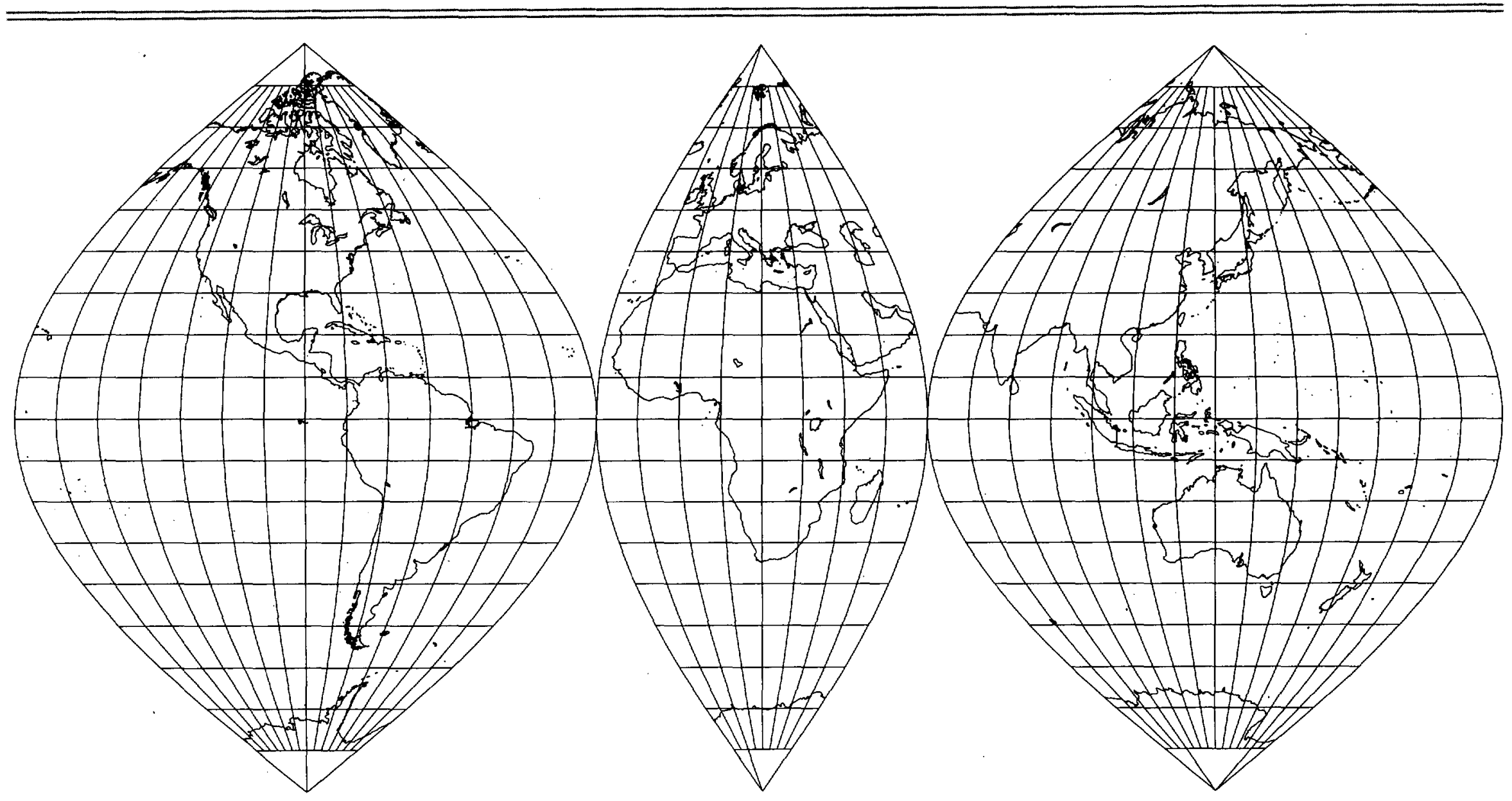




\section{McBRYDE-THOMAS FLAT-POLAR SINUSOIDAL Projection}

\section{Classifications}

Pseudocylindrical

Equal area

\section{Graticule}

Meridians: Central meridian is a straight line half as long as the Equator. Other meridians are equally spaced sinusoids, concave toward the central meridian.

Parallels: Unequally spaced straight parallel lines, widest separation near the Equator.

Perpendicular to the central meridian.

Poles: Lines one-third as Iong as the Equator Symmetry: About the central meridian or the Equator

\section{Scale}

True along latitudes $55^{\circ} 51^{\prime} \mathrm{N}$. and $\mathrm{S}$.

Constant along any given latitude; same for the latitude of opposite sign

\section{Distortion}

Free of distortion only at latitudes $55^{\circ} 51^{\prime} \mathrm{N}$. and

$\mathrm{S}$. on the central meridian (fig. 14A).

\section{Usage}

Basis of merged projections by McBryde (see McBryde S3, p. 52)

\section{Origin}

Presented by F. Webster McBryde and Paul D. Thomas through the U.S. Coast and Geodetic Survey in 1949

\section{Similar projections}

Sinusoidal projection (p. 37) uses sinusoids for meridians, but the poles are points.

Eckert VI projection (p. 50) uses sinusoids for meridians and is equal area, but the poles are lines half as long as the Equator.

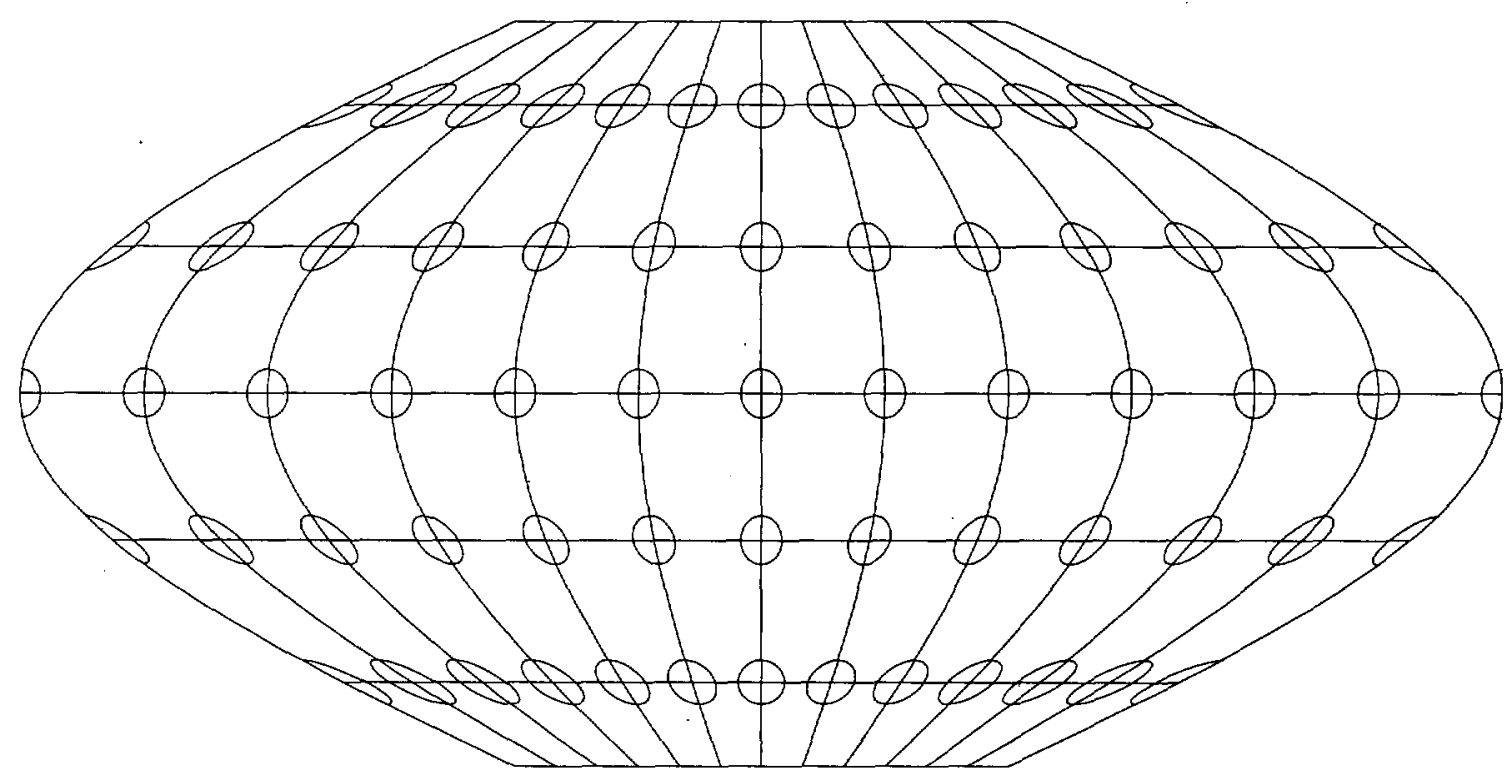

Figure 14A.-McBryde-Thomas Flat-Polar Sinusoidal projection, with Tissot indicatrices, $30^{\circ}$ graticule. 
Figure 14B.-McBryde-Thomas Flat-Polar Sinusoidal projection, with shorelines, $15^{\circ}$ graticule. Central meridian $90^{\circ} \mathrm{W}$.

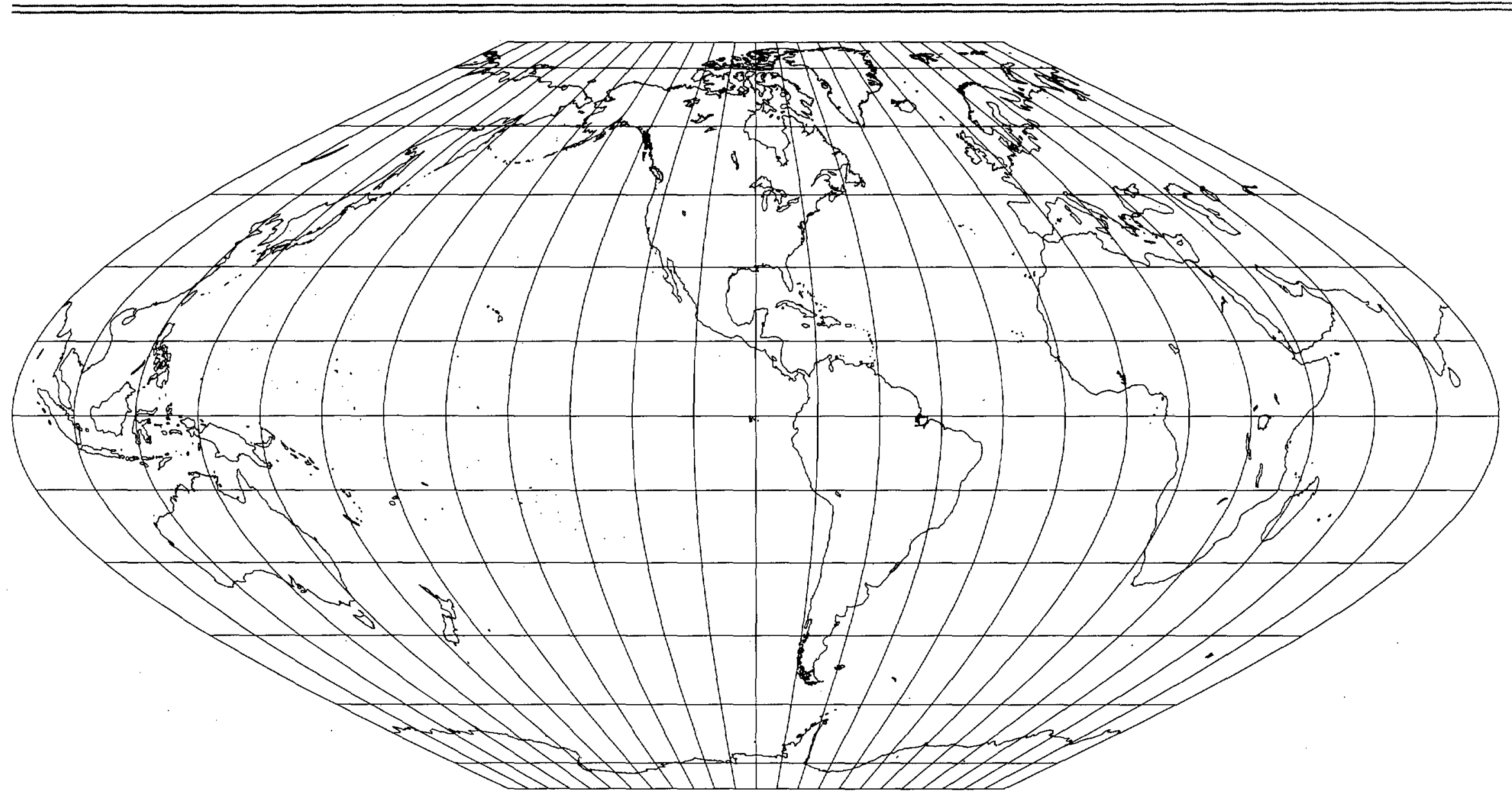




\section{Classifications}

Pseudocylindrical

Equally spaced parallels

Neither conformal nor equal area

\section{Graticule}

Meridians: Central meridian is a straight line half as long as the Equator. Other meridians are equally spaced sinusoids, concave toward the central meridian.

Parallels: Equally spaced straight parallel lines. Perpendicular to the central meridian. Poles: Lines half as long as the Equator Symmetry: About the central meridian or the Equator

\section{Scale}

True along latitudes $37^{\circ} 55^{\prime} \mathrm{N}$. and S., if the world map retains correct total area

Constant along any given latitude; same for the latitude of opposite sign

\section{Distortion}

No point is free of all distortion, but the Equator is free of angular distortion (fig. 15A).

\section{Origin}

Presented by Max Eckert (1868-1938) of Germany in 1906. The projection is an arithmetical average of the $x$ and $y$ coordinates

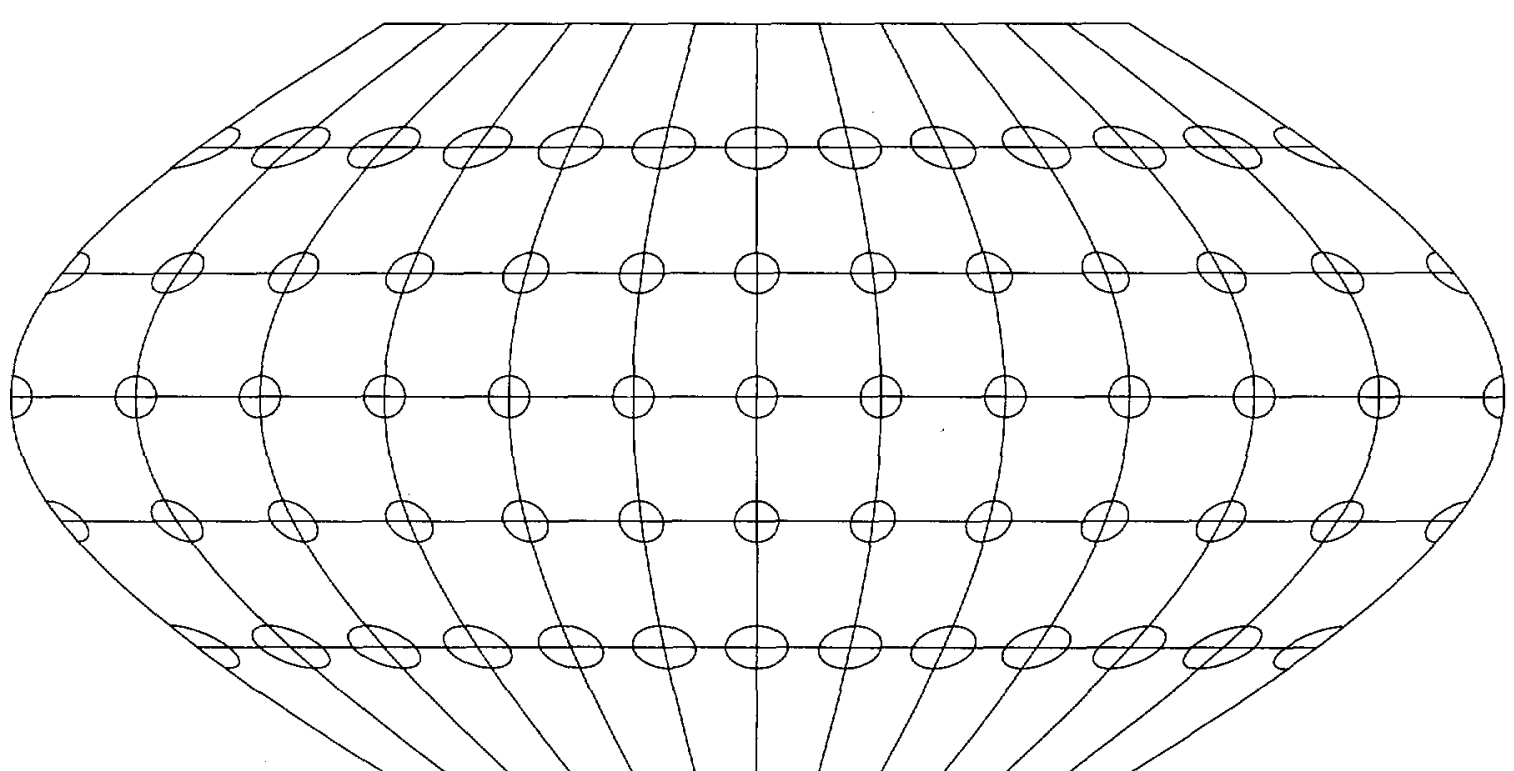

of the Sinusoidal (p. 37) and Plate Carrée (p.

22) projections.

\section{Similar projections}

Eckert VI projection (p. 50) has meridians

positioned identically, but parallels are spaced for equal area.

Winkel I (p. 48) is an average of coordinates of the Sinusoidal and Equirectangular projections.

Wagner III projection uses part rather than all of the sinusoidal curve for meridians. 
Figure 15B.-Eckert $V$ projection, with shorelines, $15^{\circ}$ graticule. Central meridian $90^{\circ} \mathrm{W}$.

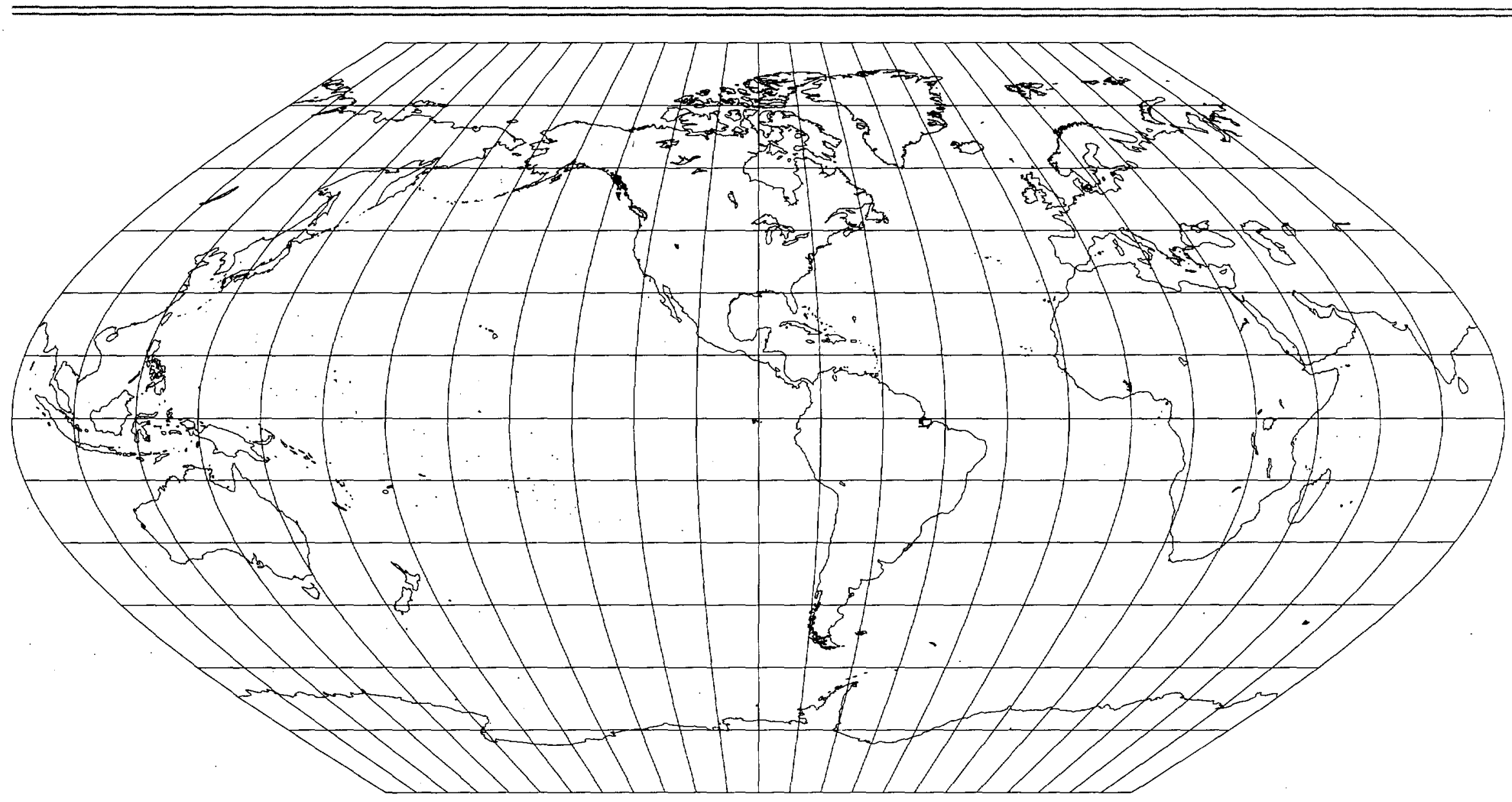




\section{WINKEL I Projection}

\section{Classifications}

Pseudocylindrical

Equally spaced parallels

Neither conformal nor equal area

\section{Graticule}

Meridians: Central meridian is a straight line 0.61 (or other value) as long as the Equator. Other meridians are equally spaced sinusoida curves, concave toward the central meridian. Parallels: Equally spaced straight parallel lines. Perpendicular to the central meridian. Poles: Lines 0.61 (or other value) as long as the Equator

Symmetry: About the central meridian or the Equator

\section{Scale}

True along latitudes $50^{\circ} 28^{\prime} \mathrm{N}$. and S. (or other chosen value)

Constant along any given latitude; same for the latitude of opposite sign

\section{Distortion}

Not free of distortion at any point

\section{Origin}

Developed by Oswald Winkel (1873-1953) of

Germany in 1914 as the average of the

Sinusoidal (p. 37) and Equirectangular (p. 24) projection in both $x$ and $y$ coordinates. When the standard parallels of the Equirectangular projection are varied, the standard parallels and appearance of Winkel I vary. Use of latitudes $50^{\circ} 28^{\prime} \mathrm{N}$. and $\mathrm{S}$. results in a map at the correct total-area scale, but the local-area scale varies.

\section{Limiting form}

Eckert $V$ (p. 46), if the Equator is the standard parallel 
Figure 16.-Winkel I projection, with shorelines, $15^{\circ}$ graticule. Central meridian $90^{\circ} \mathrm{W}$. Standard parallels $50^{\circ} 28^{\prime} \mathrm{N}$. and S

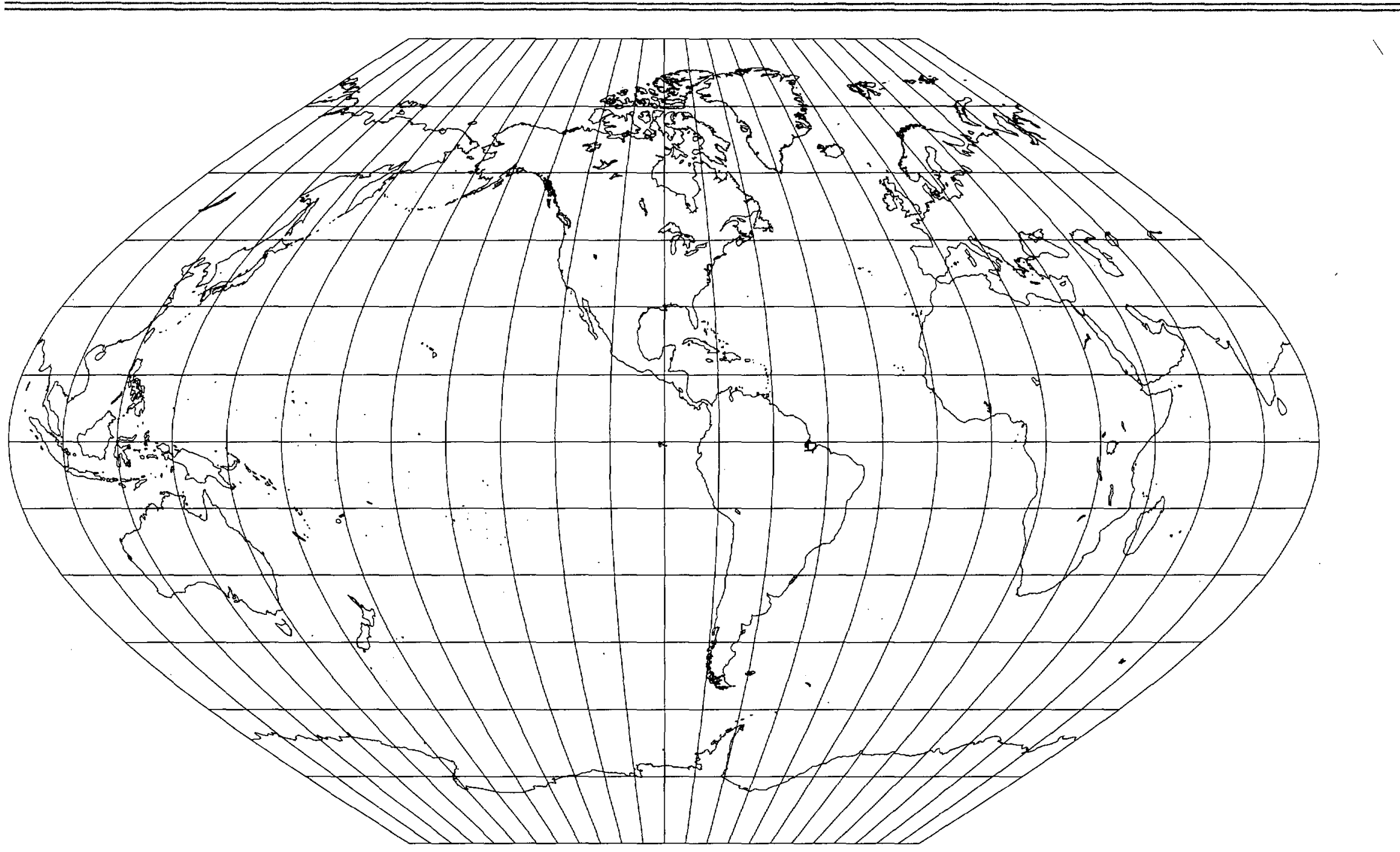




\section{Classifications}

Pseudocylindrical

Equal area

\section{Graticule}

Meridians: Central meridian is a straight line half as long as the Equator. Other meridians are equally spaced sinusoids, concave toward the central meridian.

Parallels: Equally spaced straight parallel lines, widest separation near the Equator.

Perpendicular to the central meridian.

Poles: Lines half as long as the Equator

Symmetry: About the central meridian or the Equator

\section{Scale}

True along latitudes $49^{\circ} 16^{\prime} \mathrm{N}$. and $\mathrm{S}$.

Constant along any given latitude; same for the latitude of opposite sign

\section{Distortion}

Free of distortion only at latitudes $49^{\circ} 16^{\prime} \mathrm{N}$. and S. at the central meridian (fig. 17A)

\section{Usage}

Thematic world maps in Soviet World Atlas of 1937

Some recent use for climatic maps by U.S. publishers

\section{Origin}

Presented by Max Eckert (1868-1938) of Germany in 1906.

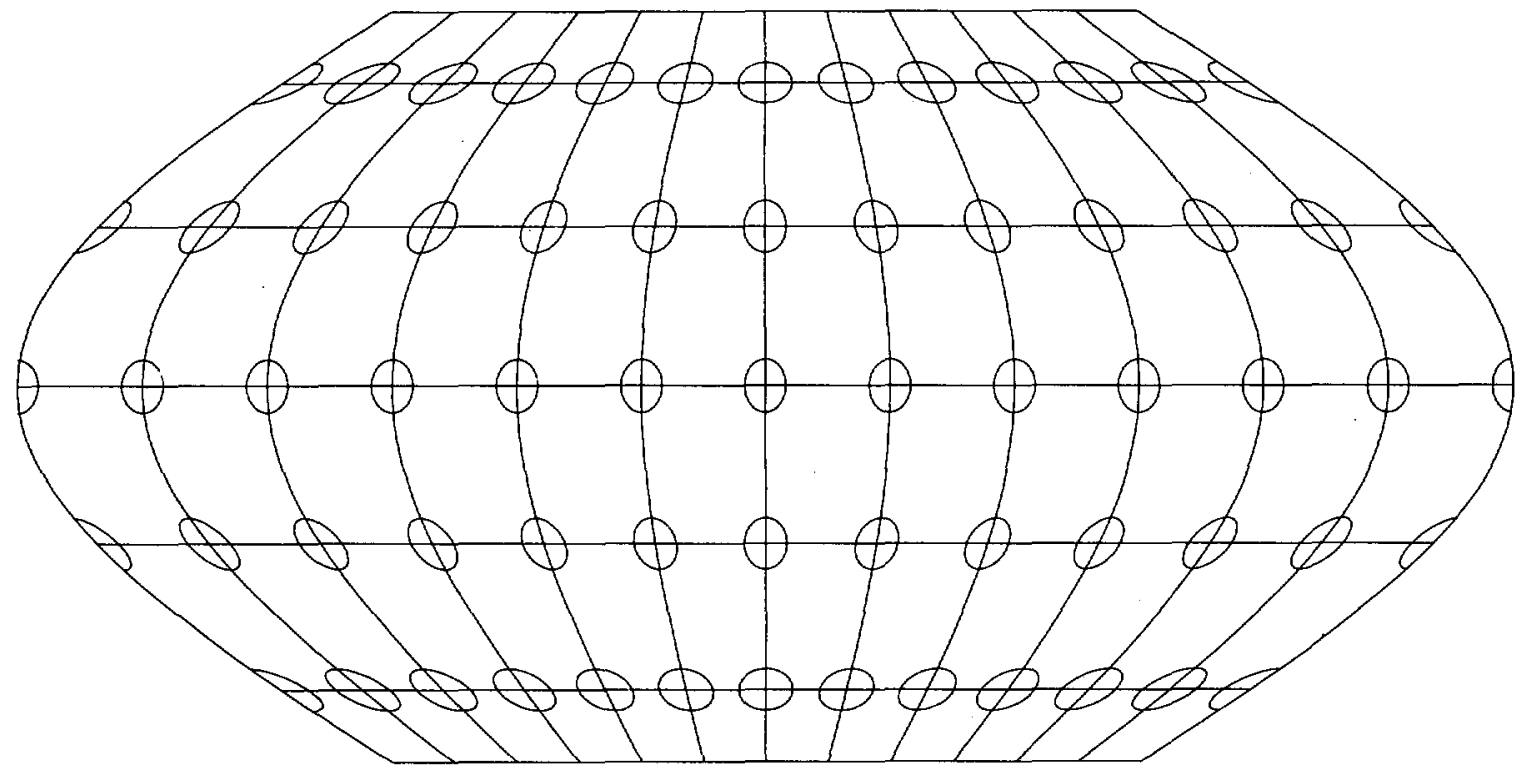

\section{Similar projections}

Eckert $V$ projection (p. 46) has meridians

positioned identically, but parallels are equally spaced.

Wagner I projection (1932) is almost identical to Eckert VI, but Wagner I uses only part of the sinusoidal curve. Kavrayskiy VI projection (1936) is identical to Wagner I. Werenskiold II projection (1944) is the same as Wagner I, except for scale.

McBryde-Thomas Flat-Polar Sinusoidal (p. 44) uses the full sinusoid and is equal area, but the poles are one-third the length of the Equator. 
Figure $17 B$.-Eckert VI projection, with shorelines, $15^{\circ}$ graticule. Central meridian $90^{\circ} \mathrm{W}$.

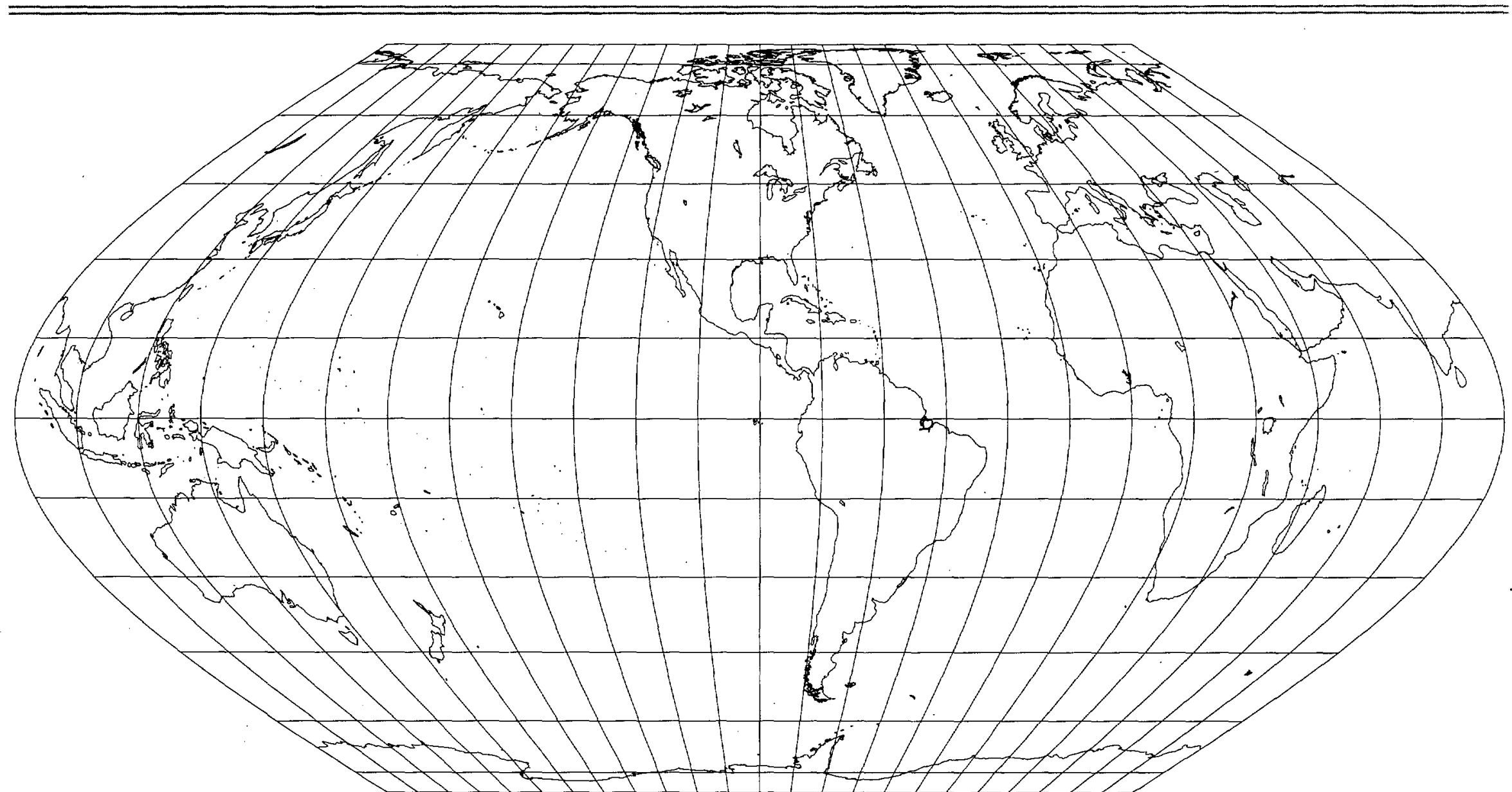




\section{McBRYDE S3 Projection}

\section{Classifications}

Pseudocylindrical composite

Equal area

\section{Graticule}

Meridians: Where the central meridian extends across the Equator, it is a straight line 0.44 as long as the Equator. Other central meridians in the usual interrupted form are straight and half as long. Other meridians are equally spaced sinusoidal curves, bending slightly at latitudes $55^{\circ} 51^{\prime} \mathrm{N}$. and S., and all are concave toward the local central meridian.

Parallels: Straight parallel lines, perpendicular to the central meridian(s). Equally spaced between latitudes $55^{\circ} 51^{\prime} \mathrm{N}$. and $\mathrm{S}$.

Gradually closer together beyond these latitudes

Poles: Interrupted straight lines totaling 0.31 the length of the Equator

Symmetry: About the central meridian or the Equator (in uninterrupted form)

\section{Scale}

True along every latitude between $55^{\circ} 51^{\prime} \mathrm{N}$. and S. and along the central meridian within the same latitude range. Constant along any given latitude; same for the latitude of opposite sign.

\section{Distortion}

Same as the Sinusoidal projection between latitude $55^{\circ} 51^{\prime} \mathrm{N}$. and S. (see fig. $13 A$ ). Same as the McBryde-Thomas Flat-Polar Sinusoidal projection beyond this range (see fig. 14A). McBryde S3 is almost always used in the interrupted form and has several central meridians.

\section{Usage}

World map interrupted to show oceans or land masses, by McBryde

\section{Origin}

Developed by F. Webster McBryde (1908- ) of Potomac, Md., in 1977 as a merging of the Sinusoidal with the McBryde-Thomas FlatPolar Sinusoidal projection at the parallels of identical scale on the two projections, latitudes $55^{\circ} 51^{\prime}$ N. and S. U.S. Patent by McBryde.

\section{Similar projections}

Identical with the Sinusoidal (p. 37) between latitudes $55^{\circ} 51^{\prime} N$. and $S$.; identical with the McBryde-Thomas Flat-Polar Sinusoidal (p. 44) poleward of those latitudes, except that those portions are closer to the Equator than they are on the McBryde-Thomas Flat-Polar

Sinusoidal projection itself.

McBryde merged projections in several other similar combinations, also in 1977. 
Figure 18.-Interrupted McBryde S3 projection, with shorelines, $10^{\circ}$ graticule, to show oceans. Projection: U.S. Patent No. 4,315,747, held by $F$. Webster McBryde and used by permission.

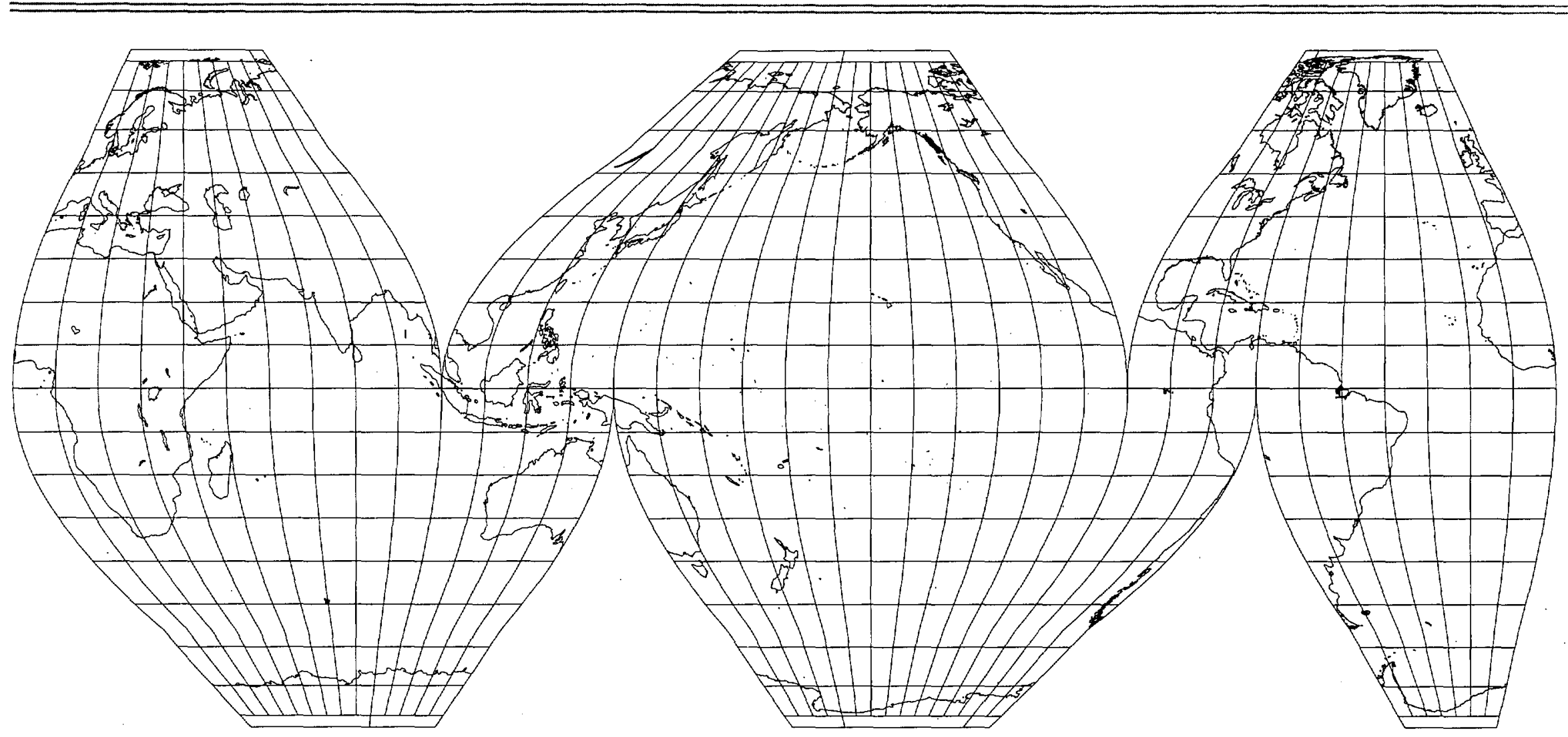




\section{MOLLWEIDE Projection}

\section{Classifications}

Pseudocylindrical

Equal area

\section{Graticule}

Meridians: Central meridian is a straight line half as long as the Equator. Meridians $90^{\circ} \mathrm{E}$. and $W$. of the central meridian form a circle. Others are equally spaced semiellipses intersecting at the poles and concave toward the central meridian.

Parallels: Unequally spaced straight parallel lines, farthest apart near the Equator; spacing changes gradually. Perpendicular to the central meridian.

Poles: Points

Symmetry: About the central meridian or the Equator

\section{Scale}

True along latitudes $40^{\circ} 44^{\prime} \mathrm{N}$. and S.

Constant along any given latitude; same for the latitude of opposite sign

\section{Distortion}

Severe near outer meridians at high latitudes (fig. 19A) but can be substantially reduced if interrupted with several central meridians (fig. $19 D$ ). Free of distortion only at latitudes $40^{\circ} 44^{\prime}$ $\mathrm{N}$. and $\mathrm{S}$. on the central meridian.

\section{Usage}

Occasional world maps, especially thematic maps. Combined with Sinusoidal projection to develop other projections such as the Goode Homolosine and the Boggs.

\section{Origin}

Presented by Carl B. Mollweide (1774-1825) of Germany in 1805

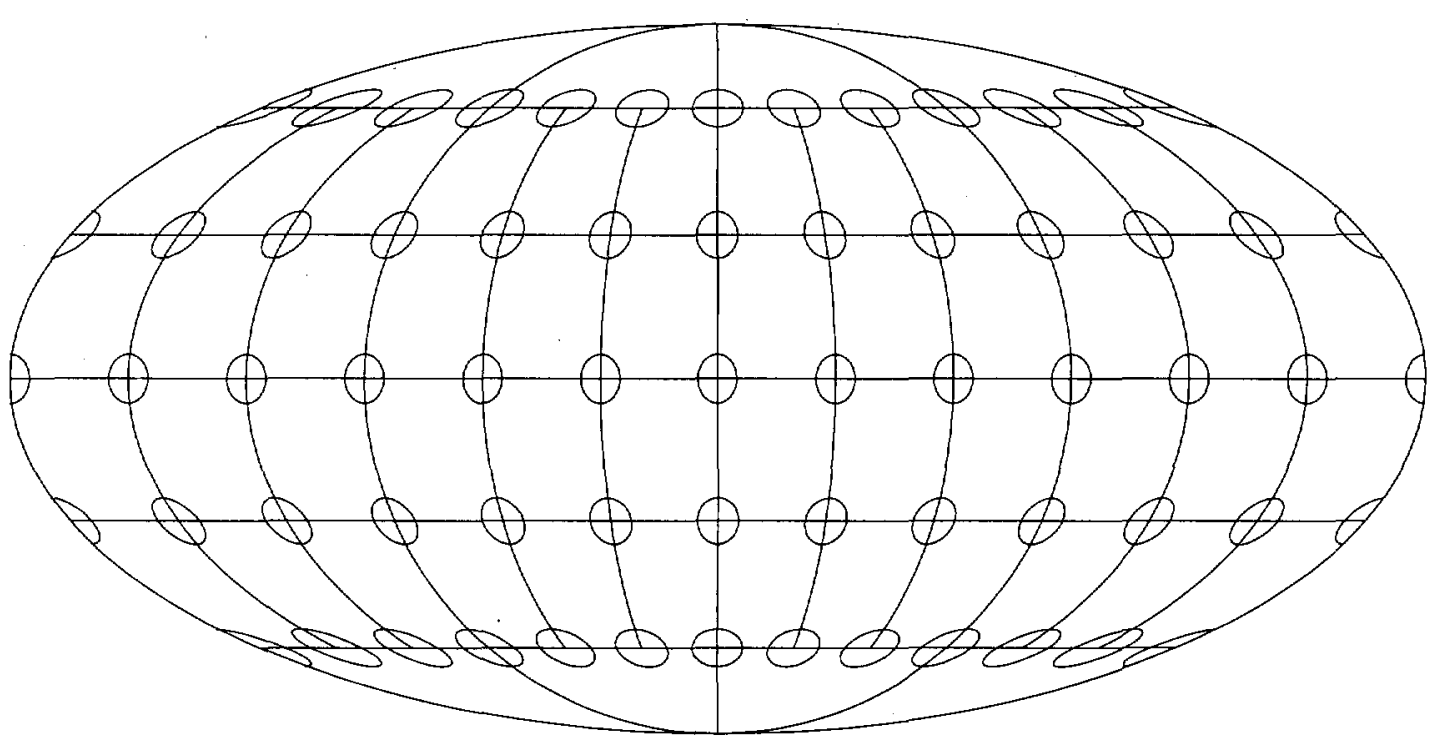

\section{Aspects}

For educational purposes, it has been shown in various aspects as examples of normal, transverse, and oblique aspects of almost any pseudocylindrical projection. The transverse aspect has also been used by John Bartholomew in The Times Atlas in England in 1958 (fig. 19C).

\section{Other names}

Homolographic projection

Homalographic projection

Babinet projection

Elliptical projection

\section{Similar projections}

Goode Homolosine (p. 66) (Mollweide merged with Sinusoidal)

Boggs Eumorphic (p. 68) (Mollweide averaged with Sinusoidal)
Bromley, by Robert H. Bromley in 1965 (Mollweide compressed from north to south with east-west expansion to achieve no distortion along the Equator)

Hyperelliptical, by Waldo R. Tobler in 1973 (equal-area pseudocylindrical having "hyperelliptical" meridians that lie between the Mollweide ellipses and a circumscribed rectangle) 


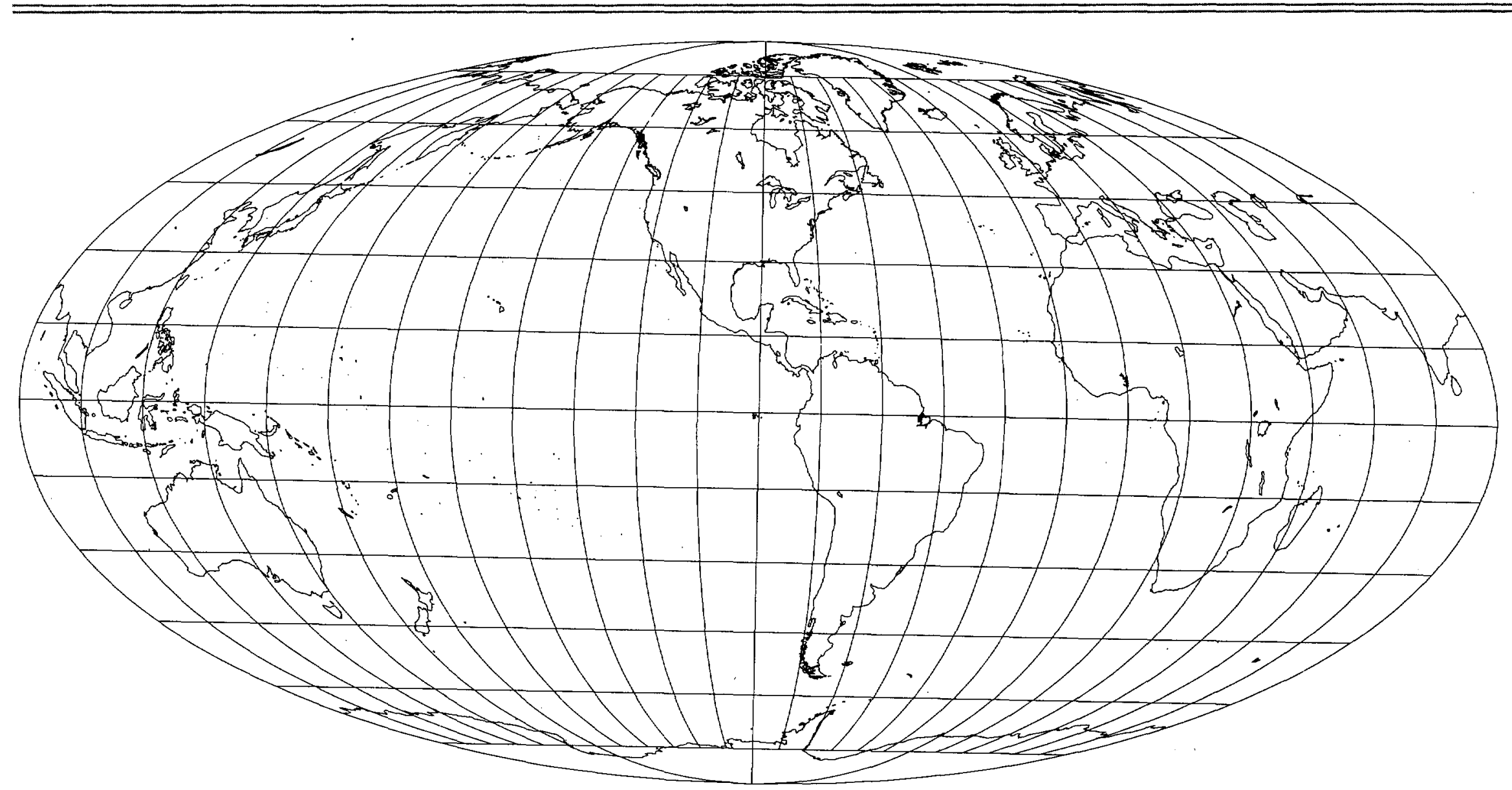




\section{MOLLWEIDE Projection}

Figure 19C.- "Atlantis" projection, a Transverse Mollweide used by John Bartholomew to highlight the Atlantic Ocean, with shorelines, $15^{\circ}$ graticule. Central meridian $30^{\circ} \mathrm{W}$. North Pole at $-45^{\circ}$ longitude on base projection.

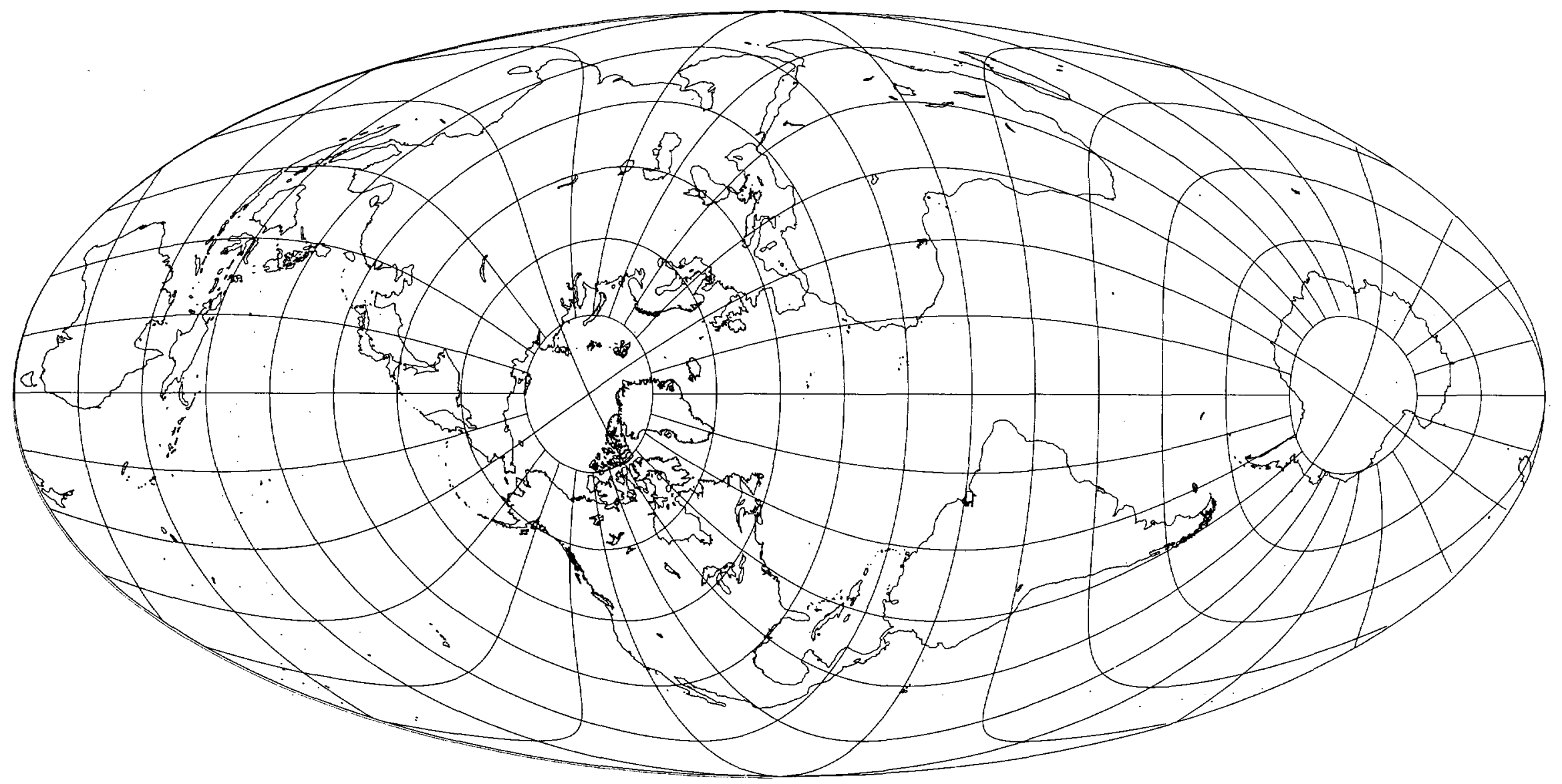


Figure 19D.-Interrupted Mollweide projection, with shorelines, $10^{\circ}$ graticule, to show oceans.

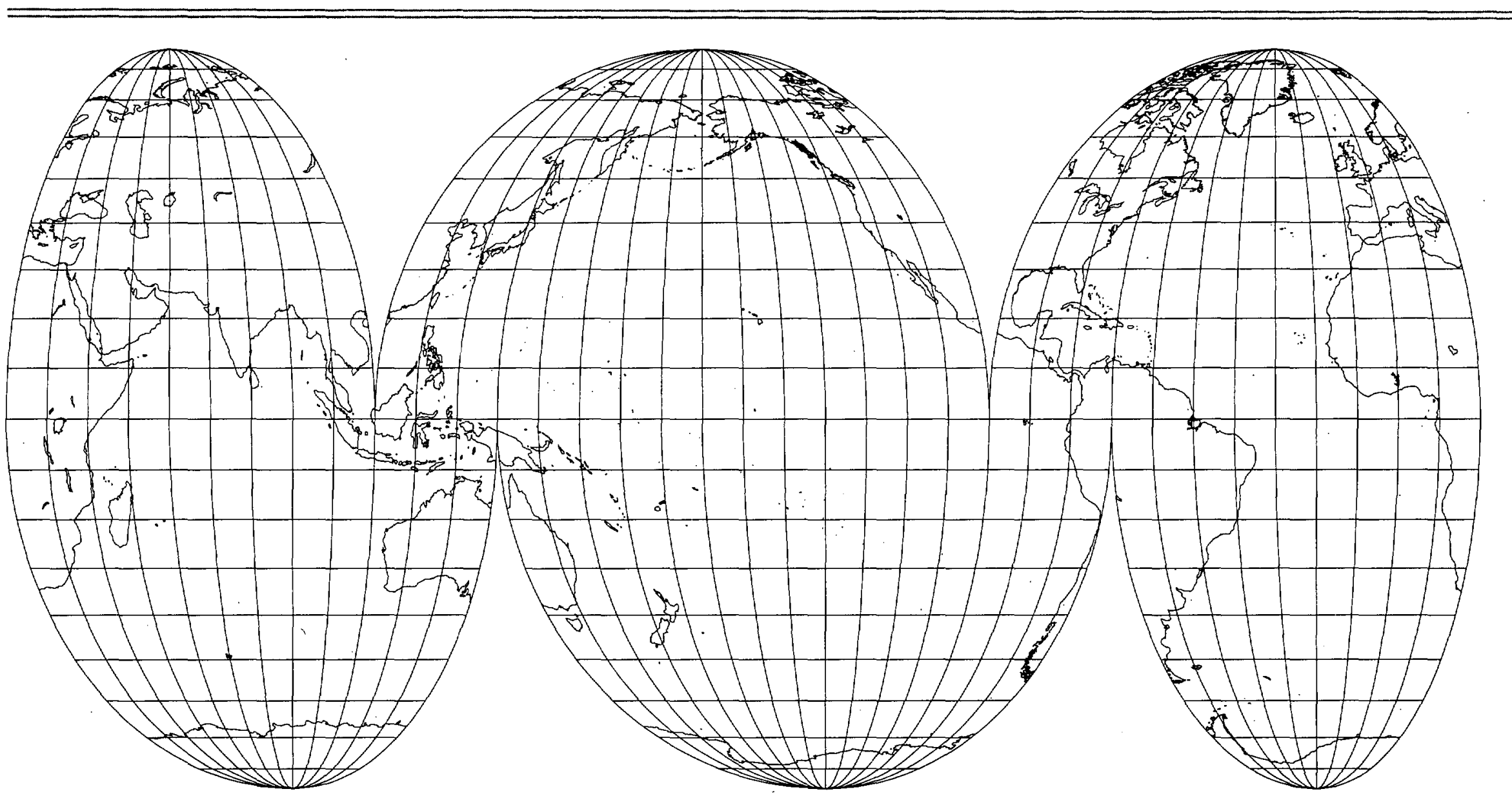




\section{ECKERT III Projection}

Figure 20A.-Eckert III projection, with Tissot indicatrices, $30^{\circ}$ graticule.

\section{Classifications}

Pseudocylindrical

Equally spaced parallels

Neither conformal nor equal area

\section{Graticule}

Meridians: Central meridian is a straight line half as long as the Equator. Other meridians are equally spaced semiellipses, concave toward the central meridian. The outer meridians $\left(180^{\circ}\right.$ east and west of the central meridian) are semicircles.

Parallels: Equally spaced straight parallel lines. Perpendicular to the central meridian. Poles: Lines half as long as the Equator Symmetry: About the central meridian or the Equator

\section{Scale}

True along latitudes $35^{\circ} 58^{\prime} \mathbf{N}$. and S., if the world map retains correct total area (area varies locally). Constant along any given latitude; same for the latitude of opposite sign.

\section{Distortion}

No point is free of all scale distortion, but the Equator is free of angular distortion (fig.

20A).

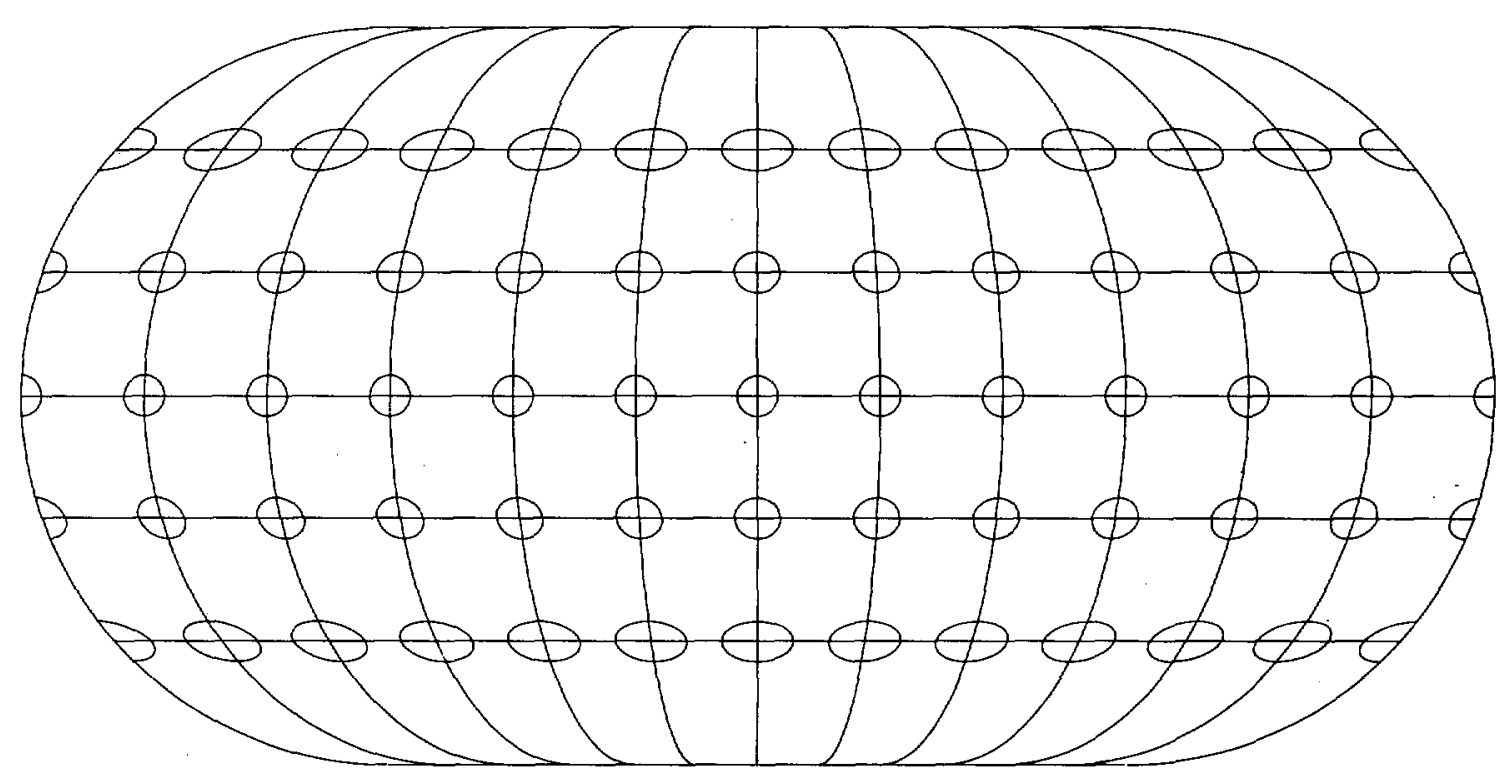

\section{Origin}

Presented by Max Eckert (1868-1938) of Germany in 1906

\section{Similar projections}

Eckert IV (p. 60) has meridians positioned

identically, but parallels are spaced for equal area.

Putniṇs $\mathrm{P}_{1}{ }^{\prime}$ has meridians that are only portions of semiellipses, but parallels are equidistant. 


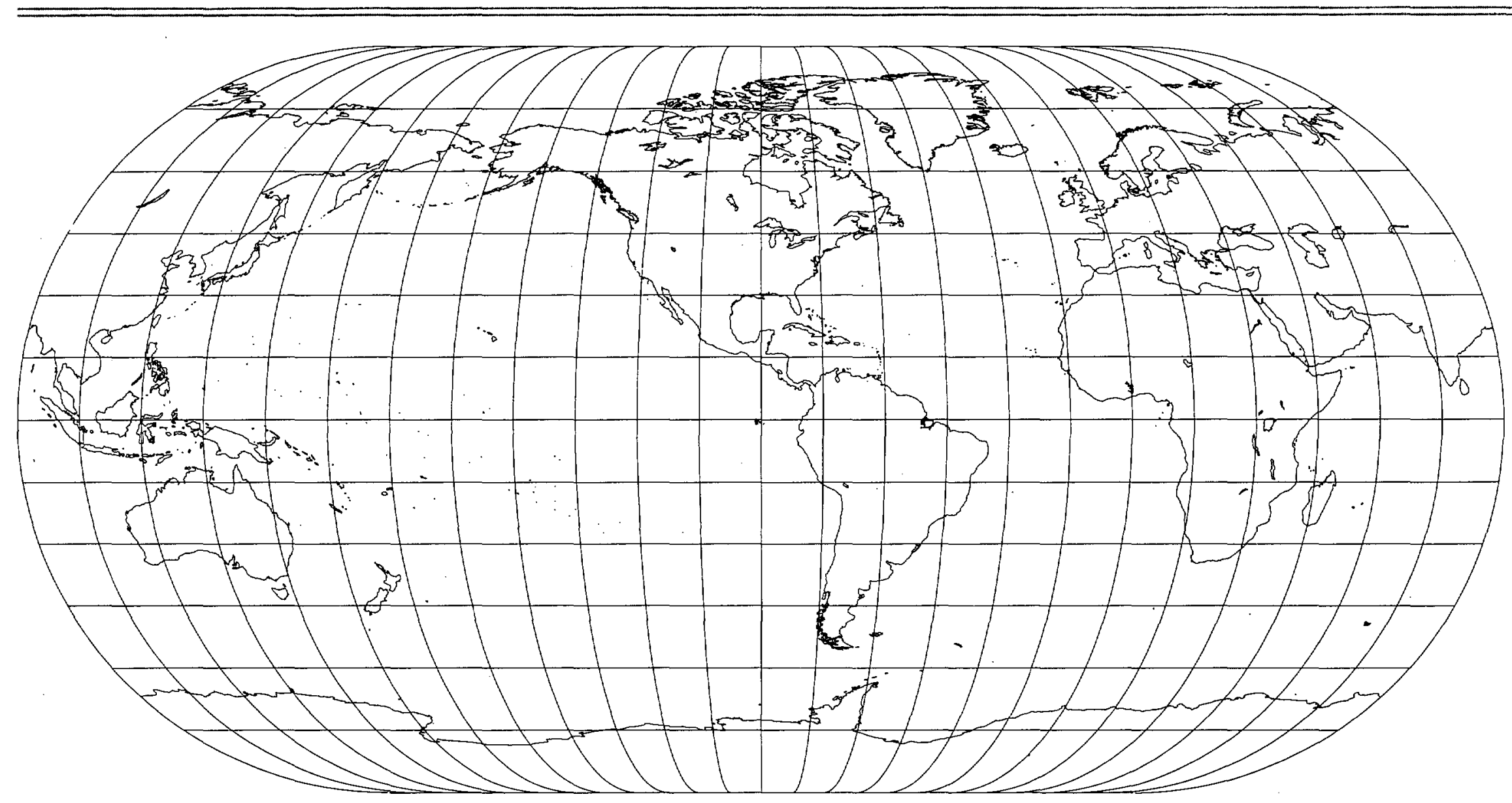




\section{Classifications}

Pseudocylindrical

Equal area

\section{Graticule}

Meridians: Central meridian is a straight line half as long as the Equator. Other meridians are equally spaced semiellipses, concave toward the central meridian. The outer meridians $\left(180^{\circ}\right.$ east and west of the central meridian) are semicircles.

Parallels: Unequally spaced straight paralle lines, widest separation near the Equator. Perpendicular to the central meridian.

Poles: Lines half as long as the Equator

Symmetry: About the central meridian or the Equator

\section{Scale}

True along latitudes $40^{\circ} 30^{\prime} \mathrm{N}$. and $\mathrm{S}$.

Constant along any given latitude; same for the latitude of opposite sign

\section{Distortion}

Free of distortion only at latitudes $40^{\circ} 30^{\prime} \mathrm{N}$. and $\mathrm{S}$. at the central meridian (fig. 21A).

\section{Usage}

Thematic and other world maps in numerous atlases and textbooks and for sheet maps

\section{Origin}

Presented by Max Eckert (1868-1938) of Germany in 1906

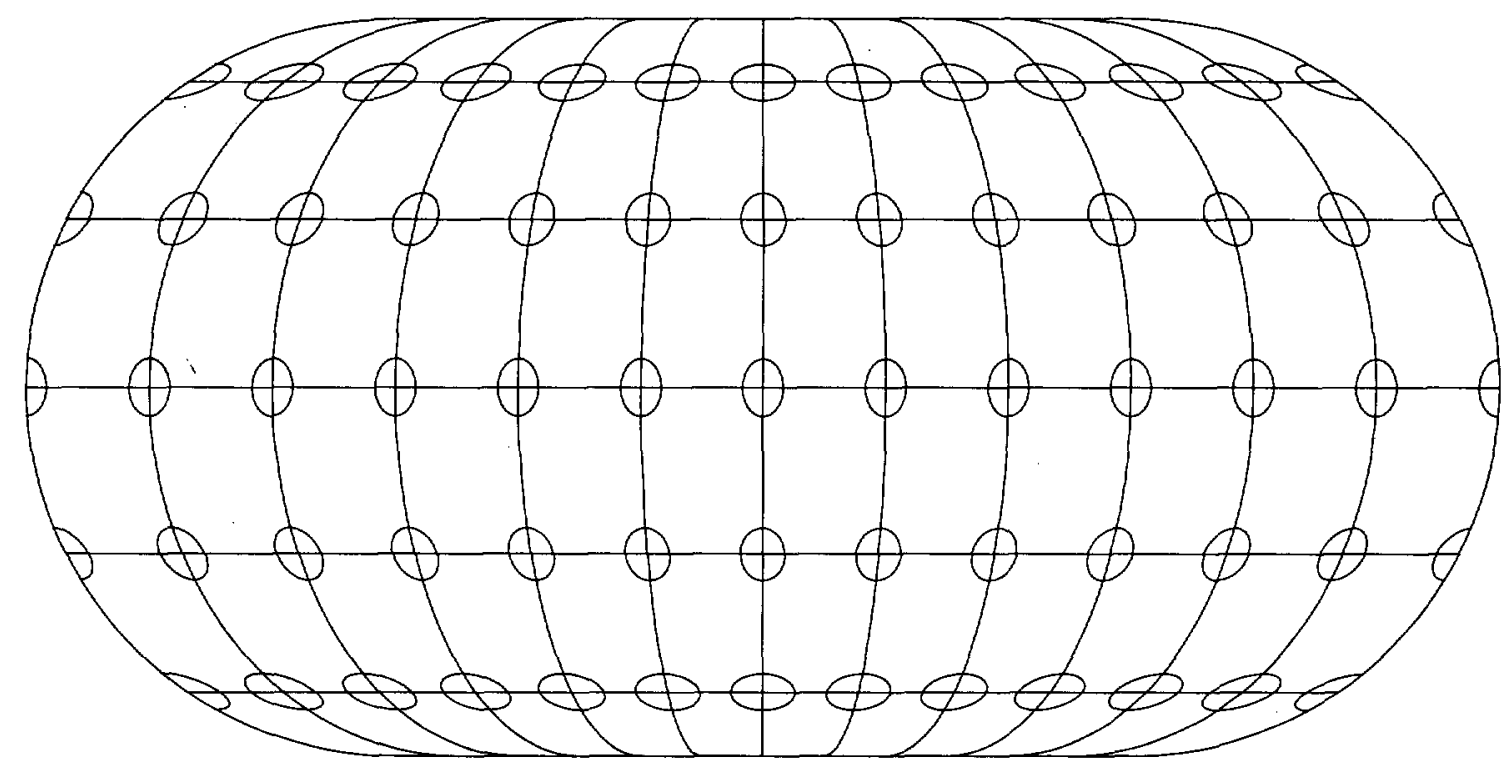

\section{Aspects}

Like most projections, transverse and oblique aspects can be prepared, although they are seldom used in this case.

\section{Similar projections}

Eckert III (p. 58) has meridians positioned

identically, but parallels are equally spaced.

Wagner IV (p. 62) has meridians that are only

portions of semiellipses, but parallels are also spaced for equal area. 
Figure 21B.-Eckert IV projection, with shorelines, $15^{\circ}$ graticule. Central meridian $90^{\circ} \mathrm{W}$.

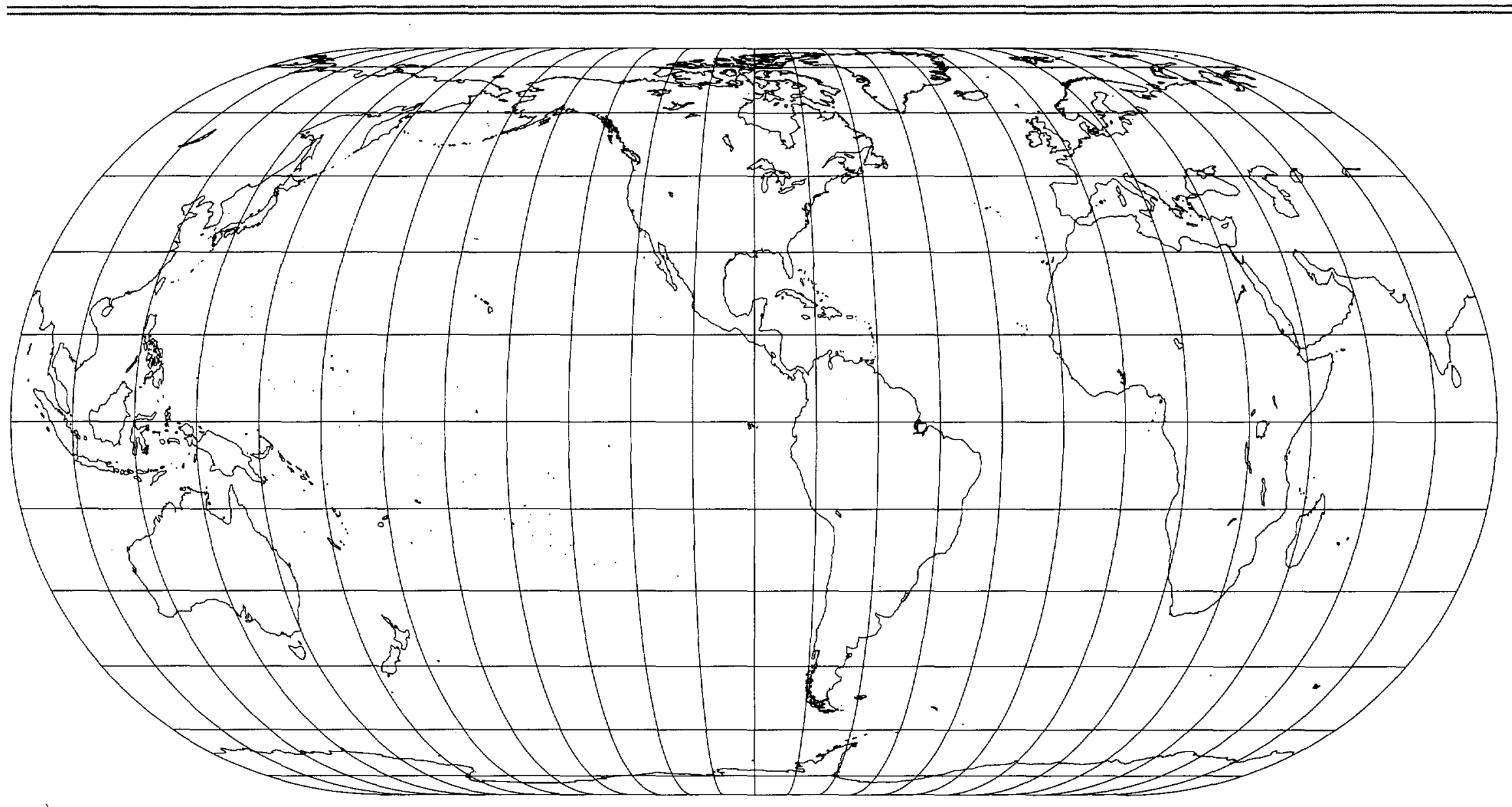




\section{Classifications}

\section{Pseudocylindrical}

Equal area

\section{Graticule}

Meridians: Central meridian is a straight line half as long as the Equator. Other meridians are equally spaced portions of ellipses (less than semiellipses) that would intersect beyond the poles and are concave toward the central meridian. The meridians at $103^{\circ} 55^{\prime}$ east and west of the central meridian are circular arcs.

Parallels: Unequally spaced straight parallel lines, widest separation near the Equator. Perpendicular to the central meridian.

Poles: Lines half as long as the Equator Symmetry: About the central meridian or the Equator

\section{Scale}

True along latitudes $42^{\circ} 59^{\prime} \mathrm{N}$. and $\mathrm{S}$.

Constant along any given latitude; same for the latitude of opposite sign

\section{Distortion}

Distortion is not as extreme near outer meridians at high latitudes as it is on pointedpolar pseudocylindrical projections, but there is considerable distortion throughout polar regions (fig. 22A). Free of distortion only at latitudes $42^{\circ} 59^{\prime} \mathrm{N}$. and $\mathrm{S}$. at the central meridian.

\section{Origin}

Presented by Karlheinz Wagner of Germany in 1932

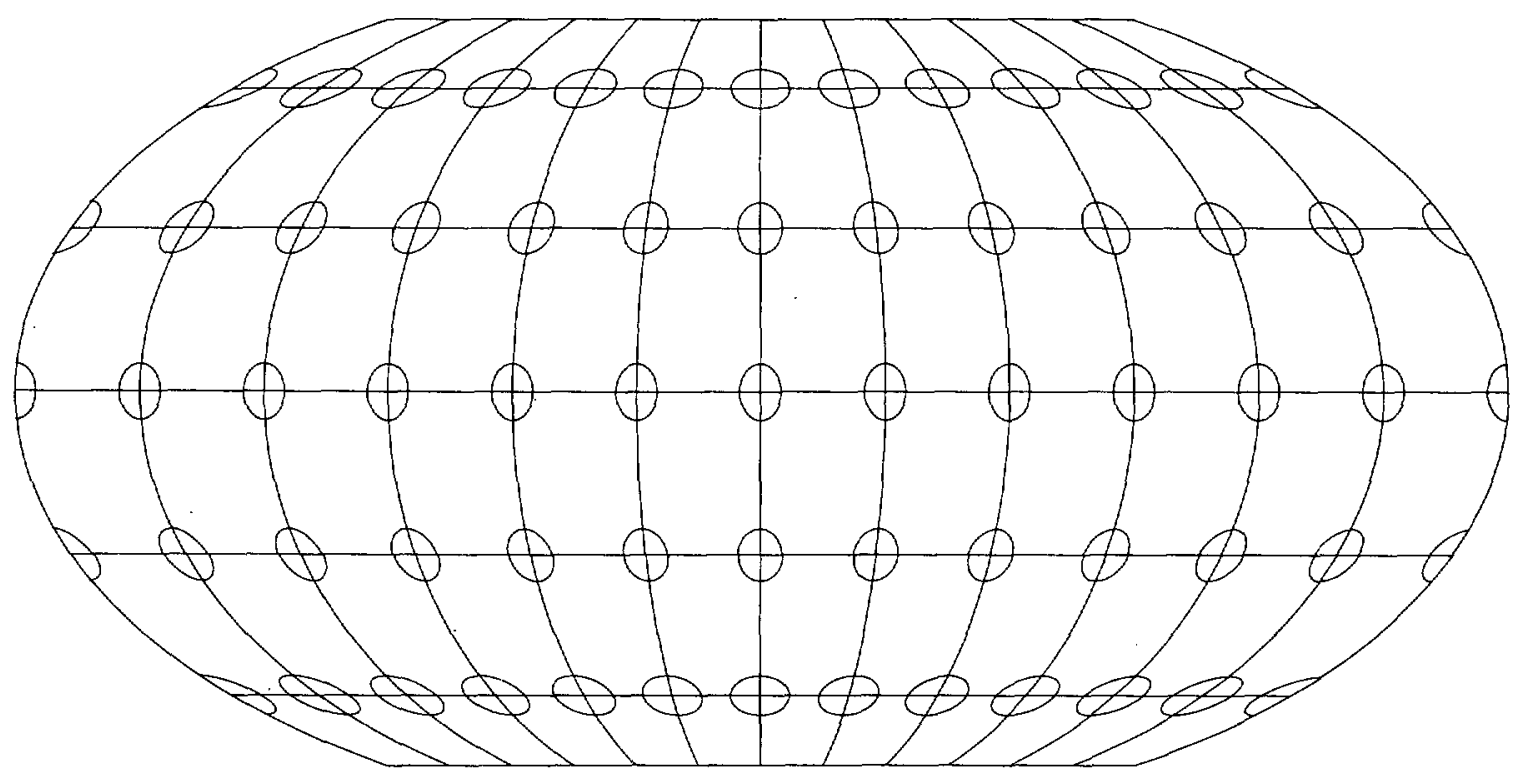

\section{Similar projections}

Putnin̄s $\mathrm{P}_{2}^{\prime}$ projection (1934) is identical.

Werenskiold III projection (1944) is identical, except that true scale is maintained along the Equator by enlarging the map.

Putnin̄s $\mathrm{P}_{2}$ equal-area projection (1934) uses the same portions of ellipses, but the poles are points.

Putnins̄ $P_{1}$ and $P_{1}^{\prime}$ projections (1934) have meridians identical in shape and position to those of Putnin̄s $P_{2}$ and $P_{2}$, respectively, but parallels are equally spaced, and the projections are not equal area.

Robinson projection (p. 82) is not equal area, meridians are not elliptical, and parallels are spaced differently. 
Figure 22B. - Wagner IV projection, with shorelines, $15^{\circ}$ graticule. Central meridian $90^{\circ} \mathrm{W}$.

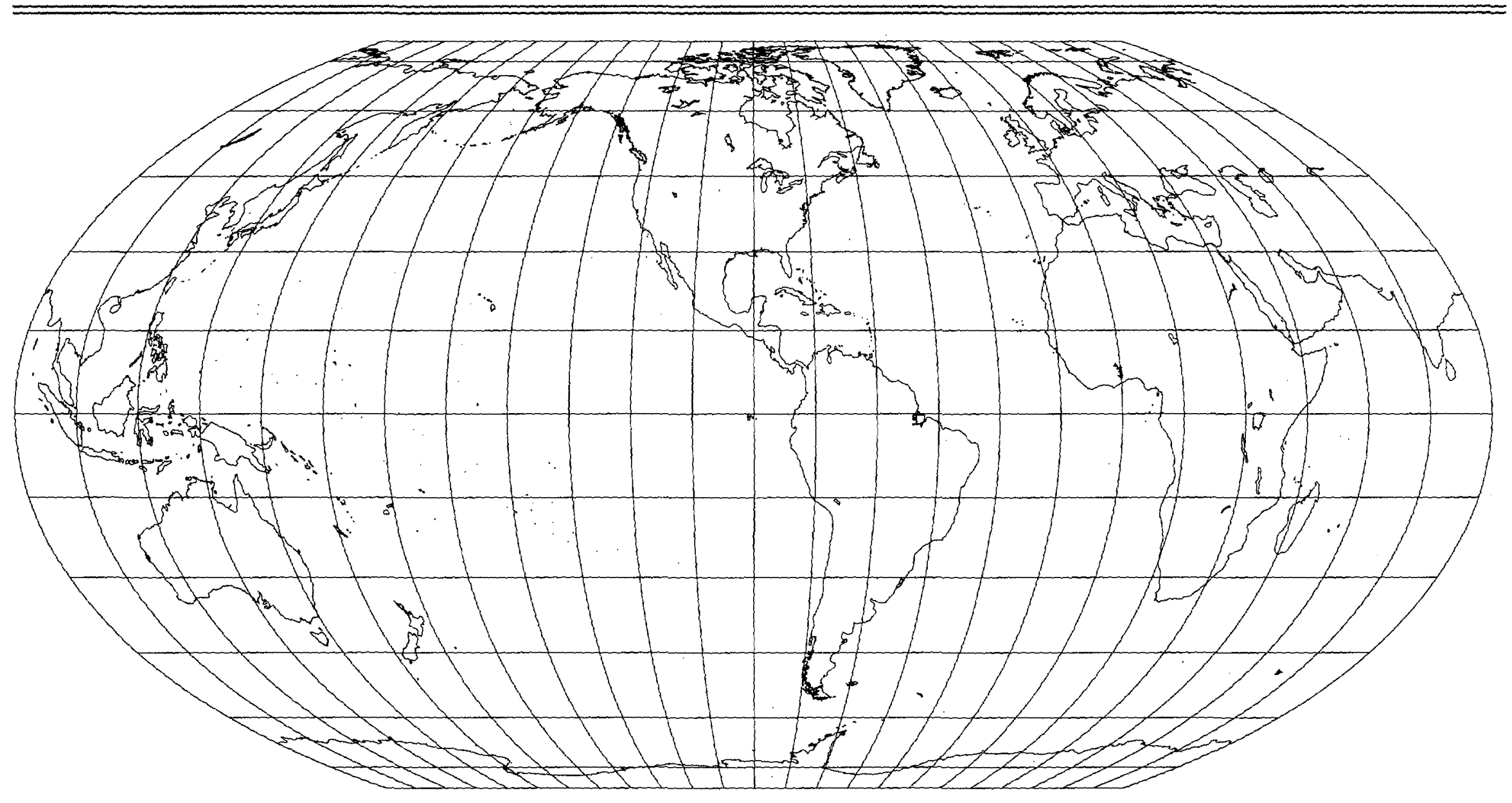




\section{HATANO ASYMMETRICAL EQUAL-AREA Projection}

\section{Classifications}

\section{Pseudocylindrical}

Equal area

\section{Graticule}

Meridians: The central meridian is a straight line 0.48 as long as the Equator. Other meridians are equally spaced elliptical arcs, concave toward the central meridian, but the eccentricity of each ellipse changes at the Equator.

Parallels: Unequally spaced straight parallel lines. Perpendicular to the central meridian. Spacing not symmetrical about the Equator. Poles: Lines. The North Pole is two-thirds as long and the South Pole is three-fourths as long as the Equator.

Symmetry: About the central meridian but not about the Equator

\section{Scale}

True along latitudes $40^{\circ} 42^{\prime} \mathrm{N}$. and $38^{\circ} 27^{\prime} \mathrm{S}$. Constant along any given latitude; normally different for the latitude of opposite sign

\section{Distortion}

Free of distortion only at latitudes $40^{\circ} 42^{\prime} \mathrm{N}$. and $38^{\circ} 27^{\prime} \mathrm{S}$. at the central meridian (fig. $23 \mathrm{~A}$ ).

\section{Origin}

Presented by Masataka Hatano of Japan in 1972 as a modification of the Mollweide and Putnins $\mathbf{P}_{2}^{\prime}$ projections

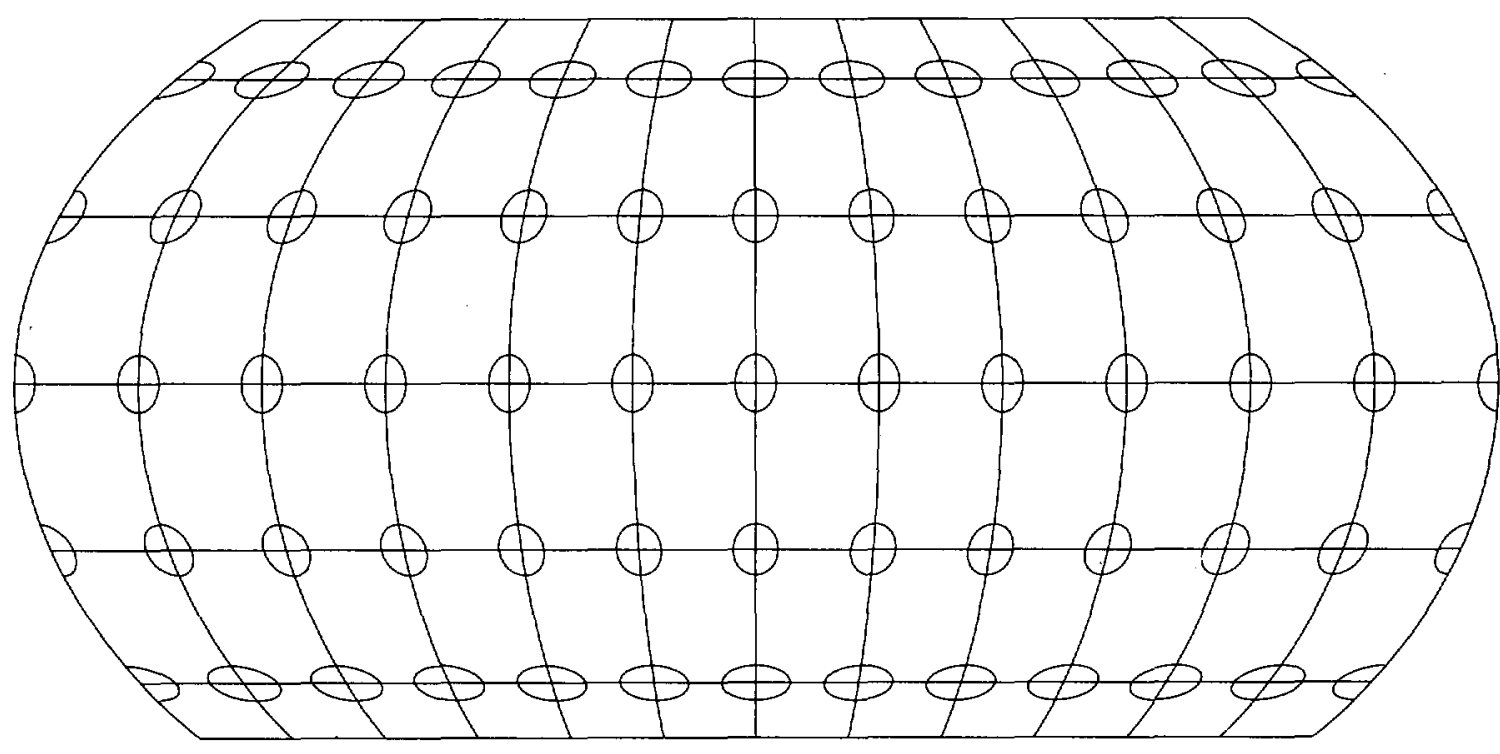

Similar projections

Mollweide projection (p. 54) uses full

symmetrical semiellipses for meridians, and poles are points.

Wagner IV projection (p. 62) uses arcs less than semiellipses for meridians, but they are symmetrical about the Equator, and poles are lines half the length of the Equator.

Hatano Symmetrical Equal-Area projection (1972) uses the same graticule for the Northern Hemisphere. The Southern Hemisphere is symmetrical (the South Pole is also two-thirds the length of the Equator). 


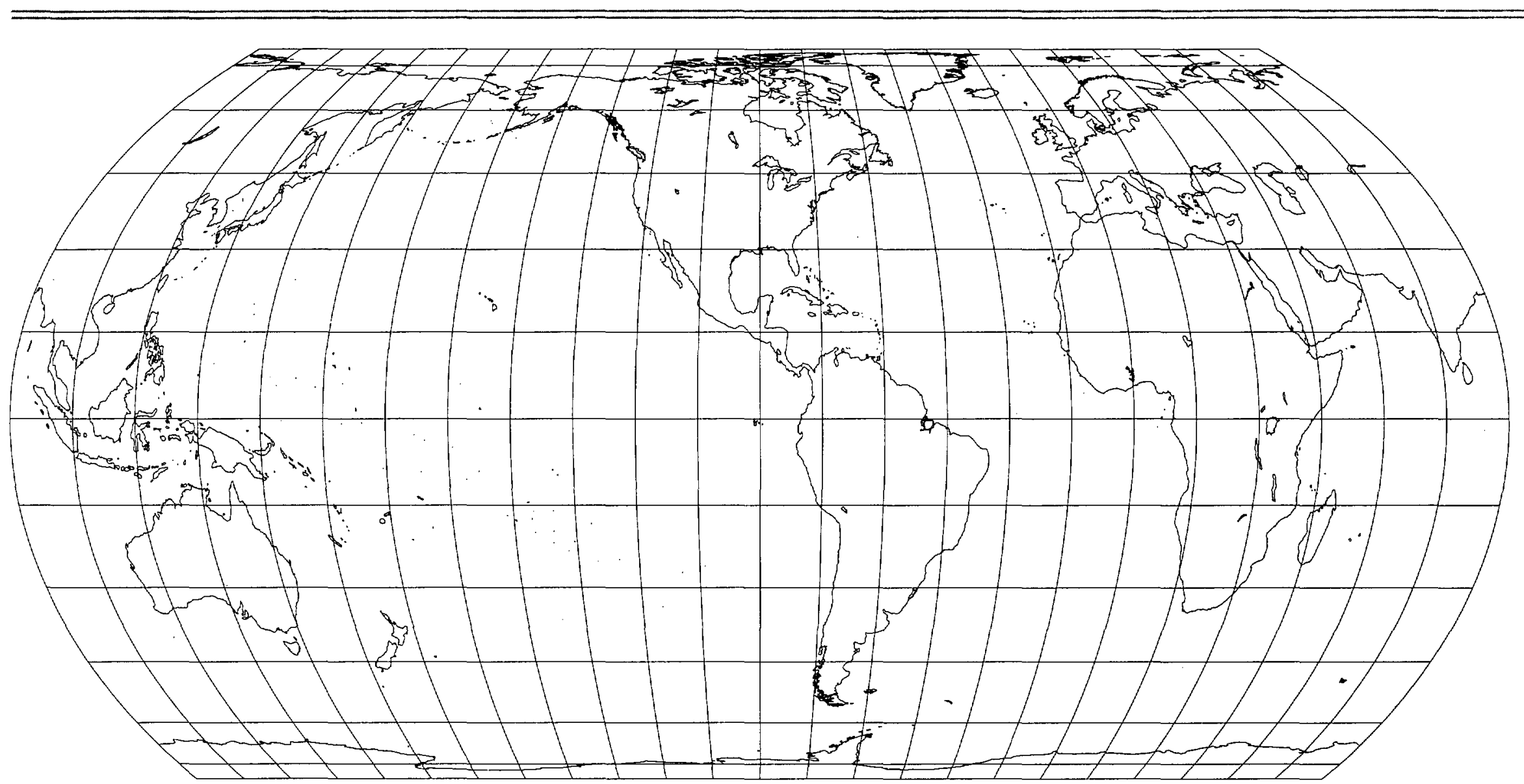




\section{GOODE HOMOLOSINE Projection}

\section{Classifications}

Pseudocylindrical composite

Equal area

\section{Graticule}

Meridians: In the interrupted form, there are six central meridians, each a straight line 0.22 as long as the Equator but not crossing the Equator. Other meridians are equally spaced sinusoidal curves between latitudes $40^{\circ} 44^{\prime} \mathrm{N}$. and $\mathrm{S}$. and elliptical arcs elsewhere, all concave toward the central meridian. There is a slight bend in meridians at the $40^{\circ} 44^{\prime}$ latitudes.

Parallels: Straight parallel lines, perpendicular to the central meridians. Equally spaced

between latitudes $40^{\circ} 44^{\prime} \mathrm{N}$. and S. Gradually closer together beyond these latitudes as a pole is approached.

Poles: Points

Symmetry: None in the usual interrupted form. If uninterrupted, there is symmetry about the central meridian or the Equator.

\section{Scale}

True along every latitude between $40^{\circ} 44^{\prime} \mathrm{N}$. and $S$. and along the central meridian within the same latitude range. At higher latitudes, scale varies but is the same for the latitude of opposite sign.

\section{Distortion}

Same as Sinusoidal projection between latitudes $40^{\circ} 44^{\prime}$ N. and S. (see fig. 13A). Same as. Mollweide projection beyond this range (see fig. 19A). Goode Homolosine projection is almost always presented interrupted, as the inventor originally intended.

\section{Usage}

Numerous world maps, especially in Goode's Atlas (Rand McNally)

\section{Origin}

Developed in 1923 by J. Paul Goode (1862-1932) of the University of Chicago as a merging of the Mollweide (or Homolographic) with the Sinusoidal at the parallels of identical scale, latitudes $40^{\circ} 44^{\prime} \mathrm{N}$. and S.; hence, the name Homolosine. Frequently plotted with some northern regions repeated on different interrupted sections to reduce discontinuities of land.

\section{Other names}

Homolosine

\section{Similar projections}

Identical with Sinusoidal (p. 37) between latitudes $40^{\circ} 44^{\prime} \mathrm{N}$. and $\mathrm{S}$. and identical with Mollweide (p. 54) beyond these latitudes, except that the Mollweide portions are closer to the Equator than they are on the original

\section{projection}

Sinu-Mollweide, devised by Allen K. Philbrick in 1953, is a merging of the Mollweide, used from the North Pole to latitude $40^{\circ} 44^{\prime}$ S., with the Sinusoidal, used only south of latitude $40^{\circ} 44^{\prime}$ S. It is equal area.

In 1968, György Érdi-Kraus used a special FlatPolar Sinusoidal projection between latitudes $60^{\circ} \mathrm{N}$. and S. and the Mollweide projection poleward. It is equal area only within each of the two projections, which are at two different area scales. 
Figure 24.-Interrupted Goode Homolosine projection, with shorelines, $10^{\circ}$ graticule, to show land masses.

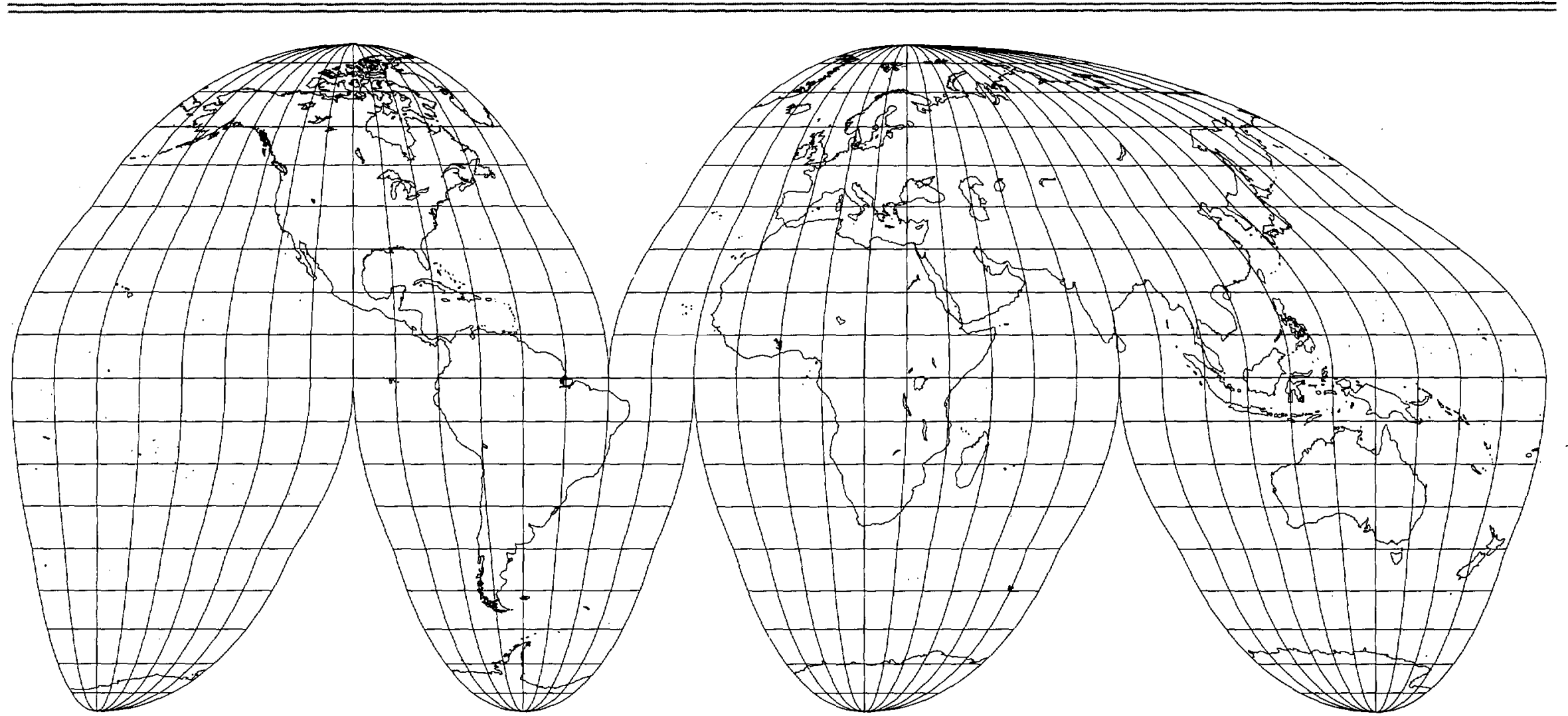




\section{Classifications}

Pseudocylindrical

Equal area

\section{Graticule}

Meridians: Central meridian is a straight line half as long as the Equator. Other meridians are equally spaced complex curves intersecting at the poles and concave toward the central meridian.

Parallels: Unequally spaced straight parallel lines perpendicular to the central meridian

Poles: Points

Symmetry: About the central meridian or the Equator

\section{Scale}

True along latitudes $40^{\circ} 15^{\prime} \mathrm{N}$. and $\mathrm{S}$.

Constant along any given latitude; same for the latitude of opposite sign

\section{Distortion}

Distortion is severe near outer meridians at high latitudes (fig. 25A) but can be

substantially reduced by interruption. Free of distortion only at latitudes $40^{\circ} 15^{\prime} \mathrm{N}$. and $\mathrm{S}$. at the central meridian.

\section{Other features}

Designed as the arithmetic mean of the Sinusoidal and Mollweide projections for north. south $(y)$ coordinates only, when drawn with the same area scale

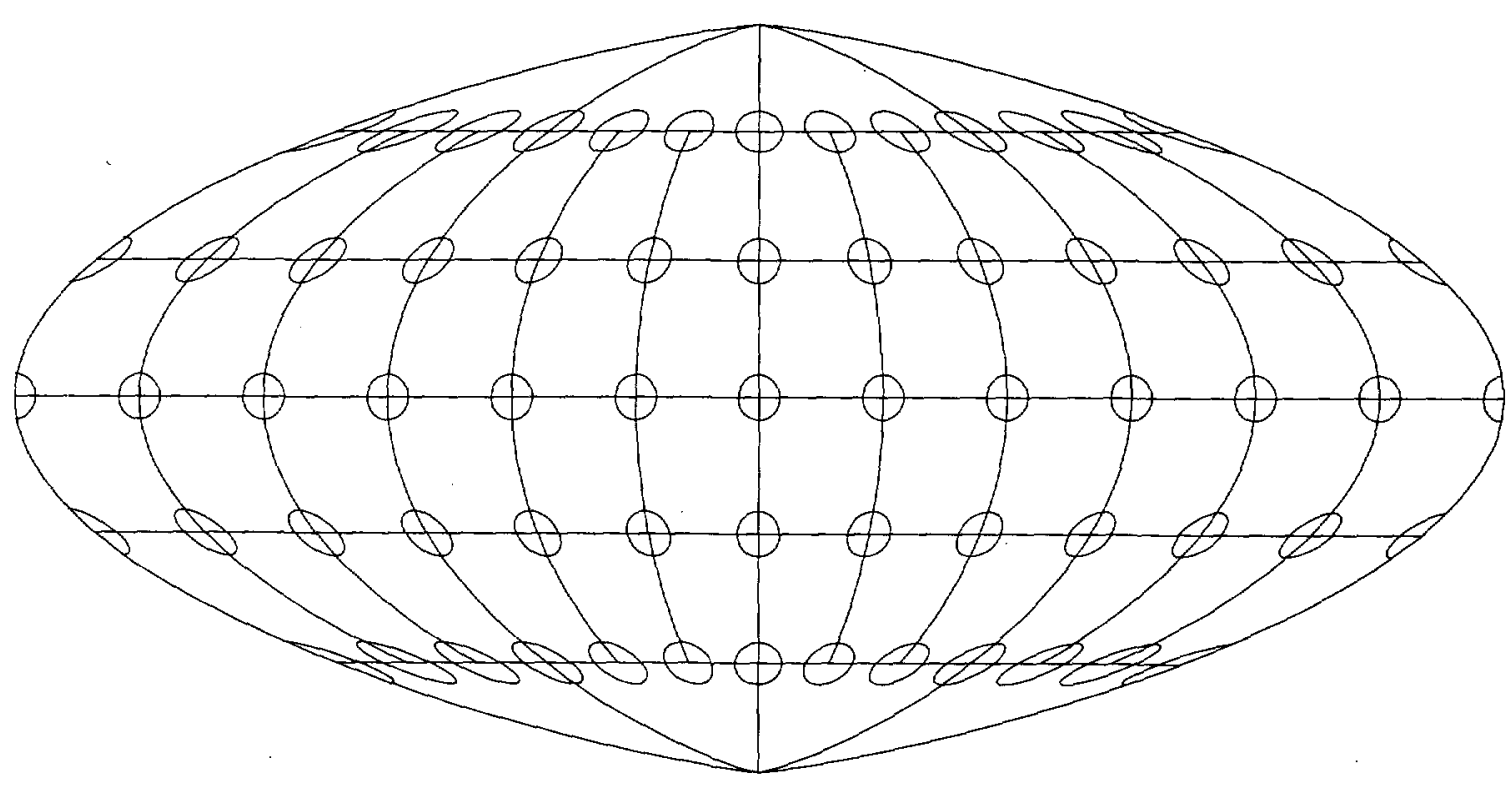

\section{Usage}

Thematic world maps in atlases and textbooks, usually in interrupted form

\section{Origin}

Presented by S. Whittemore Boggs (1884-1954)

of the U.S. Department of State in 1929

\section{Similar projections}

Several pseudocylindricals, such as the Sinusoidal (p. 37) and Craster Parabolic (p. 70). 
Figure 25B.-Boggs Eumorphic projection, with shorelines, $15^{\circ}$ graticule. Central meridian $90^{\circ} \mathrm{W}$.

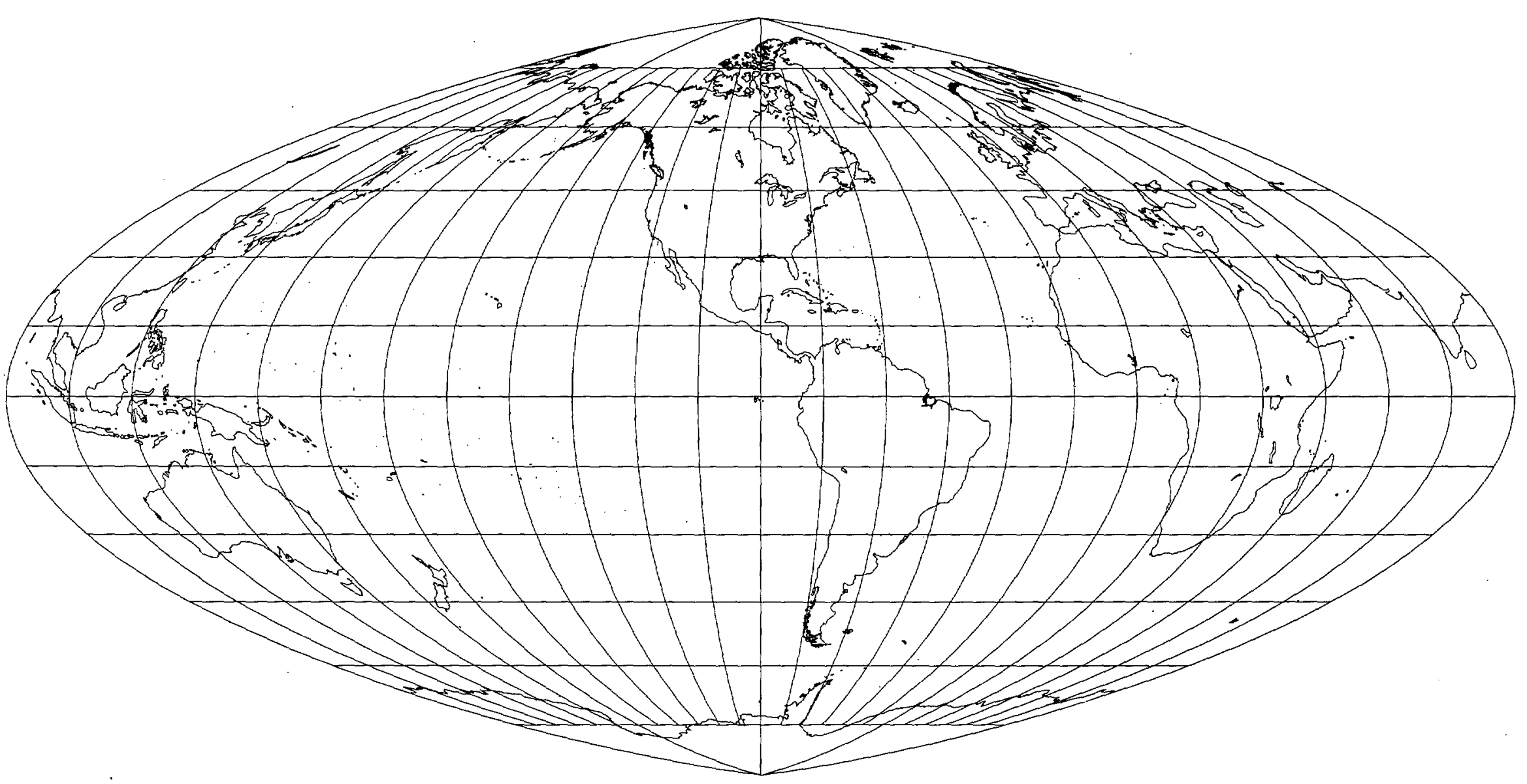




\section{Classifications \\ Pseudocylindrical \\ Equal area}

\section{Graticule}

Meridians: Central meridian is a straight line half as long as the Equator. Other meridians are equally spaced parabolas intersecting at the poles and concave toward the central meridian.

Parallels: Unequally spaced straight parallel lines, farthest apart near the Equator; spacing changes very gradually. Perpendicular to the central meridian.

Poles: Points

Symmetry: About the central meridian or the Equator

\section{Scale}

True along latitudes $36^{\circ} 46^{\prime} \mathrm{N}$. and S.

Constant along any given latitude; same for the latitude of opposite sign

\section{Distortion}

Distortion is severe near outer meridians at high latitudes (fig. 26A) but somewhat less than that of the Sinusoidal projection. Can be substantially reduced by interruption (see similar example for Sinusoidal, fig. 13G). Free of distortion only at latitudes $36^{\circ} 46^{\prime} \mathrm{N}$. and S. at the central meridian.

\section{Usage}

Thematic world maps in textbooks

\section{Origin}

Presented by John Evelyn Edmund Craster

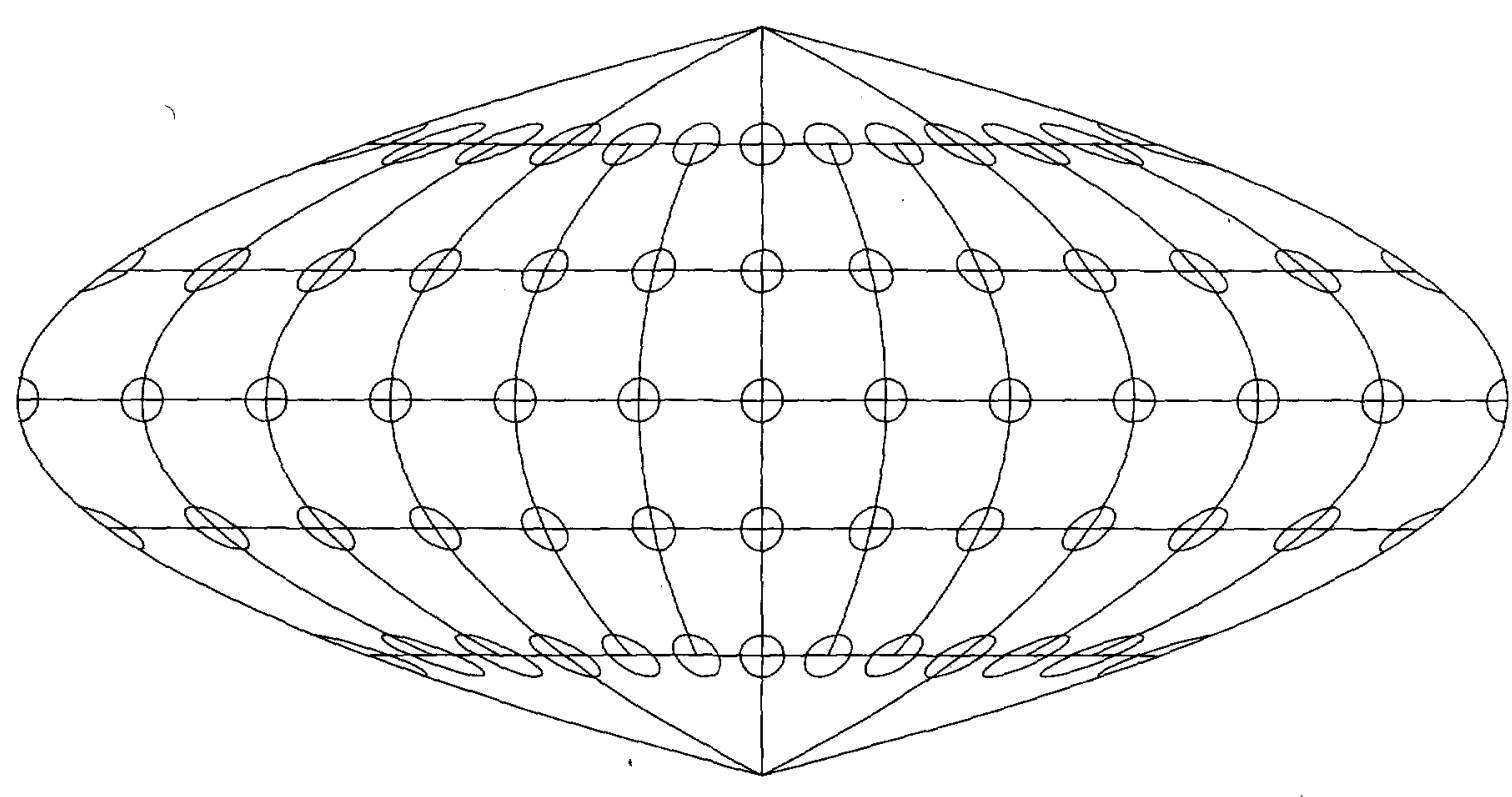

(1873-?) of England in 1929. Developed further by Charles $H$. Deetz and O.S. Adams in 1934

\section{Aspects}

Oblique aspect used for map of Asia by National Geographic Society

\section{Other names}

Putniñ $\breve{P_{4}}$, independently presented in Latvia in 1934.

\section{Similar projections}

Several pseudocylindricals, such as the Sinusoidal (p. 37) and the Boggs Eumorphic (p. 68)

Putninš $P_{3}$ projection (1934) has meridians, poles, and Equator identical to those of the Craster, but parallels are equally spaced. 


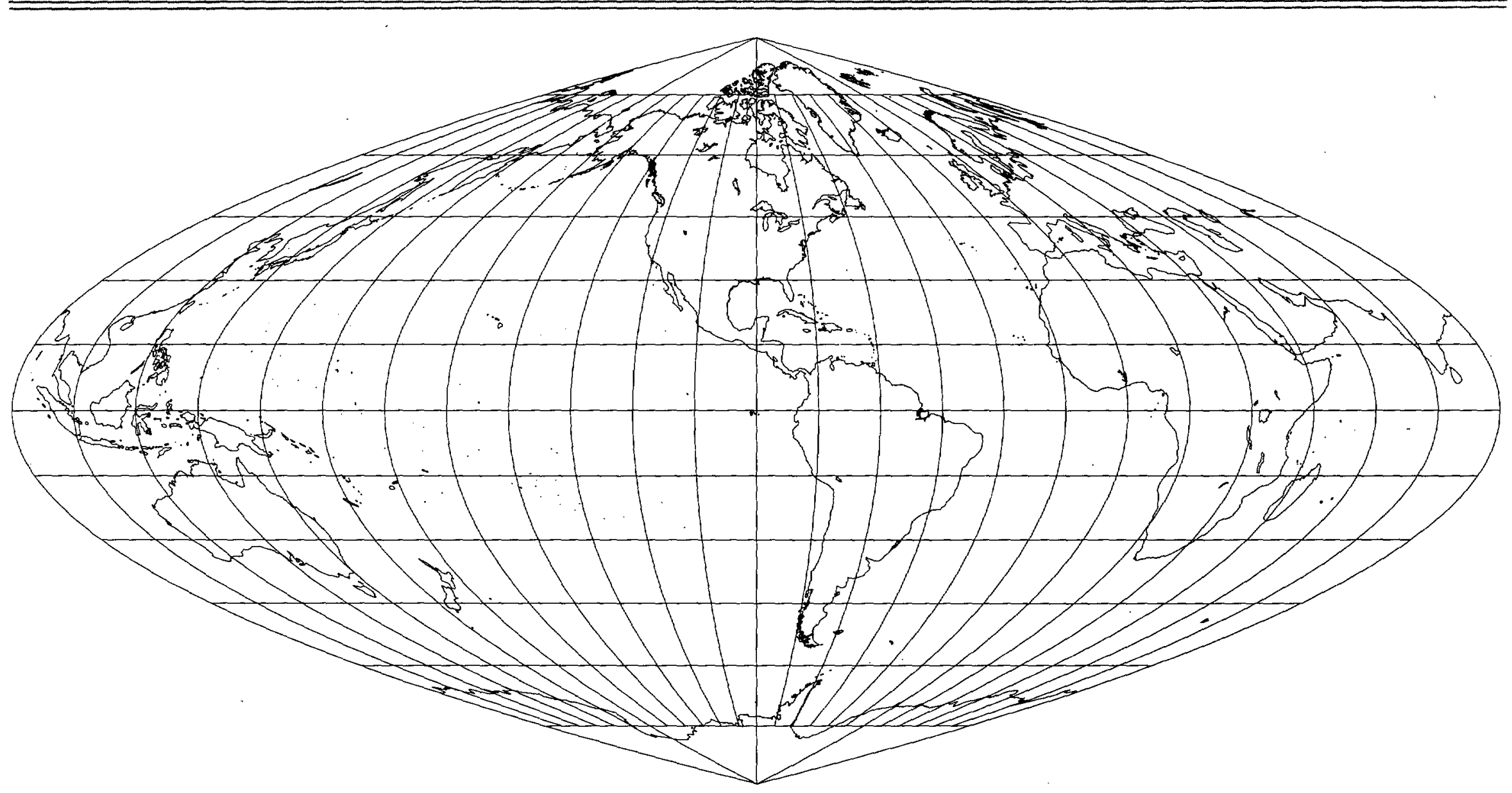




\section{McBRYDE-THOMAS FLAT-POLAR PARABOLIC Projection}

\section{Classifications}

Pseudocylindrical

Equal area

\section{Graticule}

Meridians: Central meridian is a straight line 0.48 as long as the Equator. Other meridians are equally spaced parabolic curves, concave toward the central meridian.

Parallels: Unequally spaced straight parallel lines, farthest apart near the Equator.

Perpendicular to the central meridian.

Poles: Lines one-third as long as the Equator Symmetry: About the central meridian or the Equator

\section{Scale}

True along latitudes $45^{\circ} 30^{\prime} \mathrm{N}$. and S.

Constant along any given latitude; same for the latitude of opposite sign

\section{Distortion}

Distortion is severe near outer meridians at high latitudes (fig. 27A) but less than the corresponding distortion on pointed-polar projections. Free of distortion only at latitudes $45^{\circ} 30^{\prime} \mathrm{N}$. and $\mathrm{S}$. at the central meridian.

\section{Usage}

Basis of merged projections by McBryde

\section{Origin}

Presented by F. Webster McBryde and Paul D. Thomas through the U.S. Coast and Geodetic Survey in 1949

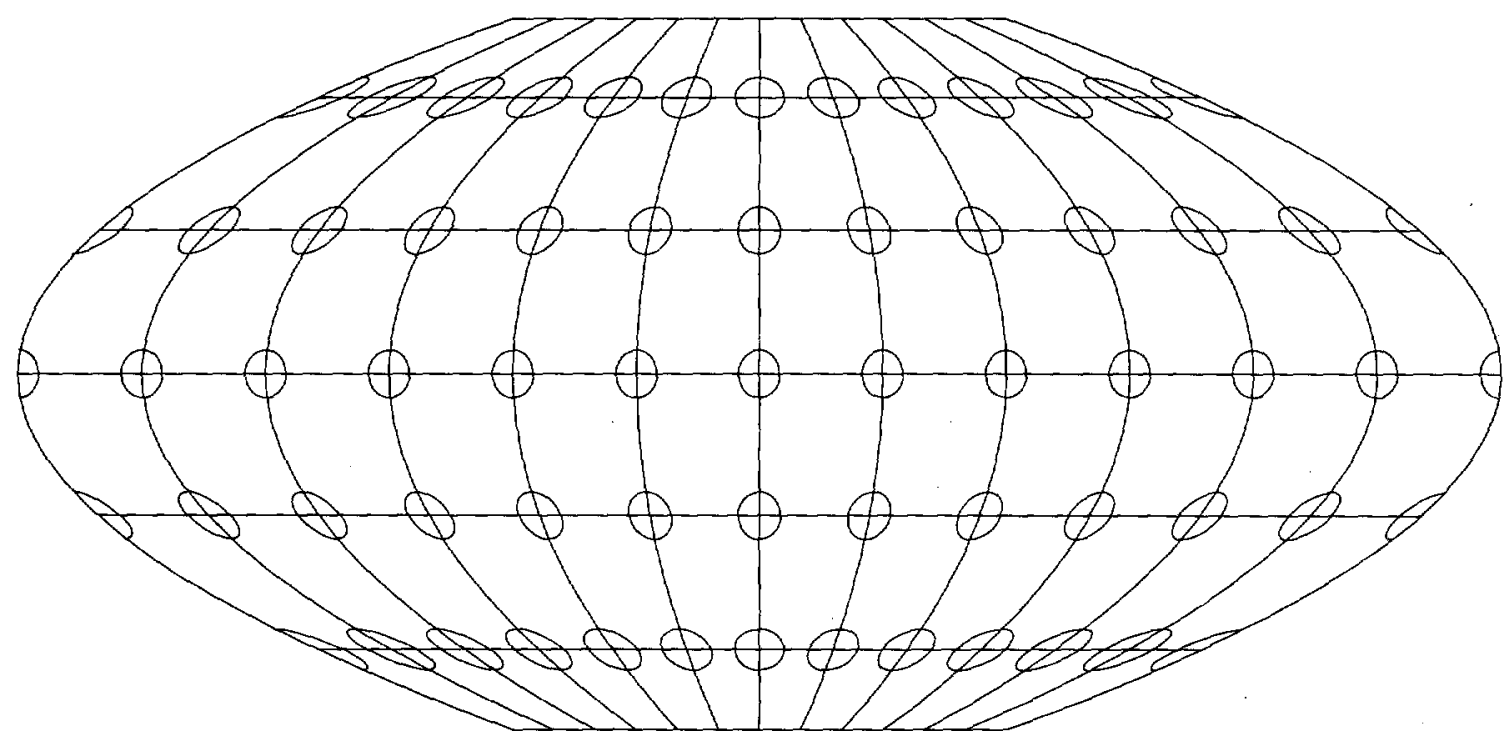

Figure 27A.-McBryde-Thomas Flat-Polar Parabolic projection, with Tissot indicatrices, $30^{\circ}$ graticule.

\section{Similar projections}

Craster Parabolic projection (p. 70), equal area with parabolic meridians but with pointed poles and a central meridian that is half the length of the Equator

Putniṇs $\mathrm{P}_{4}{ }^{\prime}$ projection (1934), equal area with parabolic meridians but with poles and a central meridian that are all half the length of the Equator. Werenskiold I (1944) is identical to Putniñs $\mathrm{P}_{4}^{\prime}$ except for the scale.

Putniṇs $\mathrm{P}_{3}$ ' projection (1934) has meridians, poles, and Equator identical to those of Putnins $\mathbf{P}_{4}{ }^{\prime}$, but parallels are equally spaced. 
Figure 27B.-McBryde-Thomas Flat-Polar Parabolic projection, with shorelines, $15^{\circ}$ graticule. Central meridian $90^{\circ} \mathrm{W}$.

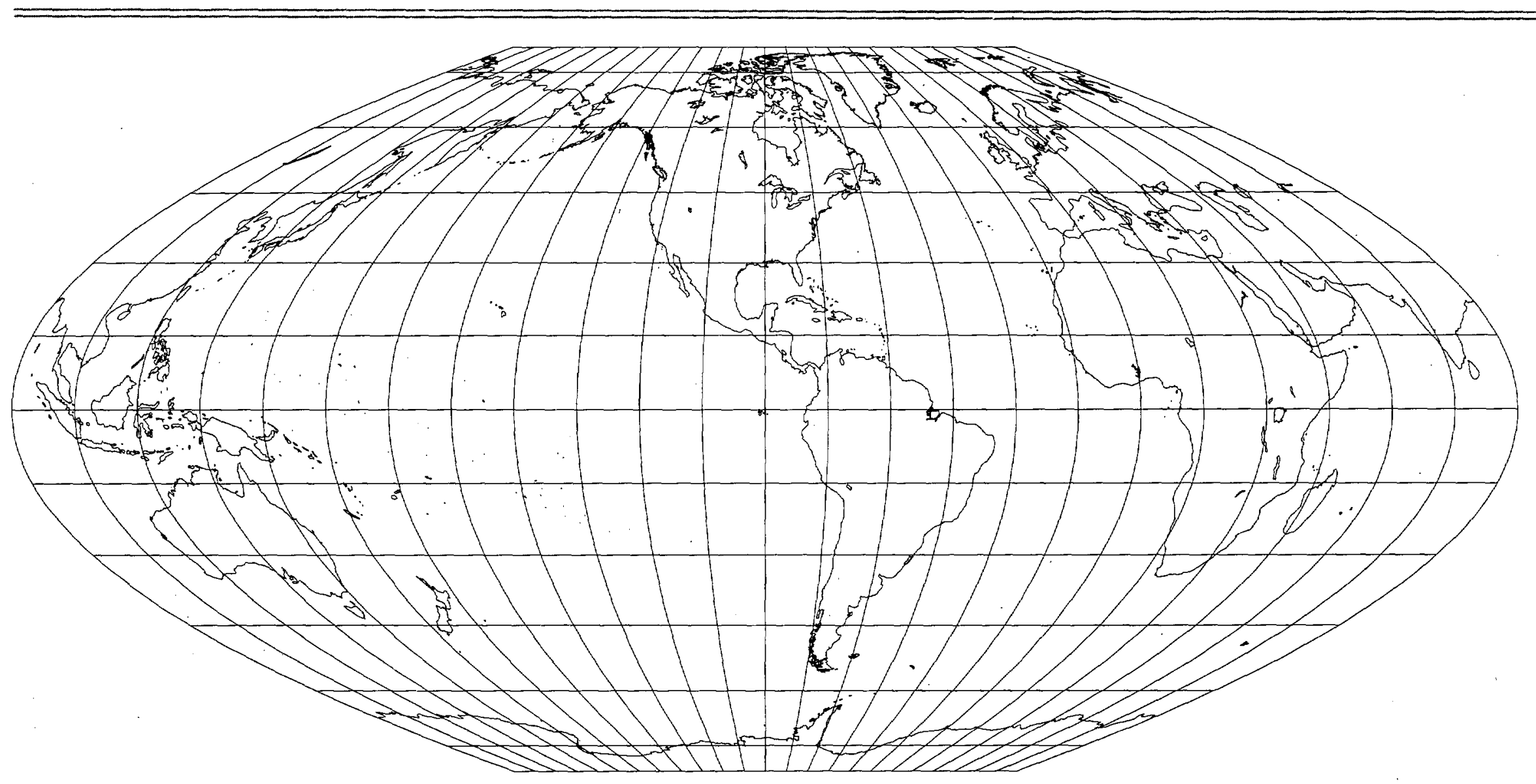




\section{QUARTIC AUTHALIC Projection}

\section{Classifications}

Pseudocylindrical

Equal area

\section{Graticule}

Meridians: Central meridian is a straight line 0.45 as long as the Equator. Other meridians are equally spaced curves fitting a fourth-order (quartic) equation and concave toward the central meridian.

Parallels: Unequally spaced straight parallel lines, farthest apart near the Equator. Spacing changes gradually. Perpendicular to the central meridian.

Poles: Points

Symmetry: About the central meridian or the Equator

\section{Scale}

True along the equator

Constant along any given latitude; same for the latitude of opposite sign

\section{Distortion}

Distortion is severe near outer meridians at high latitudes (fig. $28 A$ ) but somewhat less than that of the Sinusoidal projection. Distortion can be substantially reduced by interruption (see similar example for Sinusoidal, fig. 13G). Free of distortion along the Equator.

\section{Usage}

Basis for McBryde-Thomas Flat-Polar Quartic projection (p. 76)

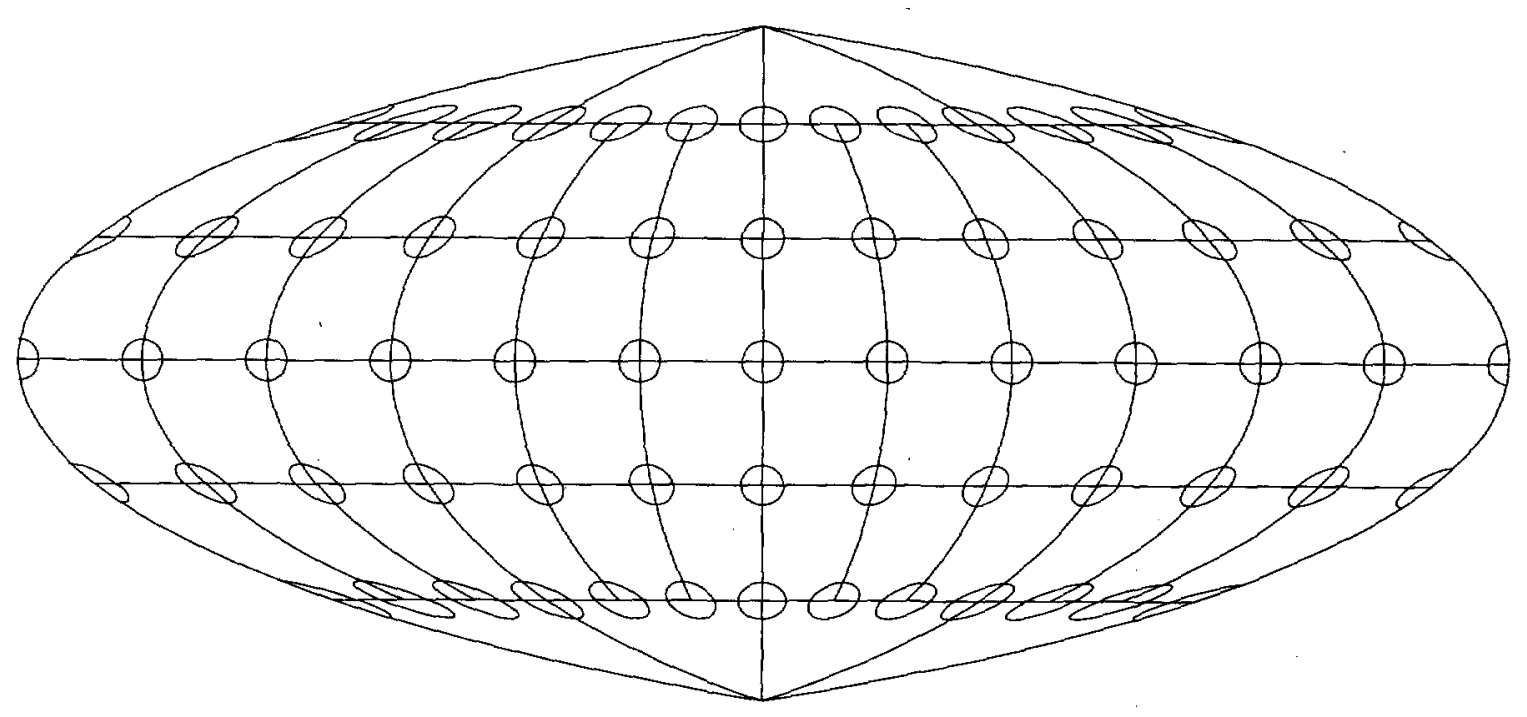

\section{Origin}

Presented by Karl Siemon (?-1937) of Germany in 1937. Independently presented by Oscar Sherman Adams (1874-1962) of the U.S. Coast and Geodetic Survey in 1945.

\section{Similar projections}

Several pseudocylindricals, such as the Sinusoidal (p. 37)

Eckert-Greifendorff (p. 166) has slightly curved parallels. 


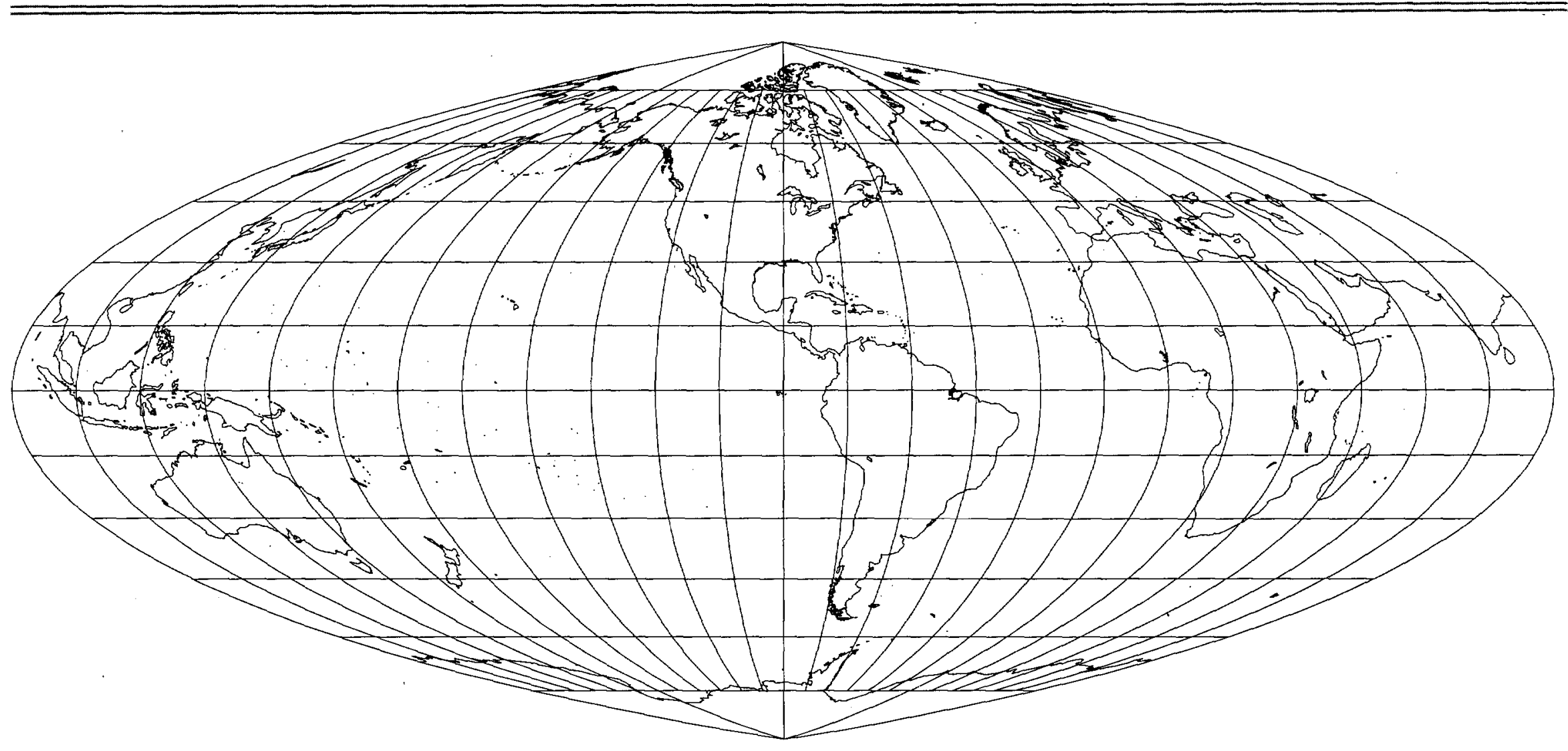




\section{MCBRYDE-THOMAS FLAT-POLAR QUARTIC Projection}

\section{Classifications}

Pseudocylindrical

Equal area

\section{Graticule}

Meridians: Central meridian is a straight line 0.45 as long as the Equator. Other meridians are equally spaced curves fitting a fourth-order (quartic) equation and concave toward the central meridian.

Parallels: Unequally spaced straight parallel lines, farthest apart near the Equator. Perpendicular to the central meridian.

Poles: Lines one-third as long as the Equator Symmetry: About the central meridian or the Equator

\section{Scale}

True along latitudes $33^{\circ} 45^{\prime} \mathrm{N}$. and $\mathrm{S}$.

Constant along any given latitude; same for the latitude of opposite sign

\section{Distortion}

Distortion is severe near outer meridians at high latitudes (fig. 29A) but less than the corresponding distortion on pointed-polar projections. Free of distortion only at latitudes $33^{\circ} 45^{\prime} \mathrm{N}$. and $\mathrm{S}$. at the central meridian.

\section{Usage}

Examples in various geography textbooks. Basis of merged projections by McBryde.

\section{Origin}

Presented by F. Webster McBryde and Paul

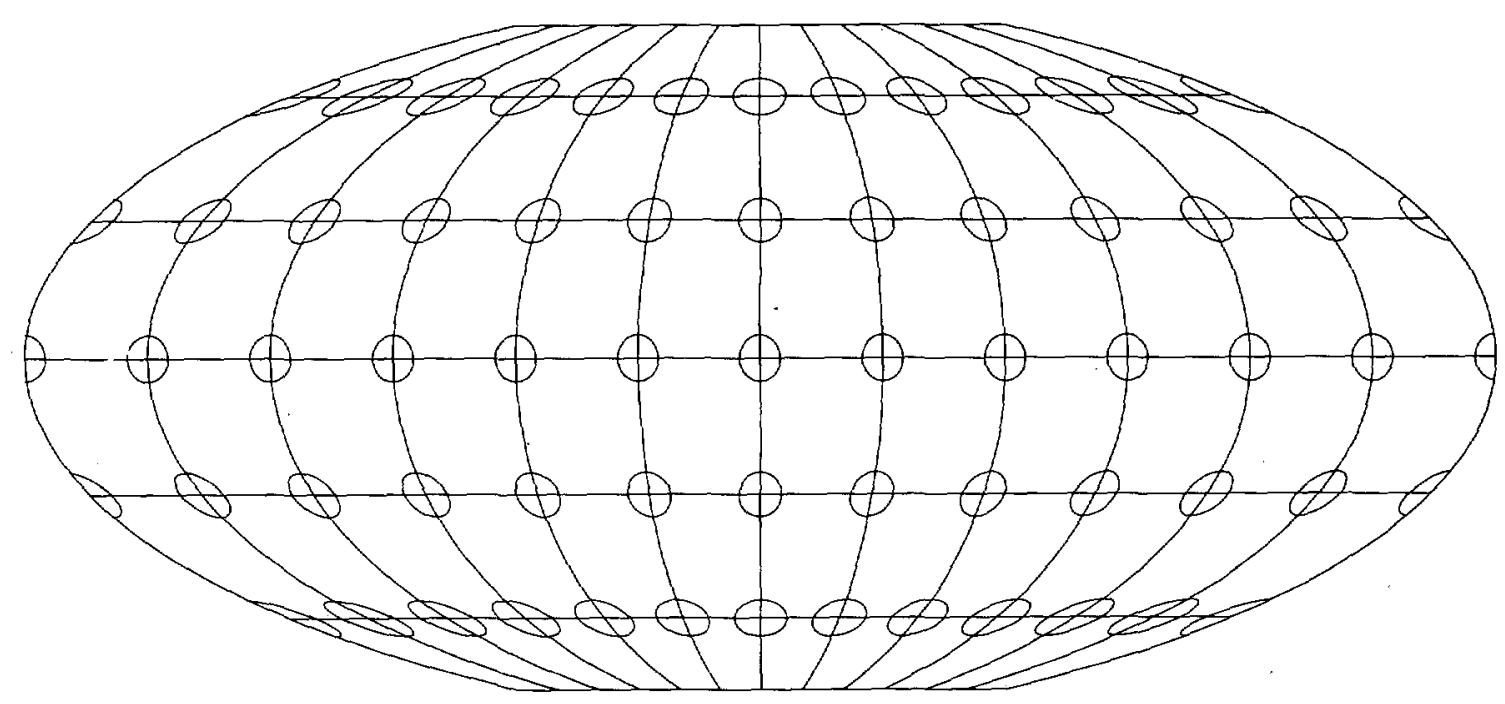

Figure 29A.-McBryde-Thomas Fiat-Polar Quartic projection, with Tissot indicatrices, $30^{\circ}$ graticule.

D. Thomas through the U.S. Coast and Geodetic Survey in 1949

\section{Other names}

Flat-Polar Quartic projection

\section{Similar projections}

Quartic Authalic projection (p. 74), the basis of this projection, has pointed poles.

Other McBryde-Thomas projections also have poles one-third the length of the Equator and otherwise resemble the projection described here. 
Figure 298.-McBryde-Thomas Flat-Polar Quartic projection, with shorelines, $15^{\circ}$ graticule. Central meridian $90^{\circ} \mathrm{W}$

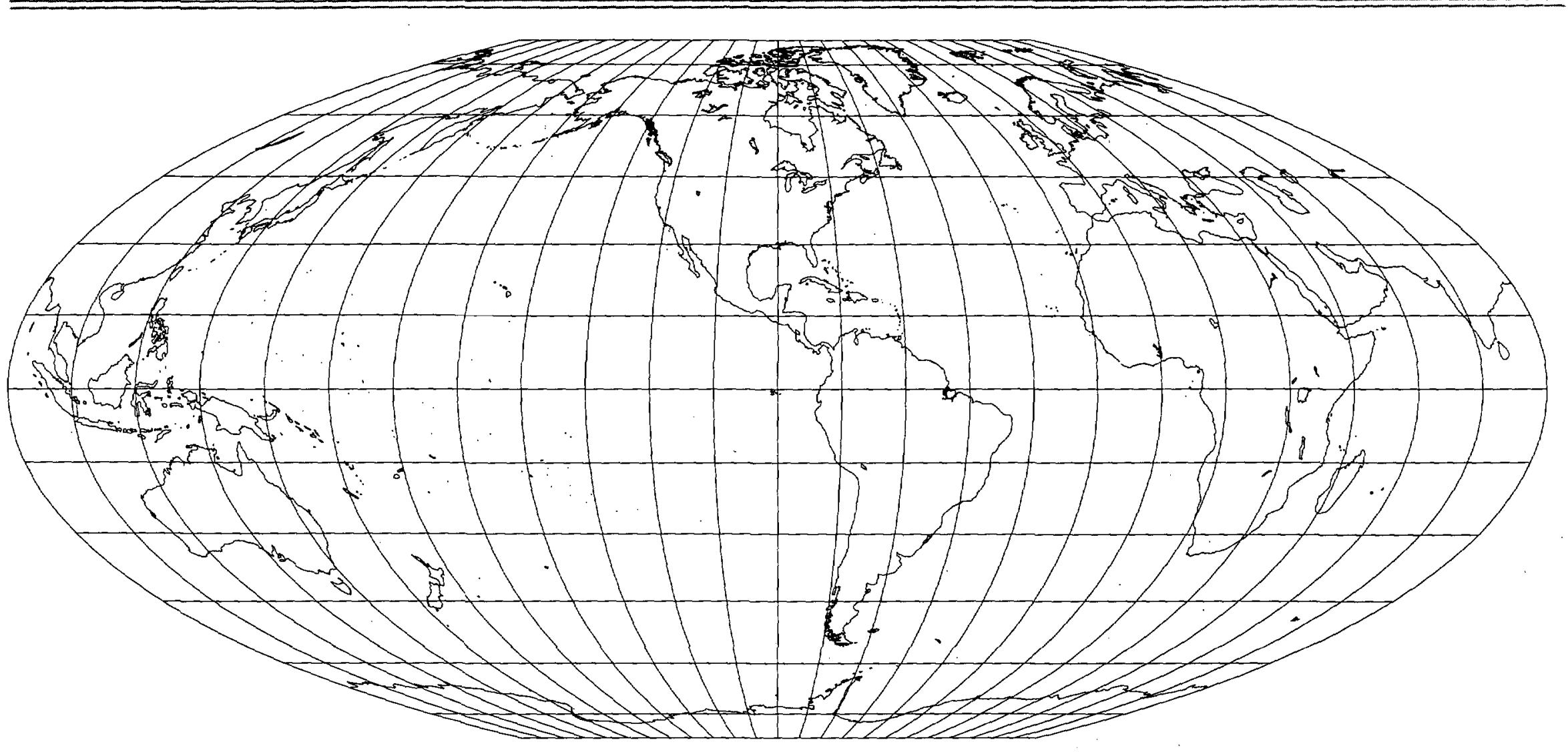




\section{PUTNINS $P_{5}$ Projection}

\section{Classifications}

Pseudocylindrical

Equally spaced parallels

Neither conformal nor equal area

\section{Graticule}

Meridians: Central meridian is a straight line half as long as the Equator. Other meridians are equally spaced portions of hyperbolas, concave toward the central meridian.

Parallels: Equally spaced straight parallel lines, perpendicular to the central meridian. Poles: Points

Symmetry: About the central meridian or the Equator

\section{Scale}

True along latitudes $21^{\circ} 14^{\prime} \mathrm{N}$. and S.

Constant along any given latitude; same for the latitude of opposite sign. Constant along the central meridian.

\section{Distortion}

Similar to other pointed-polar cylindricals but not free of distortion at any point (fig. 30A).

\section{Origin}

Presented by Reinholds V. Putniñ̆ of Latvia in 1934

\section{Similar projections}

Putniṇs $P_{6}$ equal-area projection (1934) has

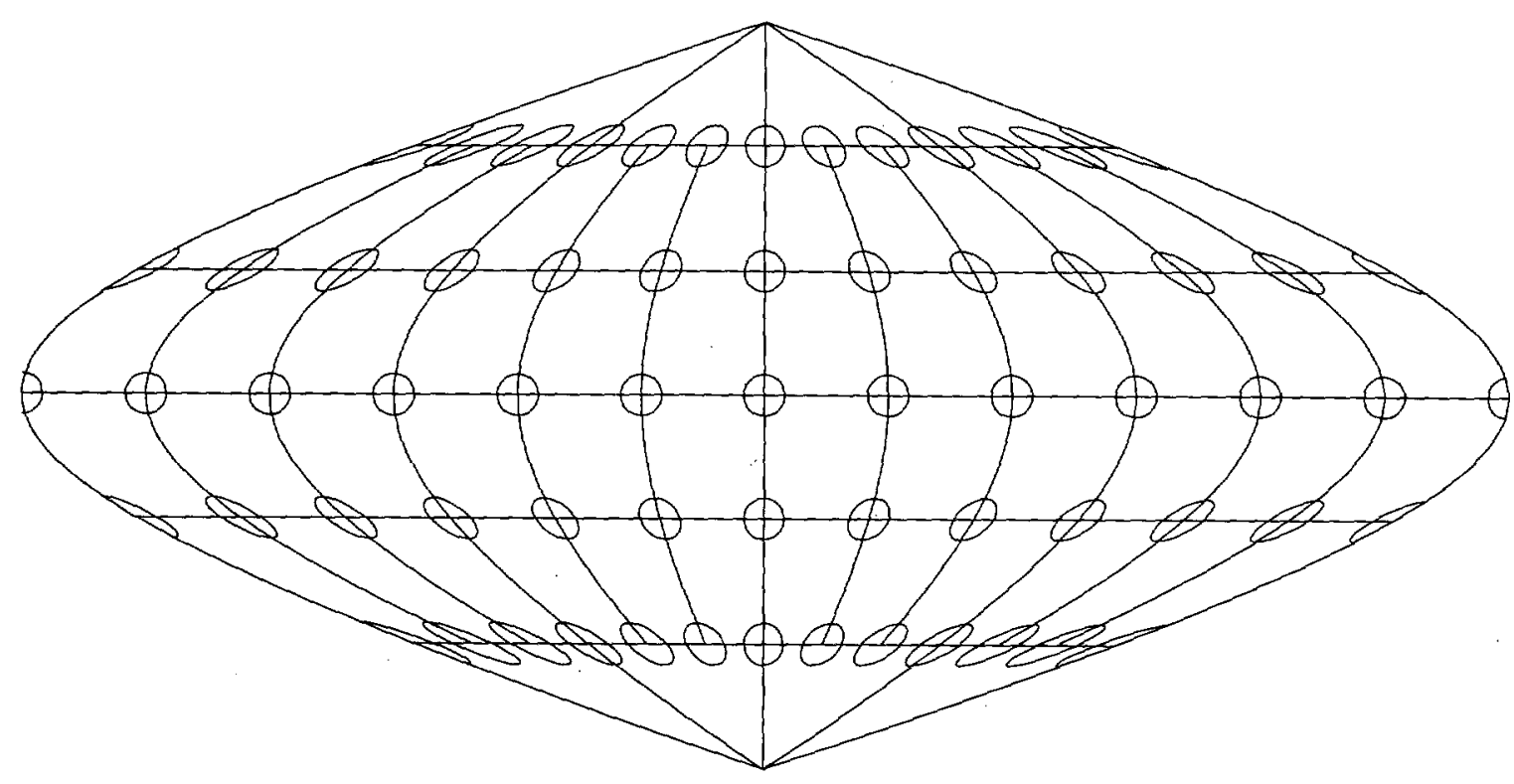

hyperbolic meridians identical in shape to those of the pointed-polar $P_{5}$; parallels are straight but are spaced for equal area.

Putnin̄ $\mathrm{P}_{5}$ ' projection (1934) has hyperbolic meridians, but the poles are straight lines half the length of the Equator. Parallels are equally spaced. The projection is not equal area.

Putnin̄ $\bar{s} P_{6}$ ' equal-area projection (1934) has hyperbolic meridians identical in shape to those of the flat-polar $\mathbf{P}_{5}{ }^{\prime}$, but parallels are straight lines spaced for equal area. 
Figure 30B.-Putnin $\bar{s} P_{5}$ projection, with shorelines, $15^{\circ}$ graticule. Central meridian $90^{\circ} \mathrm{W}$.

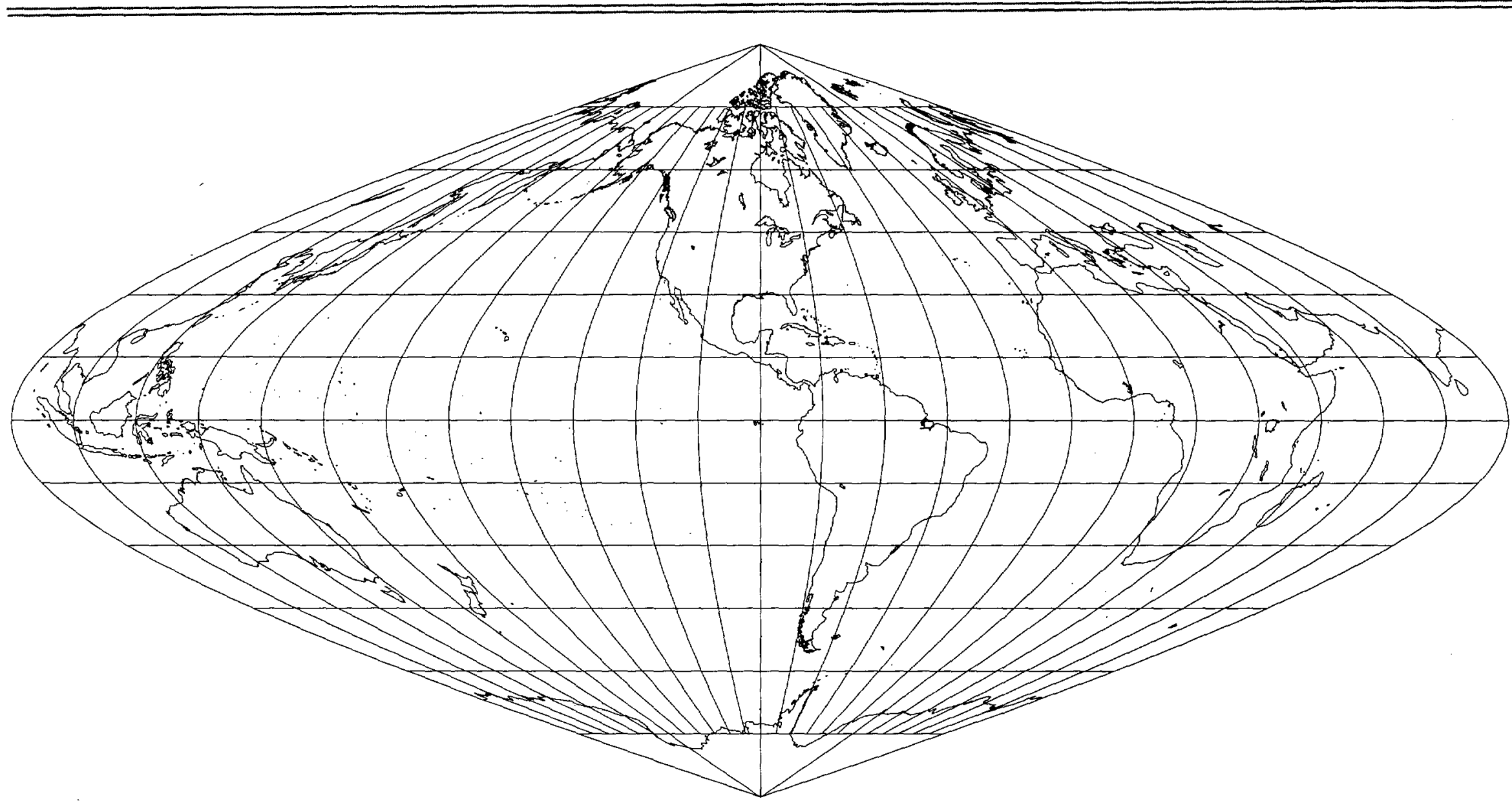




\section{DENOYER SEMI-ELLIPTICAL Projection}

\section{Classifications}

Modified pseudocylindrical

Equally spaced parallels

Neither conformal nor equal area

\section{Graticule}

Meridians: Central meridian is a straight line half as long as the Equator. Other meridians are curves that are nearly elliptical areas and nearly equally spaced, with some spreading near the outer limits; all are concave toward the central meridian.

Parallels: Equally spaced straight parallel lines. Perpendicular to the central meridian. Poles: Lines about $\mathbf{0 . 3 1}$ times the length of the Equator

Symmetry: About the central meridian or the Equator

\section{Scale}

True along the central meridian and the Equator

Constant along any given latitude; same for the latitude of opposite sign

\section{Distortion}

Free of distortion along the Equator (fig. 31A)

\section{Usage}

By Denoyer-Geppert in thematic maps and

atlases of the early and middle 20th century.

\section{Origin}

Developed by L. Philip Denoyer of Chicago about 1920. Exact design basis not known.

Empirical approximations are used for computer plots.

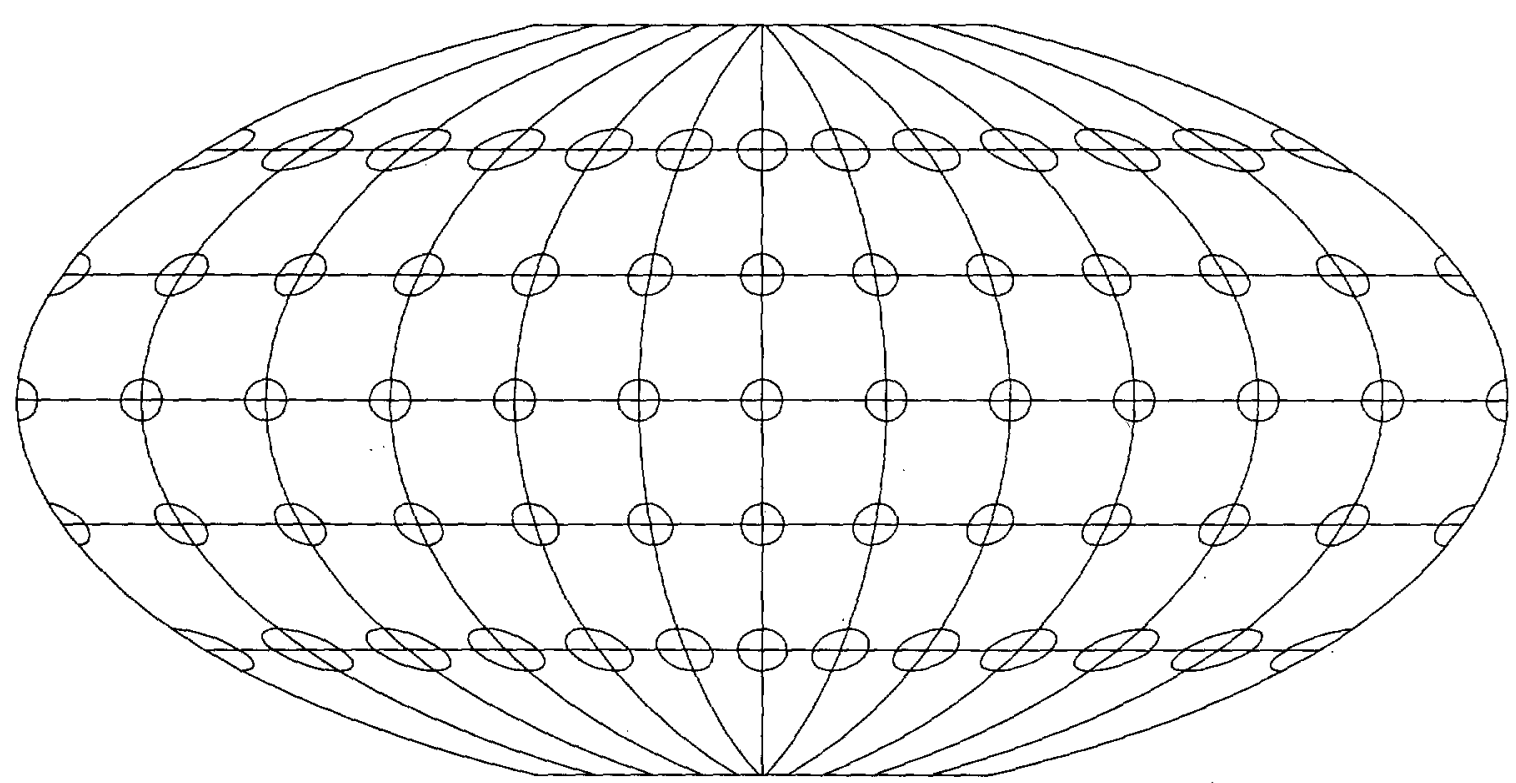

Figure 31A.-Denoyer Semi-Elliptical projection approximation, with Tissot indicatrices, $30^{\circ}$ graticule. 


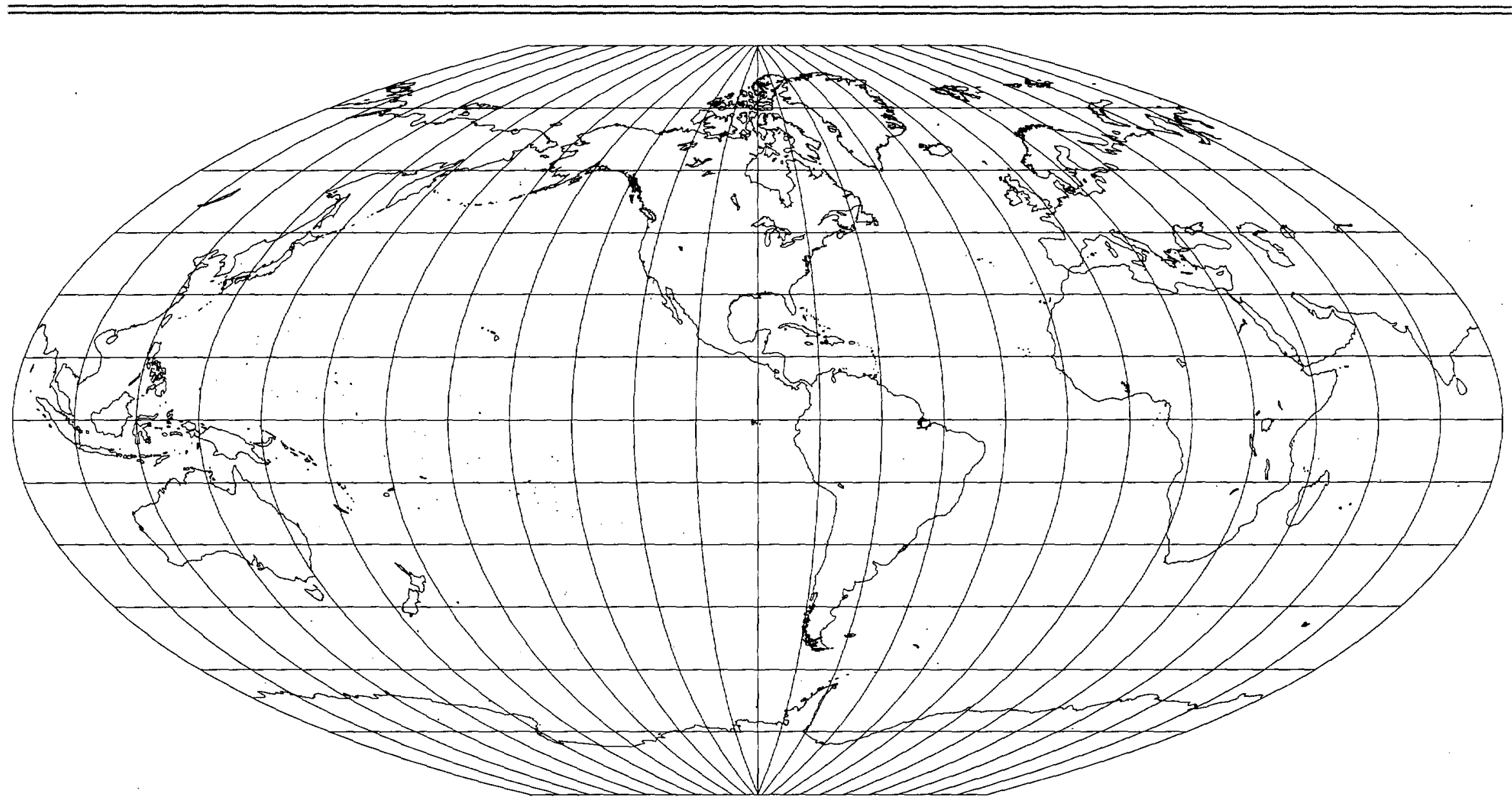




\section{ROBINSON Projection}

\section{Classifications}

Pseudocylindrical

Neither conformal nor equal area

\section{Graticule}

Meridians: Central meridian is a straight line 0.51 as long as the Equator. Other meridians resemble elliptical arcs, are equally spaced, and are concave toward the central meridian. Parallels: Straight parallel lines, equally spaced between latitudes $38^{\circ} \mathrm{N}$. and S.; space decreases beyond these limits.

Poles: Lines 0.53 as long as the Equator

Symmetry: About the central meridian or the Equator

\section{Scale}

True along latitudes $38^{\circ} \mathrm{N}$. and $\mathrm{S}$.

Constant along any given latitude; same for the latitude of opposite sign

\section{Distortion}

No point is completely free of distortion, but distortion is very low within about $45^{\circ}$ of the center and along the Equator (fig. 32B). Considerable distortion near the poles.

\section{Usage}

By Rand McNally, the National Geographic Societv, and others for numerous world maps

\section{Origin}

Presented by Arthur H. Robinson (1915- ) of the University of Wisconsin in 1963, at the request of Rand McNally and Company. Uses

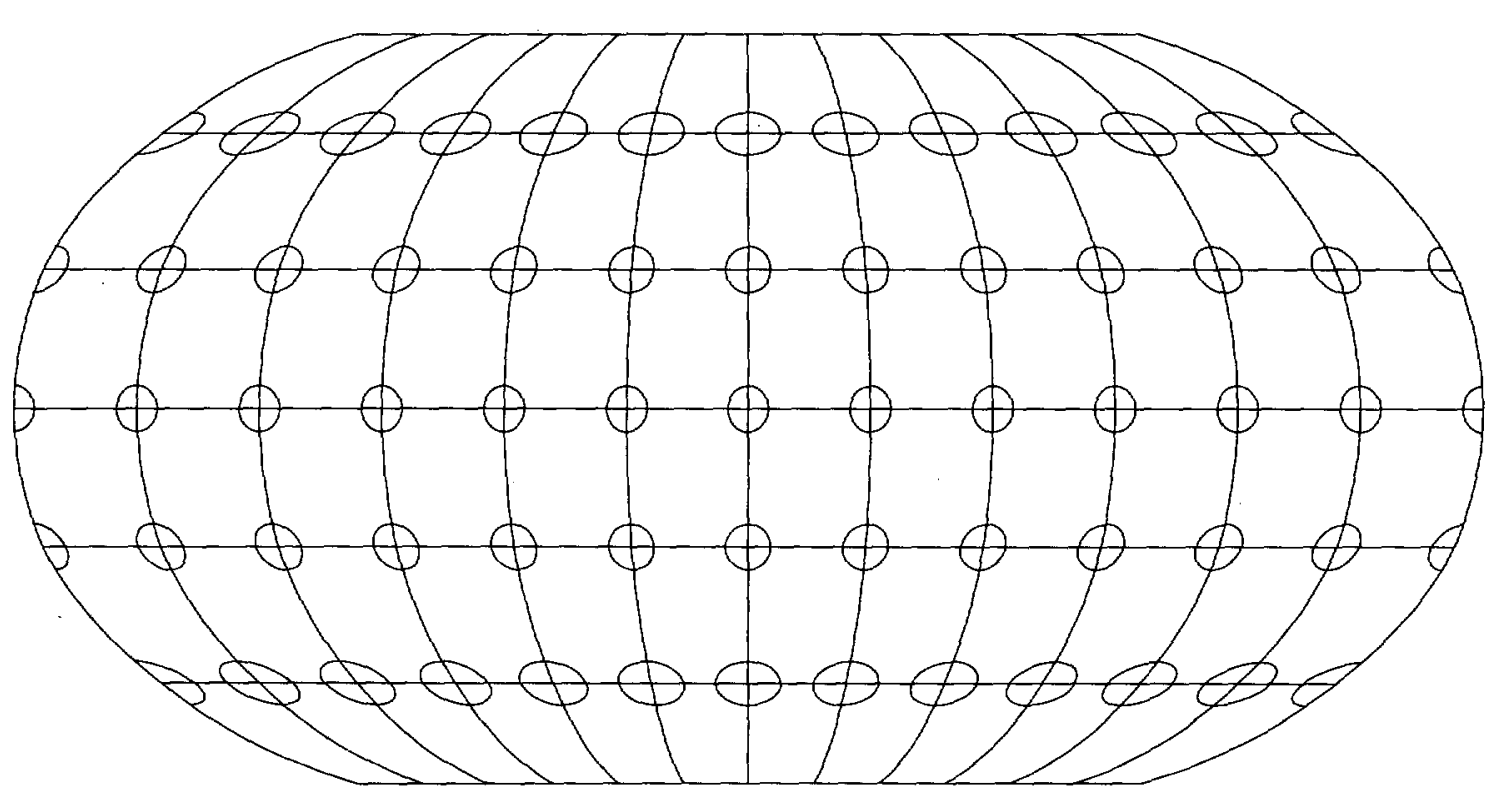

tabular coordinates rather than mathematical formulas to make the world map "look" right.

\section{Other names}

\section{Orthophanic ("right appearing")}

\section{Similar projections}

Wagner IV (p. 62), an equal-area projection on which meridians are elliptical arcs

In 1968, János Baranyi presented seven pseudocylindrical projections characterized by various graduated tabular spacings of the parallels. The poles are usually points but occasionally are lines, and the outer meridians $180^{\circ}$ from the central meridian are circular arcs combined in some cases with straight lines. His projections are neither equal area nor conformal. 
Figure $32 B$.-Robinson projection, with shorelines, $15^{\circ}$ graticule. Central meridian $90^{\circ} \mathrm{W}$.

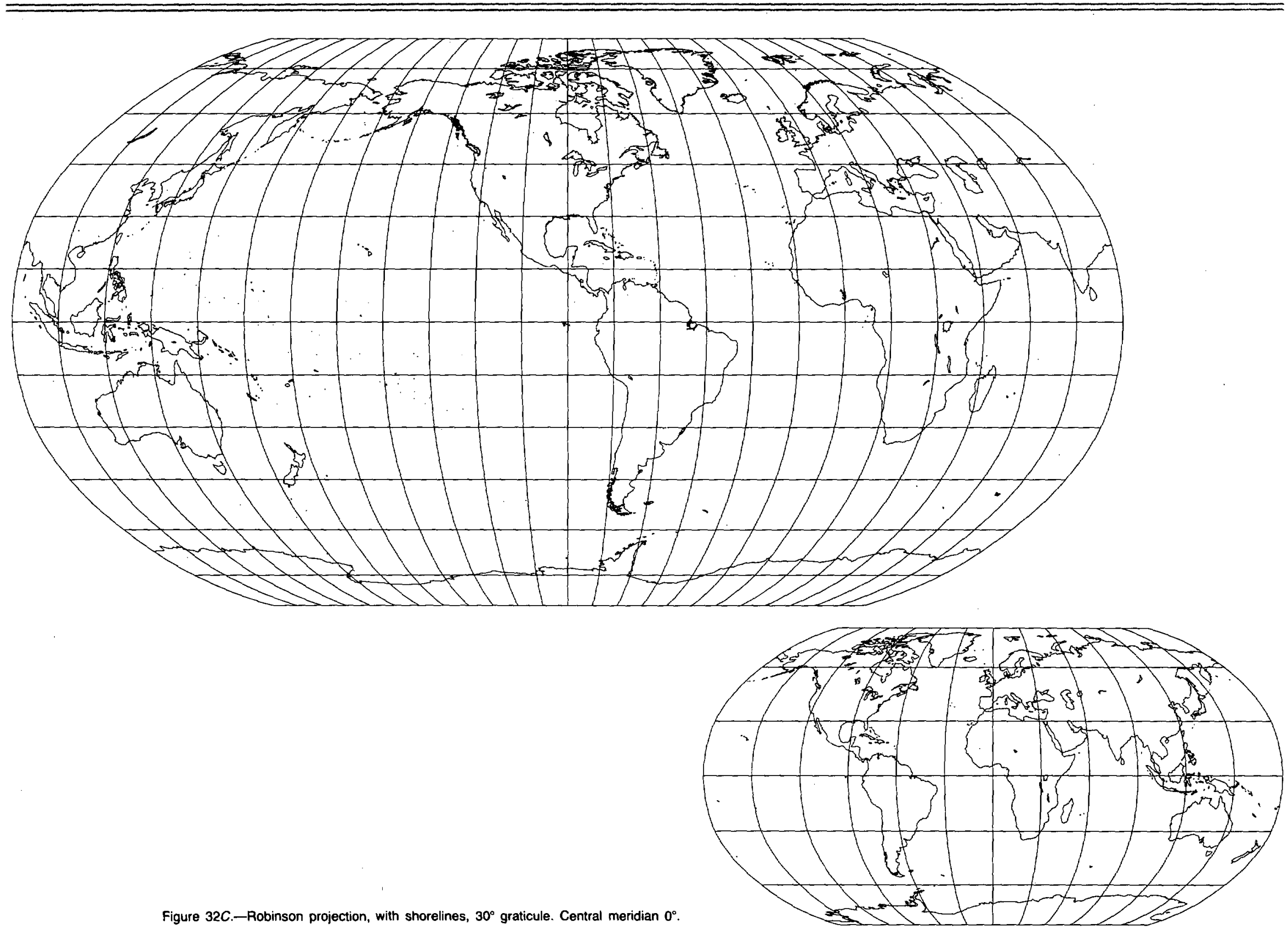




\section{COLLIGNON Projection}

\section{Classifications}

Pseudocylindrical

Equal area

\section{Graticule}

Meridians: Equally spaced straight lines converging at the North Pole

Parallels: Unequally spaced straight parallel lines, farthest apart near the North Pole, closest near the South Pole. Perpendicular to the central meridian.

Poles: North Pole is a point; South Pole is a line 1.41 times as Iong as the Equator

Symmetry: About the central meridian

\section{Scale}

True along latitudes $15^{\circ} 51^{\prime} \mathrm{N}$.

Constant along any given latitude; different for each latitude

\section{Distortion}

Severe in many regions. Free of distortion only at latitude $15^{\circ} 51^{\prime} \mathrm{N}$. at the central meridian.

\section{Usage}

Novelty showing straight-line equal-area

graticule

\section{Origin}

Presented by Édouard Collignon of France in 1865

\section{Similar projections}

Eckert I (p. 86) and II (p. 88) also use straight lines for both meridians and parallels, but meridians are broken at the Equator.

The Trapezoidal projection has equidistant straight parallel lines for parallels and converging straight meridians equally spaced along each parallel (top and bottom parallels and perpendicular central meridian true to scale). It was used for numerous maps from the 15th to the 18th centuries but is neither equal area nor conformal.

Polyhedric projection, formerly used for large. scale mapping in various parts of central

Europe. There were various forms, all resembling the Trapezoidal projection but applied to the ellipsoid. 
Figure 33.-Collignon projection, with shorelines, $15^{\circ}$ graticule. Central meridian $90^{\circ} \mathrm{W}$.

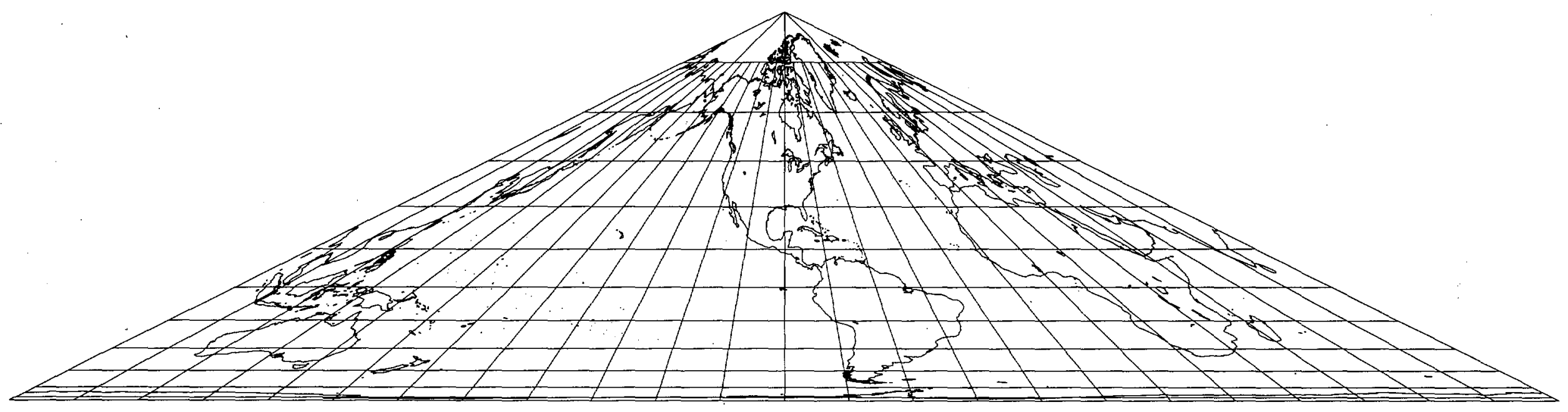

85 

graticule.

\section{Classifications}

Pseudocylindrical

Equally spaced parallels

Neither conformal nor equal area

\section{Graticule}

Meridians: Equally spaced converging straight lines broken at the Equator. Central meridian is half as long as the Equator.

Parallels: Equally spaced straight parallel lines, perpendicular to the central meridian.

Poles: Lines half as long as the Equator

Symmetry: About the central meridian or the Equator

\section{Scale}

True along latitudes $47^{\circ} 10^{\prime} \mathrm{N}$. and $\mathrm{S}$.

Constant along any given latitude; same for the latitude of opposite sign. Constant along any given meridian.

\section{Distortion}

No point free of distortion (fig. 34A). Break at the Equator introduces excessive distortion there. Discontinuous ellipses are shown there owing to the plotting algorithm; actually, the shape is indeterminate on the Equator.

\section{Usage}

Novelty showing straight-line graticule

\section{Origin}

Presented by Max Eckert (1868-1938) of

Germany in 1906

\section{Similar projections}

Eckert II (p. 88) has meridians positioned identically, but parallels are spaced for equal area.

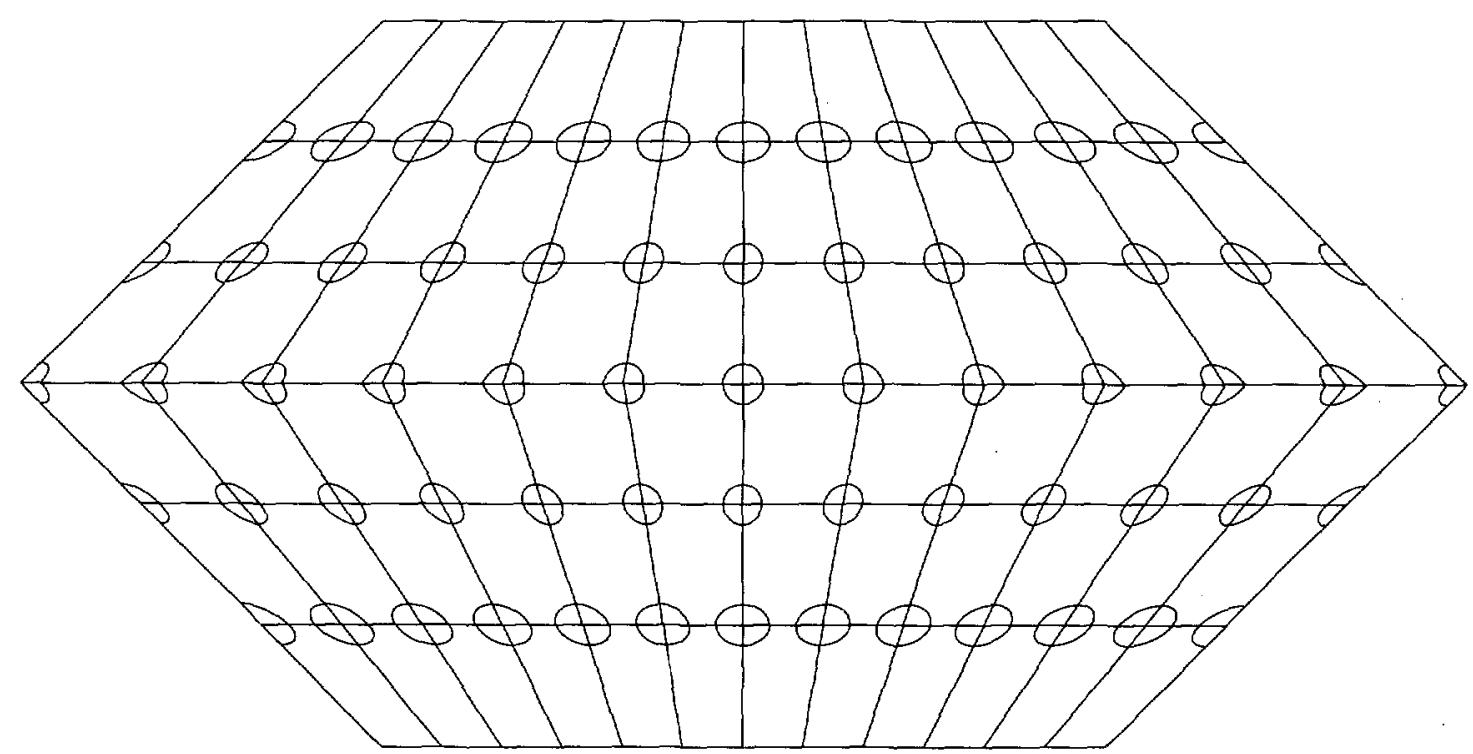


Figure 34B.-Eckert I projection, with shorelines, $15^{\circ}$ graticule. Central meridian $90^{\circ} \mathrm{W}$.

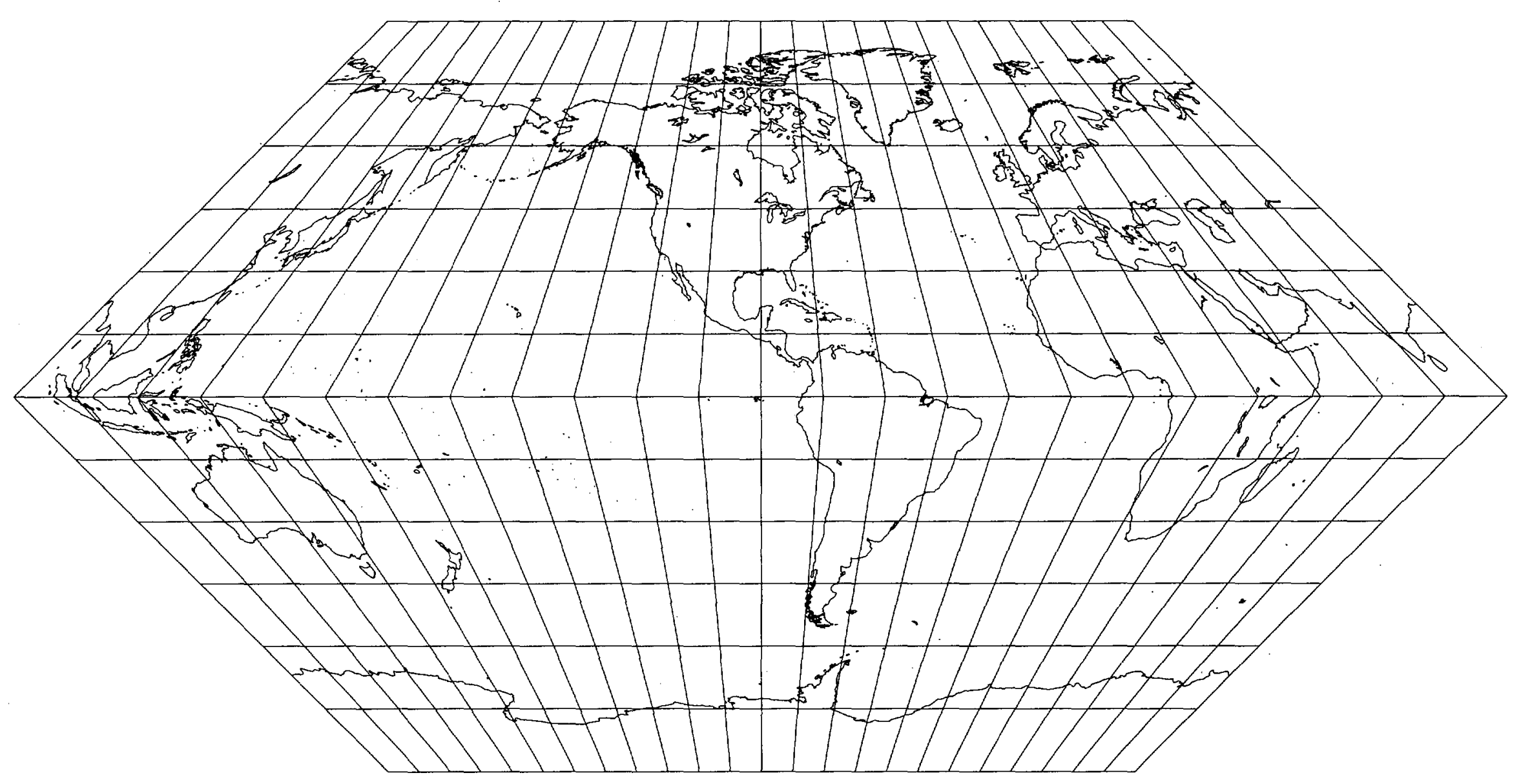




\section{Classifications}

Pseudocylindrical

Equal area

\section{Graticule}

Meridians: Equally spaced straight lines broken at the Equator. Central meridian is half as long as the Equator.

Parallels: Unequally spaced straight parallel lines, widest separation near the Equator.

Perpendicular to the central meridian.

Poles: Lines half as long as the Equator

Symmetry: About the central meridian or the Equator

\section{Scale}

True along latitudes $55^{\circ} 10^{\prime} \mathrm{N}$. and S.

Constant along any given latitude; same for

the latitude of opposite sign

\section{Distortion}

Free of distortion only at latitudes $55^{\circ} 10^{\prime} \mathrm{N}$. and S. at the central meridian (fig. $35 A$ ). Break at the Equator introduces excessive distortion there. Discontinuous ellipses are shown there owing to the plotting algorithm; actually, the shape is indeterminate on the Equator.

\section{Usage}

Novelty showing straight-line equal-area graticule

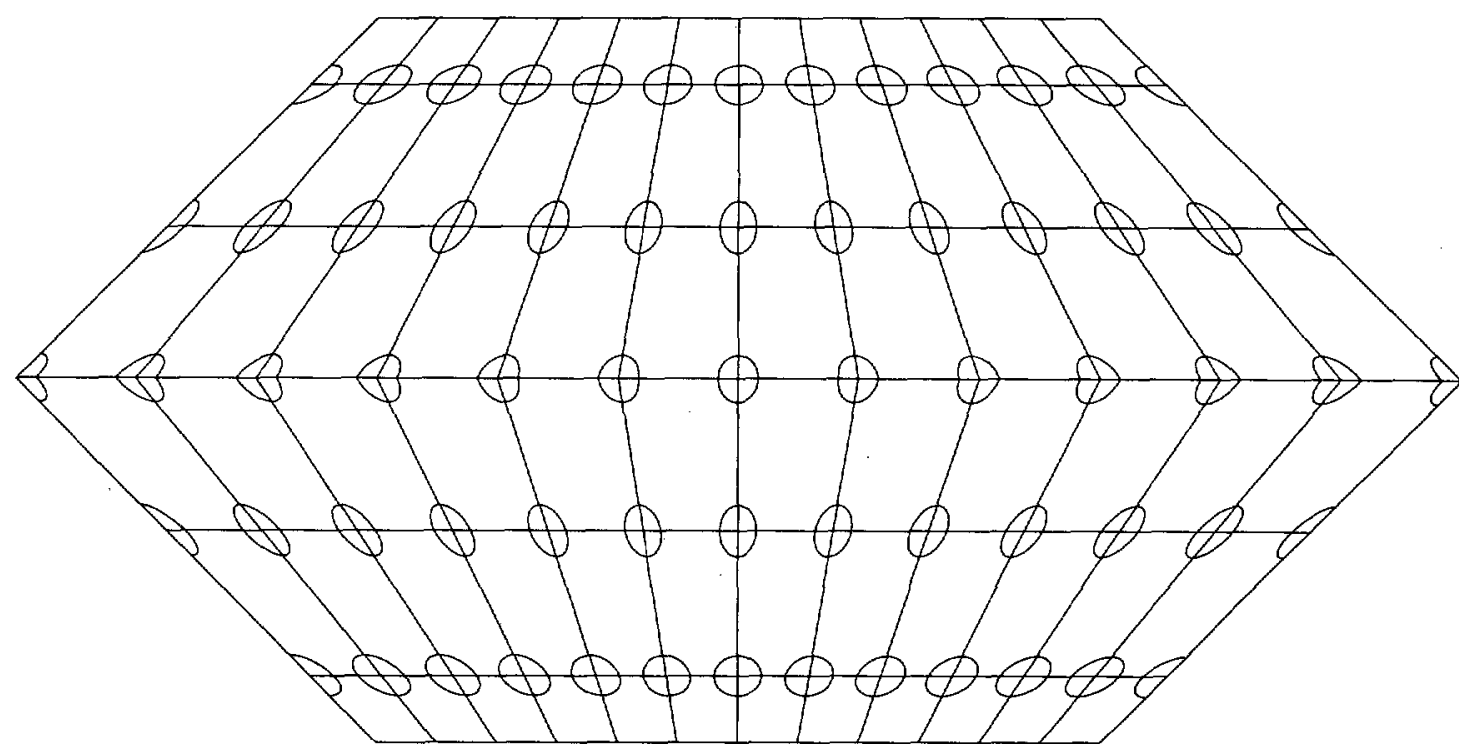

\section{Origin}

Presented by Max Eckert (1868-1938) of Germany in 1906

\section{Similar projections}

Eckert I (p. 86) has meridians positioned identically, but parallels are equidistant.

Collignon projection (p. 84) has straight

meridlans and is equal area, but meridians are

not broken at the Equator, and the poles are different. 
Figure 358.-Eckert II projection, with shorelines, $15^{\circ}$ graticule. Central meridian $90^{\circ} \mathrm{W}$.

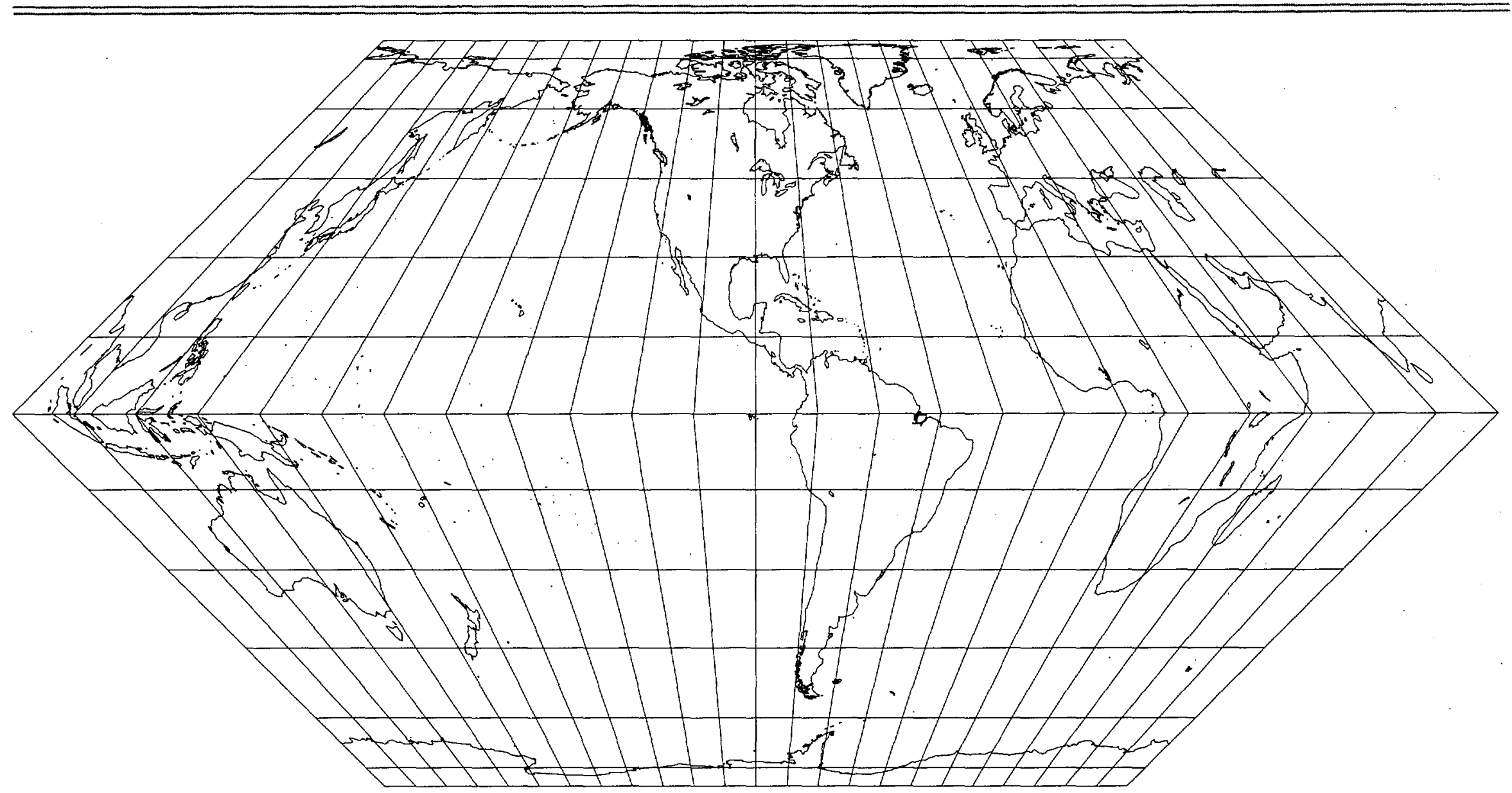




\section{LOXIMUTHAL Projection}

\section{Classifications}

Pseudocylindrical

Equally spaced parallels

Neither conformal nor equal area

\section{Graticule}

Meridians: Central meridian is a straight line generally over half as long as the Equator. depending on the central latitude. If the central latitude is the Equator, the ratio is 0.5 ; if it is $40^{\circ} \mathrm{N}$. or $\mathrm{S}$., the ratio is 0.65 . Other meridians are equally spaced complex curves intersecting at the poles and concave toward the central meridian.

Parallels: Equally spaced straight parallel lines. Perpendicular to the central meridian.

Poles: Points

Symmetry: About the central meridian. Symmetry occurs about the Equator if the specified central latitude is the Equator.

\section{Scale}

True along the central meridian

Constant along any given latitude; normally different for the latitude of opposite sign

\section{Distortion}

Distortion is moderate to extreme, but the projection is intended for the special property noted below, not as a normal world map. Free of distortion only at the central latitude on the central meridian.

\section{Special feature}

Loxodromes (rhumb lines) from the central point (at the intersection of the central meridian and the central parallel) are shown straight, true to scale, and correct in azimuth from the center. Because of angular distortion on the map projection, the azimuths with respect to other points along a rhumb line are not shown correctly.

\section{Origin}

Presented by Karl Siemon (?-1937) of Germany in 1935. Independently presented as "Loximuthal" by Waldo R. Tobler (1930- ) of the University of Michigan (1966).

\section{Similar projections}

Loxodromes are shown straight and at the correct azimuth between any two points on the regular Mercator projection, but they are not at true scale.

The Bordone Oval projection of 1520 is similar to the Loximuthal projection having the

Equator as the central latitude. 
<smiles>C=[As]CC[As]</smiles> 


\section{EQUIDISTANT CONIC Projection}

\section{Classifications}

Conic

Equally spaced parallels

Neither conformal nor equal area

\section{Graticule}

Meridians: Equally spaced straight lines converging at a common point, which is normally beyond the pole. The angles between them are less than the true angles.

Parallels: Equally spaced concentric circular arcs centered on the point of convergence of the meridians, which are therefore radii of the circular arcs

Poles: Normally circular arcs enclosing the same angle as that enclosed by the other parallels of latitude for a given range of longitude

Symmetry: About any meridian

\section{Scale}

True along each meridian and along one or two chosen standard parallels, usually but not necessarily on the same side of the Equator. As a rule of thumb, these parallels can be placed at one-sixth and five-sixths of the range of latitudes, but there are more refined means of selection. Scale is constant along any given parallel.

\section{Distortion}

Free of distortion only along the two standard parallels (fig. 37A). Distortion is constant along any given parallel. Compromise in distortion between equal-area and conformal conic projections.

\section{Usage}

The most common projection in atlases for small countries

Also used by the Soviet Union for mapping that nation

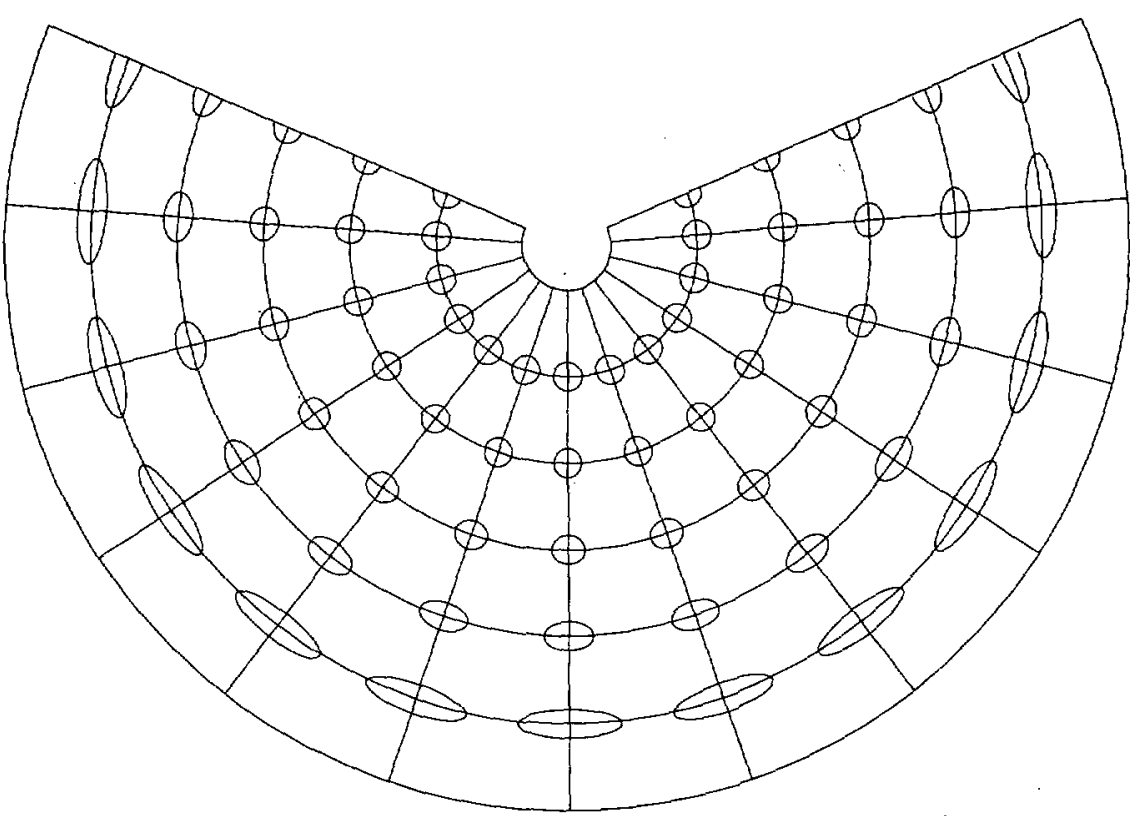

\section{Origin}

Rudimentary forms developed by Claudius Ptolemy (about A. D. 100). Improvements by Johannes Ruysch in 1508, Gerardus Mercator in the late 16th century, and Nicolas de I'Isle in 1745.

\section{Other names}

Simple Conic

Conic

\section{Limiting forms}

Polar Azimuthal Equidistant projection (fig. $37 \mathrm{C}$ and p. 133), if a pole is made the single standard parallel. The cone of projection thereby becomes a plane.

Plate Carree (fig. 37D, also p. 22), if the single standard parallel is the Equator. The cone of projection thereby becomes a cylinder.

Equirectangular (Cylindrical) projection (p. 24) if two standard parallels are symmetrically placed north and south of the Equator.
Standard conic formulas must be rewritten for the second and third limiting forms.

\section{Similar projections}

Various methods of determining optimum standard parallels have been proposed by Patrick Murdoch (projections I, III) in 1758, Leonhard Euler in 1777, British Ordnance in the late 19th century, Dmitri I. Mendeleev in 1907, Wilhelm Schjerning (projection 1) in 1882, V.V. Vitkovskiy (projection I) in 1907, and V.V. Kavrayskiy (projections II, IV) in 1934. Once the standard parallels are selected all these projections are constructed by using formulas used for the Equidistant Conic with two standard parallels.

John Bartholomew combined the Equidistant Conic projection with the Bonne projection (see p. 112). 
Figure 378 . -Equidistant Conic projection, with shorelines, $15^{\circ}$ graticule. Central meridian $90^{\circ} \mathrm{W}$. Standard parallels: $20^{\circ}$ and $60^{\circ} \mathrm{N}$.

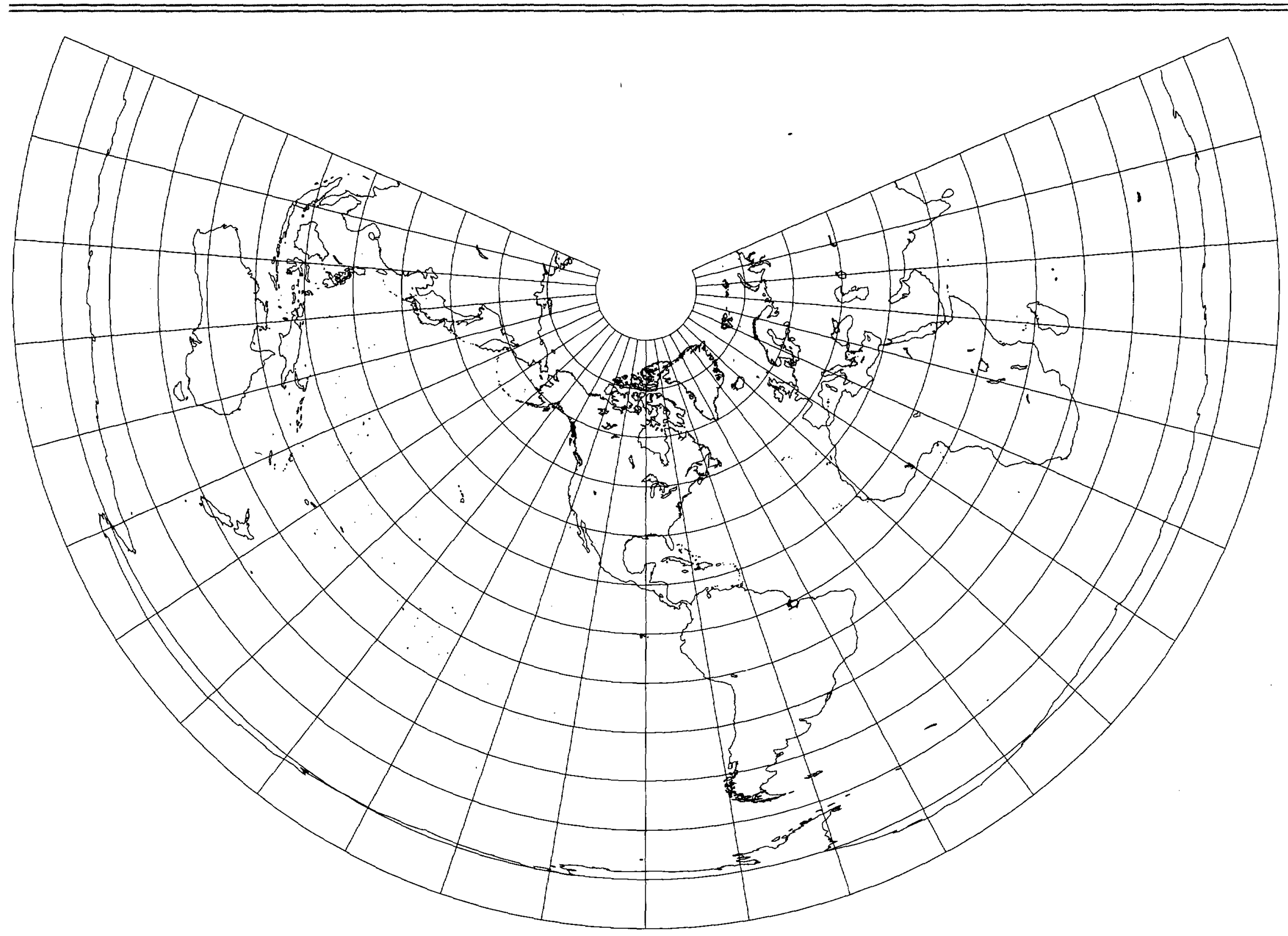




\section{EQUIDISTANT CONIC Projection}

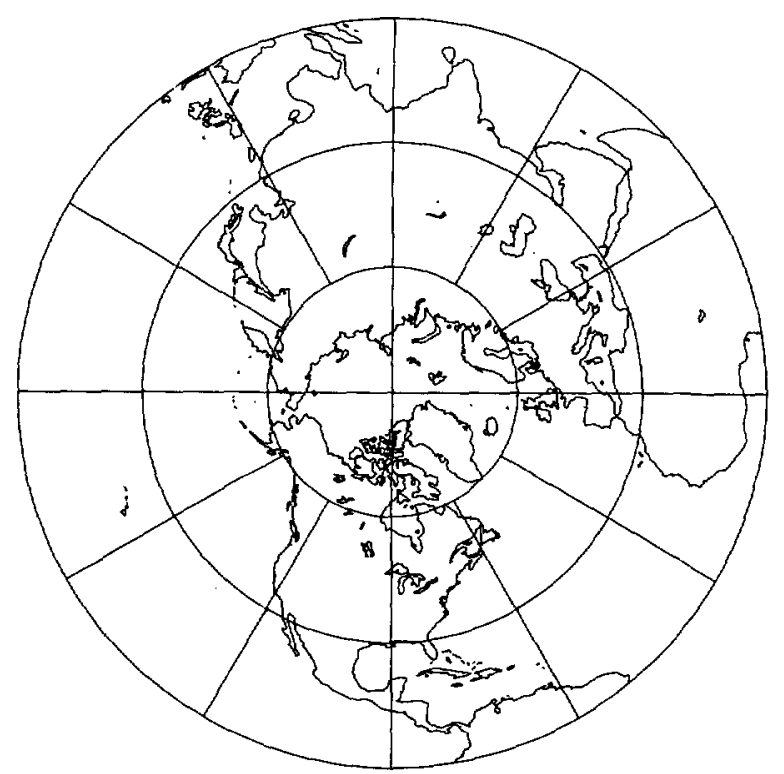

Figure 37C.-North Polar Azimuthal Equidistant projection, with shorelines, $30^{\circ}$ graticule. Central meridian $90^{\circ} \mathrm{W}$. Polar and planar limit of Equidistant Conic projection.

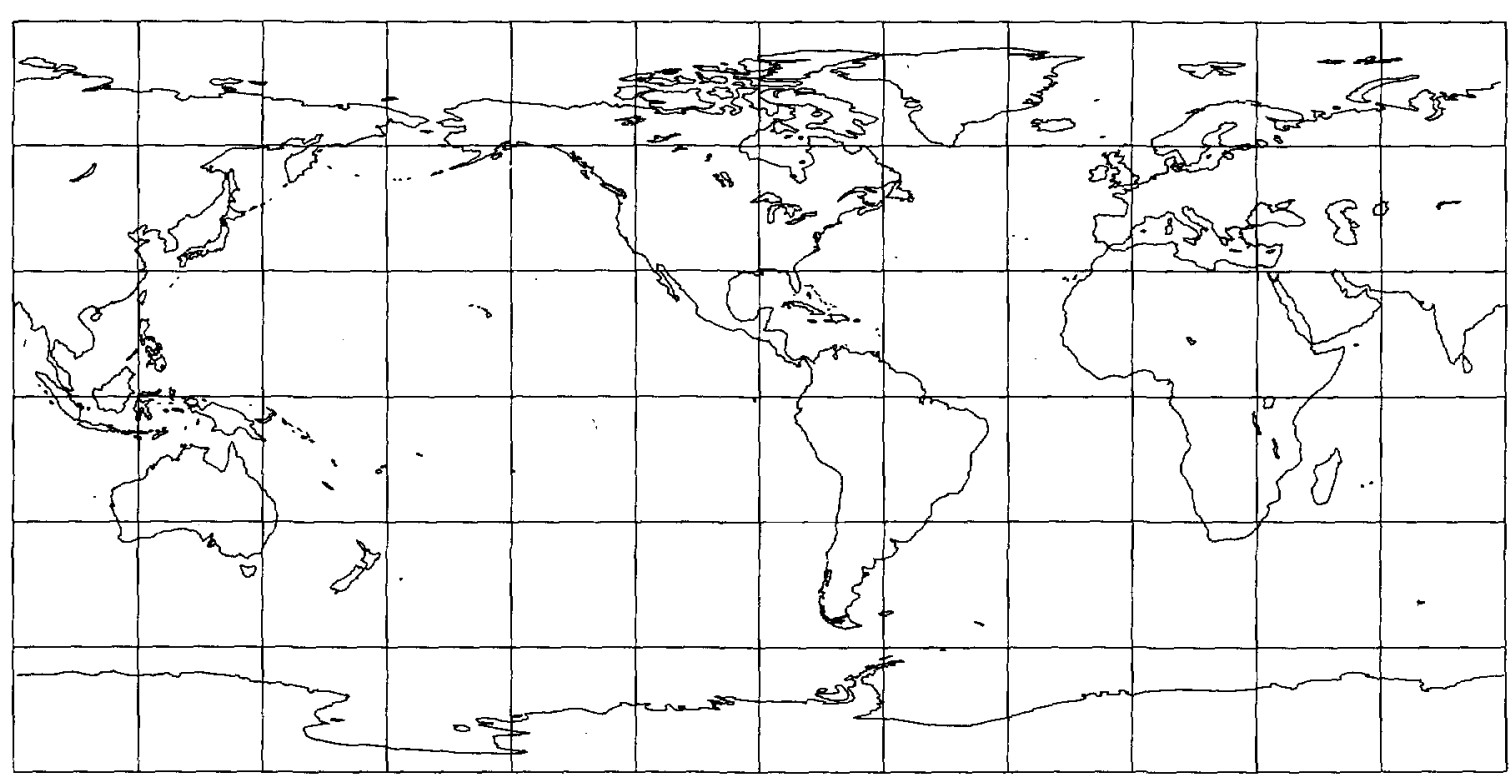

Figure $37 D$.-Plate Carrée projection, with shorelines, $30^{\circ}$ graticule. Central meridian $90^{\circ} \mathrm{W}$. Equatorial and cylindrical limit of Equidistant Conic projection. 


\section{LAMBERT CONFORMAL CONIC Projection}

\section{Classifications \\ Conic}

Conformal

\section{Graticule}

Meridians: Equally spaced straight lines converging at a common point, which is one of the poles. The angles between them are less than the true angles.

Parallels: Unequally spaced concentric circular arcs centered on the pole of convergence of the meridians, which are therefore radii of the circular arcs. Spacing of parallels increases away from the central latitudes.

Poles: The pole nearest a standard parallel is a point; the other pole cannot be shown. Symmetry: About any meridian

\section{Scale}

True along one or two chosen standard parallels, usually but not necessarily on the same side of the Equator. As a rule of thumb, these parallels can be placed at one-sixth and five-sixths of the range of latitudes, but there are more refined means of selection.

Scale is constant along any given parallel and is the same in all directions at a given point.

\section{Distortion}

Free of distortion only along the one or two standard parallels (fig. 38A). Distortion is constant along any given parallel. Conformal everywhere except at the poles.

\section{Usage}

Extensively used in ellipsoidal form for large scale mapping of regions of predominantly east-west extent, including topographic quadrangles $(1: 24,000$ and $1: 62,500$ scale) for many of the U.S. State Plane Coordinate

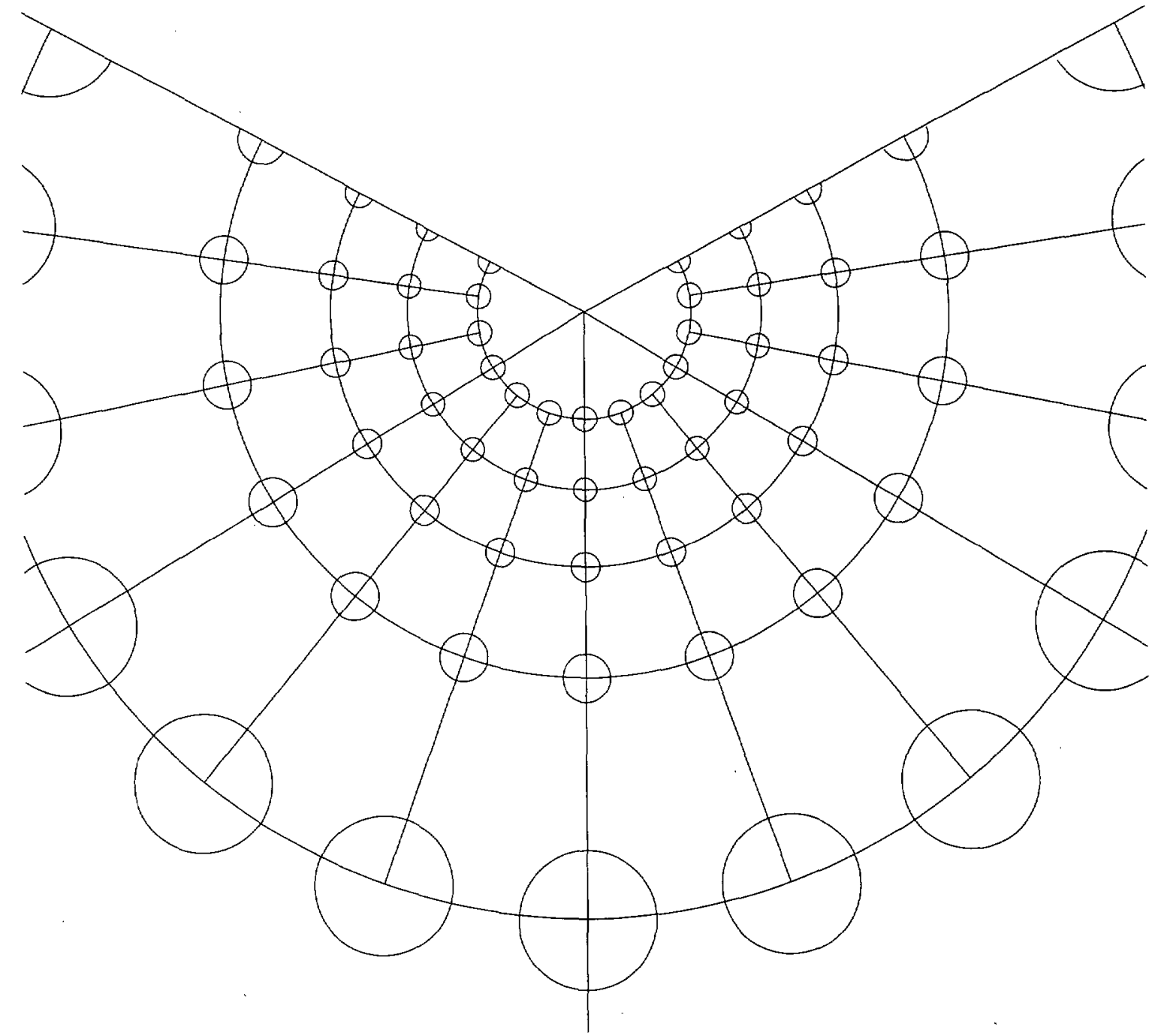

Figure 38A.--Lambert Conformal Conic projection, with Tissot indicatrices, $30^{\circ}$ graticule. Standard parallels: $20^{\circ}$ and $60^{\circ} \mathrm{N}$

System zones, many maps in the International Map of the World $(1: 1,000,000$ scale) series, the U.S. State Base Maps (1:500,000 scale),

Also used for atlas maps of some countries Recommended for conformal mapping of regions of predominantly east-west extent and topographic mapping in many other nations 


\section{LAMBERT CONFORMAL CONIC Projection}

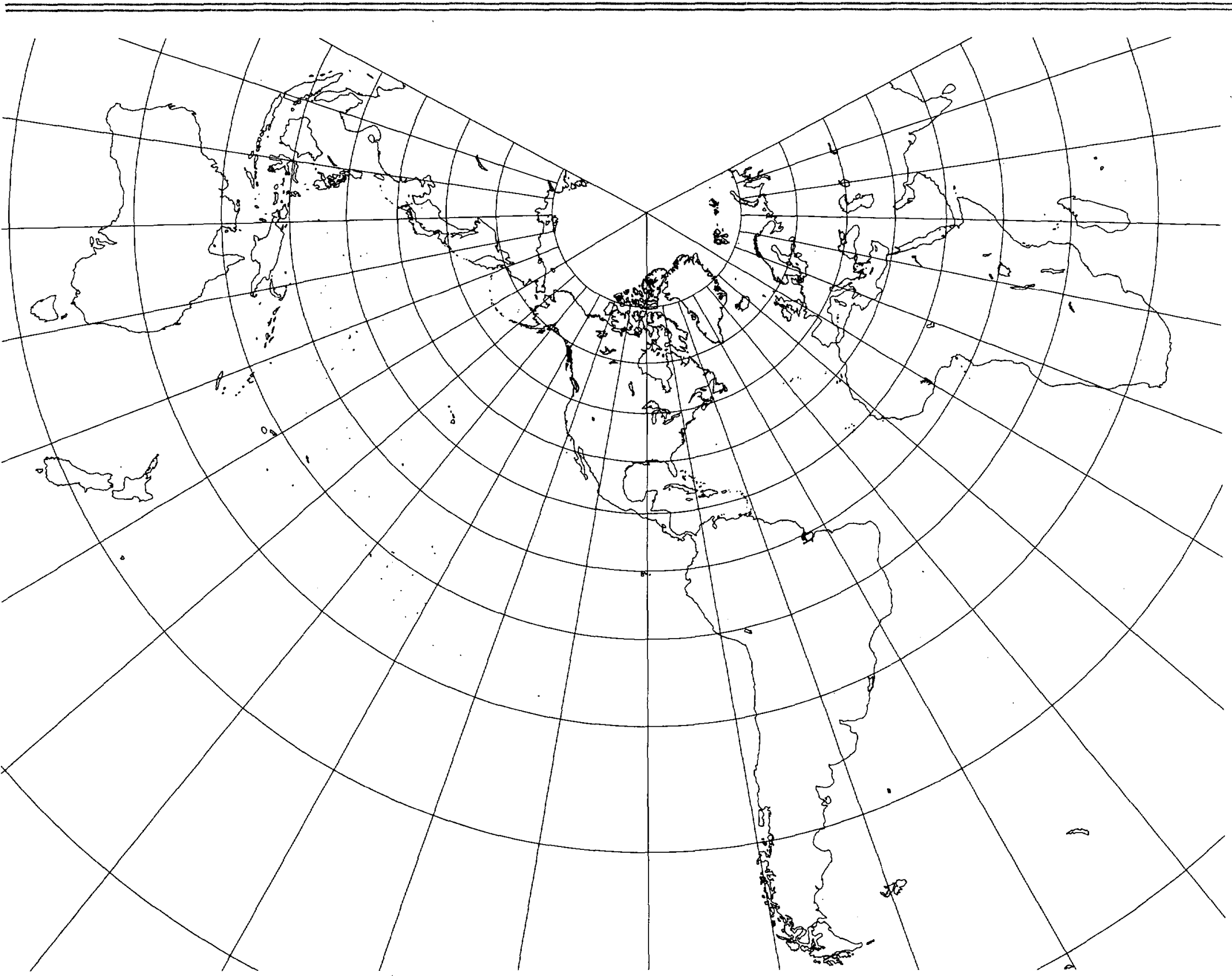

Figure 388.-Lambert Conformal Conic projection, with shorelines, $15^{\circ}$ graticule. Central meridian $90^{\circ} \mathrm{W}$. Standard parallels: $20^{\circ}$ and $60^{\circ} \mathrm{N}$. 


\section{Origin}

Presented by Johann Heinrich Lambert (172877) of Alsace in 1772

\section{Aspects}

Bipolar Oblique Conic Conformal projection (p. 99) is the only common oblique aspect. See figure $38 E$ for another example applied to the world.

\section{Other names}

Conical Orthomorphic

\section{Limiting forms}

Polar Stereographic projection (fig. $38 \mathrm{C}$ and p. 121), if a pole is the single standard parallel. The cone of projection thereby becomes a plane.

Mercator projection (fig. 38D, also p. 10), if the single standard parallel is the Equator or if two standard parallels are symmetrically placed north and south of the Equator. The cone of projection thereby becomes a cylinder. Standard conic formulas must be rewritten.

\section{Similar projections}

Various methods of determining optimum standard parallels have been proposed by John Herschel in 1860, V.V. Vitkovskiy

(projection III) in 1907, N.Ya. Tsinger in 1916, and V.V. Kavrayskiy (projection III) in 1934. Once the standard parallels are selected, all

these projections are constructed by using the same formulas used for the Lambert Conformal Conic with two standard parallels.

Bipolar Oblique Conic Conformal projection

(p. 99) consists of oblique aspects of two

Lambert Conformal Conic projections laid side by side.

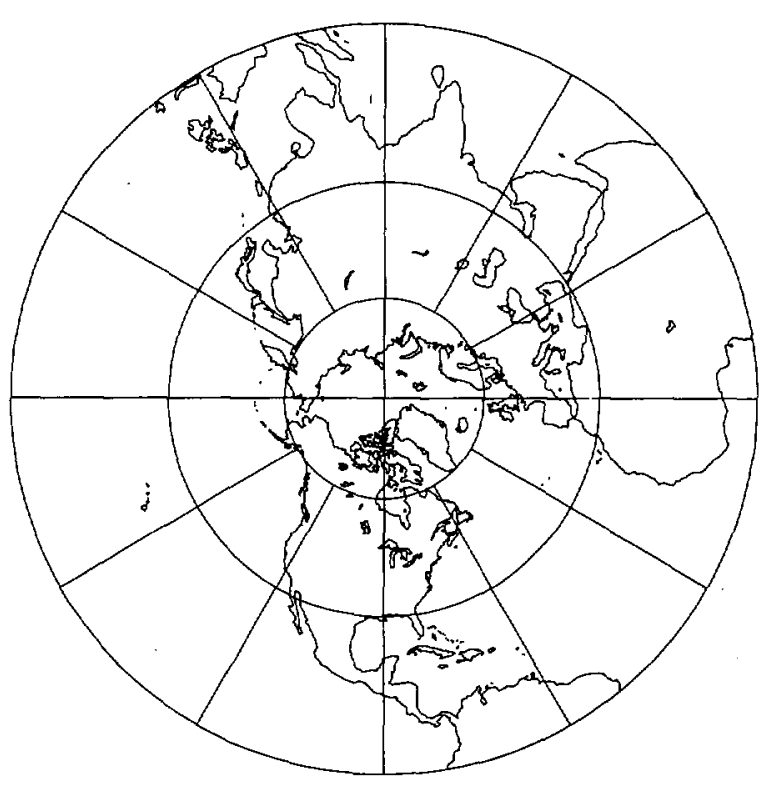

Figure 38C.-North Polar Stereographic projection, with shorelines $30^{\circ}$ graticule. Central meridian $90^{\circ} \mathrm{W}$. Polar and planar limit of Lambert Conformal Conic projection.

Figure 38D.-Mercator projection, with shorelines, $30^{\circ}$ graticule. Central meridian $90^{\circ} \mathrm{W}$. Equatorial and cylindrical limit of Lambert Conformal Conic projection

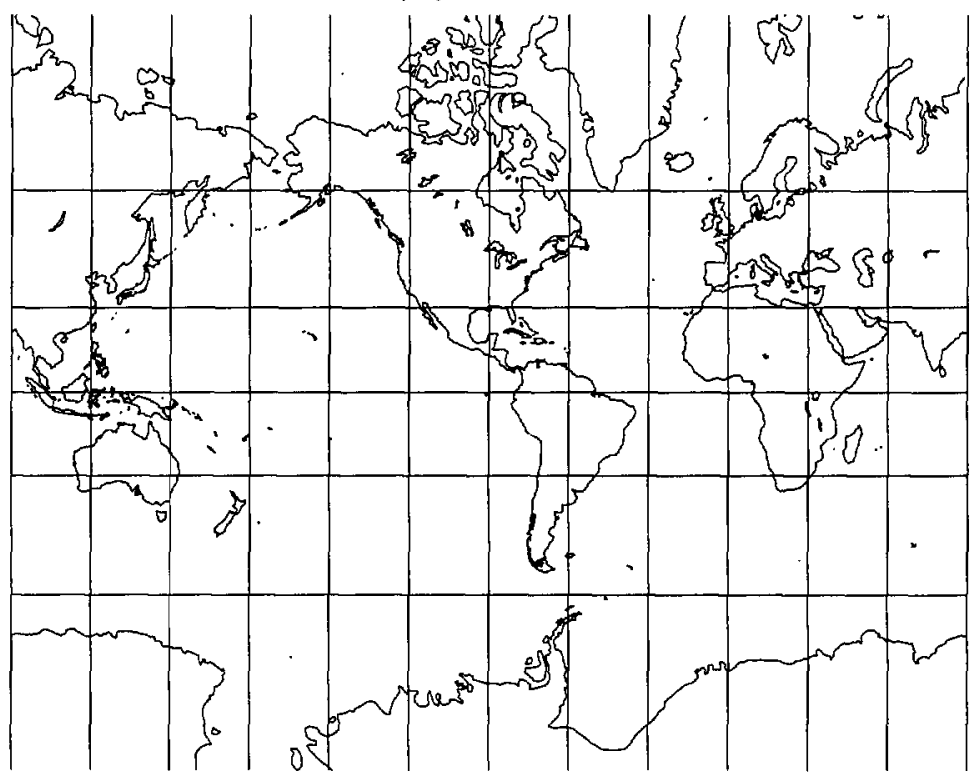




\section{LAMBERT CONFORMAL CONIC Projection}

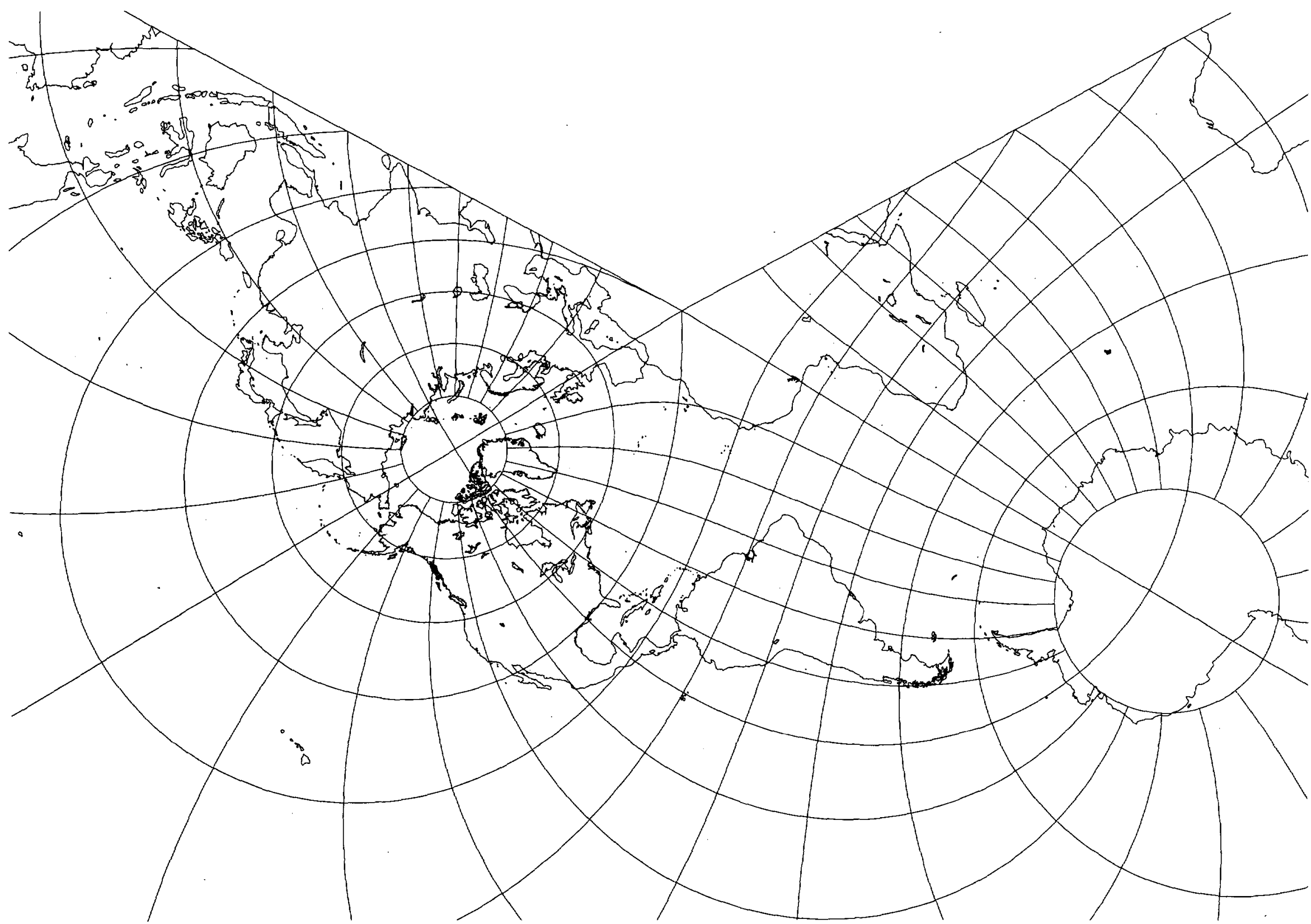

Figure 38E.-Oblique Lambert Conformal Conic projection, with shorelines, $15^{\circ}$ graticule. Central meridian $90^{\circ} \mathrm{W}$. Standard parallels $+20^{\circ}$ and $+60^{\circ}$ on base projection. North Pole at $+30^{\circ}$ latitude, $-90^{\circ}$ longitude on base projection. 


\section{BIPOLAR OBLIQUE CONIC CONFORMAL Projection}

\section{Classifications}

Conic

Conformal

\section{Graticule}

Meridians: Unequally spaced curved lines, except for two straight meridians from the transformed poles to the nearest geographic pole

Parallels: Unequally spaced curved lines Poles: Points

Symmetry: None

\section{Scale}

True along four circular arcs joined to form two $S$ curves. These arcs do not follow any parallel or meridian.

Scale is constant along circular arcs centered on each transformed pole for part of the map. The pole for most of North America is in the North Atlantic, and that for South America is in the South Pacific.

\section{Distortion}

Low throughout North and South America. None along the lines of true scale and constant along circular arcs centered on the transformed pole for the given part of the map. Slight nonconformality near the line joining the two transformed poles.

\section{Usage}

Designed specifically for a low-error map of North and South America constructed by the American Geographical Society. Other organizations, including the U.S. Geological Survey, have used the American Geographical Society map as a base for maps of North or South America alone, but, since the advent of computer plotting, other projections are preferable and practical for regional maps.

\section{Origin}

Presented by Osborn Maitland Miller (1897-

1979) and William A. Briesemeister of the

American Geographical Society in 1941

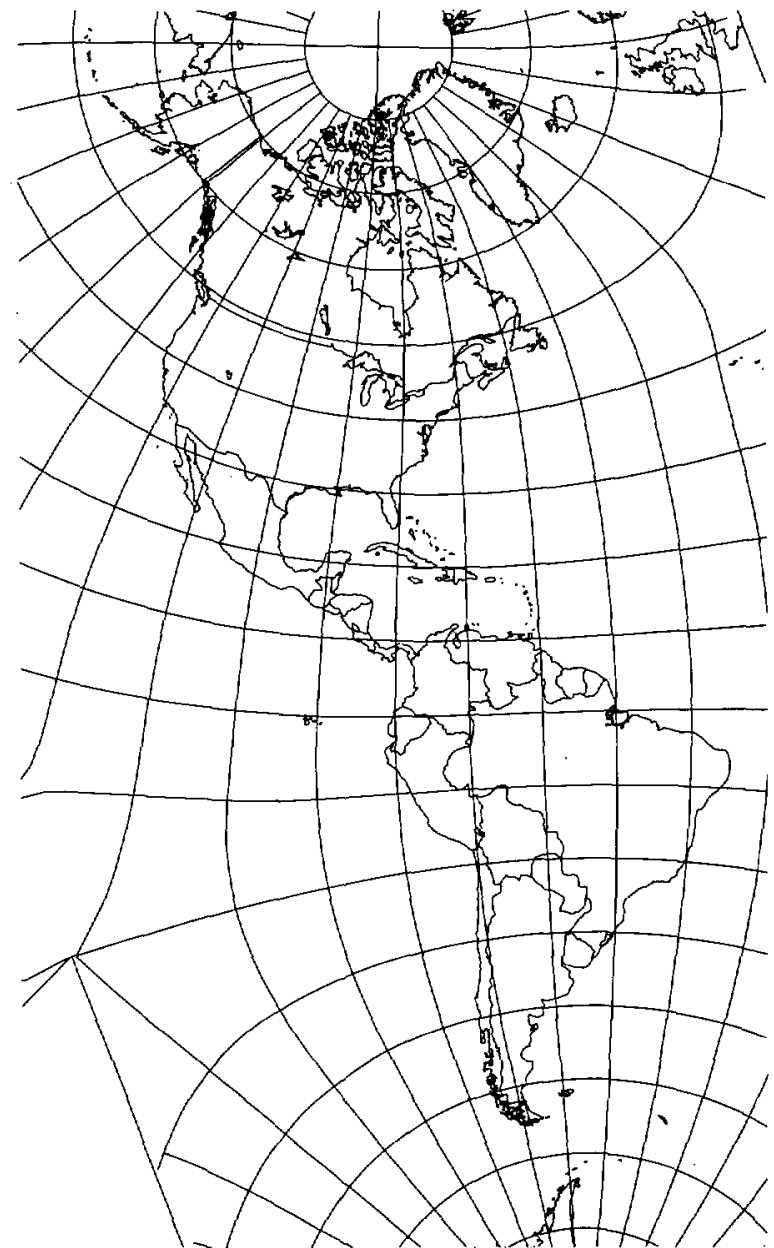

Figure 39.-Bipolar Oblique Conic Conformal projection, with

shorelines, $10^{\circ}$ graticule. Specially designed for North and South America. 


\section{Classifications}

Conic

Equal area

\section{Graticule}

Meridians: Equally spaced straight lines converging at a common point, which is normally beyond the pole. The angles between them are less than the true angles.

Parallels: Unequally spaced concentric circular arcs centered on the point of convergence of the meridians. The meridlans are therefore radii of the circular arcs. Spacing of parallels decreases away from the central latitudes.

Poles: Normally circular arcs enclosing the same angle as that enclosed by the other parallels of latitude for a given range of longitude

Symmetry: About any meridian

\section{Scale}

True along one or two chosen standard parallels, usually but not necessarily on the same side of the Equator. As a rule of thumb these parallels can be placed at one-sixth and five-sixths of the range of latitudes, but there are more refined means of selection.

Scale is constant along any given parallel. The scale factor at any given point along the meridian is the reciprocal of that along the parallel, to preserve area.

\section{Distortion}

Free of angular and scale distortion only along the one or two standard parallels (fig. 40A)

Distortion is constant along any given parallel.

\section{Usage}

Frequently used in the ellipsoidal form for maps of the United States in the National
Atlas of the United States, for thematic maps, and for world atlases

Also used and recommended for equal-area maps of regions that are predominantly eastwest in extent

\section{Origin}

Presented by Heinrich Christian Albers (17731833) of Germany in 1805

\section{Aspects}

Rarely used in oblique aspect but occasionally proposed in journal articles

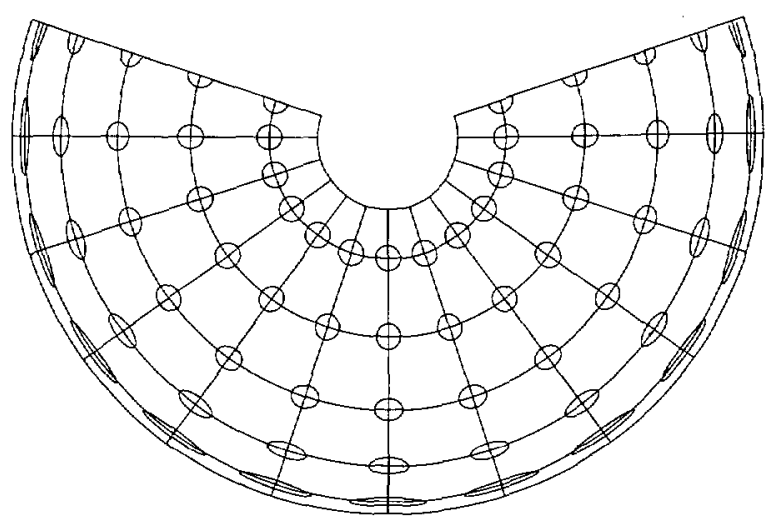

\section{Limiting forms}

Polar Lambert Azimuthal Equal-Area projection (fig. $40 C$ and p. 137), if a pole is made the single standard parallel. The cone of projection thereby becomes a plane.

Lambert Equal-Area Conic projection (p. 102), If the pole and another parallel are made the two standard parallels

Lambert Cylindrical Equal-Area projection (fig. $40 D$ and p. 17), if the Equator is the single standard parallel. The cone of projection thereby becomes a cylinder.

Behrmann or other cylindrical equal-area projections ( $p$. 19), if the two standard parallels are symmetrically placed north and south of the Equator.

Standard conic formulas must be rewritten for the third and fourth limiting forms.

\section{Similar projections}

Various methods of determining optimum standard parallels have been proposed by N.A. Tissot in 1881, V.V. Vitkovskiy (projection II) in 1907, N.Ya. Tsinger in 1916, and F.N. Krasovskiy (projection I) in 1922. Once the standard parallels are selected, all these projections are constructed by using the same formulas used for the Albers Equal-Area Conic with two standard parallels. 
Figure 40B.-Albers Equal-Area Conic Projection, with shorelines, $15^{\circ}$ graticule. Central meridian $90^{\circ} \mathrm{W}$. Standard parallels $20^{\circ}$ and

$60^{\circ} \mathrm{N}$.

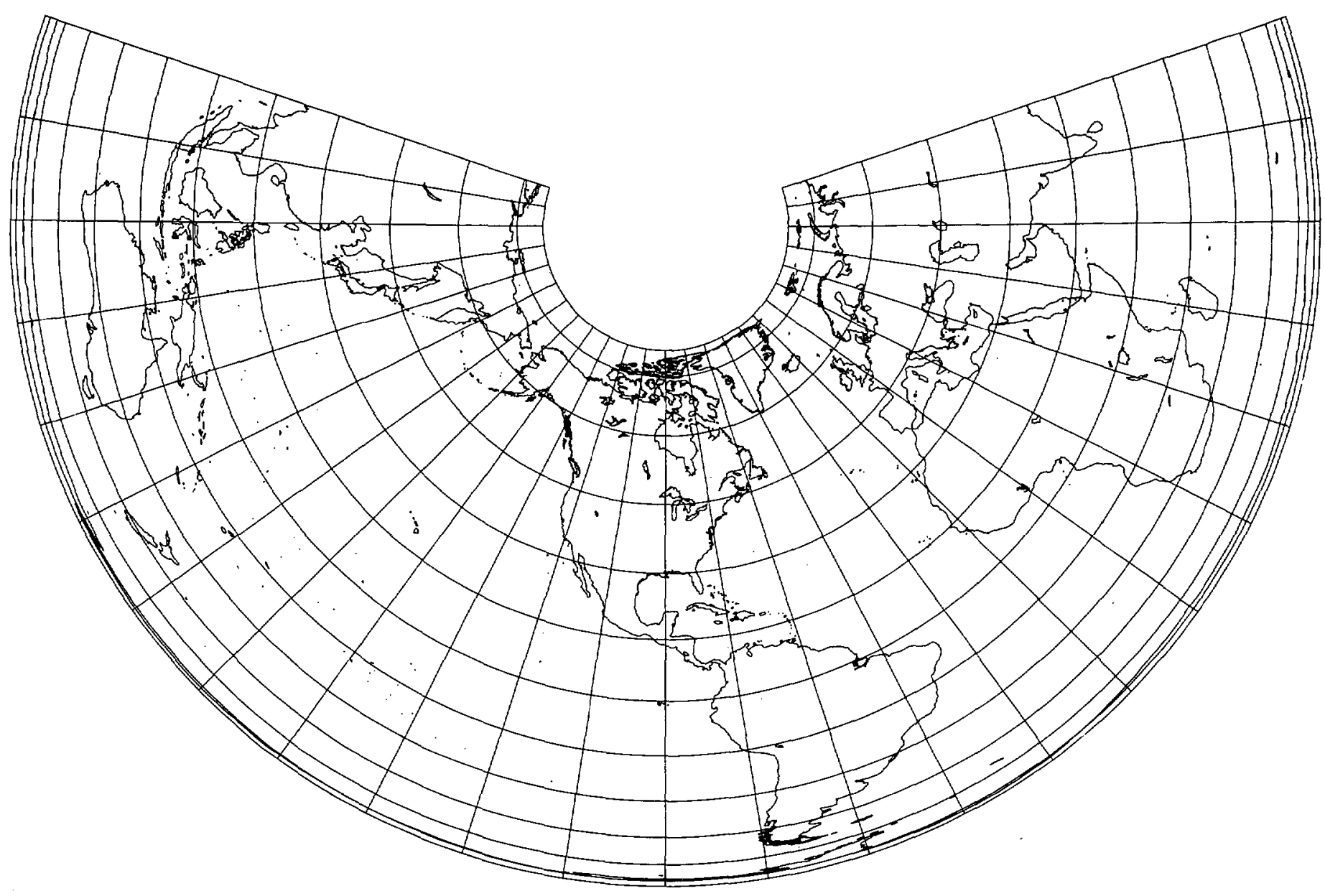

Figure 40G.-North Polar Azimuthal Equal-Area projection, with shorelines, $30^{\circ}$ graticule. Central meridian $90^{\circ} \mathrm{W}$. Polar and planar limit of Albers Equal-Area projection.

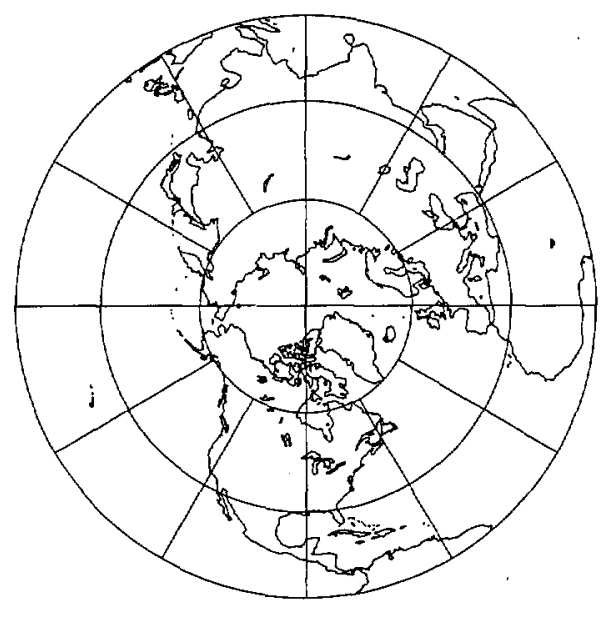

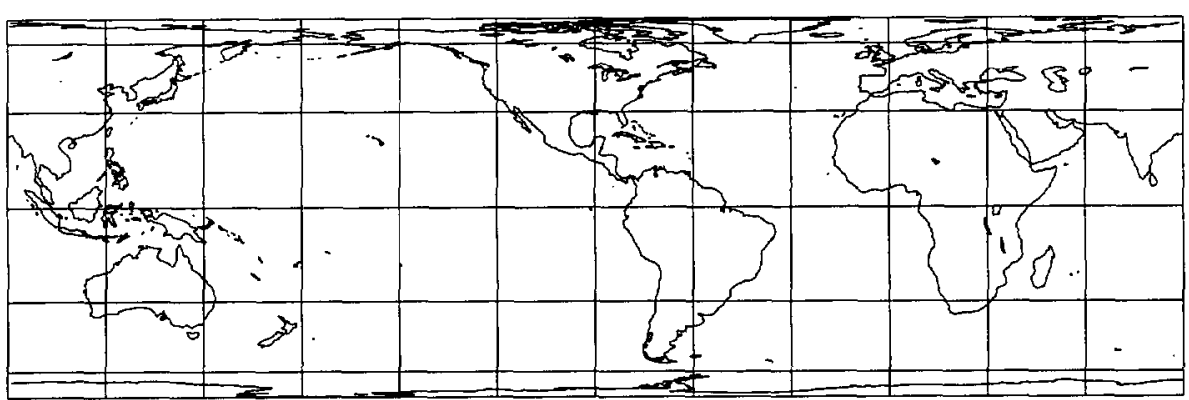

Figure 400.-Lambert Cylindrical Equal-Area projection, with shorelines, $30^{\circ}$ graticule. Standard parallet $0^{\circ}$. Central meridian $90^{\circ} \mathrm{W}$. Equatorial and cylindrical limit of Albers Equal-Area projection. 


\section{LAMBERT EQUAL-AREA CONIC Projection}

\section{Classifications}

Conic

Equal area

\section{Graticule}

Meridians: Equally spaced straight lines converging at a common point, which is one of the poles. The angles between them are less than the true angles.

Parallels: Unequally spaced concentric circular arcs centered on the pole of convergence of the meridians. The meridians are therefore radii of the circular arcs. Spacing of parallels decreases away from the pole of convergence.

Poles: One pole is a point; the other pole is a circular arc enclosing the same angle as that enclosed by the other parallels of latitude for a given range of longitudes.

Symmetry: About any meridian

\section{Scale}

True along the chosen standard parallel

Scale is constant along any given parallel. The scale factor at any given point along the meridian is the reciprocal of that along the parallel, to preserve area.

\section{Distortion}

Free of scale and angular distortion only along the standard parallel. Severe stretching near each pole.

Scale distortion and angular distortion are constant along any given parallel.

\section{Origin}

Presented by Johann Heinrich Lambert (1728-

77) of Alsace in 1772

\section{Limiting form}

Polar Lambert Azimuthal Equal-Area projection (p. 136), if the central pole is the standard parallel. The cone of projection thereby becomes a plane.

\section{Similar projections}

Albers Equal-Area Conic projection (p. 100)

also has concentric circular arcs for parallels and straight meridians, but it has two standard parallels close to the latitudes of interest. If just one of its standard parallels is made a pole, it becomes the Lambert Equal-Area Conic projection, but that pole is not free of distortion, as the usual standard parallel is. 
Figure 41.-Lambert Equal-Area Conic projection, with shorelines, $15^{\circ}$ graticule. Central meridian $90^{\circ} \mathrm{W}$. Standard parailel $0^{\circ}$.

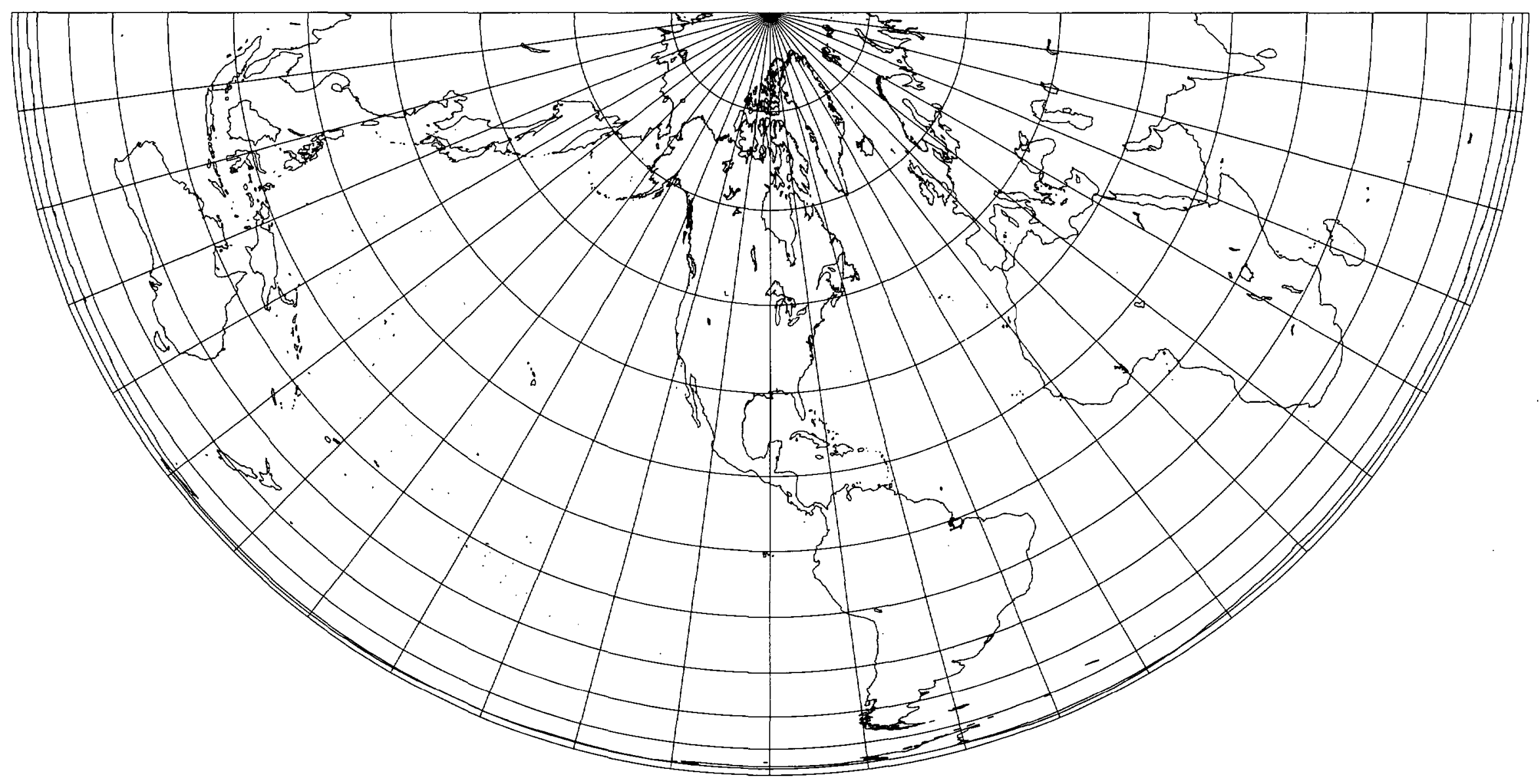




\section{PERSPECTIVE CONIC Projection}

\section{Classifications}

Conic

\section{Perspective}

Neither conformal nor equal area

\section{Graticule}

Meridians: Equally spaced straight lines converging at a common point, which is one of the poles. The angles between them are less than the true angles.

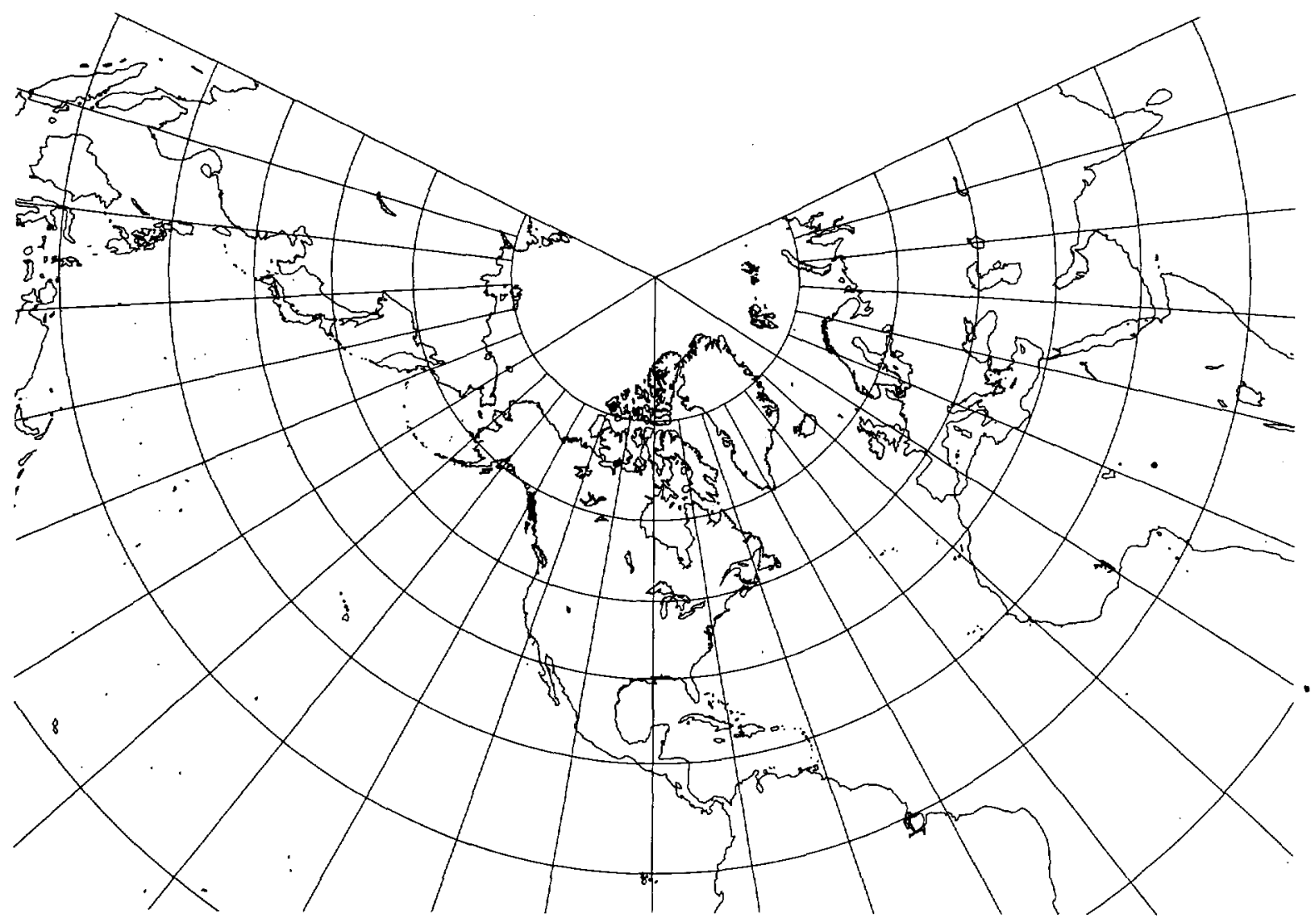

Figure 42A.-Perspective Conic projection, with shorelines, $15^{\circ}$ graticule. Central meridian $90^{\circ} \mathrm{W}$. Standard parallels $20^{\circ}$ and $60^{\circ} \mathrm{N}$.

Poles: One pole is a point; the other pole
Parallels: Unequally spaced concentric circular arcs centered on the pole of convergence of the meridians. The meridians are therefore radii of the circular arcs. Spacing of parallels increases away from the central latitudes. cannot generally be shown, but, under some conditions, it is a circular arc.

Symmetry: About any meridian

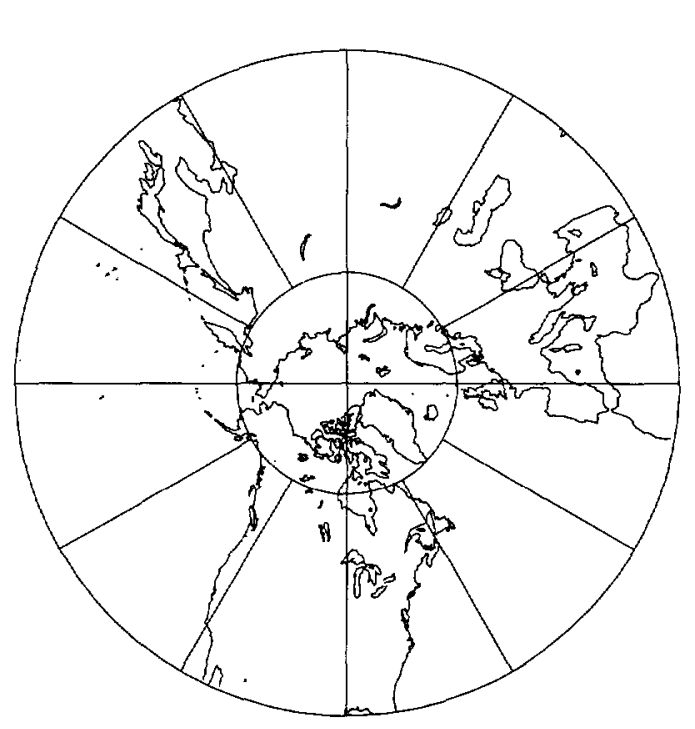




\section{Scale}

True along one or two chosen standard parallels, which may be on the same side of or both sides of the Equator

Scale is constant along any given parallel.

\section{Distortion}

Free of distortion only along the one or two standard parallels. Distortion is constant along any given parallel but changes more rapidly in a north-south direction than it does on conformal or equal-area conics, and the Perspective Conic has no compensating advantage. Other projections should be used instead.

\section{Other features}

Projection is produced geometrically by projecting the Earth perspectively from the center (or from some other point) onto a cone tangent or secant along the standard parallels.

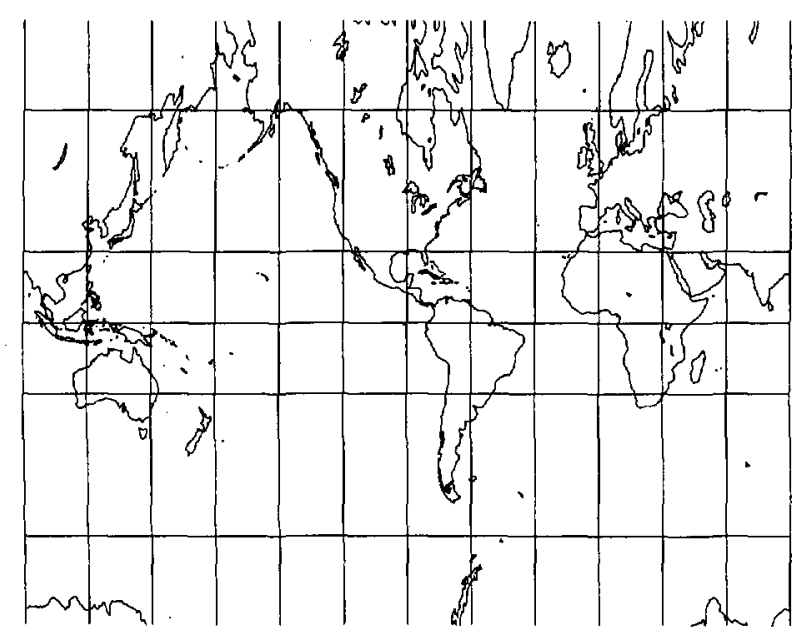

Figure $42 \mathrm{C}$. - Central Cylindrical projection, with shorelines, $30^{\circ}$ graticule. Central meridian $90^{\circ} \mathrm{W}$. Equatorial and cylindrical limit of Perspective Conic projection.

\section{Limiting forms}

Polar Gnomonic projection (fig. $42 B$ and $p$.

117), if the pole is the single standard parallel.

The cone of projection thereby becomes a

plane.

Central Cylindrical projection (fig. $42 \mathrm{C}$ and p. 30), if the Equator is the single standard parallel. The cone of projection thereby

becomes a cylinder. Standard conic formulas must be rewritten.

\section{Similar projection}

A stereographic perspective conic was presented by Carl Braun in 1867. The map is projected from the South Pole onto a cone tangent at latitude $30^{\circ} \mathrm{N}$. 


\section{Classifications}

Polyconic

Neither conformal nor equal area

\section{Graticule}

Meridians: Central meridian is a straight line. All other meridians are complex curves, spaced equally along the Equator and along each parallel and concave toward the central meridian.

Parallels: Equator is a straight line, poles are points, and all other parallels are nonconcentric circular arcs spaced at true distances along the central meridian. Each parallel has a curvature identical to its curvature on a cone tangent at that latitude. Many cones are involved; hence, the name "Polyconic projection."

Symmetry: About the central meridian and the Equator

\section{Scale}

True along the central meridian and along each parallel

\section{Distortion}

Free of distortion only along the central meridian (fig. $43 A$ ). Extensive distortion if the range extends very far east or west.

\section{Usage}

The sole projection used for large-scale mapping in the ellipsoidal form (topographic quadrangles) of the United States by the U.S. Geological Survey until the 1950's

Basis for Progressive Military Grid used by the U.S. Army until the 1940's

Projection for many early coastal charts by the U.S. Coast and Geodetic Survey

Not recommended for regional maps, because other projections are better

\section{Origin}

Apparently originated about 1820 by Ferdinand Rudolph Hassler (1770-1843), first director of the Survey of the Coast (later the U.S. Coast and Geodetic Survey)

\section{Aspects}

Transverse aspect (fig. 43D) was proposed by Charles H. Deetz of the U.S. Coast and Geodetic Survey in the early 20th century. The National Geographic Society has used the transverse aspect for maps of Eurasia and the Soviet Union.

\section{Other names}

American Polyconic

Ordinary Polyconic

\section{Similar projections}

Rectangular Polyconic projection (p. 110) has identical parallels of latitude, but meridians are plotted to intersect parallels at right angles.

The Modified Polyconic projection for the International Map of the World (p. 111) series has two meridians that are true to scale.

An "Equidistant Polyconic" projection was used by the U.S. Coast and Geodetic Survey for coastal charts in the 19th century. Its construction is unclear, but it was apparently different from other polyconics.

Equal-area polyconic projections were presented by Hans Maurer in 1935 in Germany and by Albert H. Bumstead of the National Geographic Society in 1937.

Other modified Polyconics have been presented by G.T. McCaw in England in 1921, by G.A. Ginzburg and T.D. Salmanova in the Soviet Union in 1949 and 1951, and by G.E. Bousfield in Canada in 1950.

"Polyconic" is sometimes applied to all

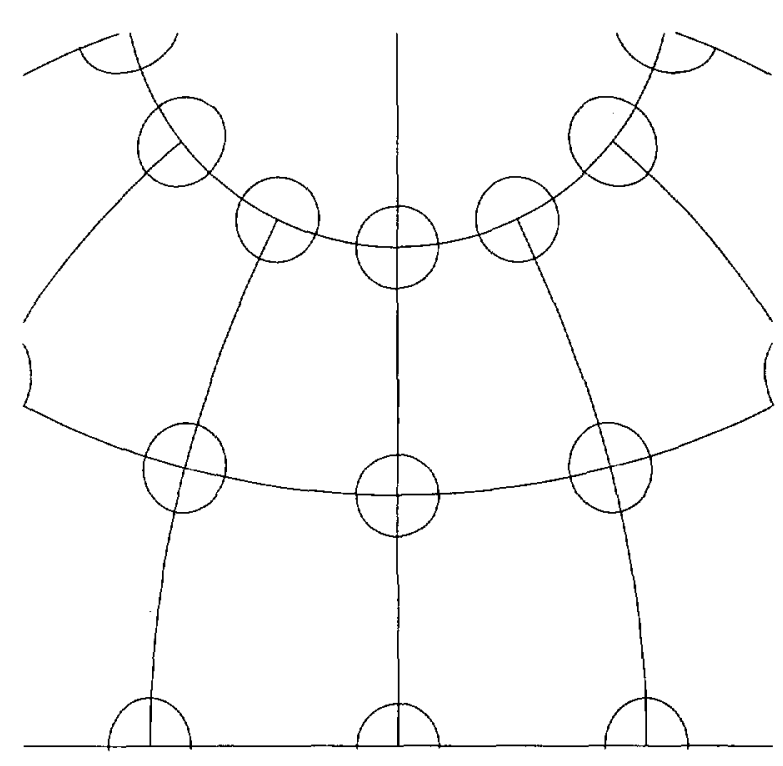

projections having circular arcs for parallels of latitude, including regular conics and the Stereographic, but this usage is not common. 


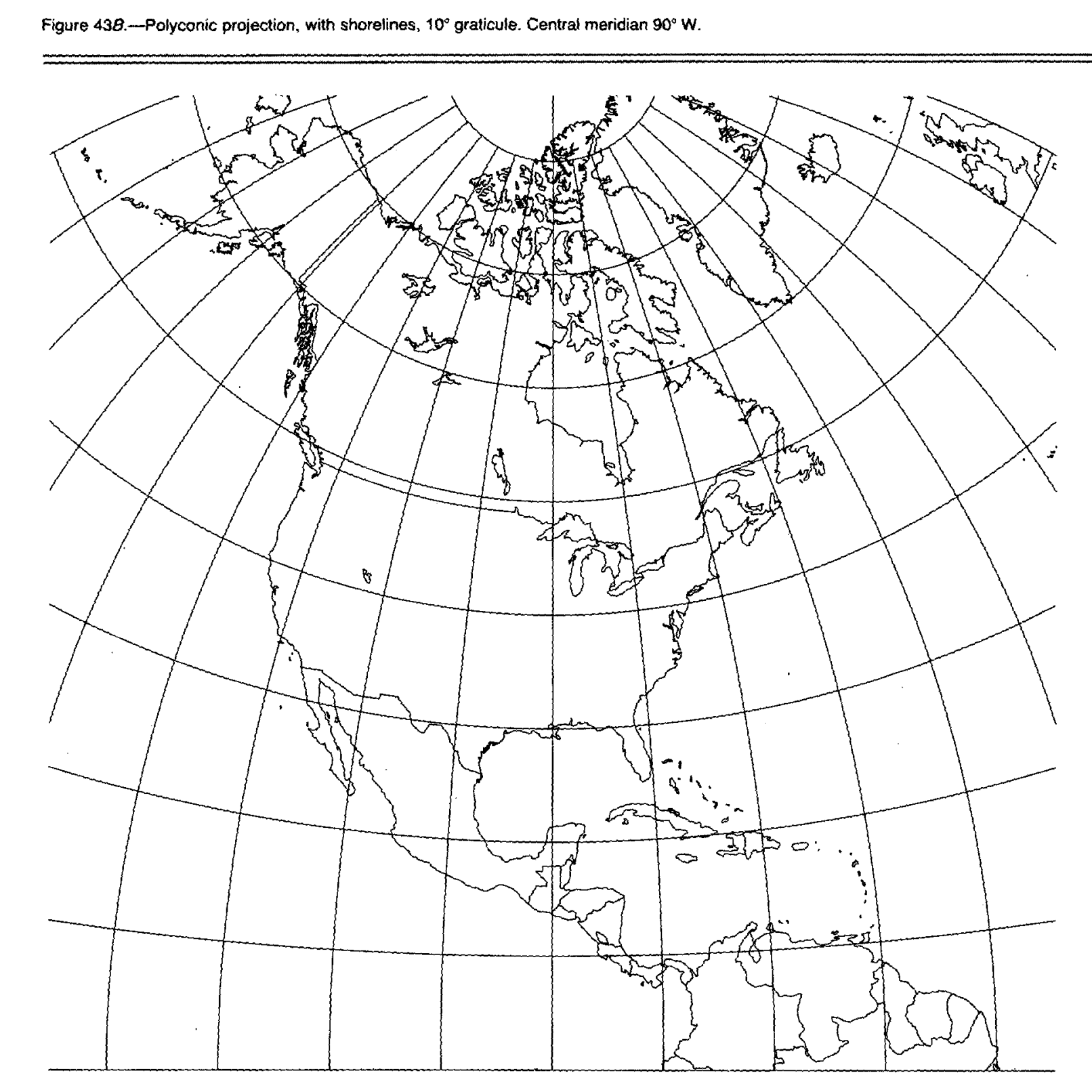




\section{POLYCONIC Projection}

Figure $43 \mathrm{C}$.-Polyconic projection, with shorelines (world map), $30^{\circ}$ graticule. Central meridian $90^{\circ} \mathrm{W}$.

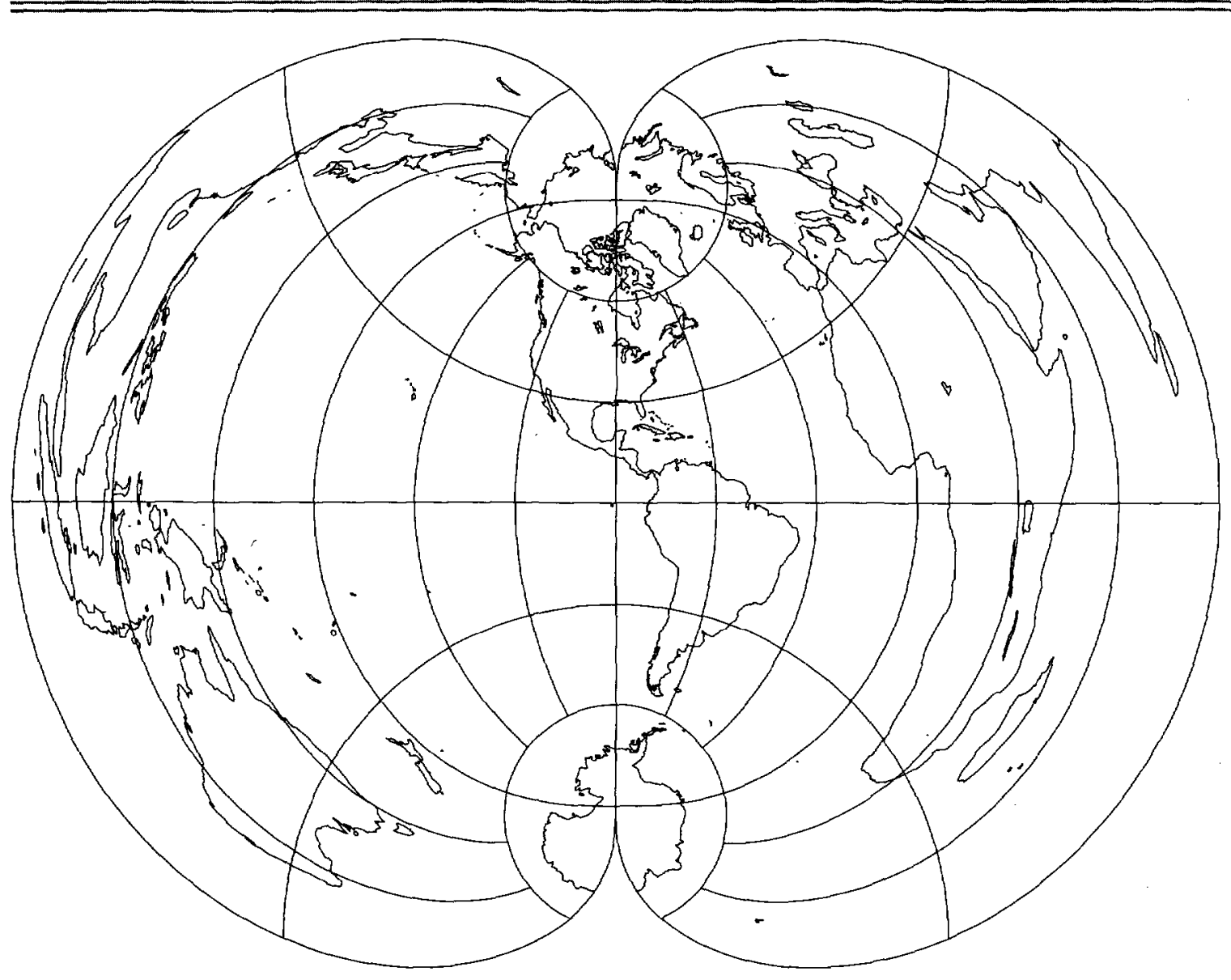


Figure 43D.-Transverse Polyconic projection, with shorelines, $10^{\circ}$ graticule. Central meridian $160^{\circ} \mathrm{W}$. North Pole at $-40^{\circ}$ Iongitude on base projection, showing North Pacific Ocean.

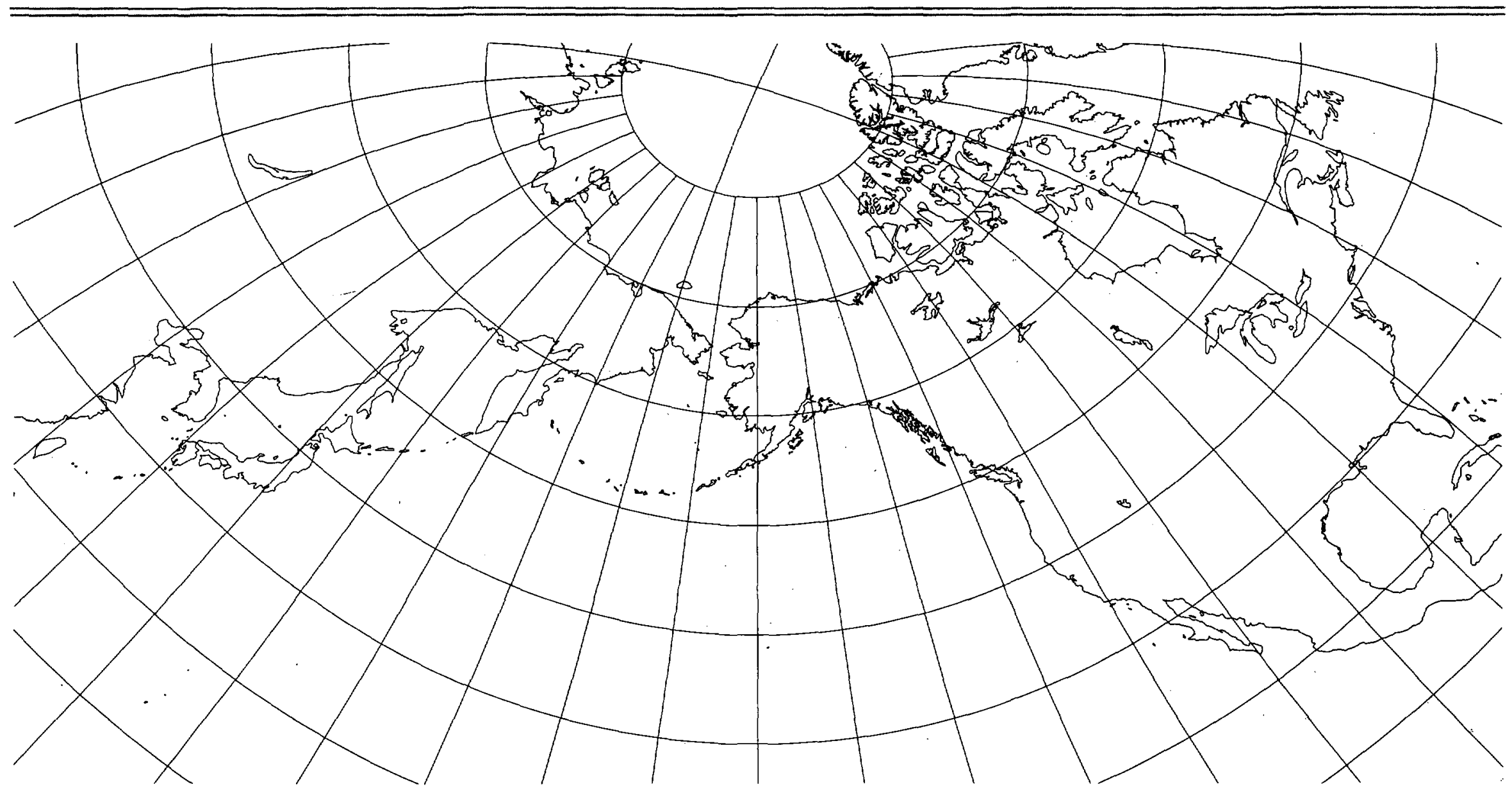




\section{RECTANGULAR POLYCONIC Projection}

\section{Classifications}

Polyconic

Neither conformal nor equal area

\section{Graticule}

Meridians: Central meridian is a straight line. All other meridians are complex curves, equally spaced along the Equator or along two chosen parallels symmetrical about the Equator. Meridians intersect all parallels at right angles.

Parallels: Equator is a straight line, poles are points, and all other parallels are nonconcentric circular arcs spaced at true distances along the central meridian. Each parallel has a curvature identical to its curvature on a cone tangent at that latitude. Many cones are involved; hence, the name "Polyconic projection."

Symmetry: About the central meridian or the Equator

\section{Scale}

True along the central meridian and along the Equator or two chosen parallels of true scale

\section{Distortion}

Free of distortion only along the central meridian. Although meridians intersect parallels at right angles, there is some angular distortion at all points other than along the central meridian.

\section{Usage}

Large-scale military mapping in Great Britain

\section{Other names}

War Office (British)

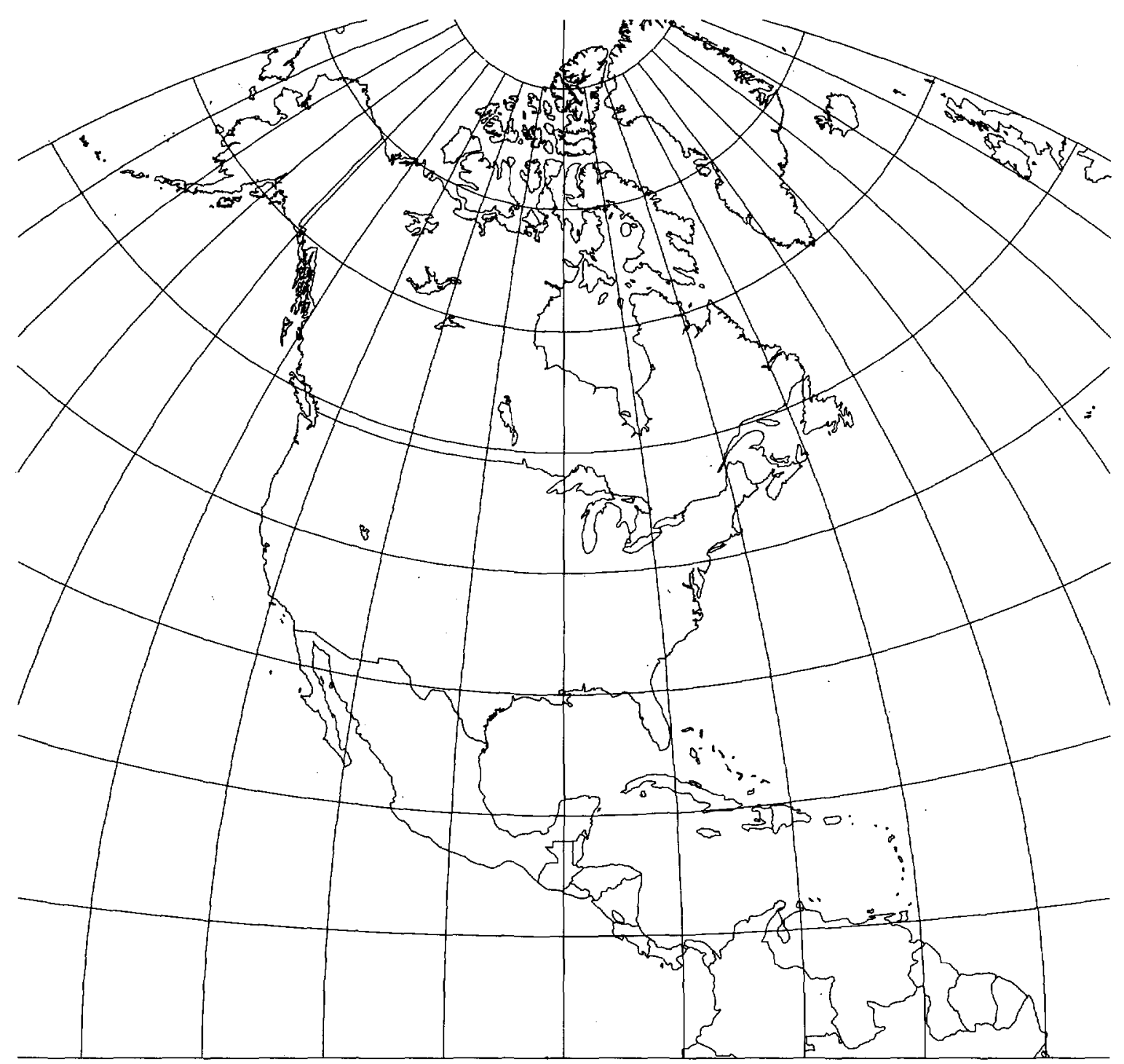

Similar projections

Listed under Polyconic projection (p. 106) 


\section{MODIFIED POLYCONIC Projection for the International Map of the World series}

\section{Classifications}

Polyconic

Neither conformal nor equal area

\section{Graticule}

Meridians: Straight lines converging but not to a common point

Parallels: Equator is a straight line, poles are points, and all other parailels are nonconcentric circular arcs spaced at true distances along the two meridians $2^{\circ}$ on either side of the central meridian (or $4^{\circ}$ or $8^{\circ}$ at latitudes beyond $60^{\circ} \mathrm{N}$. or S.). Each paralle has a curvature identical to its curvature on a cone tangent at that latitude.

Poles: Points

Symmetry: About the central meridian. Theoretically about the Equator, but the use is confined to zones that do not cross the Equator.

\section{Scale}

True along two meridians $2^{\circ}$ on either side of the central meridian (or $4^{\circ}$ or $8^{\circ}$ at latitudes beyond $60^{\circ} \mathrm{N}$. or $\mathrm{S}$.) and along two parallels at the northern and southern edges of the quadrangles, which extend $4^{\circ}$ in latitude, beginning at the Equator

\section{Distortion}

Not completely free of distortion anywhere, but distortion for the small quadrangles Involved is very low

\section{Other features}

Quadrangles in the International Map of the World series using this projection can be joined at the edges from north to south or from east to west but not in all directions at once without creating gaps.

\section{Usage}

The official projection for all new quadrangle maps in the International Map of the World series from 1909 to 1962 . It is still in use for existing quadrangles and for some new quadrangles. These quadrangles extend $4^{\circ}$ in latitude and $6^{\circ}$ in longitude, except that, beyond latitudes $60^{\circ} \mathrm{N}$. or S., the width is $12^{\circ}$ or $24^{\circ}$ of longitude.

\section{Origin}

Presented by Charles Lallemand of France in 1909 specifically for the International Map of the World series

\section{Similar projections}

Listed under Polyconic projection (p. 106)

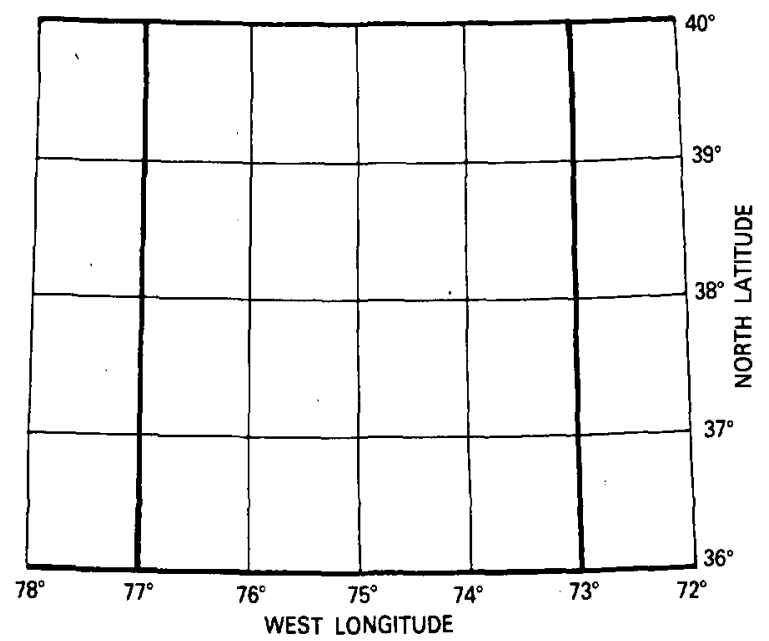

Figure 45.-Modified Polyconic projection for the International Map of the World series. Typical quadrant with $1^{\circ}$ graticule. Lines of true scale are heavy lines. 


\section{Classifications}

Pseudoconic

Equal area

\section{Graticule}

Meridians: Central meridian is a straight line. Other meridians are complex curves connecting points equally spaced along each parallel of latitude and concave toward the central meridian.

Parallels: Concentric circular arcs spaced at true distances along the central meridian. The curvature of the central or standard parallel is identical to its curvature on a cone tangent at that latitude.

Poles: Points

Symmetry: About the central meridian

\section{Scale}

True along the central meridian and along each parallel

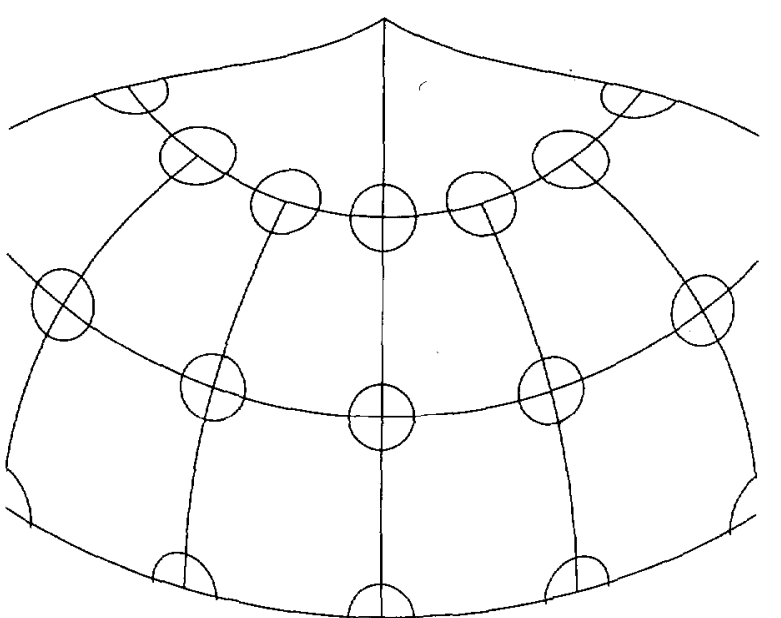

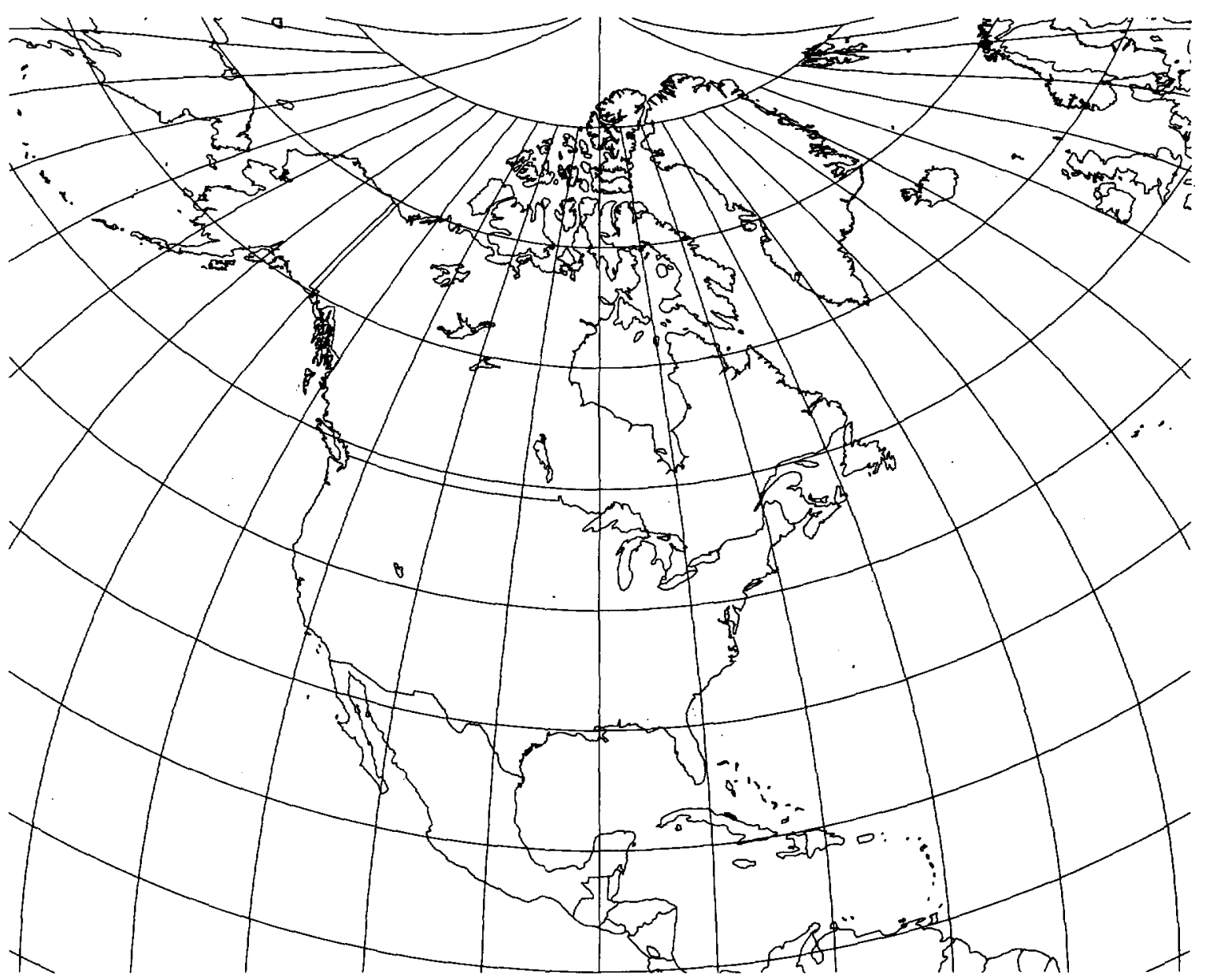

Distortion

Free of all distortion along the central meridian and the central parallel (fig. $46 A$ )

Figure $46 \mathrm{~A}$.-Bonne projection, with Tissot indicatrices, $30^{\circ}$ graticule. Central parallel $40^{\circ} \mathrm{N}$. 


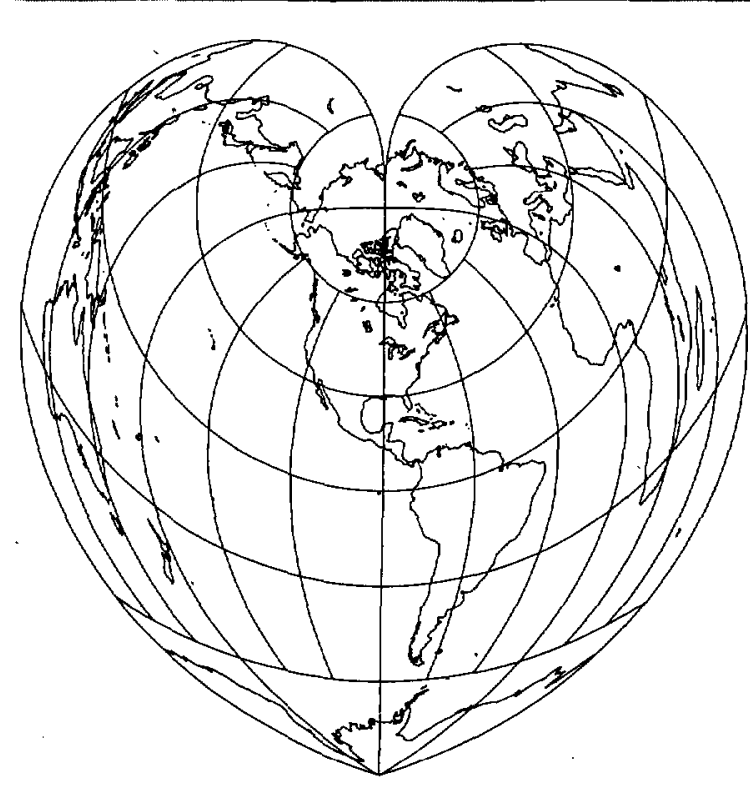

Usage

Frequently used until recently for atlas maps of continents. Used in the ellipsoidal form for topographic mapping of France in the early 19th century.

\section{Origin}

Developed in rudimentary form by Claudius Ptolemy (about A. D. 100). Further developed by Bernardus Sylvanus in 1511. Used

considerably by Rigobert Bonne (1727-95) of France, especially in 1752; the name is taken from him.

\section{Limiting projections}

Werner projection (fig. $46 C$ and p. 114) if a pole is made the standard parallel

Sinusoidal projection (fig. $46 \mathrm{D}$ and p. 37) if the Equator is made the standard parallel.
The pseudoconic projection thereby becomes a pseudocylindrical projection, but the Bonne formulas must be rewritten.

\section{Similar projection}

John Bartholomew combined the Bonne projection with the Equidistant Conic projection with two standard parallels (p. 92). In his 1942 "Kite" and 1958 "Regional" projections, the Equidistant Conic is used in the north temperate zone, and the Bonne is used south of the Tropic of Cancer in interrupted form. The more northern regions are based on the Bonne ("Kite") and Conic ("Regional") projections. These two projections emphasize land masses, and Bartholomew's 1958 "Lotus" projection emphasizes the oceans by using a southern version of the "Regional" projection.

Fure $46 D$. -Sinusoidal projection, with shorelines, $30^{\circ}$ graticule. Central meridian $90^{\circ} \mathrm{W}$. Equatorial and pseudocylindrical limit of Bonne projection.

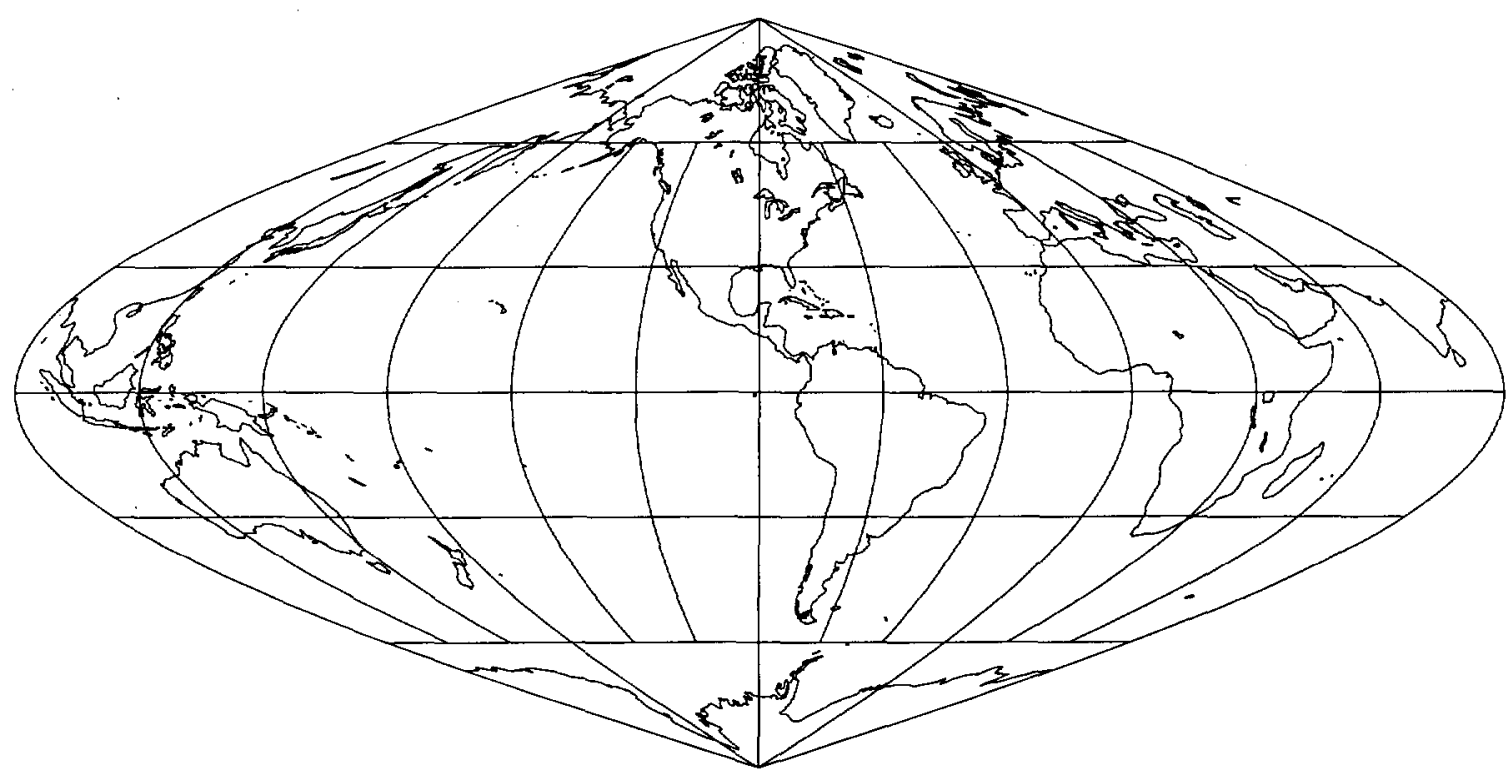




\section{Classifications}

Pseudoconic

Equal area

Cordiform (heart shaped)

\section{Graticule}

Meridians: Central meridian is a straight

line. Other meridians are complex curves connecting points equally spaced along each parallel of latitude and concave toward the central meridian.

Parallels: Concentric circular arcs spaced at true distances along the central meridian. One pole (usually the North Pole) is the center for these circular arcs.

Poles: Points

Symmetry: About the central meridian

\section{Scale}

True along the central meridian and along each parallel

\section{Distortion}

Free of all distortion only along the central meridian (fig. 47A)

\section{Usage}

Frequently used for world and some continental maps of the 16th and 17th centuries. Replaced by the Bonne projection for continental maps. No longer used except as a novelty.

\section{Origin}

Developed by Johannes Stabius (Stab) (?-1522) of Vienna about 1500 and promoted by Johannes Werner (1466-1528) of Nuremberg in 1514

\section{Other names}

Stab-Werner

\section{Similar projections}

Bonne projection is a general form on which the parallels are also circular arcs but are not generally centered on a pole. The Werner is the polar limiting form.

In 1904, Wilhelm Schjerning interrupted the Werner projection, centered on the South Pole, with three central meridians and three "petals" for a world map emphasizing the oceans.

Goode Polar Equal-Area projection, presented by John Paul Goode in 1928, is an interrupted Werner projection, centered on the North Pole and using several central meridians to display continents in a flowerlike pattern.

"Tetrahedral" projection, presented by John Bartholomew by 1942, is a combination of the Polar Azimuthal Equidistant projection (p. 133) from the pole to the tropic line of the same hemisphere and an interrupted Werner from there to the opposite pole, some adjustment being made to obtain a fit. The North Pole is the center for a map of land masses, and the South Pole is the center for an ocean map.

In 1968, W. William-Olsson of Sweden combined the northern Polar Lambert Azimuthal EqualArea projection, extending to latitude $20^{\circ} \mathrm{N}$., with four identical lobes extending to the South Pole. The lobes are based on the Werner projection but are compressed north-south and expanded east-west to fit the Lambert and to retain equality of area.

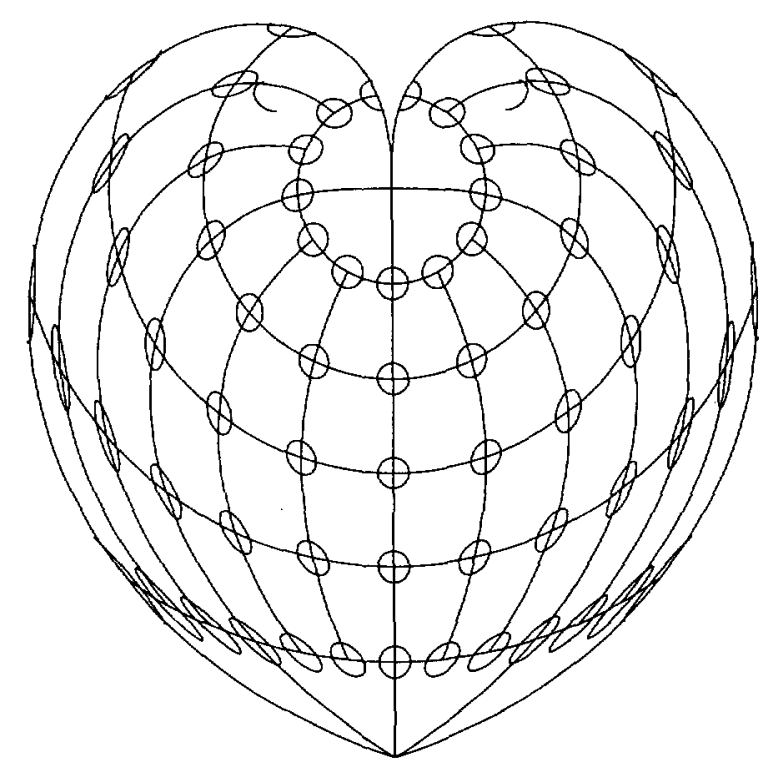


Figure 478 .-Werner projection, with shorelines, $15^{\circ}$ graticule. Central meridian $90^{\circ} \mathrm{W}$.

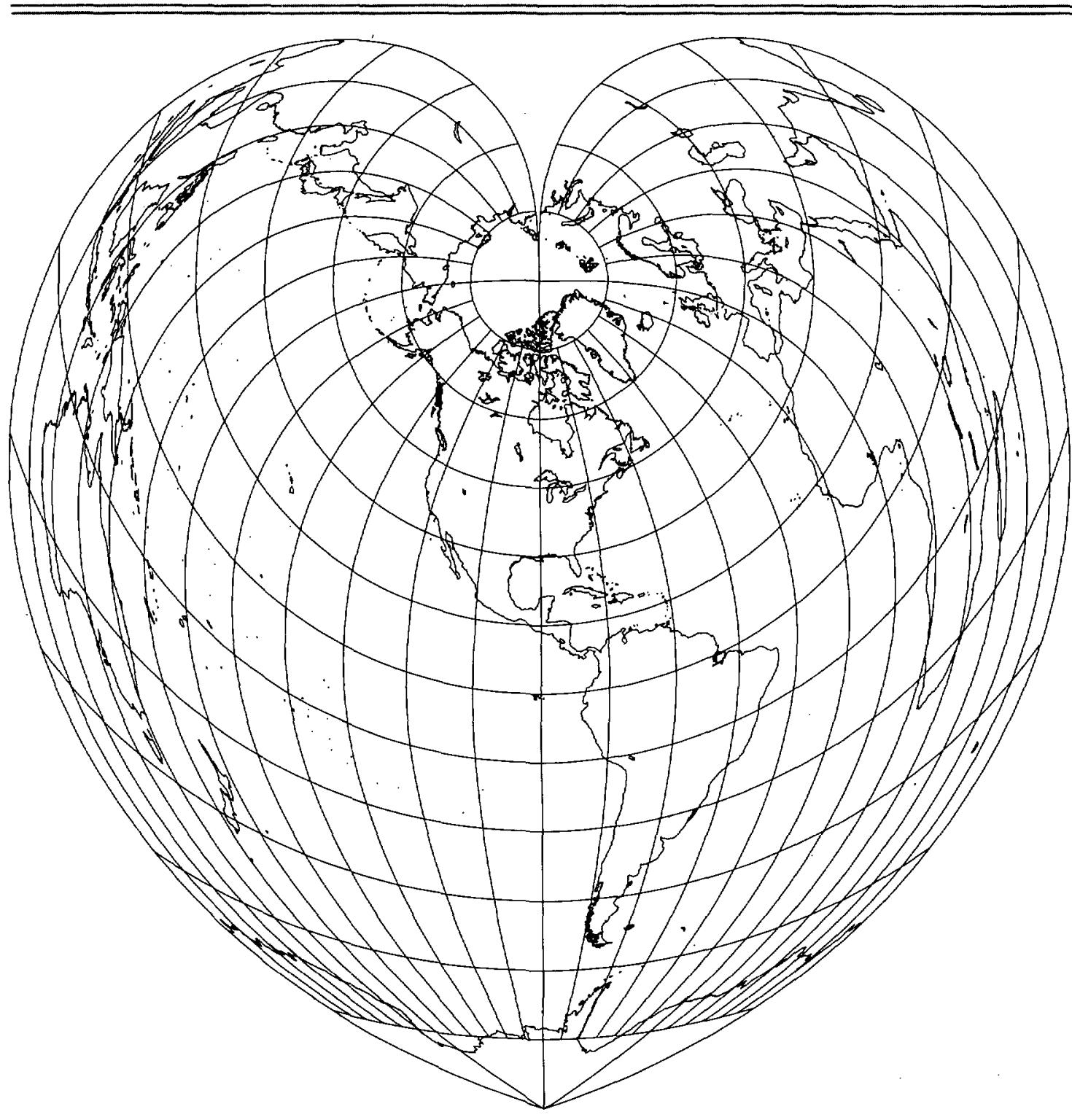




\section{Classifications}

Azimuthal

Perspective

Neither conformal nor equal area

\section{Graticule}

Polar aspect (fig. 48B):

Meridians: Equally spaced straight lines intersecting at the central pole. Angles

between them are the true angles.

Parallels: Unequally spaced circles centered at the pole, which is a point. The Equator and the opposite hemisphere cannot be shown. Spacing increases rapidly away from the pole.

Symmetry: About any meridian

Equatorial aspect (fig. $48 \mathrm{C}$ ) :

Meridians: Unequally spaced straight parallel lines. Only meridians within $90^{\circ}$ of the central meridian can be shown. Spacing increases away from the central meridian.

Parallels: Equator is a straight line perpendicular to the meridians. Other parallels are hyperbolic arcs concave toward the nearest pole. Spacing increases rapidly away from the Equator. Poles cannot be shown.

Symmetry: About the central meridian or the Equator

Oblique aspect (fig. 48D):

Meridians: Unequally spaced straight lines intersecting at the pole nearest the center of the projection

Parallels: Equator is a straight line perpendicular to the central meridian. If the central parallel is a north latitude, its colatitude $\left(90^{\circ}\right.$ - central latitude) is shown as a parabolic arc, more northern parallels are ellipses, and more southern parallels are hyperbolas, all concave toward the nearest pole. If the central parallel is a south latitude, opposite signs apply.

Symmetry: About the central meridian

\section{Range}

Less than one hemisphere centered on a given pole of projection or map origin

\section{Scale}

True only where the central parallel crosses the central meridian. Increases rapidly with the distance from the center.

\section{Distortion}

Figure 48A shows distortion for the polar aspect. Other aspects have identical distortion at the same distance from the projection center. Only the center is free from distortion. Distortion increases rapidly away from the center.

\section{Special features}

All great circles (including all meridians and the Equator) are shown as straight lines. Therefore, the path of the shortest distance between any two points on the map is a straight line.

Perspective projection from the center of the globe onto a plane tangent at the central point of projection

Less than a hemisphere can be shown on one map.

\section{Usage}

To show great-circle paths as straight lines and thus to assist navigators and aviators in determining appropriate courses. Too much distortion of many kinds for regular geographic maps.

Used in the past for some star maps and for world globes in the shapes of polyhedra

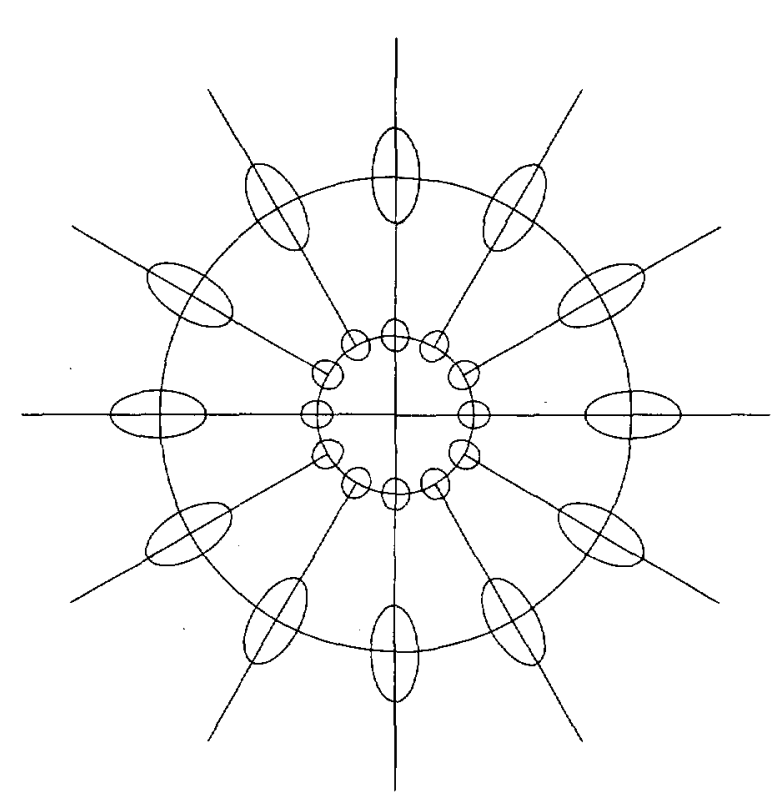

Origin

Possibly developed by Thales (c. 580 B.C.) of Greece. Name derived from the gnomon of a sundial, since meridians radiate like the hour markings on a sundial.

\section{Other names}

Gnomic

Central

\section{Similar projection}

Two-Point Azimuthal projection (p. 144) is a Gnomonic projection that has been compressed in one direction. 


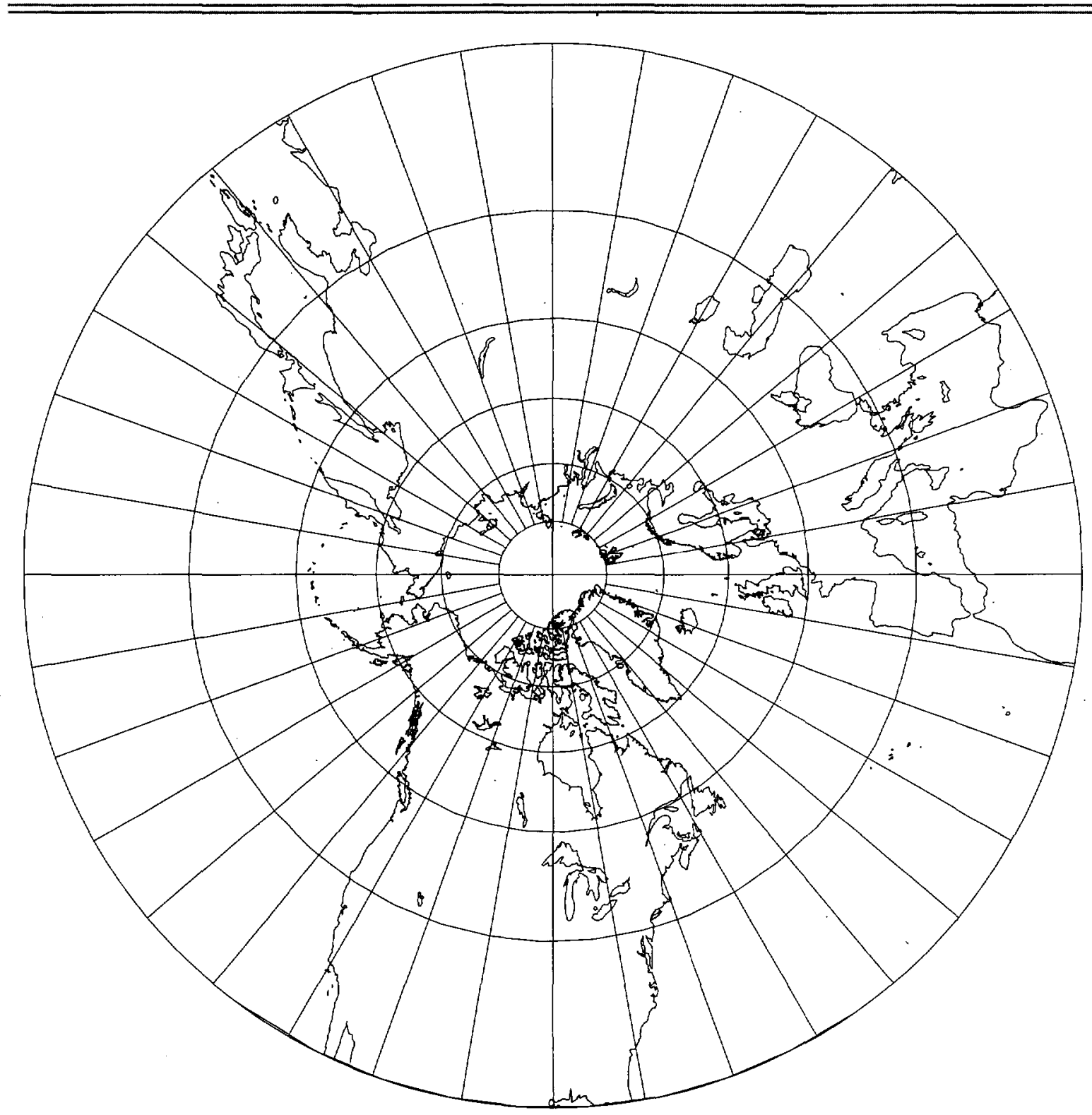




\section{GNOMONIC Projection}

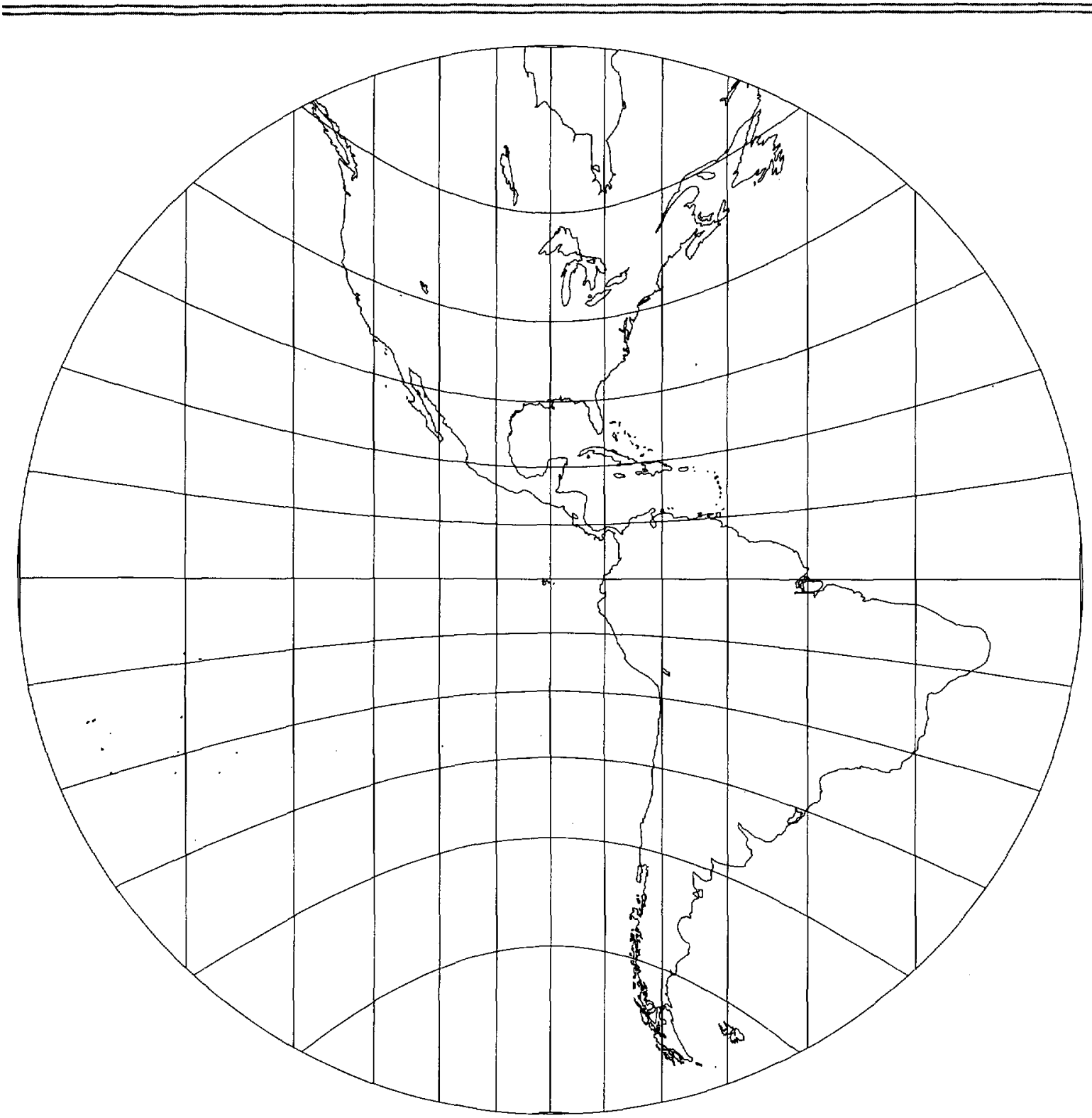

Figure $48 \mathrm{C}$.-Equatorial Gnomonic projection, with shorelines, $10^{\circ}$ graticule. Central meridian $90^{\circ} \mathrm{W}$. Range $60^{\circ}$. 


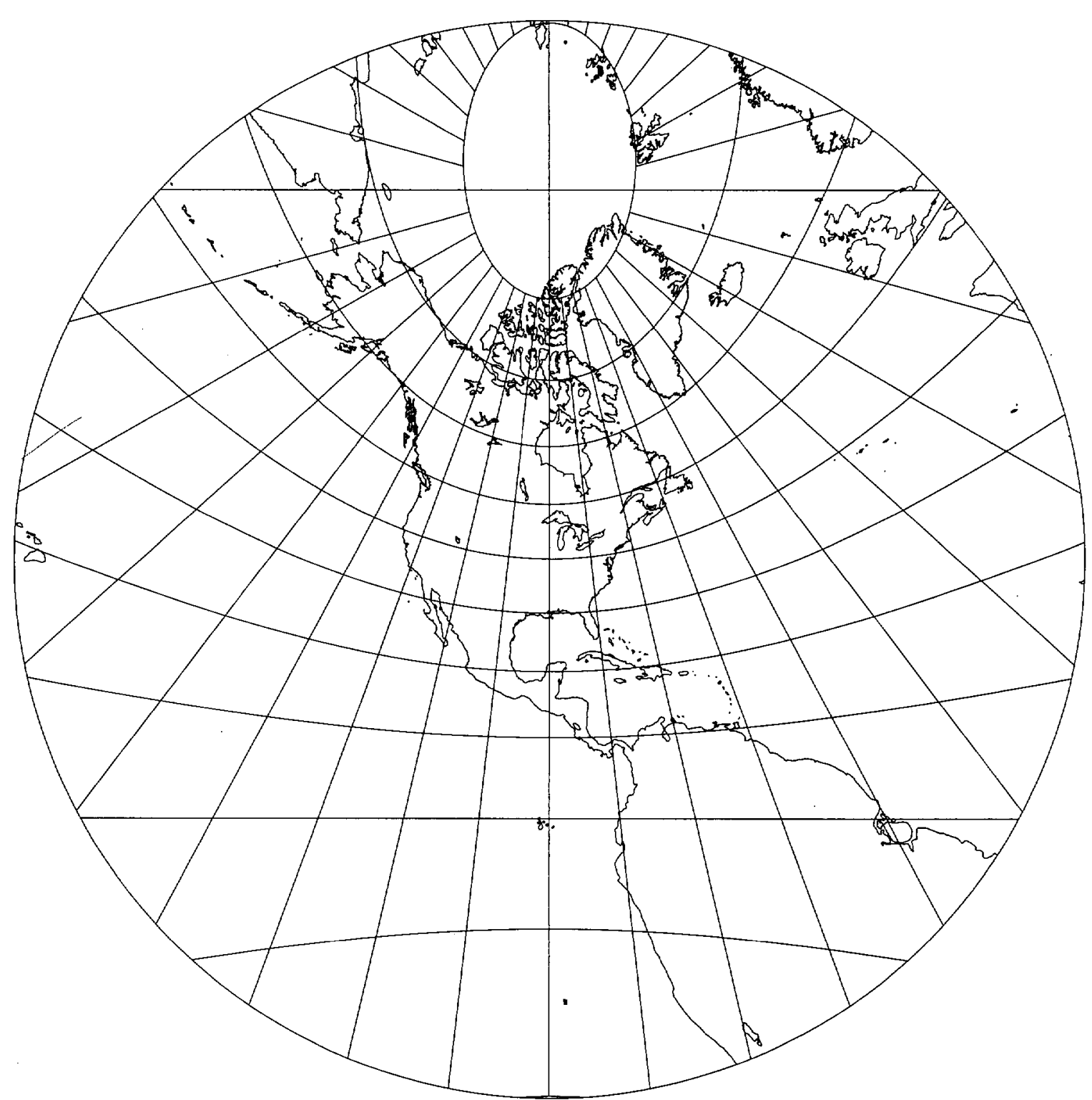

Figure 48D.-Oblique Gnomonic projection, with shorelines, $10^{\circ}$ graticule, central latitude $40^{\circ} \mathrm{N}$., central meridian $90^{\circ}$ W. Range $60^{\circ}$. 


\section{Classifications}

Azimuthal

Conformal

Perspective

Polyconic (general)

\section{Graticule}

Polar aspect (fig. 49B):

Meridians: Equally spaced straight lines

intersecting at central pole. Angles between them are the true angles.

Parallels: Unequally spaced circles centered at the pole, which is a point. Opposite pole cannot be shown. Spacing gradually increases away from the pole. Symmetry: About any meridian

Equatorial aspect (fig. 49C):

Meridians: Central meridian is a straight line. Other meridians are unequally spaced circular arcs intersecting at each pole. Spacing gradually increases away from the central meridian.

Parallels: Equator is a straight line. Other parallels are unequally spaced circular arcs concave toward the nearest pole. Spacing gradually increases away from the Equator along the central meridian, but spacing is equal along the meridians $90^{\circ}$ from the central meridian.

Symmetry: About the central meridian or the Equator

Oblique aspect (fig. 49D):

Meridians: Central meridian is a straight line. Other meridians are circular arcs intersecting at each pole and having their centers on the straight parallel opposite in sign to the central latitude. Spacing gradually increases away from the central meridian.
Parallels: The parallel opposite in sign to the central latitude is a straight line perpendicular to the central meridian. All other parallels are circular arcs concave toward the pole on the same side of the straight parallel.

Symmetry: About the central meridian

\section{Range}

One hemisphere conveniently; most but not all of the other hemisphere at accelerating scale

\section{Scale}

True only where the central latitude crosses the central meridian or, alternatively, along a circle concentric about the projection center (or a parallel on the polar aspect). Scale is constant along any circle having its center at the projection center, but scale increases moderately with distance from the center within a hemisphere.

\section{Distortion}

Figure $49 \mathrm{~A}$ shows distortion for the polar aspect. Other aspects have identical distortion at the same distance from the projection center. Only the center or the circle of true scale (if not the center) is free from all distortion.

\section{Special features}

All great or small circles (including all meridians and parallels) on the globe are shown as circular arcs or straight lines.

Perspective projection of the globe onto a tangent (or secant) plane from a point on the surface just opposite the point of tangency or the projection center. The straight parallel of latitude passes through this point.

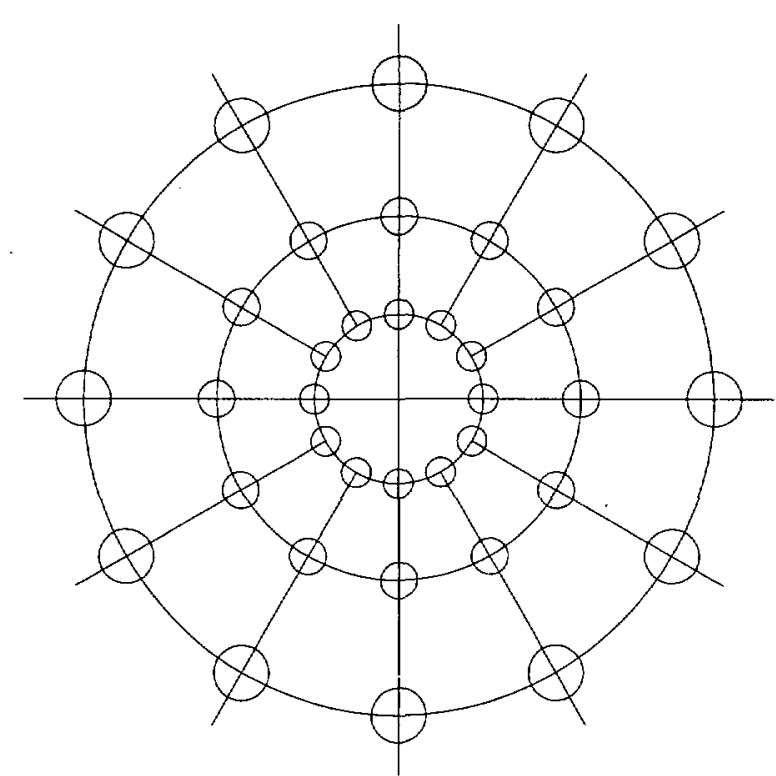

\section{Usage}

Commonly used in the polar aspect for topographic maps of polar regions. The equatorial aspect was used regularly for maps of the Eastern and Western Hemispheres in the 17th and 18th centuries. Oblique aspects are used to show paths of solar eclipses.

Recommended for conformal mapping of regions approximately circular in shape

Basis of modified Stereographic conformal projections using complex algebra, such as the Miller Oblated Stereographic (p. 148)

\section{Origin}

Apparently developed in polar aspect by Egyptians and Greeks by the $2 d$ century B.C. 
Figure 498.-Polar Stereographic projection, with shorelines, $10^{\circ}$ graticule. Central meridian $90^{\circ} \mathrm{W}$. Range $90^{\circ}$.

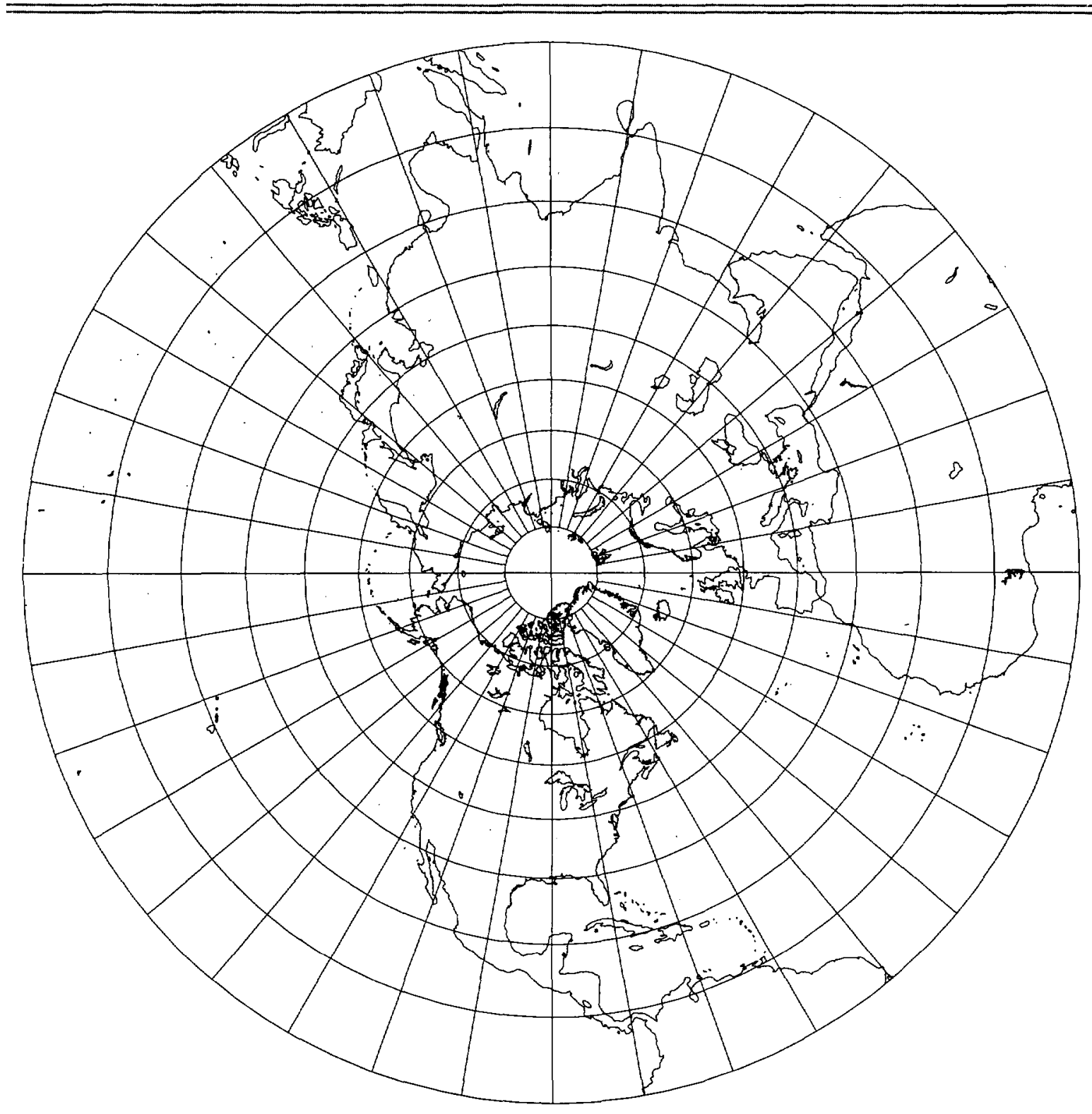




\section{STEREOGRAPHIC Projection}

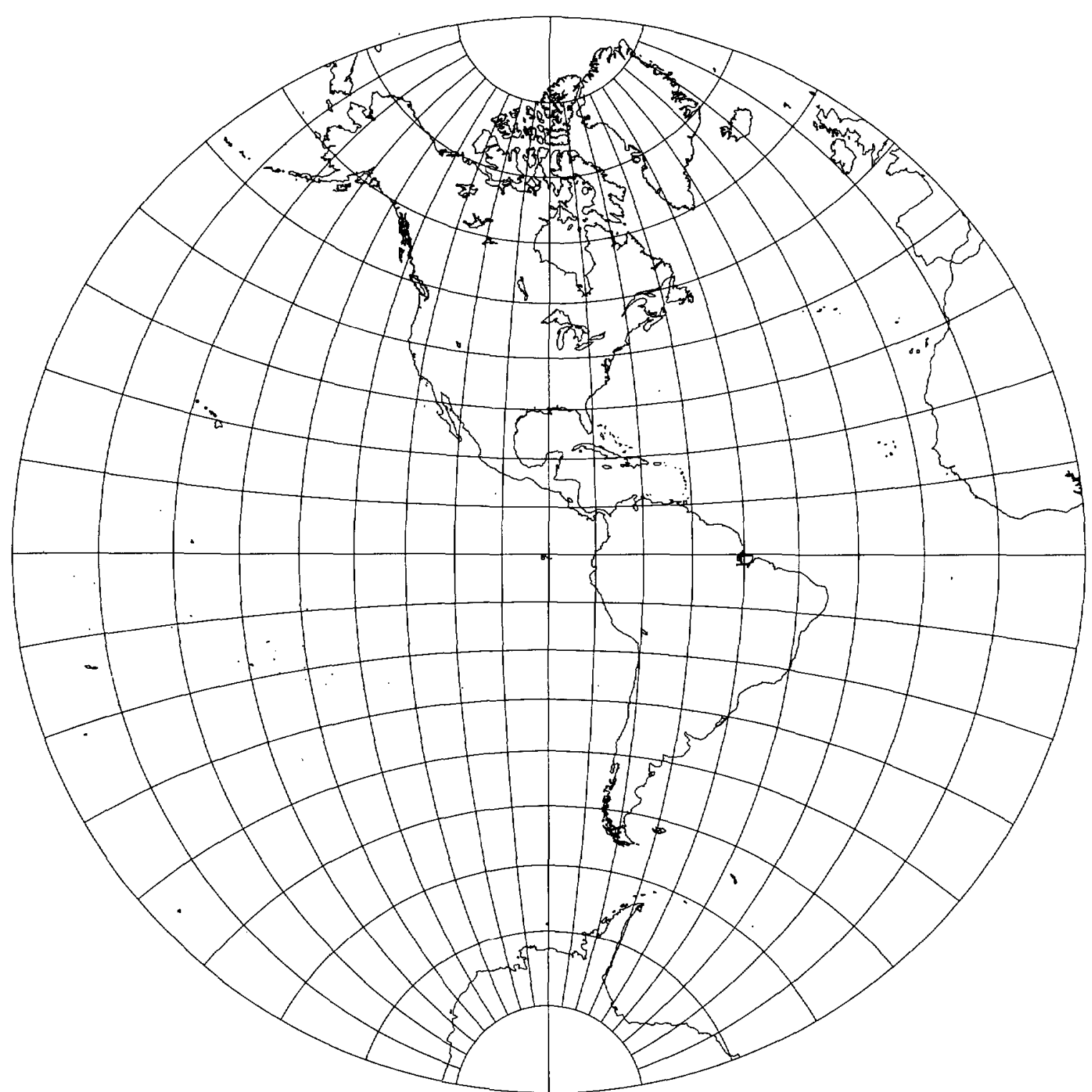

Figure $49 \mathrm{C}$.-Equatorial Stereographic projection, with shorelines, $10^{\circ}$ graticule. Central meridian $90^{\circ} \mathrm{W}$. Range $90^{\circ}$ 


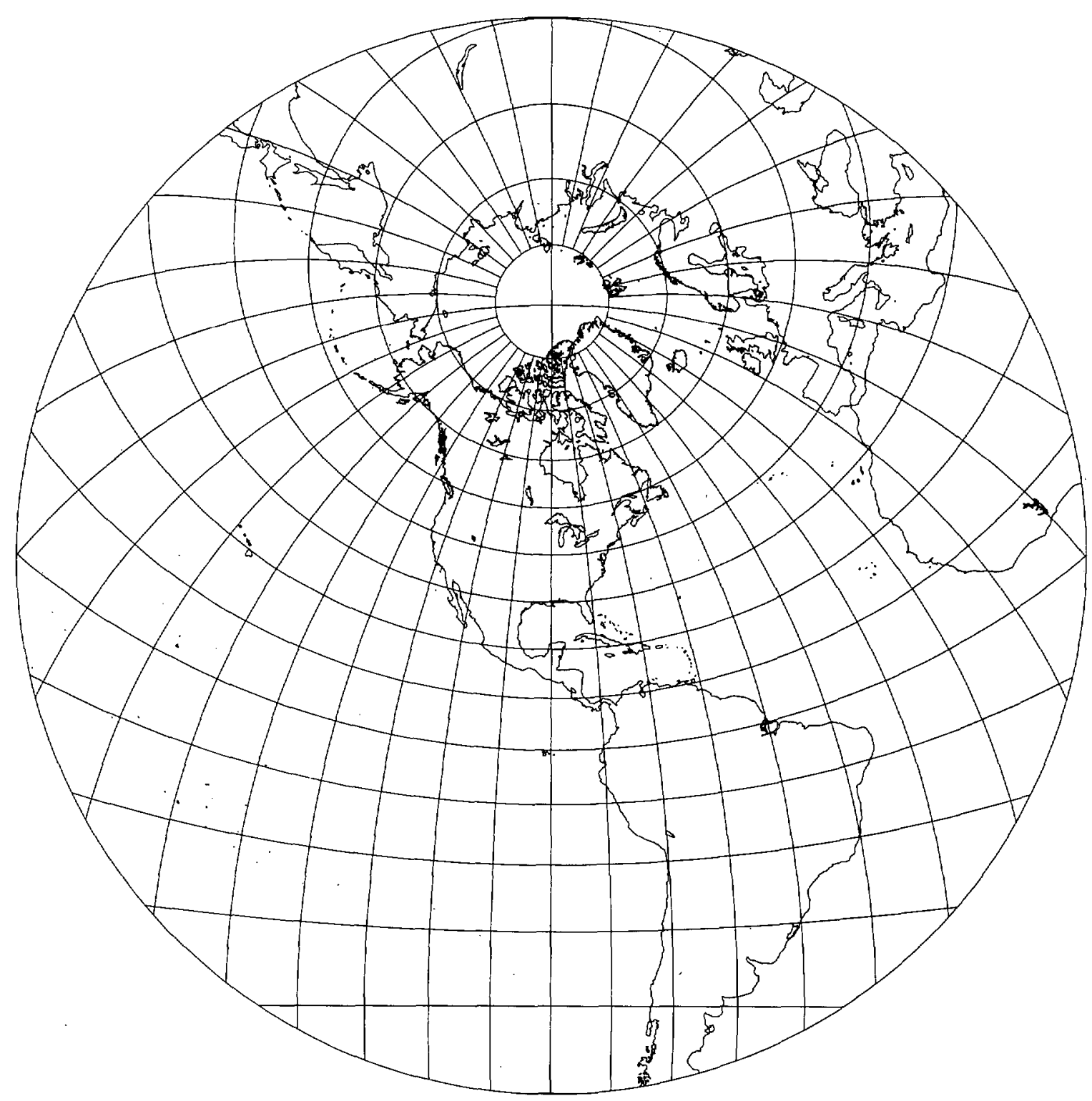

Figure $49 \mathrm{D}$.-Oblique Stereographic projection, with shorelines, $10^{\circ}$ graticule, central latitude $40^{\circ} \mathrm{N}$. Central meridian $90^{\circ} \mathrm{W}$. Range $90^{\circ}$. 


\section{ORTHOGRAPHIC Projection}

\section{Classifications}

Azimuthal

Perspective

Neither conformal nor equal area

\section{Graticule}

Polar aspect (fig. 50B):

Meridians: Equally spaced straight lines intersecting at the central pole. Angles between them are the true angles.

Parallels: Unequally spaced circles centered at the pole, which is a point. Spacing decreases away from the pole. Symmetry: About any meridian

Equatorial aspect (fig. 50C):

Meridians: Central meridian is a straight line. Outer meridians $90^{\circ}$ away are circles. Other meridians are unequally spaced elliptical arcs intersecting at each pole and concave toward the central meridian Spacing decreases away from the central meridian.

Parallels: Unequally spaced straight parallel lines perpendicular to the centra meridian. Spacing decreases away from the Equator. Parallels intersect the outer meridian, however, at equal intervals. Symmetry: About the central meridian or the Equator

Oblique aspect (fig. 50D):

Meridians: Central meridian is a straight line. Other meridians are semiellipses intersecting at each pole. Spacing

\section{decreases away from the central}

meridian.

Parallels: Complete or partial ellipses, all of the same shape (or eccentricity); their minor axes lie along the central meridian. Spacing decreases away from the center of projection.

Symmetry: About the central meridian

\section{Range}

No more than one hemisphere at a time

\section{Scale}

True at the center and along any circle having its center at the projection center but only in the direction of the circumference of the circle. Scale decreases radially with distance from the center.

\section{Distortion}

Figure 50A shows distortion for the polar aspect. Other aspects have identical distortion

at the same distance from the projection center. Only the center is free of distortion, which increases rapidly away from the center. Usage

Extreme distortion near the edge of the hemisphere.

\section{Special features}

Perspective projection of the globe onto a tangent plane from an infinite distance (that is, orthogonally); thus, the map has the look of a globe.

All great or small circles (including all meridians and parallels) are shown as elliptical arcs or straight lines.

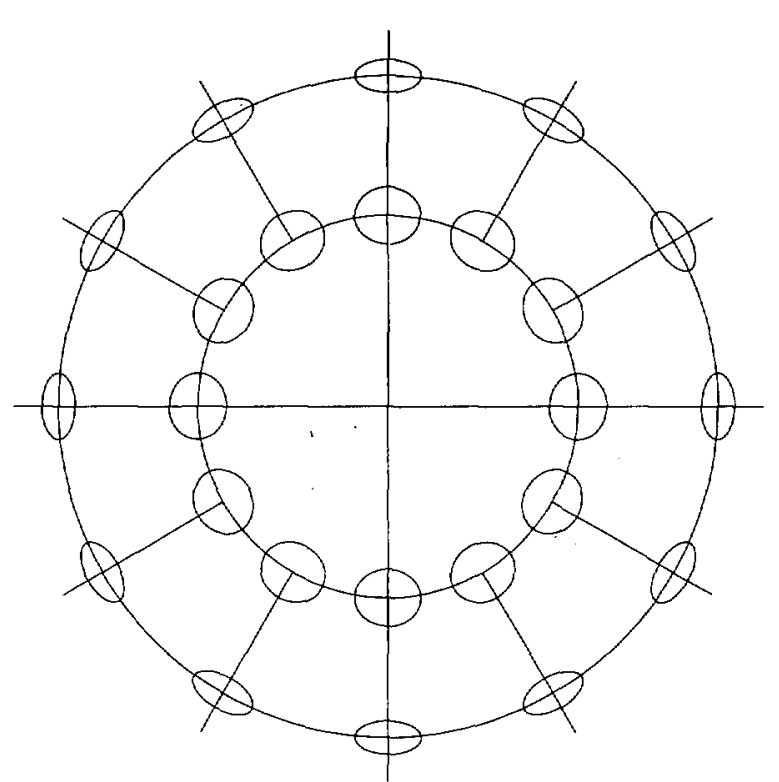

Figure 50A.-Polar Orthographic projection, with Tissot indicatrices, $30^{\circ}$ graticule. Range $80^{\circ}$

Pictorial views of the Earth, resembling those seen from space

\section{Origin}

Apparently developed by Egyptians and Greeks by the $2 d$ century B.C.

\section{Similar projections}

General Vertical Perspective (p. 128), when projected from more than a few thousand kilometers above the Earth 


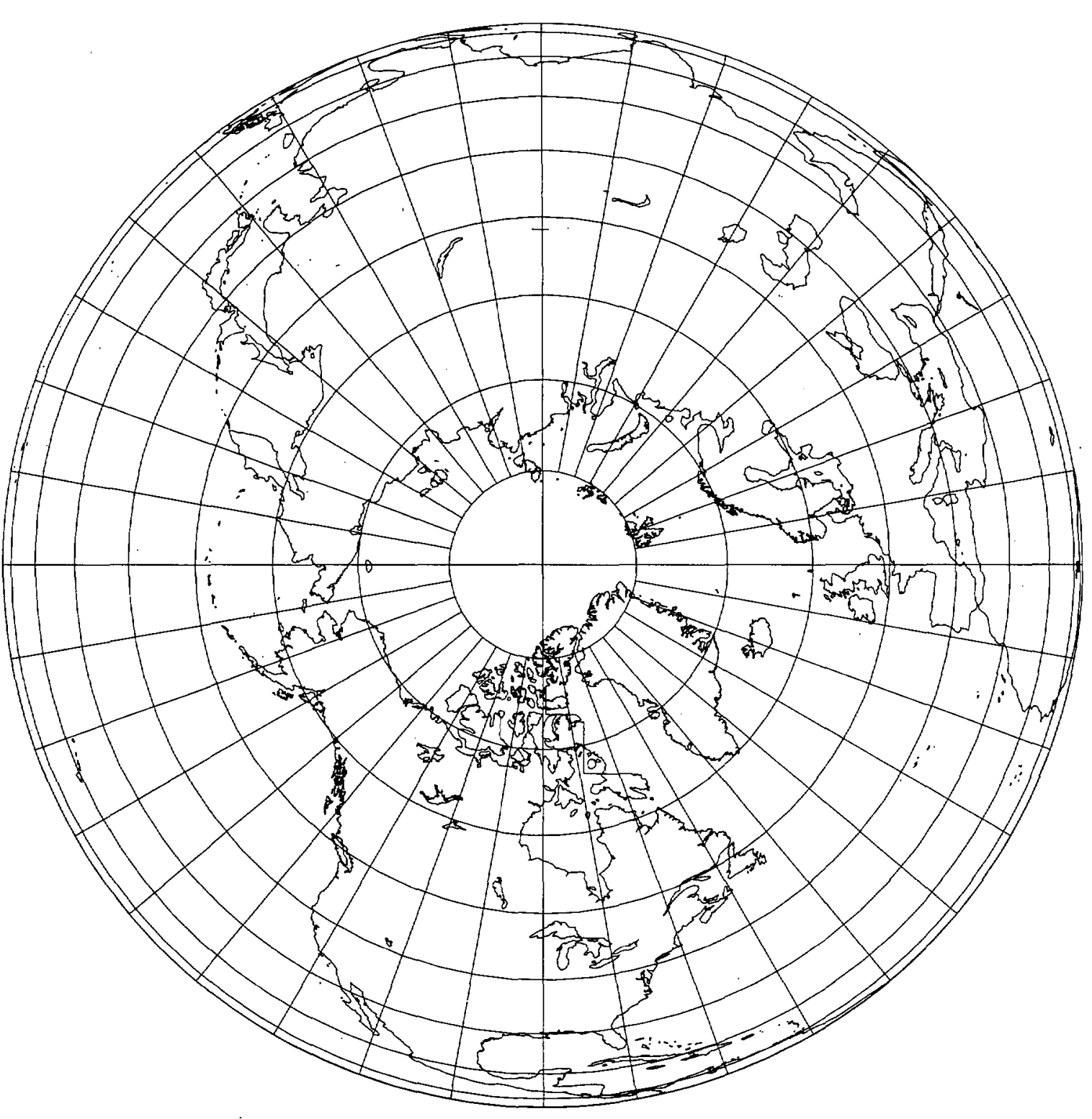




\section{ORTHOGRAPHIC Projection}

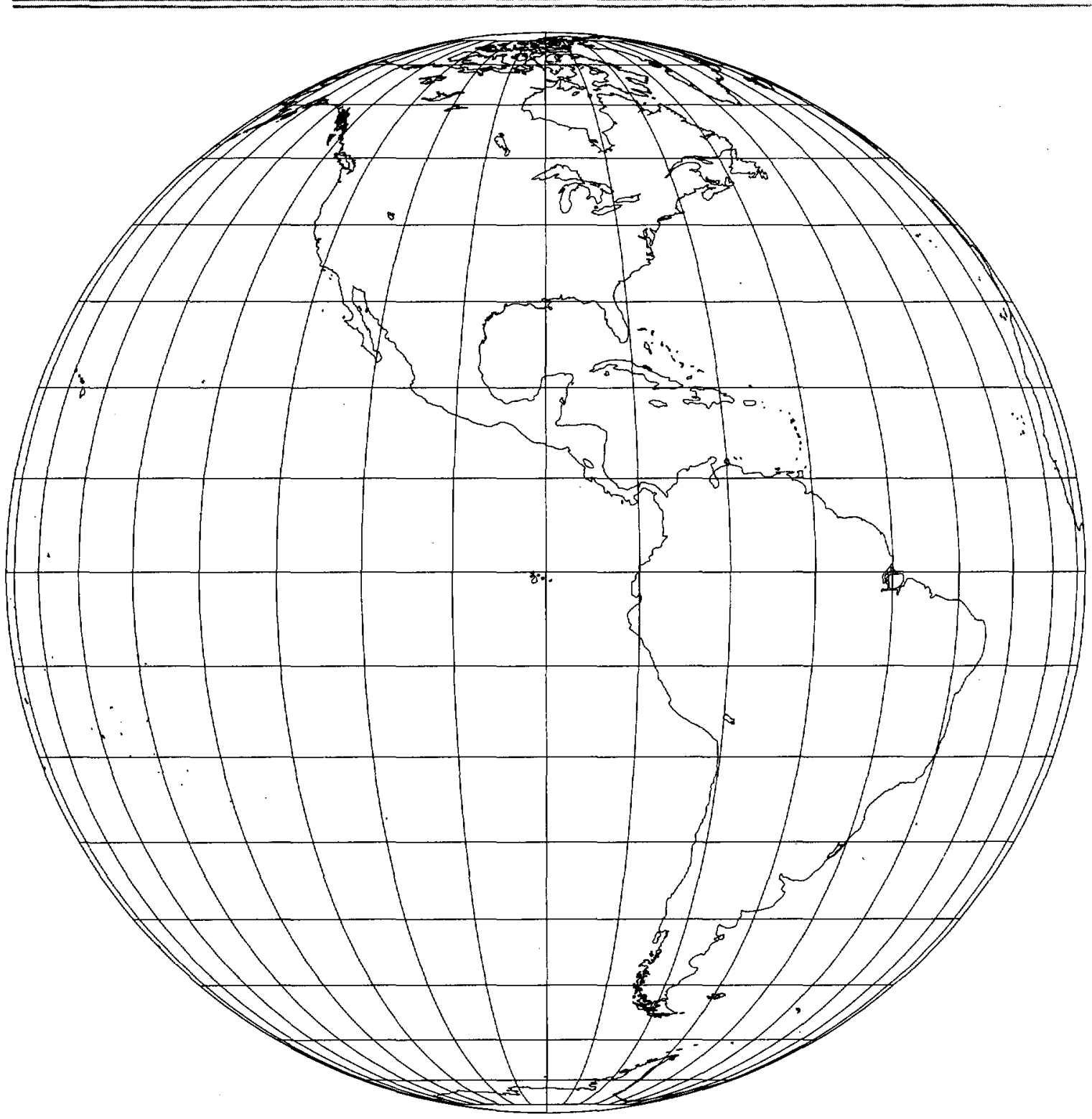

Figure 50C.-Equatorial Orthographic projection, with shorelines, $10^{\circ}$ graticule. Central meridian $90^{\circ} \mathrm{W}$ 


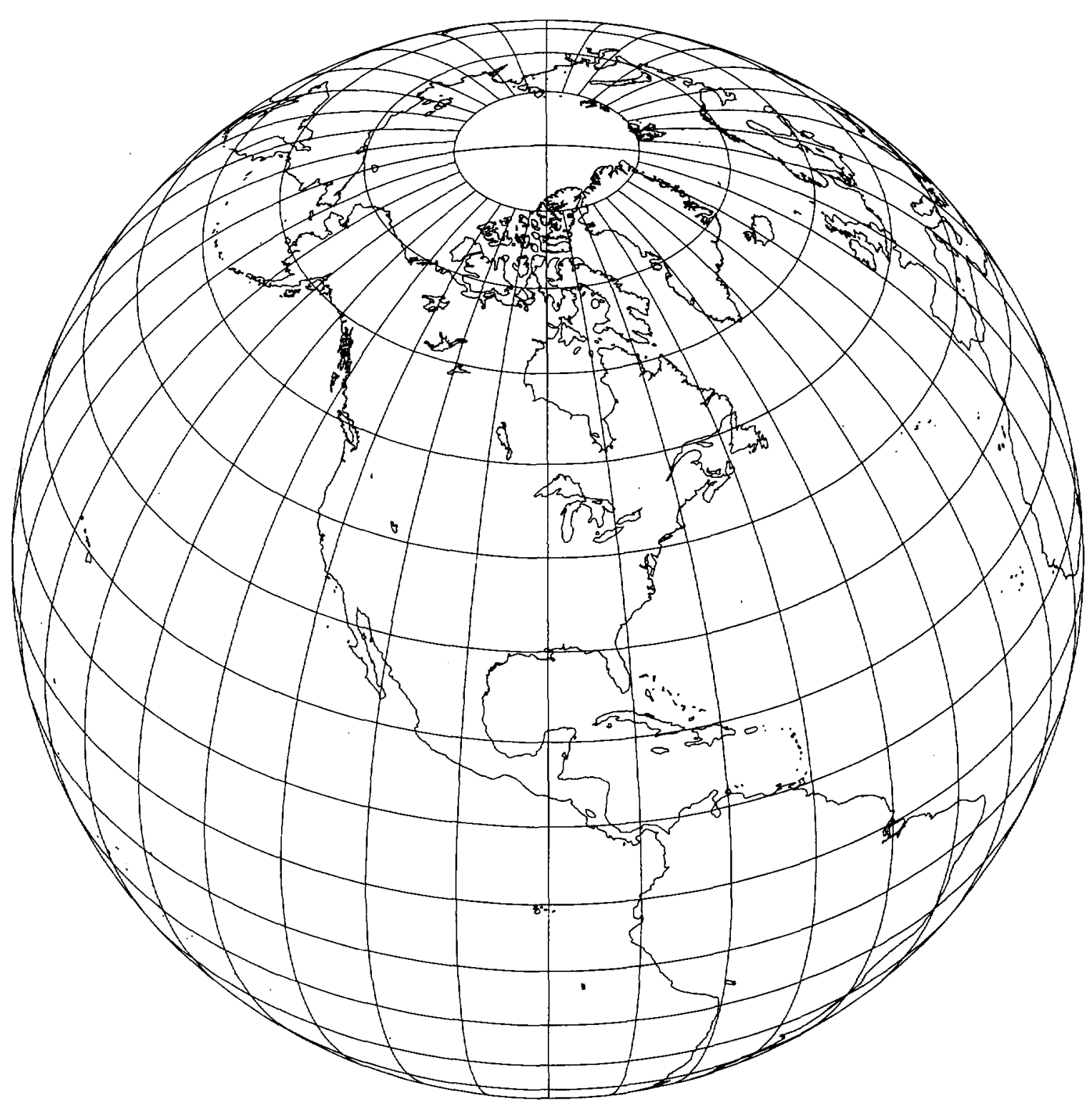

Figure 500.-Oblique Orthographic projection, with shorelines, $10^{\circ}$ graticule, central latitude $40^{\circ} \mathrm{N}$. Central meridian $90^{\circ} \mathrm{W}$. 


\section{GENERAL VERTICAL PERSPECTIVE Projection}

\section{Classifications}

\section{Azimutha}

Perspective

Neither conformal nor equal area (except in Stereographic form)

\section{Graticule}

Polar aspect (fig. 51A):

Meridians: Equally spaced straight lines intersecting at the central pole. Angles between them are the true angles.

Parallels: Unequally spaced circles centered at the pole, which is a point. If the usual perspective from above the surface is projected, the spacing of parallels decreases away from the pole and the Equator cannot be shown. If point of perspective is beneath the surface, the spacing may increase or decrease away from the pole, and part of the opposite hemisphere can be shown.

Symmetry: About any meridian

Equatorial aspect (fig. 51B):

Meridians: Central meridian is a straight line. Other meridians are generally unequally spaced elliptical arcs intersecting at the poles (which may not be visible).

Parallels: Equator is a straight line. Other parallels are usually elliptical arcs. If point of perspective is beneath the surface, the parallels can be elliptical, parabolic, or hyperbolic arcs.

Symmetry: About the central meridian or the Equator

Oblique aspect (figs. $51 C, 51 D$ ):

Meridians: Central meridian is a straight line. Other meridians are generally unequally spaced elliptical arcs intersecting at each pole.

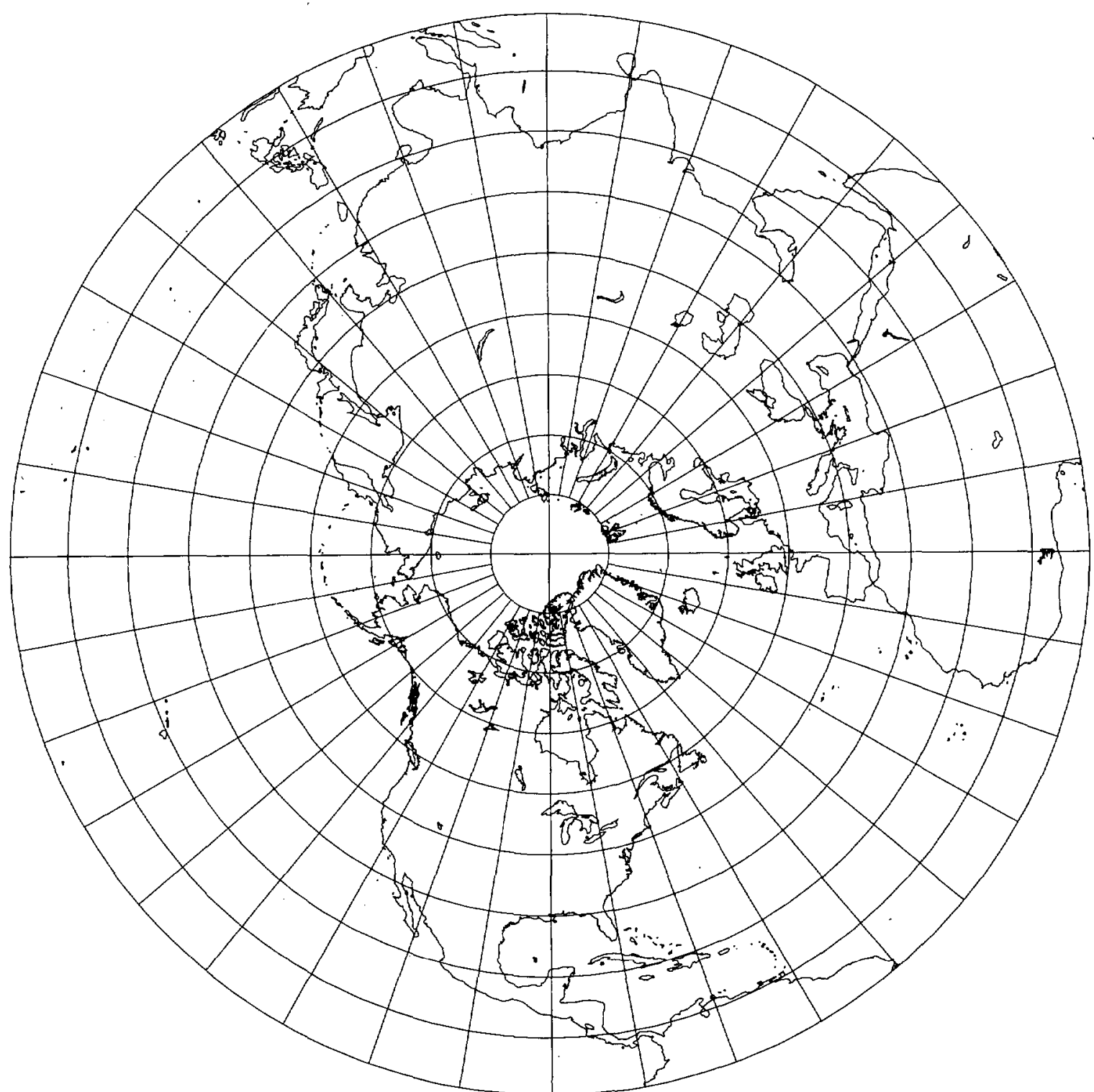

Figure 51A.-Polar Vertical Perspective projection (La Hire form), with shorelines, $10^{\circ}$ graticule. Central meridian $90^{\circ}$ W. Depth below surface $17,250 \mathrm{~km}$. 
Parallels: One parallel, normally nearer the pole than is the center of projection, depending on the position of the point of perspective, is a straight line. Other parallels are normally elliptical arcs.

Symmetry: About the central meridian

\section{Range}

If point of perspective is above the surface, less than one hemisphere

If point of perspective is on the opposite side of the Earth's center, more than one hemisphere

\section{Scale}

True at the center, if the projection is onto a tangent plane. Scale varies away from the center, depending on the point of perspective.

\section{Distortion}

Only the center is free of distortion, if projection is onto a tangent plane. Distortion is severe near the outer limit of the projection, although special locations for the points of projection on the opposite side of the Earth's center result in moderate distortion within a wide range of the center.

\section{Special features}

The General Vertical Perspective projection shows the Earth geometrically as it appears from any point above or below the surface when it is projected onto a tangent or secant plane perpendicular to the straight line connecting the point of perspective with the center of the Earth.

\section{Usage}

Pictorial views of the Earth, resembling those seen from space

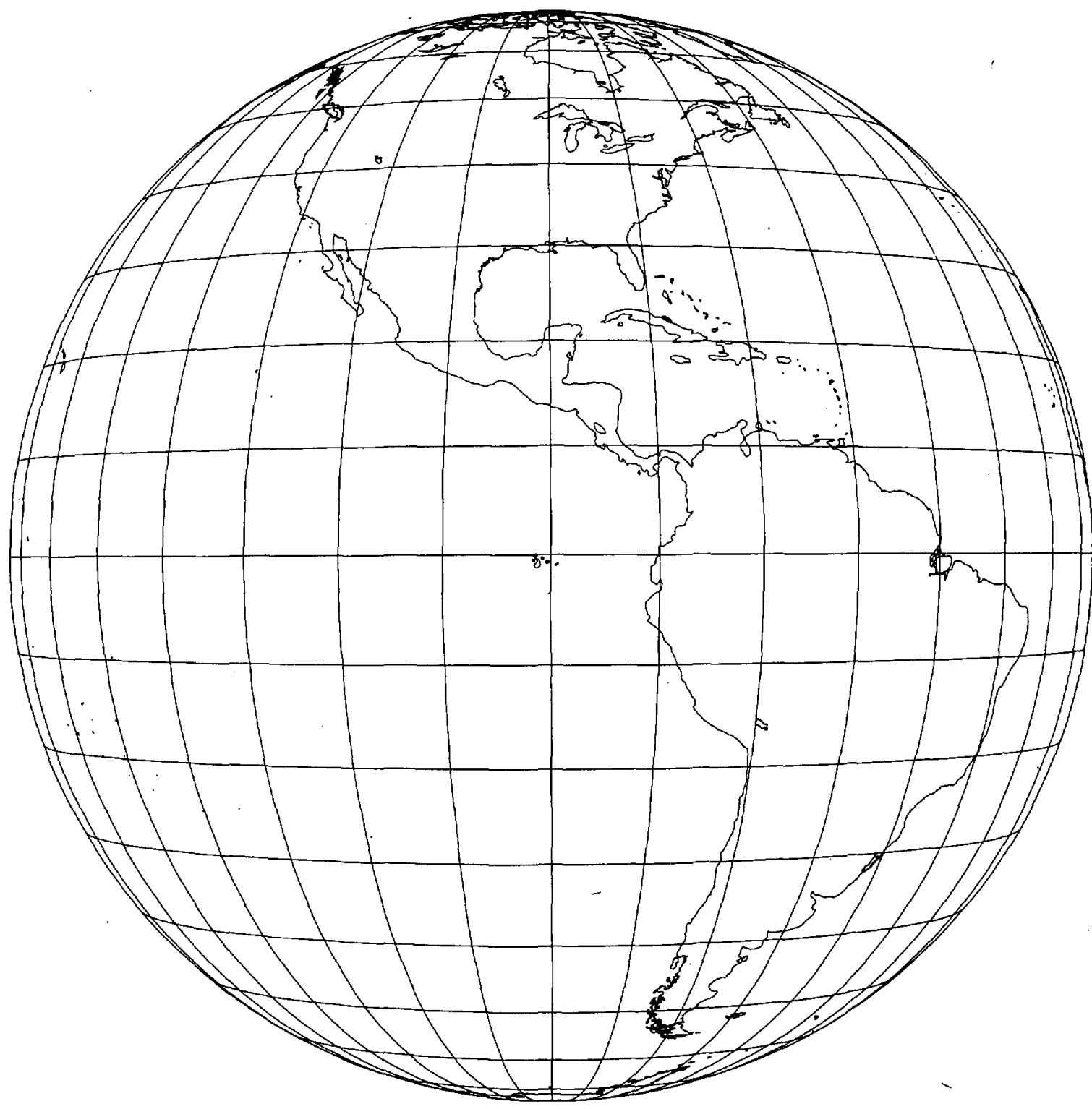

Figure 51B.-Equatorial Vertical Perspective projection, with shorelines, $10^{\circ}$ graticule. Central meridian $90^{\circ} \mathrm{W}$. Height above surface $35,900 \mathrm{~km}$ (geosynchronaus satellite position). 


\section{GENERAL VERTICAL PERSPECTIVE Projection}

\section{Origin}

Aside from the earlier limiting forms listed below, the perspective projection from a point on the opposite side of the Earth's center was studied in the 18th and 19th centuries as a source of low-distortion projections having geometric construction.

\section{Limiting forms}

Gnomonic projection (p. 116), if the point of perspective is at the center of the Earth Stereographic projection (p. 120), if the point of perspective is on the surface of the Earth opposite the center of projection

Orthographic projection (p. 124), if the point of perspective is at infinity

In turn, the Vertical Perspective is a limiting form of the Tilted Perspective projection ( $p$. 172).

\section{Other specific forms}

In 1701, Philippe de la Hire of France suggested a point of projection at 1.7071 times the radius (fig. 51A). This distance is measured from the

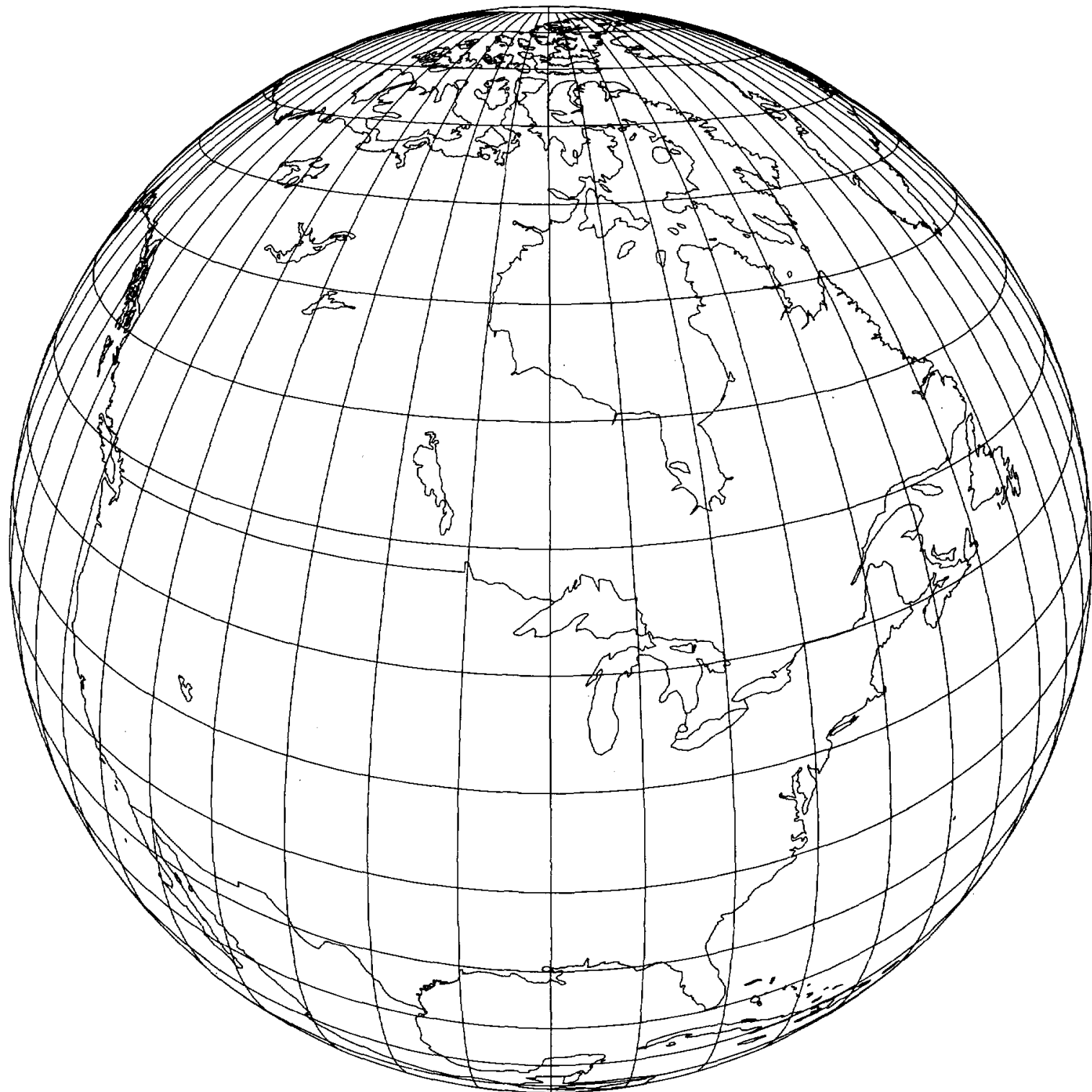

Figure $51 \mathrm{C}$-Oblique Vertical Perspective projection, with shorelines, $5^{\circ}$ graticule, central latitude $50^{\circ} \mathrm{N}$. Central meridian $90^{\circ} \mathrm{W}$. Height above surface $2,000 \mathrm{~km}$. 
center in a direction away from the surface of projection and gives the Equator of the polar aspect twice the radius of $45^{\circ}$ latitude.

Antoine Parent in 1702, Ernst Hammer about 1890, H.F. Gretschel in 1873, and Hans Maurer in 1935 were among others proposing points of projection for low-error perspectives.

A.R. Clarke and Henry James, in 1862 and later, calculated points of projection (on the far side, below the surface of projection) for minimum-error perspective azimuthal projections of regions, such as continents, bounded by a given great or small circle on the Earth. Figure $51 D$ is Clarke's Twilight projection of 1879 , bounded by a circle $108^{\circ}$ in radius.

The Army Map Service AMS Lunar projection is a far-side perspective selected by Albert $L$. Nowicki in 1963 to show slightly more than a hemisphere of the Moon, as seen over a period of time from the Earth. The point of projection is $\mathbf{1 . 5 3 7 4 8}$ times the radius from the center. See also the Airy nonperspective azimuthal projection (p. 140).

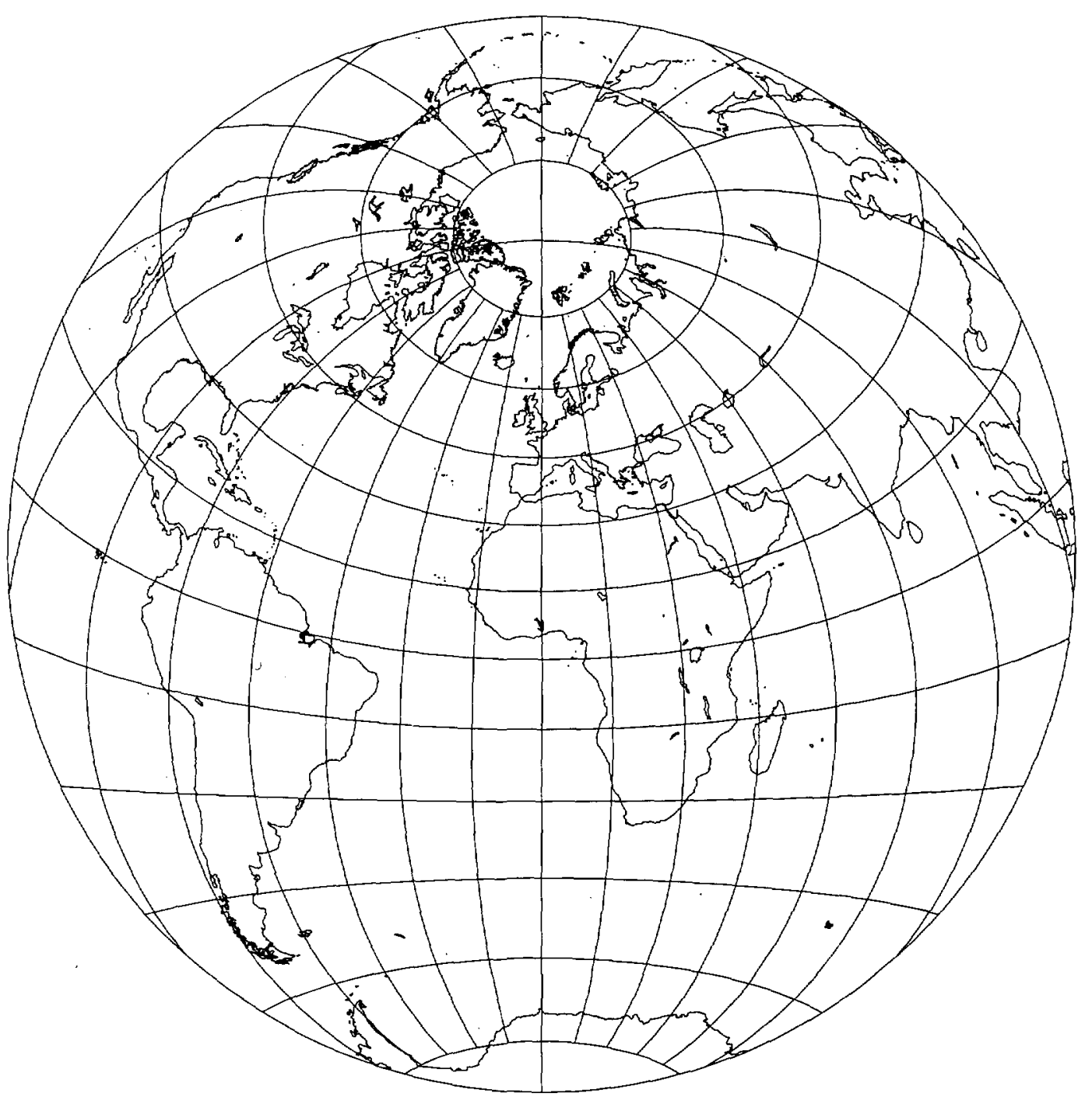

Figure 51D.-Clarke's Twilight projection, with shorelines, $15^{\circ}$ graticule, central latitude $23.5^{\circ} \mathrm{N}$. Central meridian $0^{\circ}$. Depth below surface $15,290 \mathrm{~km}$. Range $10 \mathrm{~B}^{\circ}$. 


\section{AZIMUTHAL EQUIDISTANT Projection}

\section{Classifications}

\section{Azimuthal}

Equidistant

Nonperspective

\section{Graticule}

Polar aspect (fig. 52B):

Meridians: Equally spaced straight lines

intersecting at the central pole. Angles

between them are the true angles.

Parallels: Equally spaced circles, centered at the pole, which is a point. The entire

Earth can be shown, but the opposite pole

is a bounding circle having a radius twice that of the Equator.

Symmetry: About any meridian

Equatorial aspect (fig. 52C):

Meridians: Central meridian is a straight line. Meridian $90^{\circ}$ away is a circle. Other meridians are complex curves, equally

spaced along the Equator and intersecting at each pole.

Parallels: Equator is a straight line. Other parallels are complex curves concave toward the nearest pole and equally spaced along the central meridian and the meridian $90^{\circ}$ from the central meridian.

Symmetry: About the central meridian or the Equator

Oblique aspect (fig. 52D):

Meridians: Central meridian is a stralght line. Other meridians are complex curves intersecting at each pole.

Parallels: Complex curves equally spaced along the central meridian

Symmetry: About the central meridian

\section{Range}

Entire Earth

\section{Scale}

True along any straight line radiating from the center of projection. Increases in a direction perpendicular to the radius as the distance from the center increases.

\section{Distortion}

Figure 52A shows distortion for the polar aspect. Other aspects have identical distortion at the same distance from the projection center. Only the center is free from distortion. Distortion is moderate for one hemisphere but becomes extreme for a map of the entire Earth.

\section{Special features}

The distance between any two points on a straight line passing through the center of projection is shown at true scale; this feature is especially useful if one point is the center. Compromise in distortion between

Stereographic (conformal) and Lambert Azimuthal Equal-Area projections

\section{Usage}

Commonly used in the polar aspect for maps of polar regions, the Northern and Southern Hemispheres, and the "aviation-age" Earth. The oblique aspect is frequently used for world maps centered on important cities and occasionally for maps of continents. The ellipsoidal form is used for topographic mapping of Micronesia and Guam.

\section{Origin}

Possibly developed in the polar aspect by Egyptians for star charts. Several users during the 16th century.

\section{Other names}

Postel (in France and Russia, for Guillaume
Postel, who was considered an originator, although he first used it in 1581)

Zenithal Equidistant

\section{Similar projections}

Two-Point Azimuthal (p. 144) shows correct azimuths (but not distances) from either of two points to any other point.

Two-Point Equidistant (p. 146) shows correct distances (but not azimuths) from either of two points to any other point.

Chamberlin Trimetric (p. 170) shows approximately true distances from three chosen points to any other points (cannot be exact). The three points are placed near the edges of the region being mapped to reduce overall distortion.

Airy (p. 140) and Breusing (p. 143) azimuthal projections have spacings very similar to those of the Azimuthal Equidistant if the extent is less than one hemisphere.

Berghaus Star projection (p. 156) uses the Polar Azimuthal Equidistant projection for the Northern Hemisphere.

"Tetrahedral" projection (p. 114) combines the Polar Azimuthal Equidistant projection with an interrupted Werner projection. 
Figure 52A.-Polar Azimuthal Equidistant projection, with Tissot

indicatrices, $30^{\circ}$ graticule. Range $170^{\circ}$.
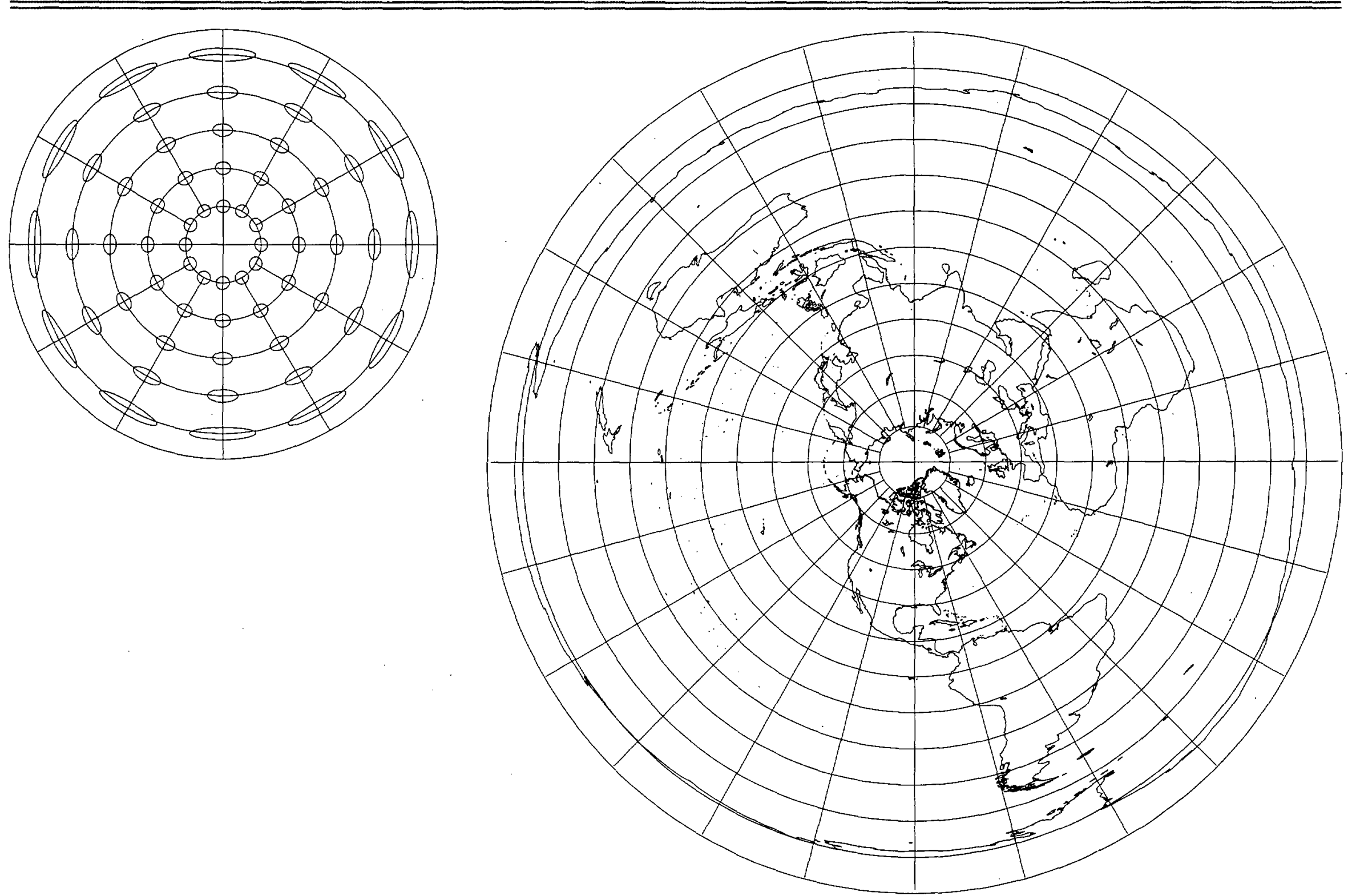

Figure 52B.-Polar Azimuthal Equidistant projection, with shorelines, $15^{\circ}$ graticule. Central meridian $90^{\circ}$ W. Range $180^{\circ}$ (world map). 


\section{AZIMUTHAL EQUIDISTANT Projection}

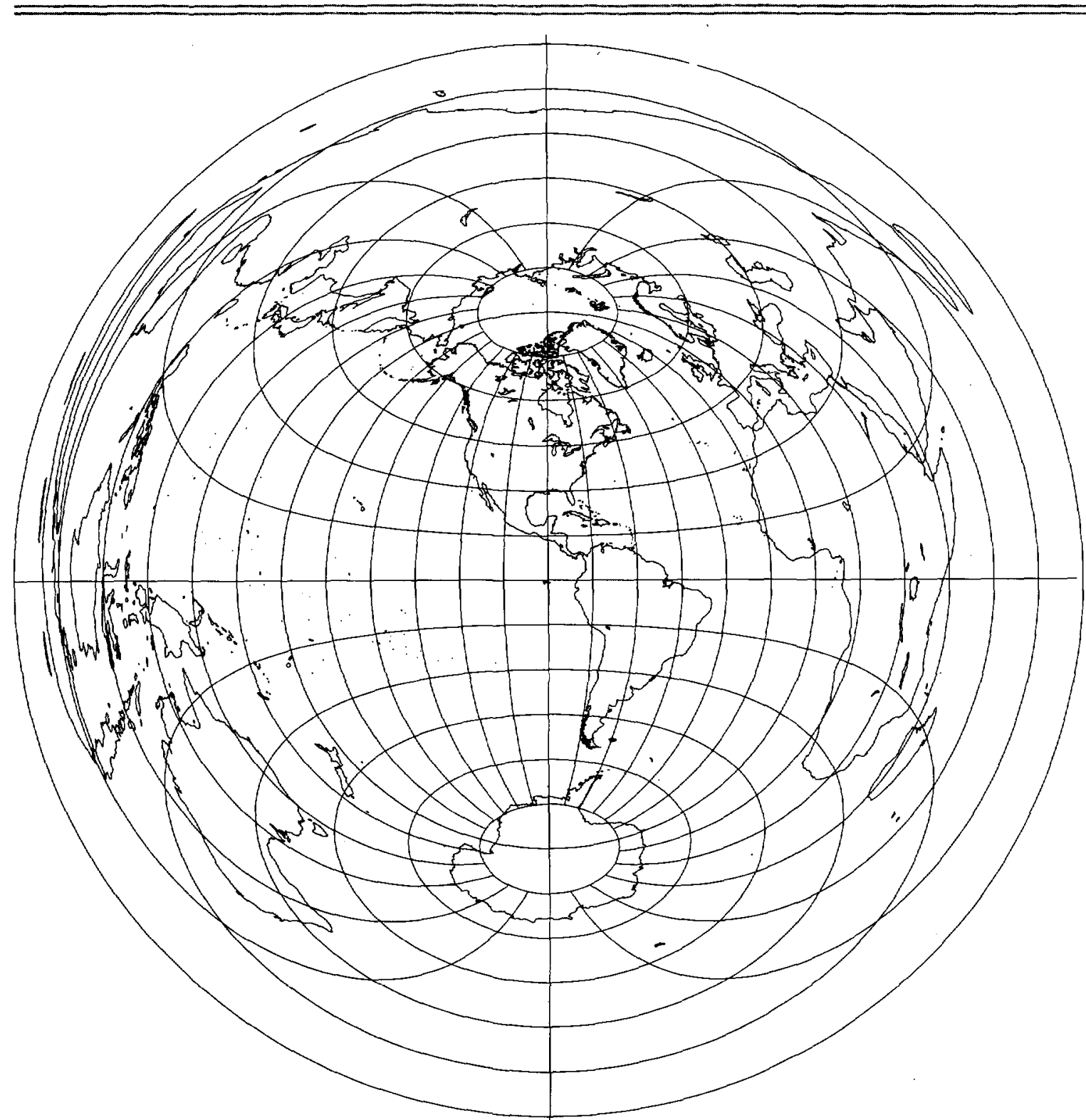

Figure 52C.-Equatorial Azimuthal Equidistant projection, with shorelines, $15^{\circ}$ graticule. Central meridian $90^{\circ} \mathrm{W}$. Range $180^{\circ}$ (world map). 


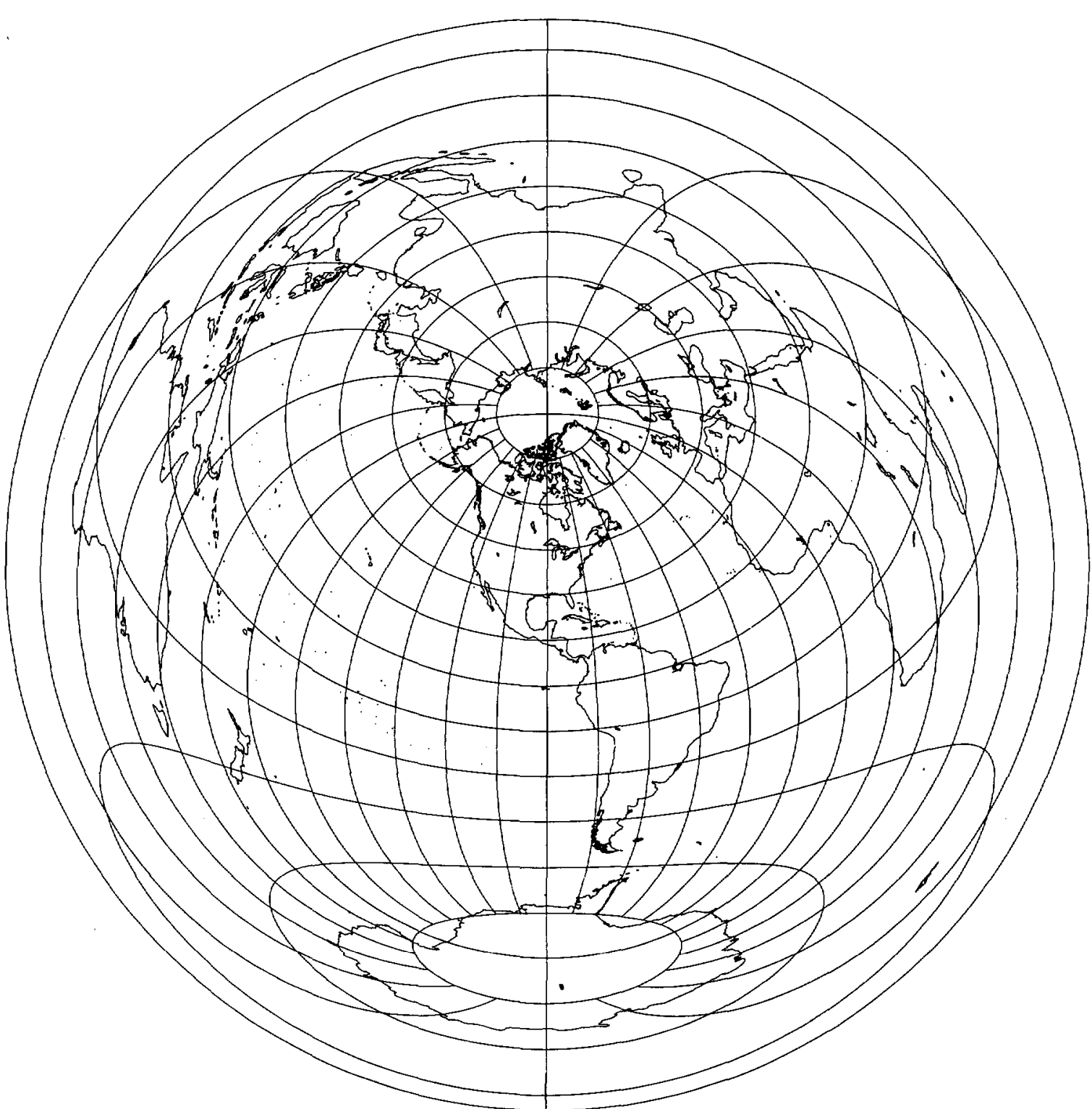

Figure 52D.-Oblique Azimuthal Equidistant projection, with shorelines, $15^{\circ}$ graticule, central latitude $40^{\circ} \mathrm{N}$. Central meridian $90^{\circ} \mathrm{W}$. Range $180^{\circ}$ (world map). 


\section{LAMBERT AZIMUTHAL EQUAL-AREA Projection}

\section{Classifications}

Azimuthal

Equal area

Nonperspective

\section{Graticule}

Polar aspect (fig. 53B):

Meridians: Equally spaced straight lines

intersecting at the central pole. Angles

between them are the true angles.

Parallels: Unequally spaced circles,

centered at the pole, which is a point.

Spacing of the circles gradually decreases

away from the pole. The entire Earth can

be shown, but the opposite pole is a

bounding circle having a radius 1.41 times

that of the Equator.

Symmetry: About any meridian

Equatorial aspect (fig. $53 C$ ):

Meridians: Central meridian is a straight line. Meridian $90^{\circ}$ away is a circle. Other meridians are complex curves, unequally spaced along the Equator and intersecting at each pole. Spacing decreases away from the central meridian.

Parallels: Equator is a straight line. Other parallels are complex curves concave toward the nearest pole. They are unequally spaced along the central meridian, and spacing decreases away from the Equator. Along the meridian $90^{\circ}$ from the central meridian, parallels are equally spaced.

Symmetry: About the central meridian or the Equator

Oblique aspect (fig. 53D):

Meridians: Central meridian is a straight line. Other meridians are complex curves intersecting at each pole shown.
Parallels: Complex curves unequally spaced along the central meridian; spacing decreases away from the center of projection

Symmetry: About the central meridian

\section{Range}

Entire Earth

\section{Scale}

True only at the center in all directions.

Decreases with distance from the center along radii. Increases with distance from the center in a direction perpendicular to radii.

\section{Distortion}

Figure $53 A$ shows distortion for the polar aspect. Other aspects have identical distortion at the same distance from the projection center. Only the center is free from distortion. Distortion is moderate for one hemisphere but becomes extreme for a map of the entire Earth.

\section{Usage}

Frequently used in the polar aspect in atlases for maps of polar regions and of Northern and Southern Hemispheres. The equatorial aspect is commonly used for atlas maps of the Eastern and Western Hemispheres. The oblique aspect is used for atlas maps of continents and oceans. The equatorial and oblique aspects are used by the U.S. Geological, Survey in cooperation with others for maps of the Circum-Pacific Map Project.

Recommended for equal-area maps of regions approximately circular in extent

\section{Origin}

Presented by Johann Heinrich Lambert (172877) of Alsace in 1772

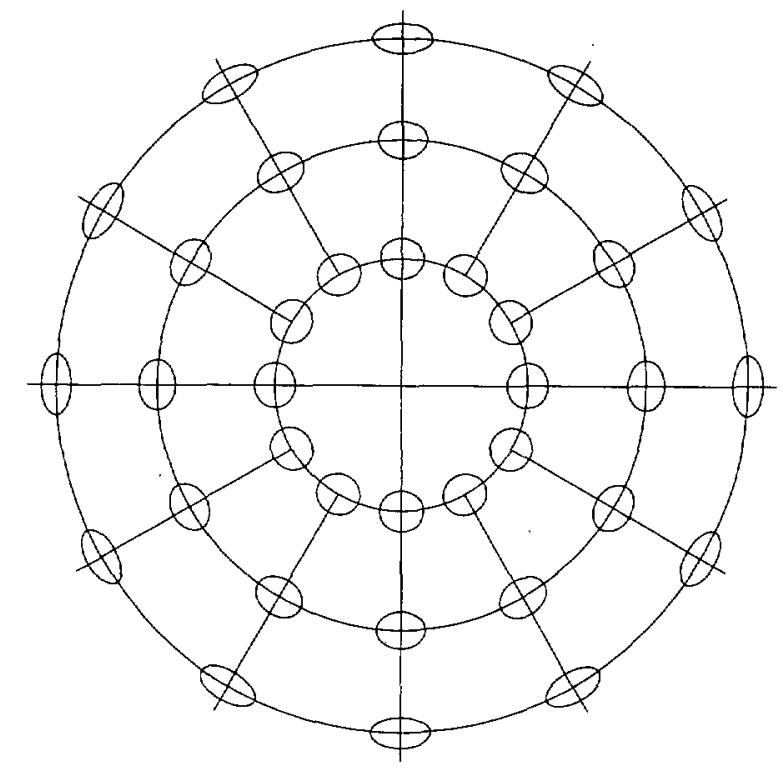

Figure 53A.-Polar Azimuthal Equal-Area projection, with Tissot indicatrices, $30^{\circ}$ graticule. Range $100^{\circ}$.

\section{Other names}

Lorgna (for polar aspect, due to independent derivation, 1789)

Zenithal Equal-Area

Zenithal Equivalent

\section{Similar projections}

W. William-Olsson's projection (p. 114) combines the Lambert Azimuthal Equal-Area with a modified Werner. 


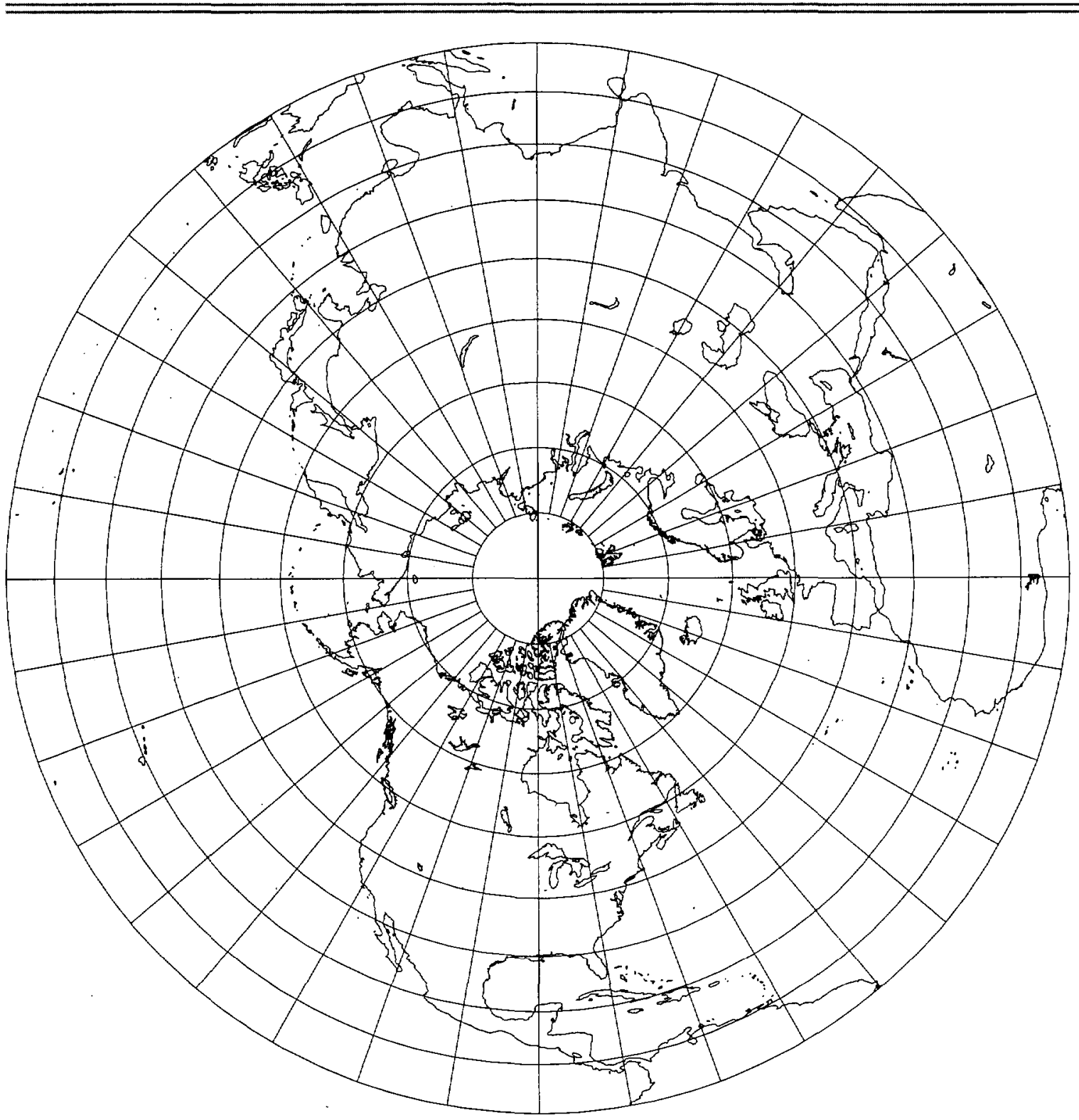




\section{LAMBERT AZIMUTHAL EQUAL-AREA Projection}

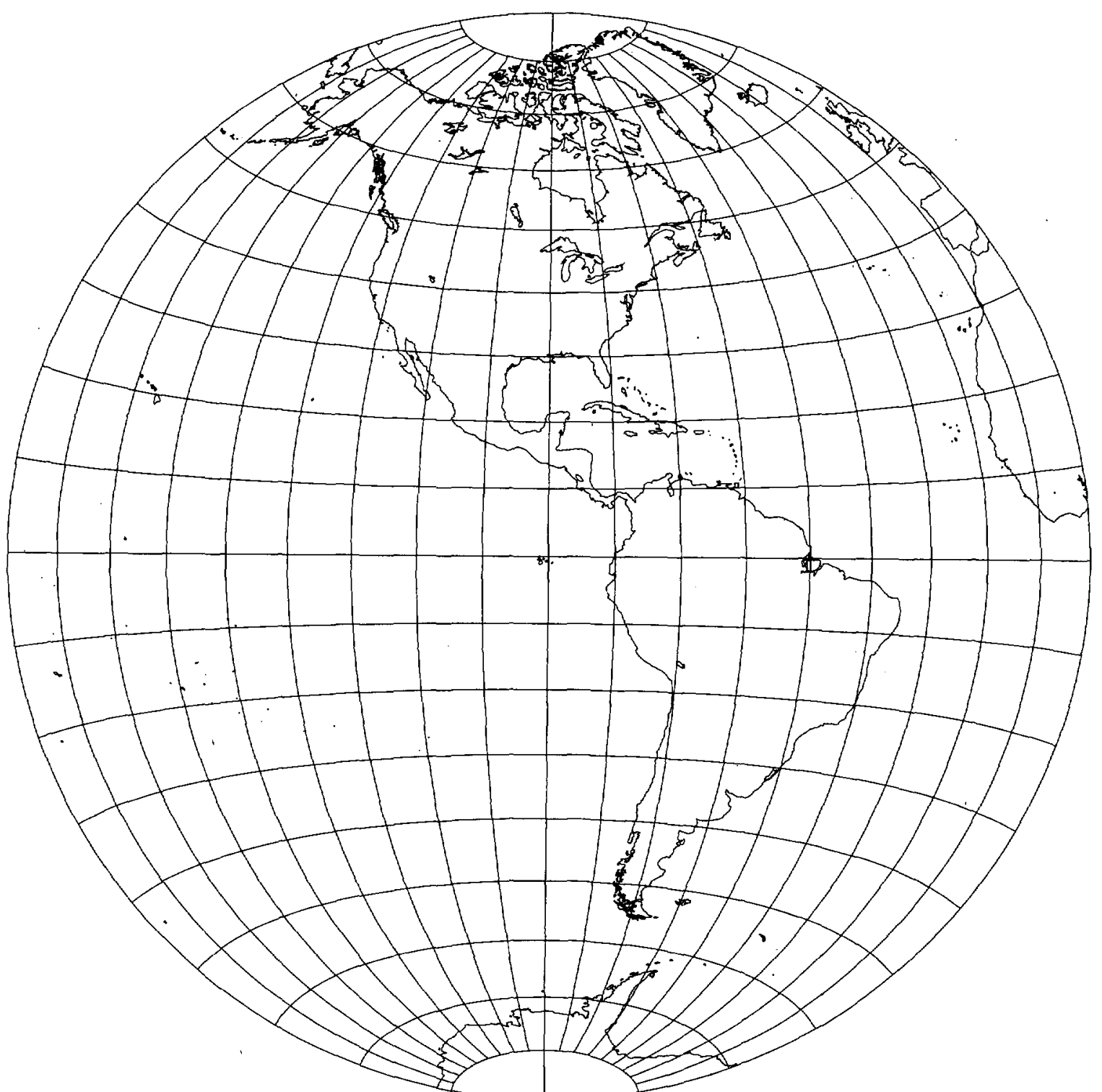

Figure 53C.-Equatorial Lambert Azimuthal Equal-Area projection, with shorelines, $10^{\circ}$ graticule. Central meridian $90^{\circ} \mathrm{W}$. Range $90^{\circ}$. 

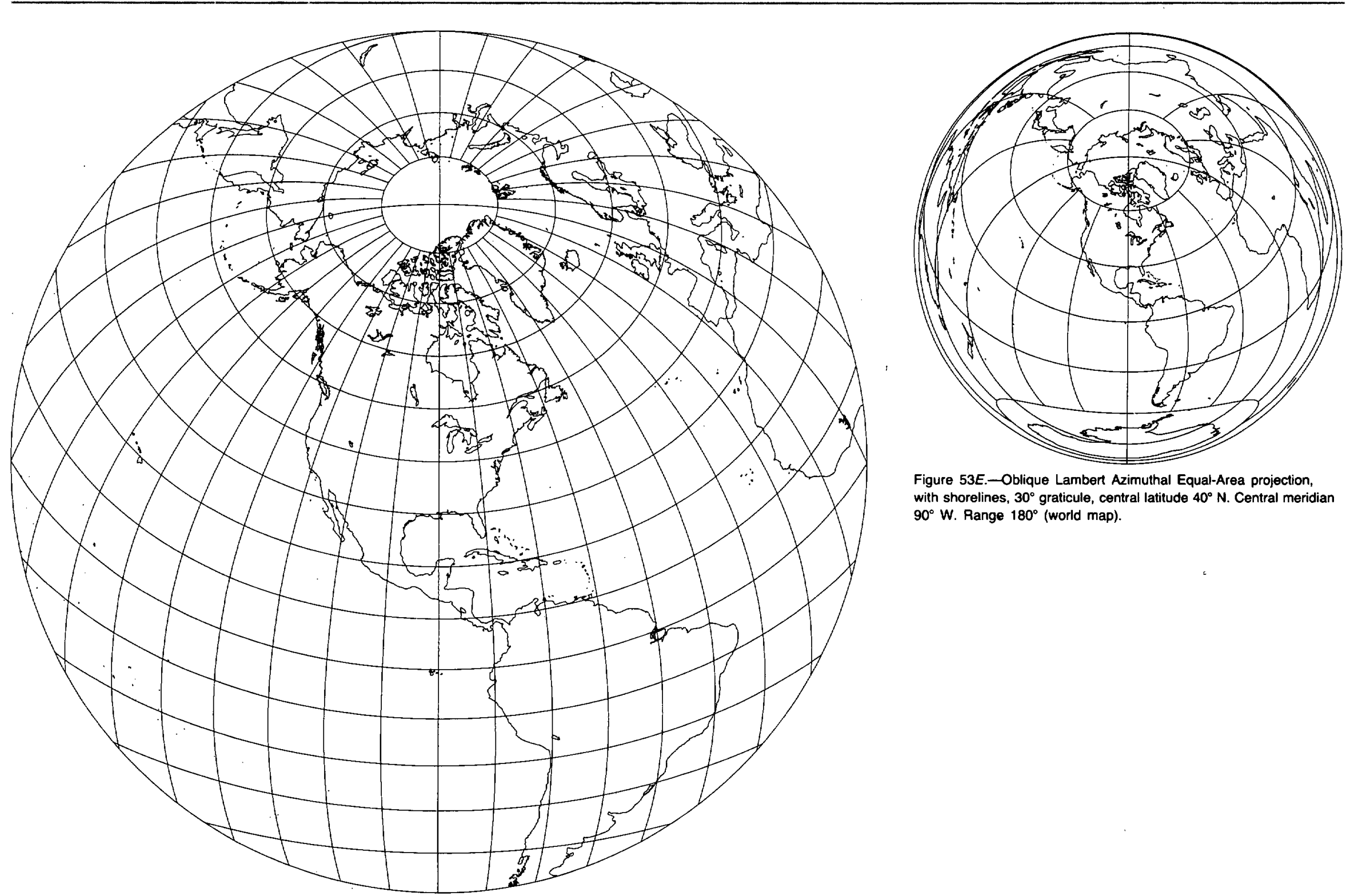

Figure 53E.-Oblique Lambert Azimuthal Equal-Area projection, with shorelines, $30^{\circ}$ graticule, central latitude $40^{\circ} \mathrm{N}$. Central meridian $90^{\circ} \mathrm{W}$. Range $180^{\circ}$ (world map).

Figure 530.-Oblique Lambert Azimuthal Equal-Area projection, with shorelines, $10^{\circ}$ graticule, central latitude $40^{\circ} \mathrm{N}$. Central meridian $90^{\circ} \mathrm{W}$. Range $90^{\circ}$ 


\section{Classifications}

Azimuthal

Minimum error

Nonperspective

Neither conformal nor equal area

\section{Graticule}

Polar aspect (fig. 54B):

Meridians: Equally spaced straight lines intersecting at the central pole. Angles between them are the true angles.

Parallels: Nearly but not quite equally spaced circles, centered at the pole, which is a point. Spacing increases slightly away from the center.

Symmetry: About any meridian

Equatorial aspect (fig. 54C):

Meridians: Central meridian is a straight line. Meridians $90^{\circ}$ away are circles. Other meridians are complex curves, unequally spaced along the Equator and intersecting at each pole. Spacing increases slightly away from the central meridian.

Parallels: Equator is a straight line. Other parallels are complex curves concave toward the nearest pole. They are unequally spaced along the central meridian, and spacing increases slightly away from the Equator. At the meridian $90^{\circ}$ from the central meridian, parallels are equally spaced.

Symmetry: About the central meridian or the Equator

Oblique aspect (fig. 54D):

Meridians: Central meridian is a straight line. Other meridians are complex curves intersecting at each pole.

Parallels: Complex curves unequally spaced along the central meridian; spacing increases slightly away from the center of projection

Symmetry: About the central meridian

\section{Range}

Should be limited to about a hemisphere or less

\section{Scale}

Scale increases gradually in all directions with distance from the center, but there is no point at which the scale is correct in all directions.

\section{Distortion}

Figure 54A shows distortion for the polar aspect extending $95^{\circ}$ or slightly more than one hemisphere. Distortion is moderate throughout the range chosen. No point is completely free of distortion, although the center is free of shape distortion.

\section{Usage}

Medium-scale Ordnance Survey map of the United Kingdom

\section{Special feature}

The minimum-error azimuthal projection of the region enclosed by a great or small circle of chosen radius from a given center

\section{Origin}

Presented by George Biddell Airy (1801-92), British astronomer and geodesist, in 1861

\section{Similar projections}

Clarke and James minimum-error perspective azimuthal projections were inspired by Airy's work.

The Azimuthal Equidistant projection (p. 132) closely resembles the Airy, but scale is

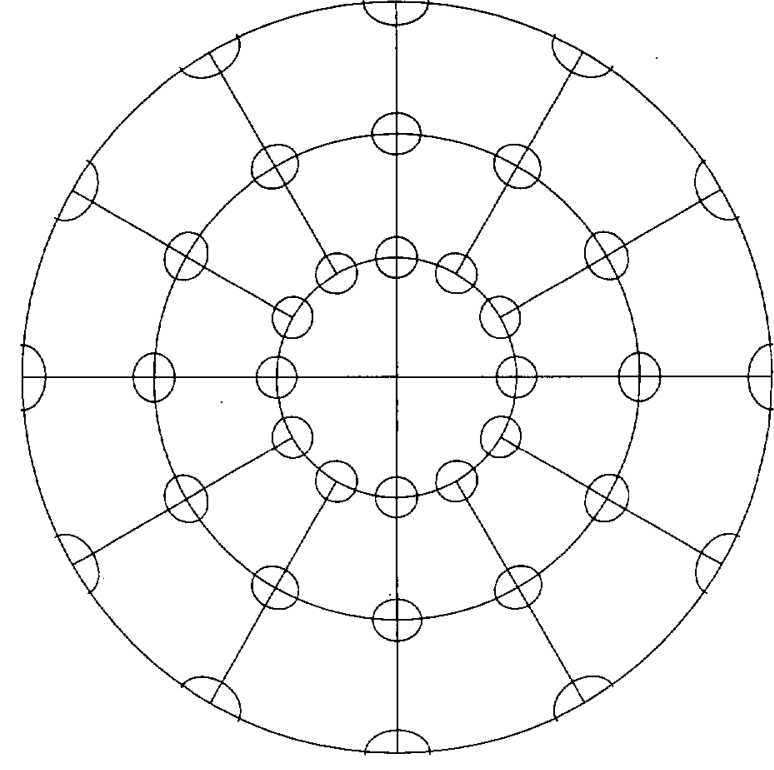

constant along radii from the center.

In 1892, A. Breusing proposed the low-error geometric mean of the Stereographic and Lambert Azimuthal Equal-Area projections.

In 1920, A.E. Young of England modified the Breusing (geometric mean) projection to provide a balance between the Stereographic and Azimuthal Equal-Area projections, called the Breusing Harmonic projection (fig. 54E).

In 1950, N.A. Urmayev of the Soviet Union proposed two other low-error azimuthal projections. 


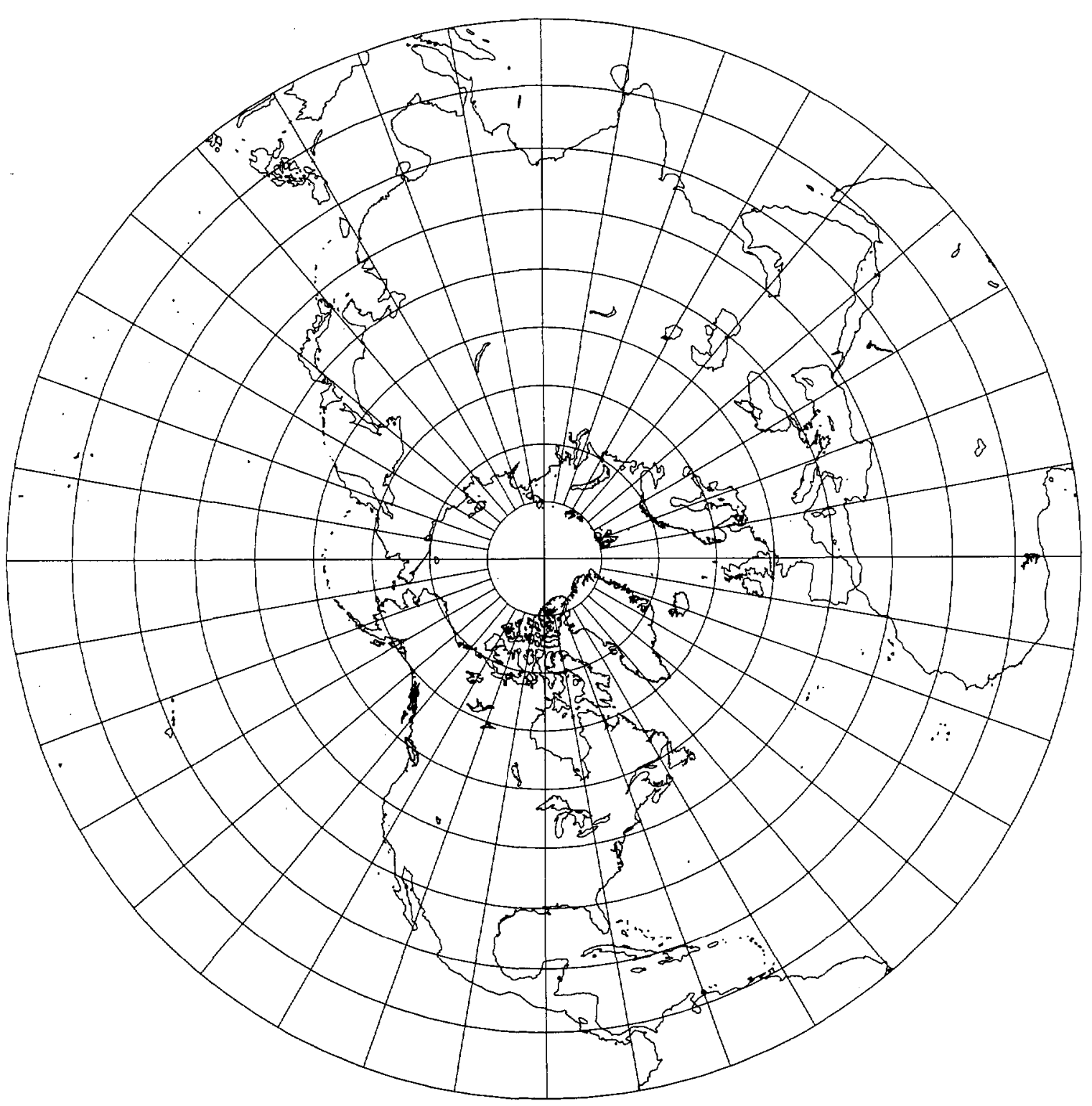




\section{AIRY Projection}

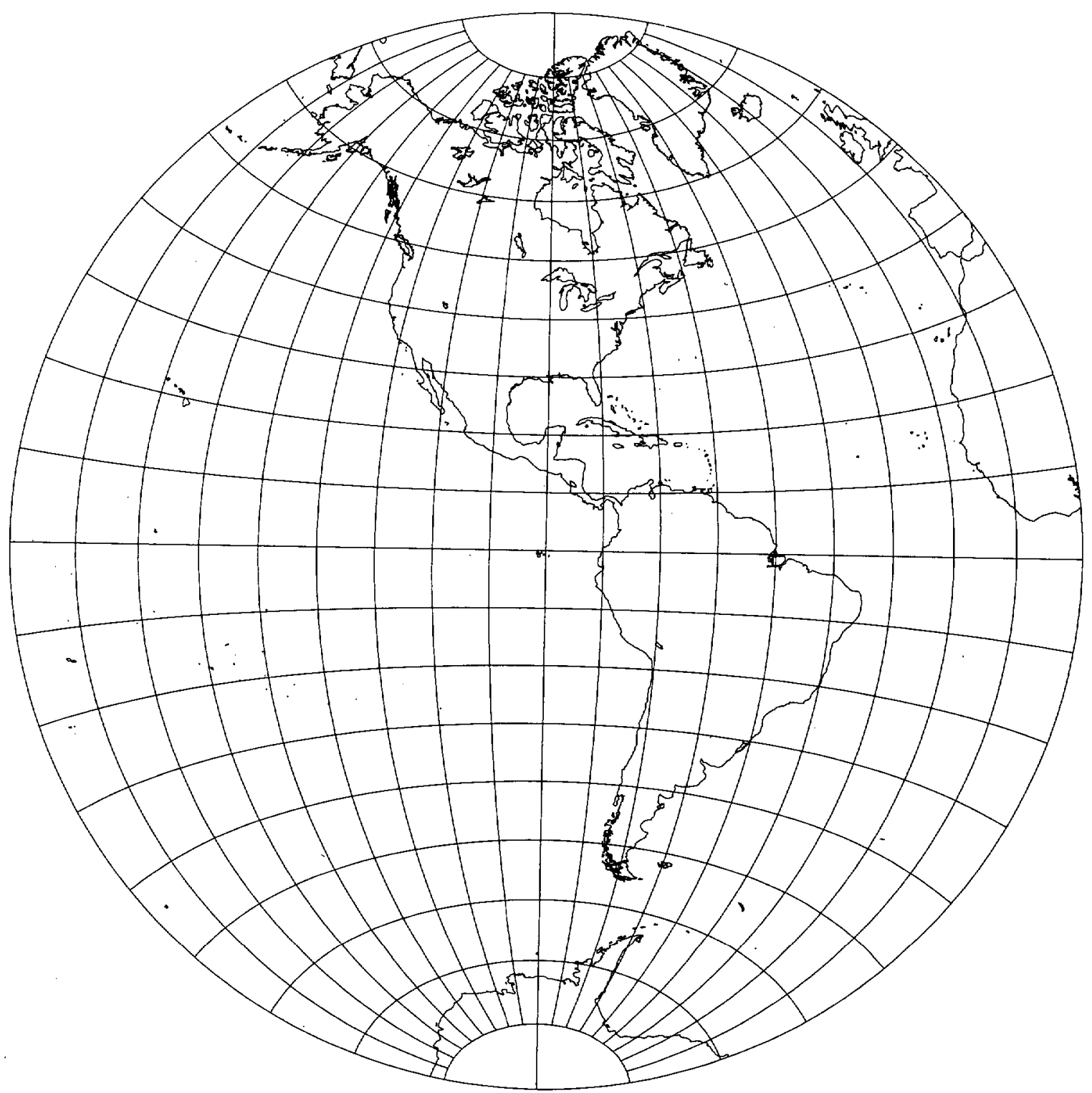

Figure 54C.-Equatorial Airy projection, with shorelines, $10^{\circ}$ graticule. Central meridian $90^{\circ} \mathrm{W}$. Range $90^{\circ}$ 

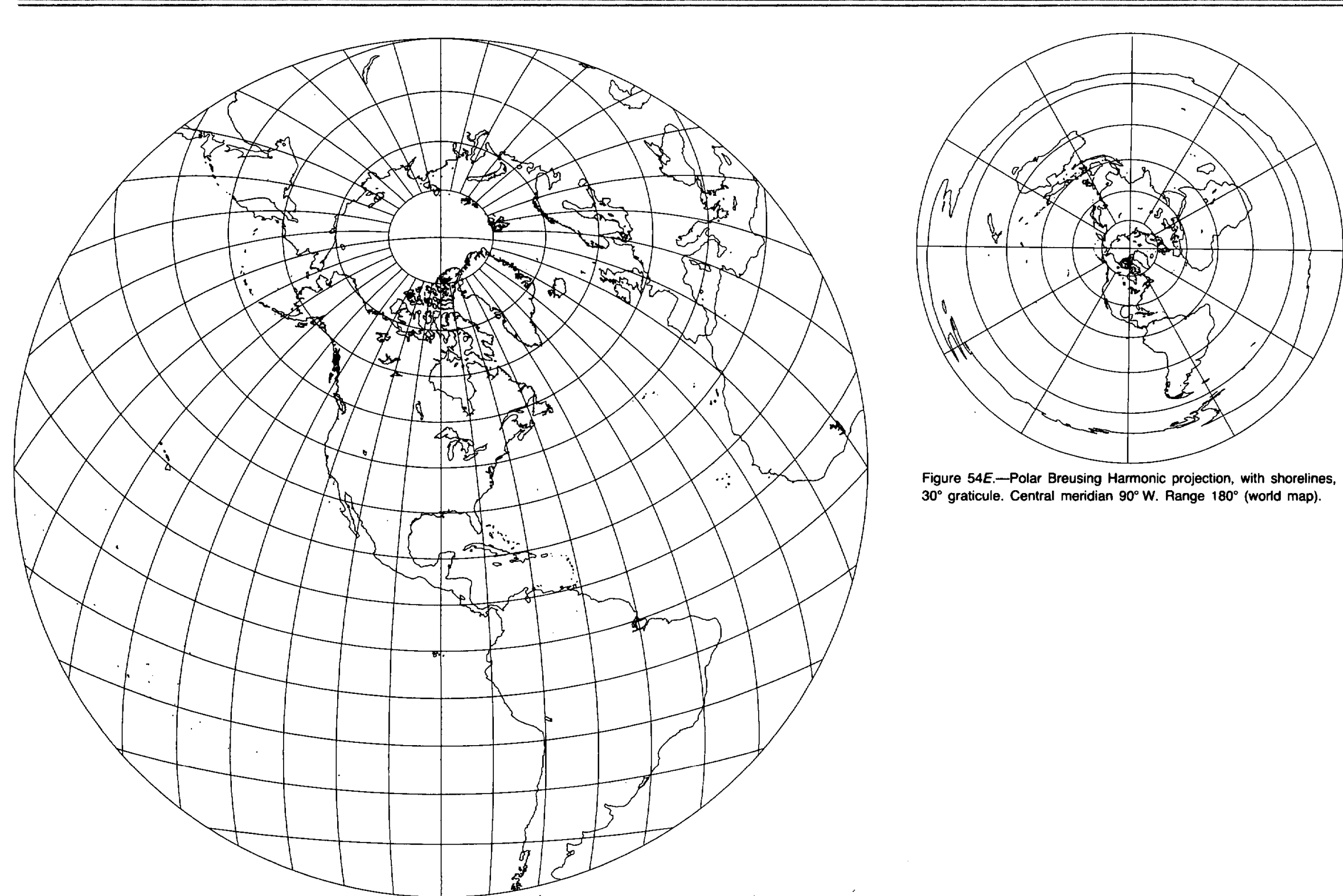

Figure $54 D$. - Oblique Airy projection, with shorelines, $10^{\circ}$ graticule, central latitude $40^{\circ} \mathrm{N}$. Central meridian $90^{\circ} \mathrm{W}$. Range $90^{\circ}$ 


\section{TWO-POINT AZIMUTHAL Projection}

\section{Classifications}

Modified azimuthal

Neither conformal nor equal area

\section{Graticule}

Meridians: Straight lines

Parallels: Equator is straight. Other parallels are ellipses, parabolas, or hyperbolas.

Poles: Points (if shown)

Symmetry: Normally none

\section{Scale}

Varies considerably. Projection should not be used for measuring distance

\section{Distortion}

Direction is correct to all other points from two central points at which there is no distortion.

\section{Other features}

Great circles are shown as straight lines.

This projection can be obtained by compressing

a Gnomonic projection in the direction parallel

to the line joining the two central points.

\section{Usage}

Could be used to locate a ship at sea, for example, if the exact location of two radio transmitters and the direction from the ship to the transmitters are known

\section{Origin}

Presented by Hans Maurer (1868-1945) of Germany in 1914 and Charles F. Close (1865-1952) of England independently in 1922

\section{Aspects}

Typically oblique

\section{Other names}

Doubly Azimuthal

Orthodromic

Close

McCaw

Immler

\section{Limiting forms}

If the two central points are identical, the Gnomonic projection results.

\section{Similar projections}

Gnomonic (p. 116), which can be compressed

as described above to produce the Two-Point

Azimuthal projection 
Figure 55.-Two-Point Azimuthal projection, with shorelines, $10^{\circ}$ graticule. Central points are near Washington, D.C. (longitude

$77^{\circ} \mathrm{W}$., latitude $39^{\circ} \mathrm{N}$.) and Honolulu, Hawaii (longitude $158^{\circ} \mathrm{W}$., latitude $21.5^{\circ} \mathrm{N}$.)

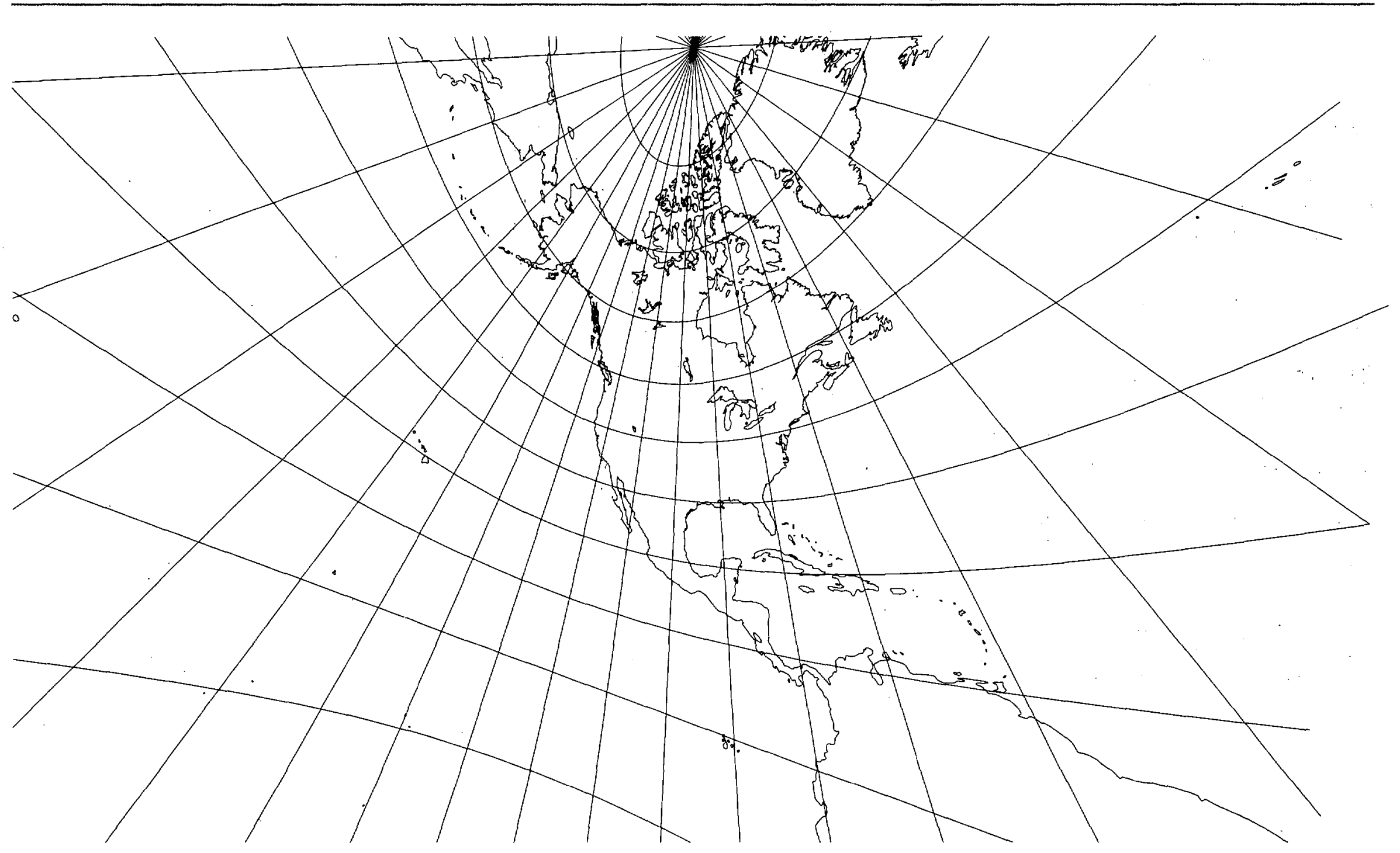




\section{TWO-POINT EQUIDISTANT Projection}

\section{Classifications}

Modified azimuthal

Neither conformal nor equal area

\section{Graticule}

Meridians: Complex curves

Parallels: Complex curves

Poles: Normally points

Symmetry: Normally none

\section{Scale}

True along a straight line from either of two central points

\section{Distortion}

No points free of distortion

\section{Usage}

Map of Asia by the National Geographic

Society

Could be used to determine the distance from

a ship at sea at a known location from the

start and end of a voyage

\section{Origin}

Presented by Hans Maurer (1868-1945) of Germany in 1919 and Charles F. Close (1865-1952) of England independently in 1921

\section{Aspects}

Typically oblique

\section{Other names}

Doubly Equidistant

\section{Limiting forms}

If the two central points are identical, the

Azimuthal Equidistant projection results.

\section{Similar projections}

Azimuthal Equidistant (p. 132), on which distance is correct from only one point

Donald Elliptical, developed by Jay K. Donald of the American Telephone and Telegraph

Company in 1956. Used by telephone companies to establish long-distance rates, the Donald is a Two-Point Equidistant projection specially modified for the ellipsoid and confined to the United States and southern Canada.

Chamberlin Trimetric ( $p .170)$, an approximate three-point equidistant projection

Two-Point Azimuthal-Equidistant, presented by Charles $\mathrm{F}$. Close in 1922, has true azimuths from one point and true distances from a second point to all other points. 
Figure 56.-Two-Point Equidistant projection, with shorelines, $10^{\circ}$ graticule. Central points are near Washington, D.C. (longitude $77^{\circ} \mathrm{W}$., latitude $39^{\circ} \mathrm{N}$.), and Honolulu, Hawaii (longitude $158^{\circ} \mathrm{W}$., latitude $21.5^{\circ} \mathrm{N}$.)

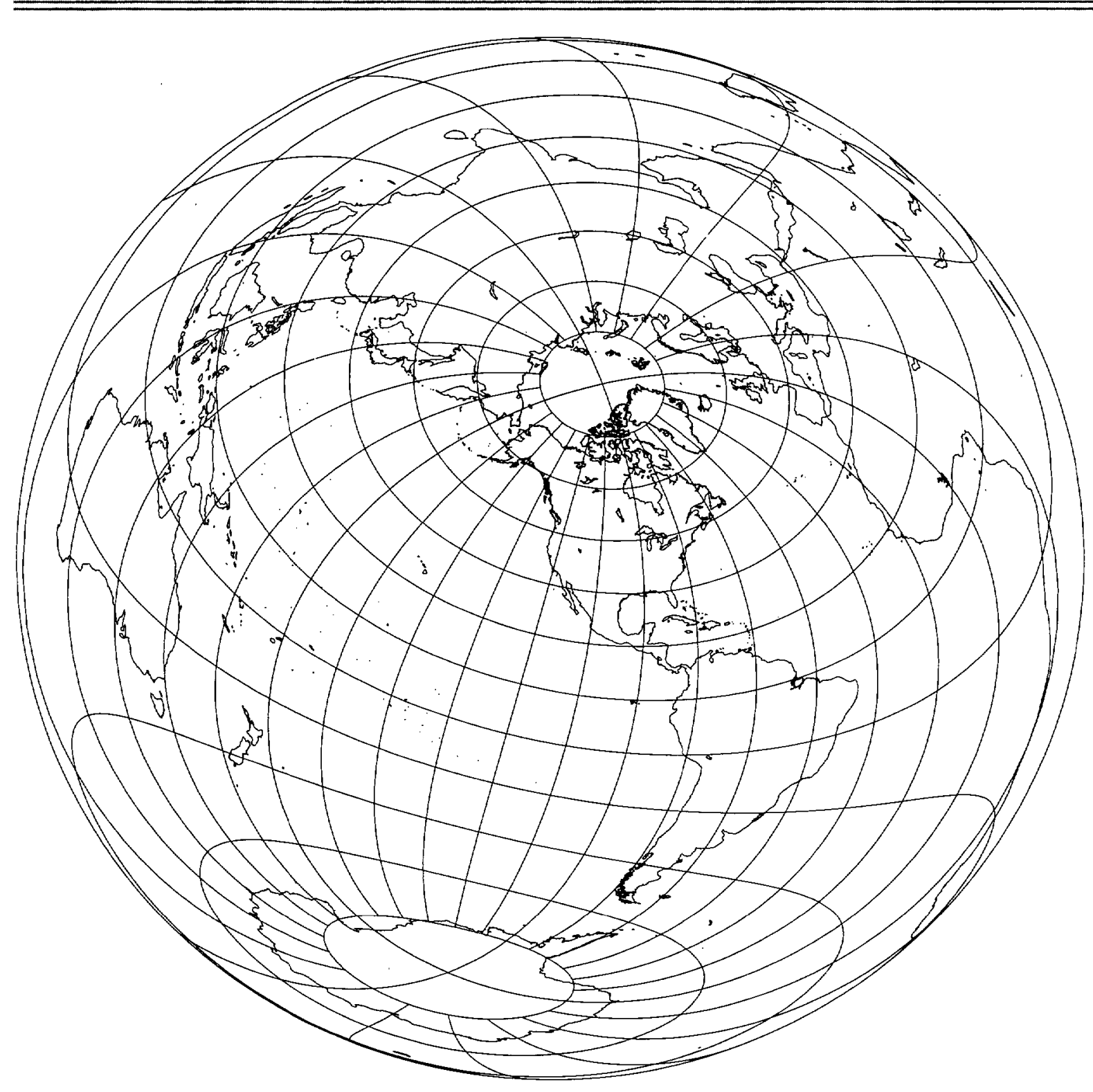




\section{MILLER OBLATED STEREOGRAPHIC Projection}

\section{Classifications}

Modified azimuthal

Conformal

\section{Graticule}

Meridians: Complex curves

Parallels: Complex curves

Poles: Points

Symmetry: About the central meridian as applied to the map of Europe and Africa. No symmetry on other prepared maps.

\section{Scale}

A line of constant scale bounds an oval region.

\section{Distortion}

Scale variations within the oval-shaped region covered are less than variations on standard conformal projections, such as an oblique Mercator or oblique Stereographic projection.

\section{Usage}

Used for an area that can be contained within an oval shape to minimize the scale distortions in the area. Applied by O.M. Miller to Africa and Europe in 1953 and to other Eastern Hemisphere regions in conjunction with several non. conformal fill-in projections in 1955 . Used by L.P. Lee for the Pacific Ocean in 1974.

\section{Origin}

Osborn Maitland Miller (1897-1979) of the American Geographical Society in 1953

\section{Other names}

Miller Prolated Stereographic

Oblated Stereographic

Prolated Stereographic

\section{Similar projections}

Stereographic projection (p. 120) has lines of constant scale that are concentric circles.

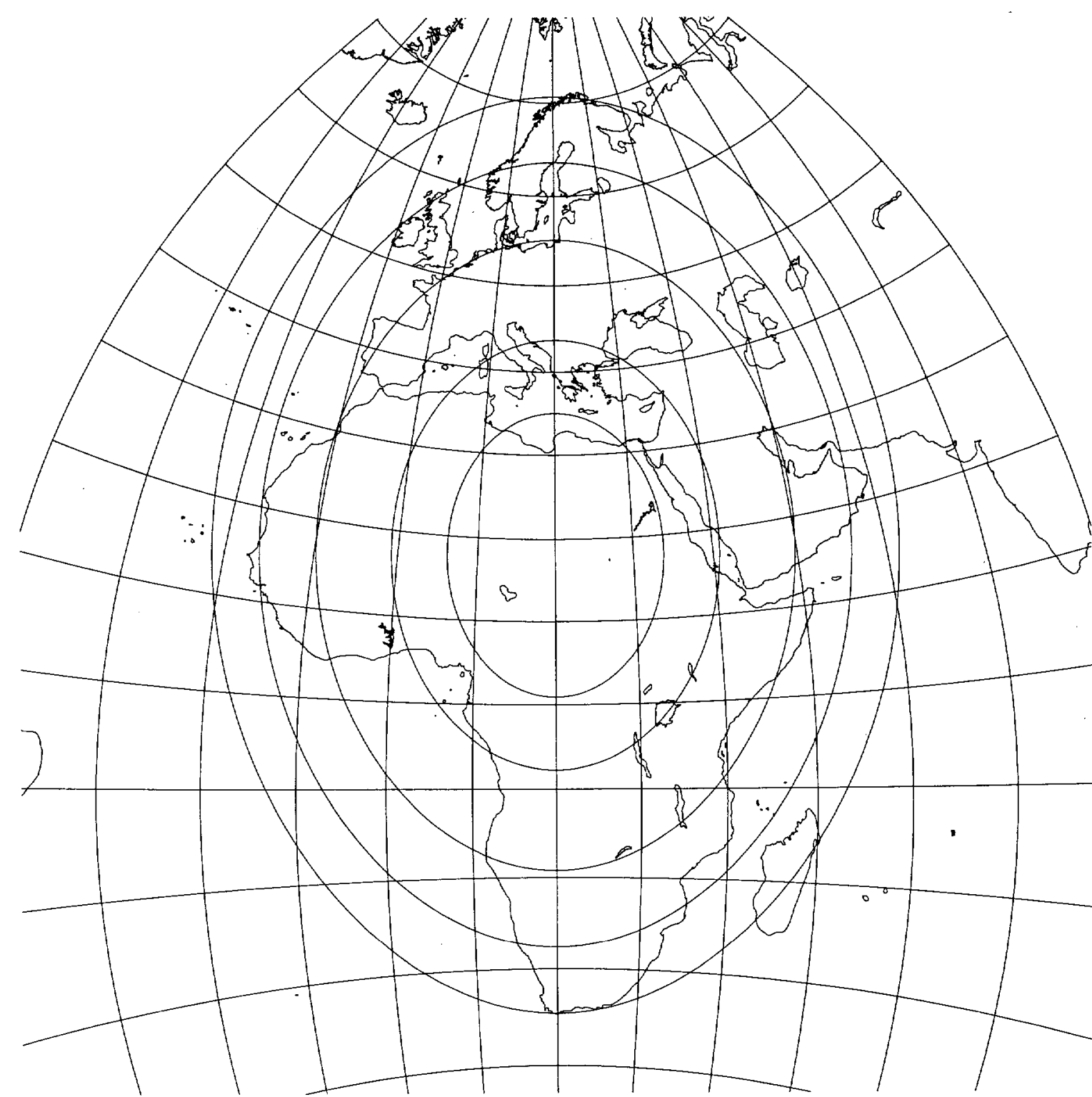

Figure 57.-Miller Oblated Stereographic projection of Europe and Africa, with shorelines, $10^{\circ}$ graticule, and lines of constant scale Scale factors are $0.94,0.96,1.00,1.04$, and 1.08 along ovals, beginning with the smallest. Scale factor at center (latitude $18^{\circ} \mathrm{N}$., longitude $20^{\circ} \mathrm{E}$.) is 0.9245 . 


\section{WIECHEL Projection}

\section{Classifications}

Modified azimuthal

Equal area

Pseudoazimuthal

\section{Graticule}

Polar aspect:

Meridians: Semicircles if extended to a world map

Parallels: Concentric circles

Poles: Central pole is a point; other pole

is a circle

Symmetry: Radial about the central pole

\section{Scale}

Correct along meridians

\section{Distortion}

Considerable distortion of shape at the map edge

\section{Usage}

A novelty map that, in its polar aspect, has semicircular meridians arranged in a pinwheel

\section{Origin}

Presented by $\mathrm{H}$. Wiechel in 1879

\section{Aspects}

Normally only polar

\section{Similar projections}

Lambert Azimuthal Equal-Area projection ( $p$. 136) has the same spacing of parallels in the polar aspect, but the meridians are straight.

Ginzburg III is a general series of

pseudoazimuthal projections developed by G.A. Ginzburg of the Soviet Union in 1952.

The lines of constant distortion are ovals rather than the usual circles of azimuthal projections.
This feature is achieved by the manner in

which the lines of constant initial azimuth are

curved. The projection series has been used only in the oblique or equatorial aspect.

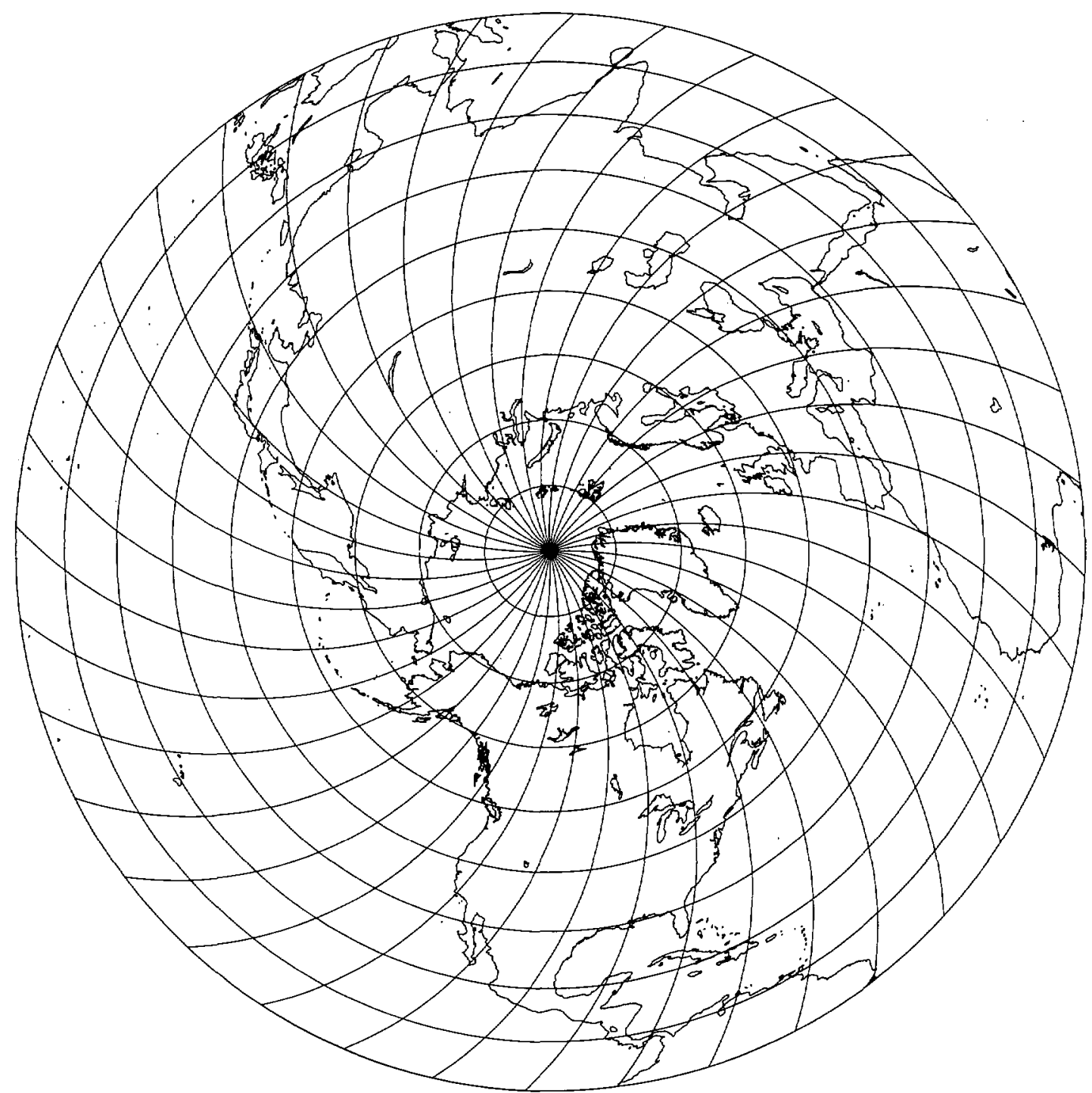




\section{CRAIG RETROAZIMUTHAL Projection}

Classifications

Retroazimuthal

Modified azimuthal

Neither conformal nor equal area

\section{Graticule}

Meridians: Equally spaced straight parallel

lines

Parallels: Curved

Poles: Curved lines

Symmetry: About the central meridian

\section{Distortion}

Extreme distortion of shape and area

\section{Other features}

The direction is correct from any location on the map to a central point.

Considerable overlapping when entire sphere is shown

\section{Usage}

To show, for example, the correct direction toward Mecca, from any point on the map, by measuring the angle from North. Because the meridians are straight parallel lines, this angle can be readily measured.

\section{Origin}

Presented by James Ireland Craig (1868-1952)

of the Survey of Egypt in 1909

\section{Other names}

Mecca

\section{Similar projections}

Hammer Retroazimuthal (p. 152) has curved, unequally spaced meridians.

Hans Maurer of Germany in 1919 and J.E. Jackson of England in 1968 devised retroazimuthal projections characterized by straight, parallel, unequally spaced meridians.

Carl Schoy presented a projection in 1913 having meridians that are straight, equidistant, and parallel but parallels that are curved, so that all great circles passing through the cen. tral point are straight lines at their true azimuths from center. It is not retroazimuthal, nor is its distortion only a function of distance from the center, as it is on most azimuthal projections. 
Figure 59.-Craig Retroazimuthal projection, centered near St. Louis, Mo. (longitude $90^{\circ} \mathrm{W}$., latitude $38.5^{\circ} \mathrm{N}$.), with shorelines, $10^{\circ}$ graticule.

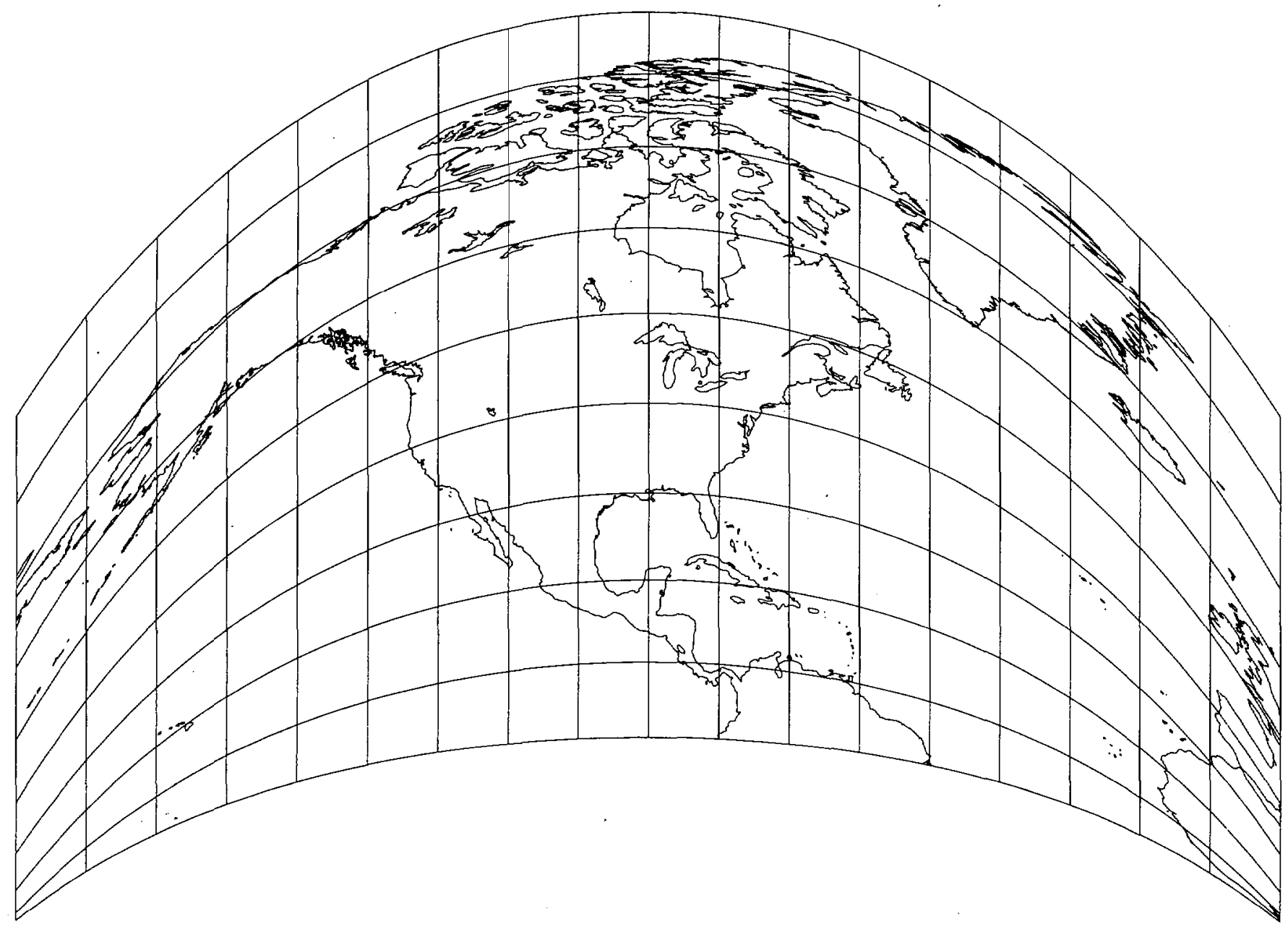




\section{HAMMER RETROAZIMUTHAL Projection}

\section{Classifications}

Retroazimuthal

Modified azimuthal

Neither conformal nor equal area

\section{Graticule}

Meridians: Central meridian is straight. Other meridians are curved.

Parallels: Curved

Poles: Curved lines

Symmetry: About the central meridian

\section{Distortion}

Extreme distortion of area and shape

\section{Other features}

The direction from any point to the center of the map is the angle that a straight line connecting the two points makes with a vertical line. This feature is the basis of the term "retroazimuthal." Scimitar-shaped boundary. Considerable overlapping when the entire sphere is shown.

\section{Usage}

To determine the direction of a central point from a given location

\section{Origin}

Presented by H.H. Ernst von Hammer (18581925) of Germany in 1910. Independently presented by Edward A. Reeves (1862-1945) and Arthur R. Hinks (1874-1945) of England in 1929.

\section{Similar projections}

Craig Retroazimuthal (p. 150) has straight, equally spaced meridians.

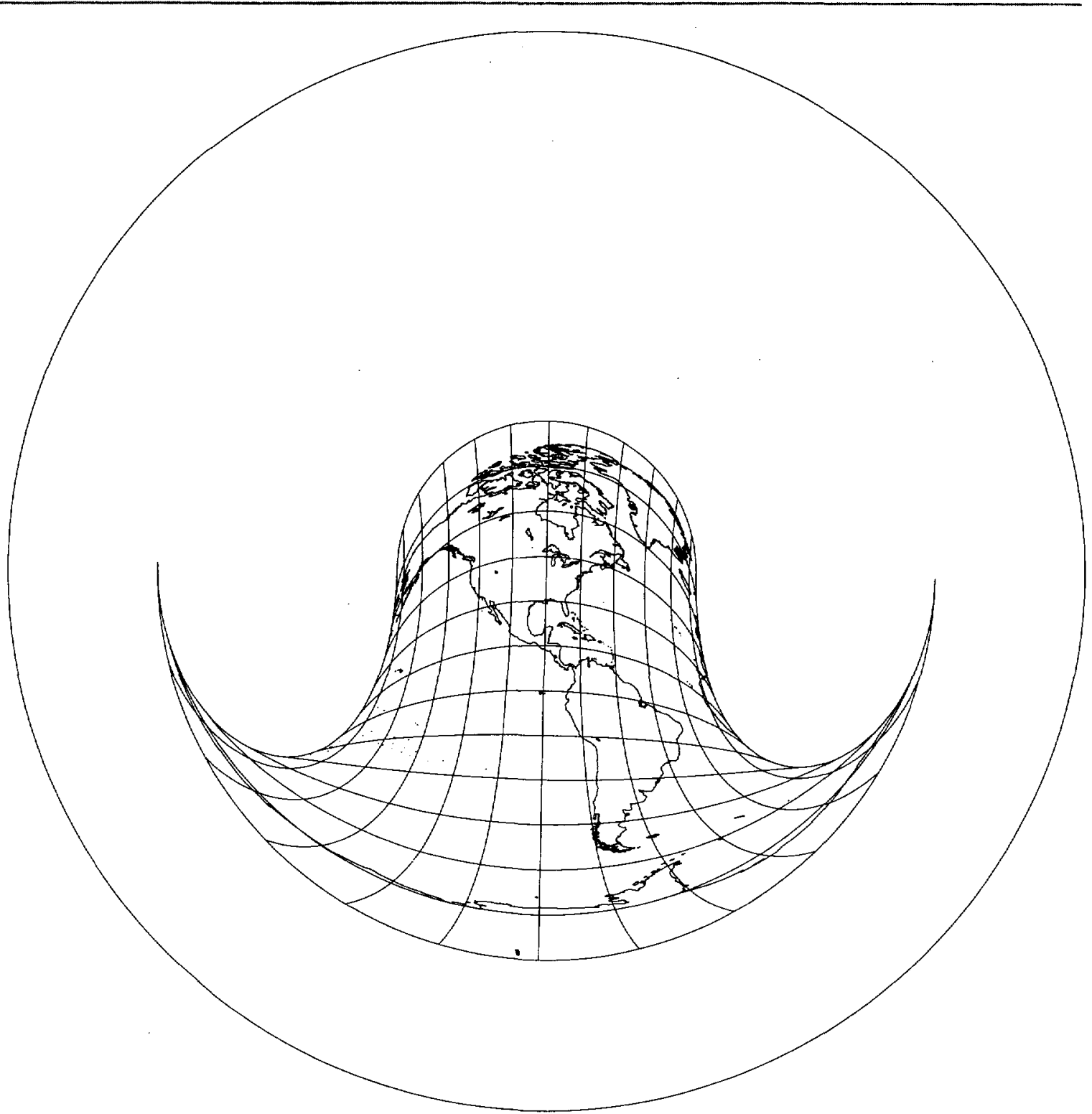

Figure 60.-Hammer Retroazimuthal projection, centered near St. Louis, Mo. (longitude $90^{\circ} \mathrm{W}$., latitude $40^{\circ} \mathrm{N}$.), with shorelines, $15^{\circ}$ graticule. Two hemispheres, one of which appears backwards; they should be superimposed for the full map. 


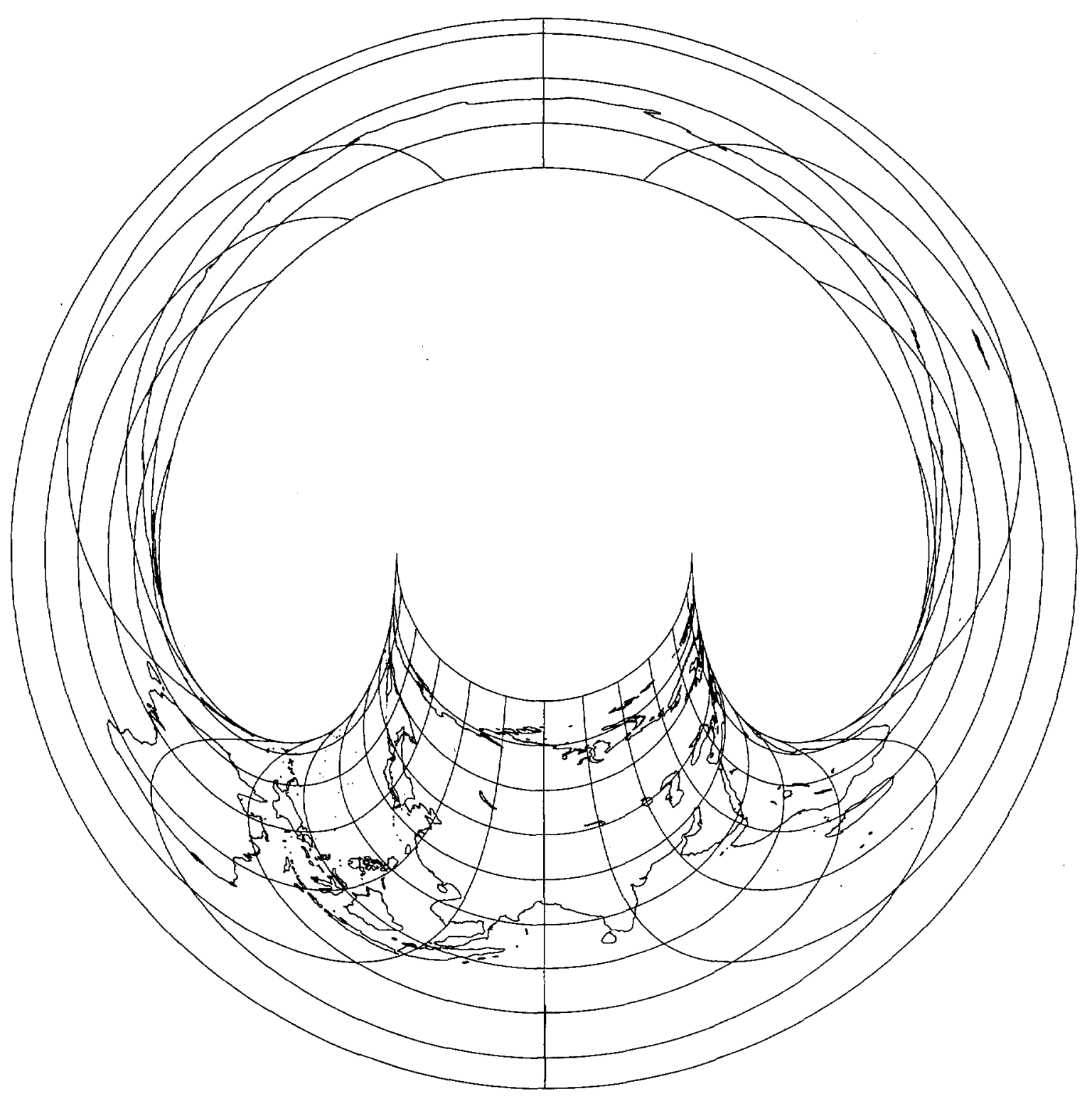




\section{LITTROW Projection}

\section{Classifications \\ Retroazimuthal \\ Conformal}

\section{Graticule}

Meridians: Central meridian and meridians $90^{\circ}$ away are straight. Others are hyperbolas convex toward the central meridian.

Parallels: Equator is straight. Other parallels are ellipses; the Equator lies along their major axes.

Poles: Not shown

Symmetry: About the central meridian and the Equator

\section{Scale}

Varies rapidly

\section{Distortion}

Extreme distortion of area

\section{Other features}

Cannot show the entire sphere. Shows the correct direction from any point on the map to any point along the central meridian as the angle between a straight line connecting the two points and a vertical straight line parallel to the central meridian.

\section{Usage}

By the British Navy in the 19th century to determine directions to a central point from other locations

\section{Origin}

Presented by J.J. Littrow of Austria in 1833

\section{Similar projections}

Littrow projection is the transverse aspect of a Lagrange projection (p. 180) having certain parameters. 


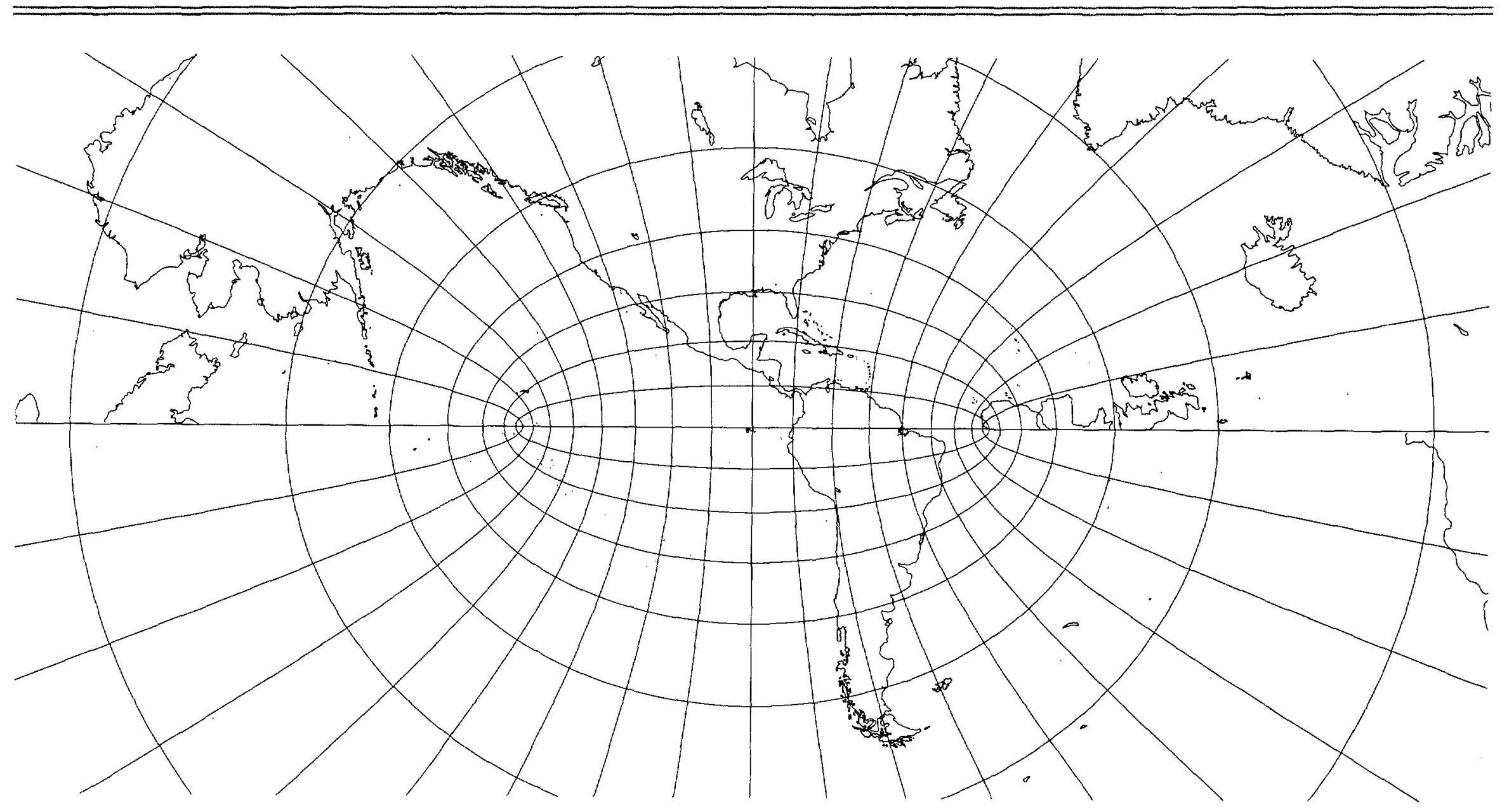




\section{BERGHAUS STAR Projection}

\section{Classifications}

Equidistant

Composite

Modified azimuthal

Interrupted

\section{Graticule}

Meridians: Straight lines, broken at the

Equator

Parallels: Concentric circular arcs; North Pole

at the center

Poles: Points

Symmetry: Radial about the North Pole

\section{Scale}

Correct along meridians in the Northern

Hemisphere and along the central meridians

of triangular points in the Southern

Hemisphere

\section{Distortion}

Moderate throughout

\section{Other features}

The Northern Hemisphere is a polar Azimuthal
Equidistant projection. The Southern

Hemisphere is divided into five equal triangular

lobes.

\section{Usage}

Largely for artistic map forms. Landforms do not readily fit into the lobes or arms of the star pattern. Used for the logo of the Association of American Geographers.

\section{Origin}

Presented by Hermann Berghaus (1828-1890) of Germany in 1879

\section{Aspects}

Polar only

\section{Similar projections}

Petermann Star (Germany, 1865) has eight unequal points; parallels are concentric circular arcs.

Jäger Star (Austria, 1865) has eight unequal points; parallels are in straight segments.

Azimuthal Equidistant (p. 132) is the projection for the Northern Hemisphere of the Berghaus Star. 


\section{Figure 62.-Berghaus Star projection, with shorelines, $18^{\circ}$ graticule. Central meridian $90^{\circ} \mathrm{W}$. World map.}

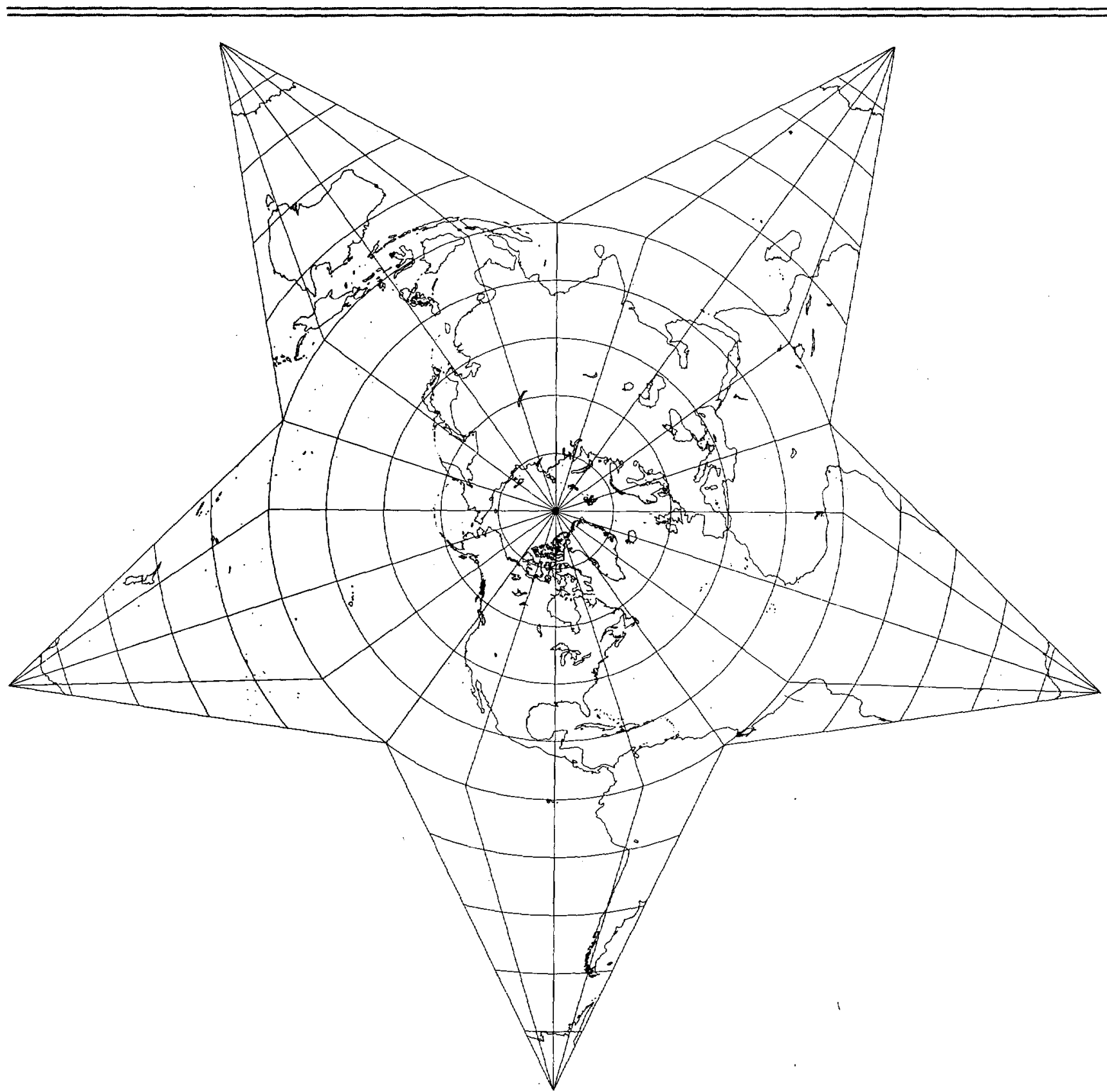




\section{Classifications}

Modified azimuthal

Neither conformal nor equal area

\section{Graticule}

Meridians: Central meridian is a straight line half the length of the Equator. Other meridians are complex curves, equally spaced along the Equator and concave toward the central meridian

Parallels: Equator is straight. Other parallels are complex curves, equally spaced along the central meridian and concave toward the nearest pole.

Poles: Points

Symmetry: About the Equator and the central meridian

\section{Scale}

True along the Equator and the central meridian

\section{Distortion}

Moderate shape and area distortion (fig. 63A)

\section{Other features}

Elliptical border. An equatorial aspect of one hemisphere of the Azimuthal Equidistant projection, on which horizontal coordinates have been doubled and meridians have been given twice their original longitudes.

\section{Usage}

Design inspired H.H. Ernst von Hammer to develop his elliptical projection (p. 160)

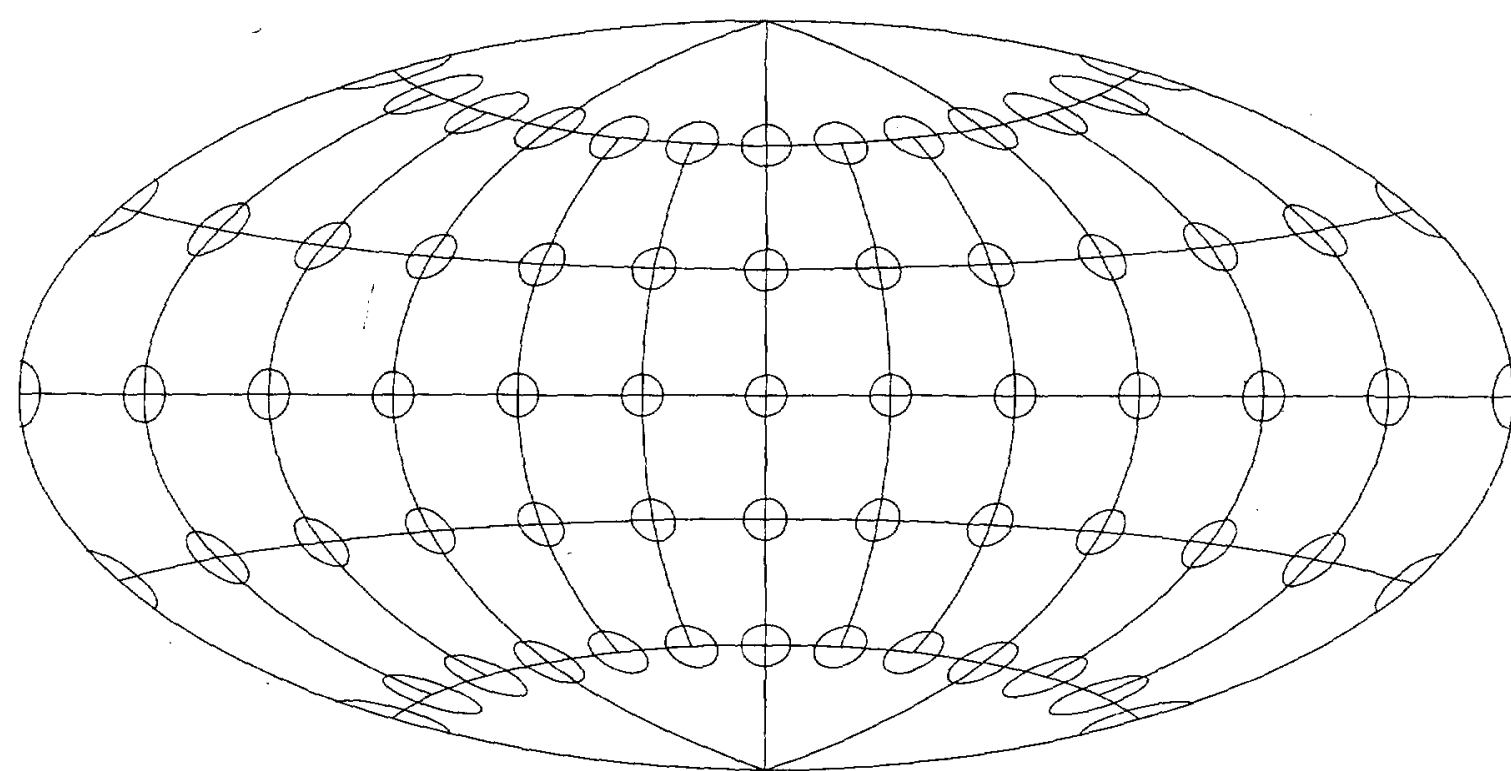

\section{Origin}

David Aitoff (or Aitow) (1854-1933) in 1889

\section{Similar projections}

Hammer (Elliptical) (p. 160), equal area and mistakenly called Aitoff in the early 20th

century

Briesemeister (p. 162), an oblique modification of the Hammer

Eckert-Greifendorff (p. 166), a modification of the Lambert Azimuthal Equal-Area projection inspired by the Hammer

Winkel Tripel (p. 164) is a combination of the Aitoff and Equidistant Cylindrical projections. 


\section{Figure 638. -Aittoff projection, with shorelines, $15^{\circ}$ graticule. Central meridian $90^{\circ} \mathrm{W}$.}

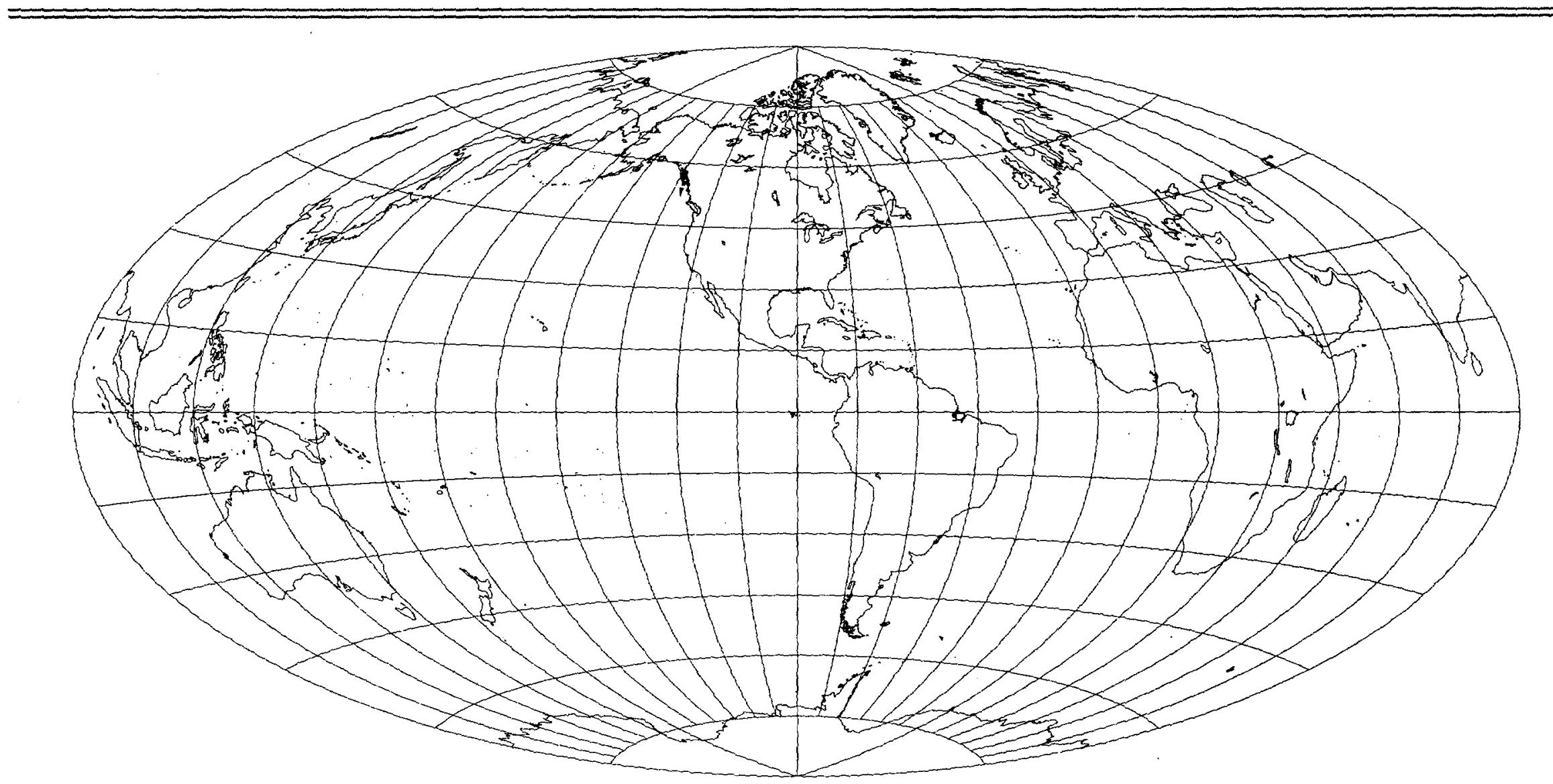




\section{HAMMER Projection}

\section{Classifications}

Modified azimuthal

Equal area

\section{Graticule}

Meridians: Central meridian is a straight line half the length of the Equator. Other meridians are complex curves, unequally spaced along the Equator and concave toward the central meridian

Parallels: Equator is straight. Other parallels are complex curves, unequally spaced along the central meridian and concave toward the nearest pole.

Poles: Points

Symmetry: About the central meridian and the Equator

\section{Scale}

Decreases along the central meridian and the Equator with distance from the center

\section{Distortion}

Moderate (fig. 64A). Less shearing action on the outer meridians near the poles than there is on pseudocylindrical projections.

\section{Other features}

Elliptical border. An equatorial aspect of one hemisphere of the Lambert Azimuthal EqualArea projection, on which horizontal coordinates have been doubled and meridians have been given twice their original longitudes.

\section{Usage}

Whole-world maps, also in interrupted and condensed forms

\section{Origin}

Presented by H.H. Ernst von Hammer (18581925) of Germany in 1892

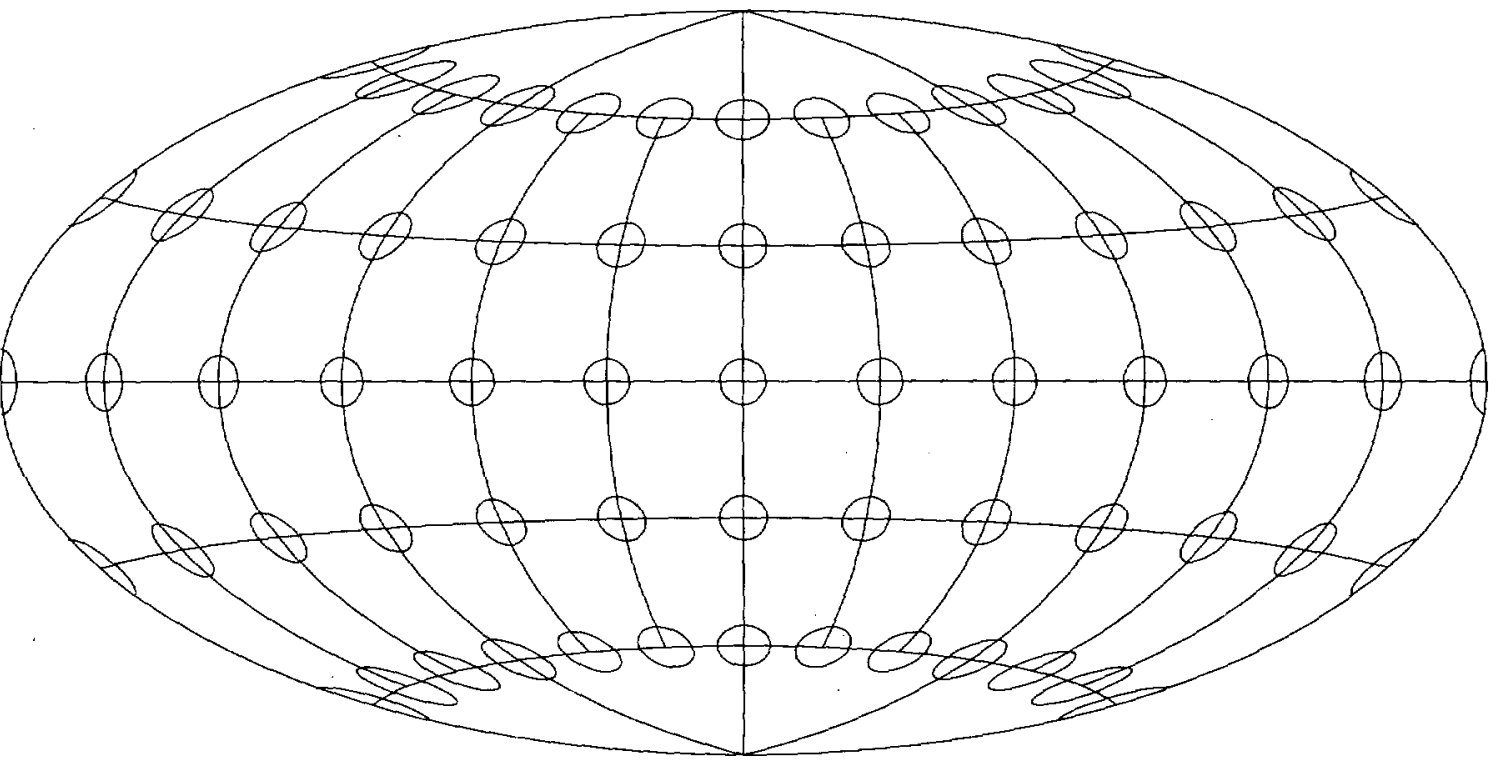

\section{Aspects}

Normal and oblique are both in use.

\section{Other names}

Hammer-Aitoff

Mistakenly called Aitoff projection in the early 20th century.

\section{Similar projections}

Briesemeister (p. 162), a modified oblique Hammer

Eckert-Greifendorff (p. 166), a further modification of the Lambert Azimuthal Equal-Area projection 


\section{Figure 648.-Hammer projection, with shorelines, $15^{\circ}$ graticule. Central meridian $90^{\circ} \mathrm{W}$.}

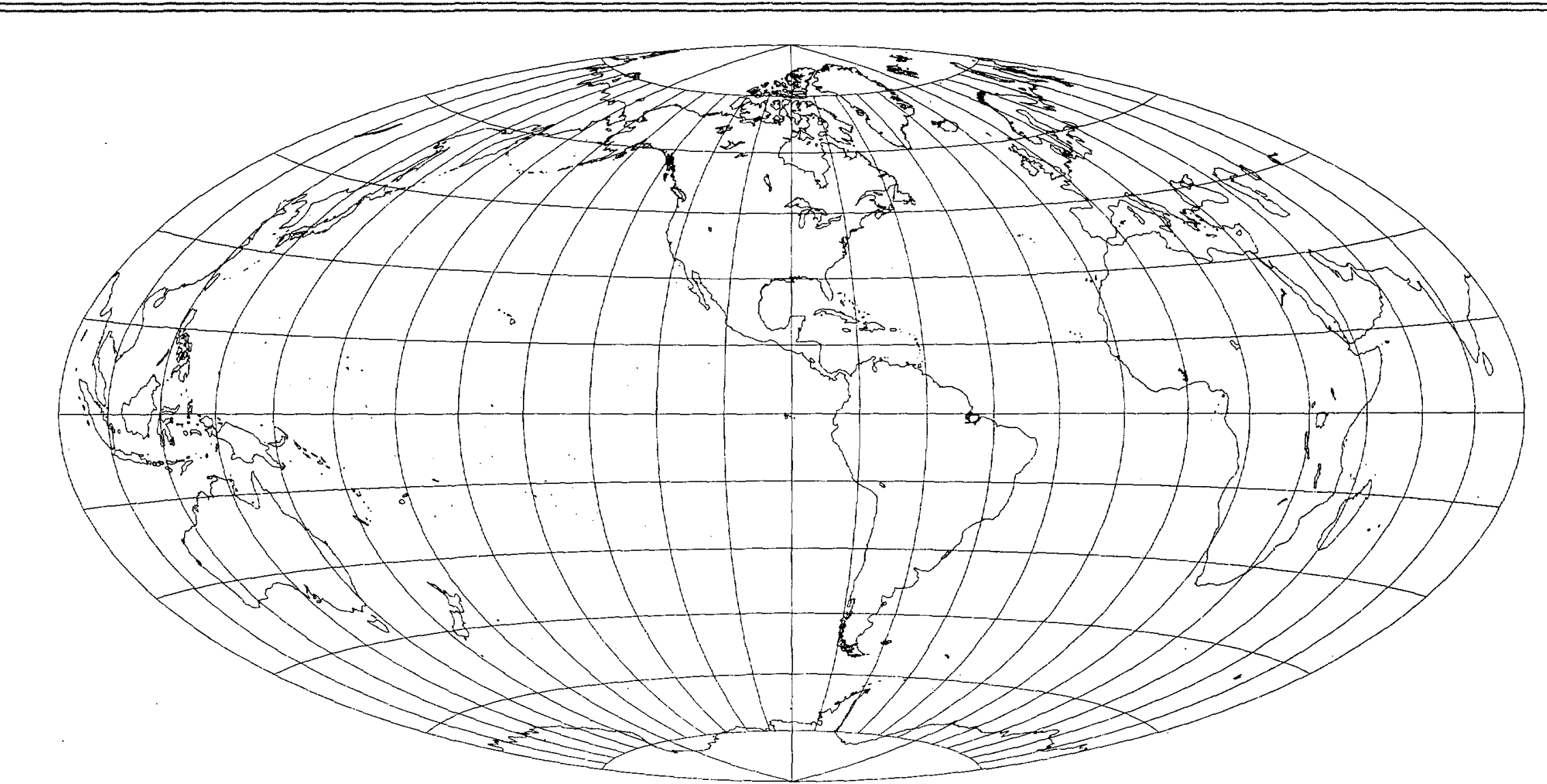




\section{BRIESEMEISTER Projection}

\section{Classifications}

Modified azimuthal

Equal area

\section{Graticule}

Meridians: Central meridian is straight. Other meridians are complex curves.

Parallels: Complex curves

Poles: Points

Symmetry: About the central meridian

\section{Scale}

True scale at latitudes $3.6^{\circ}$ and $86.4^{\circ} \mathrm{N}$. at central meridian

\section{Distortion}

None at latitudes $3.6^{\circ}$ and $86.4^{\circ} \mathrm{N}$. at central meridian (fig. $65 \mathrm{~A}$ )

\section{Other features}

An oblique Hammer projection centered at latitude $45^{\circ} \mathrm{N}$. and longitude $10^{\circ} \mathrm{E}$. and bounded by an ellipse having an axis ratio of 1.75 to 1 instead of 2 to 1 , after the vertical coordinates are expanded and the horizontal coordinates are contracted

\section{Usage}

Whole-world maps showing continents grouped near the center

\section{Origin}

Presented by William A. Briesemeister of the American Geographical Society in 1953

\section{Similar projections}

Hammer (Elliptical) (p. 160), the normal aspect of the Briesemeister, but having an axis ratio of 2 to 1

Nordic, by John Bartholomew, is the same as the Briesemeister projection, except that the axis ratio is 2 to 1 and the central meridian is $0^{\circ}$.

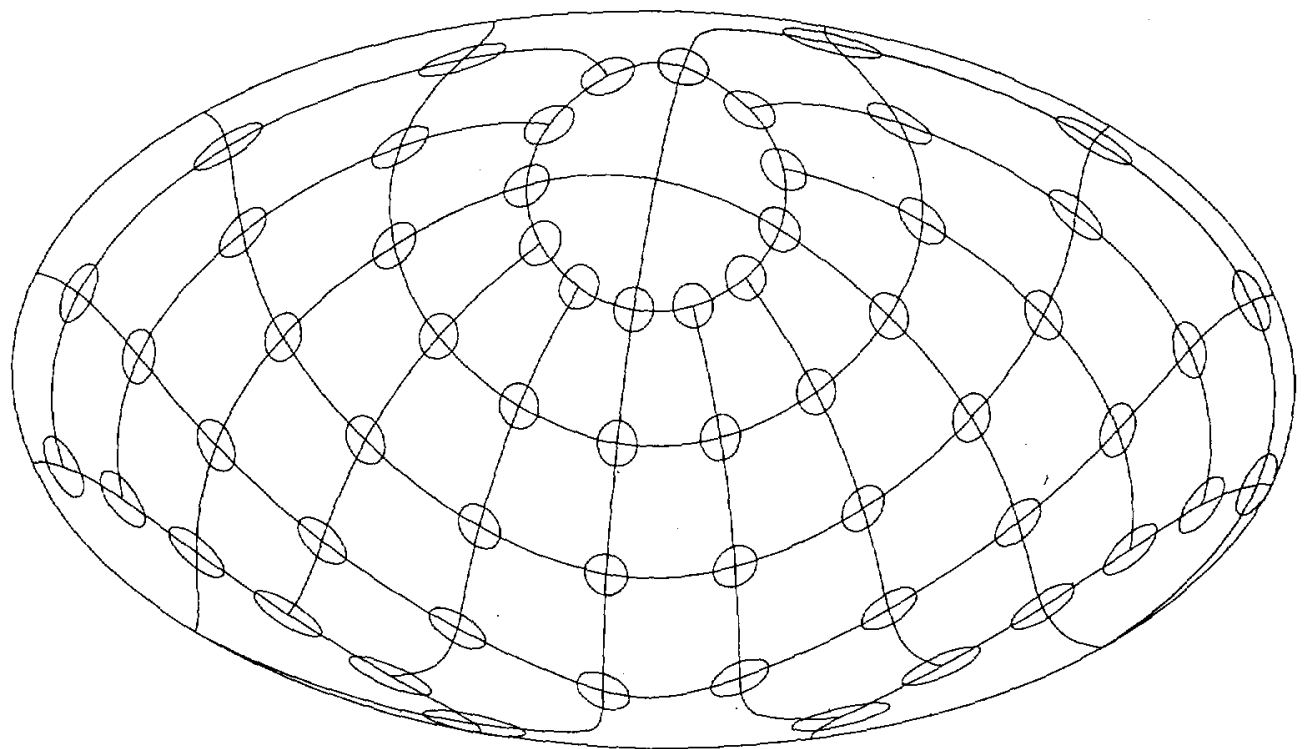

Figure 65A.-Briesemeister projection, with Tissot indicatrices, $30^{\circ}$ graticule. 
Figure 65B.-Briesemeister projection, with shorelines, $15^{\circ}$ graticule. Center at $45^{\circ} \mathrm{N} ., 10^{\circ} \mathrm{E}$.

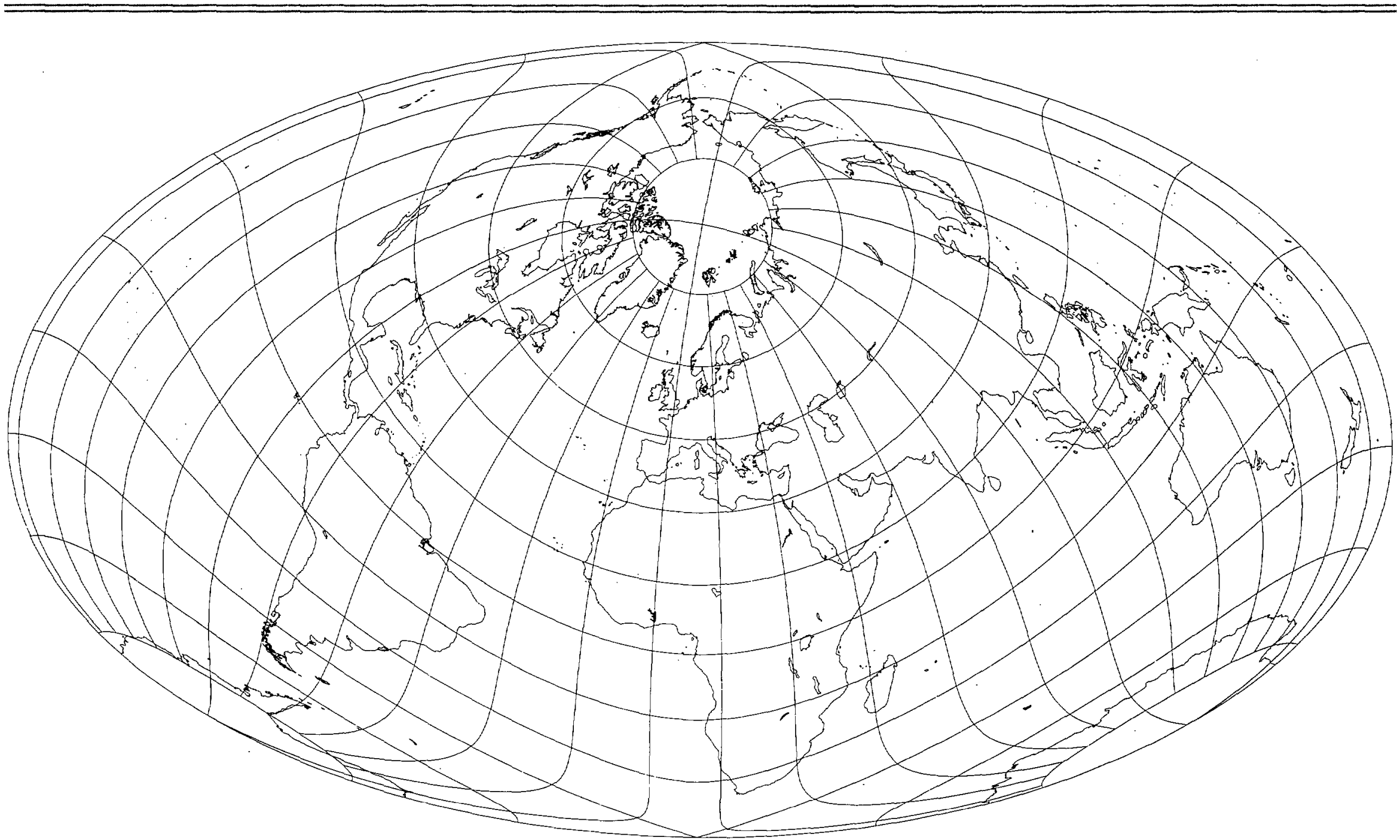




\section{WINKEL TRIPEL Projection}

\section{Classifications}

Modified azimuthal

Neither conformal nor equal area

\section{Graticule}

Meridians: Central meridian is straight. Other meridians are curved, equally spaced along the Equator and concave toward the central meridian.

Parallels: Equator and the poles are straight. Other parallels are curved, equally spaced along the central meridian and concave toward the nearest pole.

Poles: Straight lines about 0.4 as long as the Equator, depending on the latitude of the standard parallels of the base Equidistant Cylin. drical projection

Symmetry: About the central meridian or the Equator

\section{Scale}

True along the central meridian. Constant along the Equator.

\section{Distortion}

Moderate except near outer meridians in polar regions (fig. 66A)

\section{Other features}

Equally spaced central meridian and Equator

\section{Usage}

Whole-world maps

\section{Origin}

Presented by Oswald Winkel (1873-1953) of

Germany in 1921. Obtained by averaging coordinates of Equidistant Cylindrical and Aitoff (not Hammer-Aitoff) projections. Winkel applied the name "Tripel," normally meaning triple.

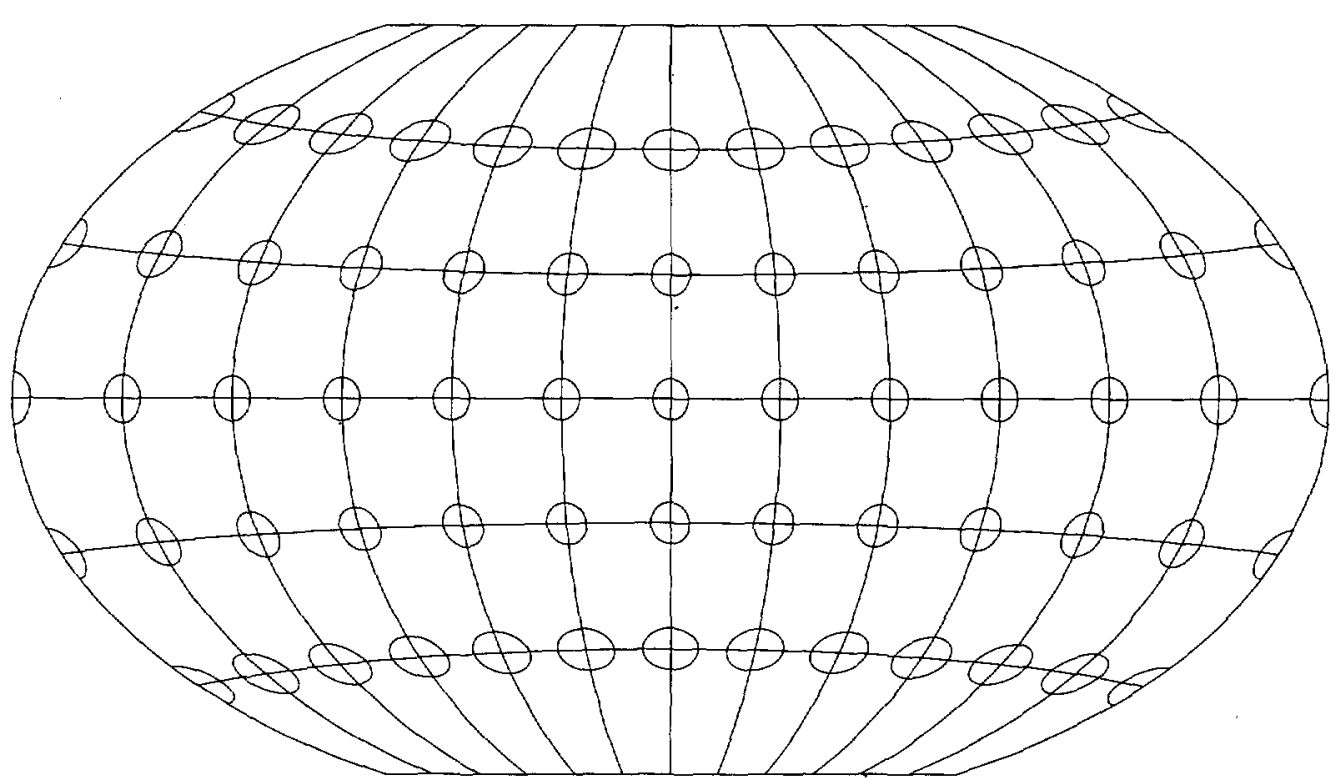

Figure 66A.-Winkel Tripel projection, with Tissot indicatrices, $30^{\circ}$ graticule. Standard parallels the same as that in figure $66 B$. 


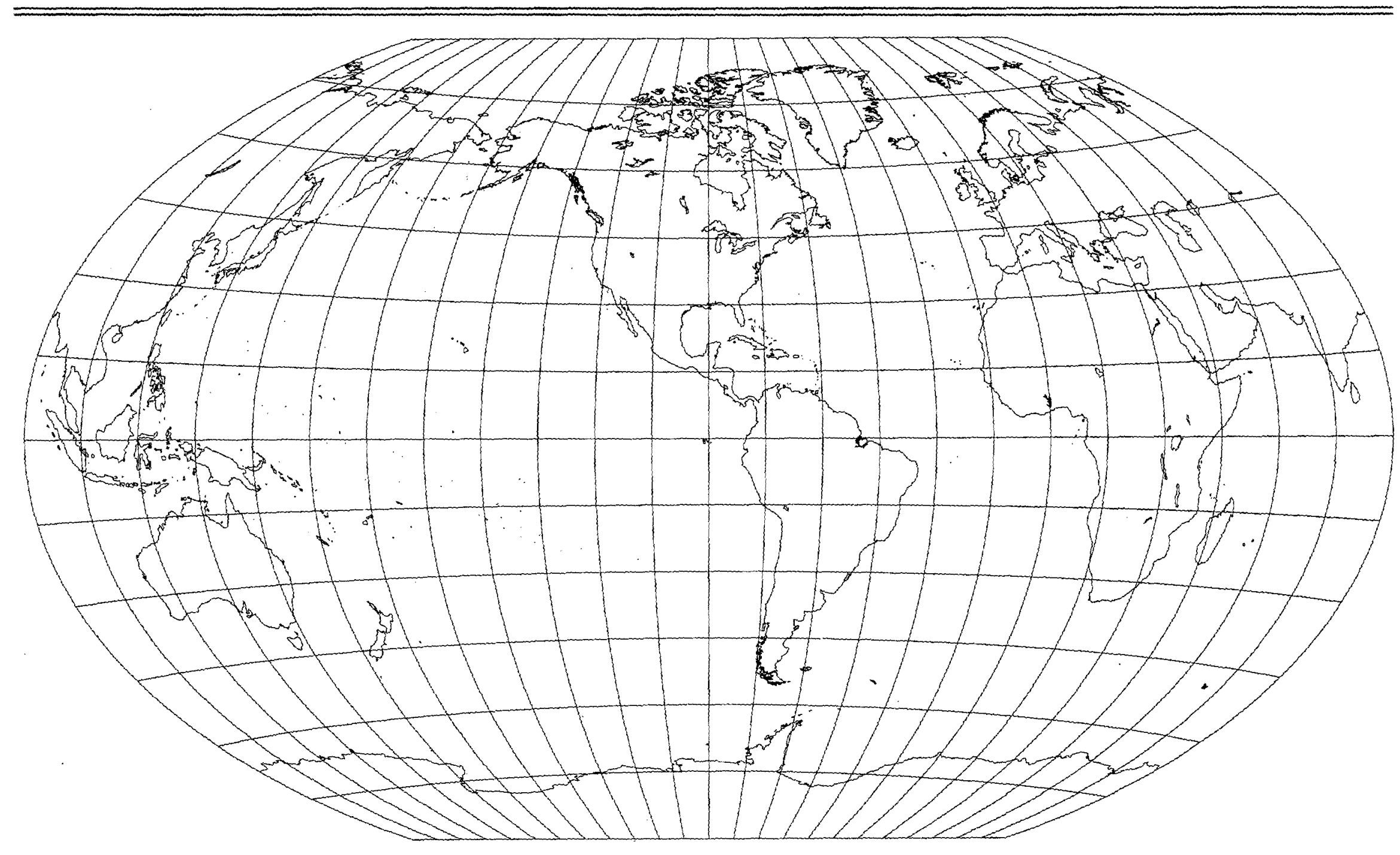




\section{ECKERT-GREIFENDORFF Projection}

\section{Classifications}

Modified azimuthal

Equal area

\section{Graticule}

Meridians: Central meridlan is a straight line 0.46 of the length of the Equator. Other merid. ians are curved, unequally spaced along the

Equator and concave toward the central meridian.

Parallels: Equator is straight. Other parallels are curved, unequally spaced along the centra meridian and concave toward the nearest pole. Poles: Points

Symmetry: About the central meridian or the Equator

\section{Scale}

Decreases steadily along the Equator and the central meridian with distance from the center of the projection

\section{Distortion}

Moderate near center, but considerable shape distortion near outer meridians

\section{Other features}

An equatorial aspect of the Lambert Azimuthal Equal-Area projection for $90^{\circ}$ of longitude, on which the horizontal coordinates have been quadrupled and the meridians have been given four times their original longitudes

\section{Usage}

Whole-world map

\section{Origin}

Max Eckert-Greifendorff (formerly Max

Eckert) (1868-1938) of Germany in 1935

\section{Similar projections}

Hammer (p. 160), a different modification of the Lambert Azimuthal Equal-Area projection

Quartic Authalic (p. 74) has straight parallels. 


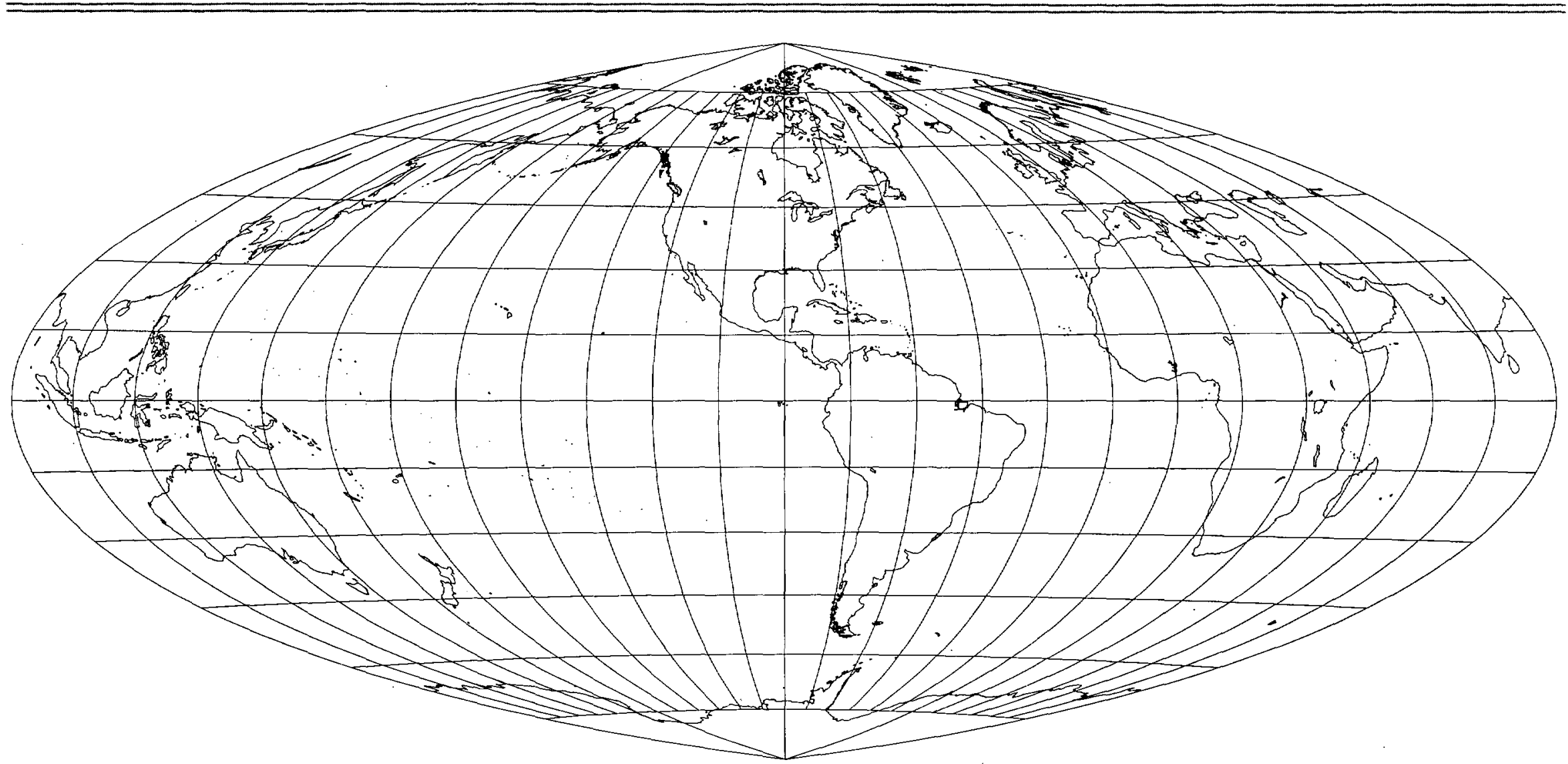




\section{WAGNER VII Projection}

\section{Classifications \\ Modified azimuthal \\ Equal area}

\section{Graticule}

Meridians: Central meridian is straight and half the length of the Equator. Other meridians are curves, unequally spaced along the Equator and concave toward the central meridian.

Parallels: Equator is straight. Other parallels are curves, unequally spaced along the central meridian and concave toward the nearest pole.

Poles: Curved lines

Symmetry: About the central meridian or the Equator

\section{Scale}

Decreases along the central meridian and the Equator with distance from the center of the projection

\section{Distortion}

Considerable shape distortion in polar areas (fig. 68A)

\section{Other features}

A modification of the Hammer projection; the poies correspond to the 65th parallels on the Hammer, and meridians are repositioned

\section{Usage}

World maps, such as climatic maps prepared by the U.S. Department of Commerce

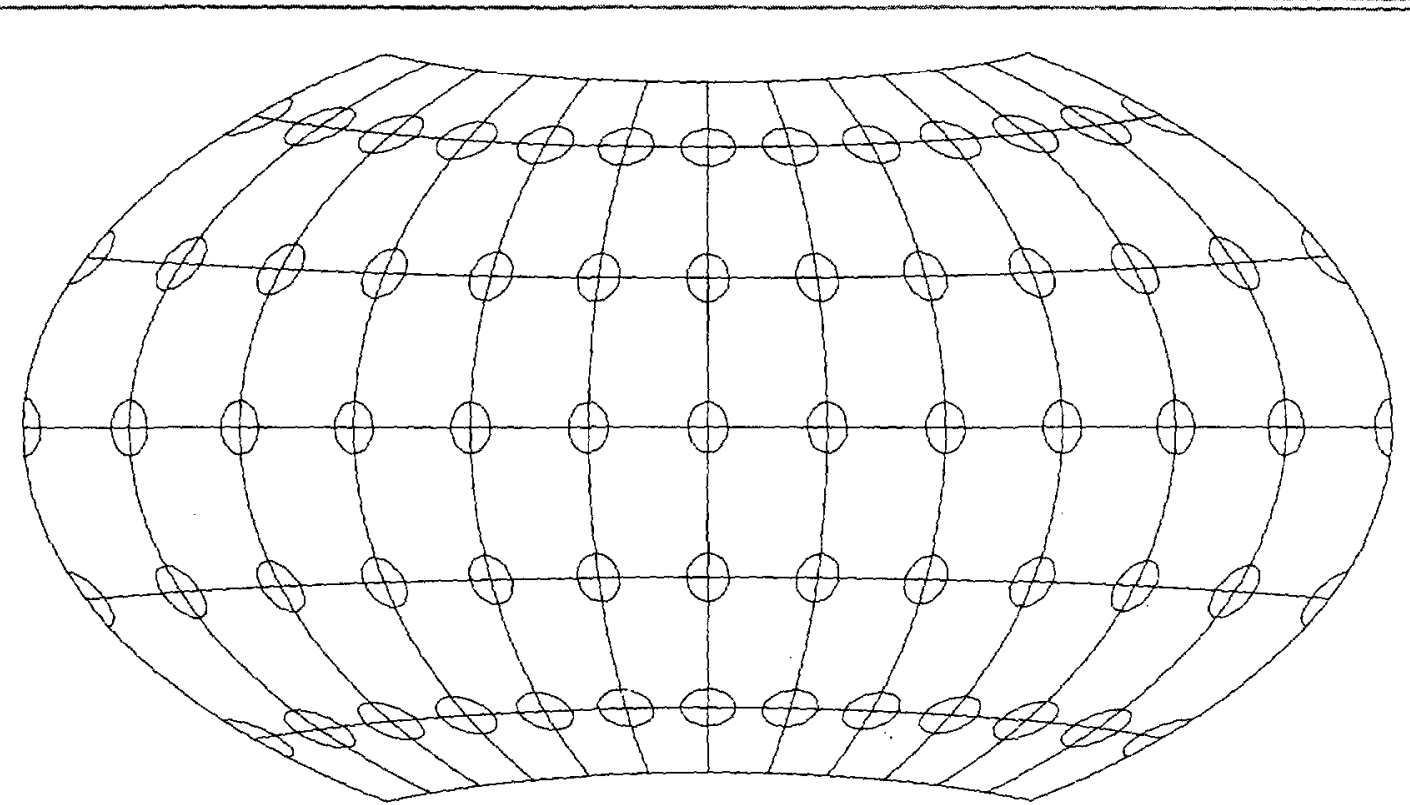

Figure $6 B A-$ Wagner VIf projection, with Tissot indicatrices, $30^{\circ}$ graticule.

\section{Origin}

Presented by Karlheinz Wagner of Germany

in 1941

\section{Other names}

Hammer-Wagner

\section{Similar projections}

Hammer (Elliptical) (p. 160), truncated and otherwise modified for the Wagner VII 


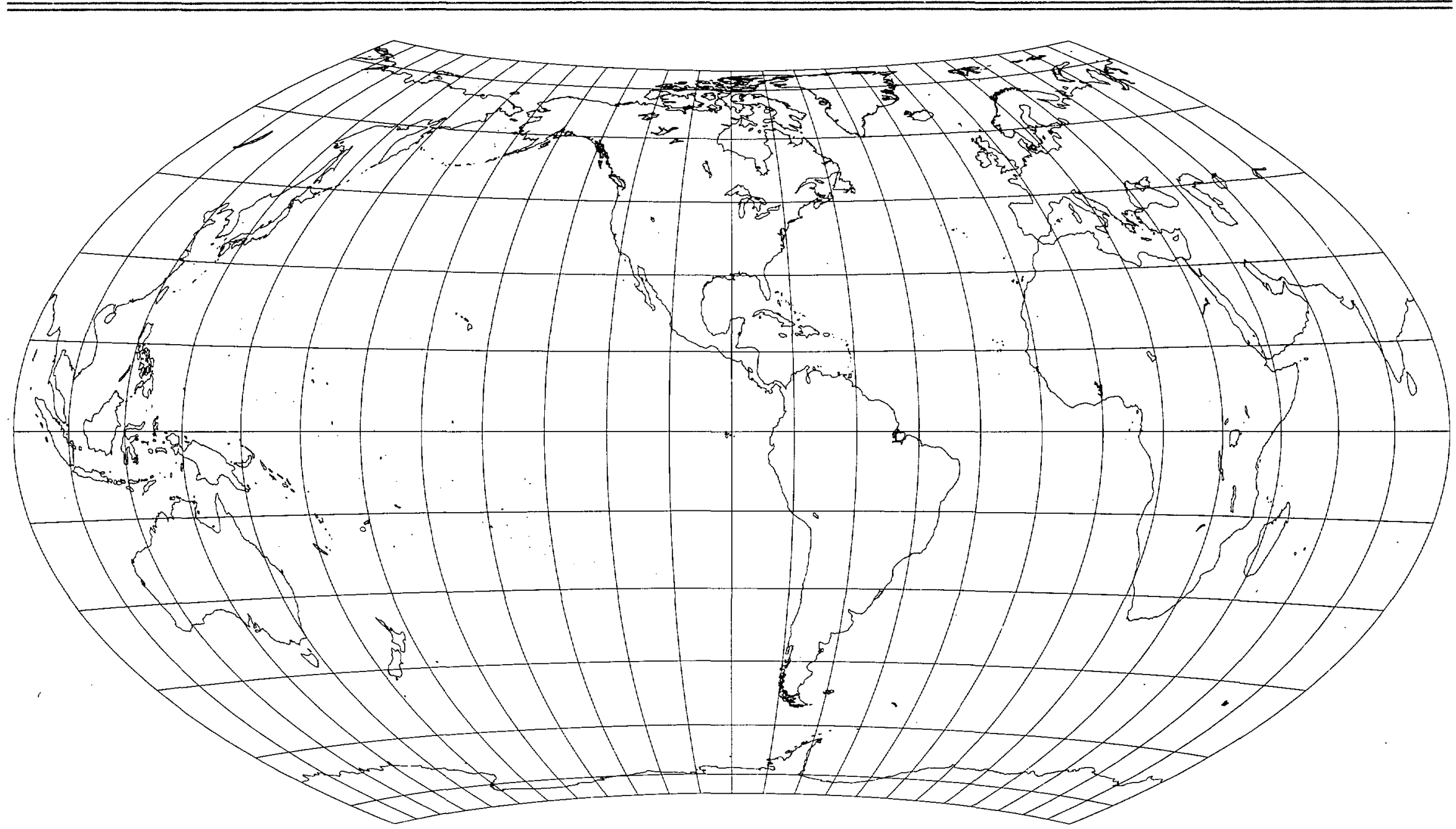




\section{CHAMBERLIN TRIMETRIC Projection}

\section{Classifications}

Modified azimuthal

Modified equidistant

Neither conformal nor equal area

\section{Graticule}

Meridians: Complex curves

Parallels: Complex curves

Poles: Points

Symmetry: Normally none

\section{Scale}

Approximately correct from three selected widely spaced points on map to any other point on map. Cannot be exactly correct.

\section{Distortion}

Low distortion throughout map if three source points are properly placed near map limits (fig. 69A)

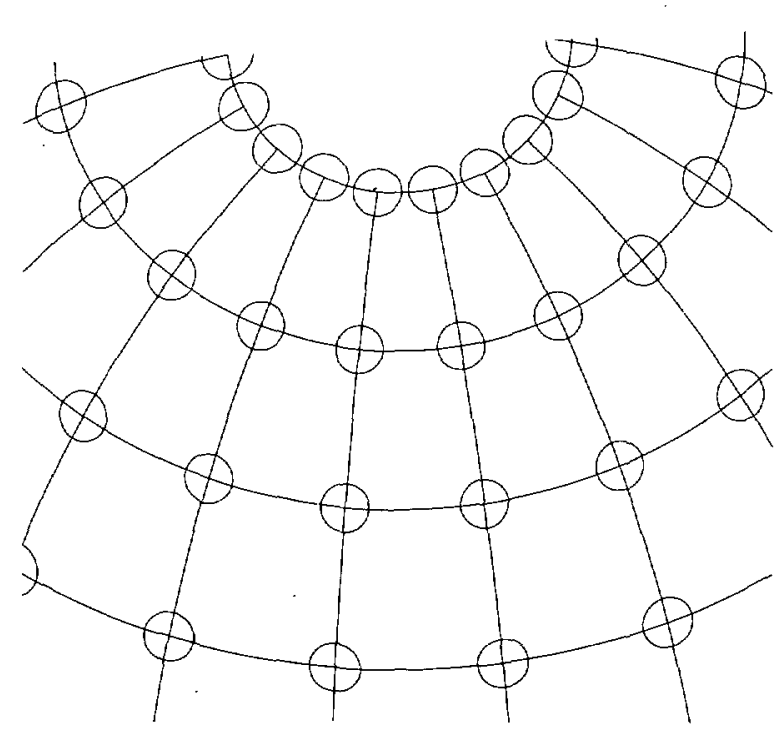

Figure 69A.-Chamberlin Trimetric projection, with Tissot indicat rices, $20^{\circ}$ graticule. Source points the same as those in figure 698 .

\section{Usage}

Atlas maps of continents by the National Geographic Society and some other atlas pub-

lishers

\section{Origin}

Presented by Wellman Chamberlin (1908-76)

of the National Geographic Society in 1946

\section{Similar projections}

Azimuthal Equidistant projection (p. 132); true distance and direction from one point

Two-Point Equidistant projection (p. 146); true distance but not direction from two points 
Figure $69 \mathrm{~b}$. - Chamberlin Trimetric projection of North America, $10^{\circ}$ graticule. Three source points for this map, as selected by the National Geographic Society: (1) latitude $55^{\circ} \mathrm{N}$., longitude $150^{\circ} \mathrm{W}$.; (2) latitude $55^{\circ} \mathrm{N}$., longitude $35^{\circ}$ W.; (3) latitude $10^{\circ} \mathrm{N}$., longitude $92^{\circ} 30^{\prime} \mathrm{W}$.

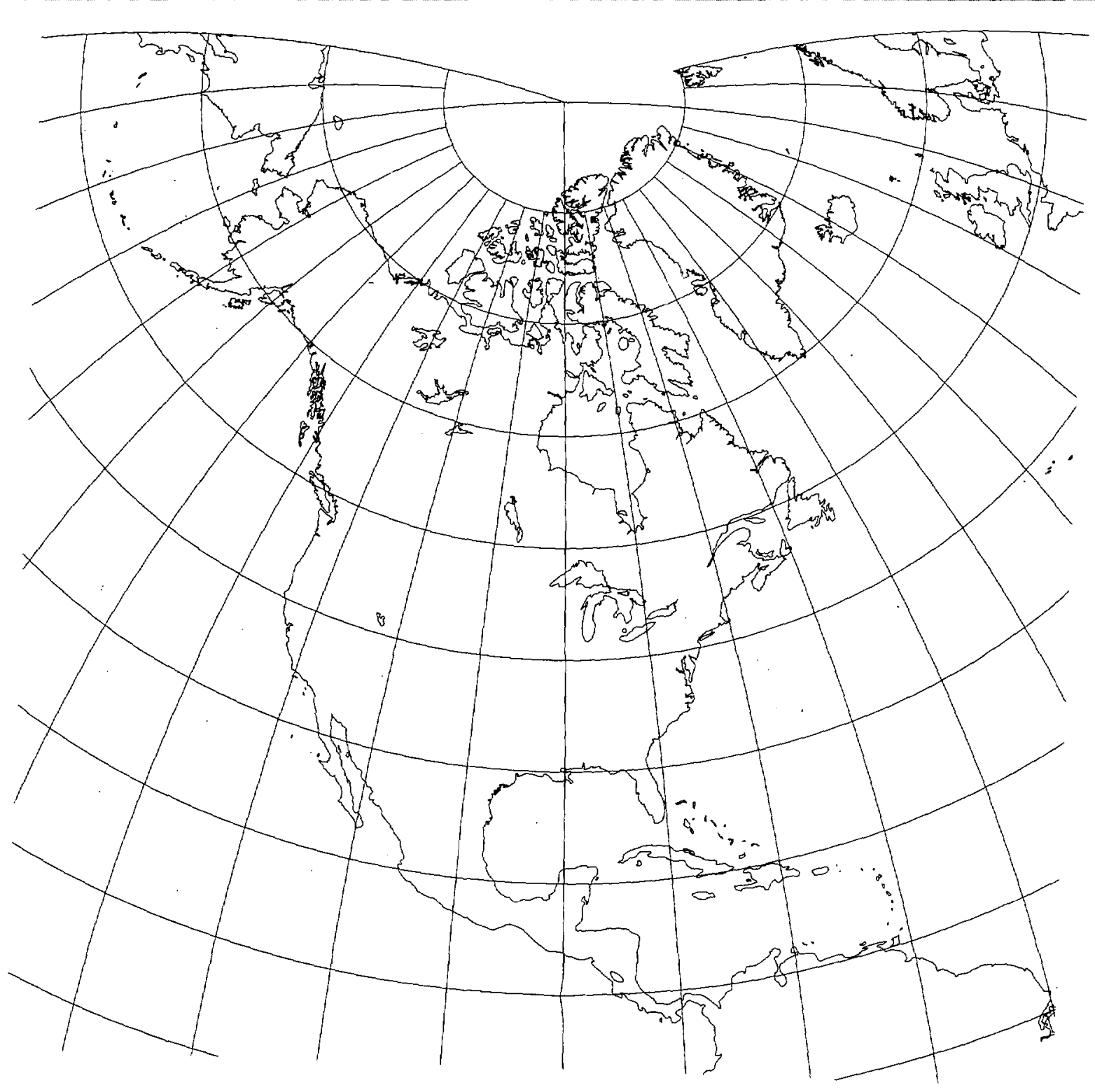




\section{TILTED PERSPECTIVE Projection}

\section{Classifications}

Modified azimuthal

Perspective

Neither conformal nor equal area

\section{Graticule}

Meridians: Central meridian is straight; the point of perspective is in its plane, but this meridian may not appear on the map. Other meridians are normally elliptical arcs, but, for certain angles of tilt or centers, they may be straight lines, parabolas, or hyperbolas.

Parallels: One parallel, normally nearer to the pole than is the center of projection (depending on the position of the point of perspective), is a straight line. Other parallels are normally elliptical arcs but, for certain angles of tilt, may be parabolas or hyperbolas.

Poles: Points

Symmetry: Normally none, but there is symmetry about the central meridian if the direc tion of view is north or south

\section{Range}

Less than one hemisphere if the point of perspective is above the surface

\section{Scale}

Normally varies widely if a large area is

covered

\section{Distortion}

Substantial distortion of shape, area, and scale if a large area is covered

\section{Special features}

Projection shows the Earth geometrically as it appears when photographed or otherwise imaged onto a plane from any point in space with any pointing of the "camera."

\section{Usage}

Pictorial views of the Earth, resembling those seen from space

\section{Origin}

Developed primarily during the 20th century to relate to aerial and space photography and photogrammetry

\section{Limiting forms}

General Vertical Perspective projection (p. 128)

Orthographic projection (p. 124), if the point of perspective is at infinite distance 
Figure 70.-Tilted Perspective projection. Eastern U.S. seaboard, viewed from a point about $160 \mathrm{~km}$ above Newburgh, N.Y., 1 graticule. Center of projection latifude $41^{\circ} 30^{\prime} \mathrm{N}$., longitude $74^{\circ} \mathrm{W}$., tilt $55^{\circ}$, rotation $210^{\circ}$.

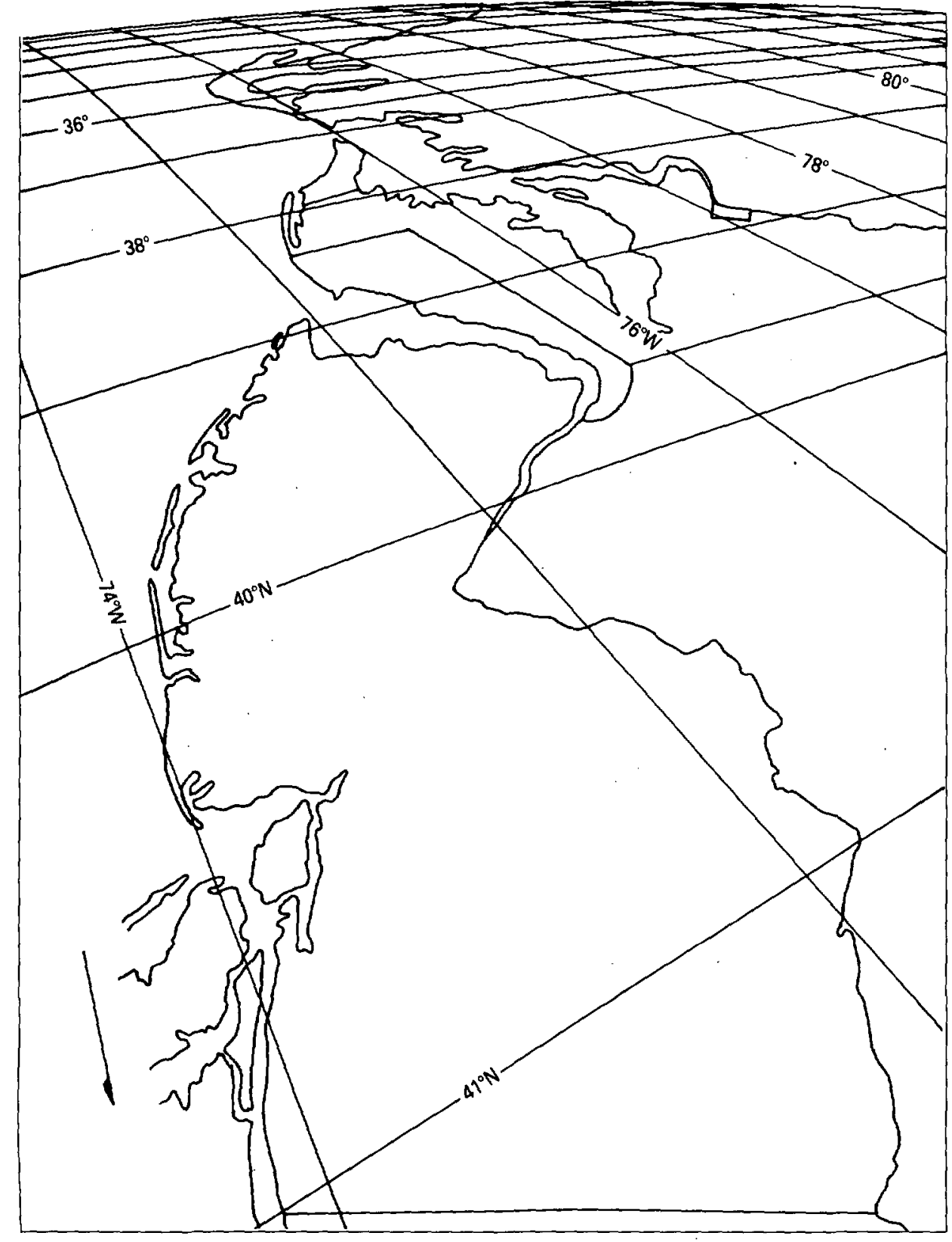




\section{BACON GLOBULAR Projection}

\section{Classification}

Globular

Neither conformal nor equal area

\section{Graticule}

Meridians: Central meridian is straight.

Other meridians are circular arcs

equally spaced along the Equator but not

along other parallels.

Parallels: Parallel straight lines equally spaced on the meridians $90^{\circ}$ from center

Poles: Points

Symmetry: About the central meridian or the Equator

Scale

True along the Equator

\section{Distortion}

Extreme area and shape distortion near the poles

\section{Usage}

Early hemispheric maps

\section{Origin}

Presented by Roger Bacon (1214-94) of

England, about 1265, but unclear

\section{Similar projections}

Glareanus (Loritz) projection of 1527 is identical.

Apian Globular I hemisphere of 1524 has straight parallels equally spaced along the central meridian.

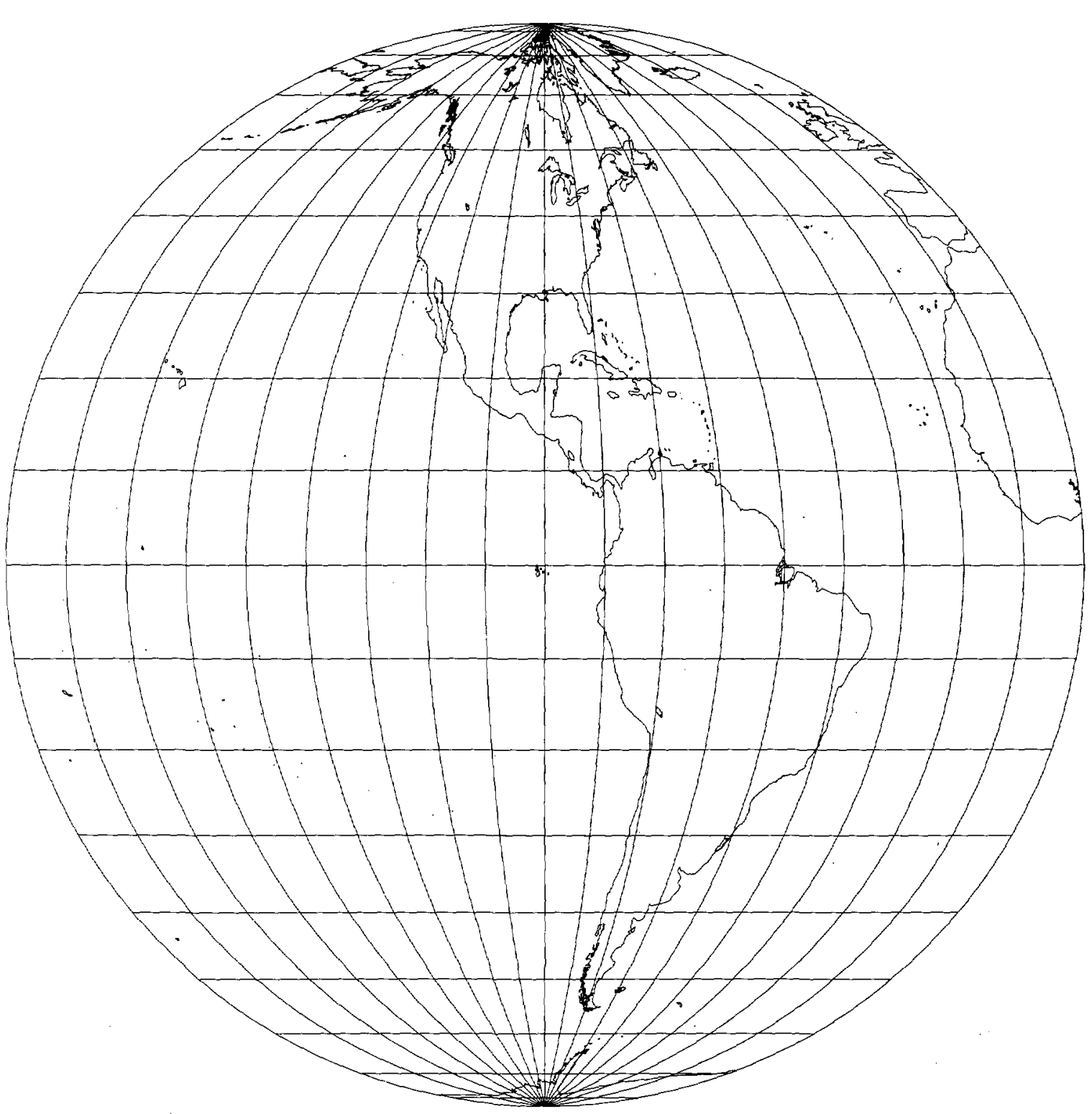




\section{FOURNIER GLOBULAR I Projection}

Figure 72.-Fournier Globular I projection, with shorelines $10^{\circ}$ graticule. Central meridian $90^{\circ} \mathrm{W}$.

\section{Classification}

Globular

Neither conformal nor equal area

\section{Graticule}

Meridians: Central meridian is straight. Other meridians are semiellipses equally spaced along the Equator but not along other parallels.

Parallels: Circular arcs equally spaced on the central and outer meridians

Poles: Points

Symmetry: About the central meridian or the Equator

\section{Scale}

True along the central meridian and the Equator

\section{Distortion}

Moderate for a hemisphere

\section{Usage}

Early whole-world or hemispheric maps

\section{Origin .}

Presented by Georges Fournier of France in 1643

\section{Similar projections}

Nicolosi Globular (p. 176) has circular arcs for meridians.

Mollweide (p. 54) has straight parallels and is used for world maps, but meridians within one hemisphere are the same as those on Fournier 1.

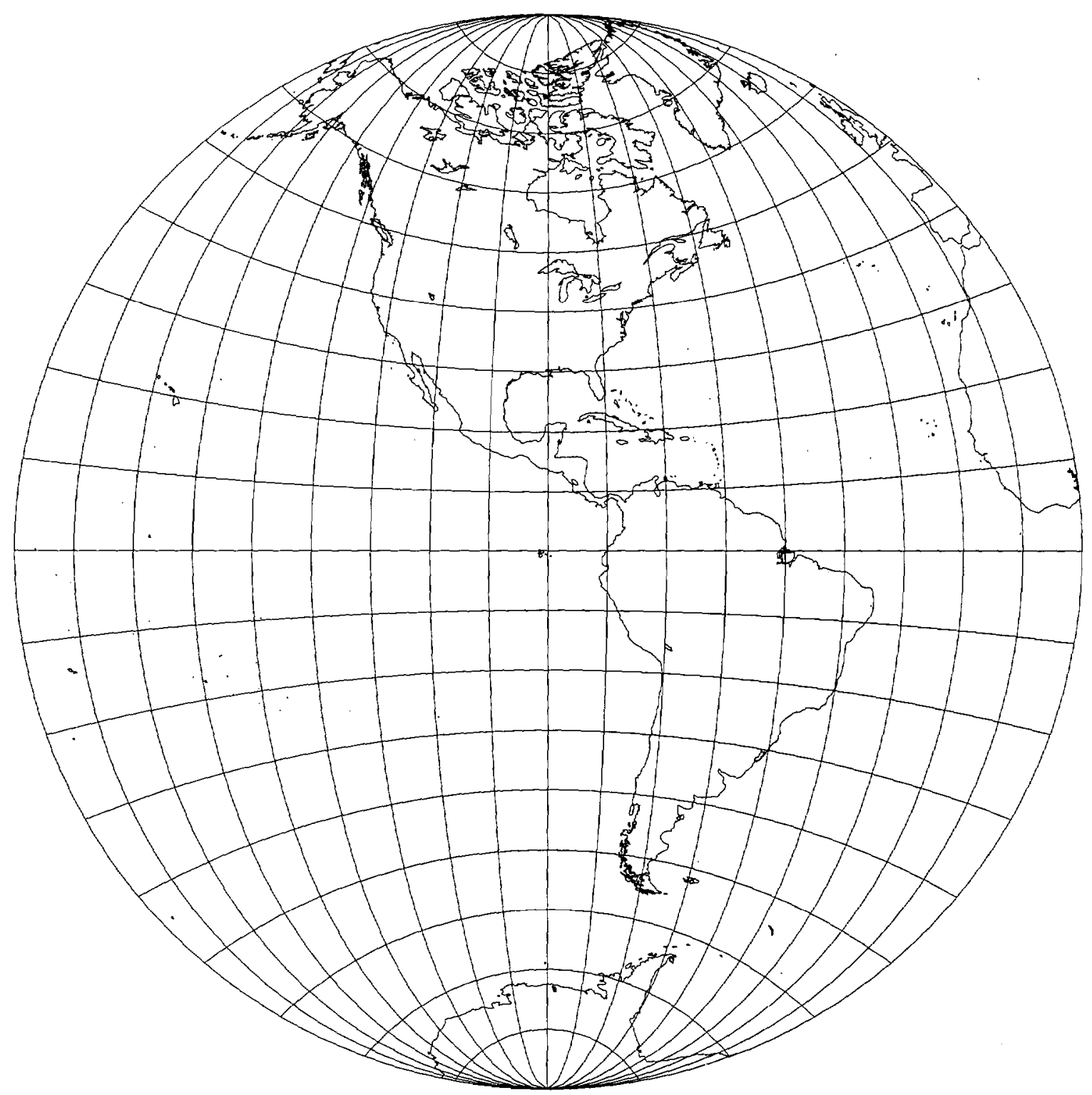

Azimuthal Equidistant (equatorial aspect)

(p. 134) has complex curves for meridians and parallels. 


\section{Classifications}

Globular

Polyconic

Neither conformal nor equal area

\section{Graticule}

Meridians: Central meridian is straight.

Other meridians are circular arcs, equally

spaced along the Equator but not along other parallels.

Parallels: Circular arcs equally spaced on the central and outer meridians

Poles: Points

Symmetry: About the central meridian or the Equator

\section{Scale}

True along the central meridian and the

Equator

\section{Distortion}

Moderate for a hemisphere

Considerable area and shape distortion if used beyond the 90th meridians

\section{Usage}

Hemispheric maps, common in atlases between 1850 and 1925

\section{Origin}

Presented by al-Bīrūnī about A.D. 1000. Reinvented by Giambattista Nicolosi in 1660

\section{Other names}

Arrowsmith

Globular

\section{Similar projections}

Van der Grinten IV (p. 204) has parallels of different curvature and is extended to a worid map.

Azimuthal Equidistant (equatorial aspect)

(p. 134) has complex curves for meridians and parallels.

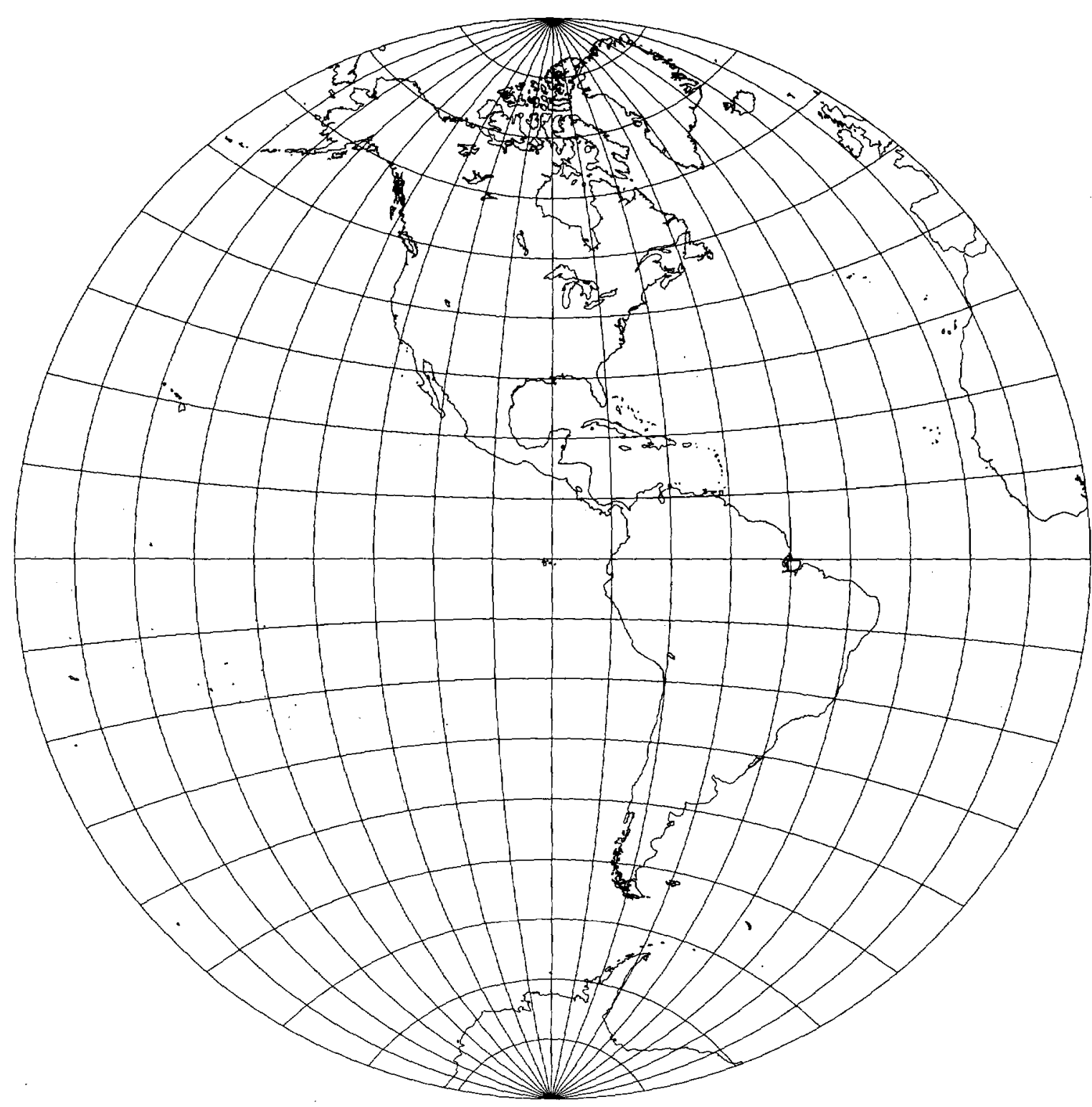

Fournier Globular I (p. 175) has elliptical arcs for meridians. 


\section{APIAN GLOBULAR I Projection}

Figure 74.-Apian Globular I projection, with shorelines, $10^{\circ}$ graticule. Central meridian $90^{\circ} \mathrm{W}$.

\section{Classifications}

Globular

Neither conformal nor equal area

\section{Graticule}

Meridians: Central meridian is straight.

Other meridians are circular arcs equally

spaced along the Equator but not along other parallels.

Parallels: Straight lines, equally spaced along the central meridian

Poles: Points

Symmetry: Along the central meridian or the Equator

\section{Scale}

True along the central meridian and the

Equator

\section{Distortion}

Moderate

\section{Usage}

Early maps of hemispheres

\section{Origin}

Presented by Peter Apian (Petrus Apianus or Peter Bienewitz) (1495-1552) of Saxony in 1524

\section{Similar projections}

Glareanus (Loritz) has identical meridians, but straight paralleis are equally spaced on the 90th meridians.

Apian Globular II has identical parallels, central meridian, and 90th meridians, but other meridians are equally spaced ellipses. Ortelius Oval (p. 178) is identical within $90^{\circ}$ of the central meridian but is used for a world

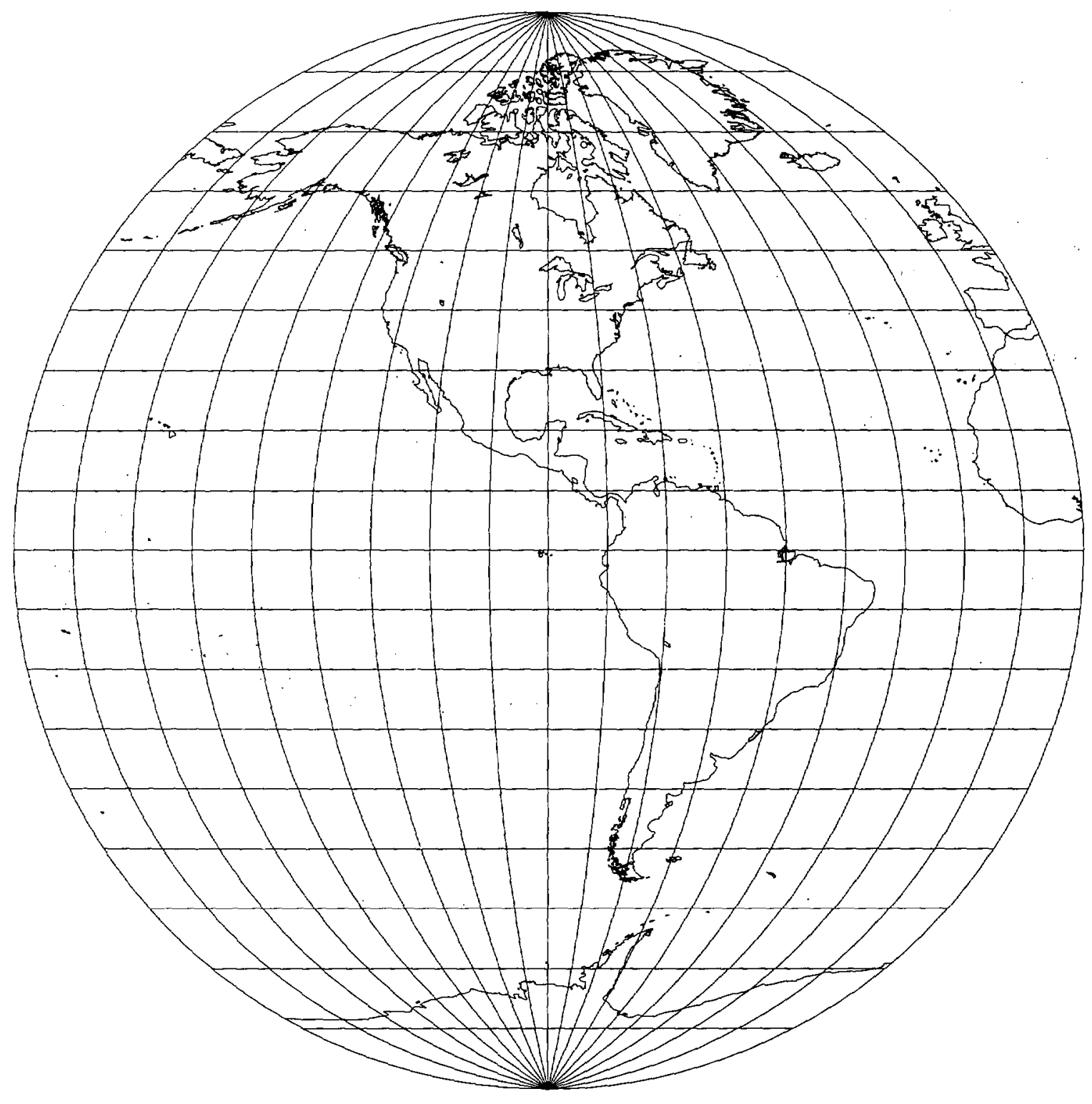
map. 


\section{ORTELIUS OVAL Projection}

\section{Classifications}

\section{Oval}

Modified pseudocylindrical

Neither conformal nor equal area

\section{Graticule}

Meridians: Central meridian is straight. Other meridians are circular arcs, becoming semicircles between the meridians $90^{\circ}$ and $180^{\circ}$ from the central meridian. Equally spaced along the Equator but not along other parallels.

Parallels: Straight lines, equally spaced on the central meridian

Poles: Lines half the length of the Equator Symmetry: Along the central meridian or the Equator

\section{Scale}

True along the central meridian and the Equator

\section{Distortion}

Moderate

\section{Usage}

16th-century whole-world maps

\section{Origin}

Battista Agnese (1514-64) used the projection in 1544, but the name comes from Abraham Ortelius (1527-98) of Flanders, who used it in 1570.

\section{Similar projections}

Often confused with Eckert 111 (p. 58), which has elliptic arcs for meridians

Apian Globular I (p. 177) is the same for one hemisphere.

Bordone Oval projection of 1528 has points for poles and near-ellipses or ovals for meridians but is otherwise very similar. 
Figure 75.-Ortelius Ovai projection, with shorelines, $15^{\circ}$ graticule. Central meridian $90^{\circ} \mathrm{W}$.

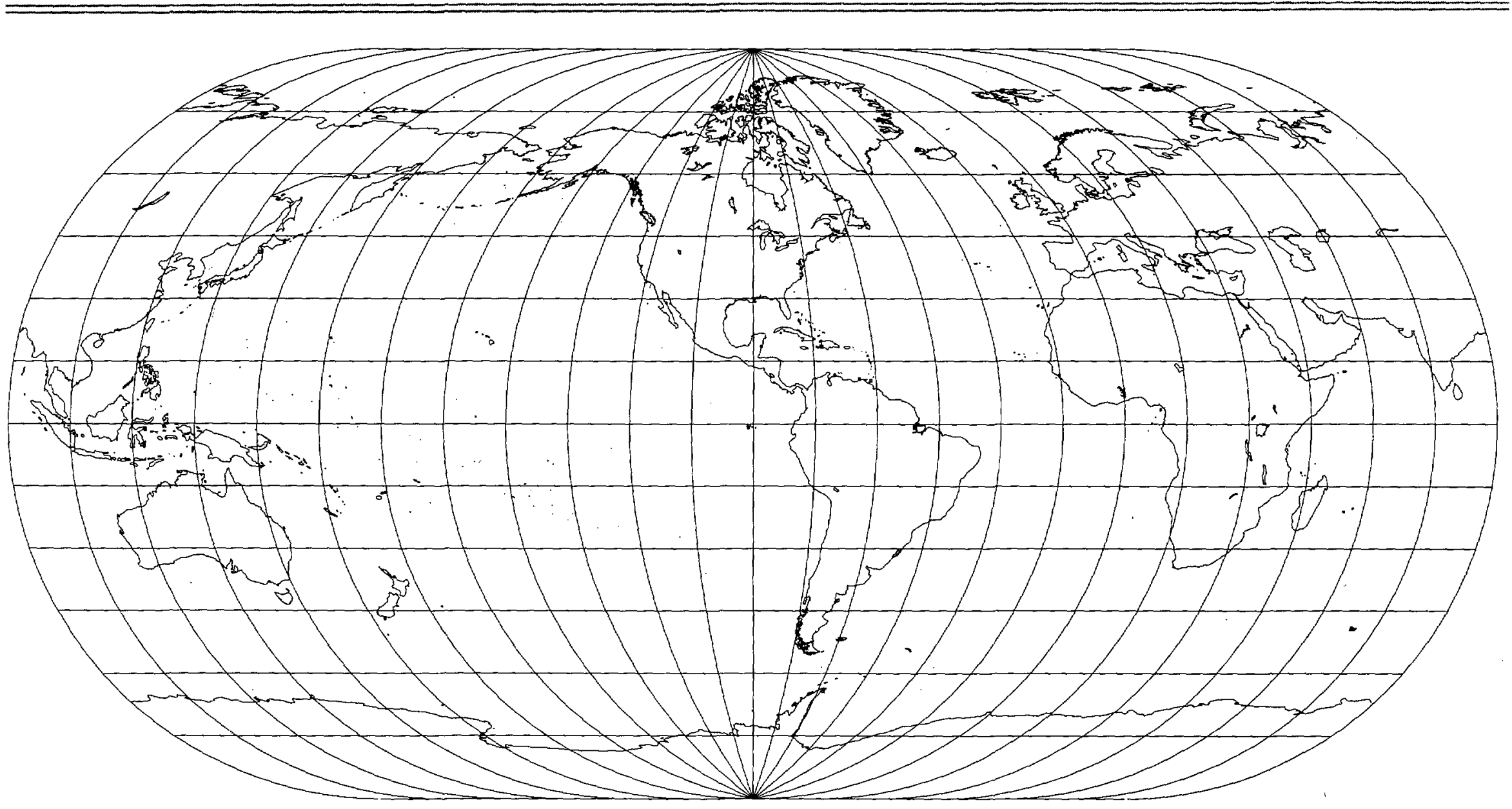




\section{LAGRANGE Projection}

\section{Classifications}

Conformal

Polyconic

\section{Graticule}

Meridians: Central meridian is straight. Other meridians are circular arcs, concave toward the central meridian.

Parallels: One parallel, often the Equator, is straight. Other parallels are circular arcs, concave toward the pole which is on the same side of the straight parallel.

Poles: Points

Symmetry: About the central meridian. Also about the Equator, if the Equator is straight.

\section{Scale}

Increases rapidly with distance from the center

\section{Distortion}

Great distortion of area and scale when the center is compared with poles or other outer limits (fig. 76A). Conformality fails at the poles.

\section{Other features}

The general formula can be used to create a variety of conformal maps.

\section{Usage}

Whole-world maps and conformal maps within a circle of smaller portions of the world

\section{Origin}

Johann Heinrich Lambert (1728-77) of Alsace presented the world conformally in a circle in 1772. This projection is usually called the Lagrange projection, however, after Joseph Louis Lagrange (1736-1813) of France, who generalized Lambert's concept in 1779.

\section{Similar projections}

Van der Grinten I (p. 200) is not conformal.

\section{Special forms}

Equatorial or oblique Stereographic projection (fig. 76D, also p. 120)

Littrow projection (p. 154)

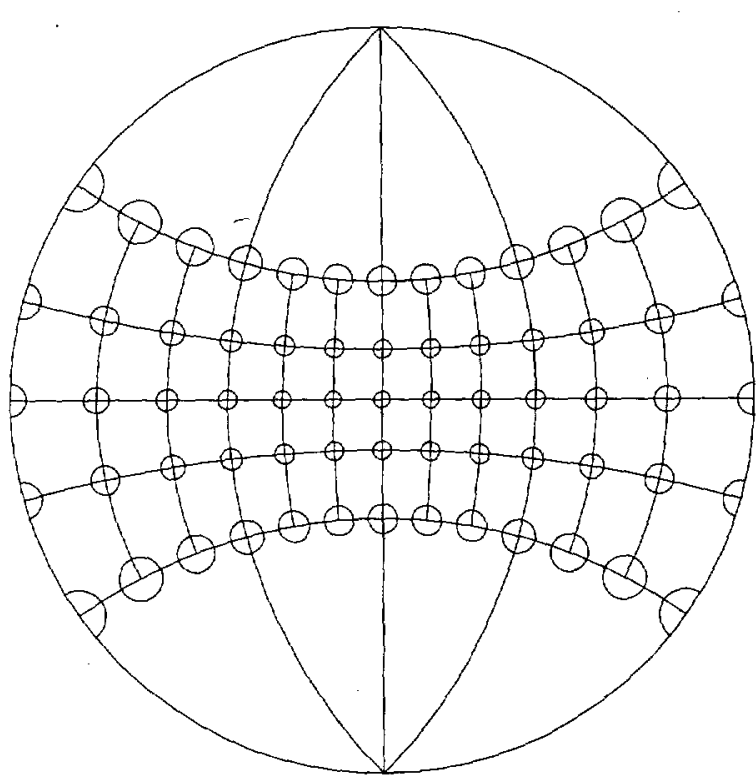




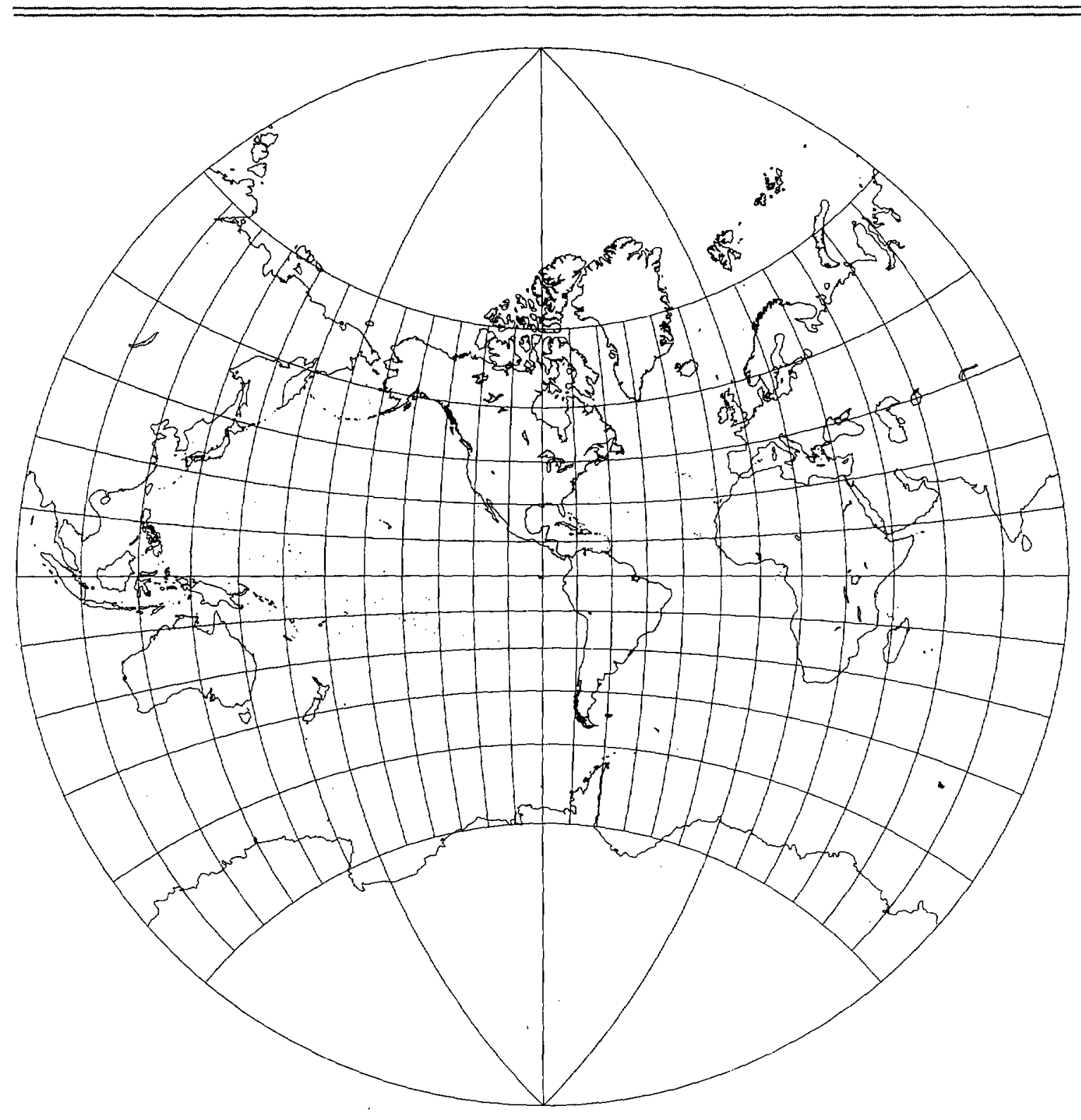




\section{LAGRANGE Projection}

Figure $76 C$.- - Lagrange projection of 0.7 of sphere in circle, with shorelines, $15^{\circ}$ graticule. Central meridian $90^{\circ} \mathrm{W}$.

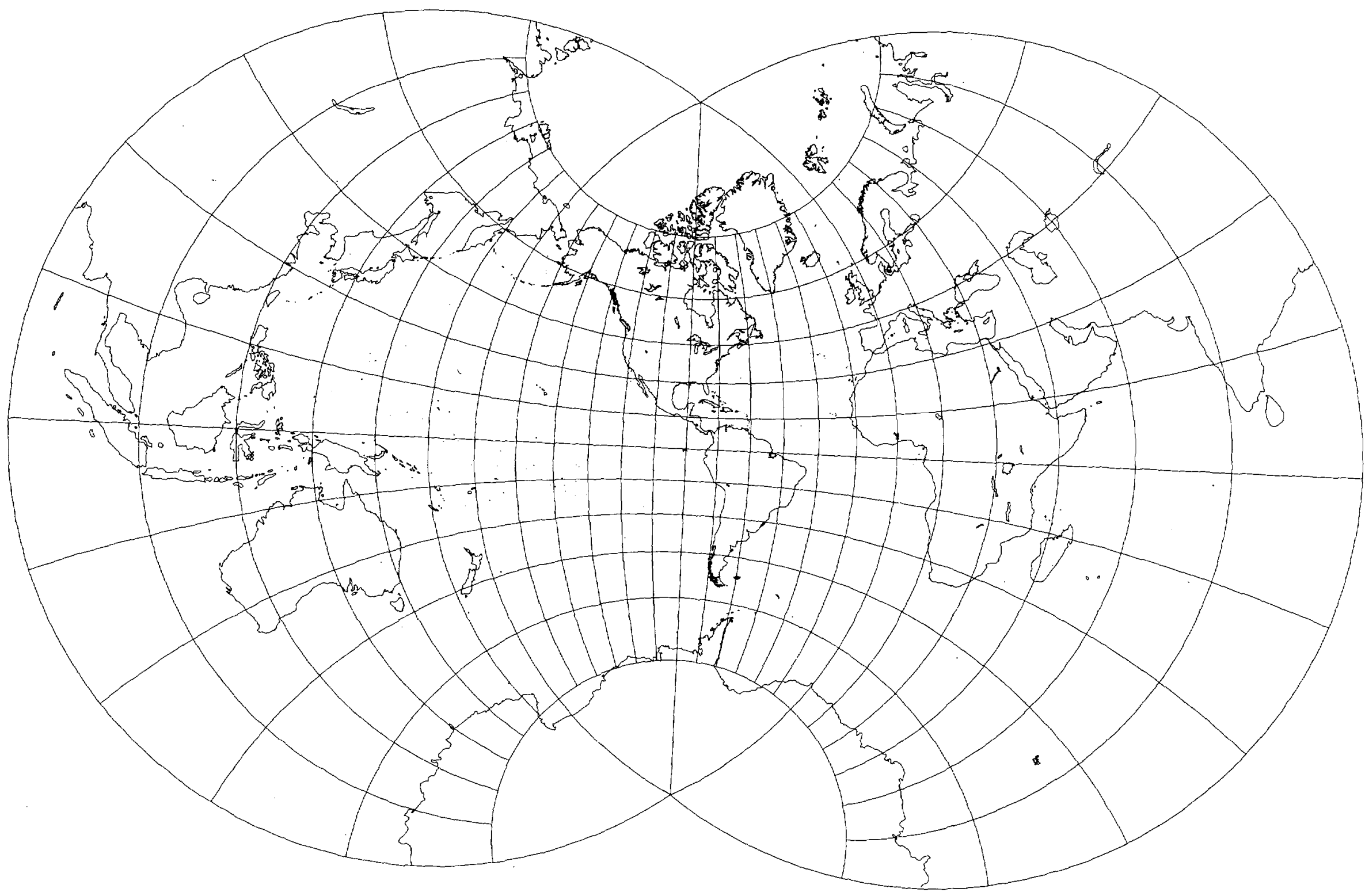


Figure 76D.-Equatorial Stereographic projection, with shorelines, $30^{\circ}$ graticule Central meridian $90^{\circ} \mathrm{W}$. Special case of Lagrange with hemisphere in circle.
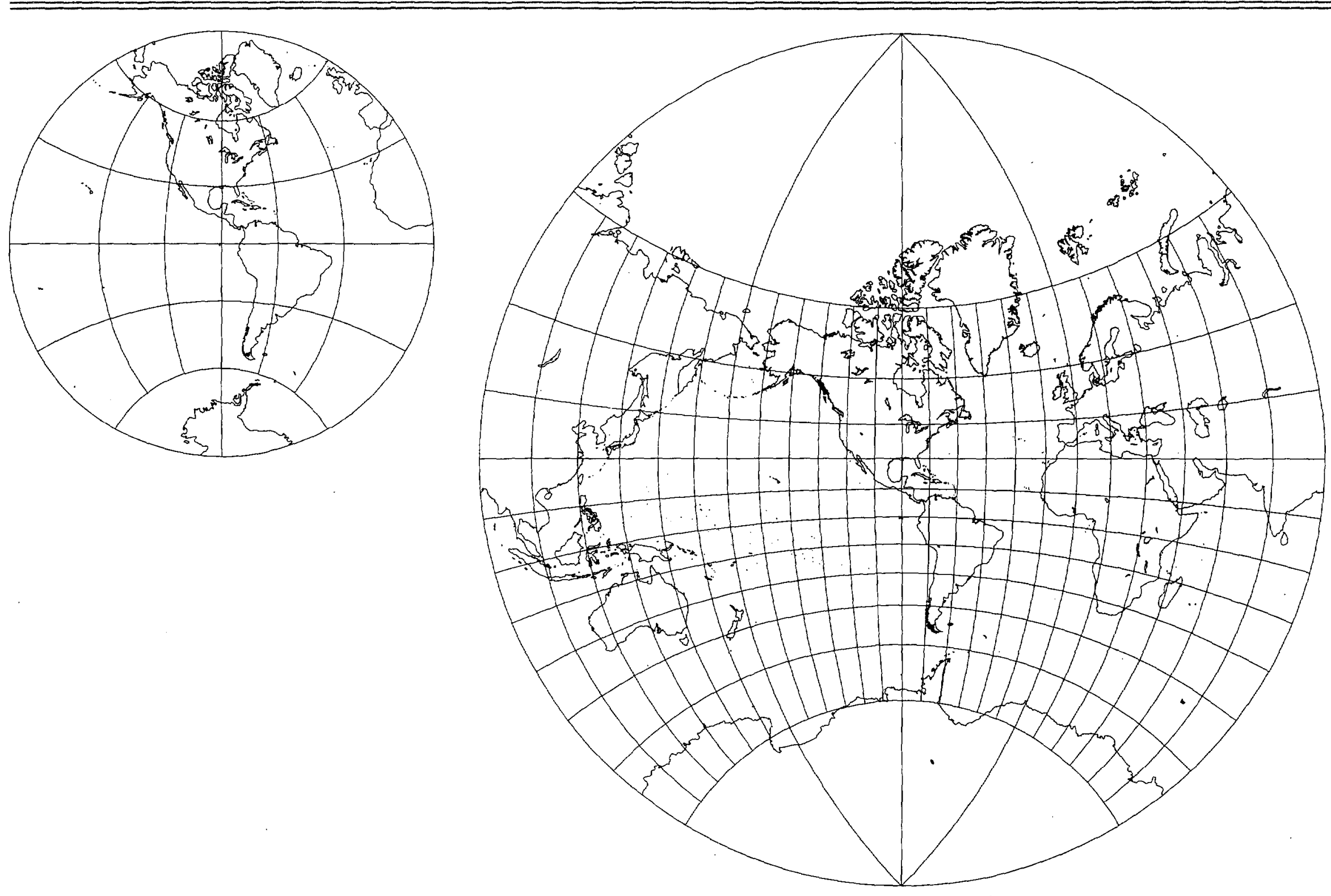

Figure $76 E$.- Lagrange projection of sphere in circle with latitude $30^{\circ} \mathrm{N}$. shown straight, with shorelines, $15^{\circ}$ graticule. Central meridian $90^{\circ} \mathrm{W}$. 


\section{EISENLOHR Projection}

\section{Classification}

Conformal

\section{Graticule}

Meridians: Central meridian is straight.

Other meridians are curved and concave

toward the central meridian.

Parallels: Equator is straight. Other parallels are curved and concave toward the nearest pole.

Poles: Points

Symmetry: About the central meridian or the Equator

\section{Scale}

Increases rapidly with distance from the center. Constant scale around the boundary of a world map.

\section{Distortion}

Great distortion of area near the 180th meridians in comparison with the center (fig. 77A).

\section{Other features}

Because the boundary of a world map is at a constant scale, the projection provides the minimum overall scale variation for a conformal world map. Like the August Epicycloidal projection (p. 186), the Eisenlohr has no "singular" points at which conformality fails.

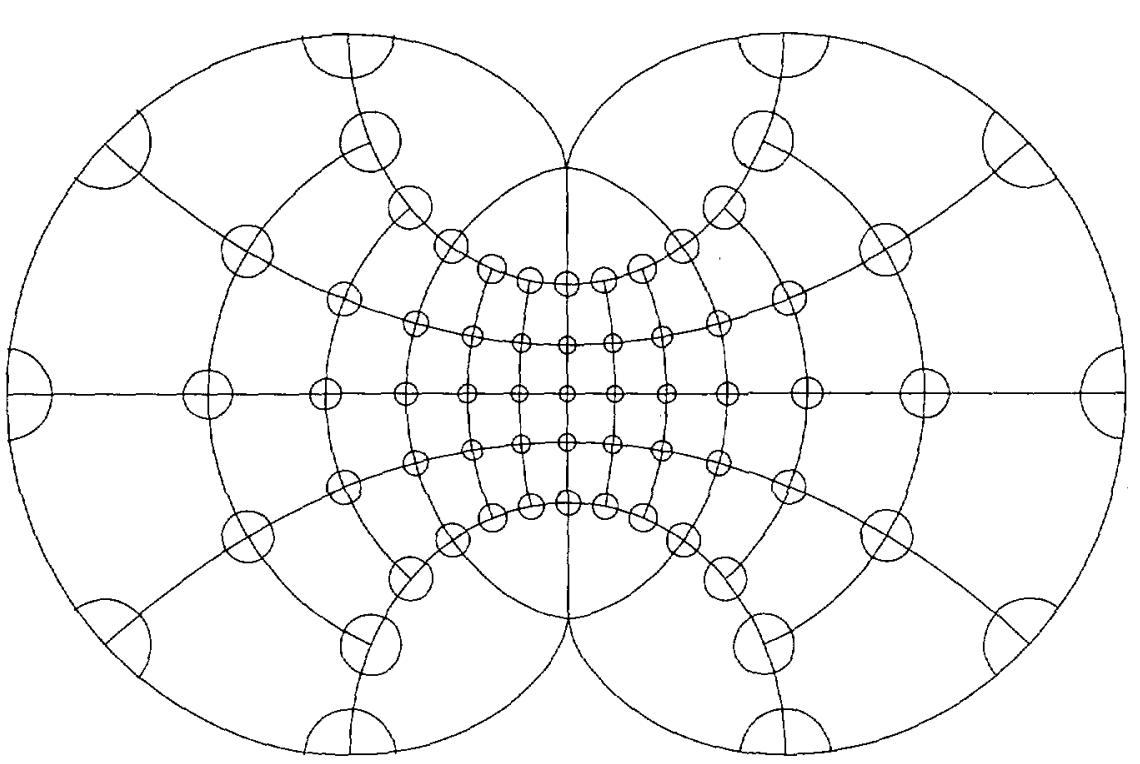

Figure 77A.-Eisenlohr projection, with Tissot indicatrices, $30^{\circ}$ graticule.

\section{Origin}

Presented by Friedrich Eisenlohr of Germany in 1870

\section{Similar projections}

August Epicycloidal (p. 186) has a boundary of varying scale.

Van der Grinten IV (p. 204) is not conformal and has circular arcs for meridians and parallels.

\section{Usage}

Novelty whole-world maps 


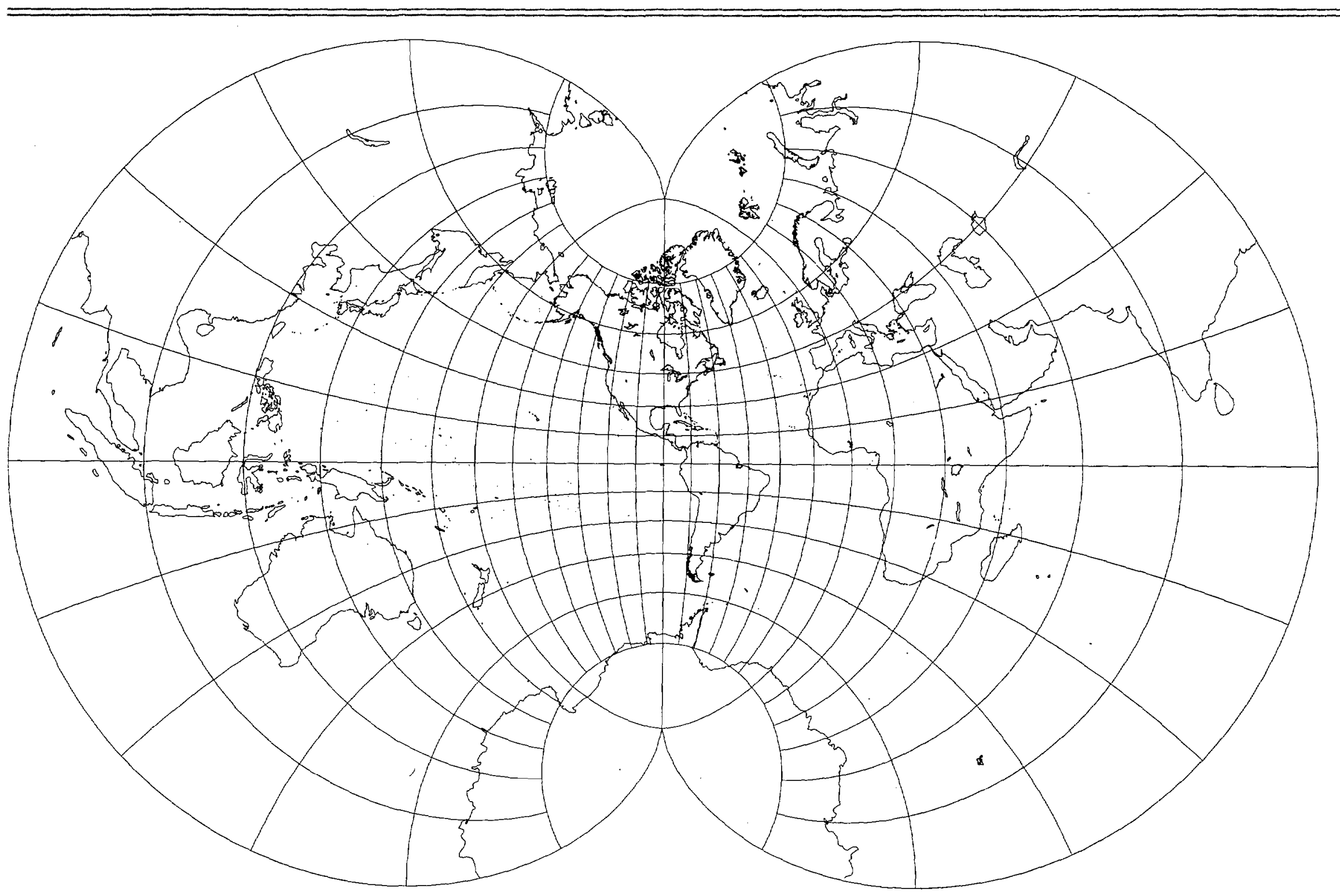




\section{AUGUST EPICYCLOIDAL Projection}

\section{Classification}

Conformal

\section{Graticule}

Meridians: Central meridian is straight. Other meridians are curved and concave toward the central meridian.

Parallels: Equator is straight. Other parallels are curved and concave toward the nearest pole.

Poles: Points

Symmetry: About the central meridian or the Equator

\section{Scale}

Increases rapidly with distance from the center

\section{Distortion}

Great distortion of area toward the 180th meridians in comparison with the center

\section{Other features}

Boundary of the world map is a two-cusped epicycloid. Like the Eisenlohr projection

(p. 184), this projection has no "singular" point at which conformality falls. Unlike the

Eisenlohr, the map boundary has a varying scale.

\section{Usage}

Novelty whole-world maps

\section{Origin}

Presented by F. August and G. Bellermann in Germany in 1874

\section{Aspects}

Transverse oblique aspect used by Athelstan F. Spilhaus in 1942 to show a map of world oceans

\section{Other names}

August

\section{Similar projections}

Eisenlohr (p. 184) has a boundary of constant scale.

Van der Grinten IV (p. 204) is not conformal and has circular arcs for meridians and parallels. 


\section{Figure 78. August Epicycloidal projection, with shorelines, $15^{\circ}$ graticule. Central meridían $90^{\circ} \mathrm{W}$.}

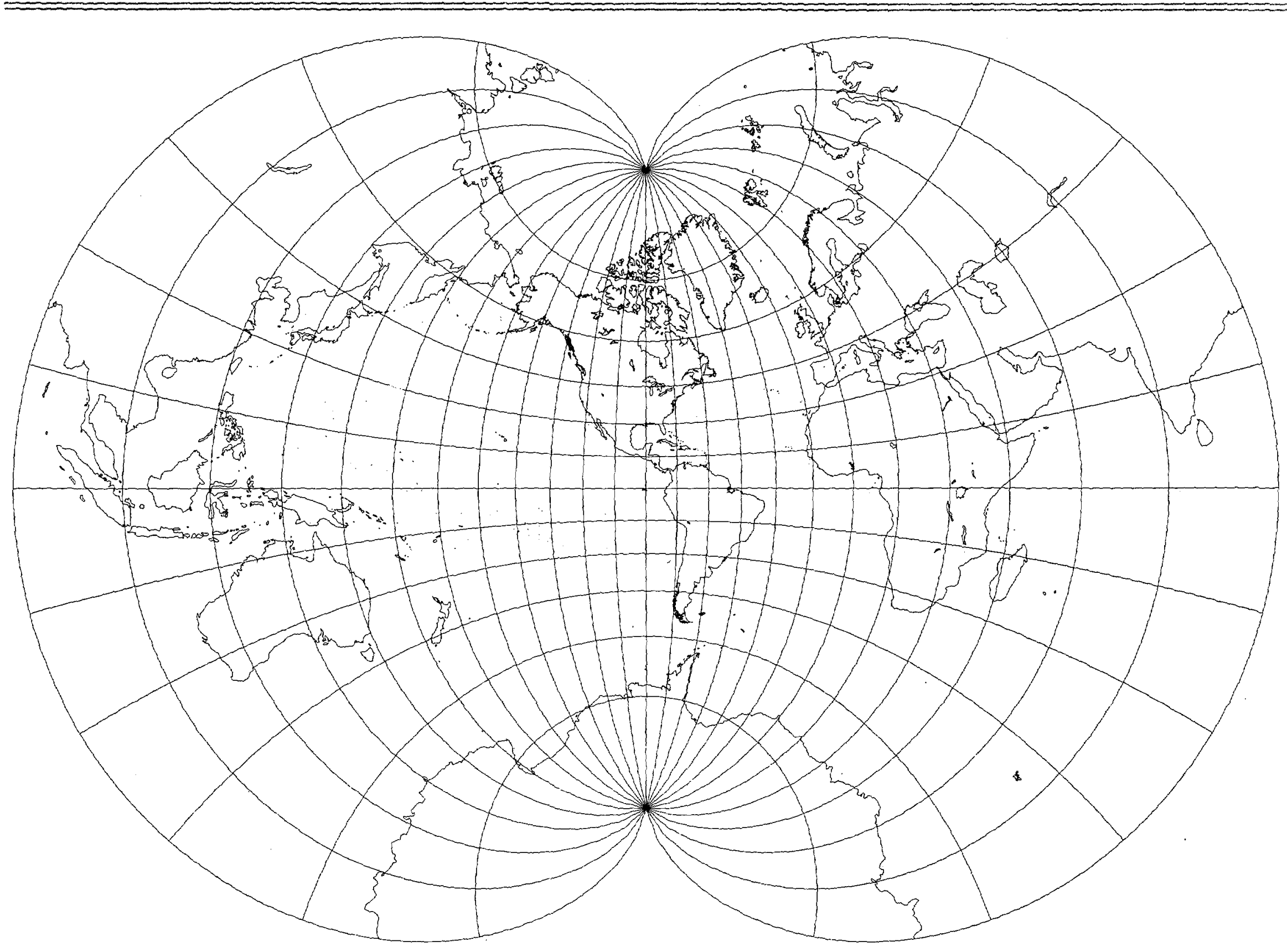




\section{GUYOU Projection}

\begin{tabular}{l|l}
\hline $\begin{array}{l}\text { Classifications } \\
\text { Conformal }\end{array}$ & $\begin{array}{l}\text { 90th meridian at latitudes } 45^{\circ} \mathrm{N} \text {. or S., where } \\
\text { conformality fails }\end{array}$
\end{tabular}

Conformal

\section{Graticule}

Meridians: Central meridian is straight. The 90th meridians are straight but bent at the 45th parallels $N$. and $S$. Other meridians are complex curves.

Parallels: Equator is straight. Other parallels are complex curves.

Poles: Points, in the midpoint of opposite sides of a square bounding a hemisphere Symmetry: About the central meridian or the Equator

\section{Scale}

Varies considerably along each meridian and paralle

\section{Distortion}

Great distortion of area, especially near the

\section{formality fails}

\section{Other features}

Shows hemisphere conformally in a square and the world in a $2 \times 1$ rectangle. Hemispheres can be mosaicked to form a continuous map indefinitely.

\section{Usage}

Novelty map of a hemisphere or of the world

\section{Origin}

Presented by Émile Guyou of France in 1887

\section{Aspects}

A transverse aspect is the Peirce Quincuncial projection (p. 190). An oblique aspect is the Adams projection of a hemisphere in a square (p. 192).

\section{Similar projections}

Listed under Aspects above 
Figure 79.-Guyou projection, with shorelines, $10^{\circ}$ graticule. Central meridian $90^{\circ} \mathrm{W}$.

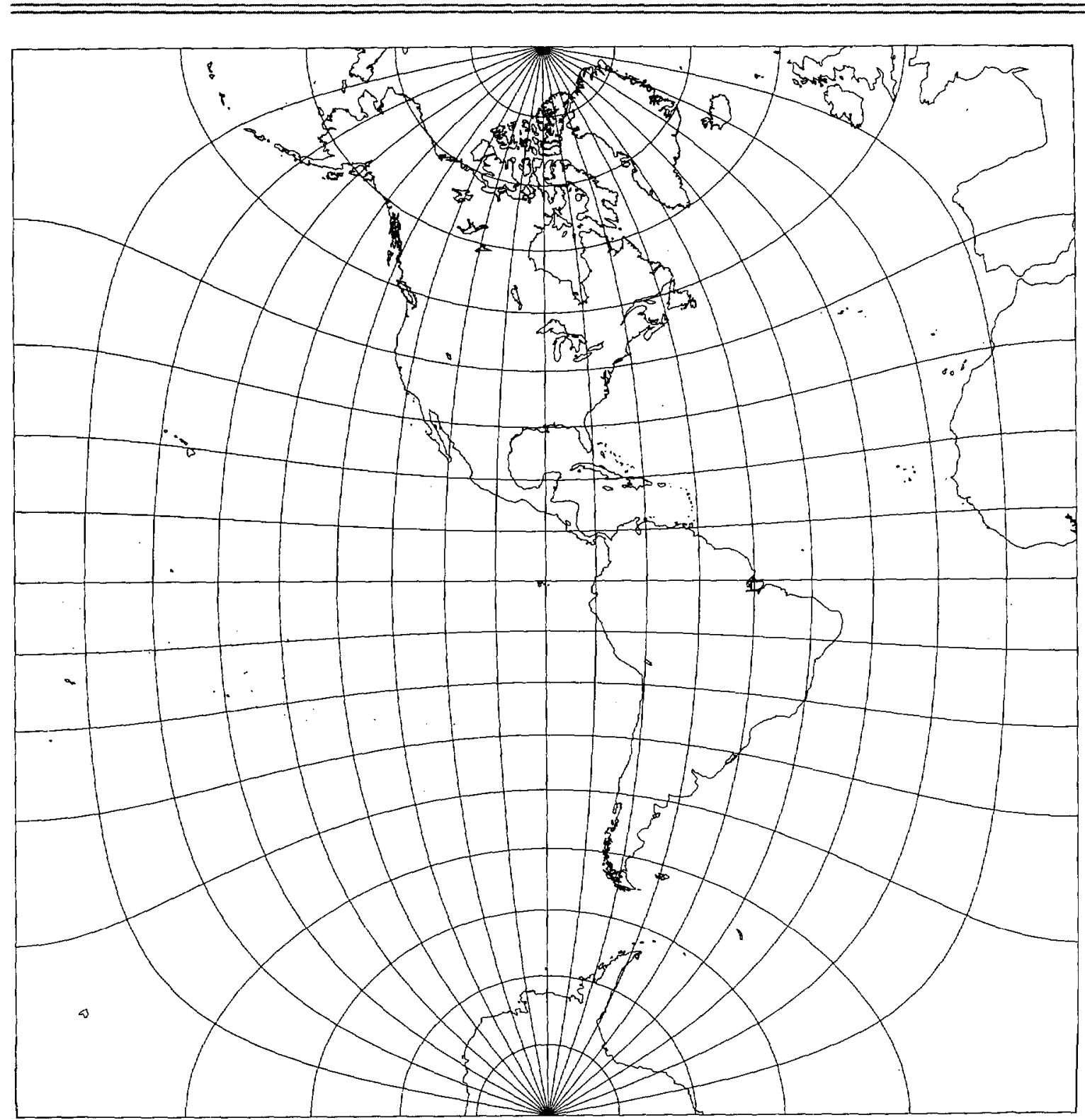




\section{PEIRCE QUINCUNCIAL Projection}

\section{Classification}

Conformal

\section{Graticule}

Meridians: Every 90th meridian is straight

but bent at the Equator. Other meridians are complex curves.

Parallels: Equator is a square, bent at the straight meridians. Other parallels are complex curves.

Poles: Points at the center of each hemispherical map

Symmetry: About the central meridian or the Equator

\section{Scale}

Varies considerably along each meridian and parallel

\section{Distortion}

Great distortion of area, especially near each 90th meridian at the Equator, where conformality fails

\section{Other features}

Actually a "star" projection in the normal aspect. Shows a polar hemisphere conformally in a square and the world in a larger square. The first use of elliptic functions for a map projection.

\section{Usage}

Novelty whole-world maps

\section{Origin}

Presented by Charles Sanders Peirce (18391914) of the U.S. Coast and Geodetic Survey in 1879

\section{Aspects}

In normal form, the pole is at the center of a square. In transverse form, placing the pole

at the midpoint of one side of a square results

in the Guyou projection (p. 188). Placing the

pole at the corner of the square results in the

Adams projection of a hemisphere in a

square (p. 192).

\section{Other names}

Quincuncial; name applied by Peirce, because there are normally five parts within a square

(the Northern Hemisphere is in the center, and four parts of the Southern Hemisphere are in the corners)

\section{Similar projections}

Listed under Aspects above 
Figure 80. Peirce Quincuncial projection, with shorelines, $15^{\circ}$ graticule. Central meridian $105^{\circ} \mathrm{W}$.

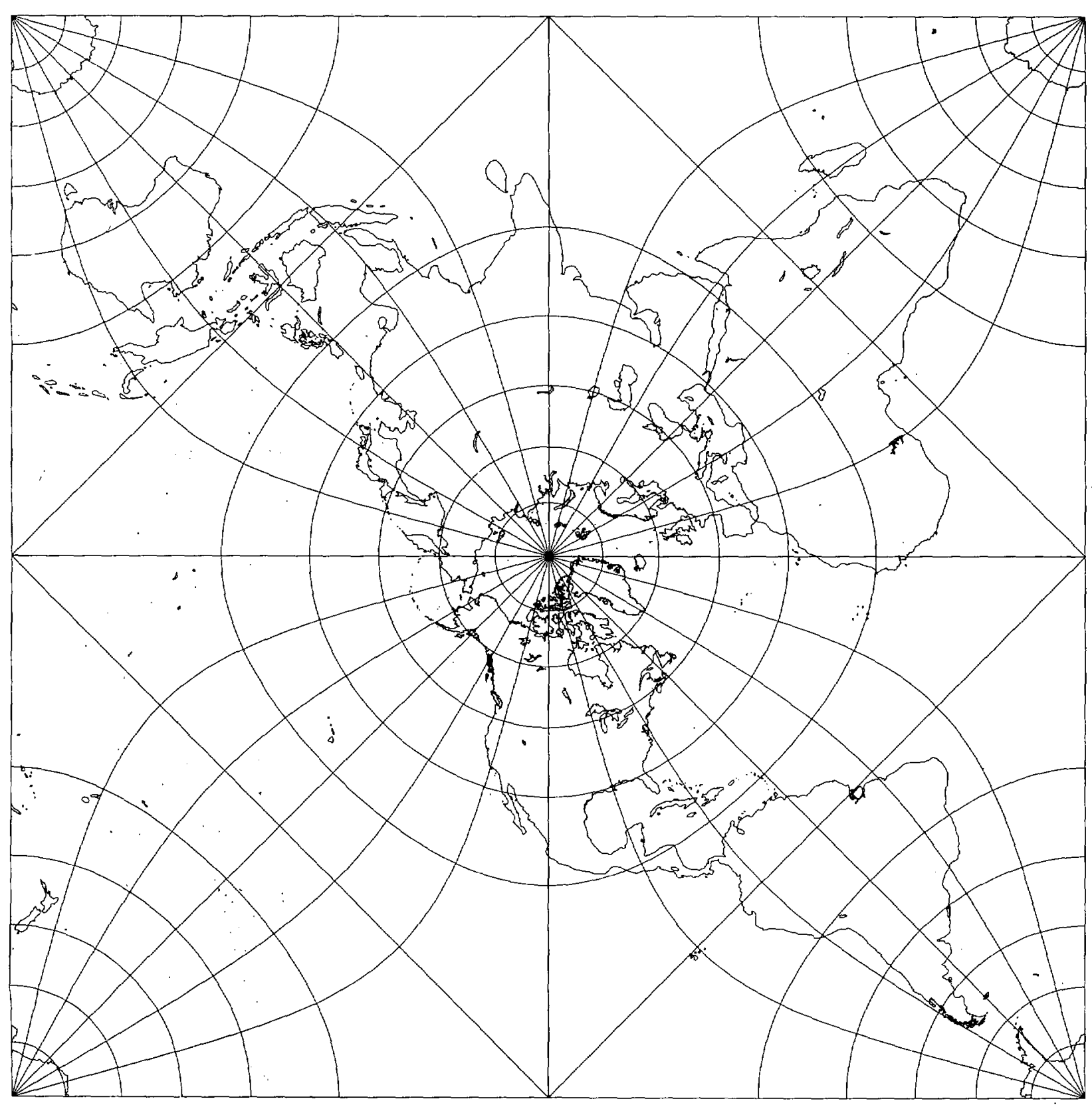




\section{ADAMS Projection of a Hemisphere in a Square}

\section{Classification}

Conformal

\section{Graticule}

Meridians: Central meridian is straight. The 90th meridians are straight lines bent at the

Equator. Other meridians are curved.

Parallels: Equator is straight. Other parallels are curved.

Poles: Points, in opposite vertices of a square Symmetry: About the central meridian or the Equator

\section{Scale}

Varies along each meridian and each parallel

\section{Distortion}

Great distortion of area at the poles or the intersection of the 90th meridians with the Equator. At these four points, conformality fails.

\section{Other features}

Hemisphere is enciosed in a square. World can be enclosed in a $2 \times 1$ rectangle.

\section{Usage}

Novelty map of a hemisphere

\section{Origin}

Presented by Oscar Sherman Adams (18741962) of the U.S. Coast and Geodetic Survey in 1925

\section{Aspects}

One transverse aspect is the Peirce Quincuncial projection (p. 190). Another transverse aspect is a rotation of the original projection. An oblique aspect is the Guyou projection (p. 188).

\section{Similar projections}

Listed under Aspects above 
Figure 81.-Adams projection of a hemisphere in a square, with shorelines, $10^{\circ}$ graticule. Central meridian $90^{\circ} \mathrm{W}$.

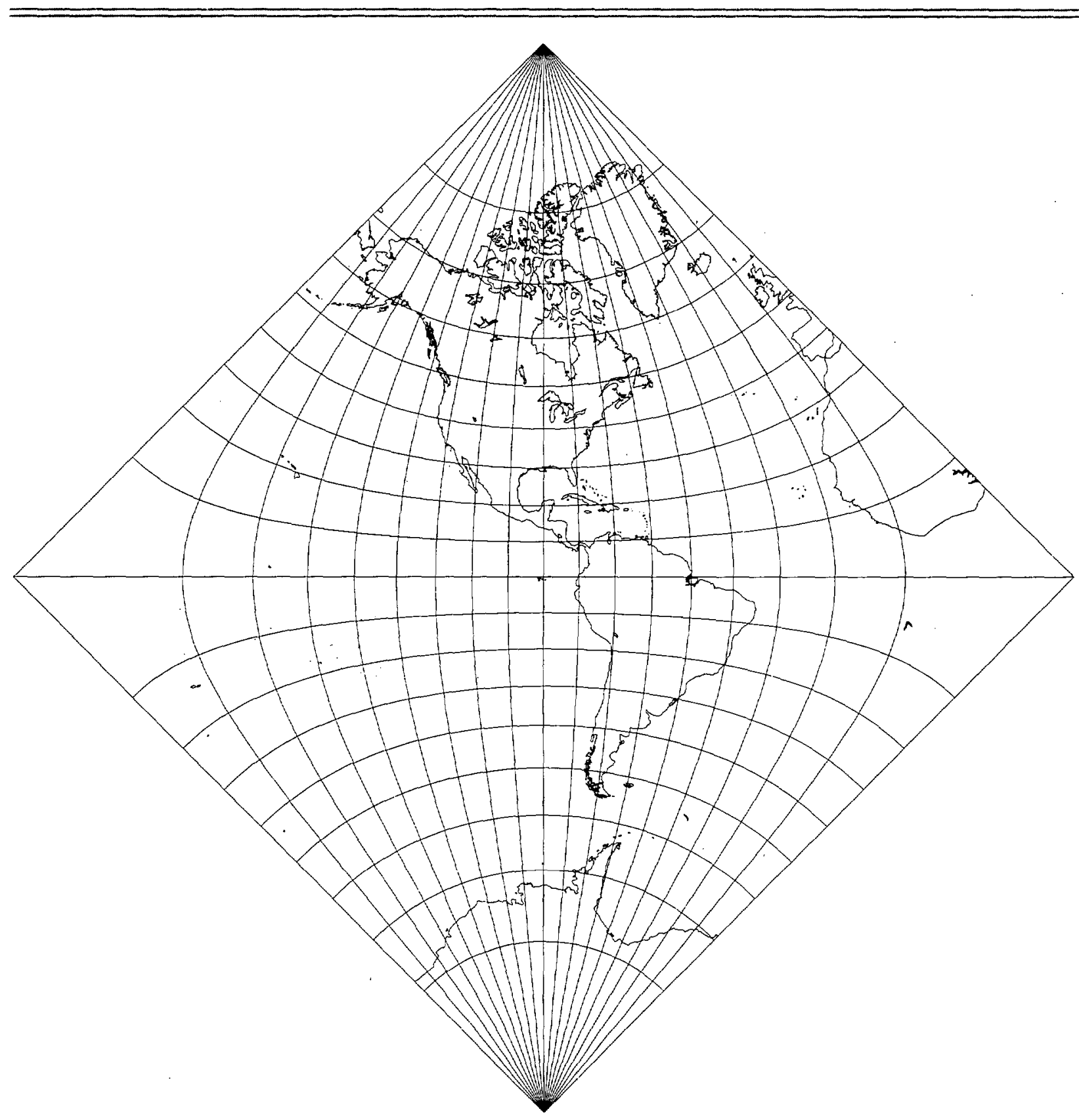




\section{ADAMS Projection of the World in a Square I}

\section{Classification}

Conforma!

\section{Graticule}

Meridians: Central meridian is straight. The 180th meridians are straight lines bent at the latitudes $70^{\circ} 31^{\prime} \mathrm{N}$. and S. Other meridians are complex curves.

Parallels: Equator is straight. Other parallels are complex curves.

Poles: Points in midpoints of opposite sides Symmetry: About the central meridian or the Equator

\section{Scale}

Varies along each meridian and each parallel

\section{Distortion}

Great area distortion at the higher latitudes. Conformality fails at the poles and at two points on each of the 180th meridians.

\section{Usage}

Novel whole-world maps

\section{Origin}

Presented by Oscar Sherman Adams (18741962) of the U.S. Coast and Geodetic Survey in 1925.

\section{Similar projections}

A general formula for representing the whole sphere or a segment of the sphere conformally in a regular polygon of three or more sides can be used to create this map and other conformal maps.

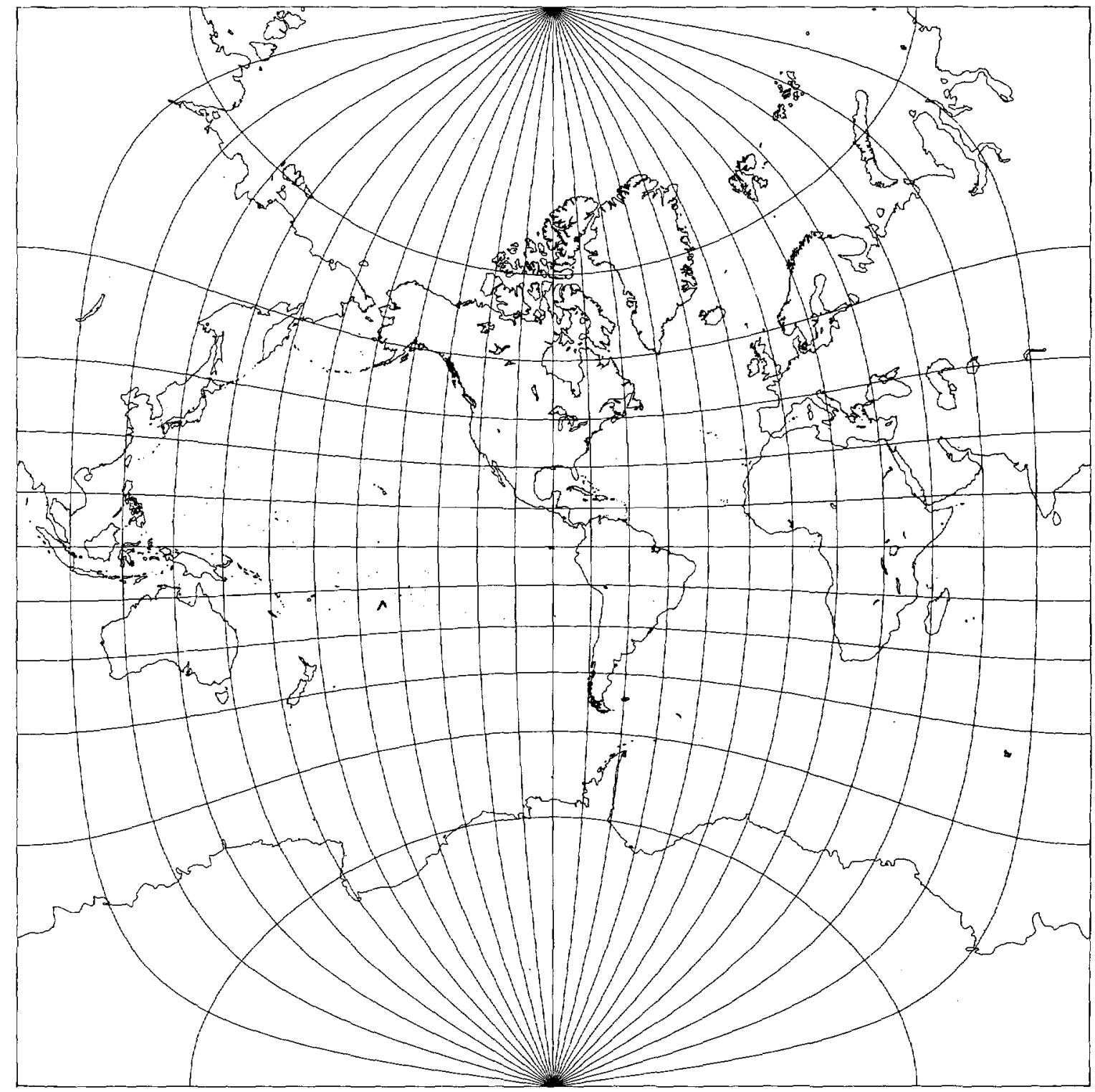

Figure 82. - Adams projection of the world in a square $\mathrm{l}$, with shorelines, $15^{\circ}$ graticule. Central meridian $90^{\circ} \mathrm{W}$. 


\section{ADAMS Projection of the World in a Square II}

\section{Classification}

Conformal

\section{Graticule}

Meridians: Central meridian is straight. The 180th meridians are straight lines bent at the Equator. Other meridians are complex curves.

Parallels: Equator is straight. Other parallels are complex curves.

Poles: Points in opposite vertices

Symmetry: About the central meridian or the Equator

\section{Distortion}

Great area distortion near the 180th meridians or at higher latitudes. Conformality fails at the poles and at the intersections of the 180th meridians with the Equator.

\section{Usage}

Novel whole-worid maps

\section{Origin}

Presented by Oscar Sherman Adams (1874 1962) of the U.S. Coast and Geodetic Survey in 1925

\section{Similar projections}

See note under Adams projection of the world in a square I (p. 194).

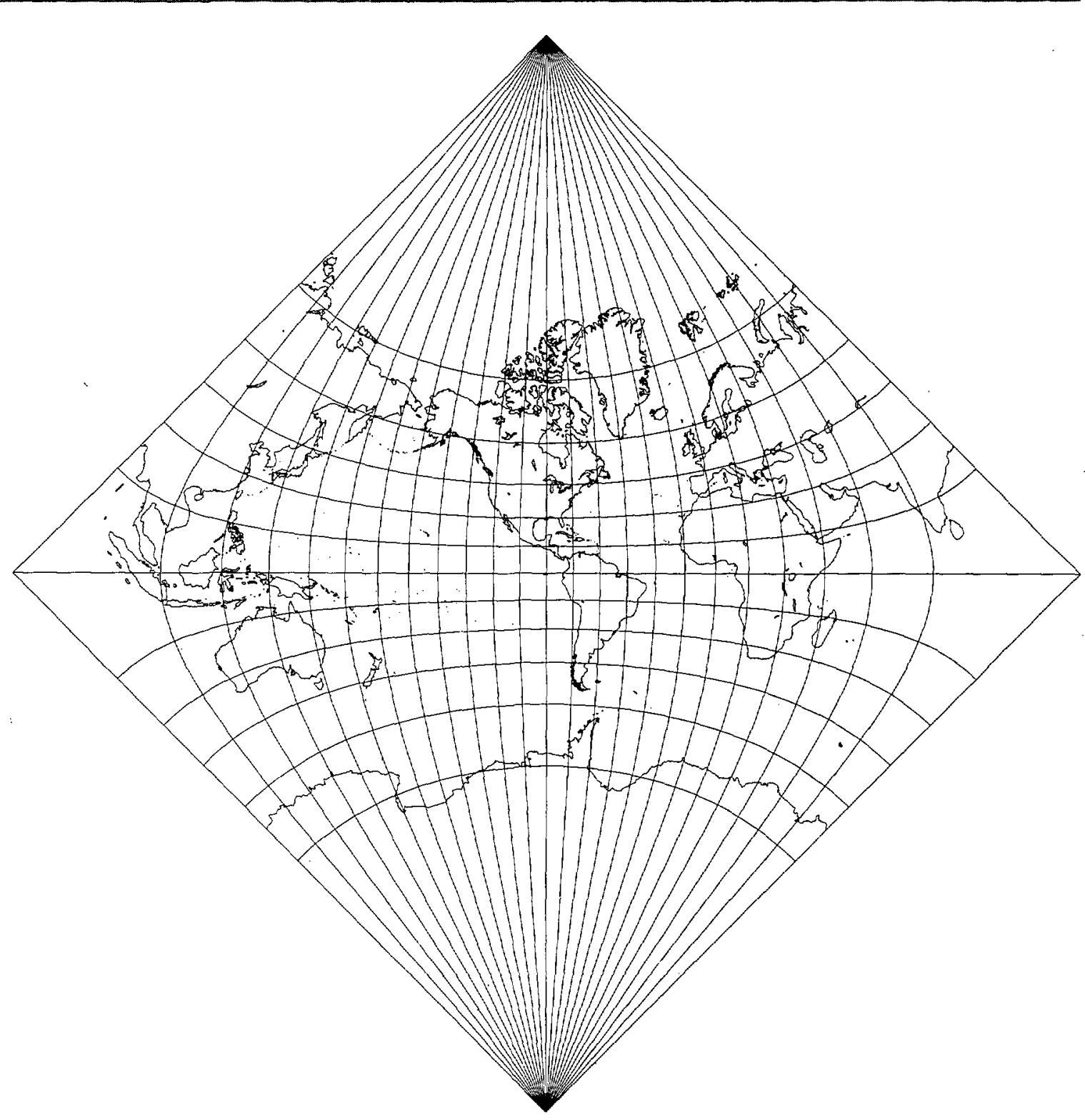

Figure 83.-Adams projection of the world in a square 11 , with shorelines, $15^{\circ}$ graticule. Central meridian $90^{\circ} \mathrm{W}$. 


\section{LEE Conformal Projection of the World in a Triangle}

\section{Classification}

Conformal

\section{Graticule}

Meridians: Central meridian is straight. The 180th meridians are straight lines bent at a southern latitude. Others are complex curves. Parallels: Complex curves.

Poles: Points, one in a vertex and the other centered on the opposite side of the triangle Symmetry: About the central meridian

\section{Scale}

Varies along each meridian and parallel

\section{Distortion}

Great area distortion near the poles and 180th meridians. Conformality fails at each pole and at one point on each of the 180th meridians.

\section{Usage}

Novel whole-world maps

\section{Origin}

Laurence Patrick Lee (1913-85) of New Zealand in 1965

\section{Similar projections}

See note under Adams projection of the world in a square I (p. 194).

Bernard J.S. Cahill, beginning in 1912, arranged conformal or nonconformal three-sided octants of the world, bounded by the Equator and two meridians $90^{\circ}$ apart, in a butterfly arrangement, called the "Butterfly Map." He promoted these interrupted arrangements for weather

maps. 


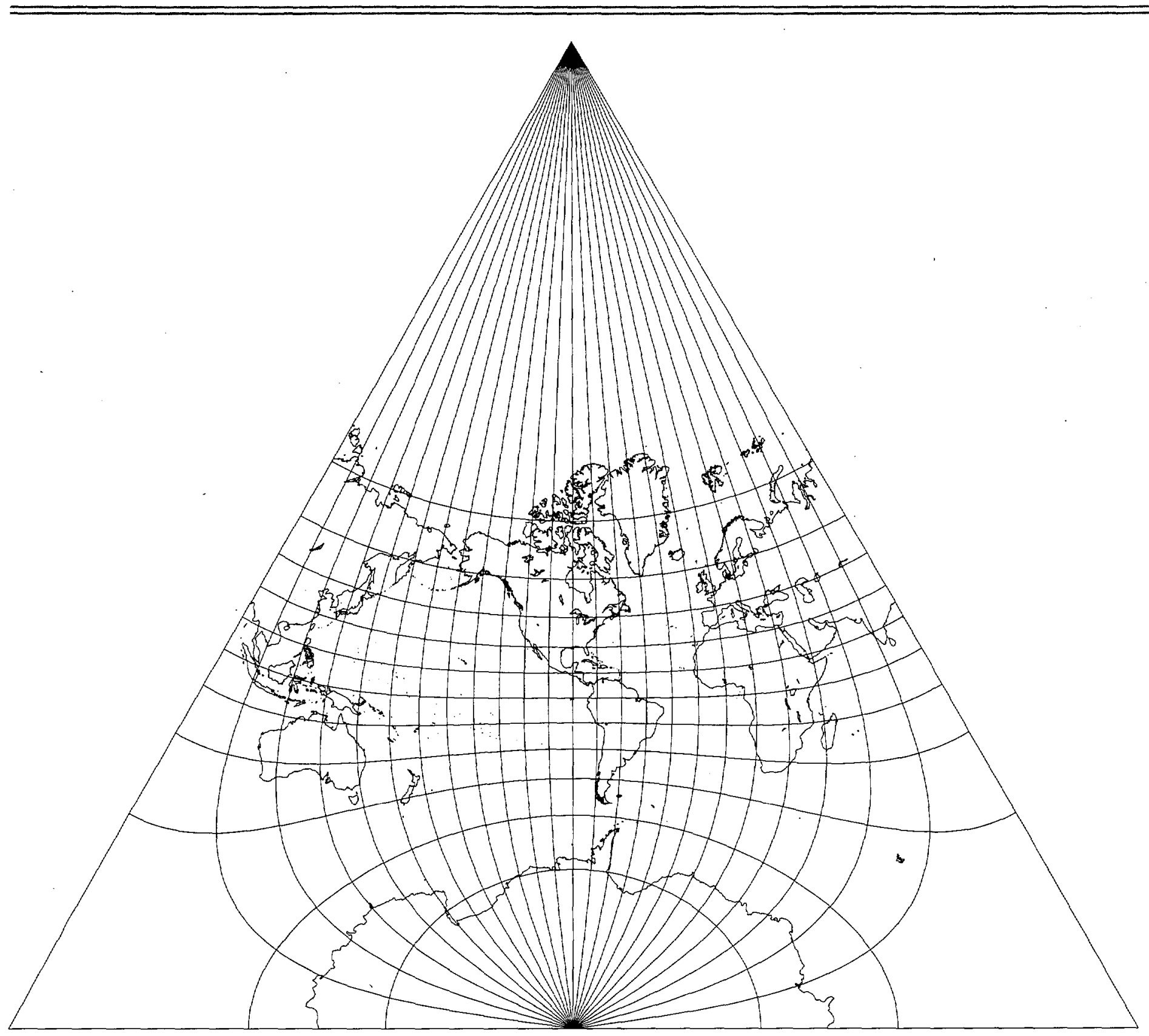




\section{Conformal Projection of the World in an Ellipse}

Classification
Conformal
Graticule
Meridians: Central meridian is straight. Other
meridians are complex curves.

Parallels: Equator is straight. Other parallels are complex curves.

Poles: Points

Symmetry: About the central meridian and the Equator

\section{Scale}

Increases rapidly with distance from the center

\section{Distortion}

Great distortion of area near the poles and the 180th meridians. Conformality fails at the poles.

\section{Other features}

The shape of the bounding ellipse can be varied. Lee uses an axial ratio of about 1.97 to 1 for convenience in using tabular values.

\section{Usage}

Novel whole-world maps

\section{Origin}

Presented by Oscar Sherman Adams (1874 1962) of the U.S. Coast and Geodetic Survey in 1925. Corrected by André Gougenheim in 1950 and Laurence Patrick Lee (1913-85) of New Zealand in 1965.

\section{Limiting form}

As the modular angle that controls the shape of the ellipse approaches $90^{\circ}$, the map increasingly resembles the Lagrange circular projection (p. 181).

\section{Similar projections}

See note under Adams projection of the world in a square 1 (p. 194). 
Figure 85. - Conformal projection of the world in an ellipse, with shorelines, $15^{\circ}$ graticule. Central meridian $90^{\circ} \mathrm{W}$.

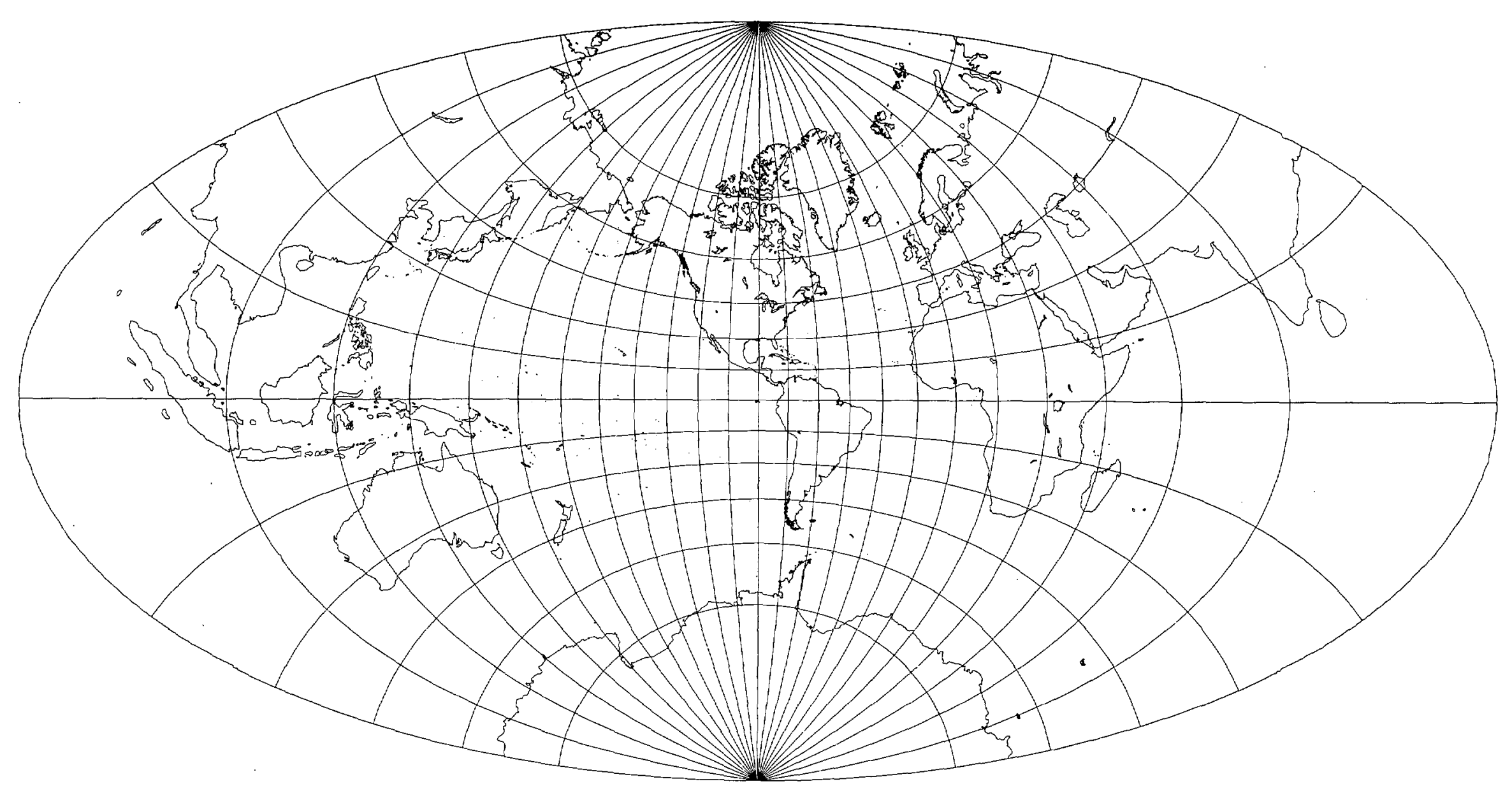




\section{VAN DER GRINTEN I Projection}

\section{Classifications}

\section{Polyconic}

Neither conformal nor equal area

\section{Graticule}

Meridians: Central meridian is straight. Other meridians are circular, equally spaced along the Equator and concave toward the central meridian.

Parallels: Equator is straight. Other parallels are circular arcs, concave toward the nearest pole.

Poles: Points

Symmetry: Along the central meridian or the Equator

\section{Scale}

True along the Equator. Increases rapidly with distance from the Equator.

\section{Distortion}

Great distortion of area near the poles (fig. 86B)

\section{Other features}

The world is enclosed in a circle.

\section{Usage}

World maps by the U.S. Department of Agriculture, the U.S. Geological Survey, the National Geographic Society, and others

\section{Origin}

Presented by Alphons J. van der Grinten (1852-1921) of Chicago in 1898. U.S. patent obtained in 1904.

\section{Other names}

Van der Grinten (without I)

\section{Similar projections}

Lagrange (p. 181) is conformal.

Van der Grinten II (p. 202) has parallels curved to intersect meridians at right angles.

Mercator (p. 10) is conformal and has straight meridians and parallels.

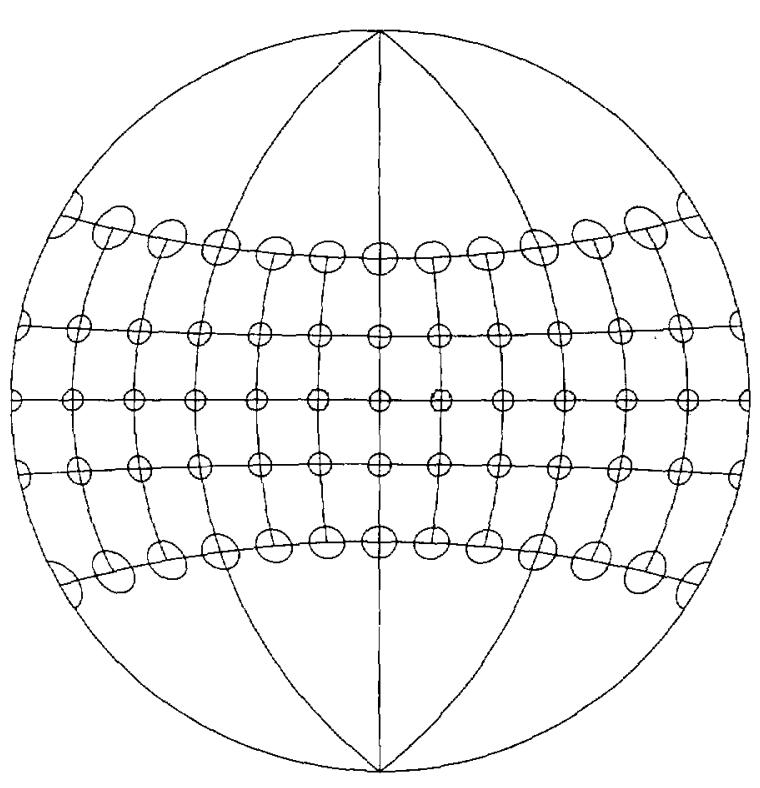




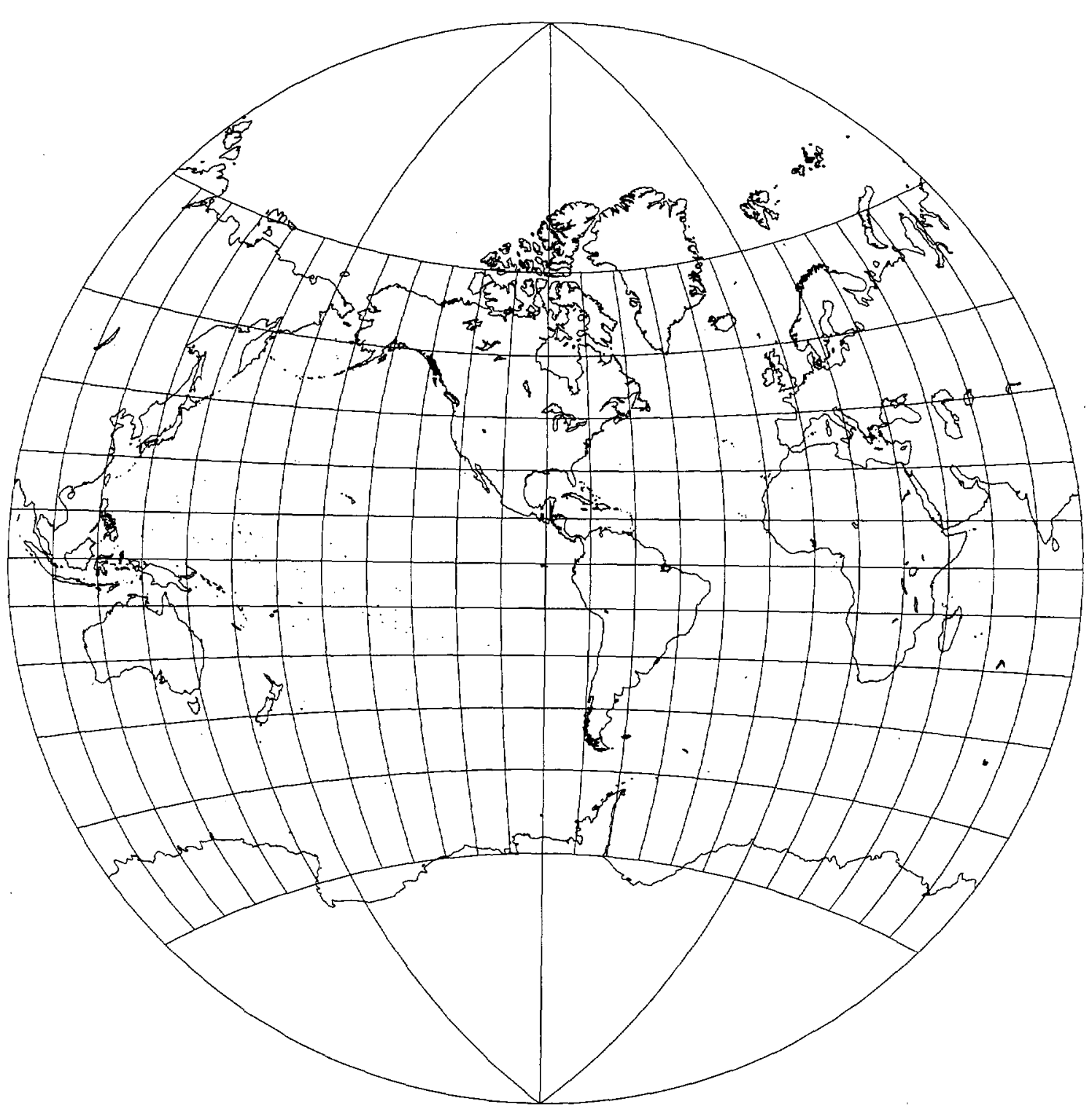




\section{VAN DER GRINTEN II Projection}

\section{Classifications}

Polyconic

Neither conformal nor equal area

\section{Graticule}

Meridians: Central meridian is straight. Other meridians are circular, equally spaced along the Equator and concave toward the central meridian.

Parallels: Equator is straight. Other parallels are circular arcs, concave toward the nearest pole.

Poles: Points

Symmetry: About the central meridian or the Equator

\section{Scale}

True on the Equator. Increases rapidly with distance from the Equator.

\section{Distortion}

Great distortion of area near the poles

\section{Other features}

The world is enclosed in a circle.

All meridians intersect parallels at right angles, but projection is not conformal. Spacing of parallels along the central meridian is the same as that for Van der Grinten I.

\section{Usage}

World maps, but rarely

\section{Origin}

Presented by Alois Bludau in 1912 as a modification of Van der Grinten I

\section{Similar projections}

Lagrange (p. 181) is conformal.

Van der Grinten I (p. 200) has parallels that do not intersect meridians at right angles.

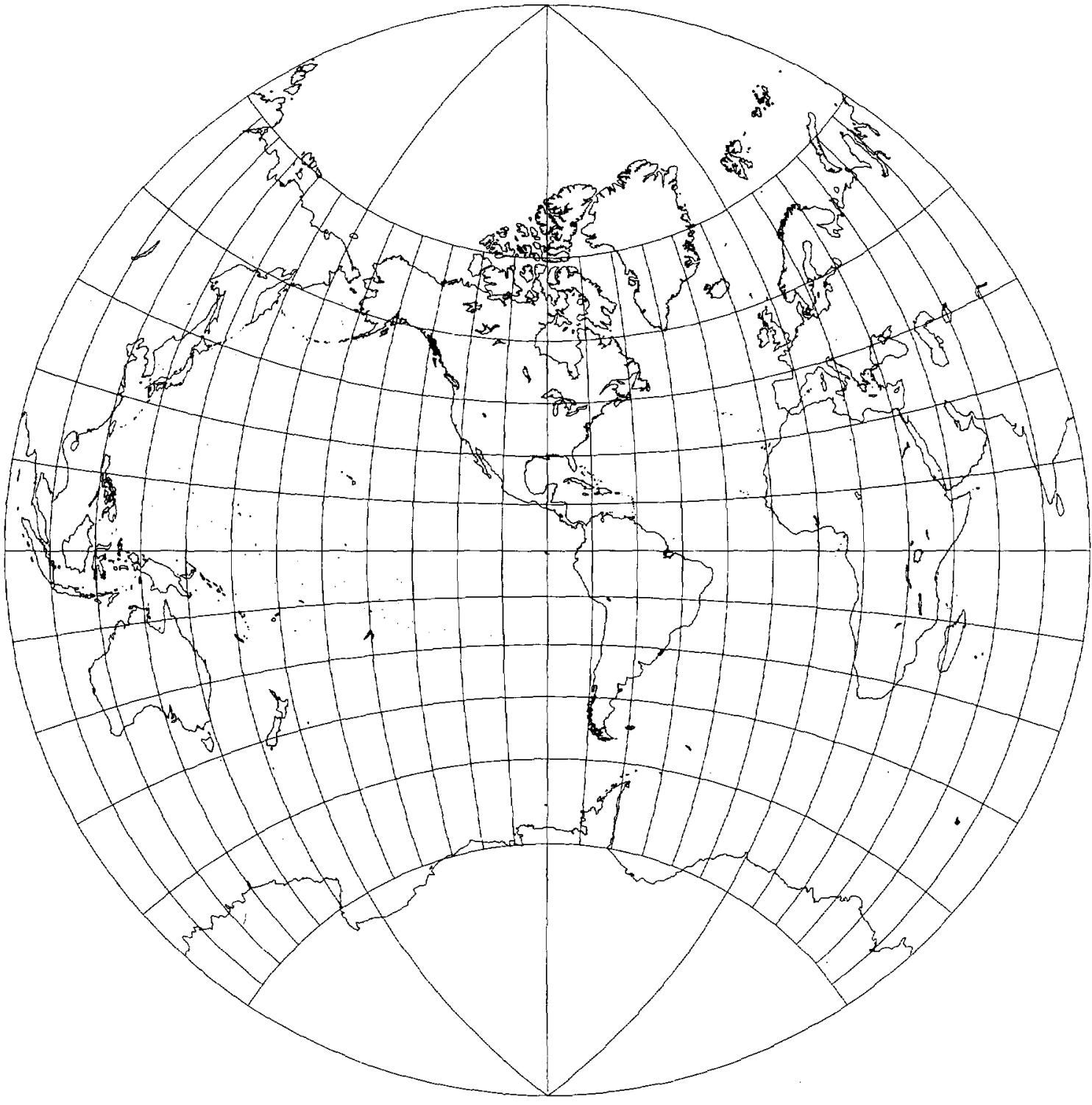

Figure 87.-Van der Grinten II projection, with shorelines, $15^{\circ}$ graticule, central meridian $90^{\circ} \mathrm{W}$ 


\section{VAN DER GRINTEN III Projection}

\section{Classification}

Modified pseudocylindrical

Neither conformal nor equal area

\section{Graticule}

Meridians: Central meridian is straight. Other meridians are circular, equally spaced along the Equator but unequally spaced along other parallels and concave toward the central meridian.

Parallels: Straight lines

Poles: Points

Symmetry: About the central meridian or the Equator

\section{Scale}

True along the Equator. Increases rapidly with distance from the Equator.

\section{Distortion}

Great distortion of area near the poles

\section{Other features}

The world is enclosed in a circle.

Spacing of parallels along the central meridian is the same as that for Van der Grinten I.

\section{Usage}

World maps, but rarely

\section{Origin}

Presented by Alois Bludau in 1912 as a modification of Van der Grinten I

\section{Similar projections}

Van der Grinten I (p. 200) has curved parallels.

"The Times," by Moir, has sinusoidal meridians, but straight parallels are spaced like those of the Gall projection (p. 33).

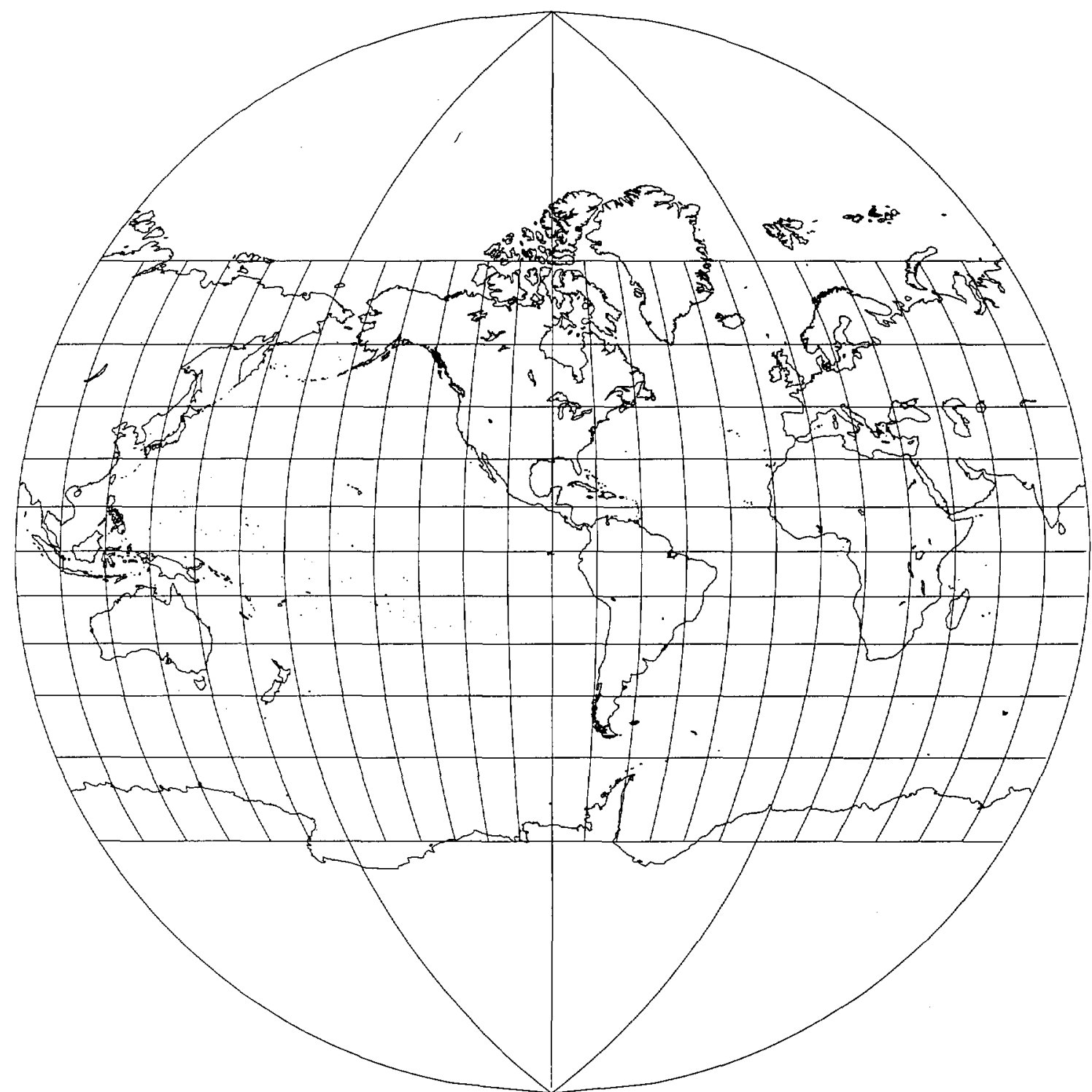

Figure 88.-Van der Grinten III projection, with shorelines, $15^{\circ}$ graticule, central meridian $90^{\circ} \mathrm{W}$. 


\section{VAN DER GRINTEN IV Projection}

\section{Classification}

Polyconic

\section{Globular}

Neither conformal nor equal area

\section{Graticule}

Meridians: Central meridian is straight. Other meridians are circular, equally spaced along the Equator and concave toward the central meridian.

Parallels: Equator is straight. Other parallels are circular, equally spaced on the central meridian and concave toward the nearest pole.

Poles: Points

Symmetry: About the central meridian or the Equator

\section{Scale}

True along the central meridian and the Equator

\section{Distortion}

Great distortion of area near the 180th

meridians at higher latitudes

\section{Other features}

The 90th meridians form a complete circle.

\section{Usage}

World maps, but rarely

\section{Origin}

Presented by Alphons J. van der Grinten

(1852-1921) of Chicago in 1904

\section{Other names}

"Apple Shaped"

\section{Similar projections}

Eisenlohr (p. 184) is conformal.

August Epicycloidal (p. 186) is conformal. 


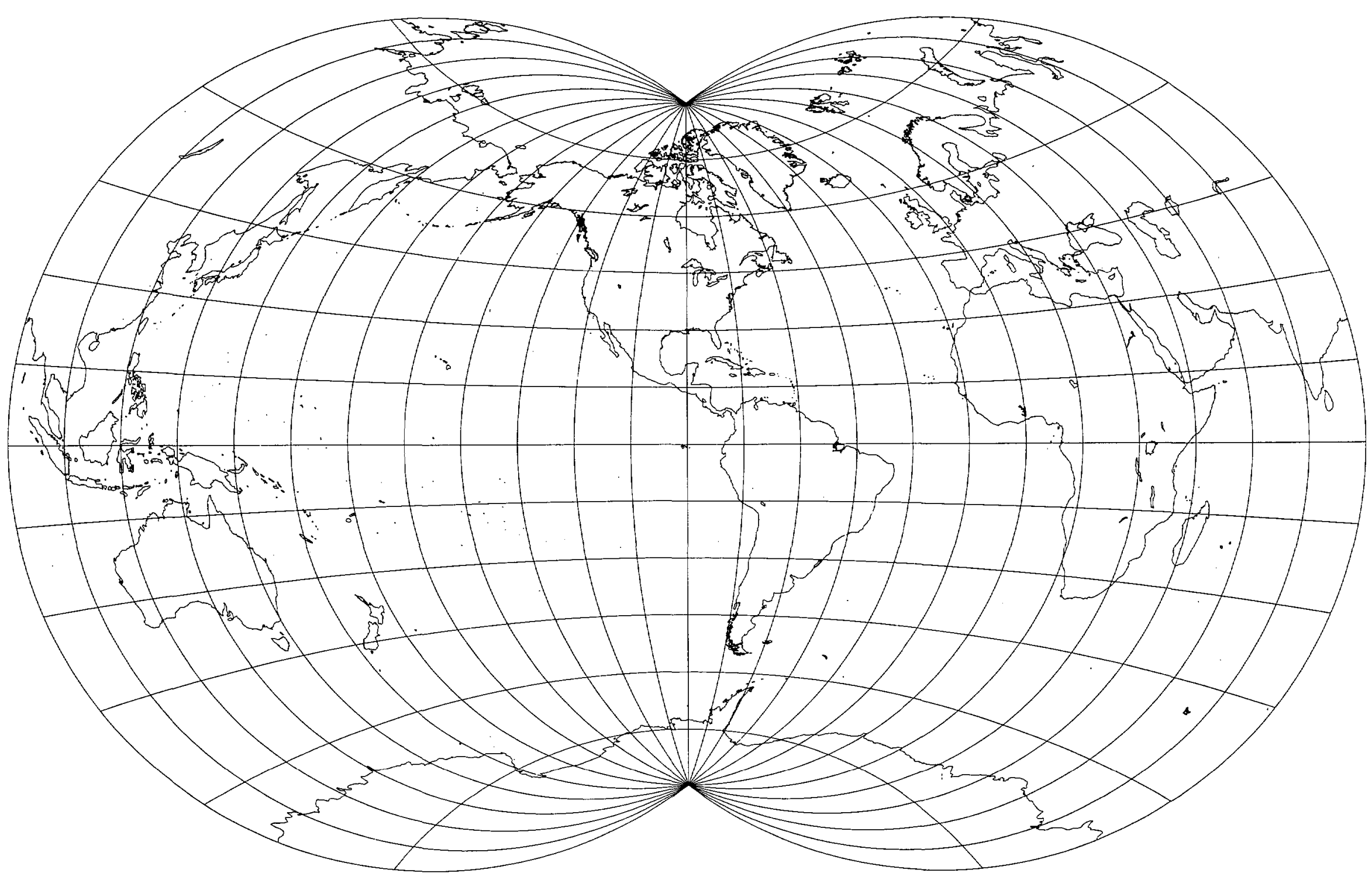




\section{ARMADILLO Projection}

\section{Classifications}

Orthoapsidal (term coined by originator)

Neither conformal nor equal area

\section{Graticule}

Meridians: Central meridian ( $10^{\circ}$ or $15^{\circ} \mathrm{E}$.) is straight. Other meridians are elliptical arcs, concave toward the central meridian.

Parallels: Elliptical arcs of the same eccentricity, concave toward the North Pole Poles: North Pole is semiellipse. South Pole cannot be shown.

Symmetry: About the central meridian

\section{Scale}

Gradually decreases with distance from the center

\section{Distortion}

Distortion is moderate in central portions.

\section{Other features}

An oblique orthographic projection of the world plotted with equidistant meridians and parallels onto a portion of a torus ring (similar to a doughnut). Antarctic region cannot be shown, but the projection was claimed to have "more land in proportion to sea than any other world map." Often plotted with New Zealand, normally hidden from view, appended to Australia as a "pigtail."

\section{Usage}

Whole-world maps

\section{Origin}

Presented by Erwin J. Raisz (1893-1968) of Harvard University in 1943

\section{Aspects}

Oblique is the basic aspect.

\section{Other names}

Raisz

\section{Similar projections}

Other "orthoapsidal" projections proposed by

Raisz in 1943. Raisz coined this term from

"orthographic" and "apsidal." 


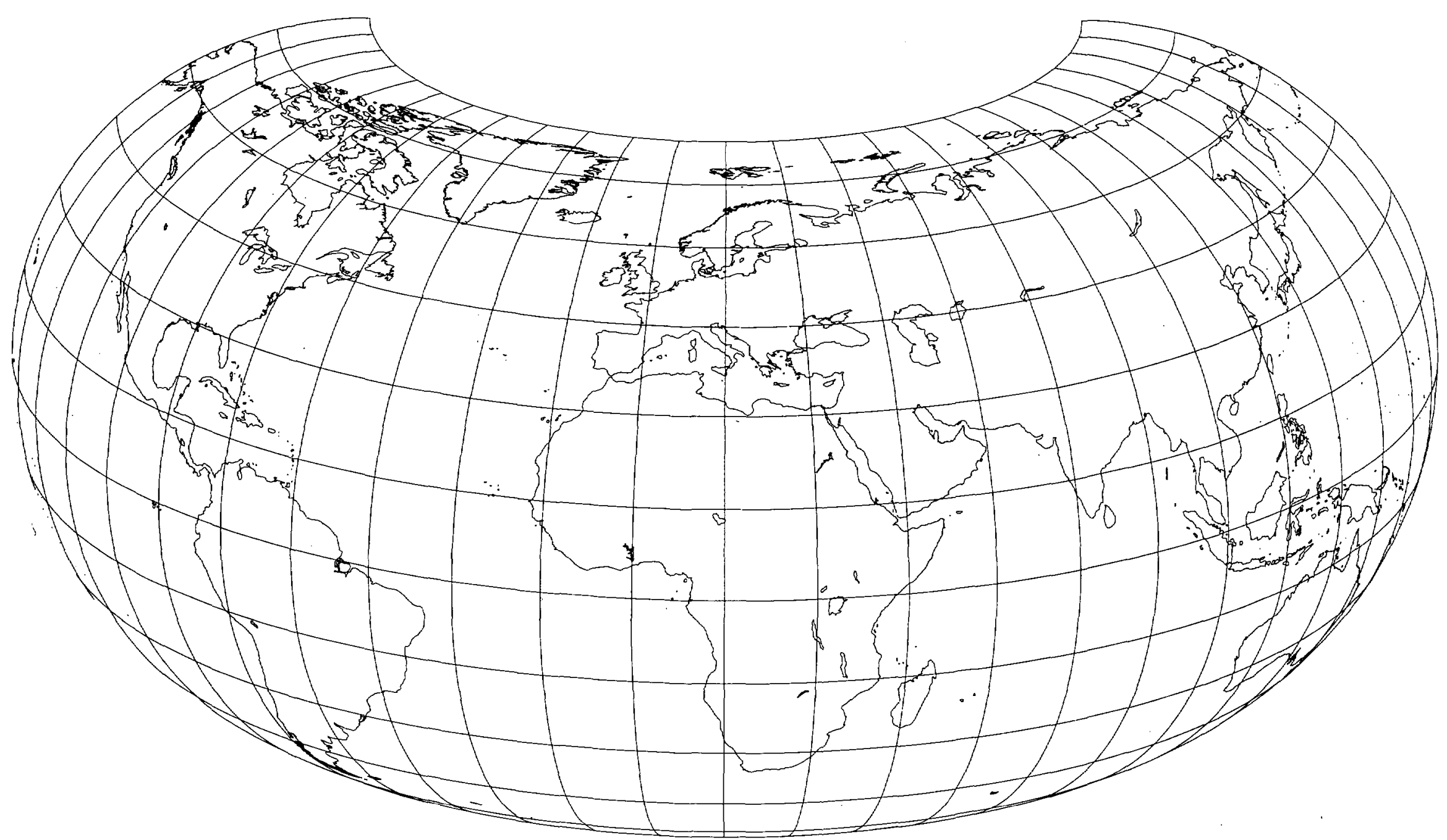




\section{GS50 Projection}

\section{Classification}

Conformal

\section{Graticule}

Meridians: Complex curves at slightly varying intervals

Parallels: Complex curves at slightly varying intervals

Poles: Points beyond the useful range of the map

Symmetry: None

\section{Scale}

True along an irregular line approximately encompassing the regions of the 50 States of the United States

Varies less than 2 percent from true scale throughout the 50 States and adjacent bodies, of water

\section{Distortion}

Shape and scale distortion very low for 50-State region (fig. 91B). Greater distortion for regions away from 50 States.

\section{Special features}

Portrays irregular region of 50 States at about one-fourth the variation in scale of the best standard projections. Uses tenth-order complex-algebra polynomials to modify the Stereographic projection and is practical only with a computer. Conformality is precise in these cases even though finlte polynomial series are used. The coefficients are useful only for this design of a 50-State map.

\section{Origin}

Developed by John P. Snyder (1926- ) of the U.S. Geological Survey in 1982

\section{Similar projections}

Oblated (or Prolated) Stereographic projections by O.M. Miller in 1953 (p. 148) and by L.P. Lee in 1974 are equivalent to third-order complex-algebra polynomial modifications of the oblique Stereographic, applied to continents or the Pacific Ocean.

New Zealand Map Grid presented by W.I.

Reilly in 1973 is a sixth-order complex-algebra polynomial modification of the Mercator, applied to New Zealand.

Modified-Stereographic Conformal projection developed by Snyder in 1983 is a sixth-order complex-algebra modification of the oblique Stereographic, applied to Alaska. 


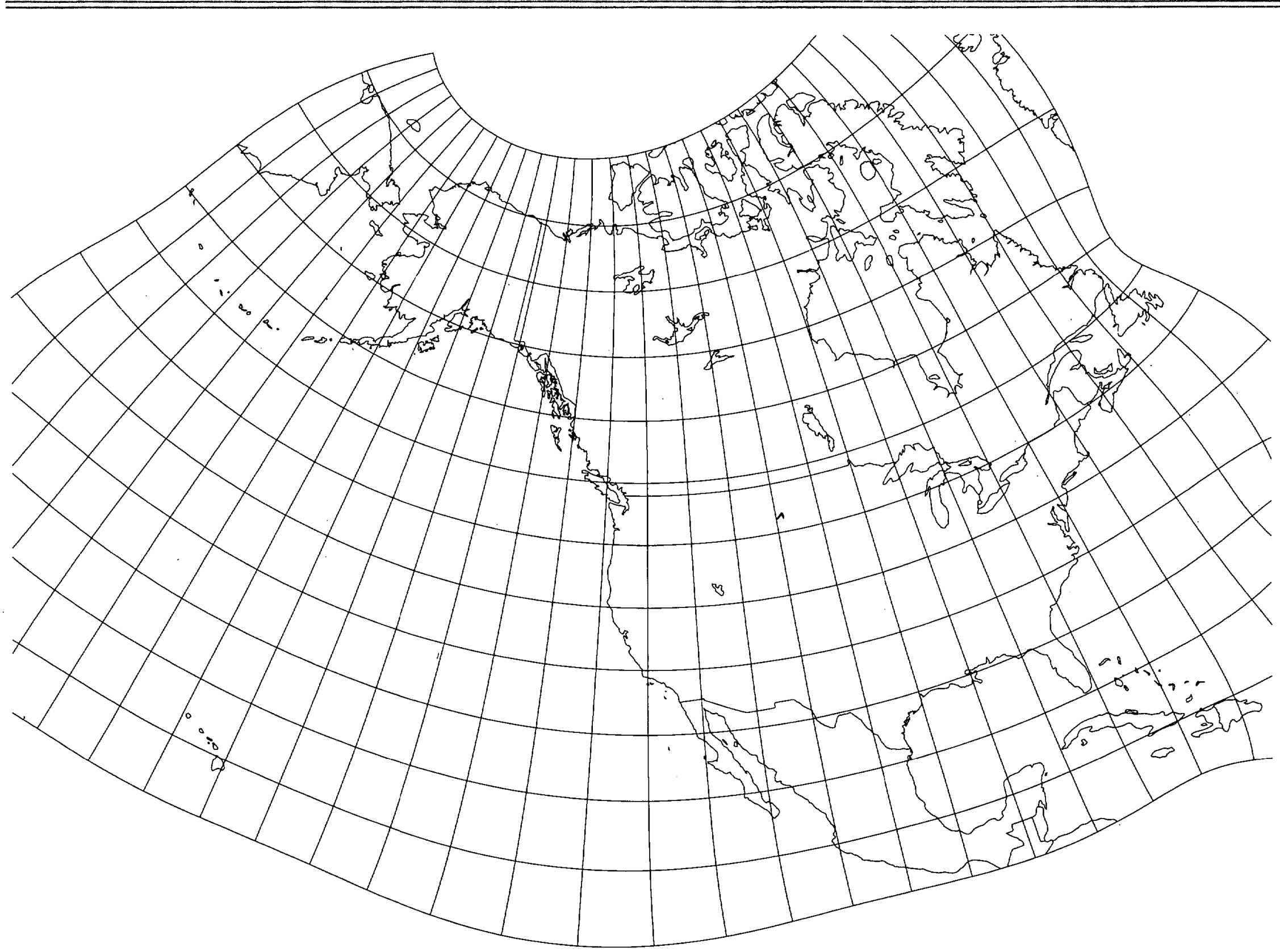


Figure $91 B .-$ GS50 projection, with lines of constant scale.

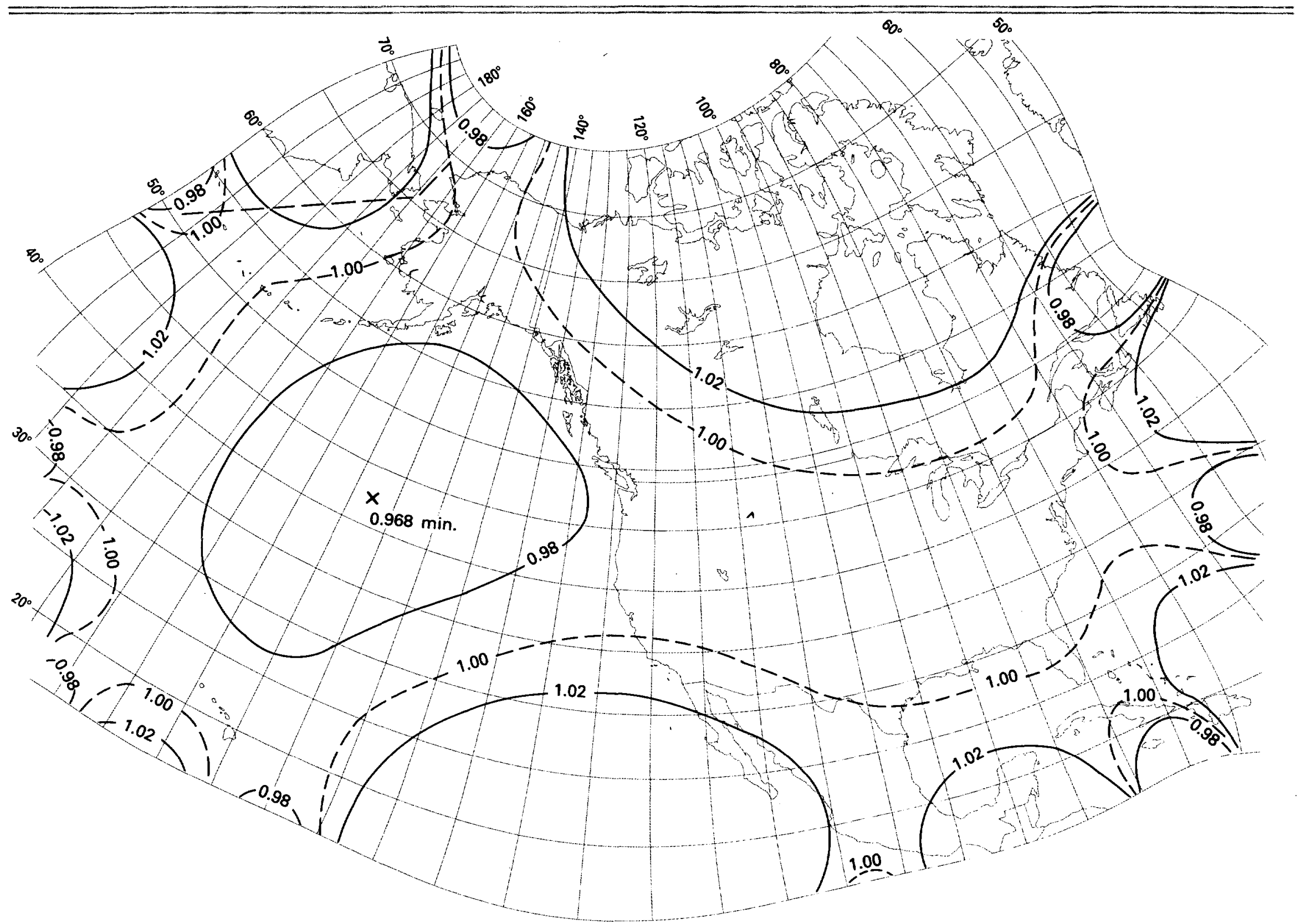




\section{SPACE OBLIQUE MERCATOR Projection}

\section{Classifications}

Modified cylindrical

Basically conformal

\section{Graticule}

Meridians: Complex curves at slightly varying intervals

Parallels: Complex curves at slightly varying intervals

Poles: Points

Symmetry: Inverse symmetry about each node (crossing of the Equator by satellite groundtrack)

\section{Scale}

True along the groundtrack

Varies about 0.01 percent within the normal

sensing range of the satellite

\section{Distortion}

No distortion along the groundtrack Distortion is essentially constant along lines

of constant distance parallel to the groundtrack.

Conformality is correct within a few parts per million for sensing range.

\section{Special features}

Provides continuous conformal mapping of swath sensed by an artificial satellite, such as Landsats 1 through 5, orbiting the Earth; no scale distortion along the groundtrack. The first projection to account for the Earth's rotation with respect to a satellite orbit.

\section{Usage}

Format for imagery from mapping satellites Landsats 4 and 5

Figure 92A.-Two orbits of the Space Oblique Mercator projection, shown for Landsat 5, paths 15 (left) and 31 (right). Designed for a narrow band along the groundtrack, which remains true to scale. Note the change in longitude at a given latitude along the groundtrack with successive orbits. $30^{\circ}$ graticule. Short, straight lines nearly perpendicular to the groundtrack are sample scan lines, extended $15^{\circ}$ from groundtrack.

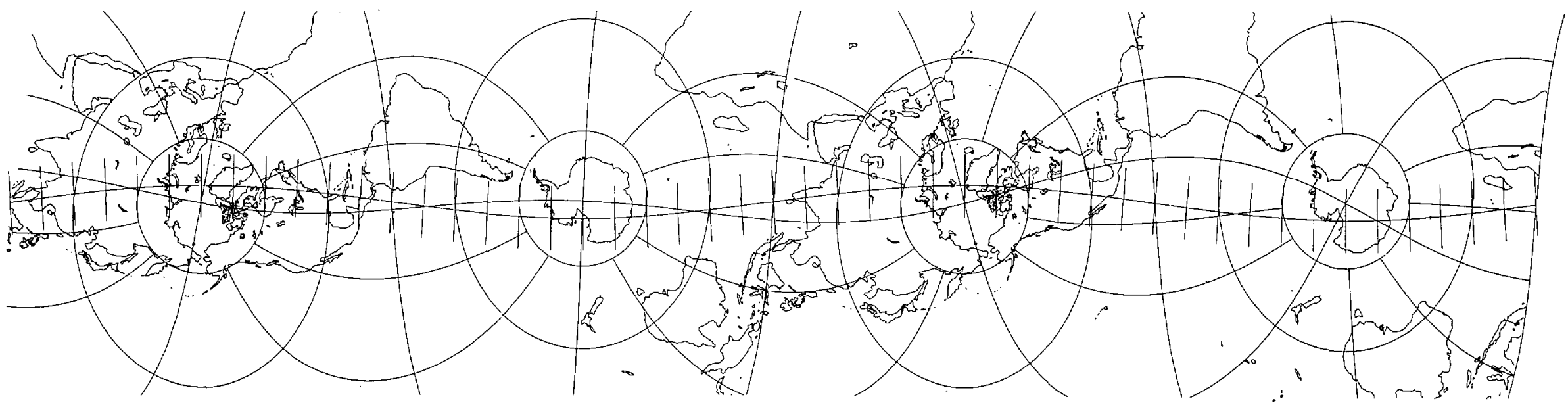

\section{Origin}

Concept presented by Alden P. Colvocoresses (1918- ) of the U.S. Geological Survey in 1973.

Mathematical development by John P. Snyder (1926- ) in 1977

\section{Similar projections}

Oblique Mercator projection (p. 14) would be suitable for a satellite orbiting a nonrotating spherical Earth and is the closest standard approximation for limited intervals involving a rotating Earth. 


\section{SPACE OBLIQUE MERCATOR Projection}

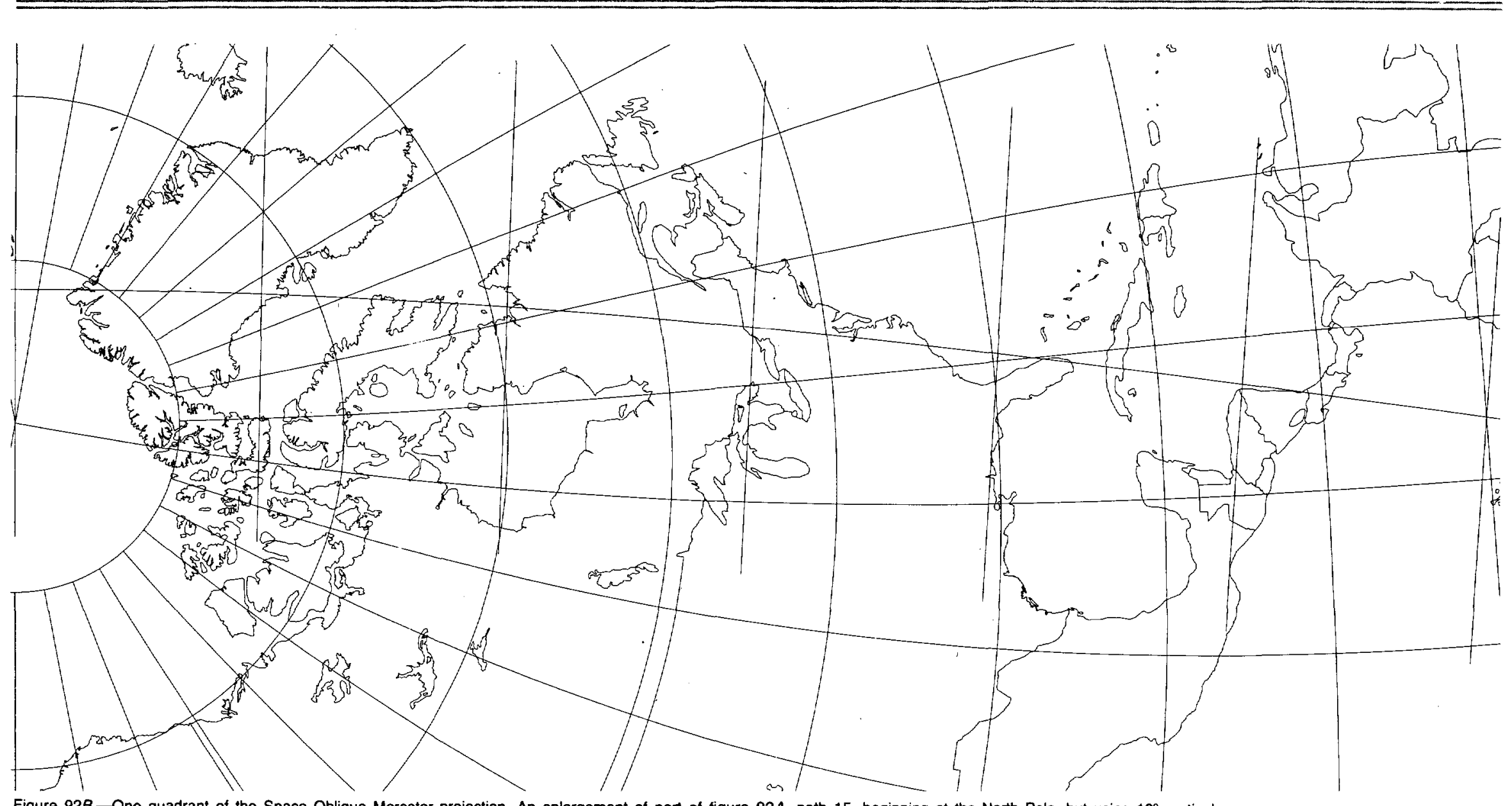

Figure 928.-One quadrant of the Space Oblique Mercator projection. An enlargement of part of figure 92A, path 15, beginning at the North Pole, but using $10^{\circ}$ graticule. 


\section{SATELLITE TRACKING Projections}

\section{Classifications}

Cylindrical or conic

Neither conformal nor equal area

\section{Graticule}

Meridians: Equally spaced straight lines, parallel on cylindrical form and converging toward common point on conic form

Parallels: Uequally spaced straight parallel lines on cylindrical form, closest near the Equator, perpendicular to meridians.

Unequally spaced concentric circular arcs on conic form, centered on the pole of convergence of the meridians, which are therefore radii of the circular arcs. Spacing of parallels increases away from the central latitudes.

Poles: Since satellite groundtracks do not pass over poles, they are not needed on the map. They can be shown in arbitrary locations, however.

Symmetry: About any meridian. On the cylindrical form, also about the Equator.

\section{Scale}

On the cylindrical form, true along the Equator or along two parallels equidistant from the Equator

On the conic form, true along one chosen parallel

\section{Distortion}

Conformality can be made to occur along two parallels equidistant from the Equator on the cylindrical form and along any two parallels within tracking range on the conic form.

\section{Special feature}

Any groundtrack for a given orbiting satellite such as Landsat is shown straight, if the map is designed for that particular satellite. The groundtrack may or may not have a sharp break at the northern or southern limit of tracking, depending on design.

\section{Origin}

Developed by John P. Snyder (1926- ) in

1977

\section{Similar projections}

Cylindrical form resembles the Mercator ( $p$.

10 ), and the conic form resembles the Lambert Conformal Conic (p. 95), but spacing of parallels is different. The Mercator and Lambert Conformal Conic are conformal throughout, except at the poles, but the Satellite Tracking projections are only conformal at two latitudes.

Figure 93A-Cylindrical Satellite Tracking projection (standard parallels $30^{\circ} \mathrm{N}$. and S.). Landsats 1,2 , and 3 orbits. Diagonal lines are groundtracks for paths $15,30,45$, and so on. $15^{\circ}$ graticule.

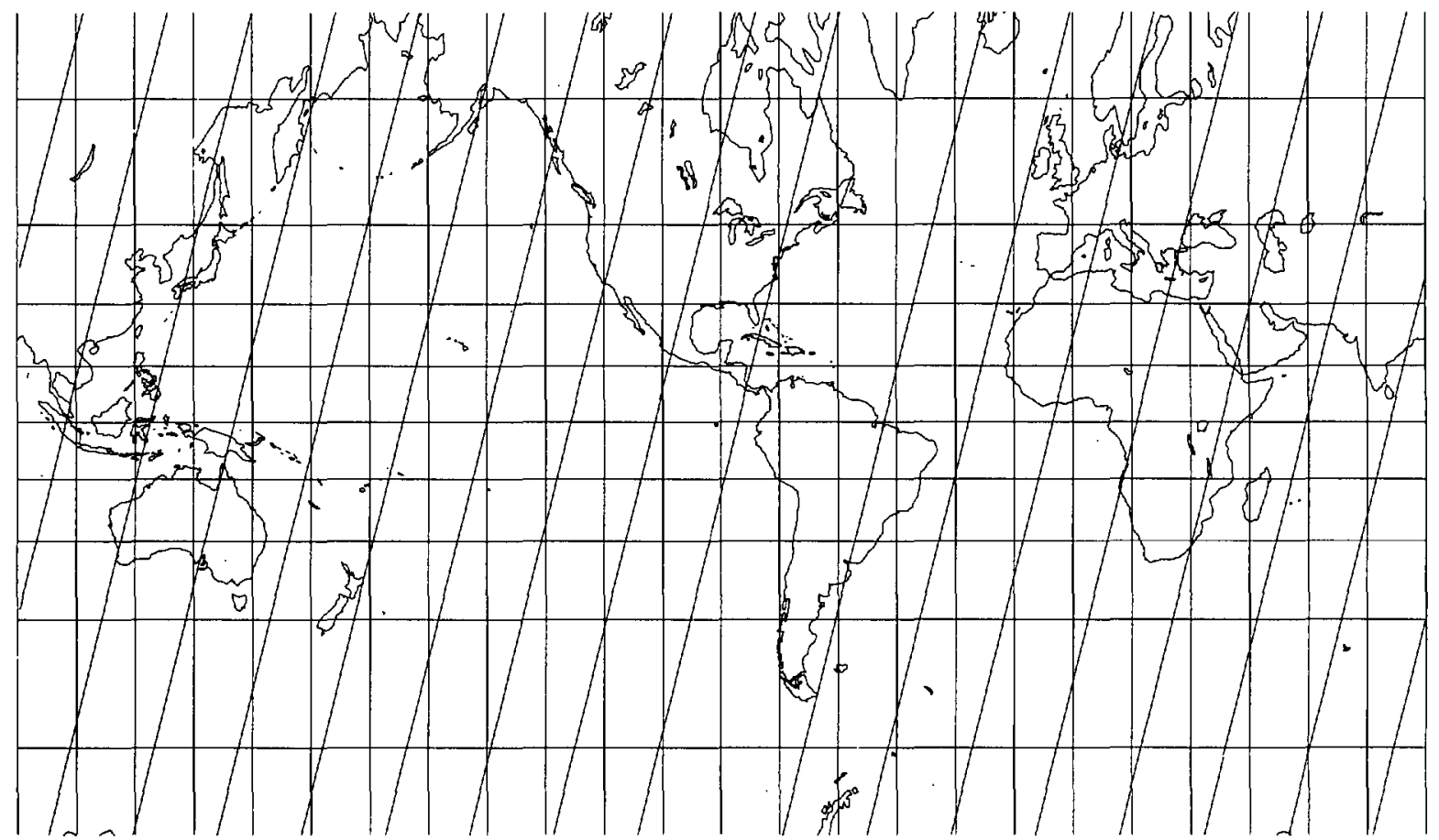




\section{SATELLITE TRACKING Projections}

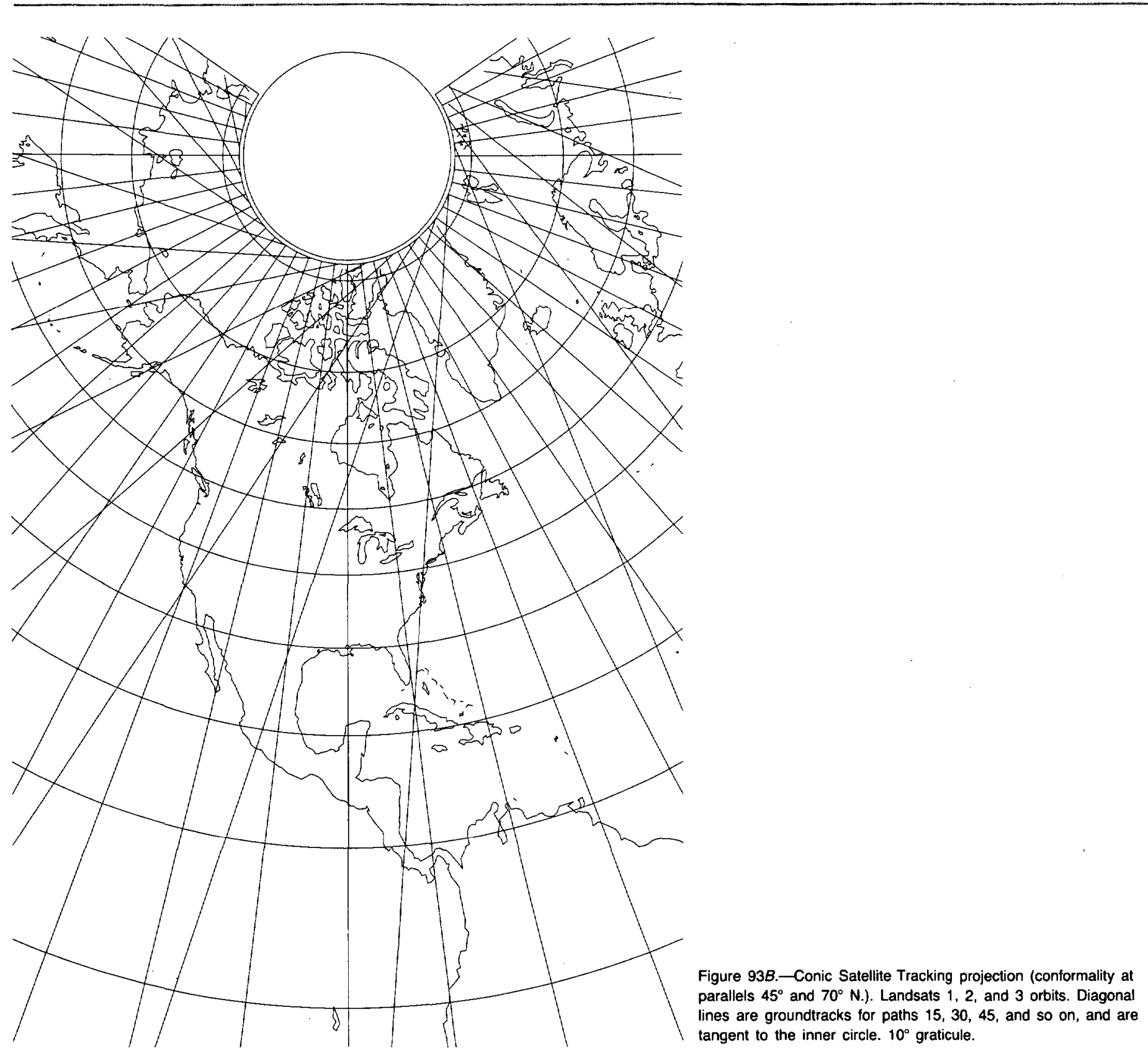




\section{APPENDIX:}

\section{FORMULAS FOR PROJECTIONS}

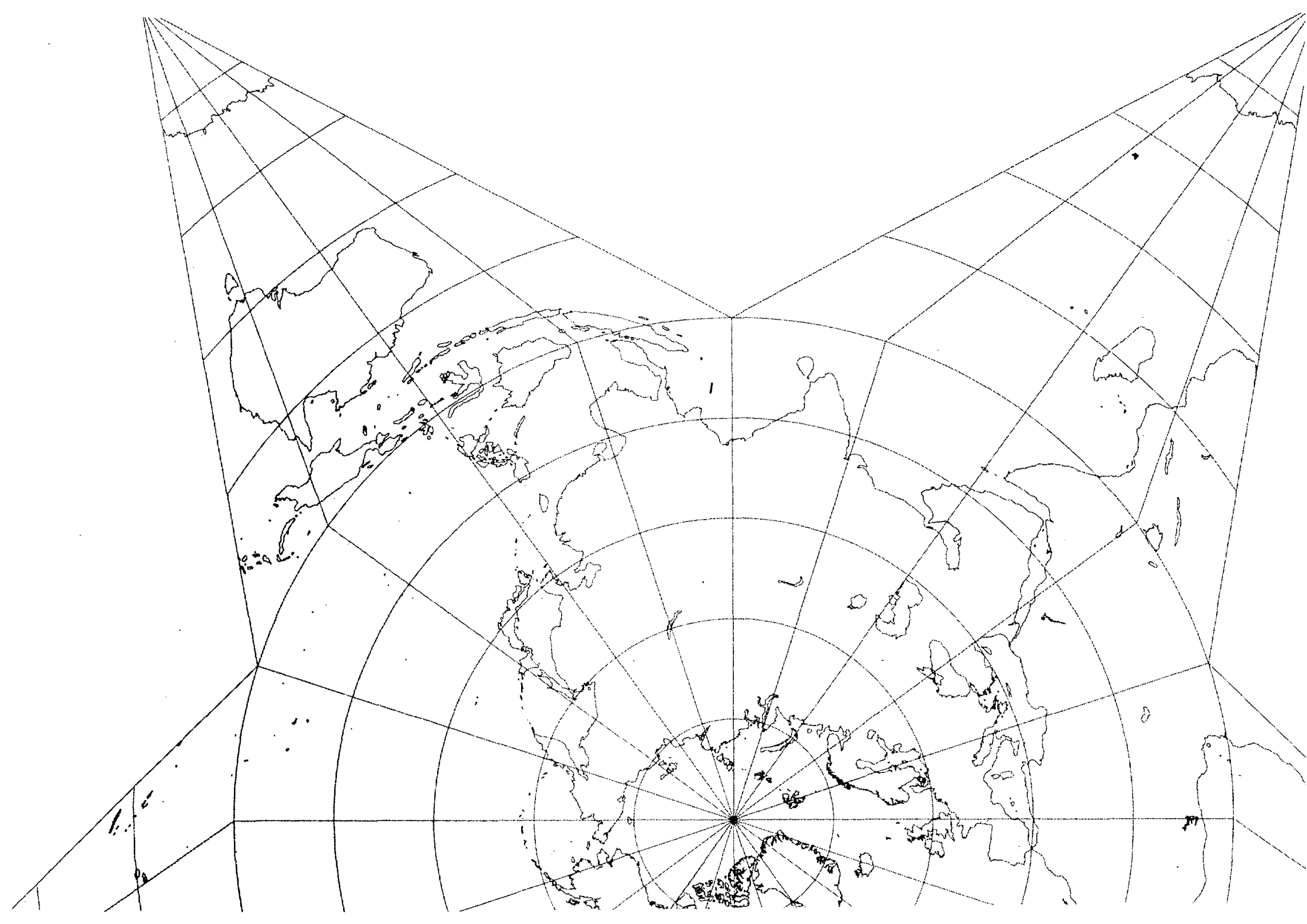




\section{APPENDIX: \\ FORMULAS FOR PROJECTIONS}

This compilation is abridged in several aspects. Only forward formulas for the sphere are given to compute rectangular coordinates from geographic latitude and longitude. For the inverse formulas and for forward or inverse formulas treating the Earth as an ellipsoid (if available), other references are given below. In addition, the reader is referred to relatively recent literature describing projections in more detail. Older sources are not listed in this volume, but they are frequently given in the literature that is referenced herein.

For a few major projections, the formulas for computing oblique, transverse, or equatorial aspects are given specifically. General transformation formulas are also given for use with any normal aspect, and most general map-plotting computer programs use these or trigonometric identities thereof rather than individual transformation formulas, unless ellipsoidal projections are to be plotted.

On the other hand, because of the importance of computer programs, the formulas below are amplified with conditional equations that should be programmed with the main formulas to avoid errors resulting from dividing by zero and so on.

In these equations, the following symbols apply:

cone constant on conic projections, or the ratio of the angle between meridians to the true angle

radius of the sphere at the scale of the map

rectangular coordinate; distance to the right of the vertical line ( $Y$ axis) passing through the origin (if negative, it is distance to the left)

rectangular coordinate; distance above the horizontal line ( $X$ axis) passing through the origin (if negative, it is distance below)

great-circle distance, as an arc of a circle natural logarithm

a special parametric variable, or an angle in polar coordinates measured generally counterclockwise from due south in these formulas

longitude east of Greenwich (for longitude west of Greenwich, a minus sign is used) $\lambda_{0}$

longitude east of Greenwich of the central meridian of the map or of the origin of the rectangular coordinates (for west longitude, a minus sign is used). If $\phi_{1}$ is a pole, $\lambda_{0}$ is the longitude of the meridian extending down on the map from the North Pole or up from the South Pole. On an interrupted projection, $\lambda_{0}$ is the central meridian of each section.

$\rho$

$\phi$

radius in polar coordinates

north latitude (if latitude is south, a minus sign is used)

$\phi_{0}$

middle latitude, or latitude chosen as the origin of rectangular coordinates for a projection, in radians. Its choice does not affect the appearance of the projection.

$\phi_{1}, \phi_{2} \quad$ standard parallels of latitude for projections having two standard parallels

$\phi_{1}$ (without $\phi_{2}$ ) single standard parallel on cylindrical or conic projections; latitude of central point on azimuthal projections. Its choice normally affects the appearance of the projection.

$\phi_{p}, \lambda_{p}$ latitude and longitude, respectively, of transformed pole of oblique, transverse, or equatorial aspect

Angles are to be expressed in radians, but degrees can be equally suitable in some cases, and some numbers are shown as degrees. Radians equal degrees times $\pi / 180^{\circ}$. The $\arctan _{2}$ function corresponds to the Fortran ATAN2 function and must therefore be adjusted for quadrant, depending on the signs of both numerator and denominator, if the function is not already programmed. The programmer should frequently apply normal techniques for computing efficiency to minimize computation of trigonometric functions; that is, a given function should be computed only once, and other functions should be derived by using common identities not listed here, since listing these techniques would often make the equations more difficult to follow.

The Newton-Raphson iteration suggested in several cases involves use of the equation

$$
\Delta v=-f(v) /[d f(v) / d v]
$$


After a suitable initial value of $v$ is used, $v$ is repeatedly adjusted with $\Delta v$ until $\Delta_{v}$ becomes less than some preset convergence

value. For example, equation 31 is transposed to develop a function of $\theta, f(\theta)$, which should approach zero:

$$
f(\theta)=\theta+\sin \theta-(1+\pi / 2) \sin \phi
$$

Differentiating, but treating $\phi$ as a constant,

$$
d f(\theta) / d \theta=1+\cos \theta
$$

Then,

$$
\Delta \theta=-[\theta+\sin \theta-(1+\pi / 2) \sin \phi] /(1+\cos \theta)
$$

If an initial value of $\theta$ equal to $\phi$ is used, convergence is rapid. 


\section{CYLINDRICAL PROJECTIONS}

Because of the common use of several transverse or oblique aspects of the cylindrical projections, formulas are given separately for them under this heading. They can also be found from the formulas for the normal aspect by using equations 130 and 131.

\section{Mercator}

$$
x=R\left(\lambda-\lambda_{0}\right)
$$

If $\phi=90^{\circ}, y=\infty$; if $\phi=-90^{\circ}, y=-\infty$. If $\phi$ is not $\pm 90^{\circ}$,

$$
y=R \ln \tan (\pi / 4+\phi / 2)
$$

The $X$ axis lies along the Equator, $x$ increasing east; the $Y$ axis lies along the central meridian, $y$ increasing north. For ellipsoidal and inverse formulas and more information, see U.S. Geological Survey (USGS) Professional Paper 1395 (Snyder, 1987, p $38-47$ ).

\section{Transverse Mercator}

Let

$$
B=\cos \phi \sin \left(\lambda-\lambda_{0}\right)
$$

If $B=1, x=\infty ;$ if $B=-1, x=-\infty ; y$ is found from equation 5. If $B$ is not \pm 1 ,

$$
\begin{gathered}
x=1 / 2 R \ln [(1+B) /(1-B)] \\
y=R \arctan _{2}\left[\tan \phi / \cos \left(\lambda-\lambda_{0}\right)\right]
\end{gathered}
$$

The $X$ axis lies along the Equator, $x$ increasing east; the $Y$ axis lies along the central meridian, $y$ increasing north. These axes are rotated $90^{\circ}$ counterclockwise from those corresponding to the equations for the Oblique Mercator below. For ellipsoidal and inverse formulas and more information, see USGS Professional Paper 1395 (Snyder, 1987, p. 48-64).

\section{Oblique Mercator}

$x=R \arctan _{2}\left[\frac{\cos \phi_{p} \sin \phi-\sin \phi_{p} \cos \phi \cos \left(\lambda-\lambda_{p}\right)}{\cos \phi \sin \left(\lambda-\lambda_{p}\right)}\right]$

Let

$$
A=\sin \phi_{p} \sin \phi+\cos \phi_{p} \cos \phi \cos \left(\lambda-\lambda_{p}\right)
$$

(1) If $A=1, y=\infty$; if $A=-1, y=-\infty$. If $A$ is not \pm 1 ,

$$
y=1 / 2 R \ln [(1+A) /(1-A)]
$$

(2) Note that the $X$ axis lies along the central line, $x$ increasing east The $Y$ axis crosses the $X$ axis at the actual Equator and a longitude $90^{\circ}$ less than $\lambda_{p}, y$ increasing north. For ellipsoidal and inverse formulas and more information, see USGS Professional Paper 1395 (Snyder, 1987, p. 66-75).

\section{Lambert Cylindrical}

Equal-Area

\section{$x=$ same as in equation 1}

$$
y=R \sin \phi
$$

For axes, see Mercator. For ellipsoidal and inverse formulas and more information for all aspects, see USGS Professional Paper 1395 (Snyder, 1987, p. 76-85)

\section{Transverse Lambert}

Cylindrical Equal-Area

$$
\begin{aligned}
& x=R \cos \phi \sin \left(\lambda-\lambda_{0}\right) \\
& y=\text { same as in equation } 5
\end{aligned}
$$

For axes, see Transverse Mercator. 


\section{Oblique Lambert}

Cylindrical Equal-Area

$x=$ same as in equation 6

Let

$$
A=\text { same as in equation } 7
$$

$$
y=R A
$$

For axes, see Oblique Mercator.

\section{Behrmann Cylindrical}

Equal-Area

$$
\begin{gathered}
x=R\left(\lambda-\lambda_{0}\right) \cos 30^{\circ} \\
y=R \sin \phi / \cos 30^{\circ}
\end{gathered}
$$

For axes, see Mercator.

\section{Plate Carrée}

$x=$ same as in equation 1

$$
y=R \phi
$$

For axes, see Mercator.

\section{Equirectangular}

$$
\begin{gathered}
x=R\left(\lambda-\lambda_{0}\right) \cos \phi_{1} \\
y=\text { same as in equation } 14
\end{gathered}
$$

For axes, see Mercator.

\section{Cassini}

$$
\begin{gathered}
x=R \arcsin \left[\cos \phi \sin \left(\lambda-\lambda_{0}\right)\right] \\
y=\text { same as in equation } 5
\end{gathered}
$$

For axes, see Transverse Mercator. For ellipsoidal and inverse formulas and more information, see USGS Professional Paper 1395 (Snyder, 1987, p. 92-95)

\section{Oblique Equidistant \\ Cylindrical}

$$
\begin{gathered}
x=\text { same as in equation } 6 \\
A=\text { same as in equation } 7 \\
y=R \arcsin A
\end{gathered}
$$

(13) For axes, see Oblique Mercator.

\section{Central Cylindrical}

$$
x=\text { same as in equation } 1
$$

If $\phi=90^{\circ}, y=\infty$; if $\phi=-90^{\circ}, y=-\infty$. If $\phi$ is not $=90^{\circ}$,

$$
y=R \tan \phi
$$

For axes, see Mercator.

\section{Transverse Central Cylindrical}

(15) Calculate $B$ from equation 3. If $B=1, x=\infty$; if $B=-1, x=$ $-\infty$. If $B$ is not \pm 1 ,

$$
x=R B /\left(1-B^{2}\right)^{4}
$$

$$
y=\text { same as in equation } 5
$$

For axes, see Transverse Mercator.

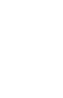

\section{Cylindrical Projections}


PSEUDOCYLINDRICAL PROJECTIONS

\section{Cylindrical Projections}

Pseudocylindrical Projections

\section{Gall (Stereographic)}

$$
\begin{gathered}
x=R\left(\lambda-\lambda_{0}\right) / 2^{1 / 2} \\
y=R\left(1+2^{k / 2} / 2\right) \tan 1 / 2 \phi
\end{gathered}
$$

For axes, see Mercator

\section{Miller Cylindrical}

$$
\begin{gathered}
x=\text { same as in equation } 1 \\
y=R[\ln \tan (\pi / 4+0.4 \phi)] / 0.8
\end{gathered}
$$

For axes, see Mercator. For more information see the article by Miller (1942) and USGS Professional Paper 1395 (Snyder, 1987, p. 86-89).

For axes, see Mercator. For transverse and oblique aspects, use the general formulas given in 130 and 131. Snyder (1977) provides original references for most of the formulas and descriptions of several other pseudocylindrical projections.

\section{Sinusoidal}

$$
x=R\left(\lambda-\lambda_{0}\right) \cos \phi
$$

$$
y=R \phi \text { (same as in equation 14) }
$$

\section{McBryde-Thomas Flat-Polar} Sinusoidal

$$
\theta / 2+\sin \theta=(1+\pi / 4) \sin \phi
$$

Find $\theta$ for the given $\phi$ by using a Newton-Raphson iteration of equation 24 , in which $\phi$ is the initial estimate of $\theta$.

$$
\begin{gathered}
x=R[6 /(4+\pi)]^{\text {/h }}(0.5+\cos \theta)\left(\lambda-\lambda_{0}\right) / 1.5 \\
y=R[6 /(4+\pi)]^{\text {h }} \theta
\end{gathered}
$$

Eckert V

$$
\begin{gathered}
x=R(1+\cos \phi)\left(\lambda-\lambda_{0}\right) /(2+\pi)^{*} \\
y=2 R \phi /(2+\pi)^{K}
\end{gathered}
$$

Winkel I

$$
\begin{gathered}
x=R\left(\lambda-\lambda_{0}\right)\left(\cos \phi_{1}+\cos \phi\right) / 2 \\
y=R \phi
\end{gathered}
$$

Eckert VI

$$
\theta+\sin \theta=(1+\pi / 2) \sin \phi
$$

Find $\theta$ for a given $\phi$ by using a Newton-Raphson iteration of equation 31 in which $\phi$ is the initial estimate of $\theta$.

$$
\begin{gathered}
x=R(1+\cos \theta)\left(\lambda-\lambda_{0}\right) /(2+\pi)^{3 / 3} \\
y=2 R \theta /(2+\pi)^{1 / 4}
\end{gathered}
$$

\section{McBryde \$3}

For latitudes between $55^{\circ} 51^{\prime} \mathrm{N}$. and S., use equations 23 and 14 . For other latitudes, use equations 24,25 , and the following:

$$
y=R\left\{[6 /(4+\pi)]^{k} \theta-0.069065 \operatorname{sign} \phi\right\}
$$

\section{Mollweide}

$$
2 \theta+\sin 2 \theta=\pi \sin \phi
$$

Find $\theta$ for the given $\phi$ by using a Newton-Raphson iteration of equation 35 in which $\phi / 2$ is the initial estimate of $\theta$.

$$
\begin{gathered}
x=2\left(2^{1 / 2}\right) R\left(\lambda-\lambda_{0}\right)(\cos \theta) / \pi \\
y=2^{1 / 2} R \sin \theta
\end{gathered}
$$




\section{Eckert III}

$$
\begin{gathered}
x=2\left\{1+\left[1-(2 \phi / \pi)^{2}\right]^{1 / 4}\right\} R\left(\lambda-\lambda_{0}\right) /\left(4 \pi+\pi^{2}\right)^{3 / 4} \\
y=4 R \phi /\left(4 \pi+\pi^{2}\right)^{1 / 4}
\end{gathered}
$$

\section{Eckert IV}

$$
\theta+\sin \theta \cos \theta+2 \sin \theta=(4+\pi)(\sin \phi) / 2
$$

Find $\theta$ for the given $\phi$ by using a Newton-Raphson iteration of equation 40 in which $\phi / 2$ is the initial estimate of $\theta$

$$
\begin{gathered}
x=2 R\left(\lambda-\lambda_{0}\right)(1+\cos \theta) /\left(4 \pi+\pi^{2}\right)^{3 /} \\
y=2 \pi^{3 / 2} R \sin \theta /(4+\pi)^{k / 2}
\end{gathered}
$$

\section{Wagner IV}

$$
2 \theta+\sin 2 \theta=\left\{\left[4 \pi+3\left(3^{h}\right)\right] / 6\right\} \sin \phi
$$

Find $\theta$ for the given $\phi$ by using a Newton-Raphson iteration of equation 43 in which $\phi / 2$ is the initial estimate of $\theta$.

$$
\begin{gathered}
x=0.86310 R\left(\lambda-\lambda_{0}\right) \cos \theta \\
y=1.56548 R \sin \theta
\end{gathered}
$$

\section{Hatano Asymmetrical}

\section{Equal-Area}

For all latitudes

$$
x=0.85 R\left(\lambda-\lambda_{0}\right) \cos \theta
$$

The parametric angle $\theta$ and $y$ are calculated differently for different latitudes. For latitudes north of the Equator,

$$
\begin{gathered}
2 \theta+\sin 2 \theta=2.67595 \sin \phi \\
y=1.75859 R \sin \theta
\end{gathered}
$$

For latitudes south of the Equator,

\section{Pseudocylindrical Projections}

$$
\begin{gathered}
2 \theta+\sin 2 \theta=2.43763 \sin \phi \\
y=1.93052 R \sin \theta
\end{gathered}
$$

Find $\theta$ for the given $\phi$ in equations 47 and 49 by using a

Newton-Raphson iteration in which $\phi / 2$ is the initial estimate of $\theta$.

\section{Goode Homolosine}

For latitudes between $40^{\circ} 44^{\prime} \mathrm{N}$. and $\mathrm{S}$., use equations 23 and 14 For other latitudes, use equations 35,36 , and the following:

$$
y=R\left[2^{k} \sin \theta-0.05280 \operatorname{sign} \phi\right]
$$

\section{Boggs Eumorphic}

If $\phi= \pm 90^{\circ}$, then $\theta=\phi, x=0$, and $y$ is found from equation 53. Otherwise, use equation 35 to find $\theta$ from $\phi$. Then,

$$
\begin{gathered}
x=2.00276 R\left(\lambda-\lambda_{0}\right) /(\sec \phi+1.11072 \sec \theta) \\
y=0.49931 R\left(\phi+2^{\hbar} \sin \theta\right)
\end{gathered}
$$

\section{Craster Parabolic}

$$
\begin{gathered}
x=3^{4 /} R\left(\lambda-\lambda_{0}\right)[2 \cos (2 \phi / 3)-1] / \pi^{3 / 6} \\
y=(3 \pi)^{4 / 6} R \sin (\phi / 3)
\end{gathered}
$$

\section{McBryde-Thomas Flat-Polar}

Parabolic

$$
\theta=\arcsin \left[(7 / 3)(\sin \phi) / 6^{\text {ths }}\right]
$$




\section{PSEUDOCYLINDRICAL PROJECTIONS}

$$
\begin{gathered}
x=6^{3 /} R\left(\lambda-\lambda_{0}\right)[2 \cos (2 \theta / 3)-1] / 7^{*} \\
y=9 R[\sin (\theta / 3)] / 7^{\text {\% }}
\end{gathered}
$$

P7) Putniṇs $\mathbf{P}_{5}$

\section{Quartic Authalic}

$$
\begin{gathered}
x=R\left(\lambda-\lambda_{0}\right)(\cos \phi) / \cos (\phi / 2) \\
y=2 R \sin (\phi / 2)
\end{gathered}
$$

\section{McBryde-Thomas Flat-Polar}

\section{Quartic}

$$
\sin (\theta / 2)+\sin \theta=\left(1+2^{k / 2} / 2\right) \sin \phi
$$

Find $\theta$ for the given $\phi$ by using a Newton-Raphson iteration of equation 61 in which $\phi$ is the initial estimate of $\theta$.

$$
\begin{gathered}
x=R\left(\lambda-\lambda_{0}\right)[1+2 \cos \theta / \cos (\theta / 2)] /\left[3\left(2^{k /}\right)+6\right]^{k /} \\
y=2\left(3^{k / 2}\right) R \sin (\theta / 2) /\left(2+2^{k / 4}\right)^{k /}
\end{gathered}
$$

(59) Denoyer Semi-Elliptical

(approximation)

(60) Let $L=\left|\lambda-\lambda_{0}\right|$.

$$
\begin{gathered}
x=R\left(\lambda-\lambda_{0}\right) \cos \left[\left(0.95-L / 12+L^{3} / 600\right)(0.9 \phi\right. \\
\left.\left.+0.03 \phi^{5}\right)\right] \\
y=R \phi(\text { same as in equation 14) }
\end{gathered}
$$

\section{Robinson}

A table is used instead of analytical formulas. The coordinates can be used in the following equations for a world map of correct total area (not equal area):

$$
x=0.8487 R X\left(\lambda-\lambda_{0}\right)
$$


Eckert I

where $X$ and $Y$ are interpolated from the following table (Robinson, 1974) with $Y$ taking the sign of $\phi$ :

$\begin{array}{ccc}\begin{array}{c}\text { lat } \boldsymbol{\phi}, \\ \text { in degrees }\end{array} & \boldsymbol{X} & \boldsymbol{Y} \\ 90 & 0.5322 & 1.0000 \\ 85 & .5722 & .9761 \\ 80 & .6213 & .9394 \\ 75 & .6732 & .8936 \\ 70 & .7186 & .8435 \\ 65 & .7597 & .7903 \\ 60 & .7986 & .7346 \\ 55 & .8350 & .6769 \\ 50 & .8679 & .6176 \\ 45 & .8962 & .5571 \\ 40 & .9216 & .4958 \\ 35 & .9427 & .4340 \\ 30 & .9600 & .3720 \\ 25 & .9730 & .3100 \\ 20 & .9822 & .2480 \\ 15 & .9900 & .1860 \\ 10 & .9954 & .1240 \\ 5 & .9986 & .0620 \\ 0 & 1.0000 & .0000\end{array}$

\section{Collignon}

$$
\begin{gathered}
x=2 R\left(\lambda-\lambda_{0}\right)(1-\sin \phi)^{k /} / \pi^{\text {h }} \\
y=\pi^{\hbar /} R\left[1-(1-\sin \phi)^{k / \hbar}\right]
\end{gathered}
$$

$$
\begin{gathered}
x=2[2 /(3 \pi)]^{\mathrm{h} /} R\left(\lambda-\lambda_{0}\right)(1-|\phi| / \pi) \\
y=2[2 /(3 \pi)]^{\mathrm{H}} R \phi
\end{gathered}
$$

\section{Eckert II}

$$
\begin{gathered}
x=2 R\left(\lambda-\lambda_{0}\right)(4-3 \sin |\phi|)^{3 / 2} /(6 \pi)^{\mathrm{K} /} \\
y=(2 \pi / 3)^{\mathrm{K}} R\left[2-(4-3 \sin |\phi|)^{\mathrm{K}}\right] \operatorname{sign} \phi
\end{gathered}
$$

\section{Loximuthal}

$x=R\left(\lambda-\lambda_{0}\right)\left(\phi-\phi_{1}\right) / \ln [\tan (\pi / 4+\phi / 2) / \tan (\pi / 4$ $\left.\left.+\phi_{1} / 2\right)\right]$

$$
y=R\left(\phi-\phi_{1}\right)
$$

where $\phi_{1}$ is the central latitude from which, at the central meridian, straight lines are loxodromes of true length and azimuth from the center. However, if $\phi=\phi_{1}$, equation 75 is replaced by

$$
x=R\left(\lambda-\lambda_{0}\right) \cos \phi_{1}
$$


For oblique aspects, use general formulas given in equations 130 and 131 .

\section{Equidistant Conic}

$$
\begin{gathered}
n=\left(\cos \phi_{1}-\cos \phi_{2}\right) /\left(\phi_{2}-\phi_{1}\right) \\
\rho=R\left[\left(\phi_{2} \cos \phi_{1}-\phi_{1} \cos \phi_{2}\right) /\left(\cos \phi_{1}-\cos \phi_{2}\right)-\phi\right] \\
\theta=n\left(\lambda-\lambda_{0}\right) \\
x=\rho \sin \theta \\
y=\rho_{0}-\rho \cos \theta
\end{gathered}
$$

where $\rho_{0}$ is calculated from equation 79 by using $\phi_{0}$ in place of $\phi$. The $Y$ axis lies along the central meridian $\lambda_{0}, y$ increasing north; the $X$ axis crosses the $Y$ axis perpendicularly at $\left(\phi_{0}, \lambda_{0}\right), x$ increasing east. For ellipsoidal and inverse formulas and more information, see USGS Professional Paper 1395 (Snyder, 1987, p. 111-115)

\section{Lambert Conformal Conic}

$n=\ln \left(\cos \phi_{1} / \cos \phi_{2}\right) / \ln [\tan (\pi / 4$

$\left.\left.+\phi_{2} / 2\right) / \tan \left(\pi / 4+\phi_{1} / 2\right)\right]$

$$
F=\cos \phi_{1} \tan "\left(\pi / 4+\phi_{1} / 2\right) / n
$$

If $\phi= \pm 90^{\circ}$ and $n$ has the same sign, $\rho=0$. If $\phi= \pm 90^{\circ}$ and $n$ has the opposite sign, $\rho=\infty$. If $\phi$ is not $\pm 90^{\circ}$,

$$
\rho=R F / \tan ^{n}(\pi / 4+\phi / 2)
$$

Use also equations 80 through $82, \rho_{0}$ being calculated from equation 85 by using $\phi_{0}$ in place of $\phi$. If only one standard parallel is desired, or $\phi_{1}=\phi_{2}$, equation 83 is indeterminate, but $n$ $=\sin \phi_{1}$. For axes, see Equidistant Conic. For ellipsoidal and inverse formulas and more information, see USGS Professional Paper 1395 (Snyder, 1987, p. 104-110).

\section{Bipolar Oblique Conic Conformal}

The formulas are too lengthy to be included here. For formulas and other information, see USGS Professional Paper 1395 (Snyder, 1987, p. 116-123).

\section{Albers Equal-Area Conic}

$$
\begin{aligned}
& n=\left(\sin \phi_{1}+\sin \phi_{2}\right) / 2 \\
& C=\cos ^{2} \phi_{1}+2 n \sin \phi_{1} \\
& \rho=R(C-2 n \sin \phi)^{x / n}
\end{aligned}
$$

Use also equations 80 through $82, \rho_{0}$ being calculated from equation 88 by using $\phi_{0}$ in place of $\phi$. For axes, see Equidistant Conic. For ellipsoidal and inverse formulas and more information, see USGS Professional Paper 1395 (Snyder, 1987, p. 98-103).

\section{Lambert Equal-Area Conic}

$$
n=\left(1+\sin \phi_{1}\right) / 2
$$

$$
\rho=R[2(1-\sin \phi) / n]^{\text {h/ }}
$$

Use also equations 80 through $82, \rho_{0}$ being calculated from equation 90 by using $\phi_{0}$ in place of $\phi$. For axes, see Equidistant Conic.

\section{Perspective Conic}

$$
\phi_{0}=\left(\phi_{1}+\phi_{2}\right) / 2
$$

If $\phi$ is less than or equal to $\left(\phi_{0}-90^{\circ}\right)$ and $\phi_{0}$ is positive or if $\phi$ is greater than or equal to $\left(\phi_{0}+90^{\circ}\right)$ and $\phi_{0}$ is negative, the point should be rejected. Otherwise,

$$
n=\sin \phi_{0}
$$


PSEUDOCONIC PROJECTIONS

$\rho=R \cos \left[\left(\phi_{2}-\phi_{1}\right) / 2\right]\left[\cot \phi_{0}-\tan \left(\phi-\phi_{0}\right)\right]$

Use also equations 80 through $82, \rho_{0}$ being calculated from equation 93 by using $\phi_{0}$ in place of $\phi$ and thus cancelling the last term. For axes, see Equidistant Conic.

\section{Polyconic (American)}

If $\phi$ is 0 ,

$$
x=R\left(\lambda-\lambda_{0}\right)(\text { same as equation 1) }
$$

$$
y=-R \phi_{0}
$$

If $\phi$ is not zero,

$$
\begin{gathered}
E=\left(\lambda-\lambda_{0}\right) \sin \phi \\
x=R \cot \phi \sin E \\
y=R\left[\phi-\phi_{0}+\cot \phi(1-\cos E)\right]
\end{gathered}
$$

For axes, see Equidistant Conic. For ellipsoidal and inverse formulas and more information, see USGS Professional Paper 1395 (Snyder, 1987, p. 124-133).

\section{Rectangular Polyconic}

If $\phi_{1}$ is 0 ,

$$
A=\left(\lambda-\lambda_{0}\right) / 2
$$

If $\phi_{1}$ is not 0 ,

$$
A=\tan \left[\left(\lambda-\lambda_{0}\right)\left(\sin \phi_{1}\right) / 2\right] / \sin \phi_{1}
$$

If $\phi$ is 0 ,

Conic Projections

$$
\begin{gathered}
x=2 R A \\
y=-R \phi_{0}
\end{gathered}
$$

If $\phi$ is not 0 ,

$$
E=2 \arctan (A \sin \phi)
$$

Use also equations 96 and 97, if $\phi$ is not 0 . For axes, see Equidistant Conic.

\section{Modified Polyconic for International Map of the World Series}

The formulas are too lengthy to be included here. For formulas and other information, see USGS Professional Paper 1395 (Snyder, 1987, p. 131, 134-137).

\section{Bonne}

Pseudoconic Projections

$$
\begin{gathered}
A=\cot \phi_{1} \hat{+} \phi_{1}-\phi \\
B=\left(\lambda-\lambda_{0}\right)(\cos \phi) / A \\
x=R A \sin B \\
y=R\left(\cot \phi_{1}-A \cos B\right)
\end{gathered}
$$

The $Y$ axis lies along central meridian $\lambda_{0}, y$ increasing north; the $X$ axis crosses the $Y$ axis perpendicularly at $\left(\phi_{1}, \lambda_{0}\right), x$ increasing east. For ellipsoidal and inverse formulas and more information, see USGS Professional Paper 1395 (Snyder, 1987, p. 138-140).

\section{Werner}

Use equations 103 through 106 , where $\phi_{1}=\pi / 2$ or $90^{\circ}$, but, if $\phi$ is also $90^{\circ}$, these equations should not be used, and $x=0$ and $y$ $=0$. 


\section{AZIMUTHAL PROJECTIONS (PERSPECTIVE)}

\section{Gnomonic}

North polar aspect:

If $\phi$ is equal to or less than zero, reject the point. Otherwise,

$$
\begin{gathered}
\rho=R \cot \phi \\
x=\rho \sin \left(\lambda-\lambda_{0}\right) \\
y=-\rho \cos \left(\lambda-\lambda_{0}\right)
\end{gathered}
$$

The origin of the axes is at the North Pole. Meridian $\lambda_{0}$ follows the negative $Y$ axis

Equatorial aspect:

If $\cos \phi \cos \left(\lambda-\lambda_{0}\right)$ is equal to or less than zero, reject the point. Otherwise,

$$
\begin{gathered}
x=R \tan \left(\lambda-\lambda_{0}\right) \\
y=R \tan \phi / \cos \left(\lambda-\lambda_{0}\right)
\end{gathered}
$$

The $Y$ axis lies along the central meridian $\lambda_{0}, y$ increasing north; the $X$ axis lies along the Equator, $x$ increasing east.

Oblique aspect (also polar and equatorial aspects):

$$
\cos z=\sin \phi_{1} \sin \phi+\cos \phi_{1} \cos \phi \cos \left(\lambda-\lambda_{0}\right)
$$

If $\cos z$ is equal to or less than zero, reject the point. Otherwise,

$$
\begin{gathered}
K=\sec z \\
x=R K \cos \phi \sin \left(\lambda-\lambda_{0}\right) \\
y=R K\left[\cos \phi_{1} \sin \phi-\sin \phi_{1} \cos \phi \cos \left(\lambda-\lambda_{0}\right)\right]
\end{gathered}
$$

The $Y$ axis lies along the central meridian $\lambda_{0}, y$ increasing north; the $X$ axis is perpendicular to the $Y$ axis at $\left(\phi_{1}, \lambda_{0}\right), x$ increasing east. For inverse formulas and other information, see USGS Professional Paper 1395 (Snyder, 1987, p. 164-168).

\section{Stereographic}

North polar aspect:

If $\phi$ is $-90^{\circ}$, reject the point. Otherwise, use equations 108 and 109 , but

$$
\rho=2 R k_{0} \tan (\pi / 4-\phi / 2)
$$

where $k_{0}$ is the scale factor at the center of the projection.

Equatorial aspect:

$$
\cos z=\cos \phi \cos \left(\lambda-\lambda_{0}\right)
$$

If $\cos z$ is -1 , reject the point. Otherwise,

$$
K=2 k_{0} /(1+\cos z)
$$

$$
x=\text { same as equation } 114
$$

$$
y=R K \sin \phi
$$

Oblique aspect (also polar and equatorial aspects):

Use equations $112,118,114$, and 115 in order, but, if $\cos z$ is -1 , reject the point. Axes for Gnomonic apply here for all aspects. For inverse and ellipsoidal formulas and other information, see USGS Professional Paper 1395 (Snyder, 1987, p. 154-163).

\section{Orthographic}

North polar aspect:

If $\phi$ is less than zero, reject the point. Otherwise, use equations 108 and 109 , but

Equatorial aspect:

$$
\rho=R \cos \phi
$$

If $\cos \phi \cos \left(\lambda-\lambda_{0}\right)$ is less than zero, reject the point. Otherwise, 
$x=$ same as in equation 114, where $K=1$

$y=R \sin \phi($ same as in equation 9)

Oblique aspect (also polar and equatorial aspects):

If $\cos z$, determined from equation 112 , is less than zero, reject the point. Otherwise,

$$
\begin{aligned}
& x=\text { same as in equation } 114, \text { where } K=1 \\
& y=\text { same as in equation } 115, \text { where } K=1
\end{aligned}
$$

Axes for Gnomonic apply here for all aspects. For inverse formulas and other information, see USGS Professional Paper 1395 (Snyder, 1987, p. 145-153). A hemisphere for any of the aspects of the Orthographic is bounded by a circle of radius $R$.

\section{General Vertical Perspective}

North polar aspect:

If $\sin \phi$ is less than $1 / P$, reject the point. Otherwise, use equations 108 and 109 , but

$$
\rho=R(P-1) \cos \phi /(P-\sin \phi)
$$

where $P$ is the distance of the point of perspective from the center of the Earth divided by the radius $R$. If the point is beneath the surface and the center of the Earth, apply a minus sign to $P$.

\section{Equatorial aspect:}

Use equations 117,114 , and 119 , but, if $\cos z$ is less than $1 / P$, reject the point. Otherwise,

$$
K=(P-1) /(P-\cos z)
$$

Oblique aspect (also polar and equatorial aspects):

Use equations $112,122,114$, and 115 in order, but, if $\cos z$ is less than $1 / P$, reject the point. Axes for Gnomonic apply here for all aspects. For inverse and ellipsoidal formulas for either Vertical or Tilted Perspective, see USGS Professional Paper 1395 (Snyder, 1987, p. 169-181). 


\section{AZIMUTHAL PROJECTIONS (NONPERSPECTIVE)}

\section{Azimuthal Equidistant}

North polar aspect:

Use equations 108 and 109 , but

$$
\rho=R(\pi / 2-\phi)
$$

Equatorial aspect:

Use equations 117,114 , and 119 , but

$$
K=z / \sin z
$$

If $\cos z=1$, equation 124 is indeterminate, but $K=1$. If $\cos z$ $=-1$, for the point opposite the center, the point is plotted as a bounding circle of radius $\pi R$.

Oblique aspect (also polar and equatorial aspects):

Use equations 112, 124 (including notes), 114, and 115 in order. Axes for Gnomonic apply here for all aspects. For inverse and ellipsoidal formulas and other information, see USGS Professional Paper 1395 (Snyder, 1987, p. 191-202).

\section{Lambert Azimuthal Equal-Area}

North polar aspect:

Use equations 108 and 109 , but

$$
\rho=2 R \sin (\pi / 4-\phi / 2)
$$

Equatorial aspect:

Use equations 117,114 , and 119 , but

$$
K=[2 /(1+\cos z)]^{\text {H }}
$$

(123) If $\cos z=-1$, for the point opposite the center, the point is plotted as a bounding circle of radius $2 R$.

Oblique aspect (also polar and equatorial aspects):

Use equations 112, 126 (including notes), 114, and 115 in order Axes for Gnomonic apply here for all aspects. For inverse and ellipsoidal formulas and other information, see USGS Professional Paper 1395 (Snyder, 1987, p. 182-190).

\section{Airy}

North polar aspect:

Use equations 108 and 109 , but

$\rho=2 R\left[\cot (z / 2) \ln \sec (z / 2)+\tan (z / 2) \cot ^{2}(\beta / 2) \ln \sec (\beta / 2)\right]$

where $z=[(\pi / 2)-\phi]$ and $\beta=$ the range from the center of the region in which distortion is to be minimized, on all aspects. For the north polar aspect, $\beta=\left(\pi / 2-\phi_{b}\right)$, where $\phi_{b}$ is the bound. ing latitude. If $z=0$, equation 127 is indeterminate, but then $\rho$ $=0$ 
Equatorial aspect:

Use equations 117,114 , and 119 , but

$$
\begin{aligned}
K=-R\{[\ln ((1+ & +\cos z) / 2)] /(1-\cos z) \\
& \left.+2[\ln \cos (\beta / 2)] /\left[\tan ^{2}(\beta / 2)(1+\cos z)\right]\right\}
\end{aligned}
$$

If $z=0$, equation 128 is indeterminate, but, then

$$
K=R\left\{0.5-[\ln \cos (\beta / 2)] / \tan ^{2}(\beta / 2)\right\}
$$

Oblique aspect (also polar and equatorial aspects):

Use equations 112,128 or 129,114 , and 115 in order. Axes for Gnomonic apply here for all aspects.

\section{General transverse and oblique transformations}

These formulas can be used with any of the above normal equations for cylindrical, pseudocylindrical, conic, or other projec-

tions to obtain transverse or oblique aspects. They can also be used with polar aspects of azimuthal projections, but the general formulas given above for oblique azimuthal projections are more easily used.

To use these formulas, change $\phi$ in the normal equations (for example, equations 54 and 55) to $\phi^{\prime}$ and $\left(\lambda-\lambda_{0}\right)$ to $\lambda^{\prime}$, and then compute $\phi^{\prime}$ and $\lambda^{\prime}$ from the following:

$$
\begin{aligned}
\phi^{\prime} & =\arcsin \left[\sin \phi_{0}^{\prime} \sin \phi+\cos \phi_{0}{ }^{\prime} \cos \phi \sin \left(\lambda-\lambda_{0}\right)\right] \\
\lambda^{\prime}=\lambda_{0}^{\prime} & +\arctan _{2}\left\{\cos \phi \cos \left(\lambda-\lambda_{0}\right) /\left[\cos \phi_{0}^{\prime} \sin \phi\right.\right. \\
& \left.\left.-\sin \phi_{0}^{\prime} \cos \phi \sin \left(\lambda-\lambda_{0}\right)\right]\right\}
\end{aligned}
$$

But, if $\phi=\phi_{0}{ }^{\prime}$ and $\left(\lambda-\lambda_{0}\right)=90^{\circ}$ or if $\phi=-\phi_{0}^{\prime}$ and $(\lambda-$ $\left.\lambda_{0}\right)=-90^{\circ}$, then equation 131 is indeterminate, and $\lambda^{\prime}$ can have any value. Under these conditions, $\phi^{\prime}=90^{\circ}$ and $-90^{\circ}$, respectively, and the "point" is a line if the corresponding pole of the basic projections is a line.

In the above formulas, $\left(\phi_{0}{ }^{\prime}, \lambda_{0}{ }^{\prime}\right)$ are the north latitude and longitude east of center, respectively, on the normal projection at which the North Pole $\left(\phi=90^{\circ}\right)$ is to be located $\left(\phi_{0}{ }^{\prime}=0\right.$ for the transverse aspect, and $90^{\circ}$ for the normal aspect).

$\left(\phi^{\prime}, \lambda^{\prime}\right)$ are the latitude and longitude east of center, respectively, on the normal projection at which point $(\phi, \lambda)$ is plotted

$\lambda_{0}$ is the meridian of the globe $90^{\circ}$ less than the meridian $\lambda_{p}$ connecting the actual North Pole to the north pole of the base projection. It is the central meridian of the transverse aspect.

For the transverse aspect, this transformation will cause the $X$ axis, rather than the $Y$ axis, to lie along the central meridian. To reverse the axes, replace $y$ with $x$ and $x$ with $-y$ after all other calculations have been completed.
Azimuthal Projections Nonperspective 


\section{MODIFIED AZIMUTHAL PROJECTIONS}

\section{Two-Point Azimuthal}

In the following formulas, the axes of the usual azimuthal projections have been rotated, so that the $X$ axis lies along the two central points $A$ and $B$ ( $B$ is more easterly, $x$ increases from $A$ to

$B$ ) from which the directions to other points are made correct. Points $A$ and $B$ are also equidistant from the $Y$ axis. Azimuth $A z_{1}$ and distance $d$ to help determine the center point are

$A z_{1}=\arctan _{2}\left[\frac{\cos \phi_{B} \sin \left(\lambda_{B}-\lambda_{A}\right)}{\cos \phi_{A} \sin \phi_{B}-\sin \phi_{A} \cos \phi_{B} \cos \left(\lambda_{B}-\lambda_{A}\right)}\right]$

$d=(1 / 2) \arccos \left[\sin \phi_{A} \sin \phi_{B}+\cos \phi_{A} \cos \phi_{B} \cos \left(\lambda_{B}-\lambda_{A}\right)\right]$

The latitude and longitude $\left(\phi_{1}, \lambda_{0}\right)$ of the point midway between $A$ and $B$ are

$$
\phi_{1}=\arcsin \left(\sin \phi_{A} \cos d+\cos \phi_{A} \sin d \cos A z_{1}\right)
$$

$$
\lambda_{0}=\lambda_{A}+\arctan _{2}\left[\sin d \sin A z_{1} /\left(\cos \phi_{A} \cos d\right.\right.
$$

$$
\left.\left.-\sin \phi_{A} \sin d \cos A z_{1}\right)\right]
$$

The azimuth $A z_{2}$ used to rotate the axes is as follows:

$A z_{2}=\arctan _{2}\left[\frac{-\cos \phi_{A} \sin \left(\lambda_{A}-\lambda_{0}\right)}{\sin \phi_{1} \cos \phi_{A} \cos \left(\lambda_{A}-\lambda_{0}\right)-\cos \phi_{1} \sin \phi_{A}}\right](136)$

The above $A z_{1}, A z_{2}, d, \phi_{1}$, and $\lambda_{0}$ apply to the entire map. For other points $(\phi, \lambda)$, find the nonrotated $(x, y)$ from equations 112 through 115 for the Gnomonic projection, and call those coordinates $\left(x, y_{t}\right)$. Rotate and adjust as follows:

$$
\begin{gathered}
x=\cos d\left(x_{f} \sin A z_{2}+y_{f} \cos A z_{2}\right) \\
y=y_{t} \sin A z_{2}-x_{f} \cos A z_{2}
\end{gathered}
$$

\section{Two-Point Equidistant}

In the following formulas, the axes of the usual azimuthal projections have been rotated so that the $X$ axis lies along the two central points $A$ and $B$ ( $B$ is more easterly, $x$ increases from $A$ to $B$ ) from which the distances to other points are correct. Points $A$ and $B$ are also equidistant from the $Y$ axis.

$$
\begin{gathered}
x=R\left(z_{A}{ }^{2}-z_{B}^{2}\right) /\left(2 z_{0}\right) \\
y= \pm R\left[4 z_{0}^{2} z_{B}^{2}-\left(z_{0}^{2}-z_{A}^{2}+z_{B}^{2}\right)^{2}\right]^{k /} /\left(2 z_{0}\right)
\end{gathered}
$$

where

$$
\cos z_{0}=\sin \phi_{A} \sin \phi_{B}+\cos \phi_{A} \cos \phi_{B} \cos \left(\lambda_{B}-\lambda_{A}\right)
$$

$$
\cos z_{A}=\sin \phi_{A} \sin \phi+\cos \phi_{A} \cos \phi \cos \left(\lambda-\lambda_{A}\right)
$$$$
\cos z_{B}=\sin \phi_{B} \sin \phi+\cos \phi_{B} \cos \phi \cos \left(\lambda-\lambda_{B}\right)
$$

and $y$ normally takes the sign of $\left[\cos \phi_{A} \cos \phi_{B} \sin \phi \sin \left(\lambda_{B}-\right.\right.$ $\left.\lambda_{A}\right)-\cos \phi_{A} \sin \phi_{B} \cos \phi \sin \left(\lambda-\lambda_{A}\right)+\sin \phi_{A} \cos \phi_{B} \cos \phi$ $\sin \left(\lambda-\lambda_{B}\right)$ ], but, if $\left(z_{0}+z_{A}+z_{B}\right)=2 \pi$, the expression for the sign of $y$ gives zero, and $y$ actually has two values at each point $(\phi, \lambda)$, one using the plus and the other using the minus. A world map is bounded by an ellipse having a semimajor axis $\pi R\left[1-z_{0} /(2 \pi)\right]$ and a semiminor axis $\pi R\left(1-z_{0} / \pi\right)^{1 / 2}$, the two axes coinciding with the $X$ and $Y$ axes, respectively.

\section{Miller Oblated Stereographic}

First $(x, y)$ are calculated for the oblique Stereographic projection by using equations $112,118,114$, and 115 in order, letting $R$ and $k_{0}$ equal 1.0 , but the coordinates are called $\left(x_{t}, y_{t}\right)$. These coordinates are then converted to $(x, y)$ as follows:

$$
\begin{aligned}
& x=R K x_{t}\left[1-Q\left(3 y_{t}^{2}-x_{t}^{2}\right) / 12\right] \\
& y=R K y_{t}\left[1+Q\left(3 x_{t}^{2}-y_{t}^{2}\right) / 12\right]
\end{aligned}
$$

For the map of Europe and Africa, Miller made $K=0.9245$ and $Q=0.2522$, and $\left(\phi_{1}, \lambda_{0}\right)$ in equations 112,114 , and 115 were made lat $18^{\circ} \mathrm{N}$., long $20^{\circ}$ (later $18^{\circ}$ ) E. If this projection is to be applied to other applications, $K$ and $Q$ are selected as follows, so that the scales at a selected arc distance $d$ from the center along the $X$ axis (left and right) equal the scales at arc distance $d_{b}$ from the center along the $Y$ axis (up and down): 


$$
\begin{gathered}
T_{a}=\left(1-\cos d_{a}\right) /\left(1+\cos d_{a}\right) \\
T_{b}=\left(1-\cos d_{b}\right) /\left(1+\cos d_{b}\right) \\
Q=\left(T_{b}-T_{a}\right) /\left(T_{a}+T_{a}^{2}+T_{b}+T_{b}^{2}\right) \\
K=1 /\left[\left(1+T_{a}\right)\left(1+Q T_{a}\right)\right]^{\text {his }}
\end{gathered}
$$

The nonconformal fill-in projections used as part of this projection in the Eastern Hemisphere are too complicated to describe here.

\section{Wiechel (north polar aspect \\ only) \\ $x=R\left[\sin \left(\lambda-\lambda_{0}\right) \cos \phi-\cos \left(\lambda-\lambda_{0}\right)(1-\sin \phi)\right](150)$ \\ $y=-R\left[\cos \left(\lambda-\lambda_{0}\right) \cos \phi+\sin \left(\lambda-\lambda_{0}\right)(1-\sin \phi)\right]$}

The origin of the axes occurs at the North Pole. The central meridian $\lambda_{0}$ starts from the pole along the negative $Y$ axis but soon deviates because it is curved.

\section{Craig Retroazimuthal}

If $\left(\lambda-\lambda_{0}\right)=0$,

$$
\begin{gathered}
x=0 \\
y=R\left(\sin \phi-\cos \phi \tan \phi_{1}\right)
\end{gathered}
$$

If $\left(\lambda-\lambda_{0}\right) \neq 0$,

$$
x=R\left(\lambda-\lambda_{0}\right)
$$

$y=R\left(\lambda-\lambda_{0}\right)\left[\sin \phi \cos \left(\lambda-\lambda_{0}\right)\right.$

$$
\left.-\cos \phi \tan \phi_{1}\right] / \sin \left(\lambda-\lambda_{0}\right)
$$

The axes pass through $\left(\phi_{1}, \lambda_{0}\right)$, the $Y$ axis following the central meridian $\lambda_{0}$ and $y$ increasing north. The direction from every point on the map to $\left(\phi_{2}, \lambda_{0}\right)$ is shown correctly with respect to the local meridian.

\section{Hammer Retroazimuthal}

Use equations 112 and 124 (including notes) to calculate $K$, and then calculate $(x, y)$ from the following alterations to equations 114 and 115 :

$$
x=R K \cos \phi_{1} \sin \left(\lambda-\lambda_{0}\right)
$$

$$
y=-R K\left[\sin \phi_{1} \cos \phi-\cos \phi_{1} \sin \phi \cos \left(\lambda-\lambda_{0}\right)\right]
$$

The axes and role of $\left(\phi_{1}, \lambda_{0}\right)$ are the same as those for the Craig Retroazimuthal.

\section{Littrow}

$$
\begin{aligned}
& x=R \sin \left(\lambda-\lambda_{0}\right) / \cos \phi \\
& y=R \tan \phi \cos \left(\lambda-\lambda_{0}\right)
\end{aligned}
$$

The $X$ axis lies along the Equator, $x$ increasing east; the $Y$ axis lies along the central meridian, $y$ increasing north.

\section{Berghaus Star}

If $\phi \geq 0$ (north latitudes),

$$
\begin{gathered}
x=R(\pi / 2-\phi) \sin \left(\lambda-\lambda_{0}\right) \\
y=-R(\pi / 2-\phi) \cos \left(\lambda-\lambda_{0}\right)
\end{gathered}
$$

If $\phi<0$ (south latitudes),

$$
\begin{aligned}
& y=R\left\{-\pi D \sin \left(\lambda-\lambda_{n}\right)+C[(\pi / 2\right. \\
& -\phi)^{2}\left(5-4 \cos \left(\lambda-\lambda_{b}\right)\right)-\pi^{2} \sin ^{2}(\lambda \\
& \left.\left.\left.-\lambda_{n}\right)\right]^{1 / 4}\right\} /\left[5-4 \cos \left(\lambda-\lambda_{n}\right)\right] \\
& x=-\left[\pi R \sin \left(\lambda-\lambda_{n}\right)+y D\right] / C
\end{aligned}
$$

where

$$
C=\cos \left(\lambda-\lambda_{0}\right)-2 \cos \left(\lambda_{n}-\lambda_{0}\right)
$$




\section{MODIFIED AZIMUTHAL PROJECTIONS}

but if $C=0$

$$
D=\sin \left(\lambda-\lambda_{0}\right)-2 \sin \left(\lambda_{n}-\lambda_{0}\right)
$$

$$
x= \pm R(\pi / 2-\phi)
$$

taking the sign of $\sin \left(\lambda_{z}-\lambda_{0}\right)$.

In the above, $\lambda_{0}$ is the central meridian for the Northern Hemisphere and extends along the negative $Y$ axis from the North Pole, which is at the origin. The central meridian of each southern point is $\lambda_{n}$, where $n$ is the number ( 1 to 5 ) of the point.

Normally, $\lambda_{1}=\lambda_{0}$

\section{Aitoff}

$$
D=\arccos \left\{\cos \phi \cos \left[\left(\lambda-\lambda_{0}\right) / 2\right]\right\}
$$

If $D=0$,

$$
\begin{aligned}
& x=0 \\
& y=0
\end{aligned}
$$

Otherwise,

$$
\begin{gathered}
C=\sin \phi / \sin D \\
x= \pm 2 R D\left(1-C^{2}\right)^{4 /}
\end{gathered}
$$

taking the sign of $\left(\lambda-\lambda_{0}\right)$.

$$
y=R D C
$$

The $X$ axis lies along the Equator, $x$ increasing east; the $Y$ axis lies along the central meridian, $y$ increasing north.

\section{Hammer (Elliptical)}

$$
\begin{gathered}
W=0.5 \\
D=2 /\left\{1+\cos \phi \cos \left[W\left(\lambda-\lambda_{0}\right)\right]\right\} \\
x=R\left(D^{\not /} / W\right) \cos \phi \sin \left[W\left(\lambda-\lambda_{0}\right)\right] \\
y=R D^{4 /} \sin \phi
\end{gathered}
$$

Axes are same as those for the Aitoff

\section{Briesemeister}

The formulas are the same as those for the Hammer, but $x$ is multiplied and $y$ is divided by $(1.75 / 2)^{3 /}=0.93541$, and the projection is recentered at lat $45^{\circ} \mathrm{N}$., long $10^{\circ} \mathrm{E}$. The equations can be combined as follows:

$$
\begin{gathered}
x=R(3.5 D)^{1 / 5} \cos \phi^{\prime} \sin \left(\lambda^{\prime} / 2\right) \\
y=R(2 D)^{3 /} \sin \phi^{\prime} / 1.75^{\text {h }}
\end{gathered}
$$

where

$$
D=2 /\left[1+\cos \phi^{\prime} \cos \left(\lambda^{\prime} / 2\right)\right]
$$

$$
\phi^{\prime}=\arcsin \left\{\left[\sin \phi-\cos \phi \cos \left(\lambda-10^{\circ}\right)\right] / 2^{\not h}\right\}
$$

$\lambda^{\prime}=\arccos \left\{\left[\sin \phi+\cos \phi \cos \left(\lambda-10^{\circ}\right)\right] /\left(2^{1 / 4} \cos \phi^{\prime}\right)\right\}$

The $Y$ axis coincides with the central meridian $10^{\circ}$ E., $y$ increasing north, and the $X$ axis crosses it perpendicularly at lat $45^{\circ} \mathrm{N}$. 
The world map is bounded by an ellipse having a semimajor axis of $7^{\text {h/ }} R$ and a semiminor axis of $4 R / 7^{\mathrm{k} / \mathrm{h}}$

\section{Winkel Tripel}

Calculate $x$ and $y$ for the Aitoff projection by using equations 166 through 169 and calling the coordinates $x_{1}$ and $y_{1}$, respectively. Then calculate $x$ and $y$ for the Equirectangular projection by using equations 14 and 15 , where $\phi_{1}=50.467^{\circ}$ for Winkel's example or $40^{\circ}$ for Bartholomew's usage, and call the coordinates $x_{2}$ and $y_{2}$, respectively. Finally, average them:

$$
\begin{aligned}
& x=\left(x_{1}+x_{2}\right) / 2 \\
& y=\left(y_{1}+y_{2}\right) / 2
\end{aligned}
$$

The axes are the same as those for the Aitoff projection.

\section{Eckert-Greifendorff}

Same formulas and axes as Hammer (equations 170-172), but $W$ $=0.25$.

\section{Wagner VII}

$$
\begin{gathered}
S=0.90631 \sin \phi \\
C_{0}=\left(1-S^{2}\right)^{\text {h }} \\
C_{1}=\left(2 /\left\{1+C_{0} \cos \left[\left(\lambda-\lambda_{0}\right) / 3\right]\right\}\right)^{1 / 5}
\end{gathered}
$$

$$
x=2.66723 R C_{0} C_{1} \sin \left[\left(\lambda-\lambda_{0}\right) / 3\right]
$$$$
y=1.24104 R^{2} C_{1}
$$

\section{Chamberlin Trimetric}

Formulas are too complicated to be provided here. They have been developed (forward and inverse) but not published by George Bynum of the Mobil Corporation.

\section{Tilted Perspective}

Calculate $x$ and $y$ for the Vertical Perspective projection by using equations $112,122,114$, and 115 in order, but, if $\cos z$ is less than $1 / P$, reject the point. Call these coordinates $x_{1}$ and $y_{1}$, respectively. Then calculate the final $(x, y)$ as follows:

$$
\begin{gathered}
x=\left(x_{1} \cos \gamma-y_{1} \sin \gamma\right) \cos \omega / A \\
y=\left(y_{1} \cos \gamma+x_{1} \sin \gamma\right) / A
\end{gathered}
$$

where

$$
A=\left\{\left(y_{1} \cos \gamma+x_{1} \sin \gamma\right) \sin \omega /[R(P-1)]\right\}+\cos \omega
$$

(180) $\gamma$ is the azimuth east of north of the $Y$ axis, the most upwardtilted axis of the plane of projection relative to the tangent

(181) . plane, and $\omega$ is the upward angle of tilt, or the angle between the $Y$ axis and the tangent plane. The $X$ axis lies at the intersec-

tion of the tangent and tilted planes. The origin is at $\left(\phi_{1}, \lambda_{0}\right)$.

\section{Modified Azimuthal Projections}




\section{MISCELLANEOUS PROJECTIONS}

\section{Bacon Globular}

$$
\begin{gathered}
y=R(\pi / 2) \sin \phi \\
F=\left[(\pi / 2)^{2} /\left|\lambda-\lambda_{0}\right|+\left|\lambda-\lambda_{0}\right|\right] / 2 \\
x= \pm R\left[\left|\lambda-\lambda_{0}\right|-F+\left(F^{2}-y^{2} / R^{2}\right)^{k_{3}}\right]
\end{gathered}
$$

taking the sign of $\left(\lambda-\lambda_{0}\right)$. If $\lambda=\lambda_{0}$, equation 189 is infinite and equation 190 is indeterminate, but $x=0$. The $X$ axis coincides with the Equator, $x$ increasing east, and the $Y$ axis coincides with the central meridian $\lambda_{0}$.

\section{Fournier Globular}

$$
\begin{aligned}
& \text { If }\left(\lambda-\lambda_{0}\right)=0 \text {, or }|\phi|=\pi / 2 \text {, } \\
& \begin{array}{c}
x=0 \\
y=R \phi
\end{array}
\end{aligned}
$$

If $\phi=0$,

$$
\begin{gathered}
x=R\left(\lambda-\lambda_{0}\right) \\
y=0
\end{gathered}
$$

If $\left|\lambda-\lambda_{0}\right|=\pi / 2$,

$$
\begin{gathered}
x=R\left(\lambda-\lambda_{0}\right) \cos \phi \\
y=R(\pi / 2) \sin \phi
\end{gathered}
$$

Otherwise,

$$
\begin{gathered}
C=\pi^{2} / 4 \\
P=|\pi \sin \phi| \\
S=\left(C-\phi^{2}\right) /(P-2|\phi|) \\
A=\left(\lambda-\lambda_{0}\right)^{2} / C-1 \\
y= \pm R\left(\left\{S^{2}-A\left[C-P S-\left(\lambda-\lambda_{0}\right)^{2}\right]\right\}^{*}-S\right) / A
\end{gathered}
$$

$$
\left.x= \pm R\left(\lambda-\lambda_{0}\right)\left[1-y^{2} / R^{2} C\right)\right]^{1 / 4}
$$

where $x$ and $y$ take the signs of $\left(\lambda-\lambda_{0}\right)$ and $\phi$, respectively. Axes are the same as those for the Bacon Globular.

\section{Nicolosi Globular}

If $\left(\lambda-\lambda_{0}\right)=0$ or $\phi=0$ or $\left|\lambda-\lambda_{0}\right|=\pi / 2$, the limiting formulas for the Fournier I apply. Also, if $|\phi|=\pi / 2$,

$$
\begin{gathered}
x=0 \\
y=R \phi
\end{gathered}
$$

Otherwise,

$$
\begin{gathered}
b=\pi /\left[2\left(\lambda-\lambda_{0}\right)\right]-2\left(\lambda-\lambda_{0}\right) / \pi \\
c=2 \phi / \pi \\
d=\left(1-c^{2}\right) /(\sin \phi-c) \\
M=[b(\sin \phi) / d-b / 2] /\left(1+b^{2} / d^{2}\right) \\
N=\left[d^{2}(\sin \phi) / b^{2}+d / 2\right] /\left(1+d^{2} / b^{2}\right) \\
x=(\pi R / 2)\left\{M \pm\left[M^{2}+\cos ^{2} \phi /\left(1+b^{2} / d^{2}\right)\right]^{3 / 3}\right\} \\
y=(\pi R / 2)\left\{N \pm\left[N^{2}-\left(d^{2}\left(\sin ^{2} \phi\right) / b^{2}\right.\right.\right. \\
\left.\left.+d \sin \phi-1) /\left(1+d^{2} / b^{2}\right)\right]^{3 / 4}\right\}
\end{gathered}
$$

in which the plus or minus sign in equation 202 takes the sign of $\left(\lambda-\lambda_{0}\right)$ and the plus or minus sign in equation 203 takes the opposite sign of $\phi$ times the sign of $b$. Axes are the same as those for the Bacon Globular.

\section{Apian Globular I$$
y=R \phi
$$

To calculate $x$, use equations 189 and 190 for the Bacon Globular, except that $y$ in equation 190 is taken from equation 14 above. If $\lambda=\lambda_{0}$, the equations are indeterminate, but $x=0$.

Axes are the same as those for the Bacon. 


\section{Ortelius Oval}

If $\left|\lambda-\lambda_{0}\right| \leq \pi / 2$, use the Apian Globular I formulas in equations 14,189 , and 190 . For $\left|\lambda-\lambda_{0}\right|>\pi / 2$, use equation 14 for $y$, but

$$
x= \pm R\left\{\left[(\pi / 2)^{2}-\phi^{2}\right]^{1 / 4}+\left|\lambda-\lambda_{0}\right|-\pi / 2\right\}
$$

taking the sign of $\left(\lambda-\lambda_{0}\right)$. Axes are the same as those for the Bacon.

\section{Lagrange}

If $|\phi|=\pi / 2$,

$$
\begin{gathered}
x=0 \\
y= \pm 2 R
\end{gathered}
$$

taking the sign of $\phi$. Otherwise, if $W$ is the range in degrees of longitude, divided by $180^{\circ}$, within a bounding circle passing through the poles and $\phi_{1}$ is the parallel shown as a straight line,

$$
\begin{gathered}
A_{1}=\left[\left(1+\sin \phi_{1}\right) /\left(1-\sin \phi_{1}\right)\right]^{1 /(2 W)} \\
A=[(1+\sin \phi) /(1-\sin \phi)]^{1 /(2 m} \\
V=A / A_{1} \\
C=(V+1 / V) / 2+\cos \left[\left(\lambda-\lambda_{0}\right) / W\right] \\
x=2 R \sin \left[\left(\lambda-\lambda_{0}\right) / W\right] / C \\
y=R(V-1 / V) / C
\end{gathered}
$$

For the usual Lagrange, $W=2$ and $\phi_{1}=0$. For the equatorial Stereographic, $W=1$ and $\phi_{1}=0$.

Eisenlohr

$$
\begin{aligned}
& S_{1}=\sin \left[\left(\lambda-\lambda_{0}\right) / 2\right] \\
& C_{1}=\cos \left[\left(\lambda-\lambda_{0}\right) / 2\right]
\end{aligned}
$$

$$
T=\sin (\phi / 2) /\left[\cos (\phi / 2)+(2 \cos \phi)^{1 / 2} C_{1}\right]
$$

$V=\left\{\left[\cos (\phi / 2)+((\cos \phi) / 2)^{\not /}\left(C_{1}+S_{1}\right)\right] /[\cos (\phi / 2)\right.$

$$
\left.\left.+((\cos \phi) / 2)^{1 / 4}\left(C_{1}-S_{1}\right)\right]\right\}^{\text {/ }}
$$

$$
x=\left(3+8^{1 / 3}\right) R[-2 \ln V+C(V-1 / V)]
$$

$$
y=\left(3+8^{1 / 4}\right) R[-2 \arctan T+C T(V+1 / V)]
$$

Axes are the same as those for the Bacon. The factor ahead of $R$ provides a scale factor of 1 at the center.

\section{August Epicycloidal}

$$
\begin{gathered}
C_{1}=\left[1-\tan ^{2}(\phi / 2)\right]^{1 / 2} \\
C=1+C_{1} \cos \left[\left(\lambda-\lambda_{0}\right) / 2\right] \\
x_{1}=\sin \left[\left(\lambda-\lambda_{0}\right) / 2\right] C_{1} / C \\
y_{1}=\tan (\phi / 2) / C \\
x=4 R x_{1}\left(3+x_{1}^{2}-3{y_{1}}^{2}\right) / 3 \\
y=4 R y_{1}\left(3+3{x_{1}}^{2}-{y_{1}}^{2}\right) / 3
\end{gathered}
$$

Axes are the same as those for the Bacon. The scale factor is 1 at the center.

\section{Guyou}

$$
\begin{aligned}
& \cos a=\left[\cos \phi \sin \left(\lambda-\lambda_{0}\right)-\sin \phi\right] / 2^{3 / 4} \\
& \cos b=\left[\cos \phi \sin \left(\lambda-\lambda_{0}\right)+\sin \phi\right] / 2^{\not 3} \\
& \sin m= \pm(1+\cos a \cos b-\sin a \sin b)^{\hbar} \\
& \sin n= \pm(1-\cos a \cos b-\sin a \sin b)^{\hbar} \\
& x=R \int_{0}^{m}\left(1-0.5 \sin ^{2} m\right)^{-x} d m \\
& y=R \int_{0}^{n}\left(1-0.5 \sin ^{2} n\right)^{-z_{1}} d n
\end{aligned}
$$


where $m$ takes the sign of $\left(\lambda-\lambda_{0}\right)$ and $n$ takes the sign of $\phi$. If $|\phi|=\pi / 2$, then $x=0$, and $y= \pm 1.85407 R$, taking the sign of $\phi$. Equations 229 and 230 are elliptical integrals of the first kind and can be computed by means of efficiently converging tech-

niques such as the Burlirsch (1965) algorithm (see p. 239). Axes are the same as those for the Bacon. *

\section{Peirce Quincuncial}

$$
\begin{aligned}
& \cos a=\cos \phi\left[\sin \left(\lambda-\lambda_{0}\right)+\cos \left(\lambda-\lambda_{0}\right)\right] / 2^{K} \\
& \cos b=\cos \phi\left[\sin \left(\lambda-\lambda_{0}\right)-\cos \left(\lambda-\lambda_{0}\right)\right] / 2^{k /}
\end{aligned}
$$

These values are then used in equations 227 through 230 for the Guyou. The axes intersect at the pole, the negative $Y$ axis coinciding with the central meridian $\lambda_{0}$ and parallel to a side of the bounding square. In equation $227, m$ takes the sign of $\sin (\lambda-$ $\left.\lambda_{0}\right)$, and, in equation $228, n$ takes the opposite sign of $\cos (\lambda-$ $\left.\lambda_{0}\right)$.

\section{Adams Hemisphere in a Square}

$$
\begin{gathered}
\cos a=\cos \phi \sin \left(\lambda-\lambda_{0}\right) \\
b=\pi / 2-\phi
\end{gathered}
$$

These values are then used in equations 227 through 230 for the Guyou, where $m$ takes the sign of $(\sin \phi+\cos a)$ and $n$ takes the sign of $(\sin \phi-\cos a)$. Axes are tilted $45^{\circ}$ with respect to the central meridian and the Equator but are parallel to the sides of the bounding square, $x$ increasing in the quadrant with positive $\phi$ and $\left(\lambda-\lambda_{0}\right)$.

Adams World in a Square II,

poles at opposite vertices

$$
\begin{gathered}
\cos a=\cos \phi^{\prime} \sin \left[\left(\lambda-\lambda_{0}\right) / 2\right] \\
\cos b=\sin \phi^{\prime}
\end{gathered}
$$

*Equations (225-234) apply only to one hemisphere of the projection at a time. The other hemisphere requires a different center in lat/long and a transposed origin on the map. where

$$
\sin \phi^{\prime}=\tan (\phi / 2)
$$

These values are then used in equations 227 through 230 for the Guyou, where $m$ takes the sign of $\left(\sin \phi^{\prime}+\cos a\right)$ and $n$ takes the sign of $\left(\sin \phi^{\prime}-\cos a\right)$. Axes are the same as those used for the Adams hemisphere in a square.

\section{Adams World in a Square I,}

poles in centers of sides

$$
\begin{aligned}
& \cos a=\left\{\cos \phi^{\prime} \sin \left[\left(\lambda-\lambda_{0}\right) / 2\right]-\sin \phi^{\prime}\right\} / 2^{\text {h/ }} \\
& \cos b=\left\{\cos \phi^{\prime} \sin \left[\left(\lambda-\lambda_{0}\right) / 2\right]+\sin \phi^{\prime}\right\} / 2^{k /}
\end{aligned}
$$

where $\phi^{\prime}$ is found from equation 237 and $a$ and $b$ are then used in equations 227 through 230 . Signs and axes are as described for the Guyou.

\section{Lee Conformal World in a Triangle}

The formulas are considered too complicated for listing here. For a discussion, see the monograph by Lee (1976).

\section{Conformal World in an Ellipse}

The shape of the bounding ellipse is established by a modular angle $\theta$ between $0^{\circ}$ and $90^{\circ}$. An angle of $23.8958^{\circ}$ gives a boundary ellipse in which the Equator is twice the length of the central meridian. If $k_{s}=\sin \theta$ and $k_{c}=\cos \theta$,

$$
K^{\prime}=\int_{0}^{\pi / 2}\left(1-k_{c}^{2} \sin ^{2} \lambda^{\prime}\right)^{-k} d \lambda^{\prime}
$$

where $\lambda^{\prime}$ is a convenient function and $K^{\prime}$ is the complete elliptic integral of the first kind. For $\theta=23.8958^{\circ}, K^{\prime}=2.34767$. Then,

$$
\begin{gathered}
u_{1}=2\left(1-k_{c}\right) \cos \phi \\
v_{1}=\left(1+k_{c}\right)\left[1+\cos \phi \cos \left(\lambda-\lambda_{0}\right)\right]
\end{gathered}
$$




$$
\begin{aligned}
& A=k_{a}^{2}\left[1-\cos \phi \cos \left(\lambda-\lambda_{0}\right)\right] \\
& B=u_{1}-v_{1} \\
& C=u_{1}+v_{1} \\
& R_{1}=A-\left(A^{2}-4 k_{c} B C\right)^{\text {h }} \\
& \lambda^{\prime}=-\arcsin \left[R_{1} /\left(2 C k_{c}\right)\right] \\
& \phi^{\prime}=\arcsin \left(\left\{1-\left[R_{1} /(2 B)\right]^{2}\right\}^{3 / /} / k_{\diamond}\right) \\
& u_{2}=\int_{0}^{\phi^{\prime}}\left(1-k_{0}^{2} \sin ^{2} \phi^{\prime}\right)^{-\xi_{0}} d \phi^{\prime} \\
& v_{2}=K^{\prime}-\int_{0}^{\lambda^{\prime}}\left(1-k_{c}{ }^{2} \sin ^{2} \lambda^{\prime}\right)^{-K_{h}} d \lambda^{\prime} \\
& u_{3}=\exp \left[\pi u_{2} /\left(4 K^{\prime}\right)\right] \\
& v_{3}=\pi v_{2} /\left(4 K^{\prime}\right) \\
& x= \pm R\left(u_{3}+1 / u_{3}\right)\left(\sin v_{3}\right) / 2 \\
& y= \pm R\left(u_{3}-1 / u_{3}\right)\left(\cos v_{3}\right) / 2
\end{aligned}
$$

(243) If $\left(\lambda-\lambda_{0}\right)=0$,

where $x$ and $y$ take the sign of $\lambda$ and $\phi$, respectively.

Equations 249 and 250 are also elliptic integrals of the first kind and, like equations 229,230 , and 240 , can be computed by means of efficiently converging techniques such as the

Burlirsch (1965) algorithm (see p. 239). Axes are the same as those for the Bacon Globular.

\section{Van der Grinten I}

$$
\begin{gathered}
B=|2 \phi / \pi| \\
C=\left(1-B^{2}\right)^{3 / 3}
\end{gathered}
$$

If $\phi=0$,

$$
\begin{gathered}
x=R\left(\lambda-\lambda_{0}\right) \\
y=0
\end{gathered}
$$

\section{Miscellaneous Projections}

$$
\begin{aligned}
& x=0 \\
& y= \pm \pi R B /(1+C) \\
& x=0 \\
& y= \pm \pi R \\
& A=\left|\pi /\left(\lambda-\lambda_{0}\right)-\left(\lambda-\lambda_{0}\right) / \pi\right| / 2 \\
& G=C /(B+C-1) \\
& P=G(2 / B-1) \\
& Q=A^{2}+G \\
& S=P^{2}+A^{2} \\
& T=G-P^{2} \\
& x= \pm \pi R\left\{A T+\left[A^{2} T^{2}-S\left(G^{2}-P^{2}\right)\right]^{k}\right\} / S \\
& y= \pm \pi R\left\{P Q-A\left[\left(A^{2}+1\right) S-Q^{2}\right]^{1 / 3}\right\} / S
\end{aligned}
$$

where $x$ and $y$ take the signs of $\left(\lambda-\lambda_{0}\right)$ and $\phi$, respectively. Axes are the same as those for the Bacon.

\section{Van der Grinten II}

Use equations 255 and 256. If $\left(\lambda-\lambda_{0}\right)=0$, use equations for this limitation under Van der Grinten I, including equation 257. (1) Otherwise, use equation 259 and

$$
x_{1}=\left[C\left(1+A^{2}\right)^{\text {H }}-A C^{2}\right] /\left(1+A^{2} B^{2}\right)
$$




\section{MISCELLANEOUS PROJECTIONS}

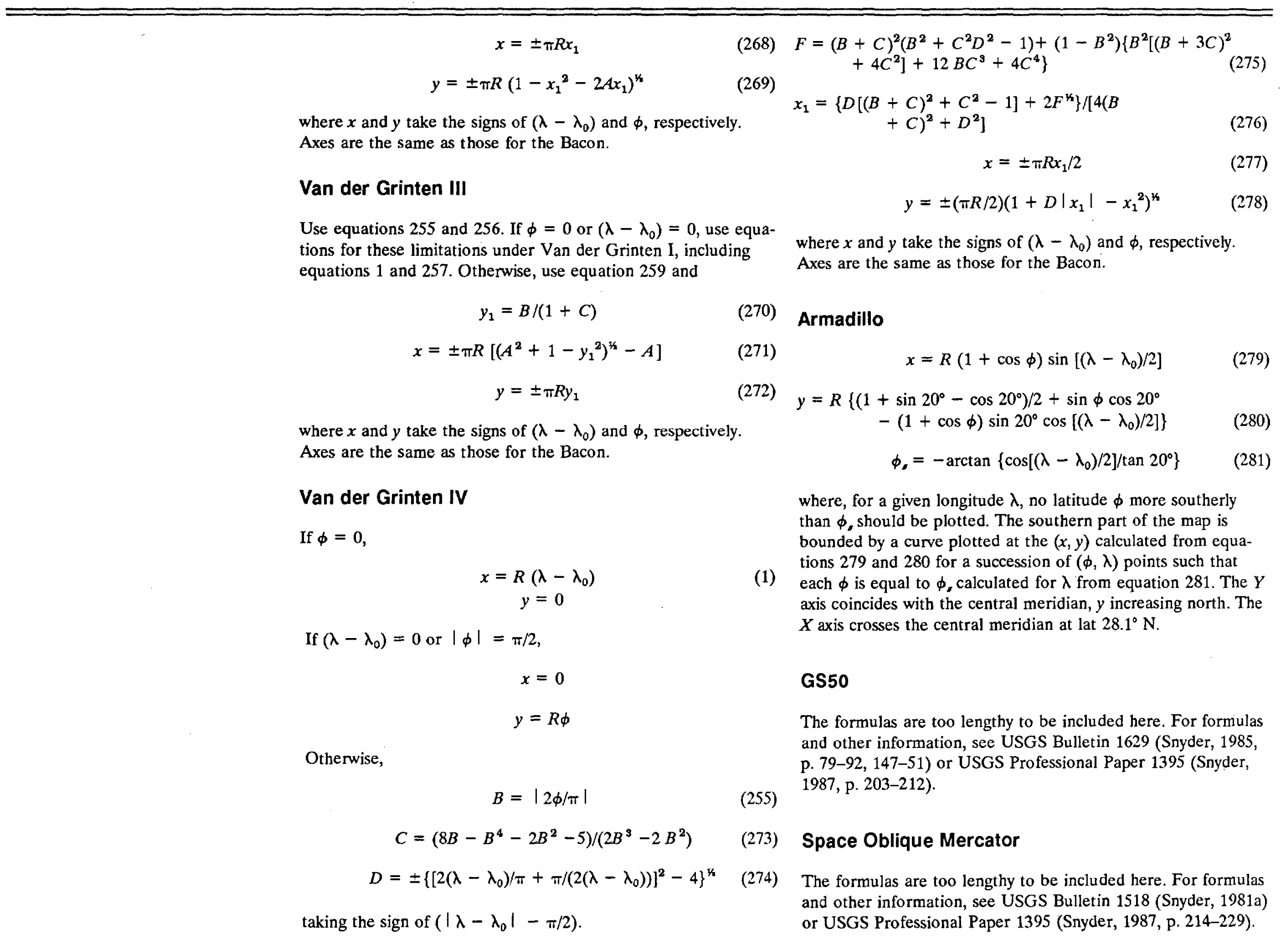




\section{Satellite-Tracking}

The formulas are too lengthy to be included here. For formulas and other information, see USGS Professional Paper 1395

(Snyder, 1987, p. 230-238).

\section{Evaluation of elliptic integrals}

Equations $229,230,240,249$, and 250 can be evaluated by using a rapidly converging algorithm by Bulirsch (1965), applicable to all elliptic integrals of the first kind; that is, to solve

$$
F=\int_{0}^{\phi}\left(1-k_{c}^{2} \sin ^{2} \phi\right)^{-k_{1}} d \phi
$$

let $S_{p}=\sin \phi$, and set convergence values $C_{\alpha}=10^{-9}$ and $C_{b}=10^{-8}$. Also set a limit $E_{p}=10^{-5}$. ( $k_{c}$ is frequently called $\sin \theta$, where $\theta$ is the modulus). Initially, let

$$
\begin{gathered}
h=S_{p}^{2} \\
k=\left(1-k_{c}^{2}\right)^{3 / 2}
\end{gathered}
$$

If $h \leq C_{b}$ (or $\phi=0$ ), then $F=0$. If $h \geq 1.0$ (or $|\phi|=\pi / 2$ ) for the "complete" elliptic integral, use equations 285 through 291 , but, if $k \leq E_{p}$, then $F= \pm \infty$, taking the sign of $S_{p}$. Let

$$
\begin{gathered}
m=1.0 \\
h=m \\
m=k+m
\end{gathered}
$$

If

$$
|h-k|>C_{a} m
$$

then let

$$
\begin{gathered}
k=(h k)^{k} \\
m=m / 2
\end{gathered}
$$

and repeat equations 286 through 290 until the condition of equation 288 is no longer met. At this point,

$$
F= \pm \pi / m
$$

taking the sign of $S_{p}$. If $h<1$ and $h>C_{b}$ (or $|\phi|<\pi / 2$ and $|\phi|>0$ ), for the "incomplete" elliptic integral, use equations 293 through 305 , but, if $k \leq E_{p}$

$$
F=\ln \left[\left(1+S_{p}\right) /\left(1-S_{p}\right)\right] / 2
$$

Otherwise, let

$$
\begin{gathered}
y=[(1-h) / h]^{1 / 3} \\
n=0 \\
m=1.0 \\
p=m k \\
g=m \\
m=k+m \\
y=y-(p / y)
\end{gathered}
$$

If $|y| \leq 0$, then let

$$
y=C_{b} p^{*}
$$

If $|g-k|>C_{p} g$, then let

$$
\begin{gathered}
k=2 p^{3} \\
n=n+n
\end{gathered}
$$

If $y<0$, then

$$
n=n+1
$$

and equations 296 through 304 are repeated until the condition of equation 301 is no longer met. At this point, if $y<0$, then $n$ $=n+1$. In any case,

$$
F= \pm[\arctan (m / y)+\pi n] / m
$$

taking the sign of $S_{p}$ and using the arctan in radians as usual.

The above results are generally accurate to 10 decimal places, if $C_{b}$ is used as given. For accuracy to 10 places or better in all cases, $C_{p}, C_{b}$, and $E_{p}$ can be made smaller as required. 
Bulirsch, R., 1965, Numerical calculation of elliptic integrals and elliptic functions: Numerische Mathematik, v. 7, p. 78-90.

Deetz, C.H., and Adams, O.S., 1934, Elements of map projection with applications to map and chart construction (4th ed.): U.S. Coast and Geodetic Survey Special Publication 68, $200 \mathrm{p}$.

Defense Mapping Agency, 1981, Glossary of mapping, charting, and geodetic terms (4th ed.): Washington, D.C., U.S. Government Printing Office, $204 \mathrm{p}$.

Hsu, M.-L., 1981, The role of projections in modern map design, in Monograph 27: Cartographica, v. 18, no. 2, p. 151-186.

Lee, L.P., 1944, The nomenclature and classification of map projections: Empire Survey Review, v. 7, p. 190-200.

-1976, Conformal projections based on elliptic functions, in Cartographica Monograph 16: Toronto, University of Toronto Press, $128 \mathrm{p}$.

Maling, D.H., 1960, A review of some Russian map projections: Empire Survey Review, v. 15 , no. 115 , p. $203-215$; v. 15 , no. 116 , p. 255-266; v. 15 , no. 117 , p. 294-303.

1973, Coordinate systems and map projections: London, George Philip, 255 p.

Maurer, Hans, 1935, Ebene Kugelbilder: Gotha, Germany, Petermanns

Mitteilungen, Ergänzungsheft 221, 87 p.

[Translated by Peter Ludwig with editing and foreword by William Warntz, 1968,

Harvard papers in theoretical geography: Cambridge, Mass., Harvard University Laboratory for Computer Graphics and Spatial Analysis, 224 p.]

Miller, O.M., 1942, Notes on cylindrical world map projections: Geographical Review, v. 32 , no. 3 , p. $424-430$.

Reignier, F., 1957, Les systèmes de projection et leurs applications: Paris, Institut Géographique National, v. I (texte), 312 p.; v. II (graphiques), 48 pls.

Robinson, A.H., 1974, A new map projection: Its development and characteristics: International Yearbook of Cartography, $v$. 14, p. 145-155.

Robinson, A.H., Sale, R.D., Morrison, J.L., and Muehrcke, P.C., 1984, Elements of cartography (5th ed.): New York, John Wiley, $544 \mathrm{p}$.

Royal Society, 1966, Glossary of technical terms in cartography: London, $84 \mathrm{p}$.

Snyder, J.P., 1977, A comparison of pseudocylindrical map projections: American Cartographer, v. 4, no. 1, p. 5981.

1981, The Space Oblique Mercator-

Mathematical development: U.S. Geological Survey Bulletin 1518, 108 p. 1982, Map projections used by the U.S.

Geological Survey: U.S. Geological Survey Bulletin 1532, $313 \mathrm{p}$.

1985, Computer-assisted map projection research: U.S. Geological Survey Bulletin $1629,157 \mathrm{p}$.

1987, Map projections: A working manual: U.S. Geological Survey Professional Paper 1395, 383 p.

Steers, J.A., 1970, An introduction to the study of map projections (15th ed.): London, University of London Press, $294 \mathrm{p}$.

Wong, F.K.C., 1965, World map projections in the United States from 1940 to 1960: Syracuse, N.Y., Syracuse University, unpublished M.A. thesis, $193 \mathrm{p}$. 


\section{INDEX}

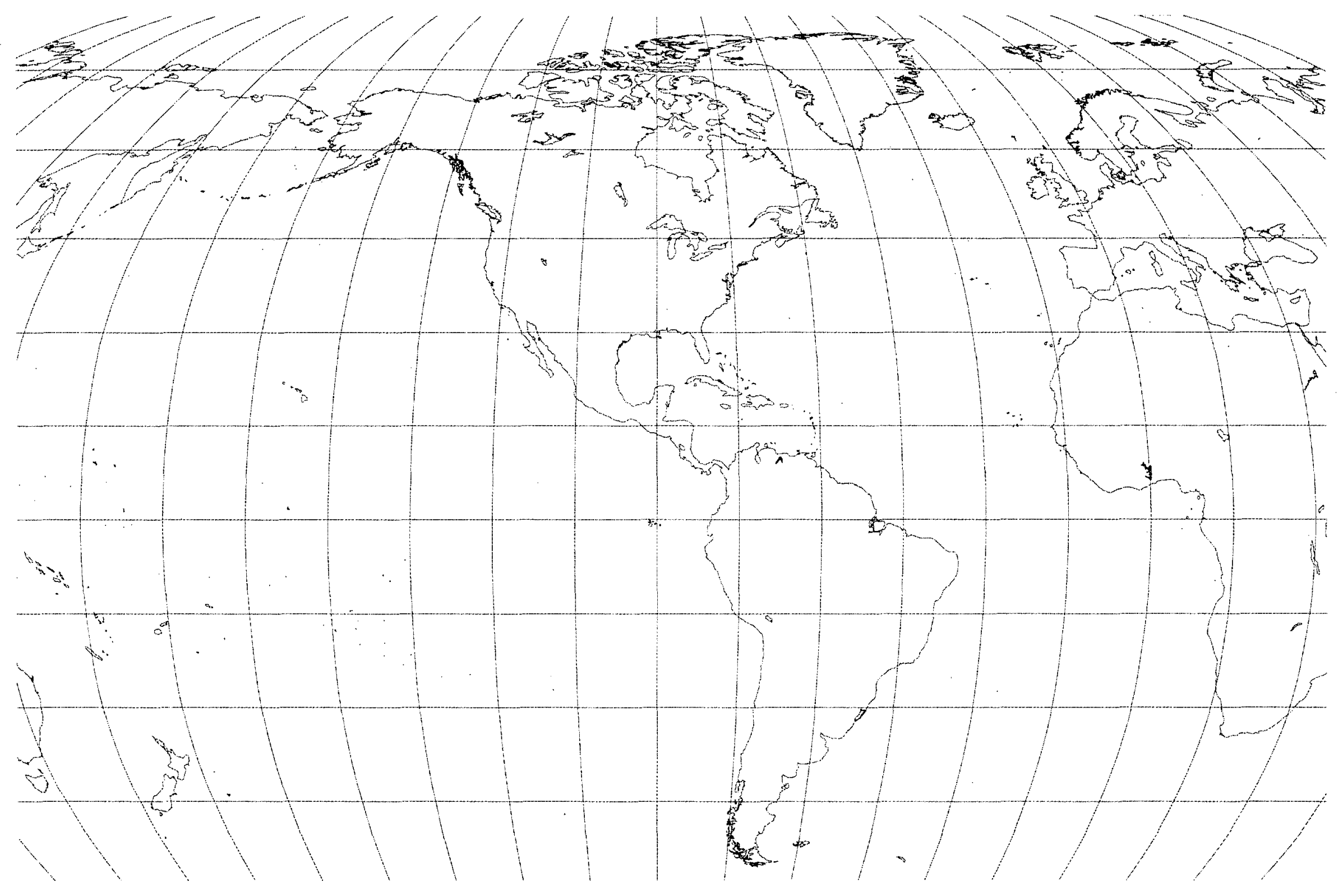




\section{INDEX}

[Main references in italics]

\section{A}

Adams, O.S. $\quad \ldots \ldots \ldots \ldots \ldots \ldots$ 70, 74, 192, 194, 195, 198 Adams projection of hemisphere in square - 188, 190, $192-193, .236$

Adams projection of world in square $1 \ldots \ldots$ 194, 236 Adams projection of world in square $11 \ldots$ 195, 236 Africa, maps of ................. . . 37, 148 Agnese, B. . . . . . . . . . . . . . . . 178 Airy, G.B. . . . . . . . . . . . . . . . . 140

Airy projection $\ldots \ldots \ldots \ldots$ 131, 132, 140-143, 228-229 Aitoff, D. . . . . . . . . . . . . . . . . . 158 Aitoff projection $\ldots \ldots \ldots \ldots$ 158-159, 160, 164, 232

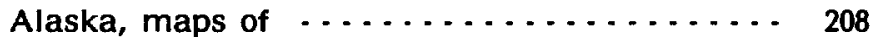

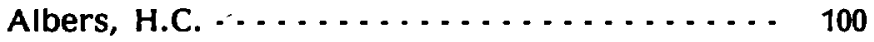

Albers Equal-Area Conic projection . . . . . . . . . 7 $100-101,102,224$

American Geographical Society .... - 35, 99, 148, 162 American Polyconic projection .......... 106 American Telephone \& Telegraph Co. . . . . . . 146 AMS Lunar projection . . . . . . . . . . . . 131

Apian(us), P. . . . . . . . . . . . . . . . . . . . . . 177

Apian Globular I projection - . . . . . 174, 177, 178, 234

Apian Globular II projection . . . . . . . . . . . . . . 177

"Apple-Shaped" projection . . . . . . . . . . . . 204 Armadillo projection $\ldots \ldots \ldots \ldots \ldots . . . .206-207,238$

Arrowsmith projection ............... . 176

Association of American Geographers - . . . . . 156

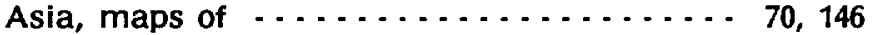

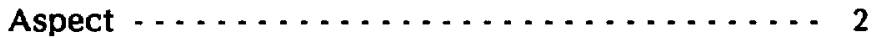

"Atlantis" projection $\ldots . . . . . . . . . . . .56$

Atlantic Ocean, maps of ............. 56

August Epicycloidal projection - 184, 186-187, 204, 235

August, F. . . . . . . . . . . . . . . . . 186

August projection $\ldots \ldots \ldots \ldots \ldots \ldots \ldots$

Authalic projection

See Quartic Authalic projection; equal-area projection

Azimuthal Equidistant projection . . . . 9 92, 94, 114, $132-135,140,146,156,170,175,176,228$

Modified .................. 158-159, 170

Azimuthal projection . . 2, 3, 4, 5, 6, 116-143, 226-229 Modified ................ 144-173, 230-233 See also specific projection
B.S.A.M. projection $\ldots \ldots \ldots \ldots \ldots \ldots \ldots \ldots$ Babinet projection ................ 54 Bacon Globular projection . . . . . . . . 174, 234 Bacon, R. . . . . . . . . . . . . . . . . . 174 Baranyi, J. ........................ . . . 82 Bartholomew, J. . . . . . . 54, 56, 92, 113, 114, 162 Behrmann Cylindrical Equal-Area

projection - ............. 16, 19-20, 100, 219 Behrmann, W. ..................... 19 Bellermann, G. ..................... 186 Berghaus, H. . . . . . . . . . . . . . 156 Berghaus Star projection ..... 132, 156-157, 231-232 Bienewitz, P. . . . . . . . . . . . . . . . . 177 Bipolar Oblique Conic Conformal projection ... 97, 99,

Boggs Eumorphic projection ... 37, 54, 68-69, 70, 221 Boggs, S.W. . . . . . . . . . . . . . . . 68 Bomford, G. ................... 33 Bonne, R. . . . . . . . . . . . . . . . . . 113 Bonne projection $\ldots \ldots \ldots \ldots$ 92, 112-113, 114, 225 Bordone Oval projection $\ldots \ldots \ldots \ldots \ldots \ldots$. . . 178

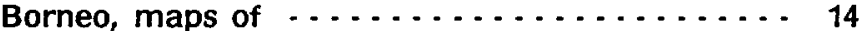
Bousfield, G.E. . . . . . . . . . . . . . 106 Braun, C. . . . . . . . . . . . . . . 105 Breusing Geometric projection $\ldots \ldots \ldots$ 132, 140 Breusing Harmonic projection ......... 140, 143 Briesemeister projection - 8, 158, 160, 162-163, 232-233 Briesemeister, W.A. . . . . . . . . . . . . . . . . . . 99, 162 British Isles, maps of $\ldots \ldots \ldots \ldots \ldots \ldots \ldots . . . . . . .26$ British Navy ..................... 154 British Ordnance . . . . . . . . . . . . . . . 92 Bromley projection $\ldots \ldots \ldots \ldots . \ldots . \ldots 54$ Bromley, R.H. . . . . . . . . . . . . . . . 54 Bulirsch algorithm $\ldots \ldots \ldots \ldots \ldots \ldots$ 236, 237, 239 Bumstead, A.H. . . . . . . . . . . . . 106

"Butterfly Map" .................... 196

Bynum, G. ...................... 233

C

Cahill, B.J.S. . . . . . . . . . . . . . . . . . 196

Carte Parallélogrammatique $\ldots \ldots \ldots . . .24$ 


\section{INDEX}

Cassini de Thury, C.F. . . . . . . . . . . 26 Cassini projection . . . . . . . . . 22, 26-27, 28, 219 Central Cylindrical projection .. 2, 10, 30-32, 105, 219 Central projection $\ldots \ldots \ldots \ldots \ldots \ldots \ldots . . \ldots 2,116$ Chamberlin Trimetric projection . . . . . . . 132, 146, 170-171, 233

Chamberlin, w. . . . . . . . . . . . . . . . . . . 170 Circum-Pacific Map Project $\ldots . . . . . . . . . .136$ Clarke, A.R. . . . . . . . . . . . . . . 131, 140 Clarke's "Twilight" projection . . . . . . . . . . . . 131

Close, C.F. . . . . . . . . . . . . . . . . . . 144, 146

Close projection . . . . . . . . . . . . . . . . 144

Collignon, E. . . . . . . . . . . . . . . . . . . 84

Collignon projection $\ldots \ldots \ldots \ldots \ldots \ldots \ldots$ 84-85, 88, 223

Colvocoresses, A.P. . . . . . . . . . . . . . . 211

Combined projections

See Composite projection

Complex algebra ...................... 2, 120

Composite projection $\ldots \ldots \ldots . .2,44,52,66,72,76,92$ $113,114,132,136,156$

Conformal projection, general $\ldots \ldots \ldots \ldots \ldots 2,5,8$

Azimuthal . . . . . . . . . . . . . . . 120-123

Conic ........................ 95-99

Cylindrical . . . . . . . . . . . . . . . . 10-15

Miscellaneous . . . . . . . . . . . 180-199, 208-212

Modified azimuthal . . . . . . . . . . . 148, 154-155

See also specific projection

Conformal projection of world in ellipse ... 198-199,

236-237

Conic projection . ............ 2, 4, 6, 7, 92-115 213-214, 224-225, 229

As specific projection ................. 92

See also other specific projections

Conical Orthomorphic projection ........... . 97

Conventional aspect . . . . . . . . . . . . . . . . 2

Cordiform projection . . . . . . . . . . . . . . . 114

Cossin, J. . . . . . . . . . . . . . . . . . . 37

Craig, J.I. . . . . . . . . . . . . . . . . . . . 150

Craig Retroazimuthal projection ... 150-151, 152, 231

Craster, J.E.E. . . . . . . . . . . . . . . . 70

Craster Parabolic projection ... 37, 68, 70-71, 72, 221

Cylindric projection .................... 6

See also Cylindrical projection
Cylindrical Equal-Area projection . ........ 10

See also Lambert Cylindrical Equal-Area projection

Cylindrical projection ........ 2,4,6, 10-36, 213,

218-220, 229

Modified ...................... 33, 211

See also specific projection

D

Deetz, C.H.

70,106

De ia Hire projection

See La Hire projection

De I'Isle, N. . . . . . . . . . . . . . . . . . . 92

Denoyer, L.P. ..................... 80

Denoyer Semi-Elliptical projection . . . . . 80-81, 222

Denoyer-Geppert .................. 80

Direct aspect $\ldots \ldots \ldots \ldots \ldots \ldots \ldots \ldots \ldots \ldots \ldots \ldots \ldots$

Distortion ..................... 2, 6, 8

See also specific projection

Donald Elliptical projection . . . . . . . . . . 146

Donald, J.K. ............... . . . . . . 146

Doubly Azimuthal projection . . . . . . . . . . . . 144

Doubly Equidistant projection ............ 146

\section{E}

Eckert (-Greifendorff), M. . . . . . . . . 46, 50,58, $60,86,88,166$

Eckert-Greifendorff projection. . . . . . . . . 74, 158, 160

$166-167,233$

\section{Eckert projections}

I $\ldots \ldots \ldots \ldots \ldots \ldots \ldots \ldots . . . .64,86-87,88,223$

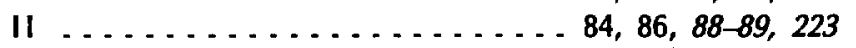

$111 \ldots \ldots \ldots \ldots \ldots \ldots \ldots \ldots 58-59,60,178,221$

IV $\ldots \ldots \ldots \ldots \ldots \ldots \ldots \ldots \ldots . \ldots 5,60-61,221$

V $\ldots \ldots \ldots \ldots \ldots \ldots \ldots . \ldots . \ldots, 46-47,48,50,220$

VI . . . . . . . . . . . . . . . 37, 44, 46, 50-51, 220

Edge matching . . . . . . . . . . . . . . . 6-7

Egypt, Survey of . . . . . . . . . . . . . . . . 150

Eisenlohr, F. ..................... 184

Eisenlohr projection $\ldots \ldots \ldots$ 184-185, 186, 204, 235

Ellipsoid, Earth as ....... 2, 3, 4, 7, 12, 14, 22, 26 $95,100,106,113,132$

Elliptic integrals . . . . . . . . . . . 236, 237, 239

Elliptical projection $\ldots \ldots \ldots \ldots . . . . . . . .54$ 


\section{INDEX}

[Main references in italics]
Equal-area polyconic projections . . . . . . . . . 106 Equal-area projection, general $\ldots \ldots \ldots \ldots \ldots 2,5,8$ Azimuthal $\ldots \ldots \ldots \ldots \ldots \ldots \ldots \ldots \ldots$ 136-139

Conic . . . . . . . . . . . . . . . . . . . . 100-103

Cylindrical $\ldots \ldots \ldots \ldots \ldots \ldots \ldots \ldots \ldots \ldots .{ }_{16-21}$ Modified azimuthal . . . . . . . . . . 160-163, 166-169

Pseudoazimuthal . . . . . . . . . . . . . . . . . 149

Pseudoconic . . . . . . . . . . . . . . . . ${ }^{112-115}$

Pseudocylindrical . . . 37-45, 50-57, 60-77, 84, 88-89 See also specific projection

Equatorial aspect ................... 2

See also specific projection

Equidistant Conic projection ..... 7, 92-94, 113, 224

Equidistant Cylindrical projection ... 22-29, 158, 164

See also Plate Carrée projection, Equirectangular projection

Equidistant Polyconic projection ........... 106

Equidistant projection, general $\ldots \ldots \ldots \ldots \ldots .2,5$

Azimuthal

See Azimuthal Equidistant projection

Conic

See Equidistant Conic projection

Cylindrical

See Equidistant Cylindrical projection

Equirectangular projection - 10, 22, 24-25, 46, 48, 92, 219

Equivalent projection

See Equal-area projection

Eratosthenes .............. 22

Erdi-Kraus, G. . . . . . . . . . . . . . . 66

Euler, L.

Eumorphic projection

See Boggs Eumorphic projection

Eurasia, maps of

Europe, maps of .................. 148

\section{$\mathbf{F}$}

Flat-Polar projection, general ............ 2

Flat-Polar Quartic projection .............. 76

See also McBryde-Thomas Flat-Polar Quartic projection

Flat-Polar Sinusoidal projection

See also McBryde-Thomas Flat-Polar Sinusoidal projection
Fournier, G. $\quad \ldots \ldots \ldots \ldots$

Fournier Globular I projection ...... 175, 176, 234

France, maps of . . . . . . . . . . . . . . . 26, 113

Gall Isographic projection $\ldots \ldots \ldots \ldots .24,33$

Gall, J. . . . . . . . . . . . . . . . . . . . 19, 13

Gall Orthographic projection . . . . . . . . 16, 19, 33

Gall projection . . . . . . . . . 10, 30, 33-34, 35, 203, 220

Modified ........................ 33

Gall Stereographic projection $\ldots \ldots \ldots \ldots \ldots \ldots$

See also Gall projection

Gauss, C.F. . . . . . . . . . . . . . . . . . . 12

Gauss Conformal projection . . . . . . . . . . . . 12

Gauss-Krüger projection . . . . . . . . . . . . . . 12

General Vertical Perspective projection . 124, 128-131,

\section{Geometric projection}

172,227

See Perspective projection

Ginzburg, G.A. ................. 106, 149

Ginzburg III projection ................... 149

Glareanus projection . . . . . . . . . . . . . . 174, 177

Globular projection . . . . . . . . . . . 2, 174-177, 204

As a specific projection $\ldots \ldots \ldots \ldots \ldots \ldots \ldots$

Glossary ...................... 2-4

Gnomic projection ..................... 116

Gnomonic projection ....... 2, 30, 104, 105, 116-119

$130,144,226$

Goode Homolosine projection . . . . . 2, 24, 66-67, 221 Goode, J.P. . . . . . . . . . . . . . . . . . . 66, 114

Goode Polar Equal-Area projection . . . . . . . . 114 Goode's Atlas . . . . . . . . . . . . . . . . 66, 83

Gougenheim, A. . . . . . . . . . . . . . . . . 198

Graticule ......................... . 2

See also specific projection

Great Britain, maps of . . . . . . . . . . 26, 110, 140 Great circle ................ . 2-3, 116, 120,124 Gretschel, H.F. . . . . . . . . . . . . . . . . . . . 131 GS50 projection . . . . . . . . . . . . . . 208-210, 238 Guam, maps of $\ldots \ldots \ldots \ldots \ldots \ldots \ldots \ldots \ldots . . \ldots 132$ Guyou, E. . . . . . . . . . . . . . . . . . . . . . 188 Guyou projection $\ldots \ldots \ldots .188-189,190,192,235-236$ 


\section{INDEX}

Hammer (Elliptical) projection ...... 158, 160-161, $162,166,168,232$

Hammer, H.H.E. von ........ 131, 152, 158, 160 Hammer Retroazimuthal projection - 150, 152-153, 231 Hammer-Aitoff projection ............ 160, 164 See also Hammer (Elliptical) projection

Hammer-Wagner projection . . . . . . . . . . 168

Harvard University . . . . . . . . . . . . . . . . . 206

Hassler, F.R. . . . . . . . . . . . . . . . . . . 106

Hatano Asymmetrical Equal-Area projection - 64-65,

Hatano, M. ....................... 64

Hatano Symmetrical Equal-Area projection ..... 64

Herschel, J. . . . . . . . . . . . . . . . . . . . . . . 97

Hinks, A.R. . . . . . . . . . . . . . . . . . . 152

Homalographic projection .............. 54

Homolographic projection ............ 54, 66

Homolosine projection ............. 37, 54, 66

See also Goode Homolosine projection

Hondius, J. ....................... 37

Hotine, M. ........................ 14

Hotine Oblique Mercator projection ... . . . . . . . 14

Hsu, M.-L., quoted ................... 6

Hyperelliptical projection .............. 54

\section{I}

Imnıler projection $\ldots . . . . . . . . . . . . .144$ Indicatrix ......................

See also specific projection

International Map of the World (IMW) . . . . 95, 111 Interrupted projection .... $3,43,54,57,66,68,70$,

$74,113,114,132,156$

$\mathbf{J}$

Jackson, J.E. . . . . . . . . . . . . . . . . . . 150

Jäger Star projection . . . . . . . . . . . . . . . . 156

James, H. .................... 131, 140

$\mathbf{K}$

Kamenetskiy, V.V.
Kavrayskiy, V.V. . . . . . . . . . . . . . . . . 9 92, 97

Kavrayskiy VI projection .............. 50

"Kite" projection ....................... 113

Krasovskiy, F.N. . . . . . . . . . . . . . . . 100

Krüger, L. . . . . . . . . . . . . . . . . . . . . . 12

\section{$\mathbf{L}$}

Laborde, J. .......................... . . 14

Laborde projection .................... 14

Lagrange, J.L. . . . . . . . . . . . . . . . . . . . . 180

Lagrange projection $\quad-154,180-183,198,200,202,235$

La Hire, $P$. de ...................... 130

La Hire projection . . . . . . . . . . . . . 128, 130-131

Lallemand, C. . . . . . . . . . . . . . . . . . . 111

Lambert Azimuthal Equal-Area projection ... . . 100, $101,102,114,132,136-139,149,158,160,166,228$

Lambert Conformal Conic projection . . . . . . . . . . 7, $95-98,213,224$

Lambert Cylindrical Equal-Area projection . . . 16-18

$19,100,101,218$

Lambert Equal-Area Conic projection ... . . . . 100,

Lambert, J.H. . . . . . . . . . 12, 16, 19, 97, 102, 136, 180

Landsat . . . . . . . . . . . . . . . . . . . 211, 213, 214

Large-scale mapping .................... . .

Latitude, defined . . . . . . . . . . . . . . . . 3, 216

Least squares ... . . . . . . . . . . . . . . . . 3

Lee, L.P. . . . . . . . . . . . . . . 12, 148, 196, 198, 208

Quoted ......................... 5-6

Lee conformal projection of the world

in triangle $\ldots \ldots \ldots \ldots \ldots \ldots \ldots$ 196-197, 236

L I'sle

See De l'isle

Littrow, J.J. . . . . . . . . . . . . . . . . . . . . . 154

Littrow projection ............ 154-155, 180, 231

Longitude, defined ................... 3, 216

Lorgna projection .................. . 136

Loritz projection '.................. 174, 177

"Lotus" projection ..................... 113

Loximuthal projection ............ 90-91, 223

Loxodrome ........................ . 3, 10, 90 


\section{INDEX}

[Main references in italics]

Madagascar, maps of

M

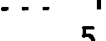

Marinus, Projection of .................. . . 24

Marinus of Tyre ... . . . . . . . . . . . . . 22, 24

Maurer, H. ............. 106, 131, 144, 146, 150

McBryde, F.W. . . . . . . . . . . . . . 44, 52, 72, 76

McBryde S3 projection ... . . . . . . . . . 52-53, 220

McBryde-Thomas Flat-Polar Parabolic

projection ................ 72-73, 221-222

McBryde-Thomas Flat-Polar Quartic

projection ............... . 74, 75, 76-77, 222 McBryde-Thomas Flat-Polar Sinusoidal

projection $\ldots . . .44-45,50,52,220$

McCaw, G.T. . . . . . . . . . . . . . . . . 106

McCaw projection .................... 144

Mecca projection ................... 150

Mendeleev, D.I. . . . . . . . . . . . . . . . . . . . 92

Mercator Equal-Area projection . . . . . . . . . . 37

Mercator, G. . . . . . . . . . . . . . . . . . . 10, 92

Mercator projection ...... 3, 4, 5, 7, 8, 10-11, 12, 14,

$30,33,35,90,97,200,208,213,218$

See also Oblique Mercator projection, Transverse

Mercator projection

Meridian, defined .......................

See also specific projection

Micronesia, maps of . . . . . . . . . . . . . . . 132

Miller Cylindrical projection ..... 10, 33, 35-36, 220

Miller Oblated Stereographic projection ... 120, 148,

208, 230-231

Miller, O.M. . . . . . . . . . . . . . . . . 35, 99, 148, 208 Miller Prolated Stereographic projection ... 148, 208

Minimum-error projection ............. 3, 140

Mobil Corp. ........................ . . 233

Modified Gall projection ................ 33

Modified Polyconic projection, for International

Map of the World ............ 106, 111, 225

Miscellaneous ..................... 106

Modified projections

See specific types, such as azimuthal and cylindrical Modified Stereographic Conformal projection ... 120,
Moir ........................ 33, 203

Mollweide, C.B. . . . . . . . . . . . . . . . . . . . 54

Mollweide projection - - 37, 54-57, 64, 66, 68, 175, 220

Moon, maps of the . . . . . . . . . . . . . . 131

Murdoch, P. ...................... 92

\section{$\mathbf{N}$}

National Atlas of the United States . . . . . . . . 100 National Geographic Society - 35, 70, 106, 146, 170, 200 Navigation, maps for ..... 3, 10, 90, 116, 144, 146, 154 New Zealand Map Grid ... . . . . . . . . . . . . . 208 Newton-Raphson iteration $\ldots . .216-217,220,221,222$ Nicolosi, G. 176 Nicolosi Globular projection $\ldots \ldots \ldots . . \ldots 175,176,234$ Nominal scale ....................... 3 Nordic projection ....................... 162 Normal aspect

See Direct aspect; see also specific projection

North America, maps of ... . . . . . . . . . . 99

Nowicki, A.L. ..................... . . 131

\section{0}

Oblated Stereographic projection ........ 148, 208

Oblique aspect ...................... 3, 229

See also specific projection

Oblique Cylindrical Orthomorphic projection ... 14

Oblique Equidistant Cylindrical projection

See Oblique Plate Carrée projection

Oblique Hammer projection ............... 162

Oblique Lambert Conformal Conic projection ... 98

See also Bipolar Oblique Conic Conformal projection

Oblique Lambert Cylindrical Equal-Area

projection ................... . 18, 219 Oblique Mercator projection .... 14-15, 148, 211, 218 Oblique Sinusoidal projection .......... . 37, 41, 42 Oblique Plate Carrée (Oblique Equidistant

Cylindrical) projection ............ 28-29, 219 Ordinary Polyconic projection ............ 106 Ordnance Survey . . . . . . . . . . . . . . . . . . . . 140

Ortelius, A. ........................ . . 178 Ortelius Oval projection . . . . . . . . 177, 178-179, 235 


\section{INDEX}

Orthoapsidal projection .............. 3, 206

Orthodromic projection

Orthographic projection

Orthomorphic projection

See Conformal projection

Orthophanic projection

Oval projection

Oxford University $\ldots \ldots \ldots \ldots \ldots \ldots \ldots \ldots$

\section{$\mathbf{P}$}

Pacific Ocean, maps of . . . . . . . . . 136, 148, 208

Parallel of latitude, defined

See also specific projection

Parameters ...................... 3

Parent, A. . . . . . . . . . . . . . . . . . . 131

Peirce, C.S. . . . . . . . . . . . . . . . . . . . . . . 190

Peirce Quincuncial projection . . . . . . . . 188,

$190-191,192,236$

Perspective Conic projection . . . . . . . . . 104-105

224-225

Perspective projection, general . . . . . . . . . . . 3

Azimuthal ................ 116-131, 226-227

Conic .................... . . 104-105, 224-225

Cylindrical ............. 16-21, 30-34, 219-220

Modified azimuthal . . . . . . . . . . . . . 172-173

See also specific projection

Petermann Star projection .............. 156

Peters, A. . . . . . . . . . . . . . . . . . . . . 19

Peters projection $\ldots \ldots \ldots \ldots \ldots \ldots \ldots \ldots \ldots$ 16, 19, 21

Philbrick, A.K. ...................... 66

Planar projection .................... 3

Planimetric map ... . . . . . . . . . . . . . . . 3, 12

Plate Carrée projection ...... 22-23, 24, 26, 28, 46, $92,94,219$

Polar aspect ........................ . 4

See also under specific azimuthal projection

Polyconic projection, type ........ 4, 6, 106-111, 180-183, 200-202, 204-205

As a specific projection ........ 106-109, 225

Polyhedric projection .................. 84
Postel, G. . . . . . . . . . . . . . . . . . . 132

Postel projection ... . . . . . . . . . . . . 132

Progressive Military Grid . . . . . . . . . . . . . . 106

Prolated Stereographic projection ....... 148, 208

Pseudoazimuthal projection ............ 6, 149

Pseudoconic projection ........ 4, 6, 112-115, 225

Pseudocylindric projection .............. 6

See also Pseudocylindrical projection

Pseudocylindrical projection ........ . 2, 4, 37-91,

$113,220-223,229$

Modified ...............80-81, 178-179, 203

See also specific projection

Ptolemy, C. .................... 92, 113

Putnins $\bar{s}$, R.V. . . . . . . . . . . . . . . . . . . . 62, 78

Putniṇs̄ projections

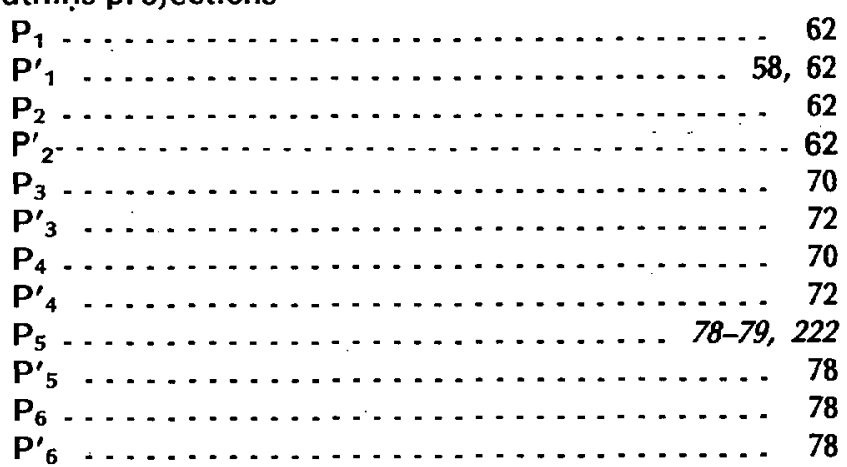

\section{Q}

Quartic Authalic projection .... 74-75, 76, 166, 222

Quincuncial projection ................ 190

$\mathbf{R}$

Raisz, E.J. . . . . . . . . . . . . . . . . . . . 206

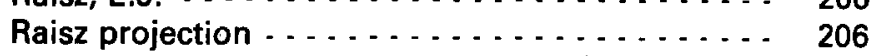

Rand McNally \& Co. . . . . . . . . . . . . . . . . 66, 82

Rechteckige Plattkarte, Die . . . . . . . . . . . . . 24

Rectangular Polyconic projection - - 106, 107, 110, 225

Rectangular projection . . . . . . . . . . . . . . . . . 24

Rectified Skew Orthomorphic projection ... . . . . . 14

Reeves, E.A. . . . . . . . . . . . . . . . . . . 152 


\section{An Album of Map Projections}

\section{INDEX}

\section{[Main references in italics]}

Regional map $\ldots \ldots \ldots$
"Regional" projection

Regular aspect

See Direct aspect

Reilly, W.l. .................... 208

Retroazimuthal projection ......... 4, 6, 150-155

Rhumb line ......................... 12

See also Loxodrome

Robinson, A.H. ..................... 82

Robinson projection . . . . . . . . 8, 62, 82-83, 222-223

Rosenmund, M. . . . . . . . . . . . . . . . . . . 14

Ruysch, J. . . . . . . . . . . . . . . . . . . . . 92

\section{$\mathbf{s}$}

Salmanova, T.D.

Sanson-Flamsteed projection .............. 37

Satellite Tracking projections $\ldots \ldots \ldots$ 213-214, 239 Satellites, artificial . . . . . . . . . . . 128, 211, 213

Scale .............................. . . 3, 4

See also specific projection

Scale factor ......................... . . 4

Schjerning, $W . \ldots \ldots \ldots \ldots \ldots \ldots \ldots$. . . . . . . . . 92, 114

Schoy, C. . . . . . . . . . . . . . . . . . . . . . 150

Secant cone, cylinder, or plane ........... 4, 24

Siemon, K. ...................... . 74, 90

Simple Conic projection . . . . . . . . . . . . . . . 92

See also Equidistant Conic projection

Simple Cylindrical projection .............. 22

See also Plate Carrée projection

Simple Perspective Cylindrical projection ...... 30

Singular points (conformality failure) $-4,180,184,186$ Sinu-Mollweide projection ............... 66 Sinusoidal projection ... . . . . . . . . . 8, 37-43, 44, $46,48,52,54,66,68,70,74,113,220$ Small circle . . . . . . . . . . . . . . . . . . . . 4, 120, 124 Small-scale mapping ... . . . . . . . . . . . . . 4 Snyder, J.P. . . . . . . . . . . . . . . . 208, 211, 213 South America, maps of . . . . . . . . . . . 37, 99 Soviet Union, maps of ............ 33, 92, 106 Space Oblique Mercator projection .... 211-212, 238

\section{Spheroid}

See Ellipsoid

Spilhaus, A.F. . . . . . . . . . . . . . . . . 186

Stabius (Stab), J. . . . . . . . . . . . . . . . . . 114

Stab-Werner projection . . . . . . . . . . . . . . 114

Standard parallel ...................... 4

See also specific projection

Standard lines $\ldots \ldots \ldots \ldots$

Star charts ..................... . 116, 132

Star projections ........... 156-157, 190, 231-232

State Base Maps ....................... . . 95

State Plane Coordinate System ... . . . . . . 12, 95

Stereographic Perspective Conic projection ..... 105

Stereographic projection .... 4, 97, 105, 106, 120-123, $128,130,132,140,148,180,183,208,226$

Sundial ......................... 116

Survey of the Coast . . . . . . . . . . . . . . 106

Switzerland, maps of . . . . . . . . . . . . . . 14

Sylvanus, B. ........................ . . 113

Tangent cone or cylinder

"Tetrahedral" projection ............ . . 114,132

Thales . .......................... 116

Thematic map ............... 4, 68, 70, 80, 100

Thomas, P.D. $\ldots \ldots \ldots \ldots \ldots \ldots \ldots \ldots \ldots \ldots$. $\ldots \ldots \ldots$. $44,72,76$

Tilted Perspective projection .. 130, 172-173, 227, 233

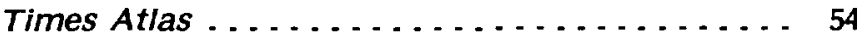

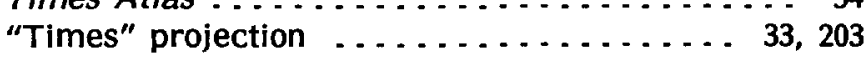

Tissot, N.A. .................. 8, 100

Tissot indicatrix

See Indicatrix

Tobler, W.R. . . . . . . . . . . . . . . . . 54, 90

Topographic map ............. $\ldots 4,12,26,95,120$

Transformed latitudes, etc. . . . . . . . . . . . . . 4

Transverse aspect .................. 4, 229

See also specific projection

Transverse Central Cylindrical

projection ..................... 30, 32, 219

Transverse Cylindrical Orthomorphic projection .. 12 


\section{INDEX}

\section{Transverse Lambert Cylindrical Equal-Area}

projection . . . . . . . . . . . . . . . . . 18, 218 Transverse Mercator projection .. . 7, 12-13, 14, 26, 218 Transverse Mollweide projection .......... 54, 56 Transverse Polyconic projection ....... . . 106, 109 Transverse Sinusoidal projection ...... 37, 39, 40 Trapezoidal projection .................. 84 True scale ........................ . . 4 Trystan Edwards projection .......... . 16, 19 Tsinger, N.Ya. .................. 97, 100 Twilight projection .................. 131 Two-Point Azimuthal projection . . . . . . . 116, 132, $144-145,230$

Two-Point Azimuthal-Equidistant projection ... 146

Two-Point Equidistant projection . . . . . . . . . . 132,

$146-147,170,230$

\section{$\mathbf{U}$}

United Kingdom, maps of See also Great Britain

United States, maps of 140

U.S. Army Map Service . . . . . . . . . . . . . . . . 131 U.S. Coast and Geodetic Survey ...... . 44, 72, 74, $76,106,190,192,194,195,198$

U.S. Department of Agriculture . . . . . . . . . . 200

U.S. Department of Commerce . . . . . . . . . 168

U.S. Department of State .......... . . . . . 68

U.S. Geological Survey _.. 99, 106, 136, 200, 208, 211 Universal Transverse Mercator (UTM) grid and

projection .......................... 12 University of Chicago ............... 66

University of Michigan ... . . . . . . . . . . . . 90

University of Wisconsin . . . . . . . . . . . 82

Urmayev, N.A. _................... 140

\section{V}

Van der Grinten, A.J. ......... 200, 202, 203, 204 Van der Grinten projection .............. 200 I $\ldots \ldots \ldots \ldots \ldots \ldots \ldots$ 180, 200-201, 202, 203, 237

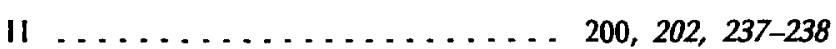

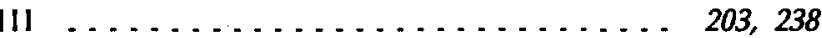

IV $\ldots \ldots \ldots \ldots \ldots \ldots \ldots 1 . \ldots \ldots, 184,186,204-205,238$ Vertical Perspective projection. 124, 128-131, 172, 227 Vitkovskiy, V.V.' . . . . . . . . . . . . . 92, 97, 100

\section{W}

Wagner, K. ....................... 168

Wagner projections

1............................. 50

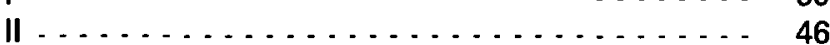

IV ................. 60. 60.63,64, 82, 221

VII . . . . . . . . . . . . . . . . . 168-169, 233

War Office projection ................. . 110

Werenskiold projections

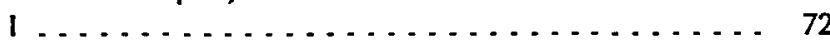

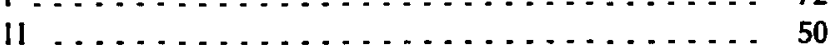

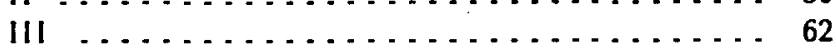

Werner, J. . . . . . . . . . . . . . . . . . . . . . . 114

Werner projection ...... 113, 114-115, 132, 136, 225

Wetch, J. ........................ 30

Wetch projection .................. 30

Wiechel, H. . . . . . . . . . . . . . . . . . . 149

Wiechel projection ................ . . 149, 231

William-Olsson, W. . . . . . . . . . . . . . 114, 136

Winkel, o. . . . . . . . . . . . . . . . . . . . 48, 164

Winkel I projection . . . . . . . . . . . 46, 48-49, 220

Winkel Tripel projection ......... 158, 164-165, 233

World Data Bank 1 ............... . . iii, 8

Wright, E. . . . . . . . . . . . . . . . . . . 10

Wright projection .................. 10

\section{$X Y Z$}

Young, A.E. . . . . . . . . . . . . . . . . . 140

Zenithal Equal-Area projection $\ldots \ldots \ldots \ldots 136$

Zenithal Equidistant projection .......... 132

See also Azimuthal Equidistant projection

Zenithal Equivalent projection . . . . . . . . . . 136

Zenithal projection

See Azimuthal projection 




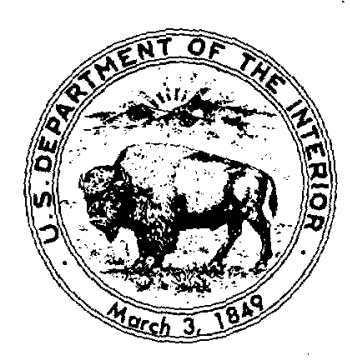

